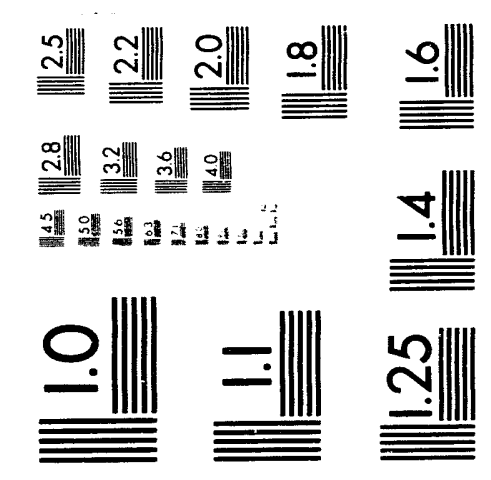



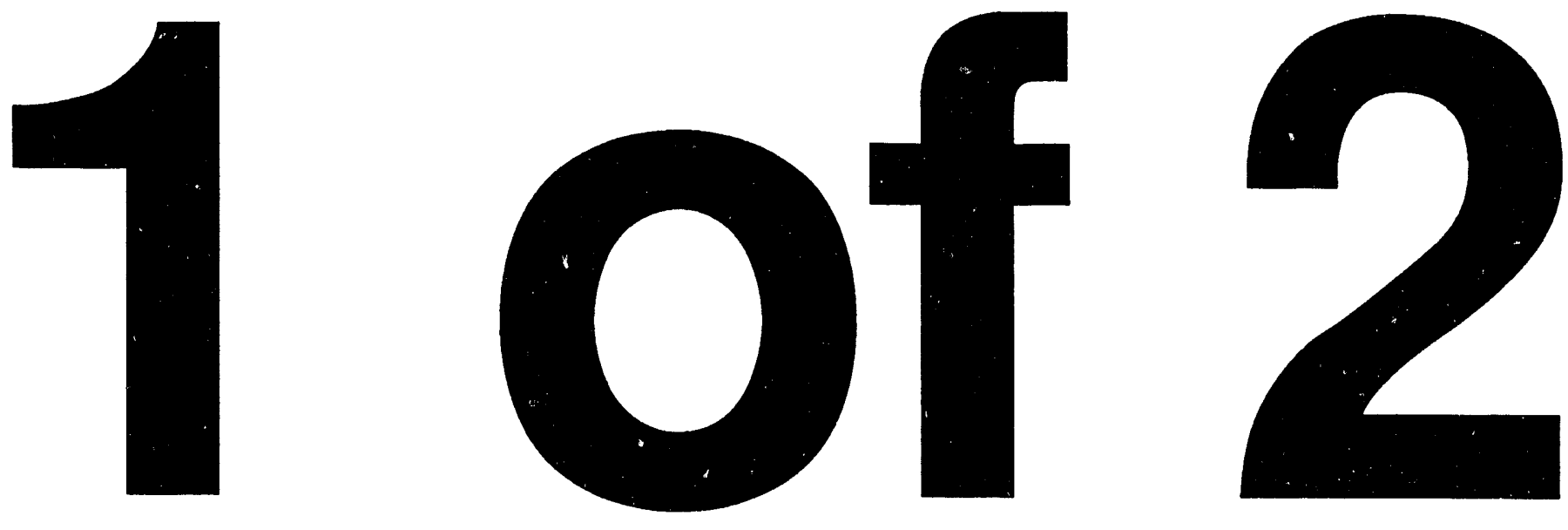
DOE/EIA-0035(93/11)

Distribution Category UC-950

\title{
Monthly Energy Review
}

\section{November 1993}

\author{
Energy Information Administration \\ Office of Energy Markets and End Use \\ U.S. Department of Energy \\ Washington, DC 20585
}

\section{MASTER}

DISTRIBUTION OF THIS DOCUMENT IS UNLIMITED

This report was prepared by the Energy Information Adiministration, the independent statistical and analytical agency within the Department of Energy. The information contained herein should not be construed as advocating or reflecting any policy position of the Department of Energy or any other organization. 


\section{Contacts}

The Monthly Energy Review is prepared by the Energy Information Administration. General information may be obtained from W. Calvin Kilgore, Director, Office of Energy Markets and End Use, 202-586-1617; Lynda T. Carlson, Director, Energy End Use and Integrated Statistics Division, 202-586-1112; and Katherine E. Seiferlein, Chief, Integrated Statistics Branch, 202-586-5692. Questions and comments concerning the contents of the Monthly Energy Review may be directed to the Principal Analyst, Chuck Allen, 202-586-5692, or to Diane D. Perrith, 202-586-2788, Carol Swiggins, 202-586-5743, or the following subject specialists:

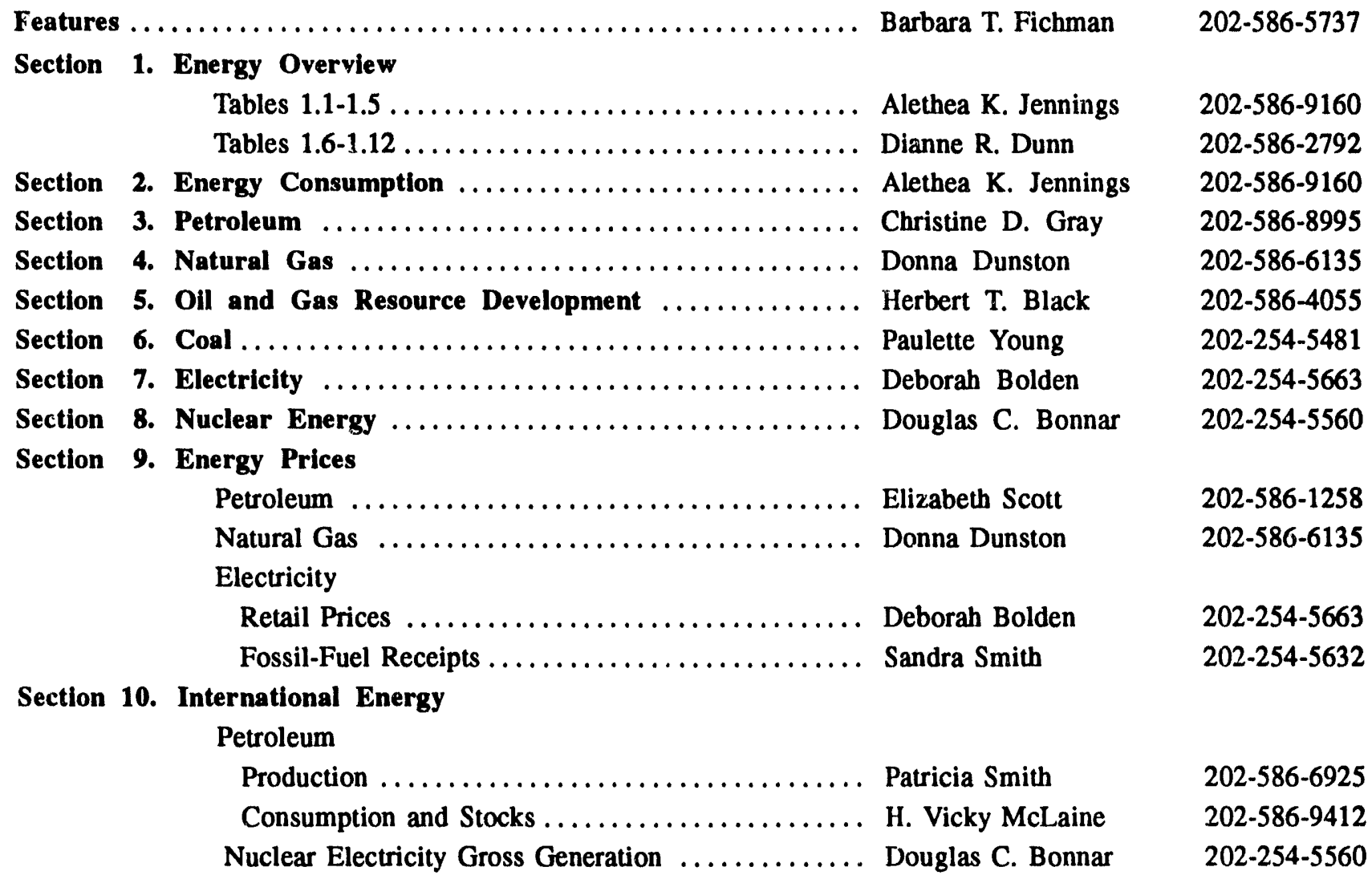

Requests for additional information on other energy statistics available from the Energy Information Administration and questions concerning subscriptions and report distribution may be directed to the National Energy Information Center, 202-586-8800 (TYY, for people who are deaf or hard of hearing, 202-586-1181). 


\section{Contents}

Highlights: The Changing Structure of the U.S. Coal Industry:

An Update ........................... 1

Section 1. Energy Overview .......................... 5

Section 2. Energy Consumption $\ldots \ldots \ldots \ldots \ldots \ldots \ldots \ldots \ldots \ldots, 25$

Section 3. Petroleum $\ldots \ldots \ldots \ldots \ldots \ldots \ldots \ldots \ldots \ldots \ldots \ldots, 43$

Section 4. Natural Gas .......................... 73

Section 5. Oil and Gas Resource Development .............. 83

Section 6. Coal .................................. 87

Section 7. Electricity ............................... 95

Section 8. Nuclear Energy ............................ 103

Section 9. Energy Prices ............................. 109

Section 10. International Energy $\ldots \ldots \ldots \ldots \ldots \ldots \ldots \ldots \ldots \ldots . \ldots \ldots$

Appendix A. Thermal Conversion Factors $\ldots \ldots \ldots \ldots \ldots \ldots \ldots \ldots$

Appendix B. Metric and Other Physical Conversion Factors ....... 153

Appendix C. List of Features ...................... 155

Glossary ...................................... 159 
Section 1. Energy Overview Page

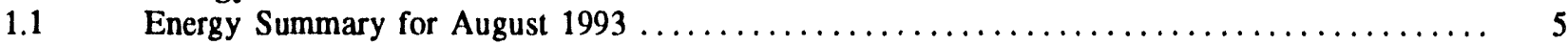

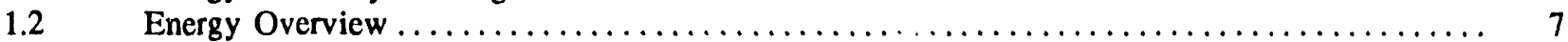

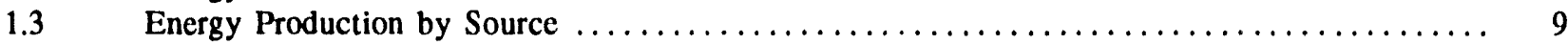

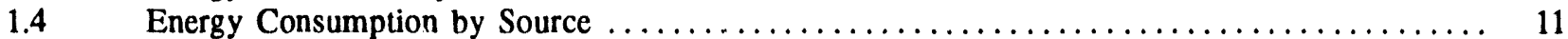

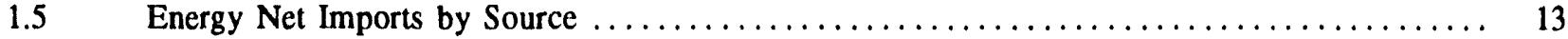

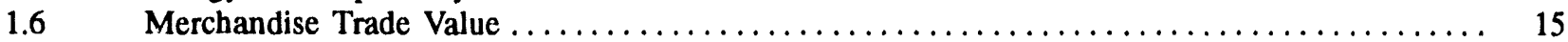

$1.7 \quad$ Energy Consumption per Dollar of Gross Domestic Product $\ldots \ldots \ldots \ldots \ldots \ldots \ldots \ldots \ldots \ldots \ldots \ldots$

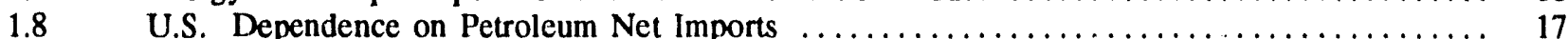

1.9 Cost of Fuels to End Users in Constant $(1982-1984)$ Dollars . . . . . . . . . . . . . . . . . 18

$1.10 \quad$ Passenger Car Efficiency . ......................................... 19

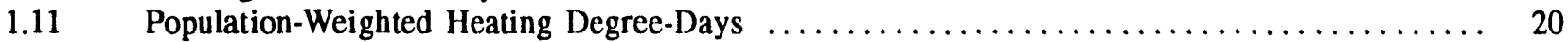

1.12 Population-Weighted Cooling Degree-Days $\ldots \ldots \ldots \ldots \ldots \ldots \ldots \ldots \ldots \ldots \ldots \ldots, 21$

Section 2. Energy Consumption

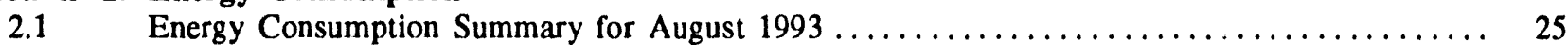

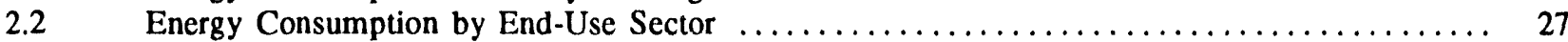

$2.3 \quad$ Residential and Commercial Energy Consumption $\ldots \ldots \ldots \ldots \ldots \ldots \ldots \ldots \ldots \ldots \ldots \ldots . \ldots \ldots$

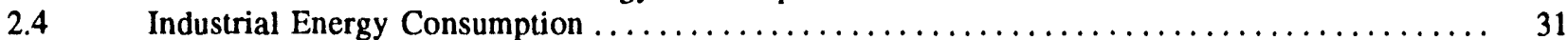

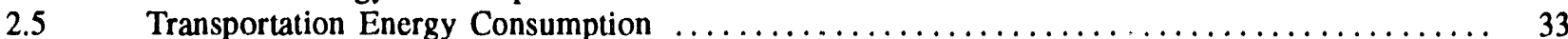

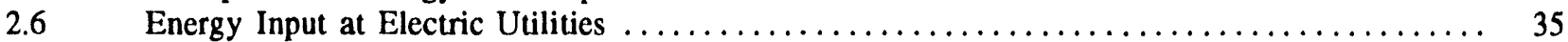

Section 3. Petroleum

3.1 Petroleum Overview

3.1a Field Production, Stock Change, Petroleum Products Supplied, and Ending Stocks ... 44

3.1b Imports, Exports, and Net Imports .............................. 45

Crude Oil Supply and Disposition
$3.2 \mathrm{a}$

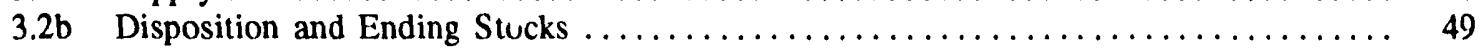

Petroleum Imports
$3.3 \mathrm{a}$ Algeria, Iraq, Kuwait, and Libya $\ldots \ldots \ldots \ldots \ldots \ldots \ldots \ldots \ldots \ldots \ldots \ldots \ldots \ldots \ldots$

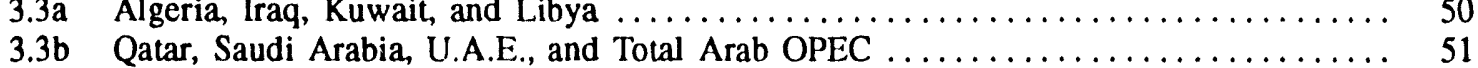

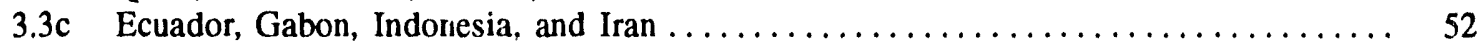

3.3d Nigeria, Venezuela, Total Non-Arab OPEC, and Total OPEC ................ 53

3.3e Angola, Australia, Bahama Islands, Brazil, Canada, and China ............... 54

$3.3 \mathrm{f}$ Colombia, Ecuador, Italy, Malaysia, Mexico, and Netherlands ............... 55

$3.3 \mathrm{~g}$ Netherland Antilles, Norway, Puerto Rico, Russia, Spain, and Trinidad and Tobago .. 56

3.3h United Kingdom, Virgin Islands, Other Non-OPEC, Total Non-OPEC, and Total

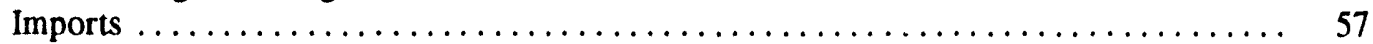

$3.4 \quad$ Finished Motor Gasoline Supply and Disposition $\ldots \ldots \ldots \ldots \ldots \ldots \ldots \ldots \ldots \ldots \ldots \ldots \ldots$

$3.5 \quad$ Distillate Fuel Oil Supply and Disposition $\ldots \ldots \ldots \ldots \ldots \ldots \ldots \ldots \ldots \ldots \ldots \ldots \ldots \ldots \ldots$

3.6 Residual Fuel Oil Supply and Disposition $\ldots \ldots \ldots \ldots \ldots \ldots \ldots \ldots \ldots \ldots \ldots \ldots \ldots \ldots \ldots \ldots$

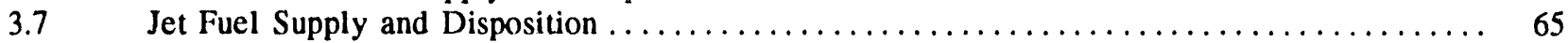

3.8 Liquefied Petroleum Gases Supply and Disposition $\ldots \ldots \ldots \ldots \ldots \ldots \ldots \ldots \ldots \ldots \ldots$

$3.9 \quad$ Propane and Propylene Supply and Disposition $\ldots \ldots \ldots \ldots \ldots \ldots \ldots \ldots \ldots \ldots \ldots \ldots$

Other Petroleum Products Supply and Disposition $\ldots \ldots \ldots \ldots \ldots \ldots \ldots \ldots \ldots \ldots \ldots$

Section 4. Natural Gas

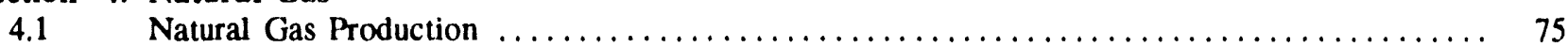

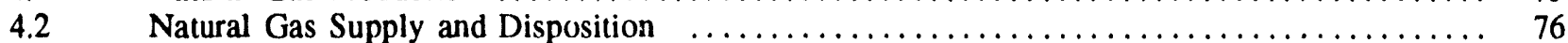

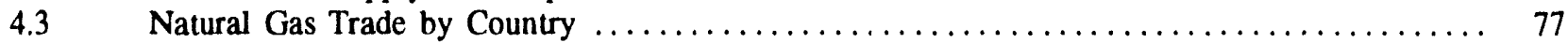

4.4 Natural Gas Consumption by End-Use Sector $\ldots \ldots \ldots \ldots \ldots \ldots \ldots \ldots \ldots \ldots \ldots \ldots \ldots$

4.5 Natural Gas in Underground Storage $\ldots \ldots \ldots \ldots \ldots \ldots \ldots \ldots \ldots \ldots \ldots \ldots \ldots \ldots$

Section 5. Oil and Gas Resource Development

$5.1 \quad$ Oil and Gas Drilling Activity Measurements $\ldots \ldots \ldots \ldots \ldots \ldots \ldots \ldots \ldots \ldots \ldots \ldots \ldots$

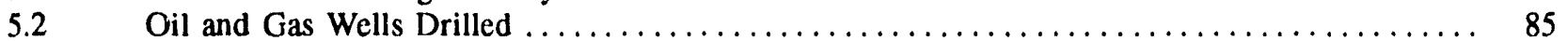




\section{Tables (Continued)}

Section 6. Coal

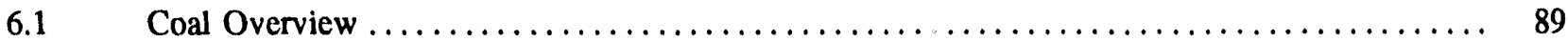

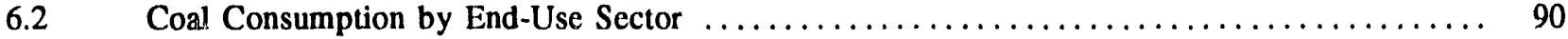

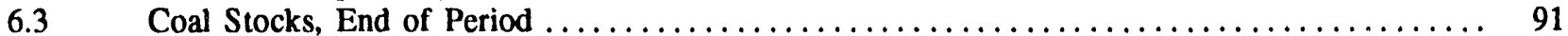

Section 7. Electricity

$7.1 \quad$ Electric Utility Net Generation of Electricity $\ldots \ldots \ldots \ldots \ldots \ldots \ldots \ldots \ldots \ldots \ldots \ldots \ldots \ldots$

7.2 Electricity Sales by End-Use Sector $\ldots \ldots \ldots \ldots \ldots \ldots \ldots \ldots \ldots \ldots \ldots \ldots \ldots \ldots \ldots \ldots \ldots$

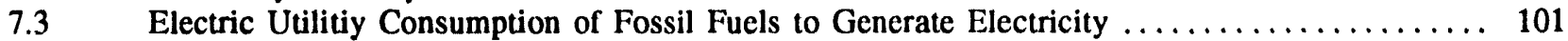

7.4 Electric Utility Stocks of Coal and Petroleum, End of Period $\ldots \ldots \ldots \ldots \ldots \ldots \ldots \ldots \ldots$

$\begin{array}{cl}\text { Section } & \text { 8. } \begin{array}{l}\text { Nuclear Energy } \\ 8.1\end{array} \\ \text { Nuclear Power Plant Operations } \ldots \ldots \ldots \ldots \ldots \ldots \ldots \ldots \ldots \ldots \ldots \ldots \ldots \ldots \ldots \ldots \ldots \ldots & 105\end{array}$

8.2 Nuclear Generating Units, End of Period $\ldots \ldots \ldots \ldots \ldots \ldots \ldots \ldots \ldots \ldots \ldots \ldots \ldots \ldots$

Section 9.
9.1 $\quad \begin{aligned} & \text { Energy Prices } \\ & \text { Crude Oil Price Summary } \ldots \ldots \ldots \ldots \ldots \ldots \ldots \ldots \ldots \ldots \ldots \ldots \ldots \ldots \ldots \ldots \ldots \ldots \ldots 111\end{aligned}$

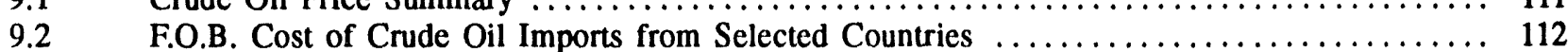

9.3 Landed Cost of Crude Oil Imports from Selected Countries ........................ 113

9.4 Motor Gasoline Retail Prices, U.S. City Average $\ldots \ldots \ldots \ldots \ldots \ldots \ldots \ldots \ldots \ldots \ldots \ldots \ldots$

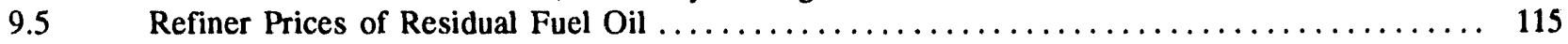

9.6 Refiner Prices of Petroleum Products for Resale $\ldots \ldots \ldots \ldots \ldots \ldots \ldots \ldots \ldots \ldots \ldots \ldots \ldots 116$

9.7 Refiner Prices of Petroleum Products to End Users $\ldots \ldots \ldots \ldots \ldots \ldots \ldots \ldots \ldots \ldots \ldots \ldots$

9.8 No. 2 Distillate Prices to Residences

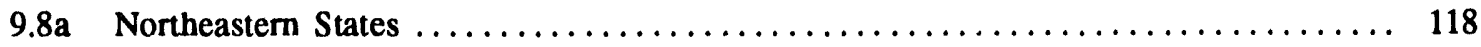

$9.8 \mathrm{~b}$ Selected South Atlantic and Midwestern States ........................ 119

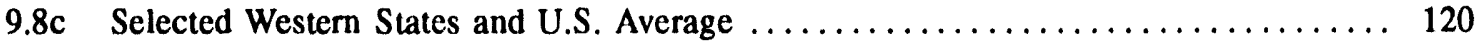

$9.9 \quad$ Electricity Retail Prices ........................................... 122

9.10 Quantity and Cost of Fossil-Fuel Receipts at Steam-Electric Utility Plants ............... 123

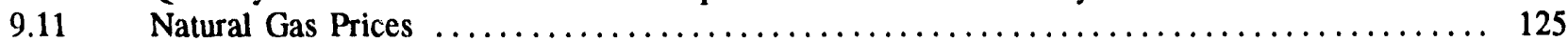

Section 10. International Energy

10.1 World Crude Oil Production

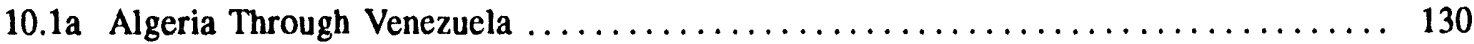

10.1b Total OPEC, Canada Through Former U.S.S.R., and World $\ldots \ldots \ldots \ldots \ldots \ldots \ldots \ldots 131$

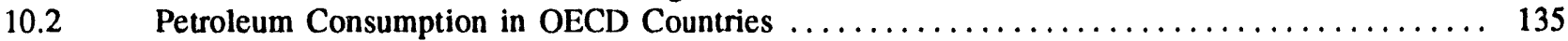

$10.3 \quad$ Petroleum Stocks in OECD Countries, End of Period $\ldots \ldots \ldots \ldots \ldots \ldots \ldots \ldots \ldots \ldots \ldots$

10.4 Nuclear Electricity Gross Generation

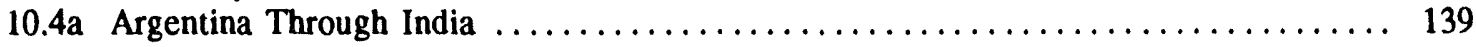

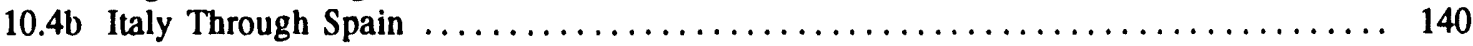

$10.4 \mathrm{c}$ Sweden Through United States and Total $\ldots \ldots \ldots \ldots \ldots \ldots \ldots \ldots \ldots \ldots \ldots \ldots 14$

Appendix A. Thermal Conversion Factors

A1. Approximate Heat Content of Petroleum Products $\ldots \ldots \ldots \ldots \ldots \ldots \ldots \ldots \ldots \ldots \ldots \ldots$

A2. Approximate Heat Content of Crude Oil, Crude Oil and Products, and Natural Gas Plant Liquids 144

A3. Approximate Heat Content of Petroleum Product Weighted Averages .................. 144

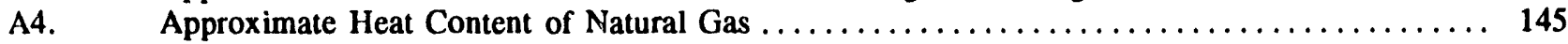

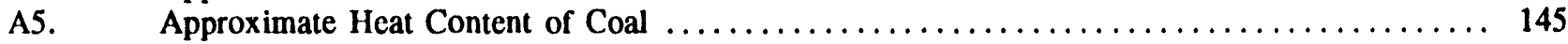

A6. Approximate Heat Content of Bituminous Coal and Lignite $\ldots \ldots \ldots \ldots \ldots \ldots \ldots \ldots \ldots \ldots$

A7. Approximate Heat Content of Anthracite and Coal Coke $\ldots \ldots \ldots \ldots \ldots \ldots \ldots \ldots \ldots \ldots$

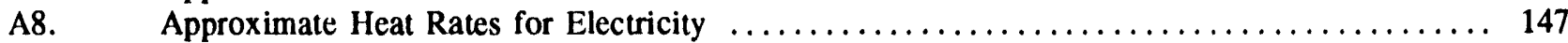

Appendix B. Metric and Other Physical Conversion Factors

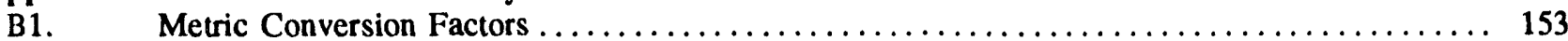

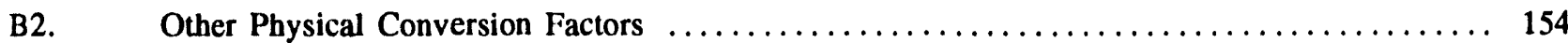

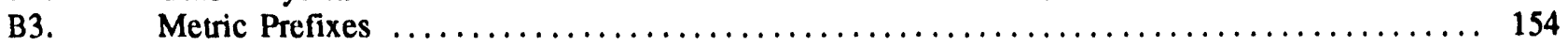


Section 1. Energy Overview

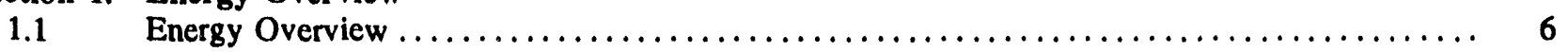

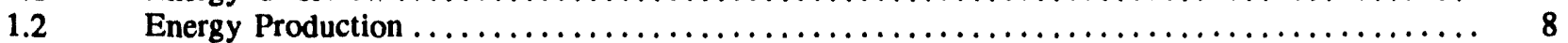

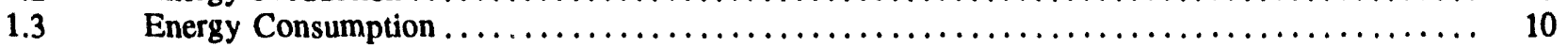

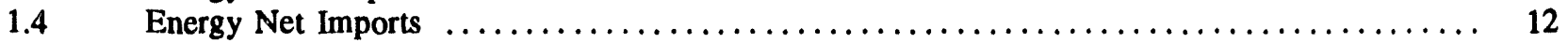

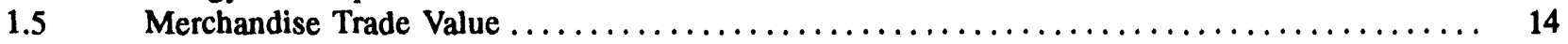

$1.6 \quad$ Energy Consumption per Dollar of Gross National Product $\ldots \ldots \ldots \ldots \ldots \ldots \ldots \ldots \ldots \ldots$

$1.7 \quad$ U.S. Dependence on Petroleum Net Imports $\ldots \ldots \ldots \ldots \ldots \ldots \ldots \ldots \ldots \ldots \ldots \ldots \ldots 17$

$1.8 \quad$ Cost of Fuels to End Users in Constant $(1982-1984)$ Dollars $\ldots \ldots \ldots \ldots \ldots \ldots \ldots \ldots \ldots \ldots . \ldots$

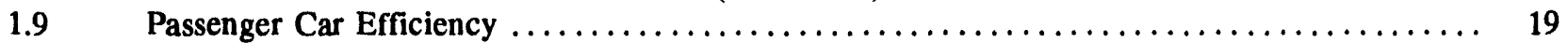

\section{Section 2. Energy Consumption}

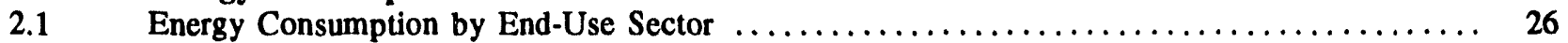

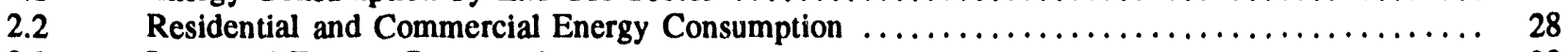

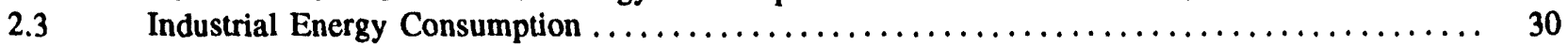

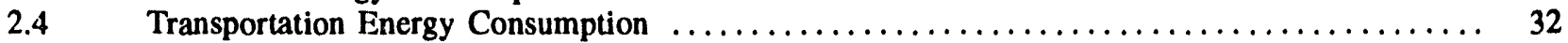

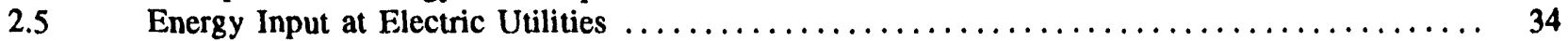

Section 3. Petroleum

3.1 Petroleum Overview ............................................ 46

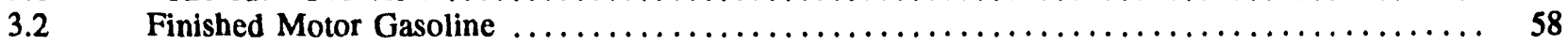

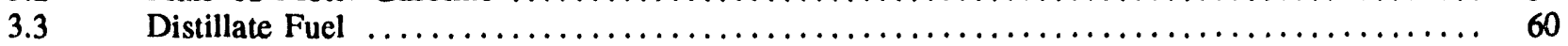

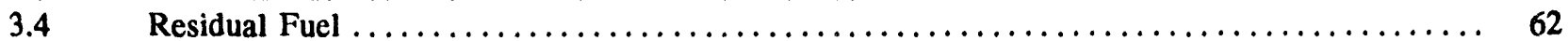

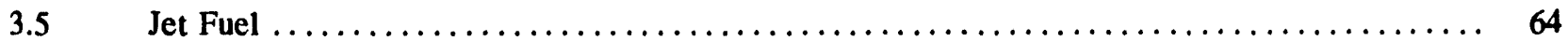

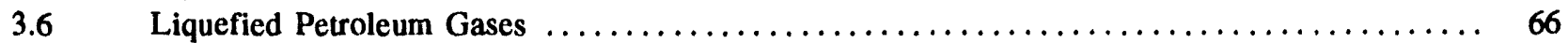

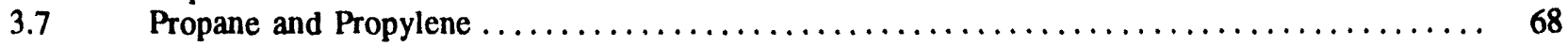

Section 4. Natural Gas

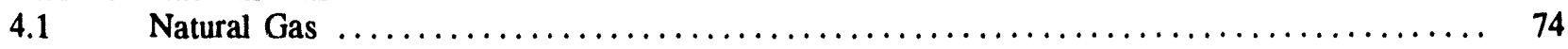

Section 5. Oil and Gas Resource Development

$5.1 \quad$ Oil and Gas Resource Development Indicators $\ldots \ldots \ldots \ldots \ldots \ldots \ldots \ldots \ldots \ldots$

Section 6. Coal

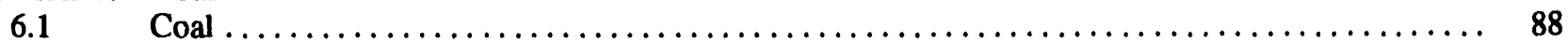

Section 7. Electricity

$7.1 \quad$ Electric Utility Net Generation of Electricity $\ldots \ldots \ldots \ldots \ldots \ldots \ldots \ldots \ldots \ldots \ldots \ldots \ldots$

$7.2 \quad$ Electricity Sales $\ldots \ldots \ldots \ldots \ldots \ldots \ldots \ldots \ldots \ldots \ldots \ldots \ldots \ldots \ldots \ldots \ldots \ldots \ldots$

Electric Utility Consumption and Stocks of Fossil Fuels $\ldots \ldots \ldots \ldots \ldots \ldots \ldots \ldots \ldots \ldots$

Section 8. Nuclear Energy

$8.1 \quad$ Nuclear Power Plant Operations $\ldots \ldots \ldots \ldots \ldots \ldots \ldots \ldots \ldots \ldots \ldots \ldots \ldots \ldots \ldots \ldots$

Section 9. Energy Prices

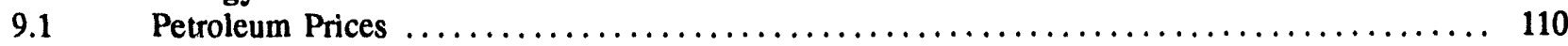

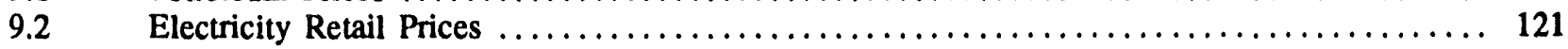

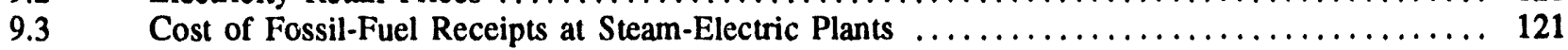

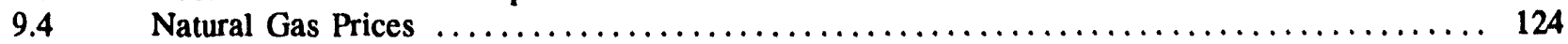

Section 10. h ternational Energy

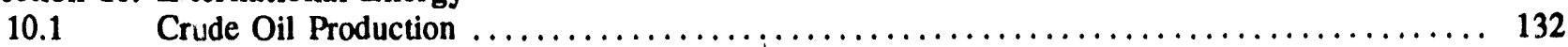

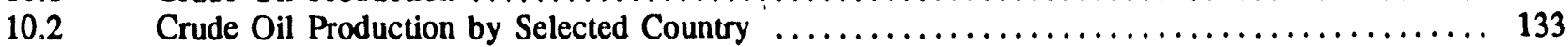

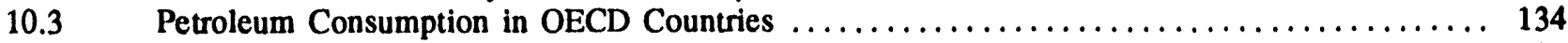

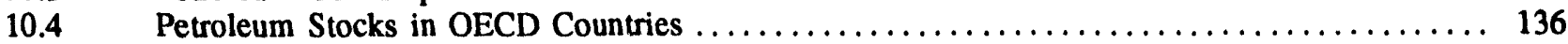

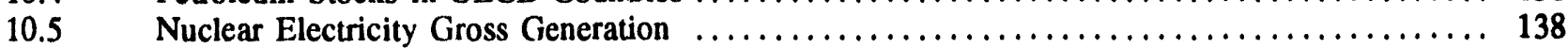




\section{Highlights:}

\section{The Changing Structure of the U.S. Coal Industry: An Update}

Although the number of active U.S. coal mines declined between 1976 and 1991, the size of the average mine more than tripled and U.S. total coal production increased by 45 percent. During the same period, mergers and increased foreign ownership of U.S. coal-producing companies significantly reshaped the industry.

These and other features of the evolving U.S. coal industry are discussed in The Changing Structure of the U.S. Coal Industry: An Update, released in July 1993 by the Energy Information Administration (EIA). The report extends an earlier EIA publication ${ }^{1}$ through 1991 (the latest year for which comprehensive data are available) and includes more recent estimates for some of the data. It analyzes the changing size distribution of U.S. mines and producers, the waves of mergers within the industry since the 1960's, the concentration of production ownership, and the possible impact of the Clean Air Act Amendments of 1990. The report has three appendices: Major U.S. Coal Producers, 1976, 1986, and 1991; Major Holders of U.S. Coal Reserves, 1990; and Regional Coal Production of Top 20 Firms, 1991.

\footnotetext{
${ }^{1}$ Energy Information Administration, The Changing Structure of the U.S. Coal Industry 1976-1986, DOE/EIA-0513 (Washington, DC, June 1988).
}

\section{Growth in Mine and Producer Size}

The average size (defined as yearly output) of U.S. coal mines increased from 105 thousand short tons per year in 1976 to 330 thousand short tons per year in 1991, while the total number of active mines fell by more than half, from 6,553 to 3,022 (Table 1). The size distribution of mines also changed radically: In 1976, only 8 percent of U.S. mines produced 200 thousand short tons or more per year, but by the end of 1991 the figure had risen to 25 percent. The number of small mines (those producing less than 50 thousand short tons per year) fell from 67 percent of the total in 1976 to 47 percent 15 years later. Moreover, the output of small mines equaled only 2.4 percent of total U.S. coal production in 1991, down from 10 percent in 1976.

Large mines, in contrast, accounted for a growing share of total coal production. Mines with annual outputs of 500 thousand tons or more accounted for 77 percent of coal production in 1991, up from 69 percent in 1986 and 54 percent in 1976. In 1991, the 7 percent of U.S. coal mines that each produced 1 million tons or more of coal yielded 66 percent of U.S. total coal production.

Table 1. Structural Elements of the U.S. Coal Industry, 1976, 1986, 1991, and 1993

\begin{tabular}{|c|c|c|c|c|}
\hline Element & 1976 & 1986 & 1991 & $1993^{a}$ \\
\hline 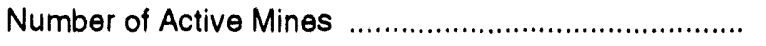 & 6,553 & 4,424 & 3,022 & NA \\
\hline Average Mine Size (thousand short tons) ................... & 105 & 201 & 330 & NA \\
\hline 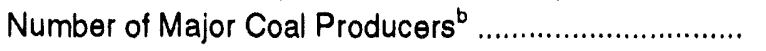 & 34 & 52 & 53 & NA \\
\hline $\begin{array}{l}\text { Foreign-Controlled Producers' Share of U.S. } \\
\text { Production (percent) }\end{array}$ & 1.4 & 6.4 & 14.3 & 15.6 \\
\hline \multicolumn{5}{|l|}{ Market Share of Four Largest Producers (percent) } \\
\hline 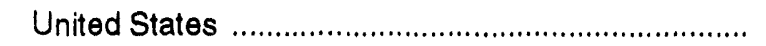 & 24.6 & 19.6 & 21.8 & 26.5 \\
\hline 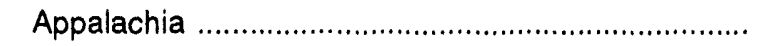 & 21.8 & 19.9 & 20.4 & 26.2 \\
\hline 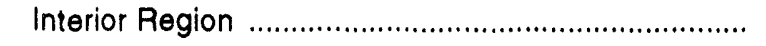 & 51.9 & 44.6 & 38.7 & 39.4 \\
\hline 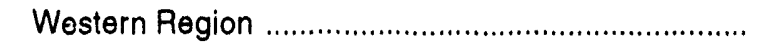 & 42.3 & 35.7 & 37.5 & 45.5 \\
\hline \multicolumn{5}{|l|}{$\begin{array}{l}\text { Share of Major Producers' Output by Company } \\
\text { Type (percent) }\end{array}$} \\
\hline 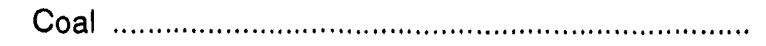 & 34.7 & 26.6 & 17.3 & 20.3 \\
\hline 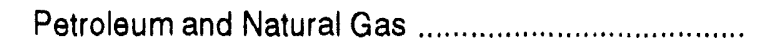 & 32.2 & 43.9 & 31.3 & 24.5 \\
\hline 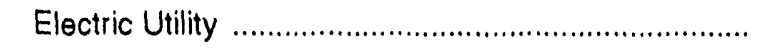 & 10.8 & 14.9 & 14.5 & 10.5 \\
\hline Steel & 8.5 & 4.5 & 3.4 & 3.5 \\
\hline 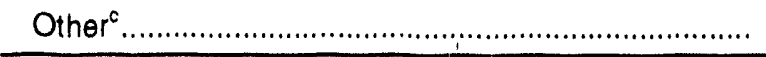 & 13.8 & 10.1 & 33.4 & 41.1 \\
\hline \multicolumn{5}{|c|}{$\begin{array}{l}\text { a Share percentages are Energy Information Administration estimates made by applying the coal company ownership as of June } 15,1993,10 \text { the } 1991 \text { coal production } \\
\text { atabase. } \\
\text { Major producers are firms with coal outputs of } 3 \text { million short tons or more per year. } \\
\text { CPrimarily conglomerates. } \\
\text { NA=Not avallable. } \\
\text { Notes: Data for } 1976 \text { and } 1986 \text { exclude anthracite, which accounted for less than } 1 \text { percent of U.S. coal production in those years. - Percentages may not add } \\
100 \text { due to independent rounding. } \\
\text { Source: Energy intormation Administration, The Changing Siructure of the U.S. Coal Industry: An Update, DOE/E(A.0513(93) (Washington, DC. July 1993), pp. VII }\end{array}$} \\
\hline
\end{tabular}


A shift toward larger coal producers accompanied the shift toward larger mines. Major coal producers (firms producing 3 million short tons or more per year) numbered 34 in 1976 and accounted for 57 percent of U.S. coal production. By the end of 1991, the number of major producers had risen to 53 and they accounted for 77 percent of domestic output. Moreover, the number of very large firms (those producing 20 million short tons or more per year) rose from three in 1976 to 12 in 1991, while their share of U.S. production nearly doubled, from 22 percent to 41 percent.

Several factors underlay the trends toward greater size in coal mines and producers:

Higher demand for low-sulfur coal. Increased interest in low-sulfur coal helped to shift production toward the thick, low-sulfur coal seams of the West. These deposits were most effectively tapped by means of vast surface mines.

Declining coal prices. Falling prices in the 1980's made mining unprofitable for many small firms. Those that could no longer cover their costs left the industry, thereby raising the average size of the remaining coal producers.

Higher demand for coal among electric utilities. Sharply rising crude oil prices and shortages of natural gas in the 1970's encouraged electric utilities to build new coal-fired generating plants, thus increasing their demand for coal. The electric utilities sought contracts with large coal producers that could meet the long-term supply needs of the new plants.

Technological change. In the West, large firms were better suited to meet the capital requirements for the huge drag lines and shovels used in the open mines prevalent in that region. Similarly, in Appalachia and the Interior, it was the larger firms that tended to install continuous mining and longwall equipment that boosted productivity in those regions.

Mergers. Among the most significant factors in the reshaping of the U.S. coal industry was the period of mergers that began in the 1960's, when major energy companies acquired coal resources as a hedge against declining domestic crude oil reserves. The international oil crises of the 1970's accelerated the process, as higher crude oil prices simultaneously yielded large cash surpluses and created expectations that the manufacture of synthetic oil and gas from coal would become profitable.

Another wave of mergers began in 1983, as several coal producers acquired other coal companies or blocks of coal reserves compatible with their existing production, handling, and processing facilities, or with their marketing strategies. Many coal companies purchased eastern low-sulfur reserves in response to tightening clean-air standards. In addition, many domestic steel companies, hurt by the recession of the carly 1980's and needing less coal due to declines in pig iron and coke production, sold their coal assets to improve their cash flows.

Finally, a number of major petroleum and natural gas companies began selling off coal properties and other assets acquired during diversification in the 1970's. They did so, in general, in order to focus more closely on their core businesses, but also because coal prices were declining and they faced the need for costly clean air-driven upgrades of their refining and distribution systems.

The waves of coal industry mergers and other acquisitions and divestitures of coal assets, which continued into 1993, altered the mix of major coal-producing company types and their relative shares of U.S. total output (Table 1). In 1976, the independent coal companies' share of the major producers' output was 35 percent, while the petroleum and natural gas companies' share was 32 percent, the electric utility-affiliated producers' share was 11 percent, and the steel companies' share was 9 percent. By the end of 1991 , the independents' share had dropped by half, to 17 percent, primarily because of the sale of a large American company to a British conglomerate. From 44 percent in 1986, the petroleum and natural gas companies' share of major producers' output fell back to 31 percent in 1991, about the same level as 10 years earlier. The share of electric utilityaffiliated producers rose to 15 percent, while the steel companies' share declined to 3 percent.

Foreign ownership of U.S. coal production assets increased during the period. In 1976, only one major coal producer, accounting for 1 percent of U.S. annual production, was foreign-controlled. By the end of 1991, the number of foreign-controlled major producers had risen to eight and they accounted for 14 percent of U.S. output (Table 1).

\section{Concentration in the U.S. Coal Industry}

Concentration (the share of production held by the largest companies) in the U.S. coal industry varied from 1976 through 1991. Measures of concentration can indicate an industry's competitiveness; generally, if the four largest firms account for more than one half of market sales, it is possible that the firms will not act independently and prices may be set above competitive levels. The report examines concentration in production nationwide and by regions.

Nationwide production concentration. Despite the trend toward larger firms and larger mines, market concentration ratios in the U.S. coal industry (defined as the market shares of the top four, eight, and 20 producers) remained low. The four biggest firms produced 25 percent of U.S. total output in 1976, 20 percent in 1986, and 22 percent in 1991. The share of the eight largest firms was 34 percent in 1976, 30 percent in 1986, and 33 percent in 1991.

Year-to-year concentration ratios varied, usually slightly, from 1965 through 1991 (Figure 1), often due to strikes by miners against the two largest companies, which are heavily unionized. The strikes had a disproportionate effect on those companies' production and thus reduced the four-firm concentration ratio. Disregarding the four major strike years $(1971,1974,1978$, and 1981), which interrupt the underlying trends, reveals the following: the four-firm concentration ratio rose from 24 percent in 1965 to 28 percent in 1969, then declined to 19 percent in 1987. From 1987 through 1991, a period when many small producers left the industry due to falling coal prices and excess production capacity, the fourfirm ratio rose again, to 22 percent. 
Regional production concentration. The three major U.S. coal producing regions-Appalachia, the Interior Region, and the Western Region (Figure 2)-differ in terms of product characteristics, production methods, distribution of mine types, and other features. Appalachia, for example, historically has heen the Nation's most important source of coal and the principal source of coal for export. In 1991, the region contained nearly 90 percent of all U.S. coal mines and accounted for 46 percent of total production (down from 60 percent in 1976). Appalachia is the dominant supplier of metallurgical (coking) coal and Central Appalachia is the primary source of high heat-content, low-sulfur coal, which is expected to figure prominently in meeting the emissions standards of the Clean Air Act Amendments of 1990 (CAAA). The region has many coal producers and its production concentration is significantly lower than that of the other two regions. The four-firm production share was 22 percent in 1976 and 20 percent in both 1986 and 1991 (Table 1).

The Interior Region has two distinct coal-producing areas, the Illinois basin (including most of Illinois and parts of Indiana and western Kentucky) and the coastal plains of Texas and Louisiand. The Illinois basin produces bituminous coal of high heat content and medium-to-high sulfur content. The mines of the Gulf Coastal Plain yield low heat-content lignite that is easily extracted by using surface mining techniques and is burned primarily in minemouth power plants in Texas.

Although the passage of the Surface Mining Control and Reclamation Act in 1977 reduced productivity and raised costs at Interior Region mines, total output increased 21 percent during the 1976-through-1991 period, as Texas lignite production nearly yuadrupled. However, the CAAA is expected to reduce demand for the region's high-sulfur coal. Despite a decline in the Interior Region's four-firm coal production concentration (from 52 percont in 1976 to 39 percent in 1991), production concentration in 1991 remained higher than in the other two regions.

Figure 1. U.S. Coal Production Concentration, 1965-1991

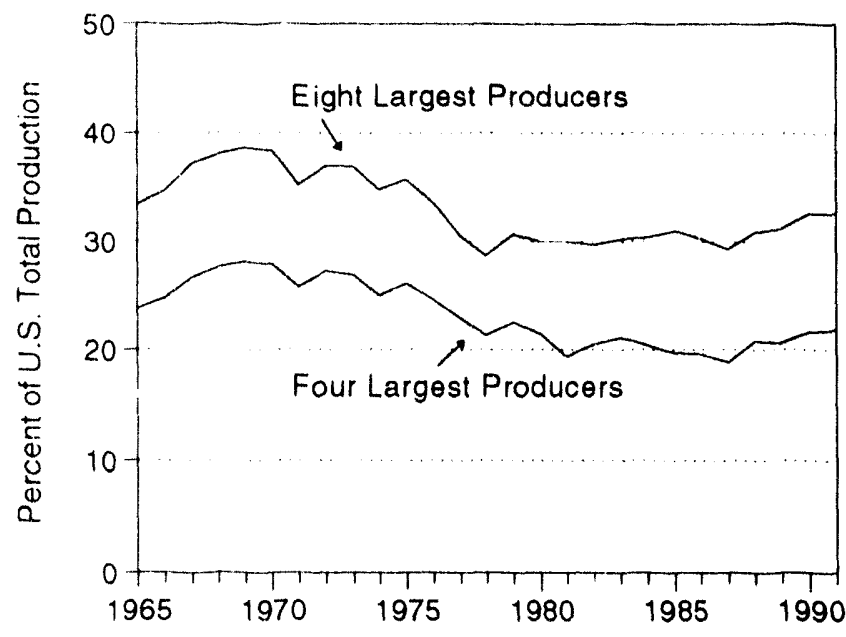

Note: Anthracite is excluded in all years except 1991, when it accounted for less than 1 percent of U.S coal production.

Source Energy Information Administration. The Changing Structure of the US Coal Industry: An Update, DOE/E/A-0513(93) (Washington, DC, July 1993).p 16
Only 3 percent of U.S. coal mines lay in the Western Region in 1991, but they accounted for 34 percent of U.S. total output, up from 16 percent in 1976. The Western Region is characterized by large, open mines with thick seams of coal lying relatively close to the surface that allow for the use of huge, efficient extraction equipment that lowers costs. Due to the large scale of mining in the region, the companies operating there tend to be large and less numerous than in the other regions. Between 1976 and 1986, however, many new firms entered the market and grew rapidly as the region's output increased. The region's four-firm production concentration consequently fell from 42 percent to 36 percent during the 10-year period. However, this trend reversed itself over the next 5 years, climbing to 38 percent in 1991.

Turnover among major coal producers. A number of important changes in company production ranking (turnover) took place in the years 1976 through 1991. The top three U.S. coal producers in $1976 \mathrm{kept}$ their rankings through 1991, but there was substantial turnover among the remaining seven of the 10 largest firms. Most of this turnover took place between 1976 and 1986 . Five of the top 10 producers in 1976 were no longer among the top 10 in 1986 or in 1991; two of those five were coal-producing steel companies.

\section{Current Developments}

The passage of the CAAA is expected to increase demand for low-sulfur coal as electric utilities seek to meet the law's tighter emissions standards for sulfur dioxide. The precise extent of the increase in demand and its effect on coal prices is, of course, uncertain. Sharp price increases at mines in Wyoming's Powder River Basin, one of the Nation's two major centers of low-sulfur coal production, seem unlikely, in part because the region has vast reserves of cheaply mined low-sulfur coal and production is not highly concentrated.

In Central Appalachia, the other major U.S. low-sulfur coal center, coal reserves are more limited. Demand for that region's output is stronger because the coal has a high heat content and is closer to most U.S. consumers and coal ports. The implementation in 1971 of New Source Performance Standards, which restricted sulfur dioxide emissions from new electric power plants, drove Central Appalachian coal prices and demand dramatically upward.

However, several factors could prevent the CAAA from having a similarly dramatic effect. For example, the law allows electric utilities to meet the emissions limits in several ways, such as burning coal with somewhat higher sulfur content than low-sulfur coal and buying emissions allowances to cover the excess emissions. Supplies of coal within the acceptable sulfur-content range are plentiful and electric utilities are expected to increase their use of coal mixtures that blend coals of different qualities.

In addition, States with extensive high-sulfur coal reserves have protected their coal industries by requiring their electric utilities to install flue-gas scrubbers, thus reducing demand for low-sulfur coal. Finally, low-sulfur coal production in Central Appalachia is increasing, even as greater "imports" of Western low-sulfur coal to the midwestern and southern 


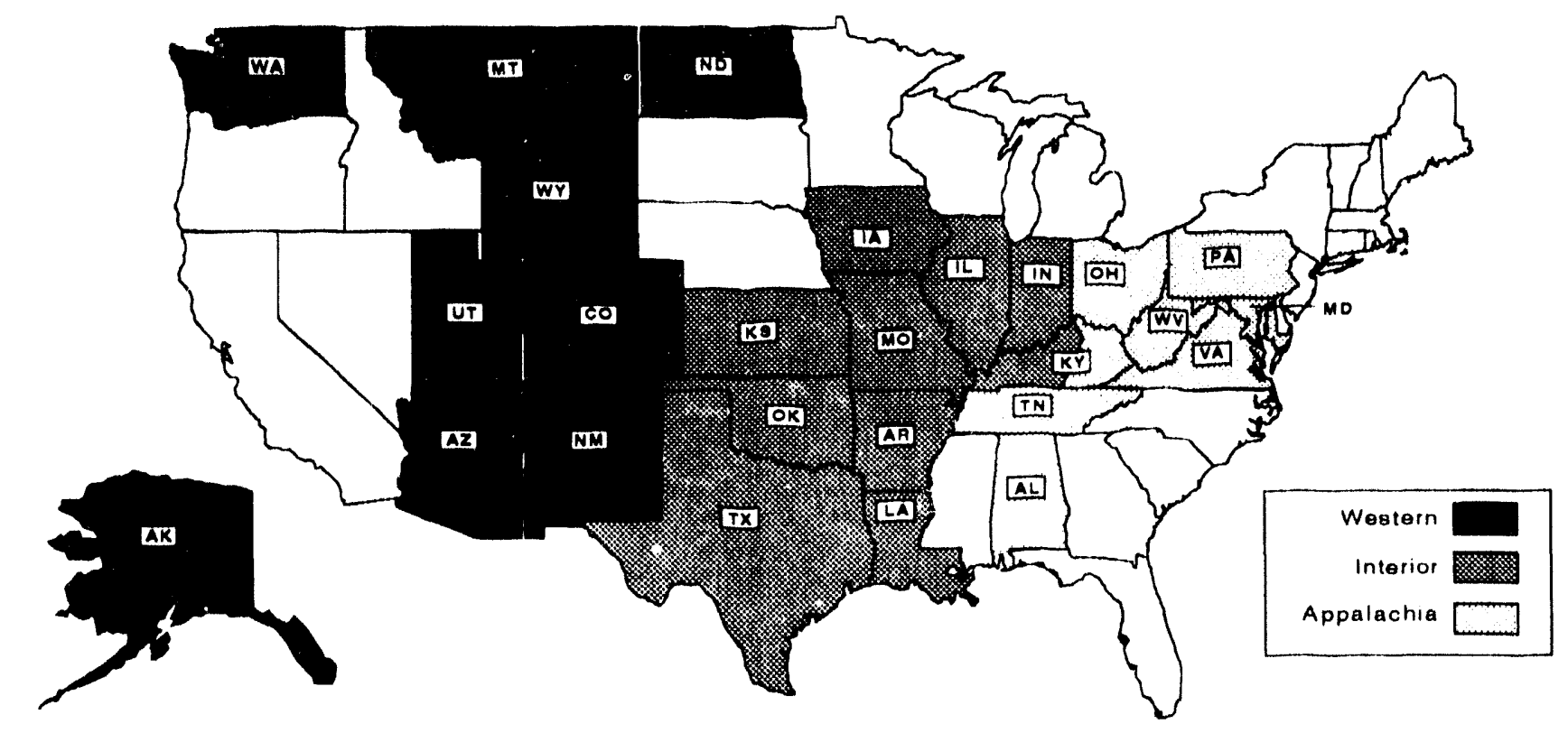

Source: Energy Intormation Administration, The Changing Structure of the U.S Coal Industry: An Update, DOE/ElA-0513(93) (Washington, DC, July 1993), p. 18.

markets are expected to temper the increase in demand for Central Appalachian coal.

Another development affecting the U.S. coal industry is the continuing wave of mergers. The mergers that took place between the beginning of 1992 and June 15, 1993, increased the estimated market share of the top four coal producers in the Nation as a whole and in all three major regions (Table 1). In absolute terms, the impact of the mergers was greatest in the Western Region, where the share of the top four firms rose eight percentage points from 38 percent in 1991 to an estimated 46 percent in mid-1993. The relative impact was greatest in Appalachia, where the share of the largest four firms increased from 20 percent to an estimated 26 percent.

In addition to their effect on production concentration, the recent mergers led to a slight increase in the share of U.S. total coal output accounted for by foreign-controlled producers, from 14 percent in 1991 to an estimated 16 percent, and raised the shares of major coal producers' output accounted for by coal and "other" companies. The shares accounted for by electric utilities and by petroleum and natural gas companies fell, while the steel companies' share remained about the same.

Considering the effects of the post-1991 mergers, key trends in the U.S. coal industry from 1976 to mid-1993 can be summarized as follows:
- Nationwide coal production concentration, expressed as the production share of the four largest producers, was lower in 1986 than it had been in 1976 but has since surpassed its 1976 level.

- The share of U.S. coal production accounted for by foreign-owned companies rose from 1 percent in 1976 to an estimated 16 percent.

- Of the major coal companies' production, the share of independent firms fell from 35 percent in 1976 to an estimated 20 percent in mid-1993. The steel companies' share fell from 9 percent to an estimated 4 percent. The petroleum and natural gas companies' share rose from 32 percent in 1976 to 44 percent in 1986, then declined to an estimated 25 percent. The production share of electric utilities likewise rose from 11 percent in 1976 to 15 percent in 1986 , then subsided to an estimated 11 percent. In the "other" category, the production share fell from the 1976 level of 14 percent to 10 percent in 1986 , but has since risen to an estimated 41 percent, largely because of acquisitions of U.S. producers, or shares of producers, by foreign conglomerates. 


\section{Section 1. Energy Overview}

Energy production during August 1993 totaled 5.5 quadrillion Btu, a 2.6-percent decrease from the level of production during August 1992. Coal production decreased 6.4 percent, petroleum production decreased 1.6 percent, and natural gas production increased 0.2 percent. All other forms of energy production combined were down 1.3 percent from the level of production during August 1992.

Energy consumption during August 1993 totaled 7.0 quadrillion Btu, 4.2 percent above the level of consumption during August 1992. Coal consumption increased 9.2 percent, natural gas consumption rose 6.2 percent, and petroleum consumption was up 1.5 percent. Consumption of all other forms of energy combined increased 0.1 percent from the level 1 year earlier.

Net imports of energy during August 1993 totaled 1.5 quadrillion Btu, 7.5 percent above the level of net imports 1 year earlier. Net imports of petroleum increased 1.0 percent, and net imports of natural gas were up 10.7 percent. Net exports of coal fell 31.0 percent from the level in August 1992.

\section{Table 1.1 Energy Summary for August 1993} (Quadrillion Btu)

\begin{tabular}{|c|c|c|c|c|c|c|c|c|}
\hline & \multicolumn{3}{|c|}{ August } & \multicolumn{5}{|c|}{ Cumulative January Through August } \\
\hline & 1093 & 1002 & $\begin{array}{l}\text { Pereent } \\
\text { Change }\end{array}$ & 1093 & $\begin{array}{l}1003 \\
\text { Dally } \\
\text { Rate }\end{array}$ & 1002 & $\begin{array}{l}1002 \\
\text { Delly } \\
\text { Rate }\end{array}$ & $\begin{array}{l}\text { Percent } \\
\text { Change }\end{array}$ \\
\hline 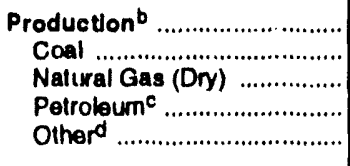 & $\begin{array}{r}8.463 \\
1.716 \\
1.498 \\
1.415 \\
.825\end{array}$ & $\begin{array}{r}6.601 \\
1.832 \\
1.495 \\
1.438 \\
.838\end{array}$ & $\begin{array}{r}-2.6 \\
-6.4 \\
.2 \\
-1.6 \\
-1.3\end{array}$ & $\begin{array}{r}43.073 \\
13.660 \\
12.452 \\
11.271 \\
6.599\end{array}$ & $\begin{array}{r}0.181 \\
.058 \\
.051 \\
.046 \\
.027\end{array}$ & $\begin{array}{r}44.867 \\
14.394 \\
12.131 \\
11.784 \\
6.248\end{array}$ & $\begin{array}{r}0.183 \\
.059 \\
.050 \\
.048 \\
.026\end{array}$ & $\begin{array}{r}-0.9 \\
-4.8 \\
3.1 \\
-4.0 \\
6.1\end{array}$ \\
\hline 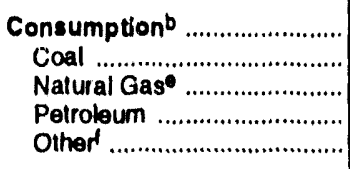 & $\begin{array}{r}6.057 \\
1.844 \\
1.382 \\
2.862 \\
.869\end{array}$ & $\begin{array}{r}6.678 \\
1.688 \\
1.302 \\
2.821 \\
.868\end{array}$ & $\begin{array}{r}4.2 \\
9.2 \\
6.2 \\
1.5 \\
.1\end{array}$ & $\begin{array}{r}56.044 \\
13.130 \\
13.950 \\
22.176 \\
6.789\end{array}$ & $\begin{array}{l}.231 \\
.054 \\
.057 \\
.091 \\
.028\end{array}$ & $\begin{array}{r}\mathbf{5 4 . 6 7 5} \\
12.560 \\
13.530 \\
22.140 \\
6.444\end{array}$ & $\begin{array}{l}.224 \\
.051 \\
.055 \\
.091 \\
.026\end{array}$ & $\begin{array}{r}2.0 \\
5.0 \\
3.5 \\
.6 \\
5.8\end{array}$ \\
\hline 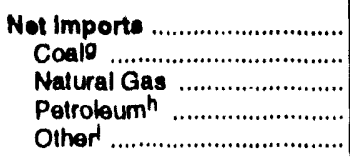 & $\begin{array}{r}1.492 \\
-.134 \\
.175 \\
1.378 \\
.043\end{array}$ & $\begin{array}{r}1.360 \\
\cdot .194 \\
.158 \\
1.364 \\
.032\end{array}$ & $\begin{array}{r}7.6 \\
-31.0 \\
10.7 \\
1.0 \\
36.2\end{array}$ & $\begin{array}{r}10.080 \\
-1.248 \\
1.386 \\
10.631 \\
.190\end{array}$ & $\begin{array}{l}.046 \\
.005 \\
.006 \\
.044 \\
.001\end{array}$ & $\begin{array}{r}0.861 \\
-1.746 \\
1.246 \\
0.856 \\
.196\end{array}$ & $\begin{array}{l}.030 \\
.007 \\
.005 \\
.040 \\
.001\end{array}$ & $\begin{array}{r}18.2 \\
-28.3 \\
11.7 \\
8.3 \\
-2.7\end{array}$ \\
\hline
\end{tabular}

\footnotetext{
- Based on dally rates prior to rounding.

- Production and consumption tolats exclude wood, waste, geothermal wind, photovoltaic, and solar thermal energy, except for small amounts used by electric utilities to generate electricily for distribution.

c Includes cnude oll, lease condensate, and natural gas plant liquids.

d "Other" is hydroelectric and nuclear electric power, and electricity generaled for distribution from wood, waste, geothermal, wind, photovoltalc, and solar themal energy.

- Includes supplemental gaseous fuels.

1 "Other" is hydroelectric and nuclear electric power; electricity generated
}

Ior distribution from wood, waste, geothermal, wind, photovollaic, and solar thermal energy; and nei imports of electricity and coal coke.

- Minus sign indicates exports are greater than imports.

h Includes crude oll, lease condensate, petroleum products, pentanes plus, unfinished olls, gasoline blending components, and imports of crude ofl for the Strategic Pelroleum Reserve.

"Other' is net imports of electricity and coal coke.

Note: Totals may not equal sum of components due to independent rounding.

Sources: Tables 1.3, 1.4, and 1.5. 
Figure 1.1 Energy Overvlew

(Quadrillion Btu)

Consumption, Production, and Imports, 1973-1992

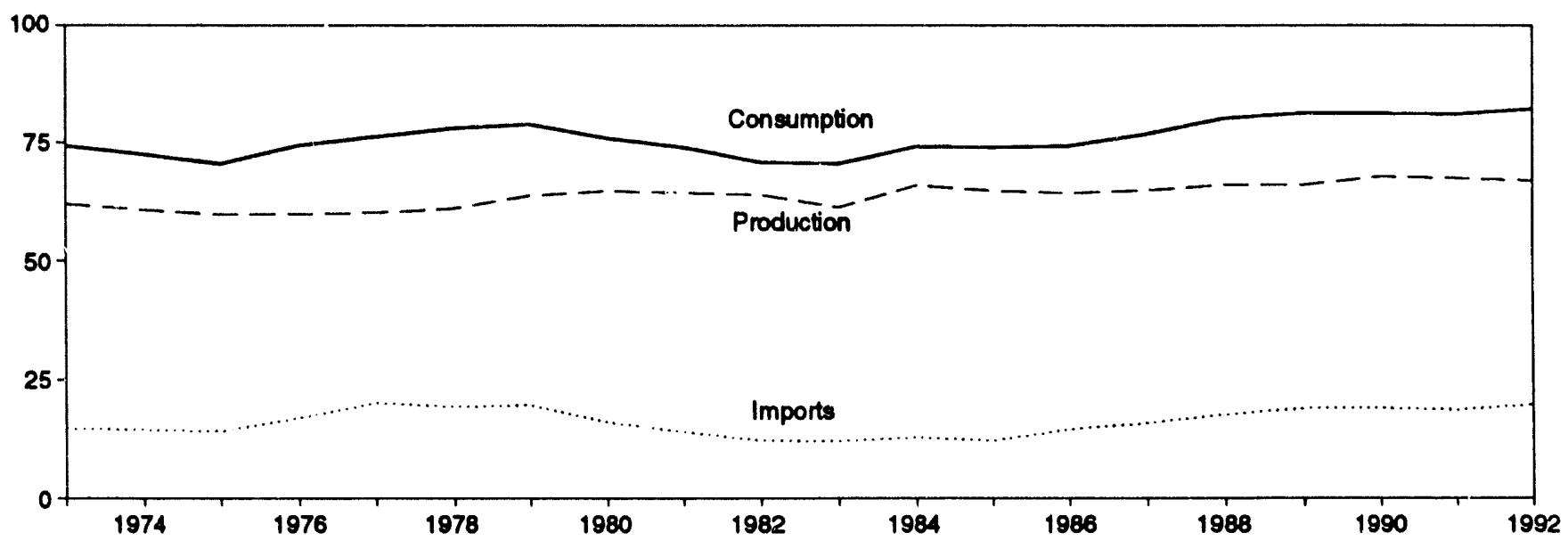

Consumption, Production, and Imports, Monthly

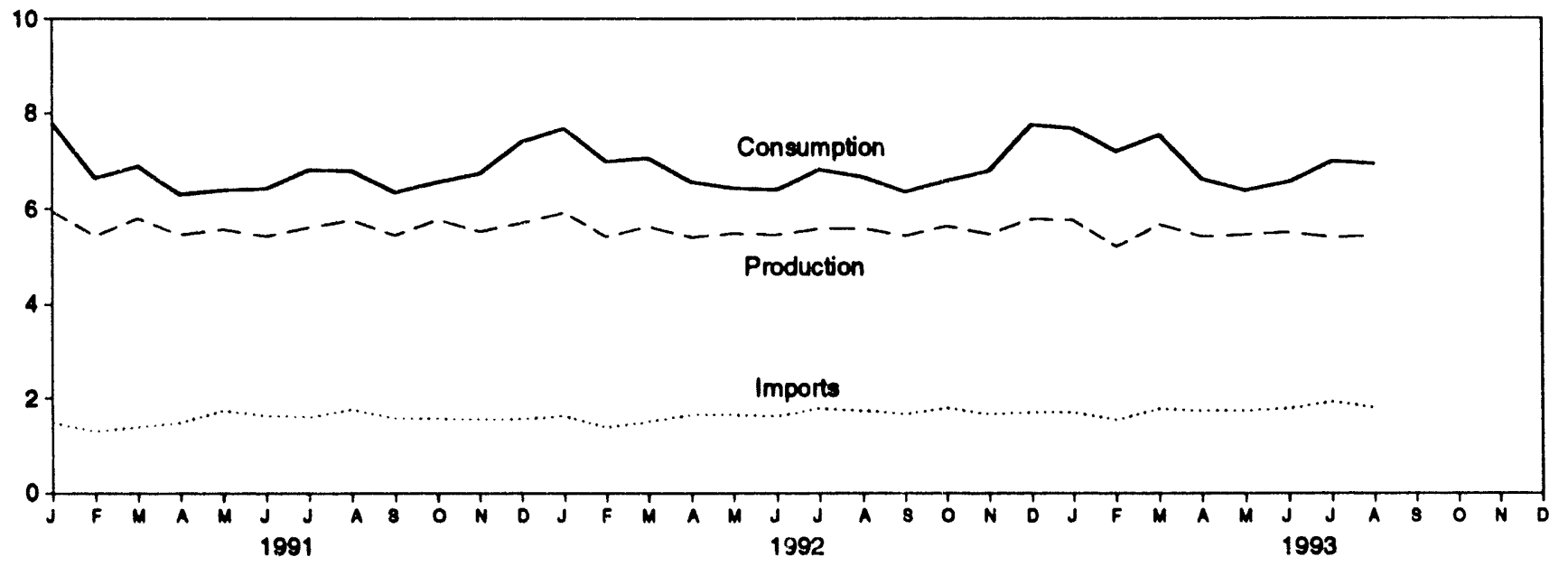

Overview, August 1993

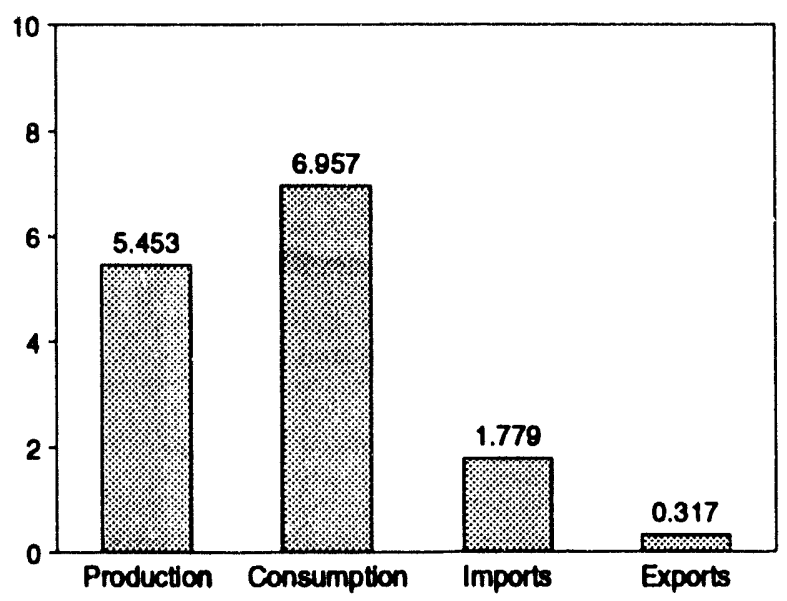

Net Imports, January-August

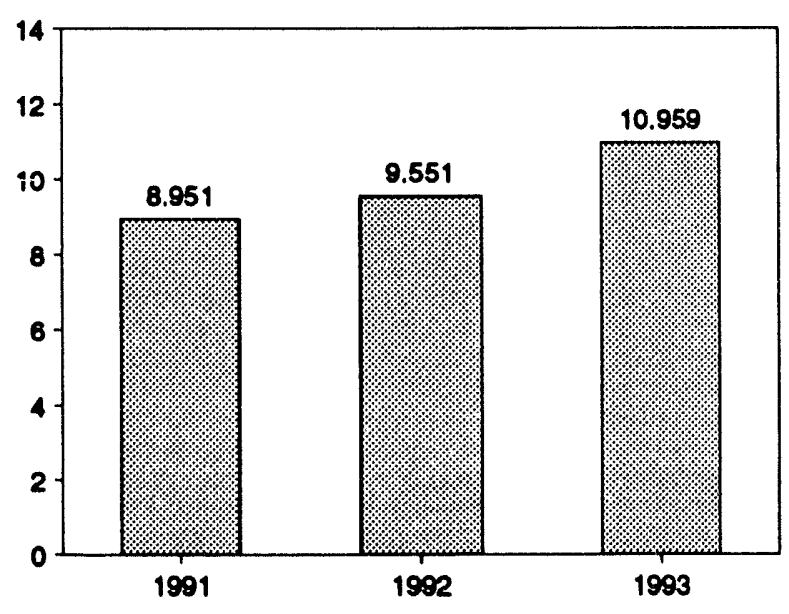

Note: Because vertical scalas differ, graphs should not be compared. Sourco: Table 1.2. 
Table 1.2 Energy Overview (Quadrillion Btu)

\begin{tabular}{|c|c|c|c|c|c|}
\hline & Producton" & Consumptona,b & Imports & Exports & Not Imports \\
\hline 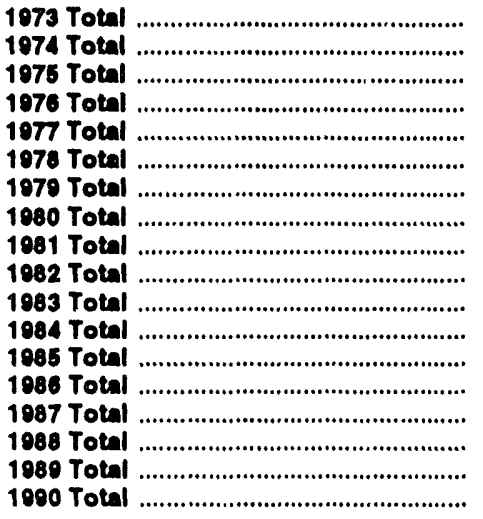 & 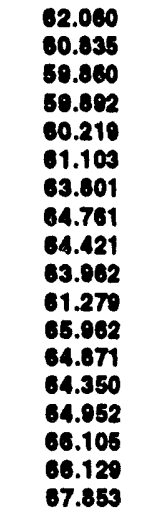 & 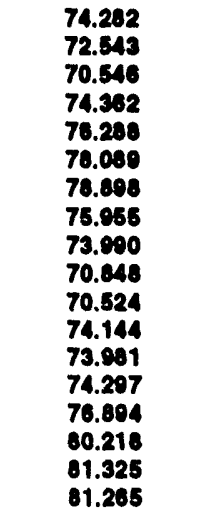 & $\begin{array}{l}14.731 \\
14.413 \\
14.111 \\
18.837 \\
20.000 \\
10.254 \\
10.816 \\
16.071 \\
13.075 \\
12.002 \\
12.027 \\
12.787 \\
12.103 \\
14.438 \\
15.764 \\
17.564 \\
18.047 \\
18.087\end{array}$ & $\begin{array}{l}2.051 \\
2.223 \\
2.350 \\
2.180 \\
2.071 \\
1.031 \\
2.070 \\
3.723 \\
4.329 \\
4.633 \\
3.717 \\
3.004 \\
4.231 \\
4.056 \\
3.053 \\
4.415 \\
4.765 \\
4.010\end{array}$ & $\begin{array}{r}12.680 \\
12.100 \\
11.752 \\
14.044 \\
18.010 \\
17.3223 \\
16.740 \\
12.247 \\
9.840 \\
7.460 \\
8.310 \\
8.063 \\
7.072 \\
10.382 \\
11.011 \\
13.140 \\
14.181 \\
14.077\end{array}$ \\
\hline 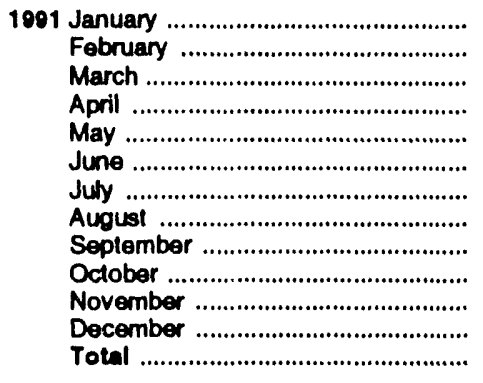 & $\begin{array}{r}\mathbf{5 . 8 4 1} \\
\mathbf{5 . 4 3 8} \\
\mathbf{5 . 8 0 3} \\
\mathbf{5 . 4 6 0} \\
\mathbf{5 . 5 7 8} \\
\mathbf{5 . 4 2 9} \\
\mathbf{5 . 6 1 3} \\
\mathbf{5 . 7 6 3} \\
\mathbf{5 . 4 5 0} \\
\mathbf{5 . 7 7 1} \\
\mathbf{5 . 5 3 0} \\
\mathbf{5 . 7 0 8} \\
\mathbf{6 7 . 4 8 4}\end{array}$ & $\begin{array}{r}7.795 \\
6.643 \\
6.893 \\
6.302 \\
6.394 \\
6.421 \\
6.818 \\
6.798 \\
6.344 \\
6.561 \\
6.740 \\
7.408 \\
81.116\end{array}$ & $\begin{array}{r}1.483 \\
1.294 \\
1.391 \\
1.482 \\
1.731 \\
1.622 \\
1.593 \\
1.754 \\
1.562 \\
1.562 \\
1.548 \\
1.556 \\
18.577\end{array}$ & $\begin{array}{l}.397 \\
.462 \\
.395 \\
.326 \\
.489 \\
.423 \\
.457 \\
.448 \\
.432 \\
.432 \\
.464 \\
.495 \\
.8 .220\end{array}$ & $\begin{array}{r}1.085 \\
.832 \\
.986 \\
1.156 \\
1.241 \\
1.199 \\
1.136 \\
1.306 \\
1.130 \\
1.130 \\
1.084 \\
1.062 \\
13.357\end{array}$ \\
\hline 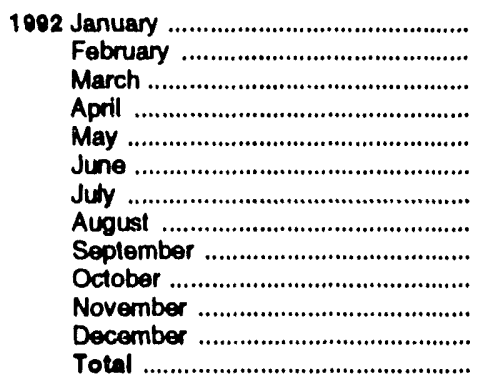 & $\begin{array}{r}\mathbf{5 . 9 2 6} \\
\mathbf{5 . 4 2 1} \\
\mathbf{5 . 6 3 7} \\
\mathbf{5 . 4 1 3} \\
\mathbf{5 . 4 9 7} \\
\mathbf{5 . 4 6 8} \\
\mathbf{5 . 5 9 4} \\
\mathbf{5 . 6 0 1} \\
\mathbf{5 . 4 4 5} \\
\mathbf{5 . 6 4 7} \\
\mathbf{5 . 4 8 5} \\
\mathbf{5 . 7 9 9} \\
\mathbf{6 6 . 9 3 3}\end{array}$ & 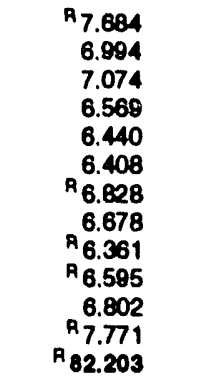 & $\begin{array}{r}1.615 \\
1.377 \\
1.500 \\
1.639 \\
1.642 \\
1.610 \\
1.770 \\
1.727 \\
1.654 \\
1.782 \\
1.650 \\
1.688 \\
10.652\end{array}$ & $\begin{array}{l}.458 \\
.372 \\
.416 \\
.413 \\
.434 \\
.426 \\
.441 \\
.367 \\
.417 \\
.383 \\
.428 \\
.462 \\
5.018\end{array}$ & $\begin{array}{r}1.157 \\
1.005 \\
1.084 \\
1.226 \\
1.207 \\
1.183 \\
1.329 \\
1.360 \\
1.237 \\
1.399 \\
1.221 \\
1.226 \\
14.634\end{array}$ \\
\hline 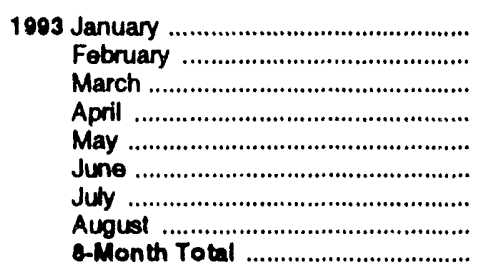 & $\begin{array}{r}R_{5} .775 \\
R_{5.218} \\
A_{5.684} \\
R_{5.434} \\
R_{5.474} \\
R_{5.521} \\
R_{\mathbf{5 . 4 1 3}} \\
\mathbf{5 . 4 5 3} \\
4 \mathbf{3 . 0 7 3}\end{array}$ & 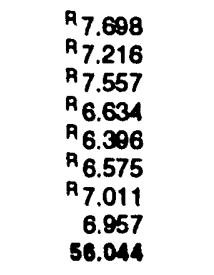 & $\begin{array}{r}1.695 \\
1.530 \\
1.763 \\
1.719 \\
A_{1.722} \\
A_{1} 1.767 \\
A_{1.914} \\
1.779 \\
13.800\end{array}$ & $\begin{array}{r}.398 \\
.362 \\
.347 \\
.344 \\
.382 \\
.406 \\
\text { ค } \\
.375 \\
.317 \\
2.031\end{array}$ & $\begin{array}{r}1.297 \\
1.168 \\
1.416 \\
1.376 \\
P_{1.340} \\
\text { ค }_{1.361} \\
\text { ค }_{1.540} \\
1.462 \\
10.950\end{array}$ \\
\hline 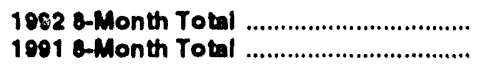 & $\begin{array}{l}44.557 \\
46.026\end{array}$ & $\begin{array}{l}54.675 \\
54.064\end{array}$ & $\begin{array}{l}12.878 \\
12.348\end{array}$ & $\begin{array}{l}3.327 \\
3.397\end{array}$ & $\begin{array}{l}9.551 \\
8.051\end{array}$ \\
\hline
\end{tabular}

- Excludes wood, waste, geothermal, wind, photovoltaic, and solar thermal energy, except for small amounts used by electric utilities to generate electricily for distribution.

$b$ The sum of domestic energy production and net imports of energy does not equal domestic energy consumption. The difference is attributed to stock changes; losses and gains in corversion, transportation, and cistribution; the addition of blending compounds; shipments of anthracite to U.S. Armed Forces in Europe; and adjustments to account lor discrepancies between reporting systems.

$R=$ Revised data

Notes: - For delinitions, see Notes 1 through 4 at end of section. - Geographic coverage is the 50 States and the District of Columbia. - Totals may not equal sum of components due to independent rounding.

Sounces: - Production: Table 1.3. - Coneumption: Table 1.4. - Imports and Exports: Tables 3.1b, 4.2, 6.1, A2-A8, and Section 2, "Energy Consumption Notes and Sources," Notes 8 and 9. - Not Imports: Table 1.5. 
Figure 1.2 Energy Production

(Quadrillion Btu)

Total Production, 1973-1992

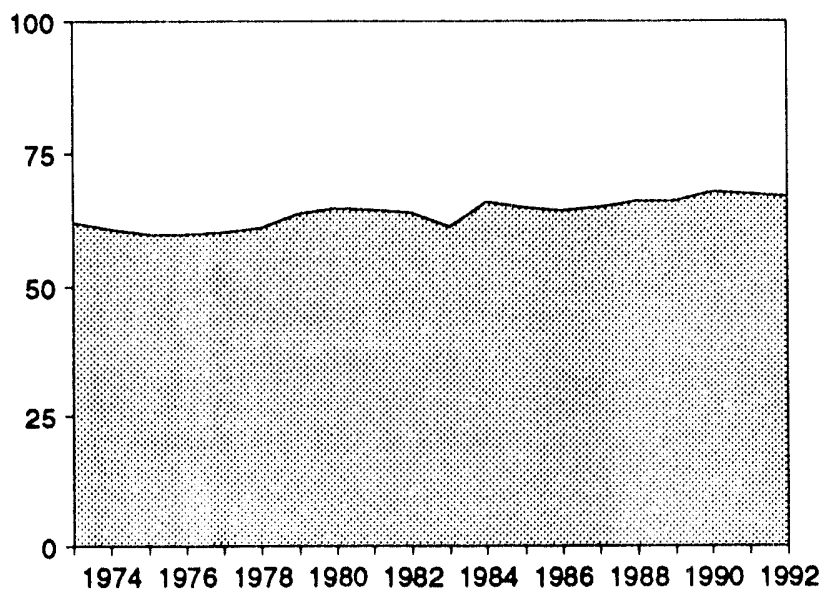

Production by Major Sources, 1973-1992

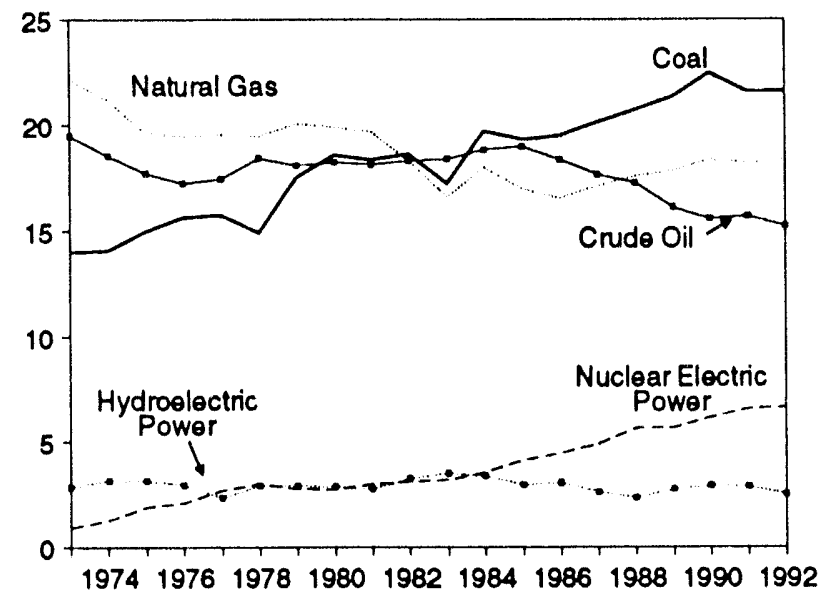

Total Production, January-August

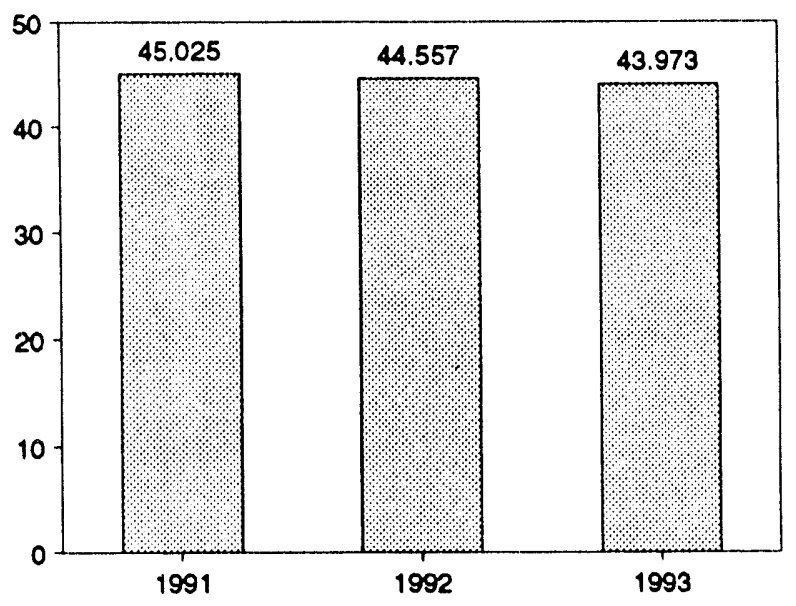

Total Production, Monthly

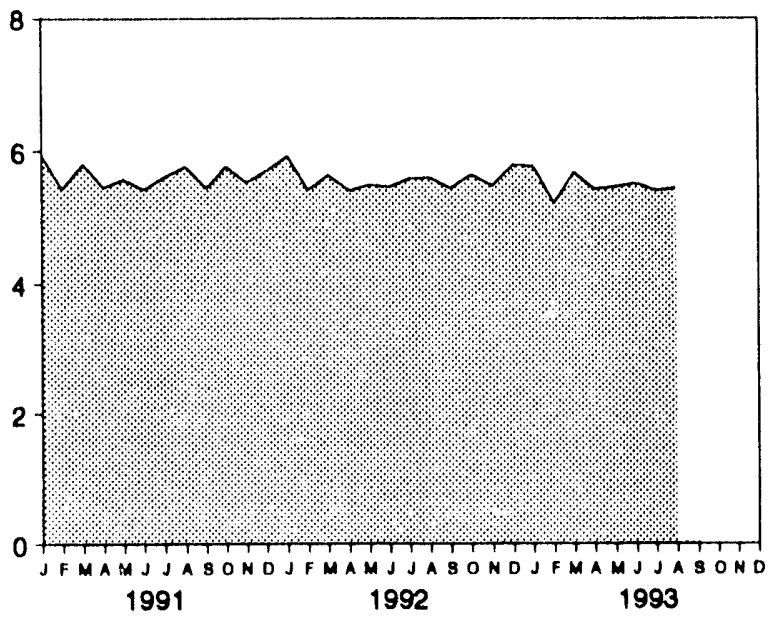

Production by Major Sources, Monthly

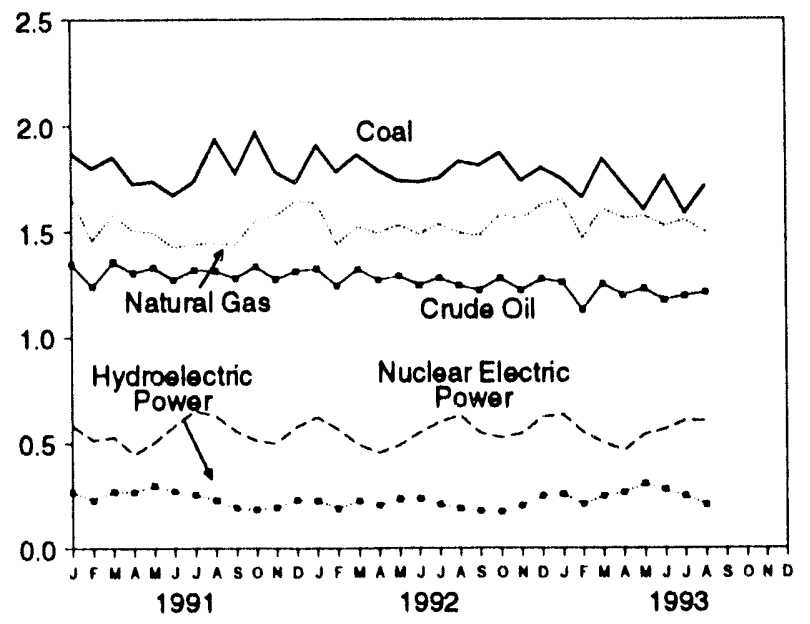

Production by Major Sources, August 1993

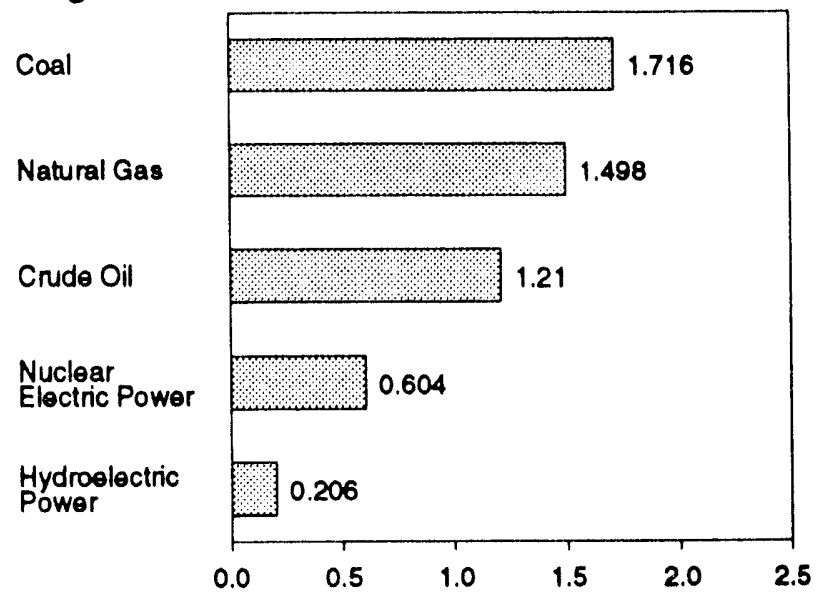

Note: Because vertical scales differ, graphs should not be compared. Source: Table 1.3. 
Table 1.3 Energy Production by Source (Quadrillion Btu)

\begin{tabular}{|c|c|c|c|c|c|c|c|c|}
\hline & Coal & $\begin{array}{l}\text { Natural } \\
\text { ane } \\
\text { (Dry) }\end{array}$ & $\begin{array}{c}\text { Crude } \\
\text { olla }\end{array}$ & $\begin{array}{l}\text { Netural } \\
\text { Cas Plant } \\
\text { Uquide }\end{array}$ & $\begin{array}{l}\text { Nuclear } \\
\text { Electrle } \\
\text { Power }\end{array}$ & $\begin{array}{l}\text { Hydro- } \\
\text { ceotrle } \\
\text { Powerb }\end{array}$ & othere & Totald \\
\hline 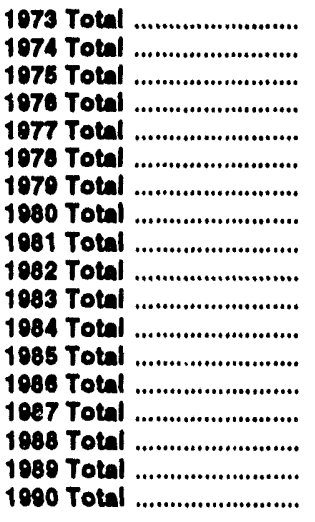 & 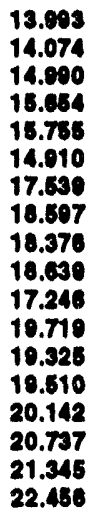 & $\begin{array}{l}22.187 \\
21.210 \\
10.840 \\
10.480 \\
10.608 \\
10.486 \\
20.070 \\
10.008 \\
19.090 \\
18.310 \\
18.593 \\
18.008 \\
18.080 \\
18.841 \\
17.136 \\
17.609 \\
17.847 \\
18.382\end{array}$ & $\begin{array}{l}19.488 \\
18.575 \\
17.720 \\
17.262 \\
17.484 \\
18.434 \\
18.104 \\
18.240 \\
18.146 \\
18.900 \\
18.392 \\
18.840 \\
18.902 \\
18.370 \\
17.075 \\
17.270 \\
18.117 \\
16.571\end{array}$ & $\begin{array}{l}2.690 \\
2.471 \\
2.374 \\
2.327 \\
2.327 \\
2.248 \\
2.286 \\
2.254 \\
2.307 \\
2.101 \\
2.184 \\
2.274 \\
2.241 \\
2.140 \\
2.215 \\
2.280 \\
2.168 \\
2.175\end{array}$ & $\begin{array}{l}0.010 \\
1.272 \\
1.000 \\
2.111 \\
2.702 \\
3.024 \\
2.770 \\
2.730 \\
3.008 \\
3.131 \\
3.203 \\
3.653 \\
4.140 \\
4.471 \\
4.008 \\
6.061 \\
6.077 \\
6.161\end{array}$ & $\begin{array}{l}2.091 \\
3.177 \\
3.166 \\
2.076 \\
2.393 \\
2.037 \\
2.031 \\
2.000 \\
2.758 \\
3.286 \\
3.827 \\
3.396 \\
2.970 \\
3.071 \\
2.036 \\
2.334 \\
2.787 \\
2.026\end{array}$ & $\begin{array}{l}0.046 \\
.058 \\
.072 \\
.081 \\
.082 \\
.088 \\
.089 \\
.114 \\
.127 \\
.106 \\
.133 \\
.174 \\
.213 \\
.232 \\
.246 \\
.238 \\
.217 \\
.202\end{array}$ & 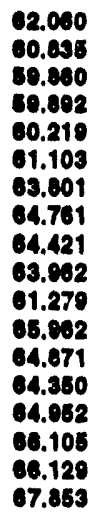 \\
\hline 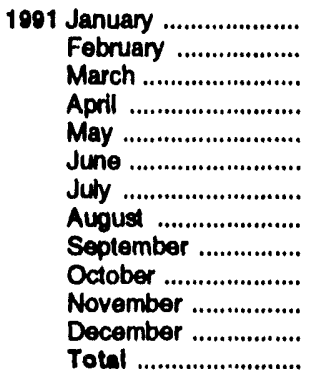 & $\begin{array}{l}1.870 \\
1.800 \\
1.853 \\
1.727 \\
1.739 \\
1.673 \\
1.738 \\
1.937 \\
1.777 \\
1.869 \\
1.782 \\
1.730 \\
21.504\end{array}$ & $\begin{array}{r}1.658 \\
1.459 \\
1.581 \\
1.506 \\
1.487 \\
1.427 \\
1.441 \\
1.447 \\
1.440 \\
1.554 \\
1.574 \\
1.645 \\
18.229\end{array}$ & $\begin{array}{r}1.348 \\
1.240 \\
1.357 \\
1.306 \\
1.332 \\
1.274 \\
1.321 \\
1.315 \\
1.282 \\
1.337 \\
1.275 \\
1.312 \\
15.701\end{array}$ & $\begin{array}{l}.184 \\
.181 \\
.199 \\
.180 \\
.196 \\
.186 \\
.191 \\
.192 \\
.185 \\
.199 \\
.194 \\
.199 \\
2.306\end{array}$ & $\begin{array}{l}.584 \\
.514 \\
.528 \\
.447 \\
.502 \\
.582 \\
.652 \\
.628 \\
.557 \\
.512 \\
.497 \\
.576 \\
\mathbf{6 . 5 7 0}\end{array}$ & $\begin{array}{l}.269 \\
.228 \\
.270 \\
.269 \\
.298 \\
.271 \\
.254 \\
.228 \\
.193 \\
.184 \\
.192 \\
.229 \\
2.085\end{array}$ & $\begin{array}{l}.017 \\
.014 \\
.016 \\
.015 \\
.015 \\
.016 \\
.016 \\
.016 \\
.015 \\
.016 \\
.017 \\
.017 \\
.101\end{array}$ & $\begin{array}{l}\mathbf{5 . 8 4 1} \\
\mathbf{5 . 4 3 8} \\
\mathbf{5 . 8 0 3} \\
\mathbf{5 . 4 6 0} \\
\mathbf{5 . 5 7 8} \\
\mathbf{5 . 4 2 9} \\
\mathbf{5 . 6 1 3} \\
\mathbf{5 . 7 6 3} \\
\mathbf{5 . 4 5 0} \\
\mathbf{5 . 7 7 1} \\
\mathbf{5 . 5 3 0} \\
\mathbf{5 . 7 0 8} \\
\mathbf{6 7 . 4 8 4}\end{array}$ \\
\hline 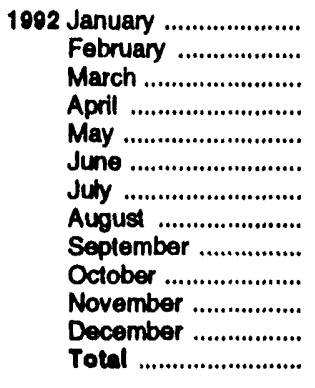 & $\begin{array}{r}1.806 \\
1.780 \\
1.861 \\
1.787 \\
1.739 \\
1.735 \\
1.753 \\
1.832 \\
1.813 \\
1.872 \\
1.741 \\
1.801 \\
21.622\end{array}$ & $\begin{array}{r}1.633 \\
1.440 \\
1.519 \\
1.491 \\
1.529 \\
1.488 \\
1.536 \\
1.495 \\
1.481 \\
1.579 \\
1.559 \\
1.628 \\
18.375\end{array}$ & $\begin{array}{l}1.323 \\
1.243 \\
1.321 \\
1.269 \\
1.269 \\
1.247 \\
1.282 \\
1.245 \\
1.223 \\
1.281 \\
1.222 \\
1.277 \\
15.223\end{array}$ & $\begin{array}{l}.189 \\
.187 \\
.200 \\
.193 \\
.200 \\
.184 \\
.198 \\
.193 \\
.189 \\
.203 \\
.200 \\
.208 \\
2.383\end{array}$ & $\begin{array}{l}.621 \\
.567 \\
.492 \\
.454 \\
.490 \\
.550 \\
.602 \\
.630 \\
.547 \\
.524 \\
.545 \\
.624 \\
.6 .646\end{array}$ & $\begin{array}{l}.226 \\
.189 \\
.226 \\
.204 \\
.234 \\
.238 \\
.207 \\
.189 \\
.177 \\
.172 \\
.202 \\
.249 \\
2.513\end{array}$ & $\begin{array}{l}.017 \\
.015 \\
.017 \\
.015 \\
.016 \\
.016 \\
.016 \\
.017 \\
.015 \\
.016 \\
.016 \\
.016 \\
.102\end{array}$ & $\begin{array}{r}\mathbf{5 . 9 2 6} \\
\mathbf{5 . 4 2 1} \\
\mathbf{5 . 6 3 7} \\
\mathbf{5 . 4 1 3} \\
\mathbf{5 . 4 9 7} \\
\mathbf{5 . 4 6 8} \\
\mathbf{5 . 5 8 4} \\
\mathbf{5 . 6 0 1} \\
\mathbf{5 . 4 4 5} \\
\mathbf{5 . 6 4 7} \\
\mathbf{5 . 4 8 5} \\
\mathbf{5 . 7 9 9} \\
\mathbf{8 6 . 0 3 3}\end{array}$ \\
\hline 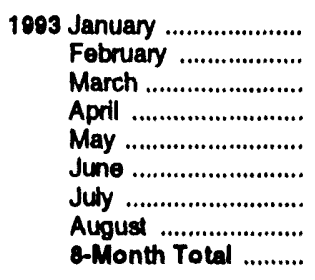 & $\begin{array}{r}1.751 \\
1.660 \\
1.844 \\
1.723 \\
1.605 \\
1.762 \\
1.588 \\
1.716 \\
13.650\end{array}$ & $\begin{array}{r}R_{1} .654 \\
A_{1.467} \\
A_{1.610} \\
A_{1.564} \\
A_{1.576} \\
A_{1.527} \\
R_{1} .556 \\
1.498 \\
12.452\end{array}$ & $\begin{array}{l}1.260 \\
1.130 \\
1.254 \\
1.200 \\
1.229 \\
1.176 \\
1.196 \\
1.210 \\
9.655\end{array}$ & $\begin{array}{r}.204 \\
.188 \\
.212 \\
.204 \\
.203 \\
.198 \\
.203 \\
.204 \\
1.616\end{array}$ & $\begin{array}{l}.634 \\
.551 \\
.501 \\
.464 \\
.541 \\
.565 \\
.607 \\
.604 \\
\mathbf{4 . 4 6 7}\end{array}$ & $\begin{array}{l}.256 \\
.207 \\
.247 \\
.264 \\
.307 \\
.279 \\
.247 \\
.206 \\
2.013\end{array}$ & $\begin{array}{l}.016 \\
.015 \\
.016 \\
.015 \\
.014 \\
.014 \\
.015 \\
.015 \\
.120\end{array}$ & $\begin{array}{r}A_{5} .775 \\
A_{5.218} \\
A_{5.684} \\
A_{5.434} \\
A_{5.474} \\
R_{5} .521 \\
A_{5.413} \\
5.453 \\
43.073\end{array}$ \\
\hline $\begin{array}{l}1002 \text { 8-Month Total ......... } \\
1001 \text { \&-Month Total ......... }\end{array}$ & $\begin{array}{l}14.304 \\
14.336\end{array}$ & $\begin{array}{l}12.131 \\
12.016\end{array}$ & $\begin{array}{l}10.210 \\
10.494\end{array}$ & $\begin{array}{l}1.585 \\
1.520\end{array}$ & $\begin{array}{l}4.408 \\
4.437\end{array}$ & $\begin{array}{l}1.714 \\
2.088\end{array}$ & $\begin{array}{l}.128 \\
.128\end{array}$ & $\begin{array}{l}44.567 \\
46.028\end{array}$ \\
\hline
\end{tabular}

- Includes lease condensate.

bectric utlity and industrial generation.

c "Oiher" production is electricity generated for distribution from wood, wagte, geothermal, wind, photovollajc, and solar thermal energy.

Excludes wood, waste, geothermal, wind, photovottaic, and solar thermal energy, except for small amounts used by electric utilities to generate electricky for distribution.

$R=$ Revised data.

Notes: - See Note 1 al end of ssction. - Geographic coverage is the 50
States and the District of Columbia. - Totals may nol equal sum of components due to independent rounding.

Sources: - Coal: Tables 6.1 and A5-A7. - Natural Ges (Dry): Tables 4.1 and A4. Crude Oll and Natural Gas Plant Luquids: Tables $3.1 \mathrm{a}$ and A2. Nuclear Electric Powor: Tablss 7.1 and A8. Hydrodectrle Power: Table 7.1; Section 2, "Energy Consumption Noles and Sources," Note 8; and Table A8. - Other: Section 2, "Energy Consumption Notes and Sources," Note 7, and Table A8. 
Figure 1.3 Energy Consumption

(Quadrillion Btu)

Total Consumption, 1973-1992

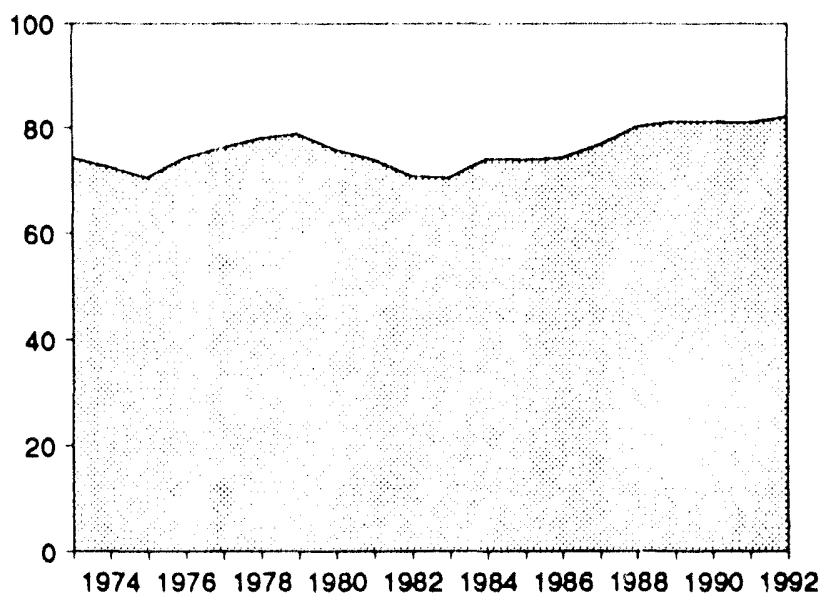

Consumption by Major Sources, 1973-1992

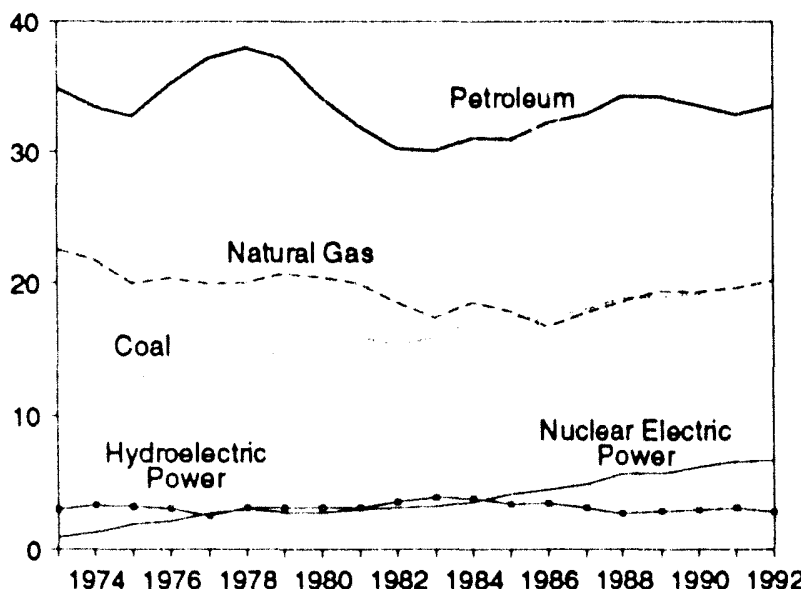

Total Consumption, January-August

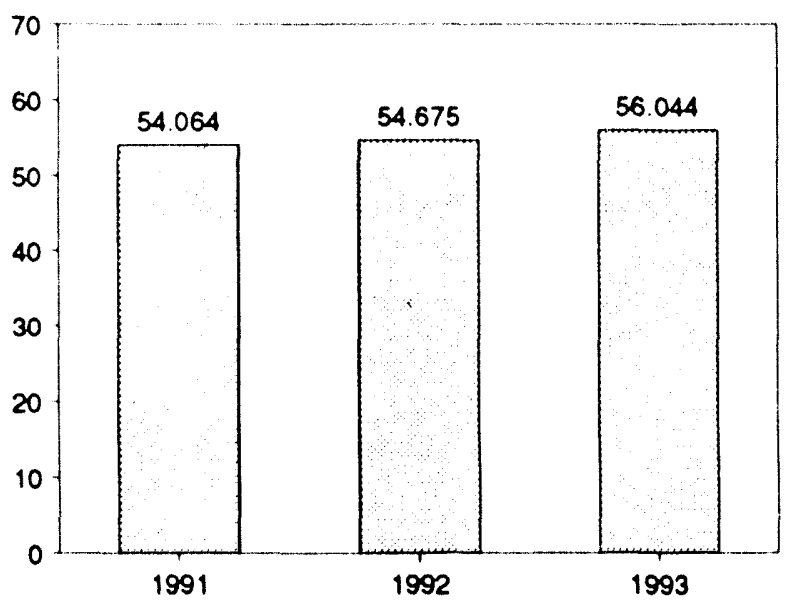

Total Consumption, Monthly

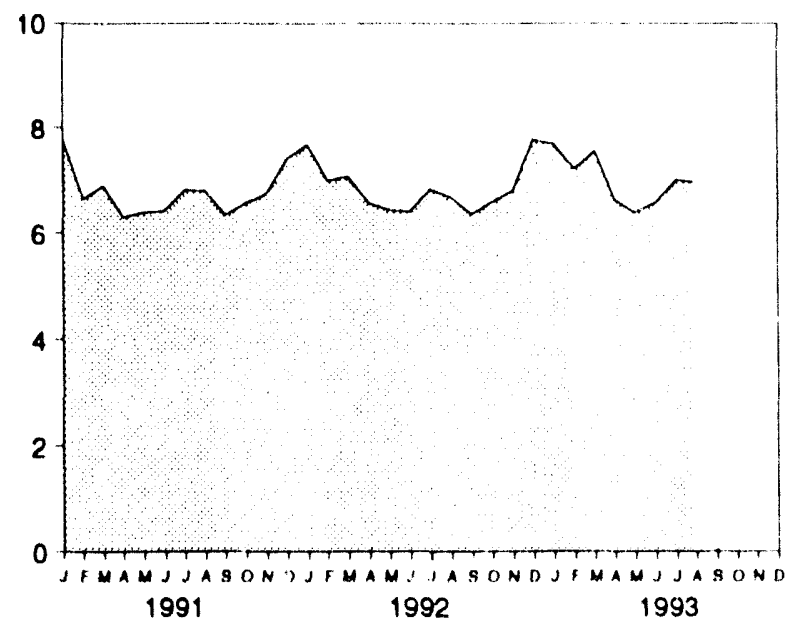

Consumption by Major Sources, Monthly

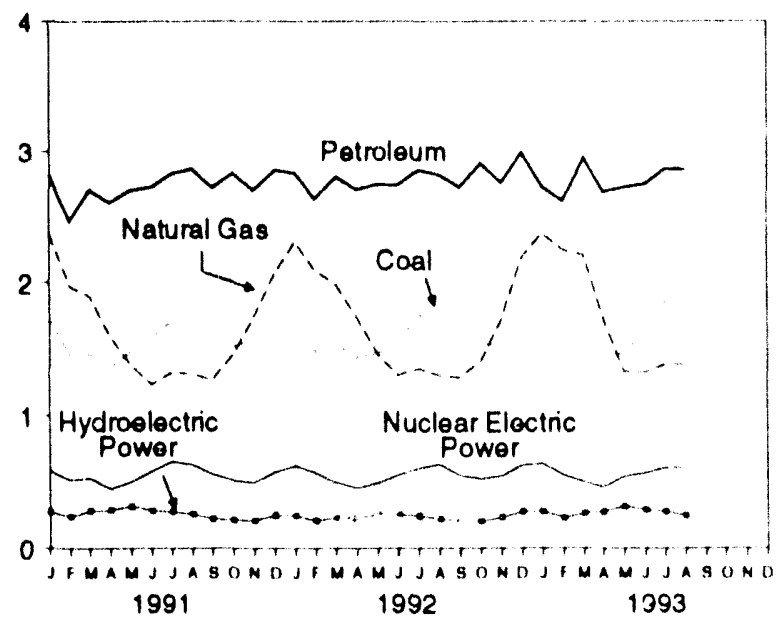

Consumption by Major Sources, August 1993

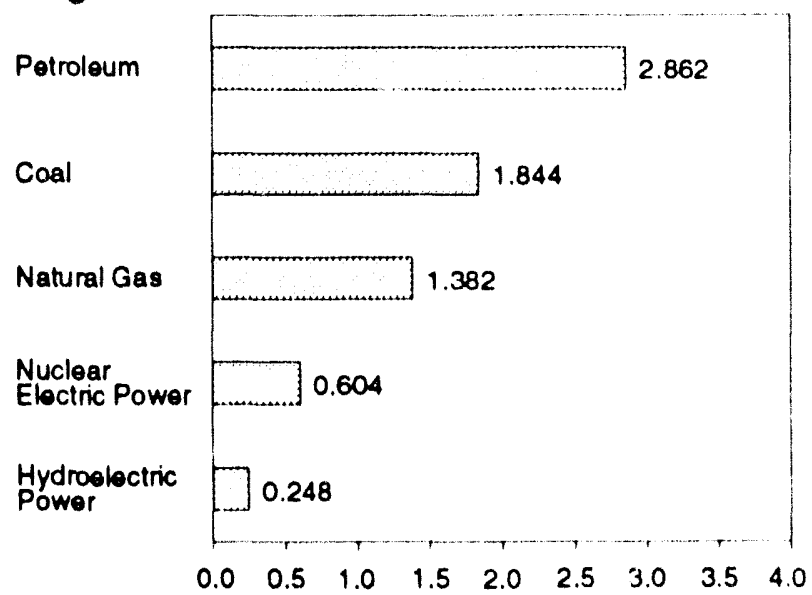

Note: Because vertical scales differ, graphs should not be compared. Source: Table 1.4. 
Table 1.4 Energy Consumption by Source

(Quadrillion Btu)

\begin{tabular}{|c|c|c|c|c|c|c|c|}
\hline & Coal & $\begin{array}{c}\text { Natural } \\
\text { Casa }\end{array}$ & Potroloum & $\begin{array}{l}\text { Nuclear } \\
\text { Electrle } \\
\text { Power }\end{array}$ & $\begin{array}{l}\text { Hydro- } \\
\text { olectrle } \\
\text { Powerb }\end{array}$ & Othere & Totald \\
\hline 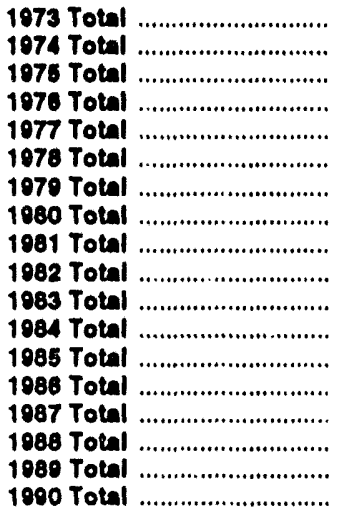 & $\begin{array}{l}12.071 \\
12.093 \\
12.083 \\
13.884 \\
13.022 \\
13.785 \\
16.030 \\
16.423 \\
18.007 \\
18.322 \\
15.804 \\
17.071 \\
17.478 \\
17.281 \\
18.008 \\
18.846 \\
18.028 \\
10.101\end{array}$ & $\begin{array}{l}22.512 \\
21.732 \\
10.048 \\
20.345 \\
19.031 \\
20.000 \\
20.686 \\
20.304 \\
10.028 \\
18.805 \\
17.357 \\
18.507 \\
17.834 \\
18.708 \\
17.744 \\
18.352 \\
10.384 \\
10.206\end{array}$ & $\begin{array}{l}34.640 \\
33.455 \\
32.731 \\
35.175 \\
\mathbf{3 7 . 1 2 2} \\
\mathbf{3 7 . 0 6 5} \\
\mathbf{3 7 . 1 2 3} \\
\mathbf{3 4 . 2 0 2} \\
31.031 \\
30.231 \\
30.054 \\
\mathbf{3 1 . 0 5 1} \\
\mathbf{3 0 . 9 2 2} \\
\mathbf{3 2 . 1 9 8} \\
\mathbf{3 2 . 8 6 5} \\
\mathbf{3 4 . 2 2 2} \\
\mathbf{3 4 . 2 1 1} \\
\mathbf{3 3 . 5 5 3}\end{array}$ & $\begin{array}{l}0.010 \\
1.272 \\
1.900 \\
2.111 \\
2.702 \\
3.024 \\
2.776 \\
2.730 \\
3.008 \\
3.131 \\
3.203 \\
3.583 \\
4.149 \\
4.471 \\
4.906 \\
5.681 \\
5.677 \\
6.161\end{array}$ & $\begin{array}{l}3.010 \\
3.300 \\
3.218 \\
3.086 \\
2.518 \\
3.141 \\
3.141 \\
3.116 \\
3.106 \\
3.672 \\
3.809 \\
3.800 \\
3.398 \\
3.446 \\
3.117 \\
2.882 \\
2.881 \\
2.046\end{array}$ & $\begin{array}{l}0.030 \\
.112 \\
.086 \\
.081 \\
.097 \\
.103 \\
.152 \\
.070 \\
.111 \\
.086 \\
.118 \\
.183 \\
.100 \\
.215 \\
.253 \\
.274 \\
.240 \\
.207\end{array}$ & 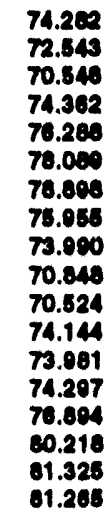 \\
\hline 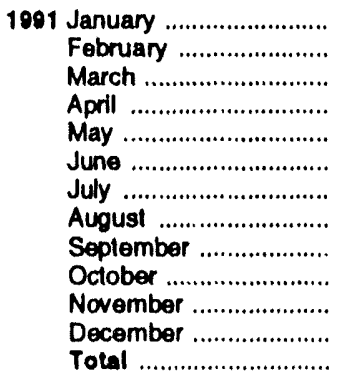 & $\begin{array}{r}1.728 \\
1.444 \\
1.463 \\
1.357 \\
1.480 \\
1.577 \\
1.718 \\
1.717 \\
1.558 \\
1.523 \\
1.570 \\
1.635 \\
18.770\end{array}$ & $\begin{array}{r}{ }^{A} 2.367 \\
1.969 \\
1.895 \\
1.589 \\
1.377 \\
1.235 \\
1.322 \\
1.312 \\
1.268 \\
1.461 \\
1.742 \\
2.069 \\
10.606\end{array}$ & $\begin{array}{r}2.819 \\
2.463 \\
2.706 \\
2.607 \\
2.702 \\
2.726 \\
2.832 \\
2.868 \\
2.721 \\
2.837 \\
2.702 \\
2.862 \\
32.845\end{array}$ & $\begin{array}{l}.584 \\
.514 \\
.528 \\
.447 \\
.502 \\
.582 \\
.652 \\
.628 \\
.557 \\
.512 \\
.497 \\
.576 \\
.5 .579\end{array}$ & $\begin{array}{l}.278 \\
.237 \\
.283 \\
.287 \\
.317 \\
.286 \\
.275 \\
.259 \\
.221 \\
.213 \\
.211 \\
.249 \\
.116\end{array}$ & $\begin{array}{l}.017 \\
.015 \\
.018 \\
.016 \\
.016 \\
.015 \\
.019 \\
.014 \\
.019 \\
.015 \\
.018 \\
.017 \\
.200\end{array}$ & $\begin{array}{r}7.795 \\
6.643 \\
6.893 \\
6.302 \\
6.394 \\
6.421 \\
6.818 \\
6.798 \\
6.344 \\
6.561 \\
6.740 \\
7.408 \\
81.118\end{array}$ \\
\hline 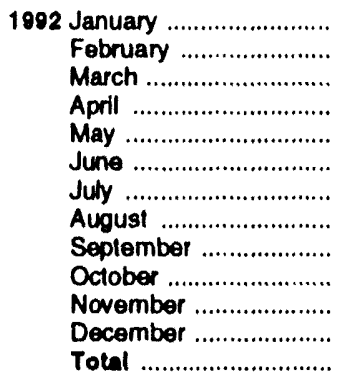 & 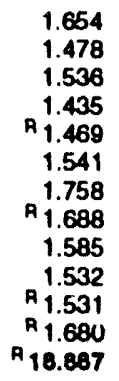 & $\begin{array}{r}2.306 \\
2.091 \\
1.884 \\
1.735 \\
1.460 \\
1.302 \\
1.351 \\
1.302 \\
1.286 \\
1.409 \\
1.722 \\
2.182 \\
20.131\end{array}$ & $\begin{array}{r}2.835 \\
2.634 \\
2.804 \\
2.704 \\
2.747 \\
2.738 \\
2.857 \\
2.821 \\
2.722 \\
2.908 \\
2.756 \\
2.988 \\
33.514\end{array}$ & $\begin{array}{l}.621 \\
.567 \\
.492 \\
.454 \\
.490 \\
.550 \\
.602 \\
.630 \\
.547 \\
.524 \\
.545 \\
.624 \\
.6 .646\end{array}$ & $\begin{array}{l}.247 \\
.206 \\
.238 \\
.223 \\
.256 \\
.258 \\
.243 \\
.221 \\
.205 \\
.203 \\
.231 \\
.276 \\
2.006\end{array}$ & $\begin{array}{l}.021 \\
.018 \\
.020 \\
.018 \\
.017 \\
.018 \\
.017 \\
.017 \\
.016 \\
.018 \\
.017 \\
.021 \\
.210\end{array}$ & $\begin{array}{r}\text { P }_{7.684} \\
6.994 \\
7.074 \\
6.560 \\
6.440 \\
6.408 \\
{ }^{\circ} 6.828 \\
6.678 \\
{ }^{\circ} 6.361 \\
{ }^{\circ} 6.595 \\
6.802 \\
{ }^{\circ} 7.771 \\
{ }^{\circ} 8.203\end{array}$ \\
\hline 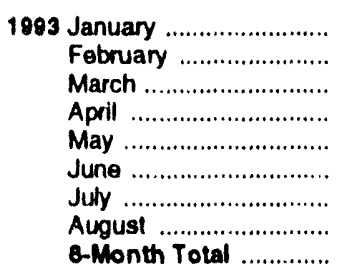 & $\begin{array}{r}1.679 \\
1.563 \\
1.620 \\
1.461 \\
1.468 \\
1.638 \\
1.858 \\
1.844 \\
13.130\end{array}$ & $\begin{array}{r}{ }_{2} 2.366 \\
A_{2.240} \\
A_{2.204} \\
R_{1} .723 \\
A_{1} .330 \\
A_{1} .318 \\
A_{1.386} \\
1.382 \\
13.950\end{array}$ & $\begin{array}{r}2.720 \\
2.619 \\
2.948 \\
2.689 \\
2.723 \\
2.747 \\
2.868 \\
2.862 \\
22.176\end{array}$ & $\begin{array}{l}.634 \\
.551 \\
.501 \\
.464 \\
.541 \\
.565 \\
.607 \\
.604 \\
.467\end{array}$ & $\begin{array}{l}.279 \\
.229 \\
.266 \\
.279 \\
.318 \\
.290 \\
.278 \\
.248 \\
2.187\end{array}$ & $\begin{array}{l}.020 \\
.015 \\
.019 \\
.018 \\
.016 \\
.016 \\
.015 \\
.017 \\
.136\end{array}$ & $\begin{array}{l}\text { A }_{7.689} \\
\text { A }_{7.216} \\
\text { A }_{7.557} \\
\text { A }_{6.634} \\
\text { A }_{6.396} \\
\text { A }_{6.575} \\
\text { R }_{7.011} \\
\mathbf{6 . 0 5 7} \\
\mathbf{5 8 . 0 4 4}\end{array}$ \\
\hline $\begin{array}{l}1902 \text { 8-Month Total ............. } \\
1091 \text { 8-Month Total ............. }\end{array}$ & $\begin{array}{l}12.580 \\
12.485\end{array}$ & $\begin{array}{l}13.530 \\
13.066\end{array}$ & $\begin{array}{l}22.140 \\
21.724\end{array}$ & $\begin{array}{l}4.406 \\
4.437\end{array}$ & $\begin{array}{l}1.892 \\
2.221\end{array}$ & $\begin{array}{l}.146 \\
.130\end{array}$ & $\begin{array}{l}54.678 \\
54.064\end{array}$ \\
\hline
\end{tabular}

- Includes supplemental gaseous fuels.

b Eectric utility and industrial generation and net imports of electricity.

c "Other" consumption is net imports of coal coke and electricity generated for distribution from wood, waste, geothermal, wind, photovoltaic, and solar thermal energy

d Excludes wood, waste, geothermal, wind, photovollaic, and solar thermal energy, except for small amounts used by electric utilities to generate electrichly for distribution.

Notes: - See Note 2 at end of section. - Geographic coverage is the 50 States and the District of Columbia. - Totals may not equal sum of components due to independent rounding.

Sources: - Cod: Tables 6.1 and A5-A7. - Natural Gas: Tables 4.2 and A4. - Potroleum: Tables 3.1a and A3. - Nuclear Electrle Power: Tables 7.1 and AB. - Hydroelectrle Power: Table 7.1; Section 2, "Energy Consumption Notes and Sources, Nole 8; and Table A8. - Other: Section 2. "Energy Consumption Noles and Sources," Note 7, and Table A8.

$R=$ Revised data. 
Figure 1.4 Energy Net Imports

(Quadrillion Btu, Except as Noted)

Total Net Imports, 1973-1992

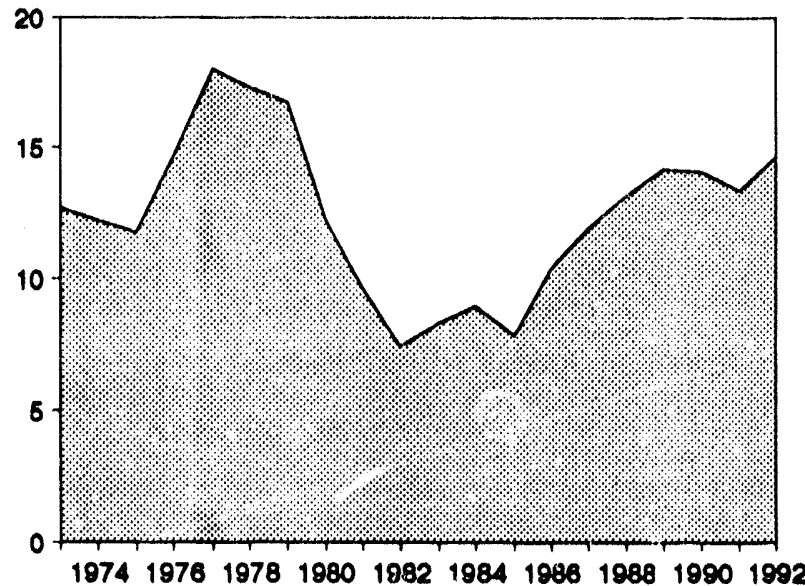

Net Imports by Major Sources, 1973-1992

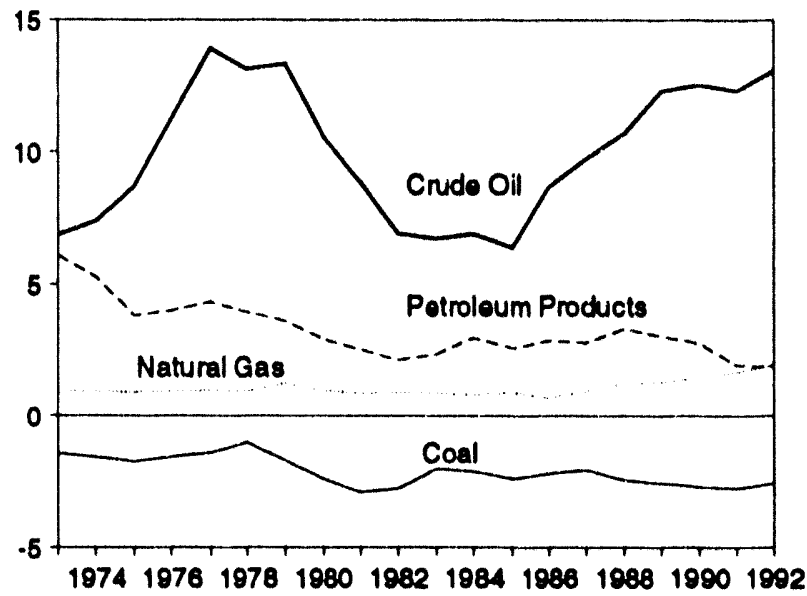

Net Imports by Major Sources,

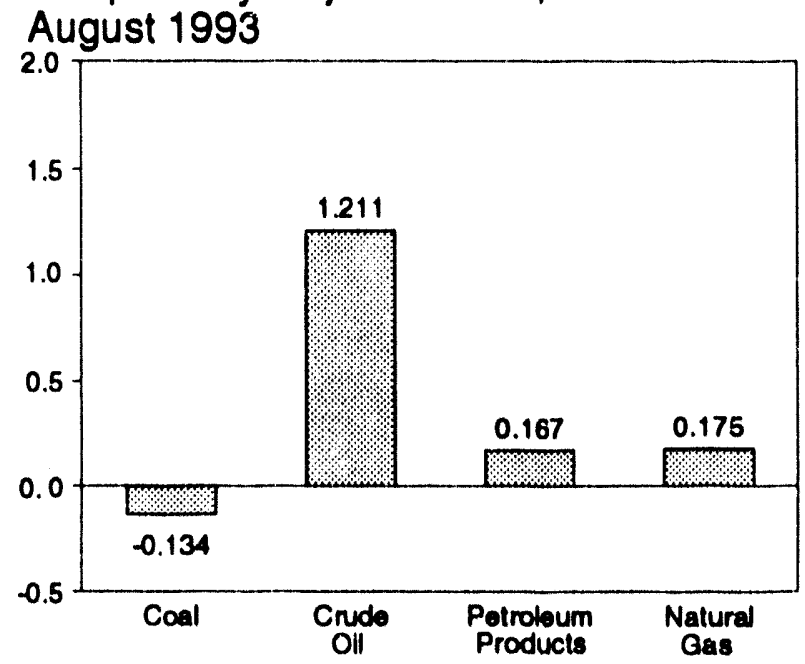

Net Imports, Monthly

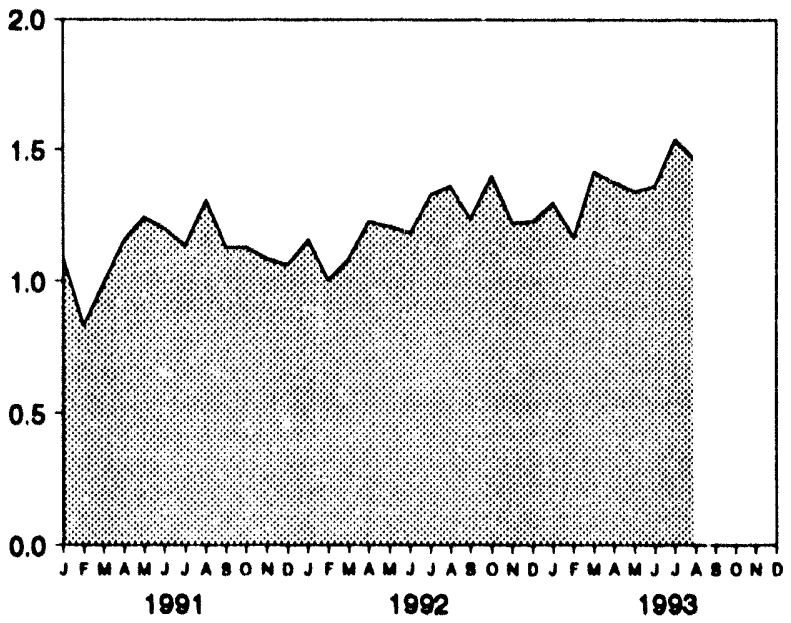

Net Imports by Major Sources, Monthly

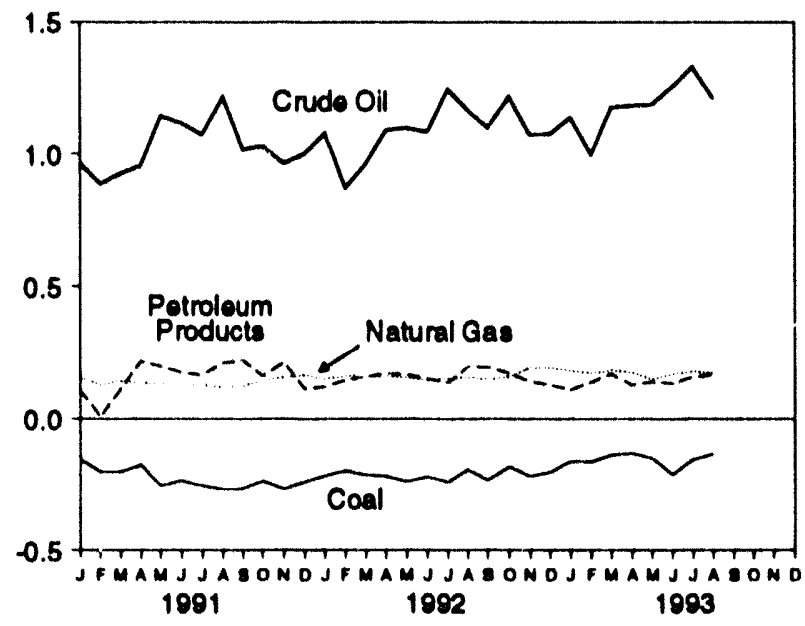

Net Imports as Share of Consumption, January-August

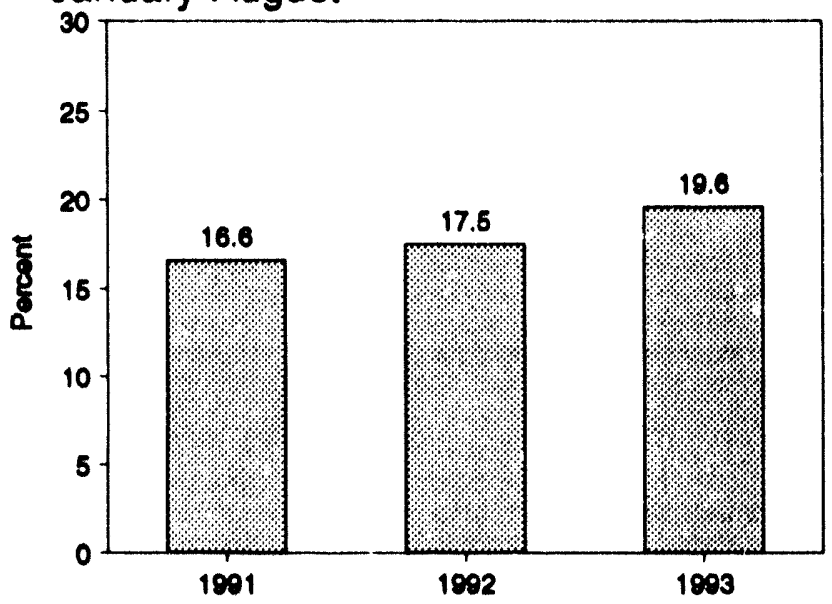

Noto: Because vertical scales differ, graphs should not be compared.

Sources: Tables 1.4 and 1.5. 
Table 1.5 Energy Net imports by Source

(Quadrillion Btu)

\begin{tabular}{|c|c|c|c|c|c|c|c|}
\hline & Cod & $\begin{array}{l}\text { Natural } \\
\text { aes }\end{array}$ & $\begin{array}{l}\text { Crude } \\
\text { olfe }\end{array}$ & $\begin{array}{l}\text { Petroleum } \\
\text { Produotb }\end{array}$ & Electribityo & $\begin{array}{l}\text { Coal } \\
\text { Coke }\end{array}$ & Toul \\
\hline 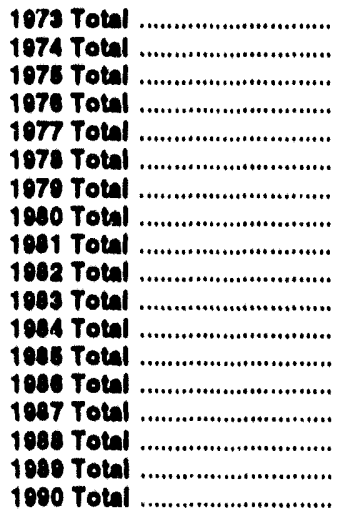 & 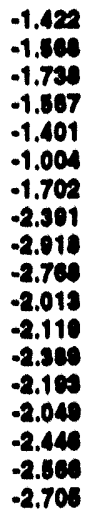 & $\begin{array}{r}0.001 \\
.007 \\
.004 \\
.020 \\
.001 \\
.041 \\
1.248 \\
.087 \\
.087 \\
.004 \\
.014 \\
.702 \\
.006 \\
.004 \\
.037 \\
1.221 \\
1.270 \\
1.464\end{array}$ & 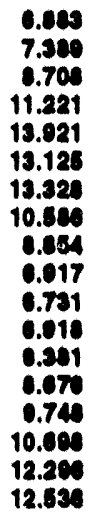 & 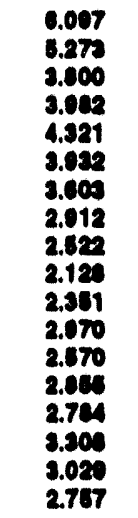 & $\begin{array}{r}0.140 \\
.139 \\
.004 \\
.000 \\
.102 \\
.204 \\
211 \\
.217 \\
.247 \\
.200 \\
.372 \\
.414 \\
.420 \\
.375 \\
.403 \\
.320 \\
.113 \\
.020\end{array}$ & 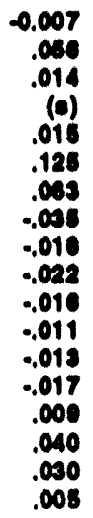 & 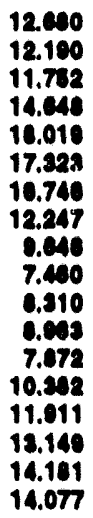 \\
\hline 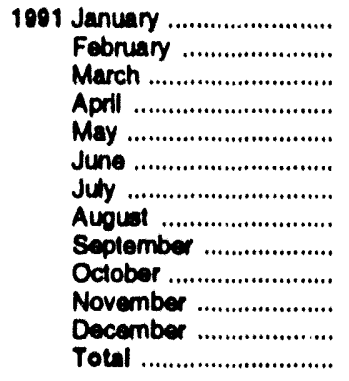 & $\begin{array}{l}. .158 \\
.202 \\
.203 \\
. .176 \\
.256 \\
.238 \\
.268 \\
-.270 \\
.267 \\
.237 \\
-.270 \\
-.240 \\
-2.700\end{array}$ & $\begin{array}{l}.156 \\
.129 \\
.143 \\
.137 \\
.135 \\
.128 \\
.129 \\
.119 \\
.126 \\
.144 \\
.158 \\
.165 \\
1.064\end{array}$ & $\begin{array}{r}.067 \\
.889 \\
.028 \\
.058 \\
1.144 \\
1.117 \\
1.073 \\
1.215 \\
1.018 \\
1.031 \\
.065 \\
1.002 \\
12.309\end{array}$ & $\begin{array}{l}.108 \\
.008 \\
.113 \\
.210 \\
.109 \\
.176 \\
.168 \\
.212 \\
.223 \\
.162 \\
.213 \\
.114 \\
1.012\end{array}$ & $\begin{array}{l}.009 \\
.007 \\
.013 \\
.018 \\
.010 \\
.016 \\
.021 \\
.031 \\
.028 \\
.029 \\
.010 \\
.021 \\
.231\end{array}$ & $\begin{array}{r}.001 \\
.001 \\
.002 \\
.001 \\
.001 \\
.001 \\
.003 \\
.002 \\
.001 \\
.001 \\
.001 \\
(0) \\
.000\end{array}$ & $\begin{array}{r}1.085 \\
.832 \\
.098 \\
1.156 \\
1.241 \\
1.190 \\
1.136 \\
1.308 \\
1.130 \\
1.130 \\
1.084 \\
1.082 \\
13.387\end{array}$ \\
\hline 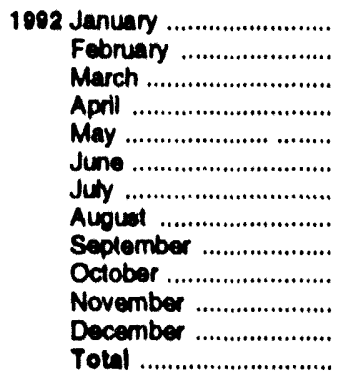 & $\begin{array}{l}. .218 \\
. .198 \\
.215 \\
. .210 \\
.240 \\
.221 \\
.241 \\
. .194 \\
-.236 \\
. .183 \\
. .210 \\
. .204 \\
-2.807\end{array}$ & $\begin{array}{l}.160 \\
.163 \\
.160 \\
.160 \\
.157 \\
.146 \\
.163 \\
.158 \\
.140 \\
.150 \\
.104 \\
.103 \\
1.041\end{array}$ & $\begin{array}{r}1.078 \\
.873 \\
.083 \\
1.000 \\
1.009 \\
1.084 \\
1.246 \\
1.168 \\
1.009 \\
1.217 \\
1.074 \\
1.076 \\
13.006\end{array}$ & $\begin{array}{l}.122 \\
.146 \\
.160 \\
.173 \\
.168 \\
.162 \\
.137 \\
.107 \\
.105 \\
.173 \\
.142 \\
.120 \\
1.095\end{array}$ & $\begin{array}{l}.021 \\
.018 \\
.012 \\
.019 \\
.022 \\
.020 \\
.038 \\
.031 \\
.028 \\
.031 \\
.029 \\
.027 \\
.203\end{array}$ & $\begin{array}{l}.004 \\
.003 \\
.003 \\
.003 \\
.001 \\
.003 \\
.001 \\
.001 \\
.001 \\
.002 \\
.001 \\
.005 \\
.027\end{array}$ & $\begin{array}{r}1.157 \\
1.006 \\
1.084 \\
1.228 \\
1.207 \\
1.183 \\
1.320 \\
1.300 \\
1.237 \\
1.300 \\
1.221 \\
1.226 \\
14.034\end{array}$ \\
\hline 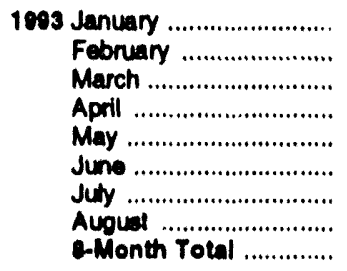 & $\begin{array}{l}-.162 \\
-.164 \\
-.137 \\
-.131 \\
-.151 \\
-.213 \\
-.156 \\
-.134 \\
-1.244\end{array}$ & $\begin{array}{r}.182 \\
.172 \\
.184 \\
.175 \\
.175 \\
.150 \\
.170 \\
\text { ค } \\
.178 \\
.176 \\
1.360\end{array}$ & $\begin{array}{r}1.138 \\
.999 \\
1.177 \\
1.184 \\
1.188 \\
1.256 \\
1.329 \\
1.211 \\
9.101\end{array}$ & $\begin{array}{l}.111 \\
.139 \\
.170 \\
.129 \\
.140 \\
.135 \\
.158 \\
.167 \\
1.180\end{array}$ & $\begin{array}{l}.023 \\
.022 \\
.010 \\
.016 \\
.011 \\
.011 \\
.031 \\
.041 \\
.174\end{array}$ & $\begin{array}{l}.004 \\
(s) \\
.003 \\
.002 \\
.002 \\
.003 \\
(s) \\
.002 \\
.018\end{array}$ & $\begin{array}{r}1.297 \\
1.168 \\
1.416 \\
1.376 \\
A_{1} 1.340 \\
A_{1.381} \\
A_{1.540} \\
1.462 \\
10.080\end{array}$ \\
\hline $\begin{array}{l}1902 \text { e-Month Total ............. } \\
1001 \text { E-Month Total ............. }\end{array}$ & $\begin{array}{l}-1.740 \\
-1.785\end{array}$ & $\begin{array}{l}1.248 \\
1.077\end{array}$ & $\begin{array}{l}8.800 \\
0.201\end{array}$ & $\begin{array}{l}1.288 \\
1.200\end{array}$ & $\begin{array}{l}.170 \\
.134\end{array}$ & .018 & $\begin{array}{l}0.881 \\
0.081\end{array}$ \\
\hline
\end{tabular}

- Crude oll, lease condensate, and imports of crude oll for the Strategle Petroloum Reserve.

Pelrobum products, untinished olls, pentanes plus, and gasoline blending componenls.

c Aseumed to be hydroelectrlctly and eatimated at the average input heat rate for lossilfluel steum-electilc power plant generation, which has ranged from 10.2 thousand Btu to 10.5 thousand Btu per klowathour since 1973. Actual heal rates applied in converting klowatthours 10 Blu are liated by yee in Table $\mathbf{A B}$.

$R=$ Revised data. Exestimate. (s) Lese than +0.5 ifllion Btu and greater than -0.5 inlilion Bqu.

Notea: - See Notes 3 and 4 if end of section. - Net imports equal imports minus exports. Minus sion indicates exports are oreater than importa. - Geographic coverage is the 50 stales and the Dibtrict of Columbia. - Total may not equal sum of components dus to independent rounding.

Sources: - Coal: Tables 8.1 and A5.A7. - Naturd Ces: Tables 4.2 and A4. Crude Oll and Potroleum Produots: Tables 3.1b and A2. - Electrlelty: Section 2. "Energy Concumplion Noles and Sources," Nole 8. and Table A8. Coal Coko: Section 2. "Energy Consumption Notes and Sources," Note 9 , and Table A7. 
Figure 1.5 Merchandise Trade Value

(Billion Dollars)

Imports and Exports, 1974-1992

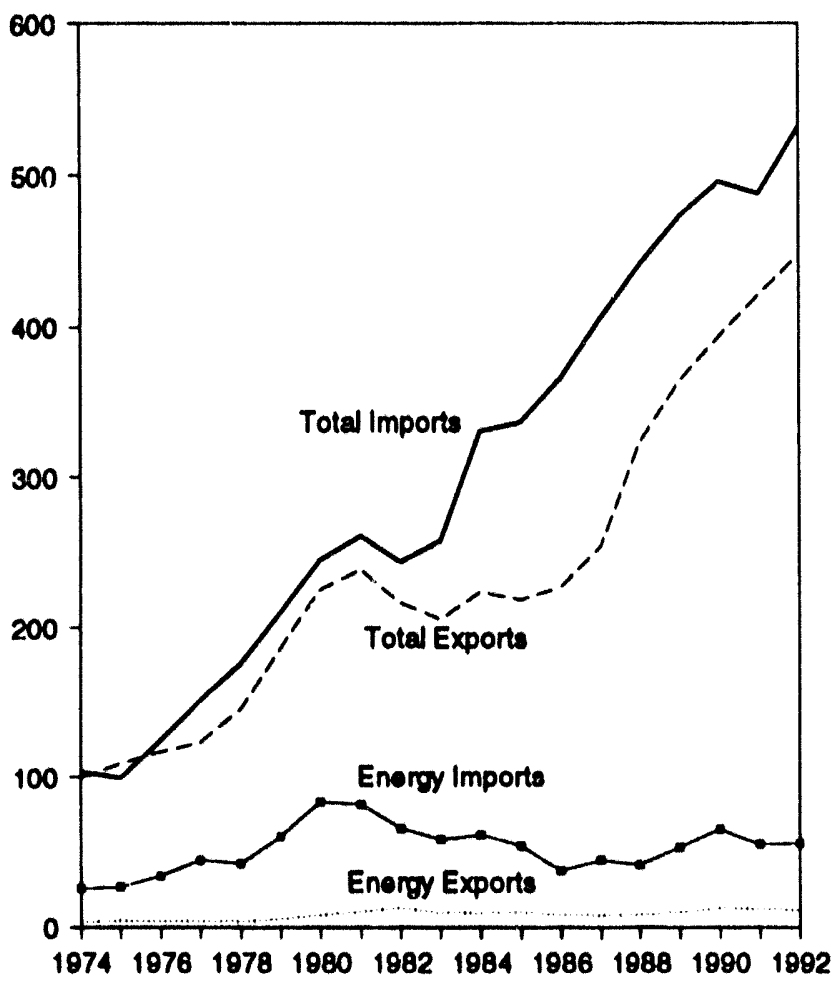

Trade Balance, 1974-1992

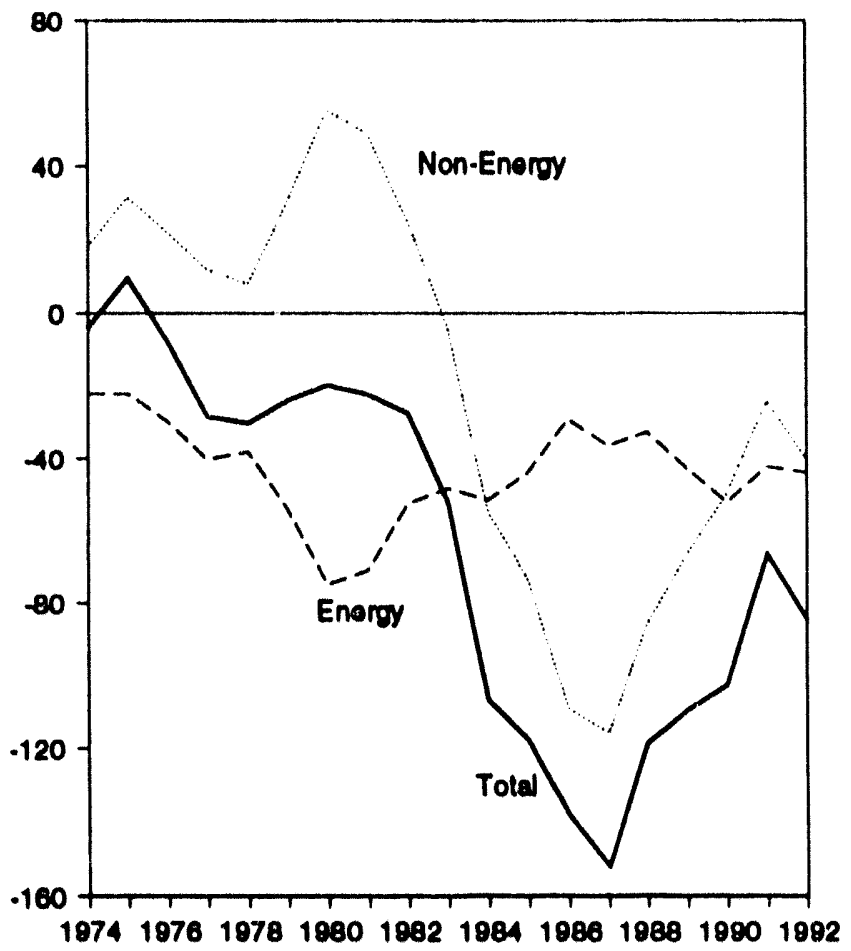

Nots: Because vertical scales differ, graphs should not be compared. Source: Table 1.8.
Imports and Exports, Monthly

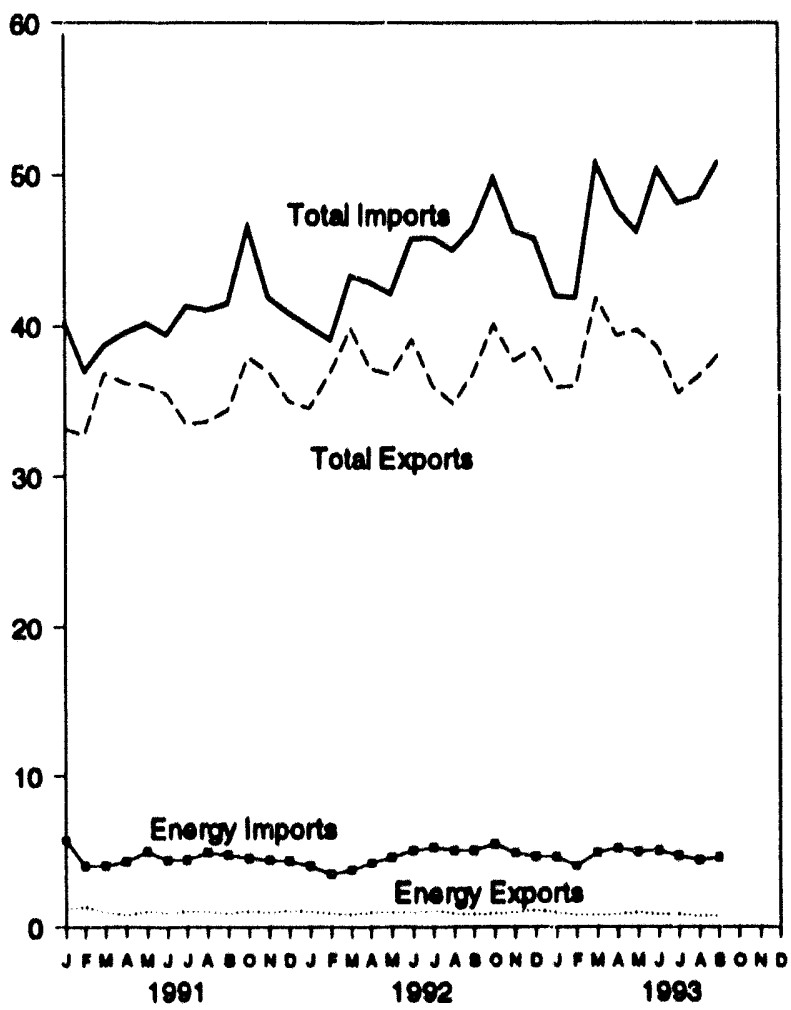

Trade Balance, Monthly

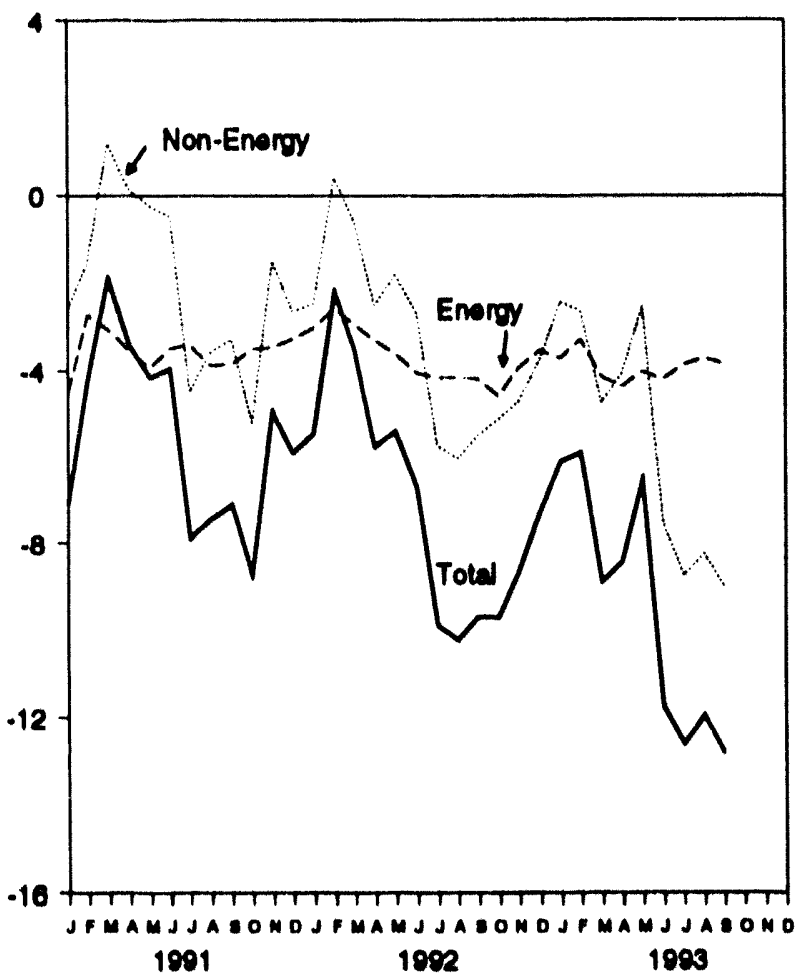


Table 1.6 Merchandise Trade Value

(Million Dollars)

\begin{tabular}{|c|c|c|c|c|c|c|c|c|c|c|}
\hline & \multicolumn{3}{|c|}{ Petroloum } & \multicolumn{3}{|c|}{ Enorgy } & \multirow{2}{*}{$\begin{array}{l}\text { Non- } \\
\text { Eneroy } \\
\text { Balaneo }\end{array}$} & \multicolumn{3}{|c|}{ Tout Merohendlee } \\
\hline & Exporte & Imports & Balance & Exporth & Imports & Balanes & & Exports & Imports & Delanes \\
\hline 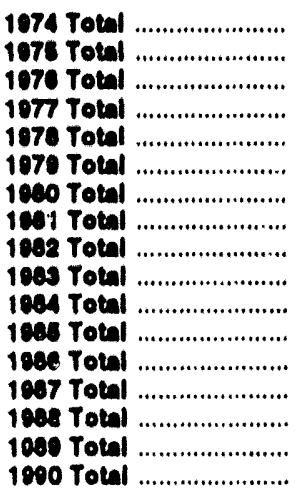 & $\begin{array}{l}702 \\
007 \\
000 \\
1,270 \\
1,801 \\
1,014 \\
2,039 \\
3,094 \\
6,047 \\
4,587 \\
4,470 \\
4,707 \\
3,040 \\
3,022 \\
3,003 \\
0,021 \\
0,001\end{array}$ & 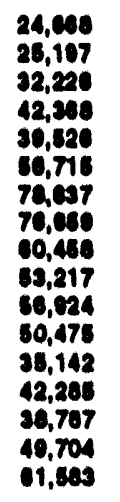 & 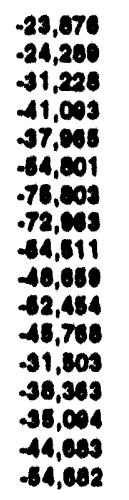 & $\begin{array}{r}3,444 \\
4,470 \\
4,220 \\
4,184 \\
3,801 \\
6,021 \\
7,092 \\
10,270 \\
12,720 \\
0,500 \\
9,311 \\
0,071 \\
9,116 \\
7,713 \\
0,236 \\
0,890 \\
12,233\end{array}$ & 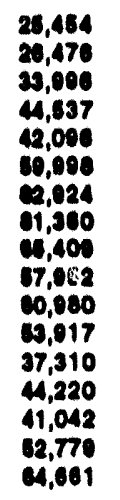 & 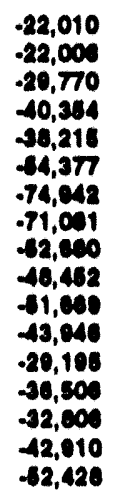 & 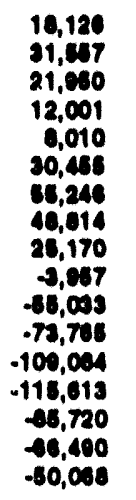 & 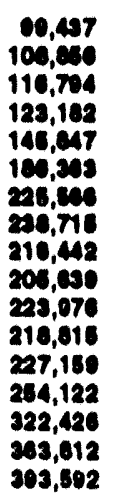 & 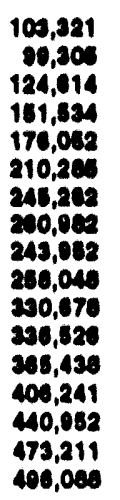 & 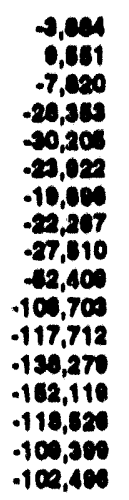 \\
\hline 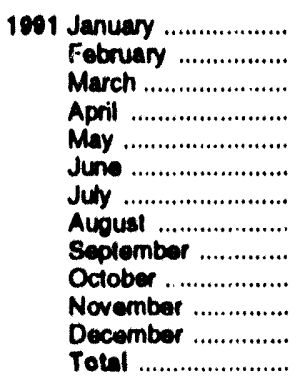 & $\begin{array}{r}881 \\
928 \\
565 \\
397 \\
562 \\
508 \\
513 \\
495 \\
415 \\
584 \\
489 \\
620 \\
6,084\end{array}$ & $\begin{array}{l}5,381 \\
3,741 \\
3,729 \\
4,030 \\
4,600 \\
4,177 \\
4,130 \\
4,641 \\
4,475 \\
4,228 \\
4,112 \\
4,028 \\
31,350\end{array}$ & $\begin{array}{l}-4,480 \\
-2,813 \\
-3,164 \\
-3,623 \\
-4,137 \\
-3,671 \\
-3,620 \\
-4,146 \\
-4,060 \\
-3,642 \\
-3,623 \\
-3,408 \\
-4,308\end{array}$ & $\begin{array}{r}1,188 \\
1,327 \\
951 \\
748 \\
1,031 \\
938 \\
987 \\
998 \\
884 \\
1,031 \\
943 \\
1,068 \\
12,081\end{array}$ & $\begin{array}{l}5,698 \\
4,032 \\
4,003 \\
4,288 \\
4,057 \\
4,408 \\
4,388 \\
4,876 \\
4,723 \\
4,533 \\
4,390 \\
4,326 \\
4,620\end{array}$ & $\begin{array}{r}-4,509 \\
\cdot 2,705 \\
\cdot 3,051 \\
\cdot 3,538 \\
\cdot 3,926 \\
\cdot 3,473 \\
\cdot 3,401 \\
\cdot 3,879 \\
-3,839 \\
\cdot 3,502 \\
\cdot 3,468 \\
\cdot 3,268 \\
-42,840\end{array}$ & $\begin{array}{r}-2,569 \\
-1,406 \\
1,163 \\
128 \\
-231 \\
-476 \\
-4,493 \\
-3,571 \\
-3,271 \\
-5,232 \\
-1,486 \\
-2,640 \\
-24,175\end{array}$ & $\begin{array}{r}33,165 \\
32,775 \\
36,820 \\
36,137 \\
36,024 \\
36,460 \\
33,444 \\
33,633 \\
34,391 \\
37,897 \\
30,970 \\
34,996 \\
421,730\end{array}$ & $\begin{array}{l}40,244 \\
36,978 \\
38,708 \\
39,548 \\
40,181 \\
39,428 \\
41,338 \\
41,062 \\
41,502 \\
46,631 \\
41,011 \\
40,904 \\
498,483\end{array}$ & $\begin{array}{l}\cdot 7,079 \\
-4,201 \\
-1,809 \\
-3,411 \\
-4,158 \\
-3,948 \\
-7,804 \\
.7,450 \\
-7,111 \\
-8,736 \\
-4,042 \\
-5,008 \\
-0,720\end{array}$ \\
\hline 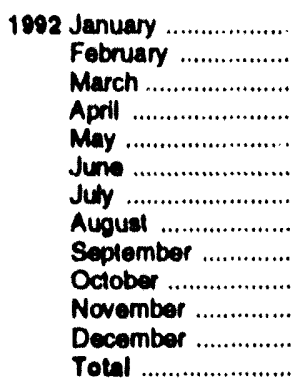 & $\begin{array}{r}602 \\
454 \\
419 \\
511 \\
536 \\
548 \\
654 \\
503 \\
428 \\
508 \\
550 \\
700 \\
6.412\end{array}$ & $\begin{array}{r}3,683 \\
3,165 \\
3,477 \\
3,931 \\
4,274 \\
4,713 \\
4,012 \\
4,702 \\
4,680 \\
5,047 \\
4,462 \\
4,172 \\
81,217\end{array}$ & $\begin{array}{r}-3,082 \\
-2,711 \\
-3,058 \\
-3,420 \\
-3,738 \\
-4,165 \\
-4,258 \\
-4,190 \\
-4,252 \\
-4,541 \\
-3,012 \\
-3,471 \\
-44,008\end{array}$ & $\begin{array}{r}1,007 \\
879 \\
831 \\
932 \\
068 \\
958 \\
1,087 \\
897 \\
830 \\
874 \\
940 \\
1,093 \\
11,284\end{array}$ & $\begin{array}{r}4,016 \\
3,452 \\
3,762 \\
4,215 \\
4,573 \\
5,007 \\
5,222 \\
5,034 \\
5,026 \\
5,456 \\
4,873 \\
4,621 \\
5,, 264\end{array}$ & $\begin{array}{r}\cdot 3,009 \\
\cdot 2,573 \\
\cdot 2,931 \\
\cdot 3,283 \\
-3,606 \\
\cdot 4,040 \\
\cdot 4,155 \\
\cdot 4,167 \\
\cdot 4,187 \\
\cdot 4,582 \\
-3,933 \\
\cdot 3,529 \\
-44,002\end{array}$ & $\begin{array}{r}-2,461 \\
396 \\
.596 \\
-2,489 \\
-1,804 \\
-2,680 \\
-5,738 \\
-6,061 \\
-5,506 \\
.5,124 \\
-4,711 \\
-3,747 \\
-40,500\end{array}$ & $\begin{array}{r}34,514 \\
36,898 \\
39,817 \\
37,154 \\
36,737 \\
39,094 \\
35,070 \\
34,838 \\
38,811 \\
40,115 \\
37,670 \\
38,537 \\
48,184\end{array}$ & $\begin{array}{r}39,884 \\
39,075 \\
43,344 \\
42,825 \\
42,146 \\
45,812 \\
45,872 \\
45,055 \\
48,503 \\
49,820 \\
46,314 \\
45,813 \\
432,865\end{array}$ & $\begin{array}{r}-5,470 \\
-2,178 \\
-3,527 \\
-5,772 \\
-5,400 \\
-6,718 \\
-0,803 \\
-10,218 \\
-0,603 \\
-0,708 \\
-8,644 \\
-7,276 \\
-4,801\end{array}$ \\
\hline 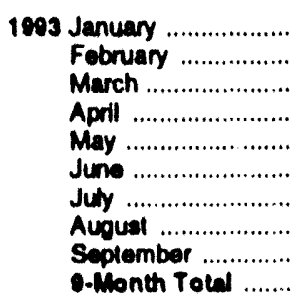 & $\begin{array}{l}817 \\
467 \\
489 \\
583 \\
647 \\
430 \\
514 \\
444 \\
438 \\
4,033\end{array}$ & $\begin{array}{r}4,254 \\
3,690 \\
4,492 \\
4,845 \\
4,614 \\
4,707 \\
4,320 \\
4,031 \\
4,171 \\
39,133\end{array}$ & $\begin{array}{l}-3,637 \\
\cdot 3,232 \\
\cdot 4,004 \\
\cdot 4,262 \\
\cdot 3,087 \\
\cdot 4,269 \\
\cdot 3,608 \\
\cdot 3,587 \\
-3,736 \\
-34,498\end{array}$ & $\begin{array}{l}936 \\
789 \\
768 \\
836 \\
944 \\
826 \\
818 \\
703 \\
723 \\
7,343\end{array}$ & $\begin{array}{l}4,642 \\
4,0 ; 0 \\
4,910 \\
5,101 \\
4,969 \\
5,023 \\
4,670 \\
4,404 \\
4,549 \\
42,438\end{array}$ & $\begin{array}{r}-3,706 \\
-3,281 \\
\cdot 4,142 \\
-4,367 \\
-4,024 \\
-4,107 \\
\cdot 3,862 \\
-3,700 \\
-3,826 \\
-38,003\end{array}$ & $\begin{array}{r}-2,407 \\
-2,625 \\
-4,745 \\
-4,072 \\
-2,518 \\
-7,552 \\
-8,747 \\
\cdot .6,240 \\
-8,974 \\
40,087\end{array}$ & $\begin{array}{r}35,022 \\
36,004 \\
41,895 \\
39,374 \\
39,751 \\
38,616 \\
35,520 \\
36,624 \\
38,038 \\
31,763\end{array}$ & $\begin{array}{r}42,036 \\
41,800 \\
50,781 \\
47,802 \\
46,293 \\
50,365 \\
48,138 \\
448,573 \\
50,837 \\
420,734\end{array}$ & $\begin{array}{r}-6,113 \\
-5,006 \\
-8,860 \\
\cdot 8,428 \\
-8,542 \\
-11,749 \\
-12,600 \\
\cdot .11,040 \\
-12,700 \\
-84,081\end{array}$ \\
\hline $\begin{array}{l}1902 \text { 9-Month Total ....... } \\
1001 \text { 9-Month Total ....... }\end{array}$ & $\begin{array}{l}4,884 \\
5,201\end{array}$ & $\begin{array}{l}37,330 \\
30,085\end{array}$ & $\begin{array}{l}-32,082 \\
-33,724\end{array}$ & $\begin{array}{l}8,340 \\
9,040\end{array}$ & $\begin{array}{l}40,300 \\
41,371\end{array}$ & $\begin{array}{r}-31,058 \\
-32,322\end{array}$ & $\begin{array}{l}-26,017 \\
-14,817\end{array}$ & $\begin{array}{l}331,842 \\
311,098\end{array}$ & $\begin{array}{l}390,717 \\
280,007\end{array}$ & $\begin{array}{l}68,875 \\
47,130\end{array}$ \\
\hline
\end{tabular}

A=Rovead data

Notes: - Monthly data are not adjusted for seasonal variations. - The U.S. Import staltatics retbet both govemment and nongovermment imports of merchandies from lorelon couniries into the U.S. customs territory, which comprises the 50 Slates, the Dbirlct of Columbia, Puerto Rico, and the Virgin tslands. See Nole 5 al end of section. Tolals may not equal sum of components due to independent rounding.

Sources: see end of section. 
Figure 1.6 Enorgy Consumptlon per Dollar of Gross Domsotlc Product

(Thousand Btu per 1987 Dollar)

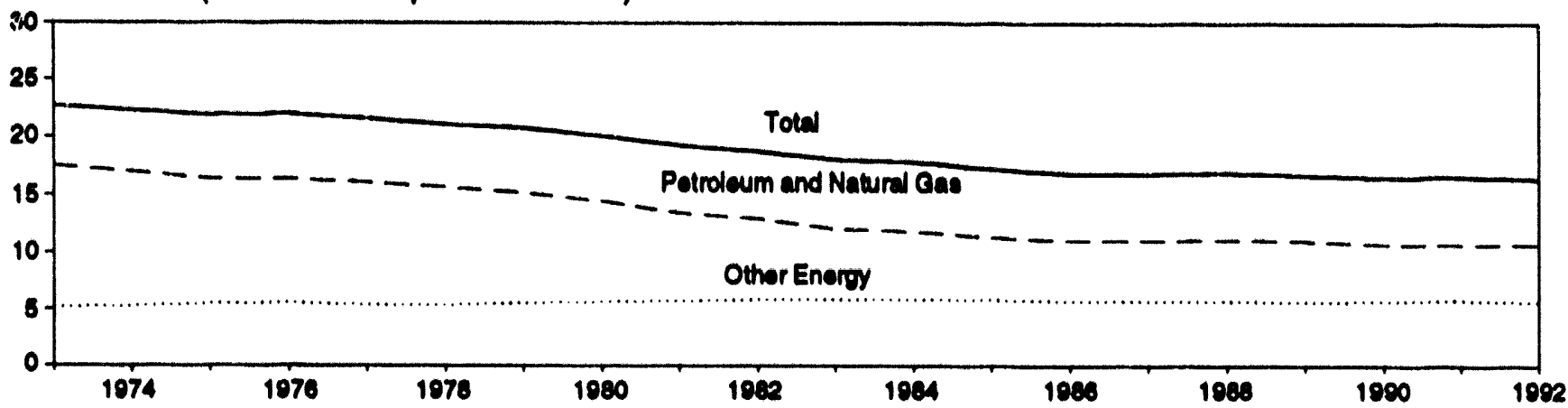

Sourco: Table 1.7 .

\section{Table 1.7 Energy Consumption per Dollar of Gross Domestic Product} (Seasonally Adjusted at Annual Rates)

\begin{tabular}{|c|c|c|c|c|c|c|c|}
\hline & \multicolumn{3}{|c|}{ Enorey Ceneumption } & \multirow{2}{*}{$\begin{array}{c}\begin{array}{c}\text { Oreas } \\
\text { Domectle } \\
\text { Produpt } \\
\text { (RDP) }\end{array} \\
\begin{array}{c}\text { Trition } \\
1087 \text { Dollen }\end{array}\end{array}$} & \multicolumn{3}{|c|}{ Inerey Cenoumption par Dollar of aDp } \\
\hline & \multicolumn{3}{|c|}{ Quaditition Bu } & & \multicolumn{3}{|c|}{ Thousand Elu per 1997 Dollar } \\
\hline 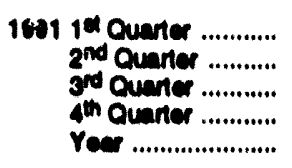 & $\begin{array}{r}n_{62.305} \\
61.034 \\
n_{62.097} \\
n_{62.000} \\
02.40\end{array}$ & 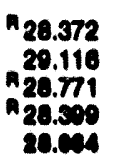 & $\begin{array}{r}9_{80.677} \\
81.050 \\
91.460 \\
981.206 \\
01.110\end{array}$ & $\begin{array}{l}4.850 \\
4.860 \\
4.873 \\
4.800 \\
4.801\end{array}$ & $\begin{array}{l}10.8 \\
10.7 \\
10.8 \\
10.8 \\
10.0\end{array}$ & $\begin{array}{l}6.0 \\
6.0 \\
5.0 \\
6.8 \\
8.0\end{array}$ & $\begin{array}{l}10.7 \\
16.7 \\
16.7 \\
16.7 \\
10.7\end{array}$ \\
\hline 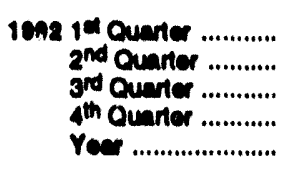 & $\begin{array}{r}9_{63.739} \\
63.003 \\
62.828 \\
9_{64.043} \\
6.040\end{array}$ & $\begin{array}{r}{ }_{28.180} \\
28.660 \\
n_{28.401} \\
n_{20.077} \\
n_{20.110}\end{array}$ & $\begin{array}{r}n_{01.024} \\
02.823 \\
n_{81.224} \\
n_{83.142} \\
n_{02.200}\end{array}$ & $\begin{array}{l}4.028 \\
4.067 \\
4.069 \\
6.000 \\
4.06\end{array}$ & $\begin{array}{l}10.9 \\
10.9 \\
10.8 \\
10.7 \\
10.8\end{array}$ & $\begin{array}{l}6.7 \\
6.8 \\
8.7 \\
6.7 \\
6.7\end{array}$ & $\begin{array}{l}16.8 \\
16.8 \\
16.3 \\
16.4 \\
10.6\end{array}$ \\
\hline
\end{tabular}

- Excludea wood, weste, geothermal, whd, photovolule, and solar themil eneroy, except for emall amounts ueed by dectite cillites to generato electriciny lor deturibution.

RoRevied date.

Noles: - Quarterty duta are ecasconaly sofueted and shown at annud

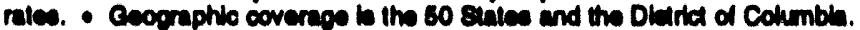
- Totale may not equal eum al componente dus to hodependent rounding.
- Yeery dela may not equal avereas of quarters dis to scasconally adpetments and independemt roundtho.

Souran: - Inergy Concumplten: Tablo 1.4. - Arese Domeatle Preduct 1072-1601-U.3. Department of Commeros, Bureeu of Economio

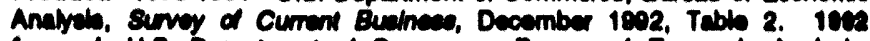

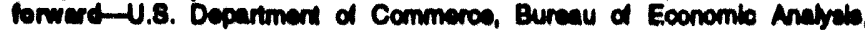
Unined Sutee Deppertment of Commeros Nowa, October 28, 1090, Table 2. 


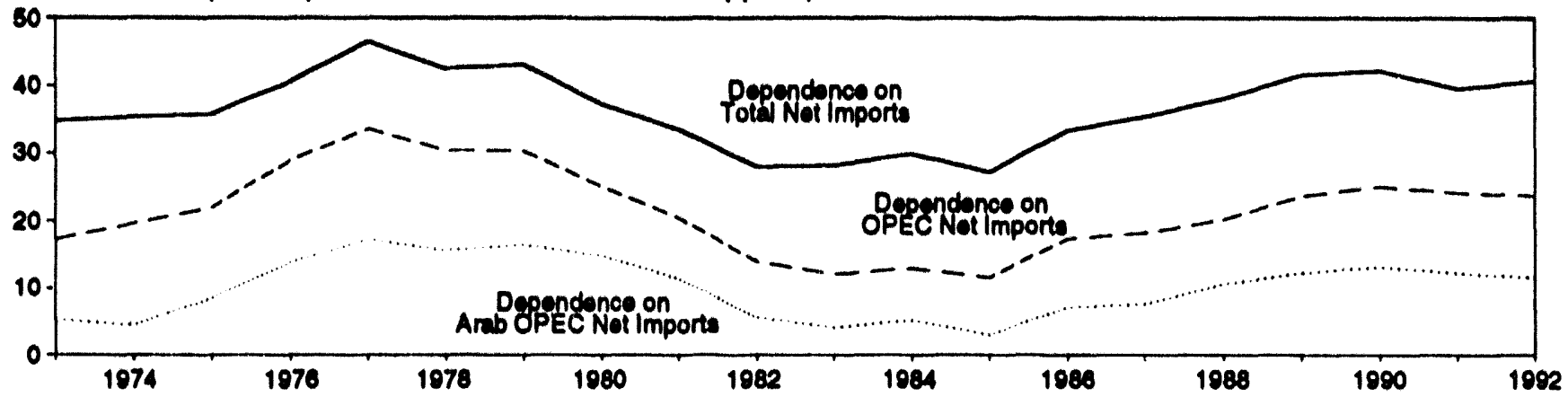

Source: Table 1.8

Table 1.8 U.S. Dependence on Petroleum Net Imports

\begin{tabular}{|c|c|c|c|c|c|c|c|}
\hline \multirow{3}{*}{ Annued Rate } & \multicolumn{3}{|c|}{ Not Imports: } & \multirow{3}{*}{$\begin{array}{l}\text { Potroloum } \\
\text { Produets } \\
\text { supplled }\end{array}$} & \multicolumn{3}{|c|}{$\begin{array}{l}\text { Not Imports ee Peroent of } \\
\text { U.s. Petroleum Produots supplled }\end{array}$} \\
\hline & $\begin{array}{l}\text { From Arab } \\
\text { Optecb }\end{array}$ & $\begin{array}{l}\text { From } \\
\text { OPEC }\end{array}$ & $\begin{array}{l}\text { From All } \\
\text { Countries }\end{array}$ & & $\begin{array}{l}\text { Prom Arab } \\
\text { OPEC }\end{array}$ & $\begin{array}{c}\text { From } \\
\text { OPECo }\end{array}$ & $\begin{array}{l}\text { From All } \\
\text { Countribe }\end{array}$ \\
\hline & \multicolumn{3}{|c|}{ Thousand Barrols per Day } & & \multicolumn{3}{|c|}{ Percent } \\
\hline 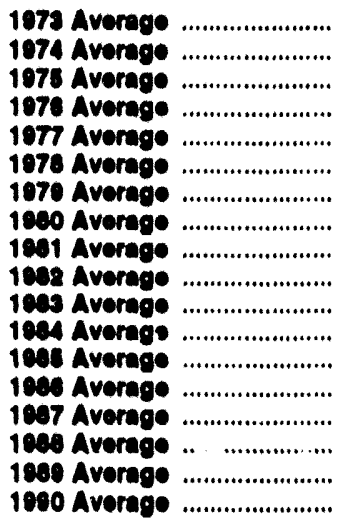 & $\begin{array}{r}014 \\
762 \\
1,302 \\
2,423 \\
3,184 \\
2,002 \\
3,084 \\
2,040 \\
1,844 \\
802 \\
030 \\
817 \\
170 \\
1,100 \\
1,272 \\
1,037 \\
2,128 \\
2,243\end{array}$ & $\begin{array}{l}2,091 \\
3,277 \\
3,609 \\
5,003 \\
6,100 \\
6,747 \\
6,033 \\
4,203 \\
3,218 \\
2,130 \\
1,043 \\
2,007 \\
1,021 \\
2,008 \\
3,063 \\
3,613 \\
4,124 \\
4,285\end{array}$ & 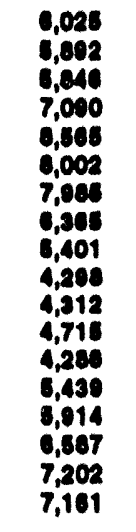 & 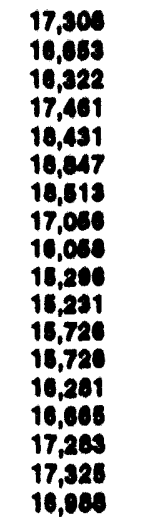 & 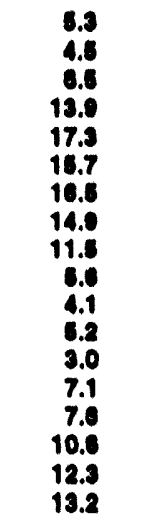 & $\begin{array}{l}17.3 \\
10.7 \\
22.0 \\
20.0 \\
39.6 \\
30.6 \\
30.4 \\
25.2 \\
20.6 \\
11.0 \\
12.1 \\
13.0 \\
11.6 \\
17.4 \\
18.3 \\
20.3 \\
23.8 \\
25.2\end{array}$ & 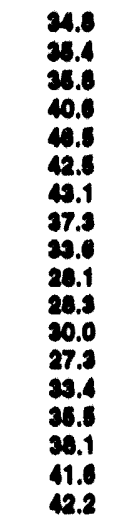 \\
\hline 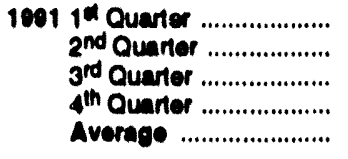 & $\begin{array}{l}1,078 \\
2,253 \\
2,026 \\
1,071 \\
2,067\end{array}$ & $\begin{array}{l}3,727 \\
4,301 \\
4,252 \\
3,974 \\
4,004\end{array}$ & $\begin{array}{l}5,686 \\
7,127 \\
7,224 \\
6,462 \\
6,020\end{array}$ & $\begin{array}{l}16,460 \\
18,400 \\
17,002 \\
16,950 \\
16,714\end{array}$ & $\begin{array}{l}12.0 \\
13.7 \\
11.9 \\
11.6 \\
12.3\end{array}$ & $\begin{array}{l}22.6 \\
26.2 \\
25.0 \\
23.4 \\
24.3\end{array}$ & $\begin{array}{l}34.5 \\
43.5 \\
42.5 \\
38.0 \\
30.6\end{array}$ \\
\hline 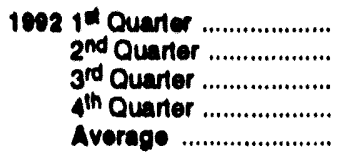 & $\begin{array}{l}2,052 \\
1,922 \\
1,910 \\
2,005 \\
1,072\end{array}$ & $\begin{array}{l}3,783 \\
4,056 \\
4,230 \\
4,210 \\
4,071\end{array}$ & $\begin{array}{l}6,239 \\
7,027 \\
7,451 \\
7,029 \\
6,090\end{array}$ & $\begin{array}{l}16,910 \\
16,740 \\
16,984 \\
17,493 \\
17,038\end{array}$ & $\begin{array}{l}12.1 \\
11.5 \\
11.2 \\
11.5 \\
11.6\end{array}$ & $\begin{array}{l}22.4 \\
24.2 \\
24.9 \\
24.1 \\
23.0\end{array}$ & $\begin{array}{l}39.9 \\
42.0 \\
43.9 \\
40.2 \\
40.7\end{array}$ \\
\hline 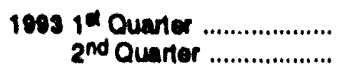 & $\begin{array}{l}2,025 \\
2,053\end{array}$ & $\begin{array}{l}4,311 \\
4,362\end{array}$ & $\begin{array}{l}7,038 \\
7,507\end{array}$ & $\begin{array}{l}17,126 \\
16,678\end{array}$ & $\begin{array}{l}11.8 \\
12.3\end{array}$ & $\begin{array}{l}25.2 \\
26.1\end{array}$ & $\begin{array}{l}41.1 \\
46.0\end{array}$ \\
\hline
\end{tabular}

- "Net Imports' are imports minus exports. Imports irom members of the Organizalion of Petroleum Exporting Countrice (OPEC) exclude indirect importa, which are pelrobum products primarly from Caribbean and Weet European areas and rellined from crude oll produced by OPEC.

- The Arab membere of OPEC we Algeria, Iraq, Kuwall, Llbya, Oatar. Saudi Arabla, and the Uniled Arab Emirales. Ned Imports Irom the Nocured Zono between Kuwalt and Saudi Arable are heluded in net imports from Arab OPEC.

- OPEC currently consiats of Gabon, Indoneala, Iran, Nigerla, and Vonezuela, as woll as the Arab members.
Notca: - Becinning in October 1977, Strateoic Potrobum Reserve are inchuded. - Geographic coverage in the 50 States and the District of Columbla. - Annul averages may not equal average of quarters due to independent rounding.

Sources: - Importa: Tables 3.3a-3.3h. - Exporta: 1073-1074-U.S. Departmen of the Interber. Bureau of Mines, Mineral Industry Surveys. 1077.10se-Energy Intormation Adminiatration (EIA). Energy Data Roports, "Petroleum statement, Amual." 1001-1002-EIA, Petroleum Supply Annual. 1909 ronward-EL, Potroleum Supply Monthy. - Potroleum Produete supplled: Table 3.1a. 
Figure 1.8 Cost of Fuels to End Users In Constant (1982-84) Dollars

(Dollars per Million Btu)

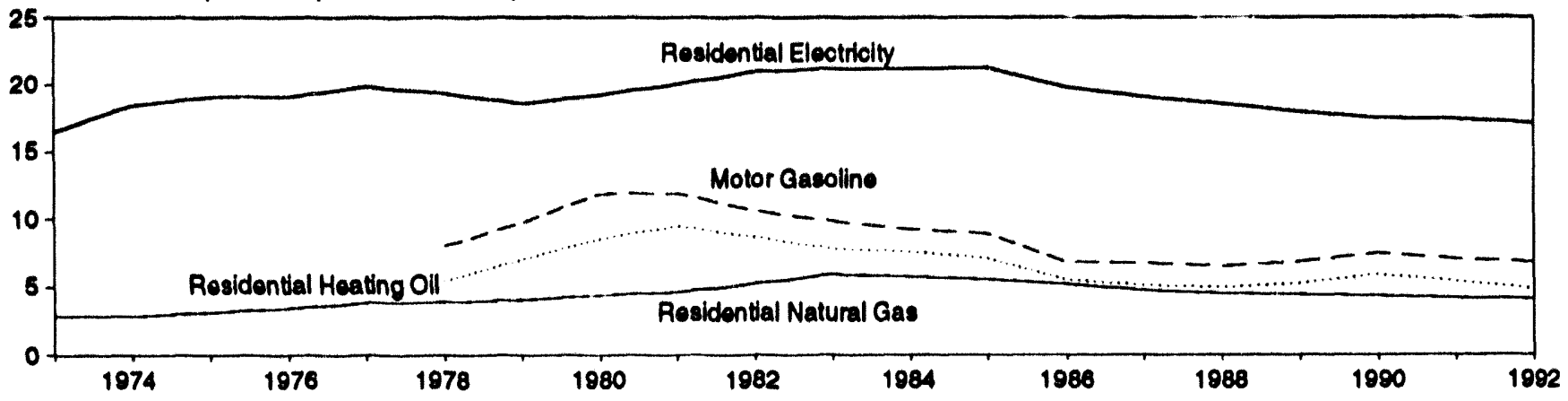

Source: Table 1.9.

Table 1.9 Cost of Fuels to End Users in Constant (1982-84) Dollars

\begin{tabular}{|c|c|c|c|c|c|c|c|c|}
\hline & \multicolumn{2}{|c|}{ Motor Guseoline } & \multicolumn{2}{|c|}{$\begin{array}{l}\text { Residential } \\
\text { Hosting Oll }\end{array}$} & \multicolumn{2}{|c|}{$\begin{array}{l}\text { Reoidential } \\
\text { Nelural Ges }\end{array}$} & \multicolumn{2}{|c|}{$\begin{array}{l}\text { Recidentiel } \\
\text { Eleotritoliy }\end{array}$} \\
\hline & $\begin{array}{l}\text { Cents per } \\
\text { Gallon }\end{array}$ & $\begin{array}{l}\text { Dollar per } \\
\text { Multon Biu }\end{array}$ & $\begin{array}{l}\text { Conts per } \\
\text { Galon }\end{array}$ & $\begin{array}{l}\text { Dollars per } \\
\text { Million Btu }\end{array}$ & $\begin{array}{c}\text { Cents per } \\
\text { Thousand Cublc Feel }\end{array}$ & $\begin{array}{l}\text { Dollars per } \\
\text { Millon Btu }\end{array}$ & $\begin{array}{l}\text { Cenls per } \\
\text { Kilowatthour }\end{array}$ & $\begin{array}{l}\text { Dollars per } \\
\text { Miltion Blu }\end{array}$ \\
\hline 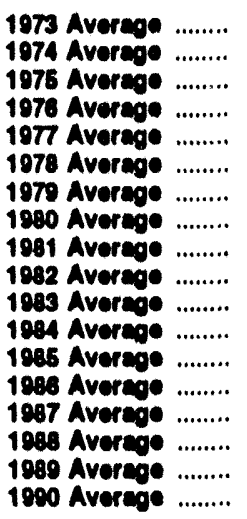 & $\begin{array}{l}\text { NA } \\
\text { NA } \\
N A \\
N A \\
N A \\
100.0 \\
121.5 \\
148.2 \\
148.8 \\
132.7 \\
123.0 \\
115.3 \\
111.2 \\
84.8 \\
84.2 \\
81.4 \\
85.5 \\
93.1\end{array}$ & $\begin{array}{l}N A \\
N A \\
N A \\
N A \\
N A \\
8.00 \\
9.71 \\
11.85 \\
11.90 \\
10.61 \\
0.83 \\
9.22 \\
8.89 \\
6.79 \\
6.74 \\
6.61 \\
6.83 \\
7.44\end{array}$ & $\begin{array}{l}\text { NA } \\
\text { NA } \\
\text { NA } \\
\text { NA } \\
\text { NA } \\
75.2 \\
97.0 \\
118.2 \\
131.4 \\
120.2 \\
108.2 \\
105.0 \\
97.8 \\
76.3 \\
70.7 \\
88.7 \\
72.6 \\
81.3\end{array}$ & $\begin{array}{l}\text { NA } \\
\text { NA } \\
\text { NA } \\
\text { NA } \\
\mathbf{5 . 4 2} \\
6.99 \\
8.52 \\
9.47 \\
8.67 \\
7.80 \\
7.57 \\
7.06 \\
5.50 \\
5.10 \\
4.96 \\
5.23 \\
5.86\end{array}$ & $\begin{array}{l}290.6 \\
290.1 \\
317.8 \\
349.0 \\
387.8 \\
392.6 \\
410.6 \\
448.6 \\
471.0 \\
535.8 \\
608.4 \\
589.0 \\
568.8 \\
531.0 \\
487.7 \\
462.4 \\
454.8 \\
443.8\end{array}$ & $\begin{array}{l}2.85 \\
2.03 \\
3.12 \\
3.41 \\
3.81 \\
3.86 \\
4.03 \\
4.36 \\
4.60 \\
6.20 \\
5.00 \\
5.72 \\
5.52 \\
5.17 \\
4.73 \\
4.49 \\
4.41 \\
4.31\end{array}$ & $\begin{array}{l}6.8 \\
6.3 \\
6.5 \\
6.5 \\
6.8 \\
6.6 \\
6.3 \\
6.6 \\
6.0 \\
7.2 \\
7.2 \\
7.2 \\
7.2 \\
6.8 \\
6.5 \\
6.3 \\
6.1 \\
6.0\end{array}$ & $\begin{array}{l}16.60 \\
18.43 \\
19.07 \\
19.08 \\
19.89 \\
19.33 \\
18.57 \\
19.21 \\
19.00 \\
20.94 \\
21.10 \\
21.16 \\
21.26 \\
19.79 \\
19.09 \\
18.58 \\
17.96 \\
17.49\end{array}$ \\
\hline 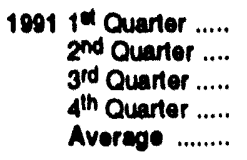 & $\begin{array}{l}90.0 \\
88.1 \\
87.3 \\
86.1 \\
87.8\end{array}$ & $\begin{array}{l}7.19 \\
7.04 \\
6.98 \\
6.88 \\
7.02\end{array}$ & $\begin{array}{l}81.7 \\
68.5 \\
64.2 \\
69.7 \\
74.8\end{array}$ & $\begin{array}{l}5.89 \\
4.84 \\
4.63 \\
5.03 \\
5.30\end{array}$ & $\begin{array}{l}413.2 \\
470.5 \\
524.5 \\
416.8 \\
427.3\end{array}$ & $\begin{array}{l}4.01 \\
4.57 \\
5.09 \\
4.04 \\
4.14\end{array}$ & $\begin{array}{l}5.6 \\
6.0 \\
6.1 \\
5.8 \\
5.9\end{array}$ & $\begin{array}{l}16.52 \\
17.72 \\
18.01 \\
17.03 \\
17.43\end{array}$ \\
\hline $\begin{array}{l}10921^{\text {th }} \text { Quarter ..... } \\
\text { 2nd Quarter .... }^{\text {3nd Quarter ..... }} \\
\text { 3th Quarter ..... }^{\text {th }} \text { Averwy ....... }\end{array}$ & $\begin{array}{l}81.1 \\
85.3 \\
87.1 \\
85.6 \\
84.8\end{array}$ & $\begin{array}{l}6.49 \\
6.82 \\
6.96 \\
6.84 \\
6.78\end{array}$ & $\begin{array}{l}67.7 \\
66.0 \\
63.7 \\
66.5 \\
66.6\end{array}$ & $\begin{array}{l}4.88 \\
4.76 \\
4.59 \\
4.79 \\
4.80\end{array}$ & $\begin{array}{l}398.0 \\
443.5 \\
517.4 \\
429.2 \\
419.8\end{array}$ & $\begin{array}{l}3.86 \\
4.30 \\
5.02 \\
4.16 \\
4.07\end{array}$ & $\begin{array}{l}5.6 \\
5.8 \\
6.1 \\
5.8 \\
5.8\end{array}$ & $\begin{array}{l}16.48 \\
17.40 \\
17.89 \\
16.94 \\
17.13\end{array}$ \\
\hline $\begin{array}{l}1093 \text { 1 Quarter ..... } \\
\text { 2nd Quarter .... }\end{array}$ & $\begin{array}{l}81.9 \\
82.3\end{array}$ & $\begin{array}{l}6.55 \\
6.58\end{array}$ & $\begin{array}{l}68.2 \\
63.0\end{array}$ & $\begin{array}{l}4.78 \\
4.54\end{array}$ & $\begin{array}{l}397.6 \\
463.2\end{array}$ & $\begin{array}{l}3.80 \\
4.40\end{array}$ & $\begin{array}{l}5.5 \\
5.8\end{array}$ & $\begin{array}{l}15.88 \\
17.28\end{array}$ \\
\hline
\end{tabular}

NA=Not avallable.

Notes: - Fual costs are calculated by wing the Utban Consumer Price Index (CPI) developed by the Bureau of Labor Statistics. See Note 6 at and of section. Geographic coverage it the 50 states and the District of Columbla. - Annual averages may not equal average of quarters due to Independent rounding.

Sources: - Annual Data: Annual prices in Tables 9.4 (All Types), 9.8c,
9.11. and 9.9 (Monthly Series), adfusted by the CPI. Quarterly Duta: Simple averages of monthly prices in Tables 9.4 (All Types), 9.8c, 8.11, and 9.9 (Monthly Series), adiusted by the CPI. - CPI: 1973-1900-Economic Report of the President, Februan 1993, Table B-56. 1991 forward-Councl of Economic Adviears, Economic Indicators, Odober 1983, "Coneumer Prices - All Uban Consumers." - Converbion Factors: Tables A1, A4, and A8. 
Figure 1.9 Passenger Car Efficlency

(Index, 1973 = 100)

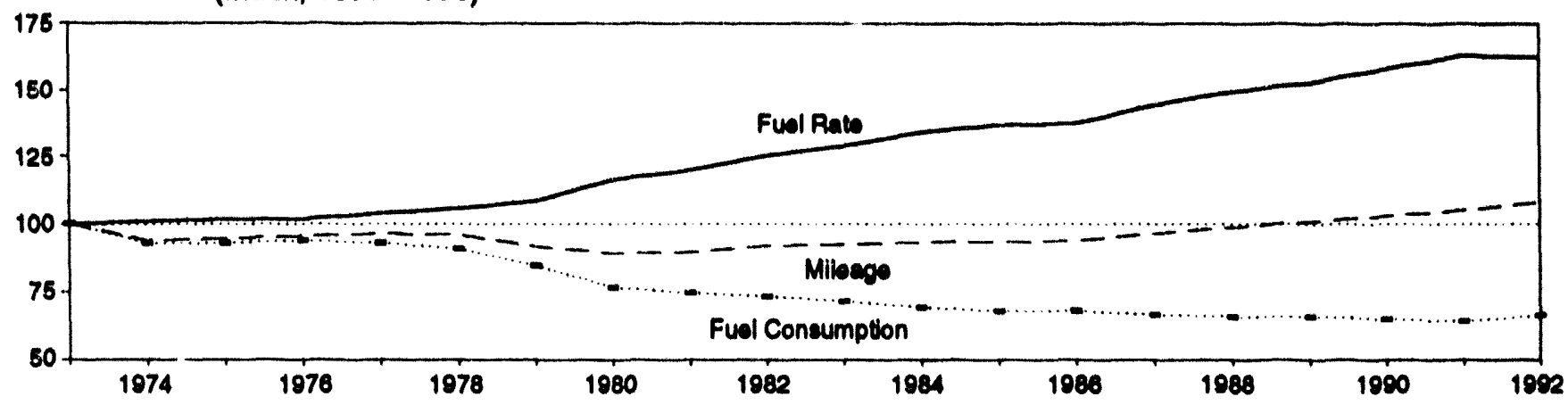

Source: Table 1.10.

Table 1.10 Passenger Car Efficlency

\begin{tabular}{|c|c|c|c|c|c|c|}
\hline & \multicolumn{2}{|c|}{ Mllewe } & \multicolumn{2}{|c|}{ Fuel Consumption } & \multicolumn{2}{|c|}{ Fud Rate } \\
\hline & $\begin{array}{l}\text { Miles } \\
\text { per Car }\end{array}$ & $\underset{1973-100.0}{\text { Index }}$ & $\begin{array}{l}\text { Gallons } \\
\text { per Car }\end{array}$ & $\underset{1073=100.0}{\operatorname{Index}}$ & $\begin{array}{c}\text { Miles } \\
\text { per Gallon }\end{array}$ & $\underset{1073=100.0}{\operatorname{lnd} x}$ \\
\hline $\begin{array}{l}1078 \\
1074 \\
1078 \\
1078 \\
1977 \\
1978 \ldots . \\
1970 \\
1980\end{array}$ & 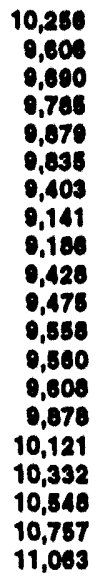 & $\begin{array}{r}100.0 \\
93.7 \\
94.5 \\
95.4 \\
94.3 \\
94.0 \\
91.7 \\
09.1 \\
89.8 \\
91.0 \\
92.4 \\
93.2 \\
03.2 \\
93.7 \\
94.3 \\
99.7 \\
100.7 \\
102.8 \\
104.9 \\
107.0\end{array}$ & 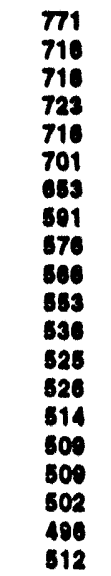 & 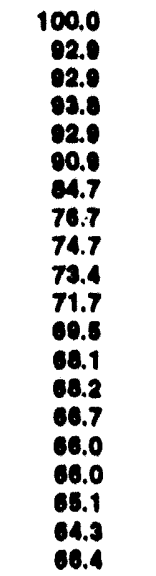 & $\begin{array}{l}19.30 \\
13.42 \\
13.82 \\
13.83 \\
13.80 \\
14.04 \\
14.41 \\
18.40 \\
18.04 \\
18.64 \\
17.14 \\
17.83 \\
18.20 \\
18.27 \\
10.20 \\
19.87 \\
20.31 \\
21.02 \\
21.00 \\
21.00\end{array}$ & $\begin{array}{l}100.0 \\
100.0 \\
101.7 \\
101.7 \\
108.8 \\
105.0 \\
104.8 \\
118.2 \\
119.4 \\
128.2 \\
126.0 \\
134.1 \\
134.8 \\
137.4 \\
144.4 \\
149.4 \\
182.7 \\
188.0 \\
183.1 \\
182.4\end{array}$ \\
\hline
\end{tabular}

- Preliminary data.

Note: Geographic coverage to the 50 States and the Disirict of Columbla.

Sources: Indices are prepared from statistics publiahed by the U.S.

Department of Tranaportalion. Federal Highway Administration. Federa
Hiohway Stalbitics Division. - 1073-108s: Hohway Statistica Summan to 1985, Table VM-201A. - 1008 lonward: Highway Statistics, annul, Table VM-1. 


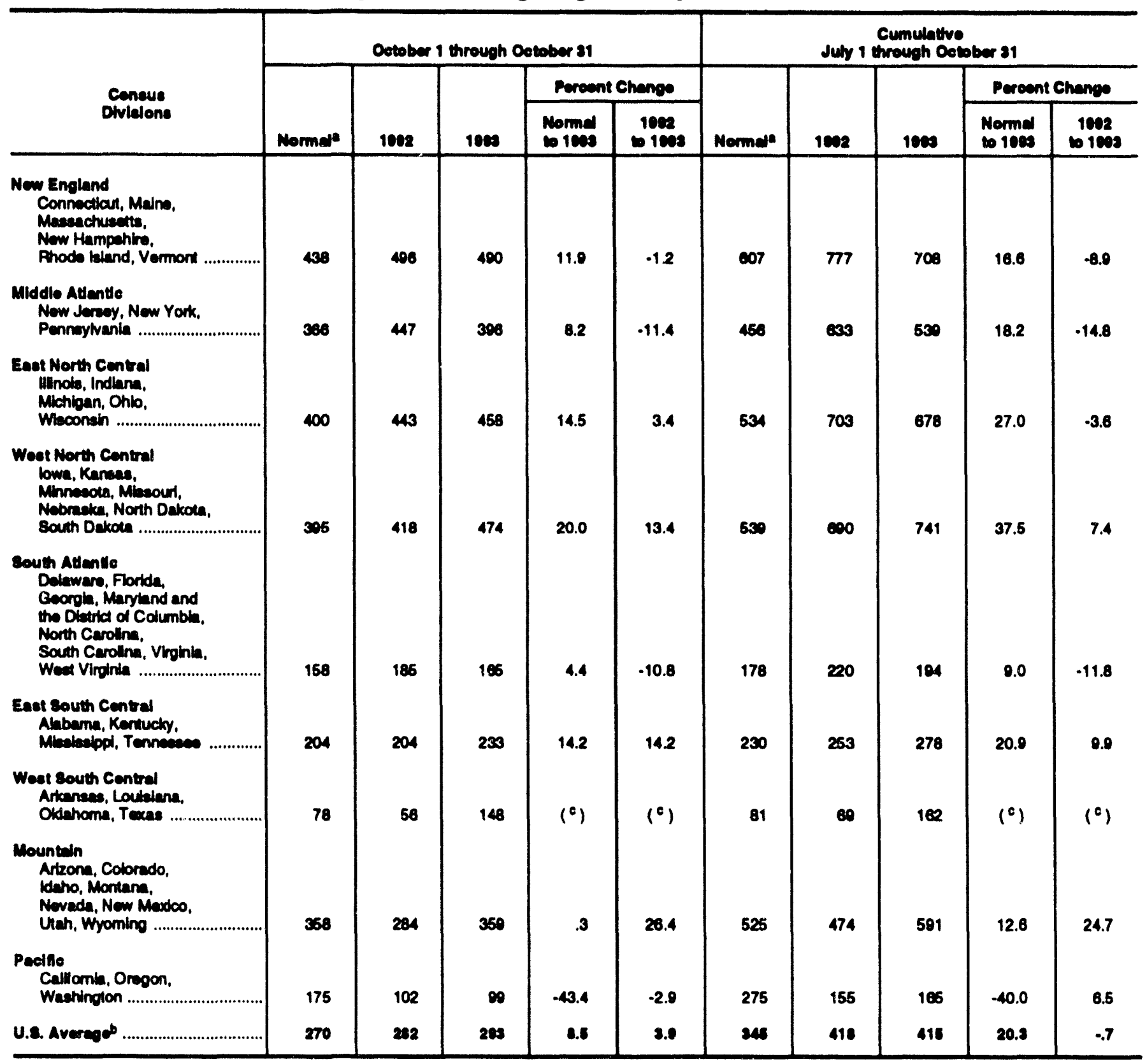

- "Normer be besed on celculations of date trom 1981 through 1900

- Excludes Alakta and Hawal.

- Percent change la not meaningtut: normal la lase than 100 or ratio is incalovinblo.

Source: Sev Nole 7 at end of section. 


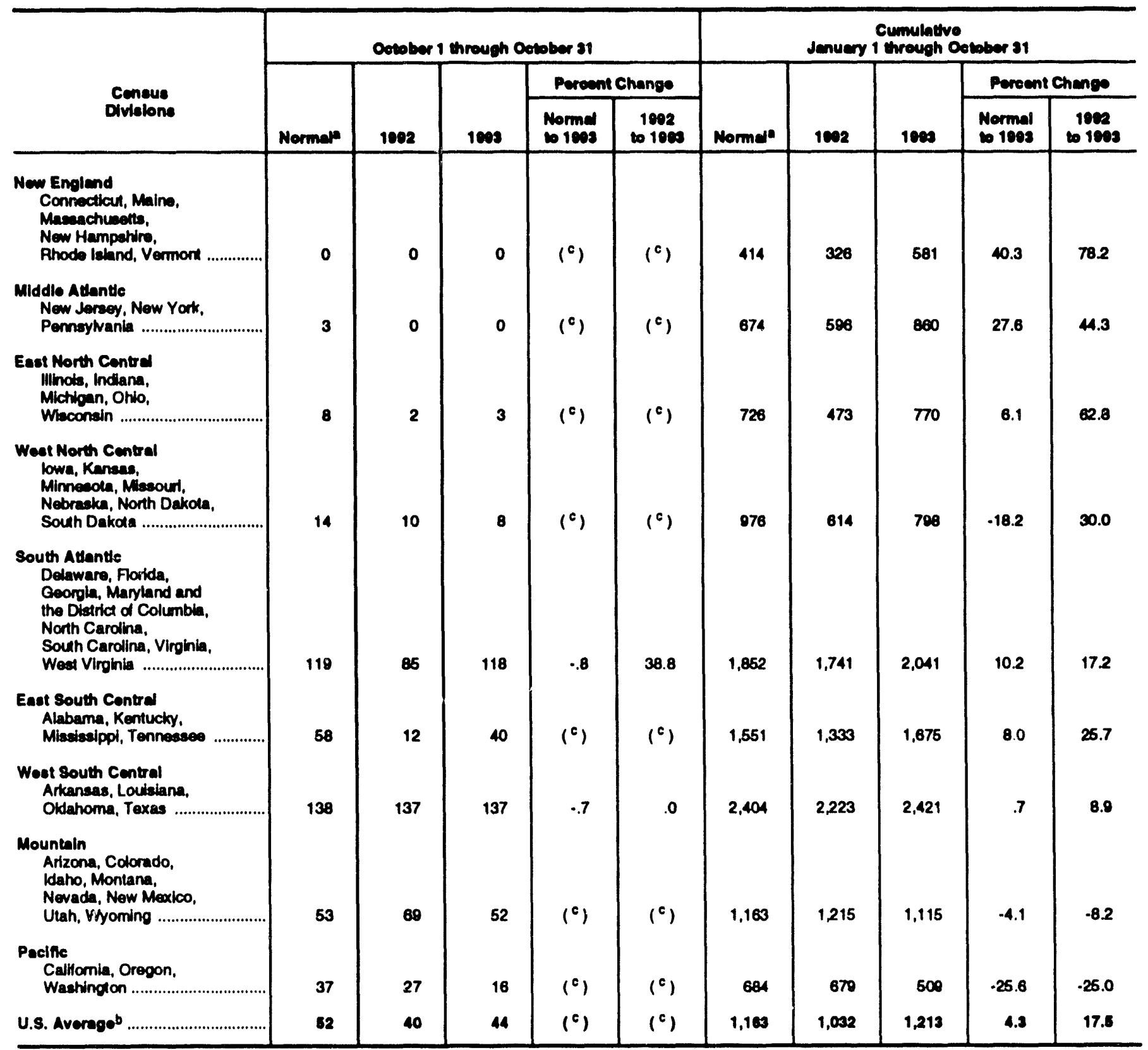

- 'Normal' is based on calculations of data from 1981 through 1990.

- Excludes Alaska and Hawall.

- Percent change bo not meaningtul: normal ts lese than 100 or ralio is incalculable.

Source: See Nole 7 at end of section. 


\section{Energy Summary Notes}

1. Energy Production: Production of energy includes production of coal, crude oil and lease condensate, natural gas plant liquids, natural gas (dry), electric utility and industrial production of hydroelectric power, and electricity generated from nuclear power. Production also includes electricity generated for distribution from wood, waste, geothermal, wind, photovoltaic, and solar thermal energy but excludes other energy obtained from those sources because consistent historical data are not available. Approximate heat contents (Btu values) are derived by using the conversion factors provided in Appendix A.

2. Energy Consumption: Consumption of energy includes consumption of coal, natural gas (including supplemental gaseous fuels), petroleum products supplied, electric utility and industrial production of hydroelectric power, net imports of electricity (assumed to be hydroelectricity), net imports of coal coke, and electricity generated from nuclear power. Consumption also includes electricity generated for distribution from wood, waste, geothermal, wind, photovoltaic, and solar thermal energy but excludes other energy obtained from those sources because consistent historical data are not available. Approximate heat contents (Btu values) are derived by using the conversion factors provided in Appendix A.

3. Energy Imports: Energy imports include imports of coal, crude oil (including crude oil imported for the Strategic Petroleum Reserve), petroleum products, natural gas, electricity (assumed to be hydroelectricity), and coal coke. Approximate heat contents (Btu values) are derived by using the conversion factors provided in Appendix A. For further information on electricity, see "Note for imports and exports of electricity" under Note 8 of the Notes and Sources for the Energy Consumption Section.

4. Energy Exports: Energy exports include coal, crude oil, petroleum products, natural gas, electricity produced from hydroelectric power, and coal coke. Approximate heat contents (Btu values) are derived by using the conversion factors provided in Appendix A. For more information on electricity, see "Note for imports and exports of electricity" under Note 8 of the Notes and Sources for the Energy Consumption Section.

5. Merchandise Trade Value: Import data presented are based on the customs value. That value does not include insurance and freight and is consequently lower than the cost, insurance, and freight (CIF) value, which is also reported by the Bureau of the Census. All export data, and import data prior to 1981, are on a free alongside ship (f.a.s.) basis.

"Balance" is exports minus imports; a positive balance indicates a surplus trade value and a negative balance indicates a deficit trade value. "Energy" includes mineral fuels, lubricants, and related material. "NonEnergy Balance" and "Total Merchandise" include foreign exports (i.e., reexports) and nonmonetary gold and Department of Defense Grant-Aid shipments. The "Non-Energy Balance" is calculated by subtracting the "Energy" from the "Total Merchandise Balance."

"Imports" consist of government and nongovernment shipments of merchandise into the 50 States, the District of Columbia, Puerto Rico, the U.S. Virgin Islands, and the U.S. Foreign Trade Zones. They reflect the total arrival from foreign countries of merchandise that immediately entered consumption channels, warehouses, the Foreign Trade Zones, or the Strategic Petroleum Reserve. They exclude shipments between the United States, Puerto Rico, and U.S. possessions, shipments to U.S. Armed Forces and diplomatic missions abroad for their own use, U.S. goods returned to the United States by its Armed Forces, and in-transit shipments.

6. The Consumer Price Index: The values for the Consumer Price Index, All Urban Consumers, All Items, $1982-84=100$, are as follows:

$\begin{array}{lrlll}1973 & 44.4 & 1988 & & 118.3 \\ 1974 & 49.3 & 1989 & & 124.0 \\ 1975 & 53.8 & 1990 & & 130.7 \\ 1976 & 56.9 & 1991 & \text { 1st Quarter } & 134.8 \\ 1977 & 60.6 & & \text { 2nd Quarter } & 135.6 \\ 1978 & 65.2 & & \text { 3rd Quarter } & 136.7 \\ 1979 & 72.6 & & \text { 4th Quarter } & 137.7 \\ 1980 & 82.4 & & \text { Year } & 136.2 \\ 1981 & 90.9 & 1992 & \text { 1st Quarter } & 138.7 \\ 1982 & 96.5 & & \text { 2nd Quarter } & 139.8 \\ 1983 & 99.6 & & \text { 3rd Quarter } & 140.9 \\ 1984 & 103.9 & & \text { 4th Quarter } & 14 ! .9 \\ 1985 & 107.6 & & \text { Year } & 140.3 \\ 1986 & 109.6 & 1993 & \text { 1st Quarter } & 143.1 \\ 1987 & 113.6 & & \text { 2nd Quarter } & 144.2\end{array}$

7. Degree-Days: Degree-days are relative measurements of outdoor air temperature. Cooling degree-days are defined as deviations of the mean daily temperature at a sampling station above a base temperature equal to $65^{\circ} \mathrm{F}$ by convention. Heating degree-days are deviations of the mean daily temperature below $65^{\circ} \mathrm{F}$. For example, if a weather station recorded a mean daily temperature of $78^{\circ} \mathrm{F}$, cooling degree-days for that station would be 13 (and heating degree-days, 0). A weather station recording a mean daily temperature of $40^{\circ} \mathrm{F}$ would report 25 heating degree-days (and 0 cooling degree-days).

There are several degree-day databases maintained by the National Oceanic and Atmospheric Administration. The information published in the Monthly Energy Review (MER) is developed by the National Weather Service Climate Analysis Center, Camp Springs, MD. The data are available weekly with monthly summaries and are based on mean daily temperatures recorded at about 200 major weather stations around the country. The temperature information recorded at those weather 
stations is used to calculate statewide degree-day averages based on population. The State figures are then aggregated into Census Divisions and into the national average. The population weights currently used represent resident State population data estimated for 1980 by the U.S. Department of Commerce, Bureau of the Census. The data shown in the MER are available sooner than the Historical Climatology Series 5-1 and 5-2 developed by the National Climatic Center, Asheville, NC, which compiles data from some 8,000 weather stations.

\section{Sources for Table 1.6}

U.S. Department of Commerce, Bureau of the Census, Foreign Trade Division:

- Petroleum Exports-1974-1987: "U.S. Exports," FT410, December issues. 1988: "Report on U.S. Merchandise Trade, 1988 Final Revisions." 1989: "Report on U.S. Merchandise Trade, 1989 Revisions." 1990: "U.S. Merchandise Trade, 1990 Final Report." 1991: "U.S. Merchandise Trade, 1991 Final Report," May 13, 1992. 1992: "U.S. Merchandise Trade, 1992 Final Report," May 12, 1993. 1993: "U.S. Merchandise Trade," FT900, monthly.

- Petroleum Imports-1974-1987: "U.S. Merchandise Trade," FT900, December issues, 1975-1988. 1988: "Report on U.S. Merchandise Trade, 1988 Final Revisions." 1989: "Report on U.S. Merchandise Trade, 1989 Revisions." 1990: "U.S. Merchandise Trade, 1990 Final Report." 1991: “U.S. Merchandise
Trade, 1991 Final Report," May 13, 1992, and "U.S. Merchandise Trade, October 1992," December 17, 1992. page 3. 1992: "U.S. Merchandise Trade, 1992 Final Report," May 12, 1993. 1993: "U.S. Merchandise Trade," FT900, monthly.

- Energy Exports and Imports-1974-1987: U.S. merchandise trade press releases and database printouts for adjustments. 1988: January-July, monthly FT900 supplement, 1989 issues. August-December, monthly FT900, 1989 issues. 1989: Monthly FT900, 1990 issues. 1990: "U.S. Merchandise Trade, 1990 Final Report." 1991: "U.S. Merchandise Trade, 1991 Final Report," May 13, 1992, and "U.S. Merchandise Trade, October 1992," December 17, 1992, page 3. 1992: "U.S. Merchandise Trade, 1992 Final Report," May 12, 1993. 1993: "U.S. Merchandise Trade," FT900, monthly.

- Total Merchandise-1974-1987: U.S. merchandise trade press releases and database printouts for adjustments. 1988: "Report on U.S. Merchandise Trade, 1988 Final Revisions," August 18, 1989. 1989: "Report on U.S. Merchandise Trade, 1989 Revisions," July 10, 1990. 1990: "U.S. Merchandise Trade, 1990 Final Report," May 10, 1991, and "U.S. Merchandise Trade, December 1992," February 18, 1993, page 3. 1991-1992: "U.S. Merchandise Trade, 1992 Final Report," May 12, 1993. 1993: "U.S. Merchandise Trade," FT900, monthly.

- Petroleum Balance, Energy Balance, and Non-Energy Balance-Calculated by the Energy Information Administration. 


\section{Section 2. Energy Consumption}

U.S. total energy consumption in August 1993 was 7.0 quadrillion Btu. Petroleum products accounted for $\mathbf{4 1}$ percent ${ }^{1}$ of the energy consumed in August 1993, while coal accounted for 27 percent and natural gas accounted for 20 percent.

Residential and commercial sector consumption was 2.4 quadrillion Btu in August 1993, up 10 percent from the August 1992 level. The sector accounted for 35 percent of August 1993 total consumption, up 2 percentage points from its 33-percent share in August 1992.

Industrial sector consumption was 2.5 quadrillion Btu in August 1993, down slightly from the August 1992 level. The industrial sector accounted for 36 percent of August 1993 total consumption, down 2 percentage points from its 38-percent share in August 1992.
Transportation sector consumption of energy was 2.0 quadrillion Btu in August 1993, up 3 percent from the August 1992 level. The sector accounted for 28 percent of August 1993 total consumption, down 1 percentage point from its 29-percent share in August 1992.

Electric utility consumption of energy totaled 3.0 quadrillion Btu in August 1993, up 9 percent from the August 1992 level. Coal contributed 54 percent of the energy consumed by electric utilities in August 1993, while nuclear electric power contributed 20 percent; natural gas 12 percent; bydroelectric power 8 percent; petroleum 4 percent; and wood, waste, geothermal, wind, photovoltaic, and solar thermal energy, about 1 percent.

\section{Table 2.1 Energy Consumption Summary for August 1993}

(Quadrillion Btu)

\begin{tabular}{|c|c|c|c|c|c|c|}
\hline \multirow[b]{2}{*}{ Energy souree } & \multicolumn{4}{|c|}{ End-Uae sectors } & \multirow[b]{2}{*}{$\begin{array}{l}\text { Ellooters } \\
\text { Unillide }\end{array}$} & \multirow[b]{2}{*}{ Totel } \\
\hline & $\begin{array}{l}\text { and } \\
\text { Commerelel }\end{array}$ & Industriel & Treneportetion & Tolen & & \\
\hline 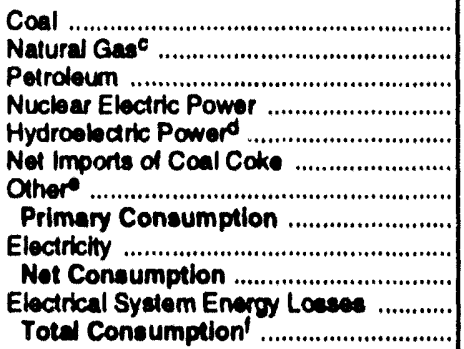 & $\begin{array}{r}0.010 \\
243 \\
.157 \\
- \\
- \\
- \\
- \\
.400 \\
.638 \\
1.047 \\
1.392 \\
2.430\end{array}$ & $\begin{array}{r}0.208 \\
.730 \\
.654 \\
-\overline{.002} \\
.002 \\
\overline{-} \\
1.500 \\
.298 \\
1.892 \\
.646 \\
2.834\end{array}$ & $\begin{array}{r}(0) \\
.044 \\
1.026 \\
- \\
= \\
- \\
1.070 \\
.001 \\
1.071 \\
.003 \\
1.074\end{array}$ & $\begin{array}{r}0.222 \\
1.018 \\
2.730 \\
-002 \\
.002 \\
- \\
3.091 \\
.038 \\
4.016 \\
2.041 \\
.057\end{array}$ & $\begin{array}{r}1.621 \\
.396 \\
.126 \\
.604 \\
.246 \\
- \\
.015 \\
2.076 \\
- \\
- \\
- \\
-\end{array}$ & $\begin{array}{r}1.844 \\
1.382 \\
2.882 \\
.604 \\
.248 \\
.002 \\
.015 \\
6.087 \\
- \\
- \\
- \\
-\end{array}$ \\
\hline
\end{tabular}

- Totala for coal and natural gas may nol equal sum of sectors due to the use of sector-specitic conversion tadors.

b small amounts of coal consumed for transportation are reported as industrial sector consumption.

c Inchudes supplemental geseous luals. Trameportation sector is plpeline luel only.

Indudes net imports of electriclly.

- Other b eledilclly generaled lo detribution from wood, waste, geothermal, wind, photovotialc, and solar themal energy.
1 Exclude wood, weate, geothermal, whd, photovoltak, and solet thomnl onergy, except for emall amounts uned by clectric vilitive to enerate electitclly for distribution.

- Not applicable. (s)=Leas than t0.5 irlition But and greater than -0.5 intion Eu.

Nole: Total may not equal sum of componerts dus to independent rounding.

Addtional Notes and Sources: See Tablas 2.2.2.6 and end of section.

'Percentage changes are based on numbers in the following tables. 
Figure 2.1 Energy Consumption by End-Use sector

(Quadrillion Btu)

Consumption by End-Use Sector, 1973-1992

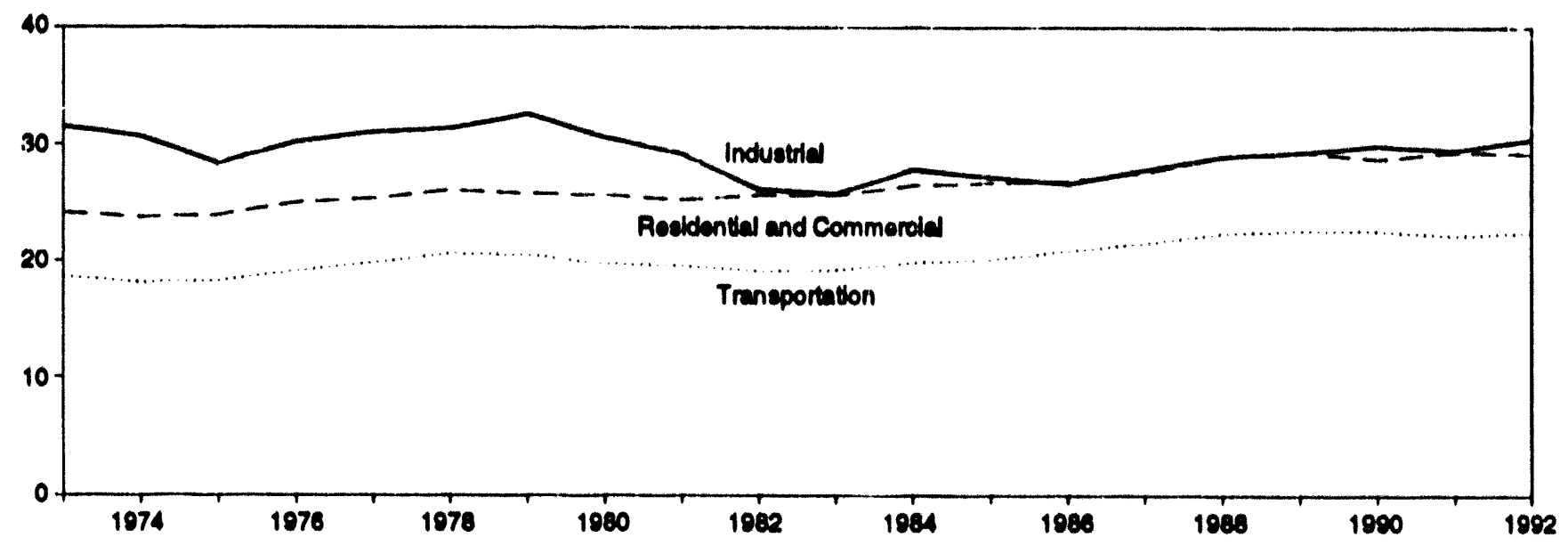

Consumption by End-Use Sector, Monthly

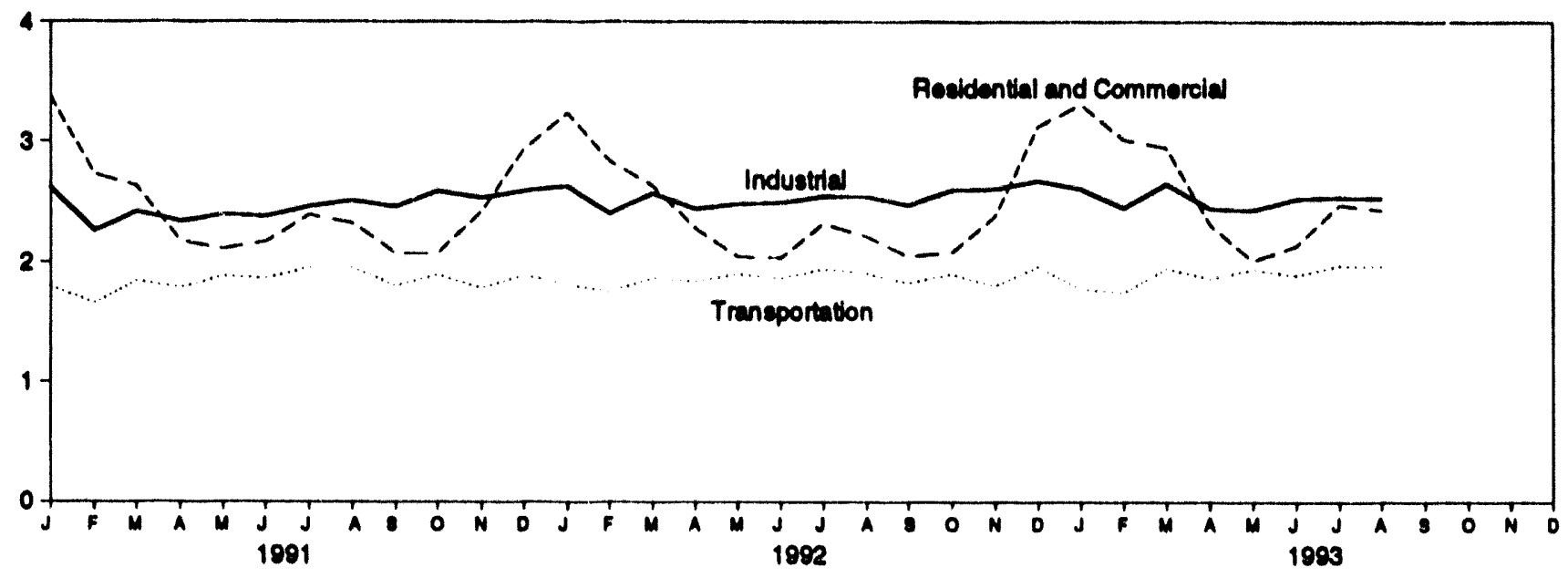

Consumption by End-Use Sector, August
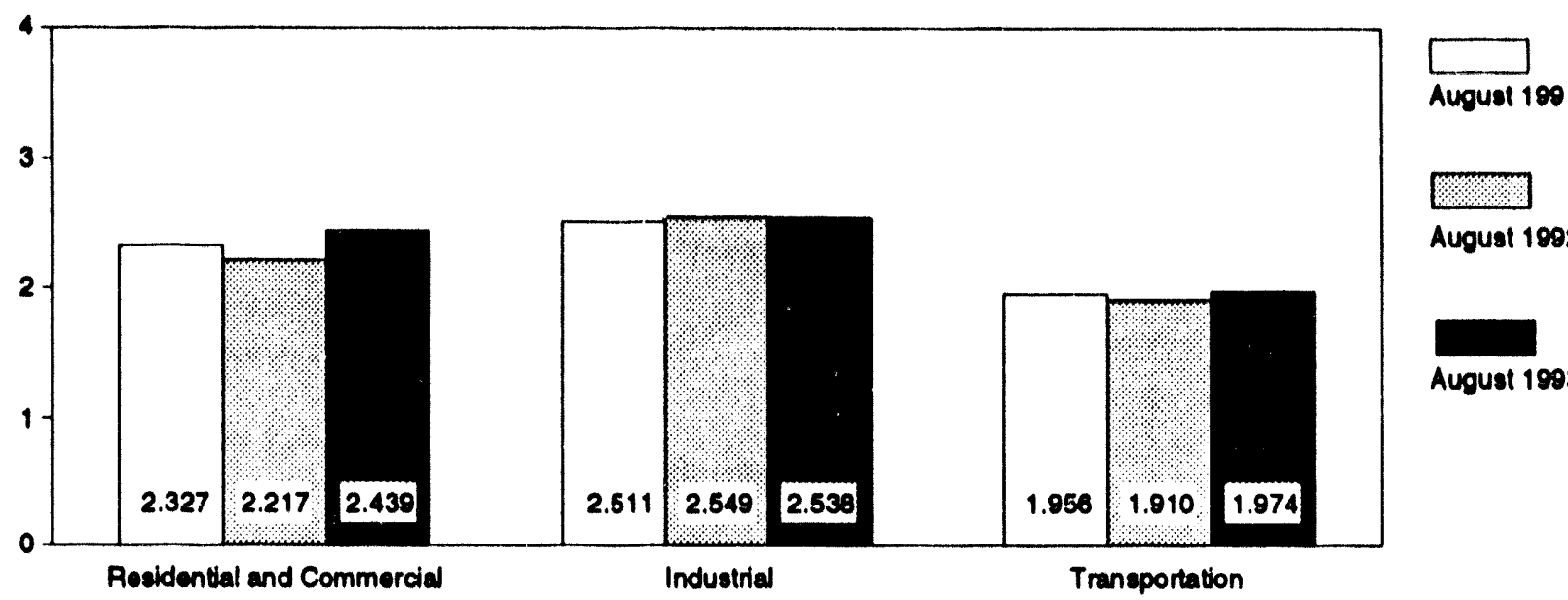

Auguat 1982

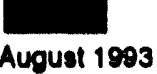

Nolo: Because vertical scales difler, grephe should not be compared.

Source: Teblo 2.2. 
Table 2.2 Energy Consumption by End-Use Sector (Quadrillion Btu)

\begin{tabular}{|c|c|c|c|c|c|c|c|c|}
\hline & \multicolumn{2}{|c|}{ Acoldential and Commerald } & \multicolumn{2}{|c|}{ Induatrid } & \multicolumn{2}{|c|}{ Trenaportation } & \multirow[b]{2}{*}{ Net } & \multirow[b]{2}{*}{ Total } \\
\hline & Not & Tolal & Not & Totel & Nol & Total & & \\
\hline 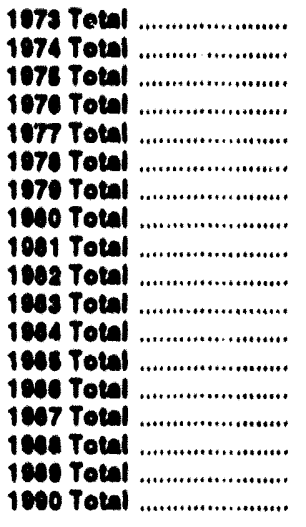 & 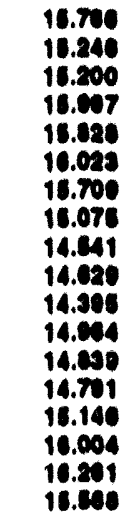 & 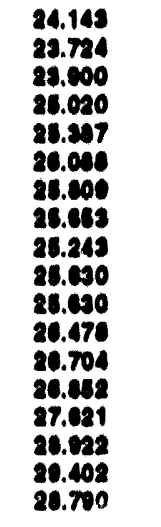 & 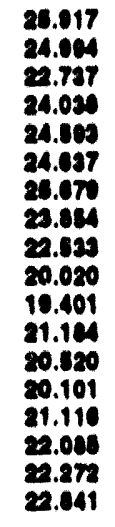 & 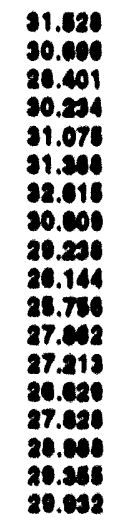 & 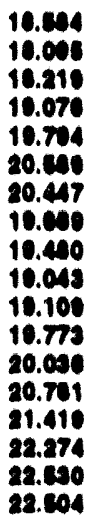 & 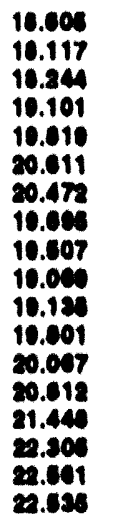 & 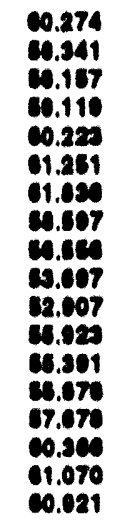 & 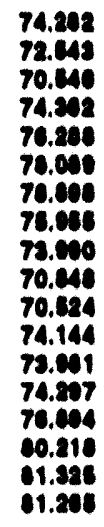 \\
\hline 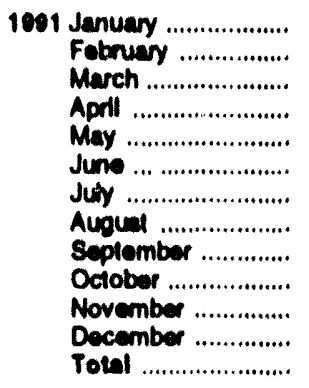 & $\begin{array}{l}2.141 \\
1.754 \\
1.505 \\
1.234 \\
1.004 \\
.072 \\
1.009 \\
1.002 \\
.002 \\
1.003 \\
1.400 \\
1.793 \\
18.000\end{array}$ & $\begin{array}{r}\text { A } 3.378 \\
2.720 \\
2.632 \\
2.170 \\
2.111 \\
2.171 \\
2.306 \\
2.327 \\
2.078 \\
2.076 \\
2.421 \\
2.028 \\
20.421\end{array}$ & $\begin{array}{l}2.048 \\
1.706 \\
1.860 \\
1.789 \\
1.757 \\
1.764 \\
1.822 \\
1.806 \\
1.906 \\
2.001 \\
1.900 \\
2.014 \\
2 . .840\end{array}$ & $\begin{array}{r}2.620 \\
2.201 \\
2.420 \\
2.339 \\
2.307 \\
2.391 \\
2.463 \\
2.511 \\
2.481 \\
2.500 \\
2.530 \\
2.501 \\
20.571\end{array}$ & $\begin{array}{l}1.705 \\
1.653 \\
1.842 \\
1.784 \\
1.882 \\
1.603 \\
1.062 \\
1.853 \\
1.602 \\
1.603 \\
1.783 \\
1.688 \\
22.000\end{array}$ & $\begin{array}{l}1.790 \\
1.056 \\
1.844 \\
1.780 \\
1.806 \\
1.868 \\
1.056 \\
1.050 \\
1.804 \\
1.600 \\
1.786 \\
1.601 \\
2.120\end{array}$ & $\begin{array}{l}5.984 \\
5.170 \\
5.280 \\
4.806 \\
4.603 \\
4.603 \\
1.800 \\
4.828 \\
4.600 \\
4.950 \\
5.146 \\
5.694 \\
0.024\end{array}$ & $\begin{array}{l}7.705 \\
6.843 \\
6.803 \\
6.302 \\
6.304 \\
6.421 \\
6.818 \\
6.706 \\
6.344 \\
6.501 \\
6.740 \\
7.400 \\
01.110\end{array}$ \\
\hline 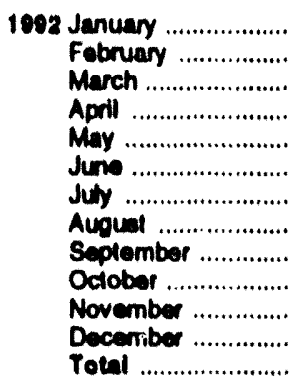 & $\begin{array}{r}2.040 \\
1.828 \\
1.610 \\
1.343 \\
1.060 \\
.943 \\
1.018 \\
.097 \\
.001 \\
1.006 \\
1.372 \\
1.010 \\
16.170\end{array}$ & $\begin{array}{r}3.237 \\
2.838 \\
2.636 \\
2.287 \\
2.049 \\
2.040 \\
2.326 \\
2.217 \\
2.051 \\
2.087 \\
2.389 \\
P_{3.127} \\
P_{20.276}\end{array}$ & 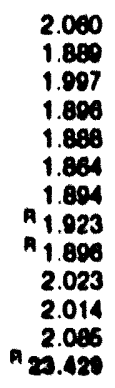 & $\begin{array}{r}A_{2} .631 \\
2.406 \\
A_{2.573} \\
2.444 \\
2.466 \\
2.408 \\
A_{2} .560 \\
A_{2.540} \\
2.477 \\
2.603 \\
2.610 \\
2.677 \\
A_{20.604}\end{array}$ & $\begin{array}{l}1.815 \\
1.750 \\
1.865 \\
1.808 \\
1.803 \\
1.606 \\
1.846 \\
1.007 \\
1.828 \\
1.002 \\
1.602 \\
1.063 \\
22.204\end{array}$ & $\begin{array}{l}1.817 \\
1.753 \\
1.668 \\
1.640 \\
1.906 \\
1.660 \\
1.940 \\
1.010 \\
1.830 \\
1.004 \\
1.804 \\
1.965 \\
2.414\end{array}$ & $\begin{array}{r}5.913 \\
5.466 \\
R^{5.470} \\
5.075 \\
4.850 \\
4.675 \\
4.862 \\
4.819 \\
44.687 \\
5.021 \\
5.187 \\
5.067 \\
01.060\end{array}$ & 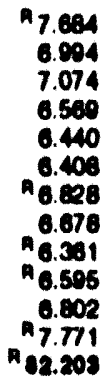 \\
\hline 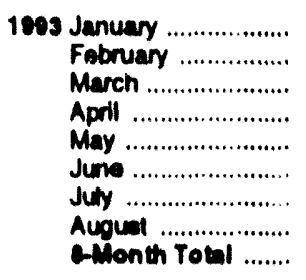 & $\begin{array}{r}A_{2} .009 \\
A_{1} .066 \\
A_{1} .853 \\
A_{1} .301 \\
1.022 \\
A_{.070} \\
A_{1} .067 \\
1.047 \\
11.414\end{array}$ & $\begin{array}{r}A_{3.310} \\
A_{3.017} \\
A_{2.050} \\
2.320 \\
A_{2.017} \\
2.144 \\
A_{2.403} \\
2.439 \\
20.000\end{array}$ & $\begin{array}{l}A_{2} .030 \\
A_{1} .021 \\
A_{2} .008 \\
A_{1} \\
A_{1} .000 \\
A_{1} .821 \\
A_{1.800} \\
1.802 \\
18.420\end{array}$ & $\begin{array}{r}A_{2} .609 \\
A_{2} .464 \\
A_{2.053} \\
A_{2.440} \\
A_{2} .437 \\
A_{2.533} \\
A_{2.544} \\
2.538 \\
20.217\end{array}$ & $\begin{array}{l}A_{1} 1.776 \\
A_{1} .743 \\
A_{1} .052 \\
A_{1} .805 \\
A_{1.011} \\
A_{1.803} \\
A_{1.975} \\
1.971 \\
18.110\end{array}$ & $\begin{array}{l}A_{1} .778 \\
A_{1.746} \\
A_{1.954} \\
A_{1.868} \\
A_{1.944} \\
A_{1.896} \\
A_{1.978} \\
1.974 \\
16.130\end{array}$ & $\begin{array}{l}95.913 \\
95.820 \\
A 5.887 \\
95.145 \\
9.783 \\
44.750 \\
4.936 \\
4.916 \\
41.000\end{array}$ & 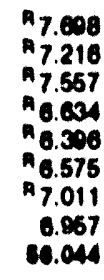 \\
\hline $\begin{array}{l}\text { 1.02 e-Month Total ....... } \\
\text { 1001 e-Month Totil ....... }\end{array}$ & $\begin{array}{l}10.021 \\
10.742\end{array}$ & $\begin{array}{l}10.020 \\
10.922\end{array}$ & $\begin{array}{l}18.411 \\
14.060\end{array}$ & $\begin{array}{l}20.130 \\
10.202\end{array}$ & $\begin{array}{l}14.000 \\
14.724\end{array}$ & $\begin{array}{l}14.010 \\
14.746\end{array}$ & $\begin{array}{l}41.120 \\
40.140\end{array}$ & $\begin{array}{l}64.070 \\
64.004\end{array}$ \\
\hline
\end{tabular}

RaRevised dala.

Noles: - Geographic covernge ts ith 50 Stules and the Destict of Columbia. - Tolals may nol equal sum of compononts dus to independent rounding and the use of sector-epectilc conversion lactors lor natural gas and coul. Additonal Noles and Sources: See end of section. 
Figure 2.2 Realdential and Commorclal Enorgy Consumptlon

(Quadrillion Btu)

Consumption by Major Sources, $1973-1992$

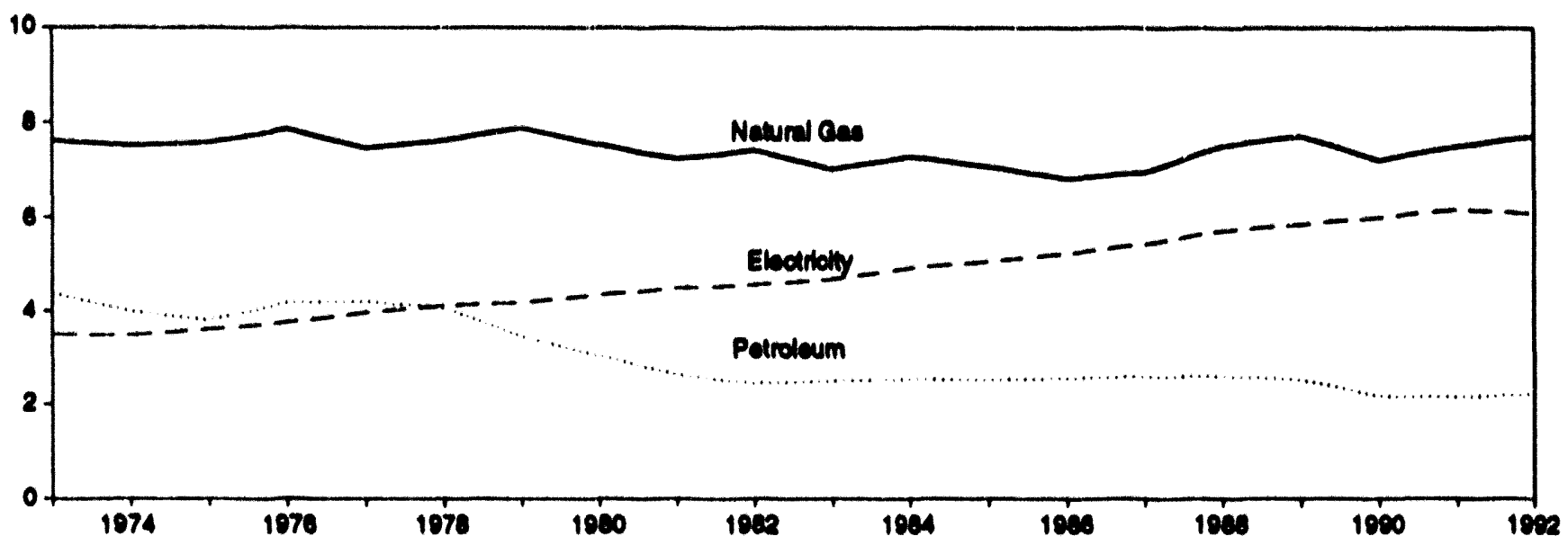

Consumption by Major Sources, Monthly

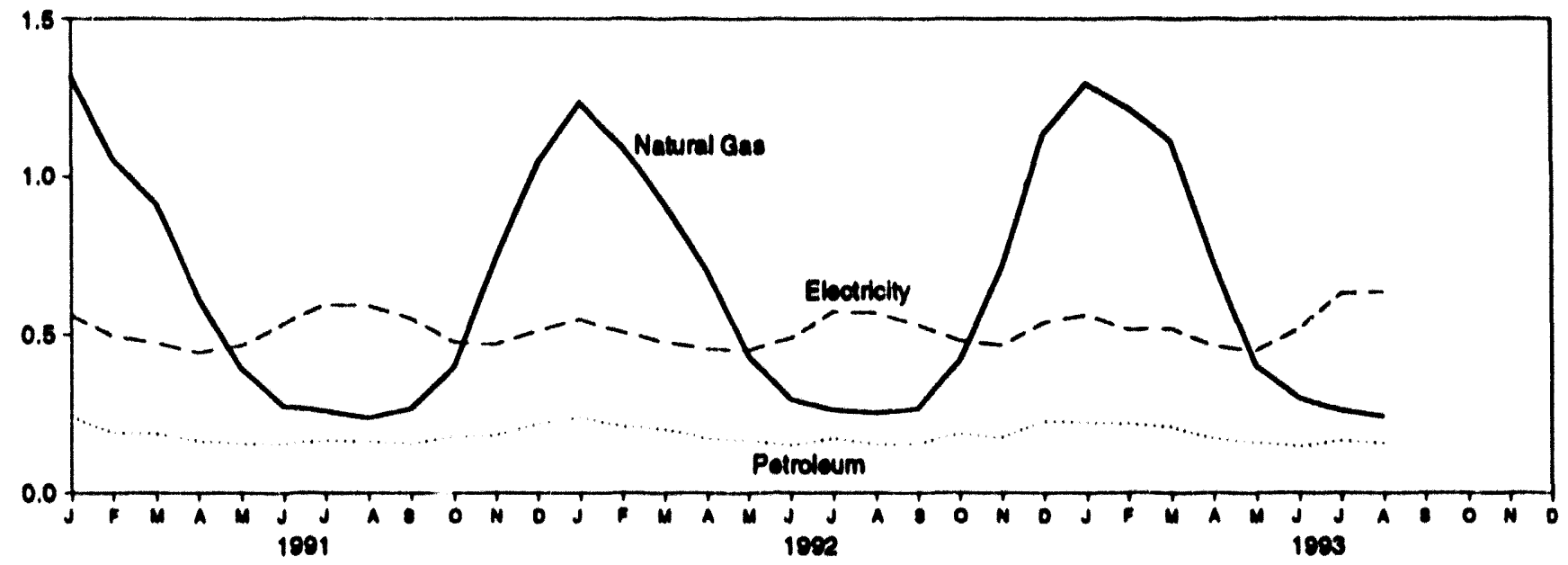

Total Consumption, January-August

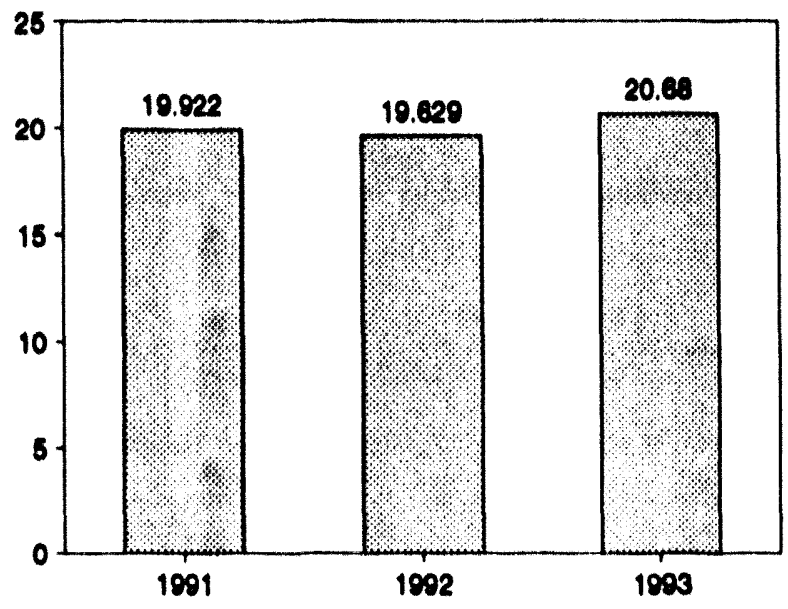

Consumption by Major Sources, August 1993

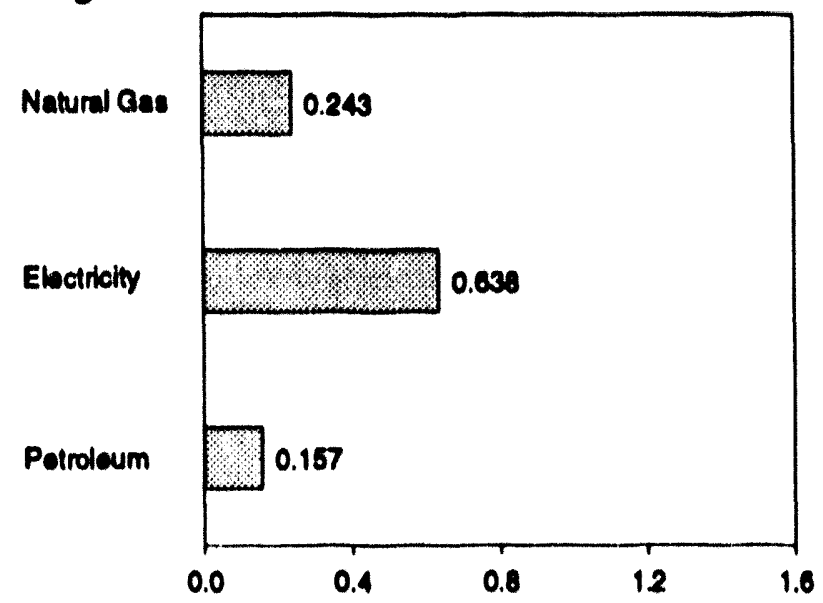

Nolo: Because vertical scales aller, graphe should not be compared. 
Tablo 2.3 Rosldentlal and Commorclal Enorgy Consumption

(Quadrillion Btu)

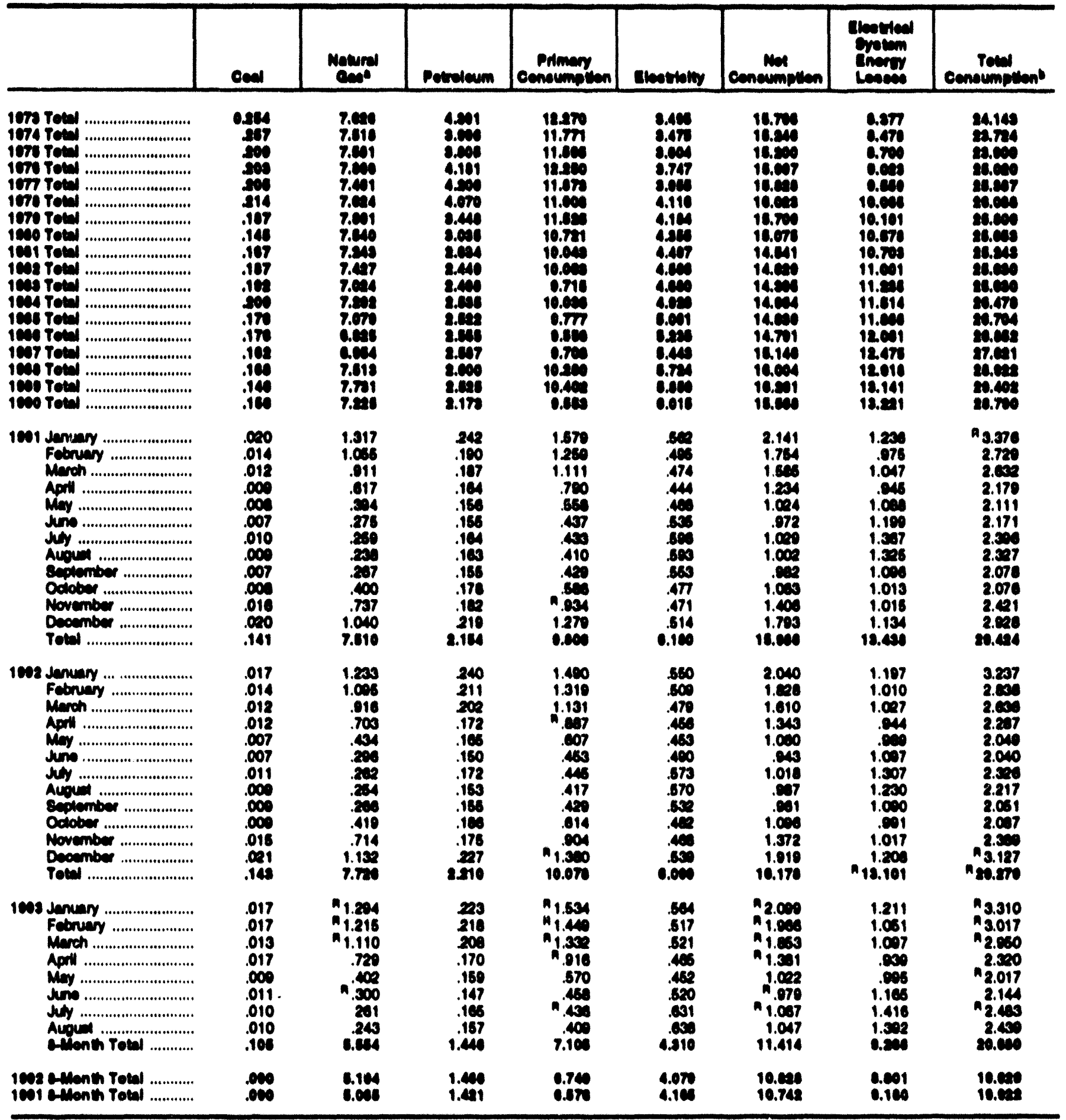

\footnotetext{
- Inchudes upplementel gecocous huel.

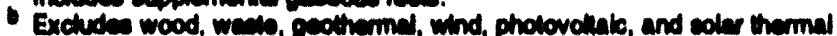

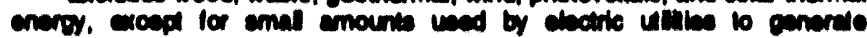
enctiviny lor diatribution.
}

Rarheviecd dela.

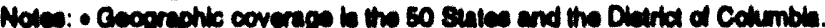

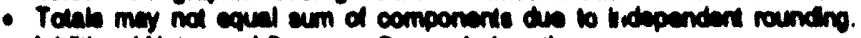
Additonal Notes end sources: seep end of ecection. 
Figure 2.3 Industrial Enorgy Consumption

(Quadrillion Biu)

Consumption by Major Sources, 1973-1992

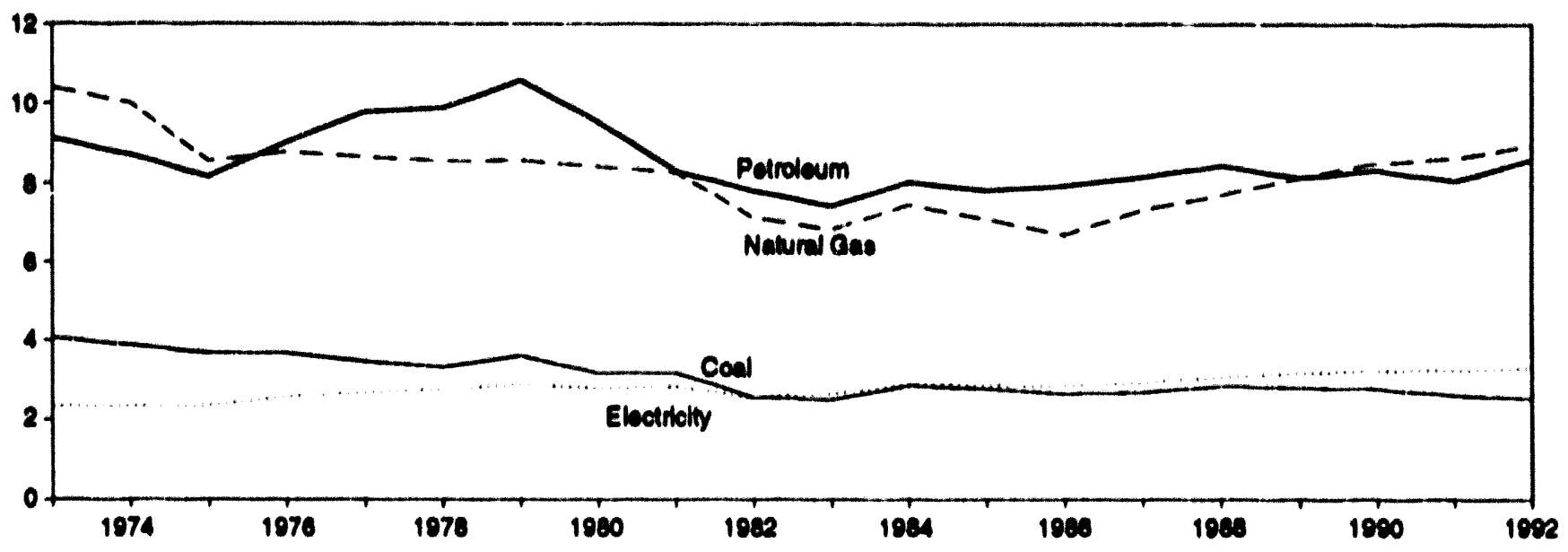

Consumption by Major Sources, Monthly

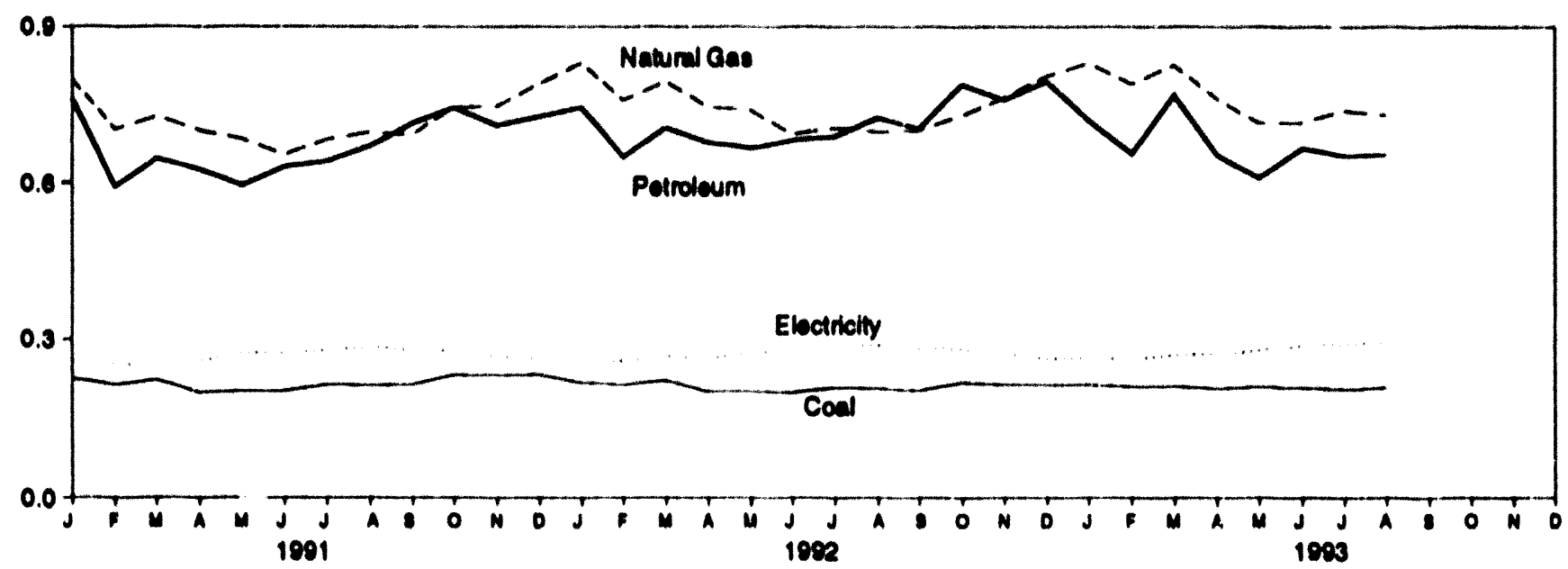

Total Consumption, January-August

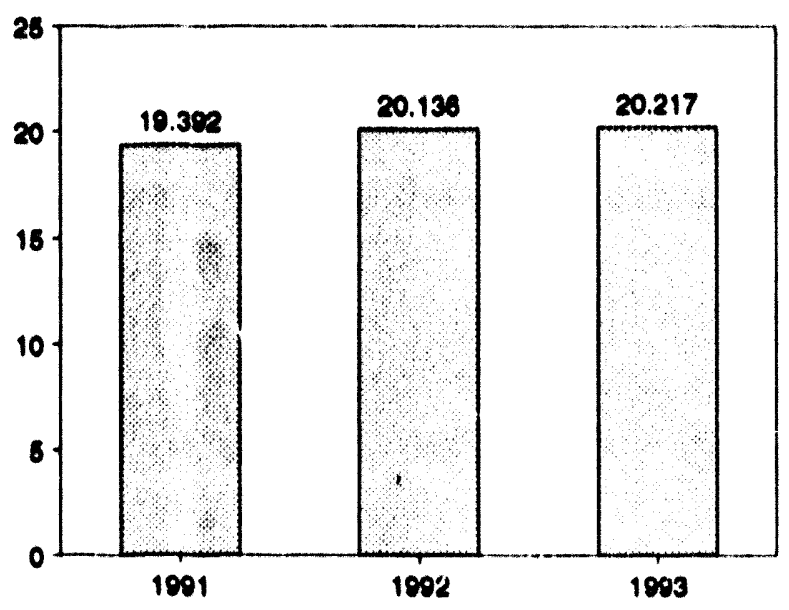

Consumption by Major Sources, August 1993

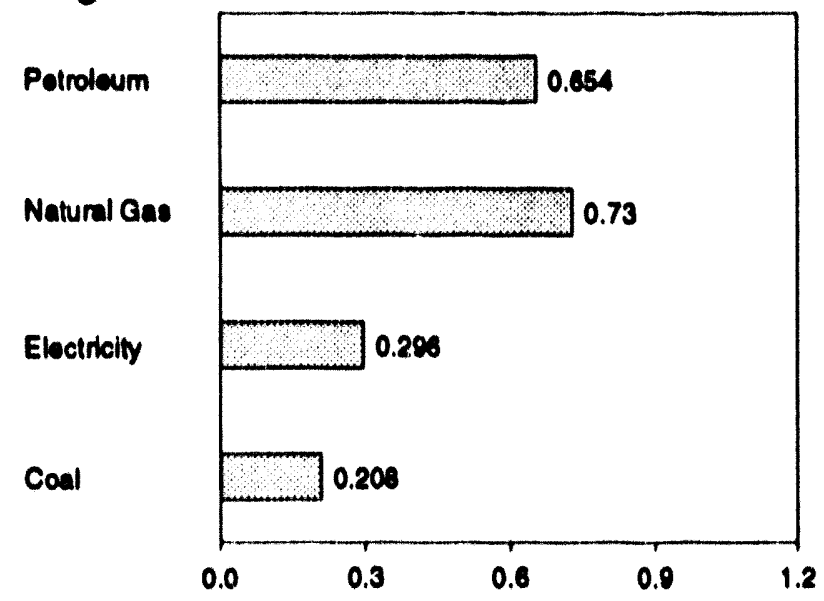

Nolo: Because vertical eceles atfor, graphe should nol be compered. Source: Tabin 2.4. 


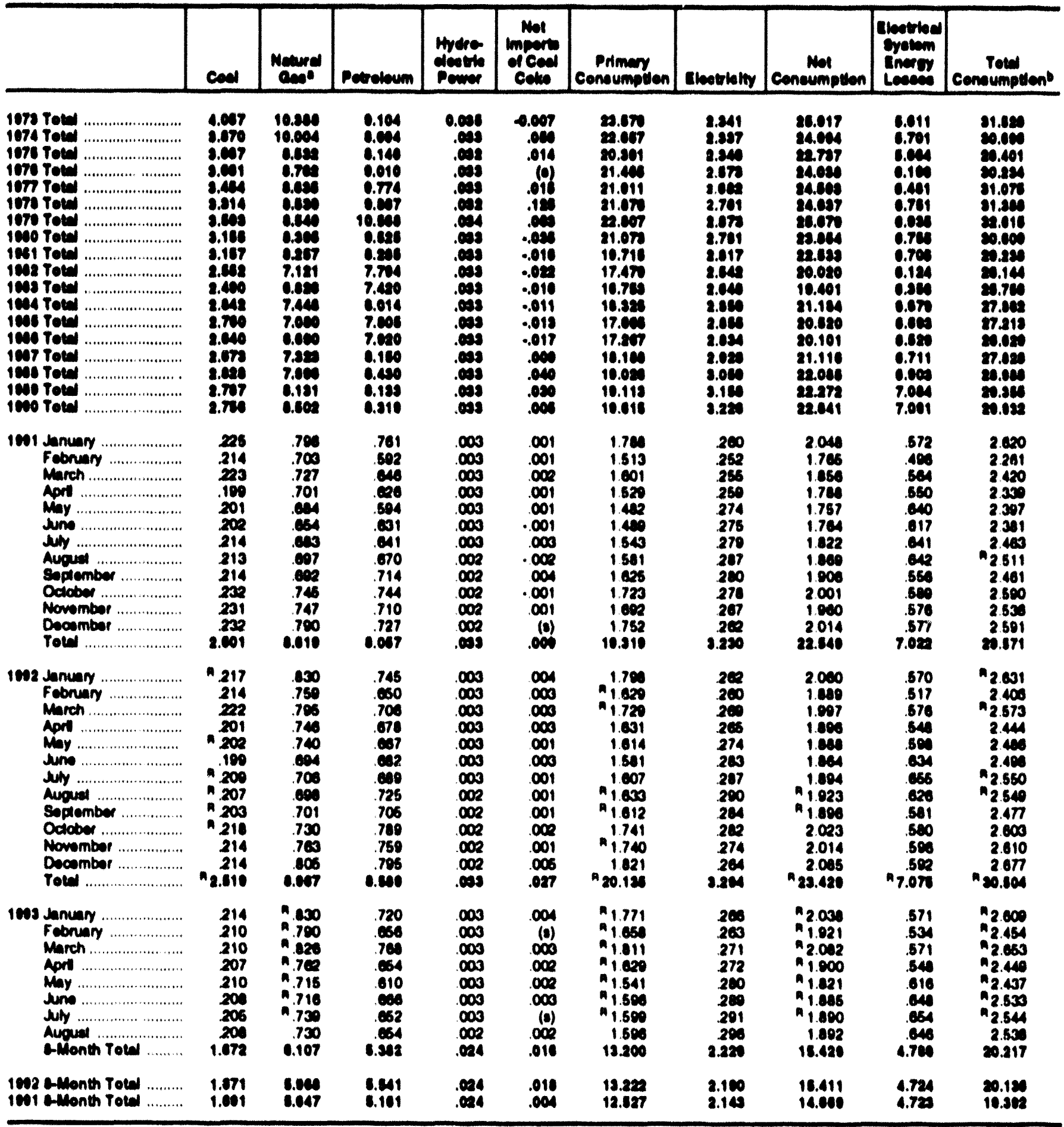

- Includes suppiementy onesore fuels.

- Excludes wood, wete, geothorme, whd, photovolaic, and solve theme onory, except lor enal emounts used by clectic lalibs 10 conarate abciriciny lor diatribution.

AmRoviesd dala. (o)=Lese than 0.5 intion Bu and grealer than 0.5 intion Bu.

Noles: - Coogrephic coversos to the 50 states and the Dititict of Columbla - Tolale moy not equal sum al componime du 10 independant rounding Additional Notes and Sources: see end of section. 
Figure 2.4 Traneportation Enorgy Conoumption

(Ouadrillion Btu)

Consumption by Major Sources, 1973-1992

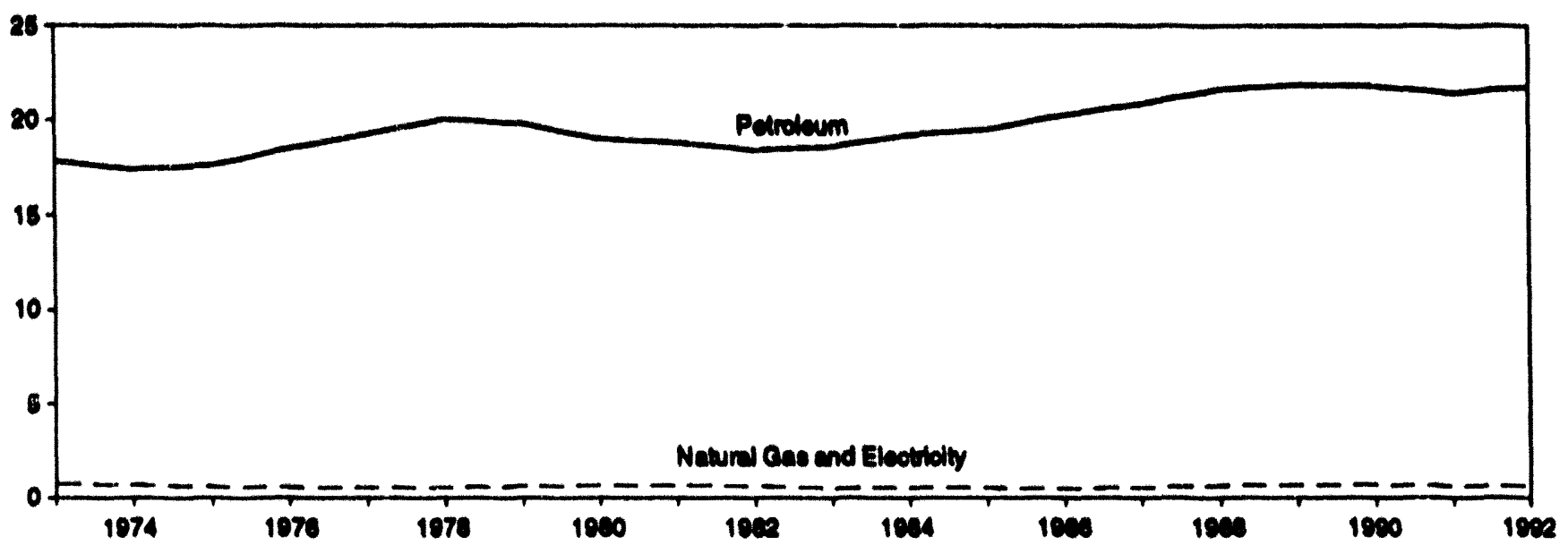

Consumption by Major Sources, Monthly

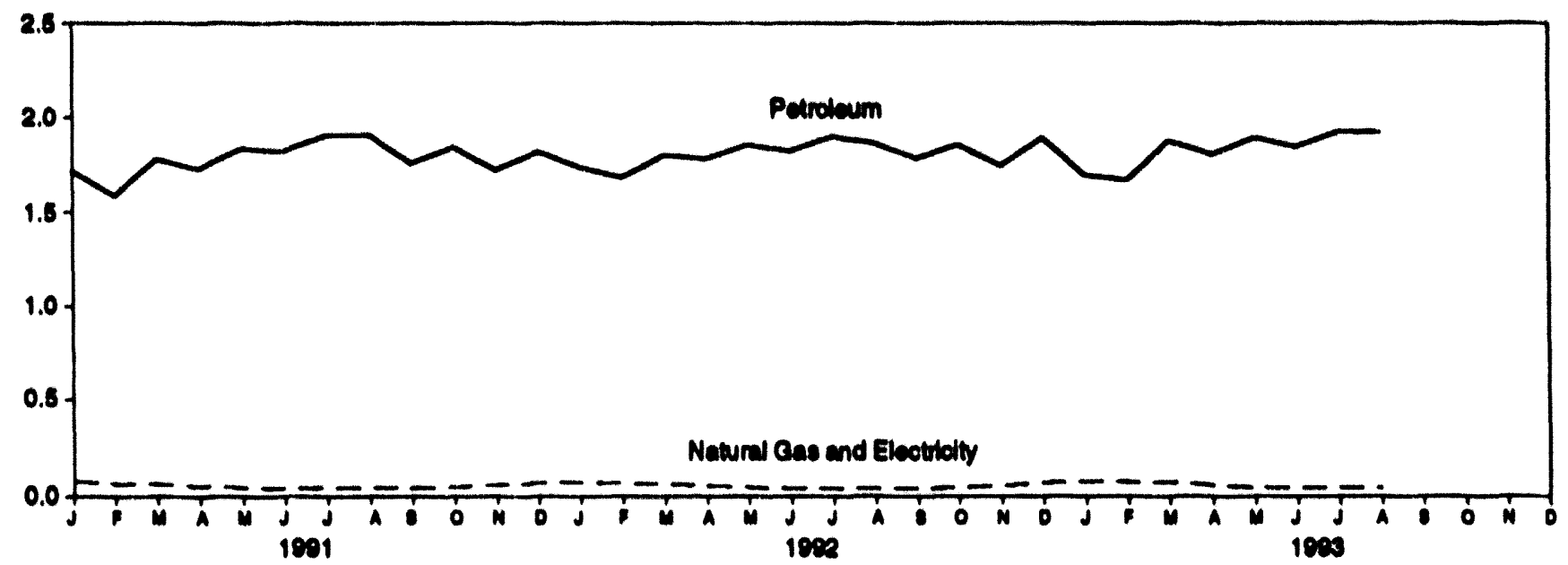

Total Consumption, January-August

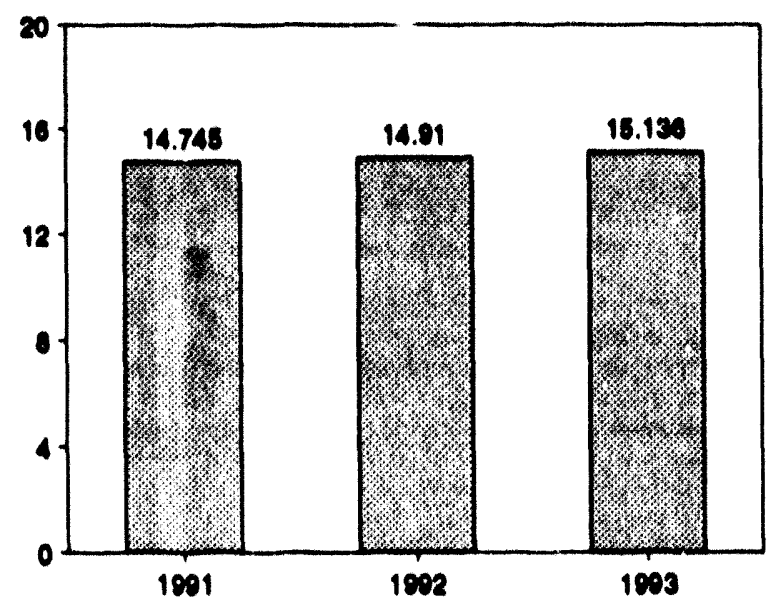

Total Consumption, Monthly

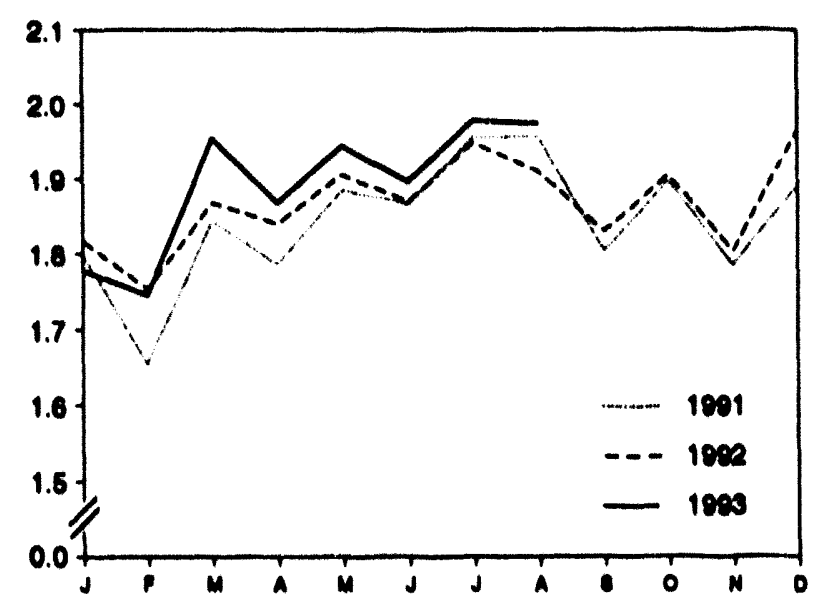

Nole: Becaues verticel eceles arter, onphe should not be compered. Sourco: Tebie 2.6 . 
Tablo 2.5 Transportation Energy Consumption (Quadrillion Btu)

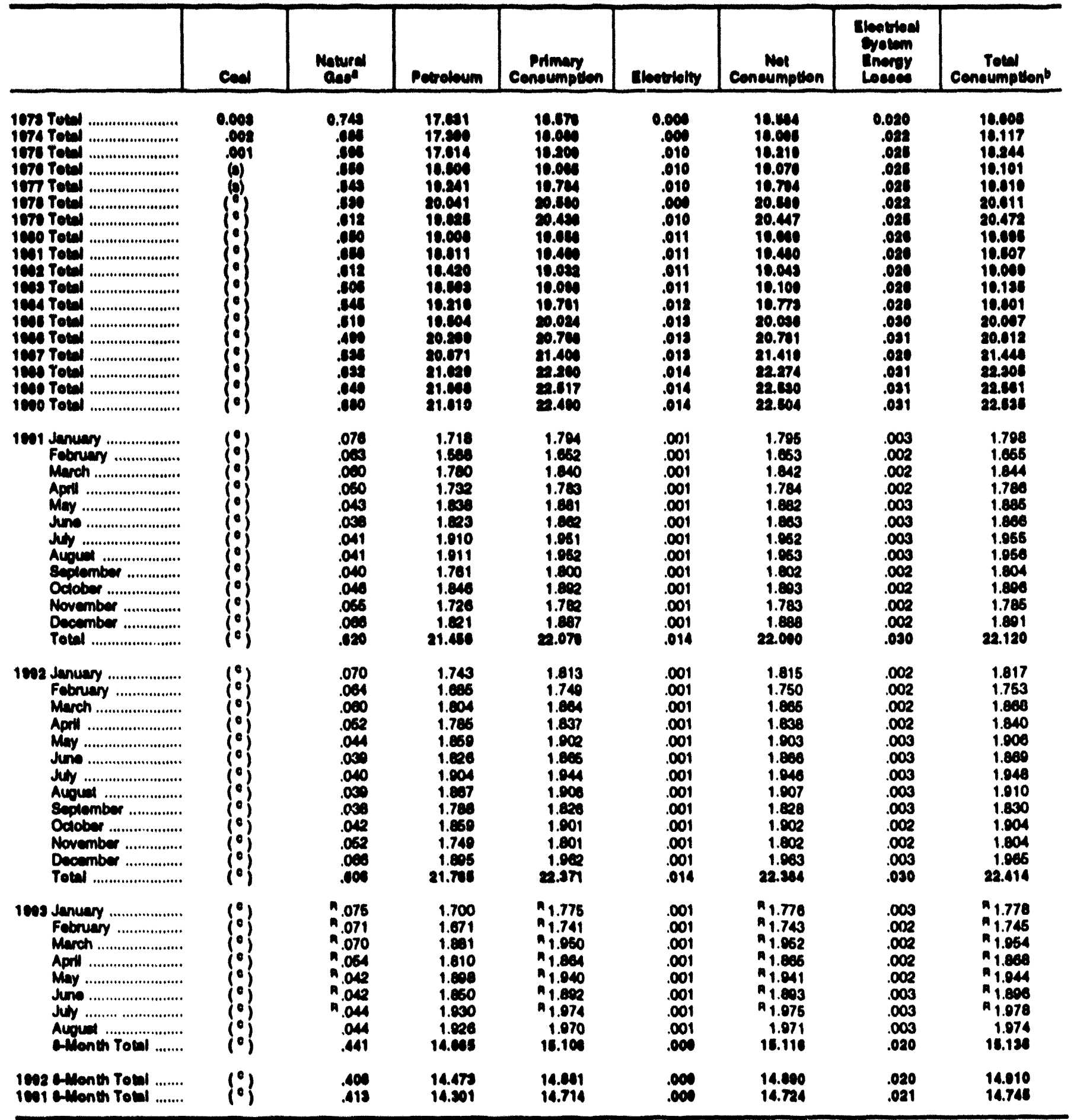

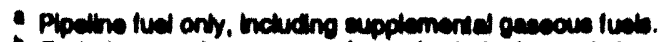

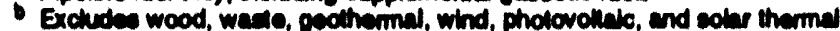

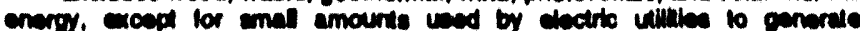
cincticily lor distribution.

- Eince 1978, the emell emounte of col coneumed for inneportation se reported es indunitel sector consumption.

ReRevind dale. (o)=leses then 0.5 intion Btu.

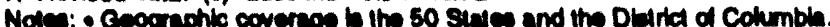

- Totels may not equal sum al components dus to independent rounding. Addilonal Notes and Sources: Ses end of exciton. 
Flgure 2.5 Energy Input at Electric Utilities (Quadrillion Btu)

Total Input, 1973-1992

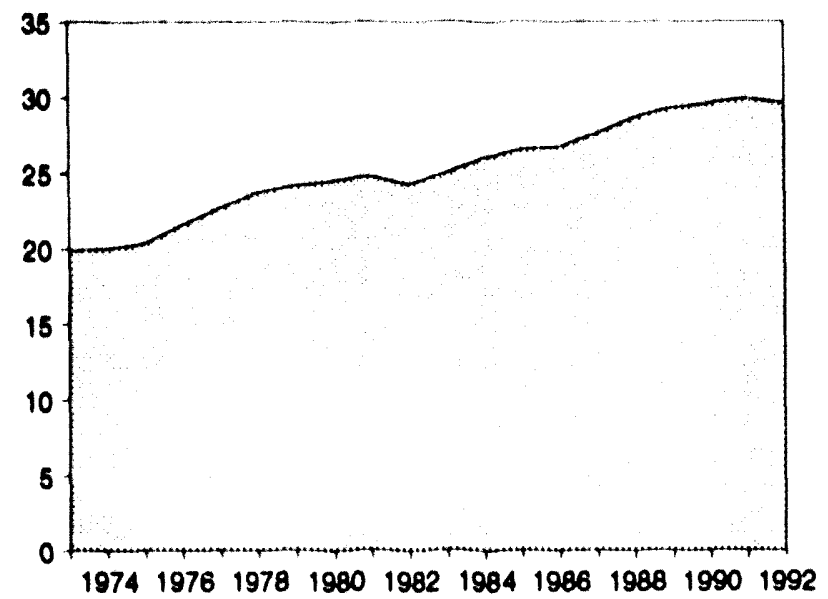

Input by Major Sources, 1973-1992

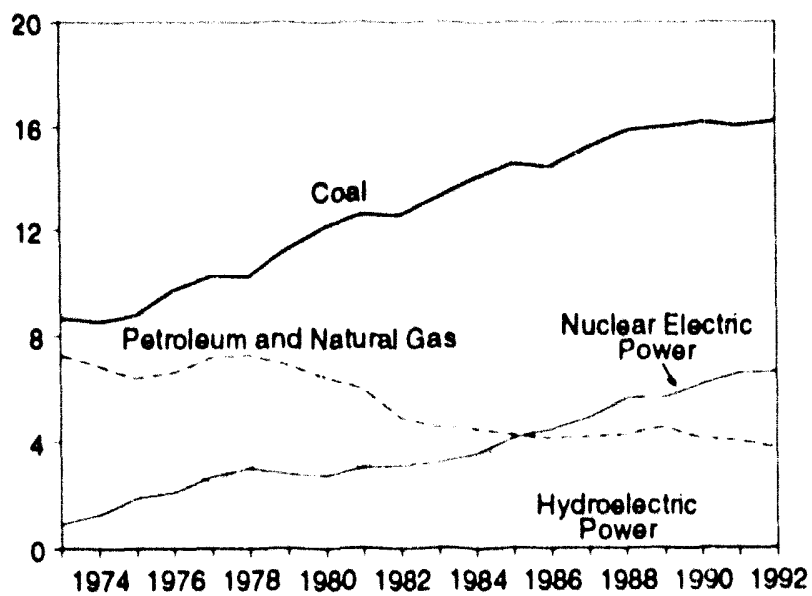

Total Input, January-August

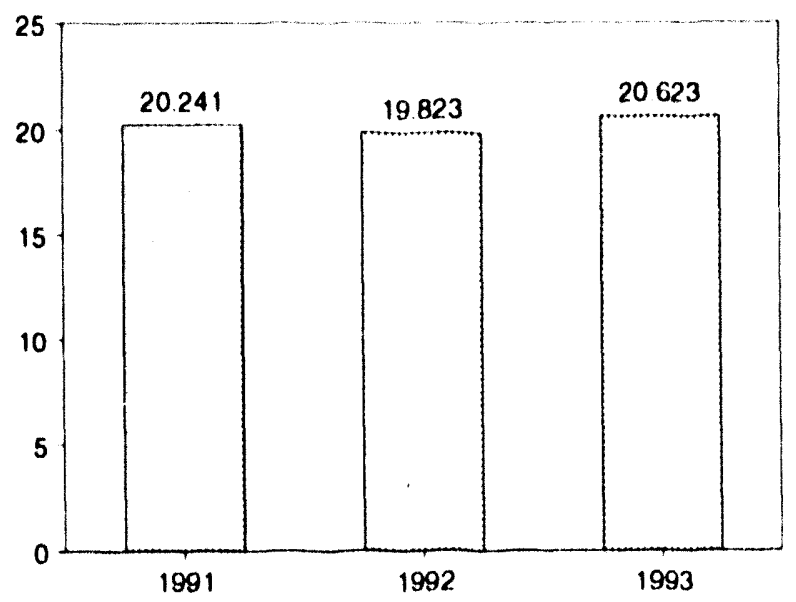

Total Input, Monthly

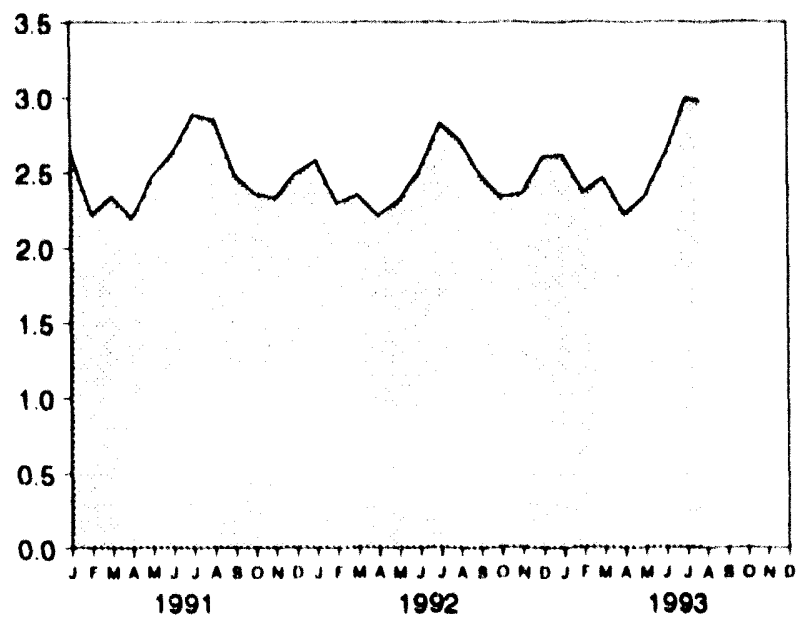

Input by Major Sources, Monthly

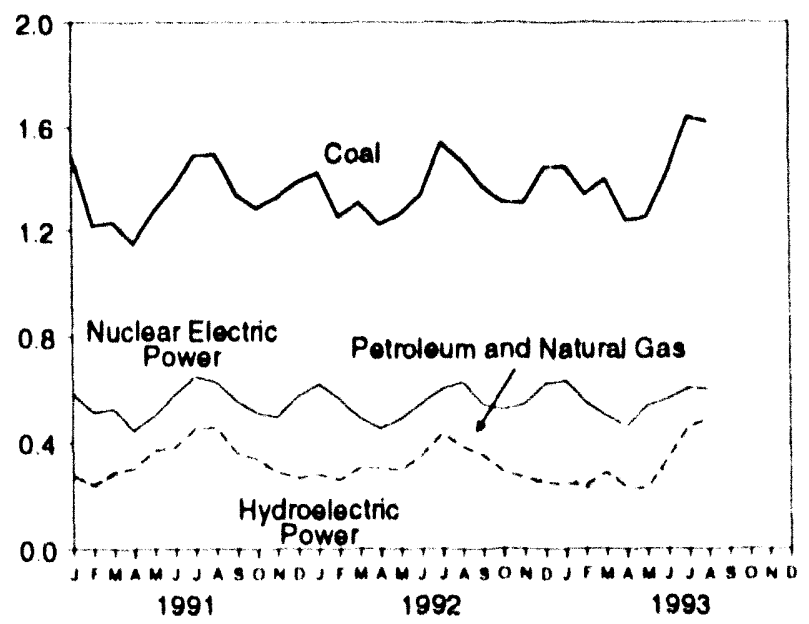

Input by Major Sources,

August 1993

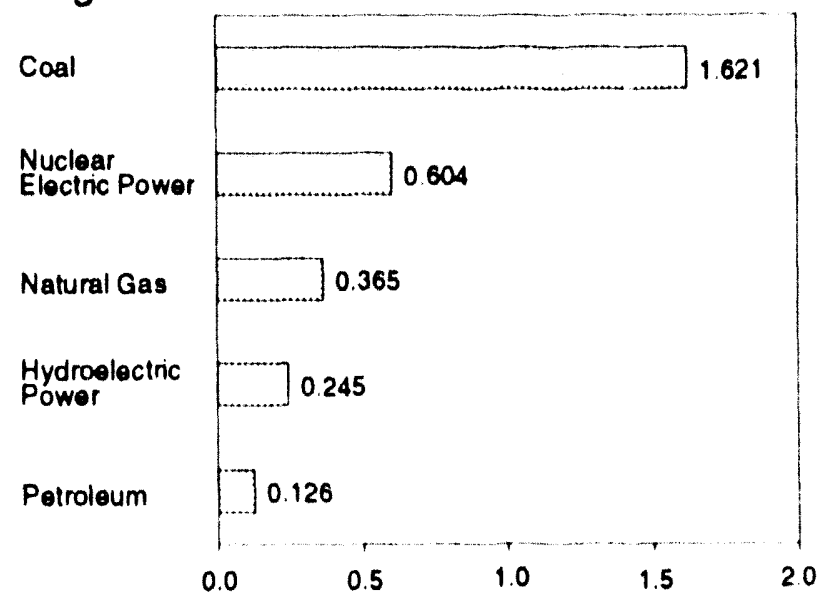

Note: Because vertical scales differ, graphs should nol be compared 
Table 2.6 Energy Input at Electric Utilities

(Quadrillion Btu)

\begin{tabular}{|c|c|c|c|c|c|c|c|}
\hline & Cod & Notured & Petroleumb & $\begin{array}{l}\text { Nucher } \\
\text { Electurte } \\
\text { Pouner }\end{array}$ & $\begin{array}{l}\text { Hydre- } \\
\text { elestule } \\
\text { Ponerc }\end{array}$ & Otherd & Total \\
\hline 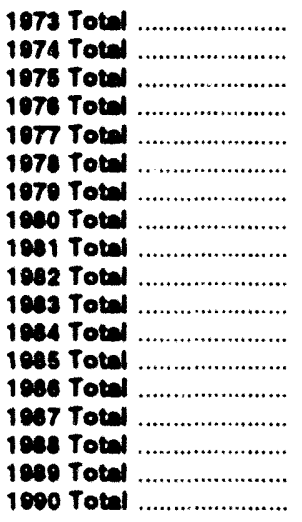 & 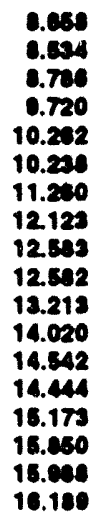 & $\begin{array}{l}3.740 \\
3.810 \\
3.240 \\
3.162 \\
3.204 \\
3.207 \\
3.619 \\
3.010 \\
3.700 \\
3.342 \\
2.009 \\
3.220 \\
3.100 \\
2.601 \\
2.038 \\
2.700 \\
2.071 \\
2.012\end{array}$ & 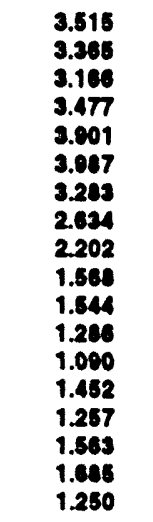 & $\begin{array}{l}0.010 \\
1.272 \\
1.000 \\
2.111 \\
2.702 \\
3.024 \\
2.770 \\
2.700 \\
3.000 \\
3.131 \\
3.203 \\
3.655 \\
4.141 \\
4.471 \\
4.009 \\
5.031 \\
6.077 \\
6.141\end{array}$ & 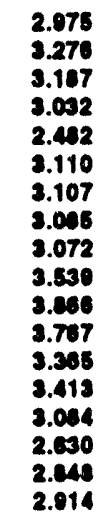 & $\begin{array}{l}0.048 \\
.056 \\
.072 \\
.001 \\
.002 \\
.068 \\
.080 \\
.114 \\
.127 \\
.108 \\
.133 \\
.174 \\
.213 \\
.232 \\
.245 \\
.236 \\
.217 \\
.202\end{array}$ & $\begin{array}{l}10.082 \\
20.022 \\
20.350 \\
21.574 \\
22.713 \\
23.724 \\
24.124 \\
24.505 \\
24.760 \\
24.270 \\
24.084 \\
26.020 \\
26.518 \\
26.704 \\
27.000 \\
28.640 \\
20.240 \\
20.500\end{array}$ \\
\hline 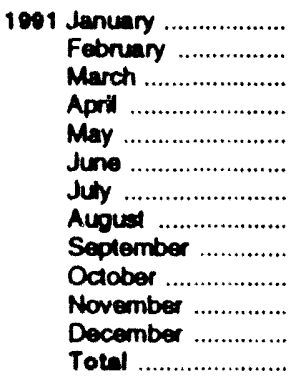 & $\begin{array}{l}1.482 \\
1.217 \\
1.230 \\
1.151 \\
1.271 \\
1.388 \\
1.491 \\
1.492 \\
1.337 \\
1.284 \\
1.324 \\
1.384 \\
10.028\end{array}$ & $\begin{array}{l}.177 \\
.150 \\
.188 \\
.221 \\
255 \\
268 \\
.338 \\
.335 \\
.269 \\
270 \\
.203 \\
.174 \\
2.050\end{array}$ & $\begin{array}{l}.099 \\
.092 \\
.092 \\
.084 \\
.115 \\
.117 \\
.118 \\
.123 \\
.091 \\
.068 \\
.084 \\
.094 \\
1.170\end{array}$ & $\begin{array}{l}.584 \\
.514 \\
.528 \\
.447 \\
.502 \\
.582 \\
.652 \\
.628 \\
.557 \\
.512 \\
.497 \\
.576 \\
.570\end{array}$ & $\begin{array}{l}275 \\
234 \\
.280 \\
.284 \\
.314 \\
283 \\
.272 \\
256 \\
218 \\
211 \\
.209 \\
247 \\
3.083\end{array}$ & $\begin{array}{l}.017 \\
.014 \\
.016 \\
.015 \\
.015 \\
.016 \\
.016 \\
.016 \\
.015 \\
.016 \\
.017 \\
.017 \\
.191\end{array}$ & $\begin{array}{r}2.634 \\
2.221 \\
2.344 \\
2.201 \\
2.472 \\
2.631 \\
2.887 \\
2.851 \\
2.488 \\
2.361 \\
2.333 \\
2.492 \\
29.016\end{array}$ \\
\hline 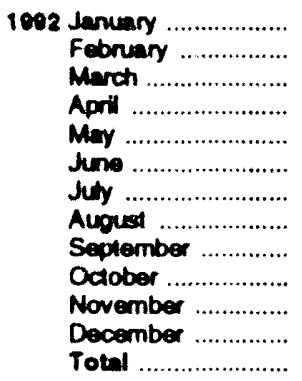 & $\begin{array}{l}1.420 \\
1.252 \\
1.304 \\
1.223 \\
1.281 \\
1.334 \\
1.536 \\
1.470 \\
1.372 \\
1.307 \\
1.303 \\
1.443 \\
1.224\end{array}$ & $\begin{array}{r}.173 \\
.174 \\
213 \\
.236 \\
242 \\
272 \\
.342 \\
.310 \\
280 \\
218 \\
.184 \\
.180 \\
2.026\end{array}$ & $\begin{array}{l}.108 \\
.087 \\
.092 \\
.069 \\
.056 \\
.080 \\
.092 \\
.076 \\
.074 \\
.073 \\
.074 \\
.070 \\
.051\end{array}$ & $\begin{array}{l}.621 \\
.587 \\
.492 \\
.454 \\
.490 \\
.550 \\
.602 \\
.650 \\
.547 \\
.524 \\
.545 \\
.624 \\
.6 .046\end{array}$ & $\begin{array}{l}243 \\
204 \\
235 \\
220 \\
252 \\
255 \\
240 \\
218 \\
.202 \\
201 \\
228 \\
274 \\
2.773\end{array}$ & $\begin{array}{l}.017 \\
.015 \\
.017 \\
.015 \\
.016 \\
.016 \\
.016 \\
.017 \\
.015 \\
.016 \\
.016 \\
.016 \\
.192\end{array}$ & $\begin{array}{r}2.583 \\
2.290 \\
2.354 \\
2.216 \\
2.317 \\
2.507 \\
2.827 \\
2.720 \\
2.491 \\
2.330 \\
2.359 \\
2.607 \\
{ }_{2} 20.613\end{array}$ \\
\hline 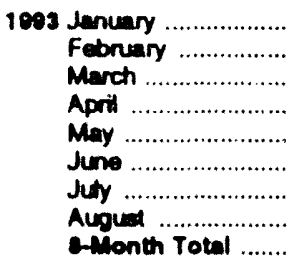 & $\begin{array}{r}1.446 \\
1.338 \\
1.395 \\
1.239 \\
1.250 \\
1.417 \\
1.638 \\
1.621 \\
11.342\end{array}$ & $\begin{array}{l}.168 \\
.168 \\
.198 \\
.178 \\
.171 \\
.261 \\
.341 \\
.365 \\
1.040\end{array}$ & $\begin{array}{l}.077 \\
.074 \\
.090 \\
.055 \\
.056 \\
.083 \\
.121 \\
.126 \\
.093\end{array}$ & $\begin{array}{l}.634 \\
.551 \\
.501 \\
.464 \\
.541 \\
.565 \\
.607 \\
.604 \\
\mathbf{4 . 4 9 7}\end{array}$ & $\begin{array}{l}276 \\
227 \\
.263 \\
276 \\
.314 \\
287 \\
.275 \\
245 \\
2.164\end{array}$ & $\begin{array}{l}.016 \\
.015 \\
.016 \\
.015 \\
.014 \\
.014 \\
.015 \\
.015 \\
.120\end{array}$ & $\begin{array}{l}2.617 \\
2.360 \\
2.464 \\
2.228 \\
2.347 \\
2.627 \\
2.996 \\
2.978 \\
20.620\end{array}$ \\
\hline $\begin{array}{l}1002 \text { - Wonth Total ....... } \\
1001 \text { E-Month Total ....... }\end{array}$ & $\begin{array}{l}10.090 \\
10.009\end{array}$ & $\begin{array}{l}1.000 \\
1.041\end{array}$ & .100 & $\begin{array}{l}4.409 \\
4.437\end{array}$ & $\begin{array}{l}1.000 \\
2.100\end{array}$ & $\begin{array}{l}.128 \\
.129\end{array}$ & $\begin{array}{l}10.020 \\
20.241\end{array}$ \\
\hline
\end{tabular}

- Includes supplemertal gaseous fuals.

- Petrobum products reponted as "oll consumed in ateam plants" through 1979 and "heavy oit" from 1980 lonward, which are sseumed to be residual luel oll; petroleum proctucis reported es "oll consumed in gas turbine and intemal combustion engine plants" throuch 1979 and "liati ofl" from 1880 tonward, which sire assumed to be distilate fuel oil, kerosene, and petroloum coke. c Includes net imports of electriclly.

$\therefore$ "Other" is electricity cenerated for distribution from wood, waste, geothermal, wind, photovoltaic, end soler thermal energy.

Notes: - Geogrephic covernge is the 50 States and the District of Columbia.

- Totals may not equal sum of components due to independent rounding.

Additional Notes and Sources: See end of section. 


\section{Energy Consumption Notes and Sources}

The data in this section of the Monthly Energy Review $(M E R)$ are obtained initially from a group of energy-related surveys, typically called "supply surveys," conducted by the Energy Information Administration (EIA). Supply surveys are those surveys directed to suppliers and marketers of specific energy sources. They measure the quantities of specific energy sources produced, or the quantities supplied to the market, or both. The data obtained from the EIA's supply surveys are integrated to yield the summary consumption statistics published in this section (and in Section 1) of the $M E R$. Users of the EIA's energy consumption statistics should be aware of a second group of energy-related surveys, typically called "consumption surveys." Consumption surveys gather information on the types of energy consumed by end users of energy, along with the characteristics of those end users that can be associated with energy use. For example, the Manufacturing Energy Consumption Survey belongs to the consumption survey group because it collects information directly from end users (the manufacturing establishments). There are important differences between the supply and consumption surveys that need to be taken into account in any analysis that uses both data sources. For information on those differences, see Energy Consumption by End-Use Sector, A Comparison of Measures by Consumption and Supply Surveys, DOE/EIA-0533, Energy Information Administration, Washington, DC, April 6, 1990. The numbered notes that follow elaborate on essential information in Section 2.

1. Total Energy Consumed: Total energy consumed includes coal, natural gas (including supplemental gaseous fuels), petroleum products supplied, electric utility and industrial generation of bydroelectric power, net imports of electricity generated from hydroelectric power, and electricity generated from nuclear power. Total energy consumed also includes electricity generated from wood, waste, geothermal, wind, photovoltaic, and solar thermal energy but excludes other energy obtained from those sources because consistent historical data are not available.

2. Economic Sectors: Energy use is assigned to the major economic sectors according to the following guidelines as closely as possible:

- Residential-All private residences, whether occupied or vacant, owned or rented, including single-family homes, multifamily housing units, and mobile homes. Secondary homes, such as summer homes, are also included. Institutional housing, such as school dormitories, hospitals, and military barracks, generally are not included in the residential sector; they are included in the commercial sector.

- Commercial-Business establishments that are not engaged in transportation or in manufacturing or other types of industrial activity (agriculture, mining, or construction). Commercial establishments include hotels, motels, restaurants, wholesale businesses, retail stores, laundries, and other service enterprises; religious and nonprofit organizations; health, social, and educational institutions; and Federal, State, and local governments. Street lights, pumps, bridges, and public services are also included if the establishment operating them is considered commercial.

- Industrial-Manufacturing industries, which make up the largest part of the sector, along with mining, construction, agriculture, fisheries, and forestry. Establishments in the sector range from steel mills to small farms to companies assembling electronic components.

- Transportation-Private and puhlic vehicles that move people and commodities. Included are automobiles, trucks, buses, motorcycles, railroads and railways (including streetcars), aircraft, ships, barges, and natural gas pipelines.

- Electric Utility-Privately and publicly owned establishments that generate, transmit, distribute, and sell electricity primarily for use by the public and meet the definition of an electric utility. Nonutility power producers are not included in the electric utility sector.

Although the end-use allocations are made according to these aggregations as closely as possible, some data are collected by using different classifications. For example, data on agricultural use of natural gas are collected and reported in the commercial sector, rather than in the industrial sector. Since agricultural use of natural gas cannot be identified separately, it is included in the commercial sector in this report. Another example is master-metered condominiums and apartments, and buildings with a combination of residential and commercial units. In many cases, the metering and billing practices cause residential energy usage of electricity, natural gas, or fuel oil to be included in the commercial sector. No adjustments for these discrepancies were made.

3. Conversion Factors: See the conversion factors listed in Appendix $\mathrm{A}$.

4. Coal: Coal is anthracite, bituminous coal (including subbituminous coal), and lignite. Sources:

- 1973-September 1977: U.S. Department of the Interior (DOI), Bureau of Mines (BOM), Minerals Yearbook and Minerals Industry Surveys.

- Electric Utilities-October 1977 forward: Energy Information Administration (EIA), Form EIA-759 (formerly Form FPC-4), "Monthly Power Plant Report."

- Other Industrial-October 1977-December 1979: EIA, Form EIA-3, "Monthly Coal Consumption Report - Manufacturing Plants"; January 1980 for- 
ward: EIA, Form EIA-3, "Quarterly Coal Consumption Report - Manufacturing Plants," and Form EIA-6, "Coal Distribution Report."

- Coke Plants-October 1977-December 1980: EIA, Form EIA-5/5A, "Coke and Coal Chemicals - Monthly/Annual"; January 1981-December 1984: EIA, Form EIA-5/5A, "Coke Plant Report Quarterly/Annual Supplement"; January 1985 forward: EIA, Form EIA-5/5A, "Coke Plant Report," quarterly.

- Residential and Commercial-October 1977. December 1979: EIA, Form EIA-2, "Monthly Coal Report, Retail Dealers - Upper Lake Docks"; January 1980 forward: EIA, Form EIA-6, "Coal Distribution Report."

5. Natural Gas: Natural gas consumption by end use is based on data presented in Table 4.3 of this report. For Section 2 calculations, lease and plant fuel consumption are added to industrial deliveries, and pipeline fuel represents transportation use of natural gas. Values in Btu are derived by using the conversion factors provided in Appendix A. Sources:

- 1973-1975: DOI, BOM, Minerals Yearbook, "Natural Gas" chapter.

- 1976-1978: EIA, "Energy Data Reports," Natural Gas, Annual.

- 1979: EIA, Natural Gas Production and Consumption 1979.

- 1980-1992: EIA, Natural Gas Annual.

- 1993: EIA, Natural Gas Monthly.

- Electric Utilities-1973-1976: Form FPC-4, "Monthly Power Plant Report"; 1977-1981: Federal Energy Regulatory Commission (FERC), Form FPC-4, "Monthly Power Plant Report"; 1982 forward: EIA, Form EIA-759, "Monthly Power Plant Report."

- American Gas Association, "Monthly Gas Utility Statistical Report," residential and commercial monthly sales data for 1973-1979, which are used to estimate monthly consumption values from EIA annual consumption values.

6. Petroleum: Petroleum consumption by end use is the sum of all individual petroleum products estimated to be consumed in each end-use sector. First, total consumption by product is determined. Petroleum consumption in this section of the Monthly Energy Review (MER) is the series called "petroleum products supplied" in Section 3. Sources for petroleum products supplied by individual products are:

- 1973-1975: DOI, BOM, Mineral Industry Surveys, "Petroleum Statement, Annual."

- 1976-1980: EIA, Energy Data Reports, "Petroleum Statement, Annual."

- 1981-1991: EIA, Petroleum Supply Annual.

- 1992 and 1993: EIA, Petroleum Supply Monthly.
Specific petroleum products' end-use allocation procedures follow:

- Avlation Gasoline-All product supplied is assigned to the transportation sector.

- Asphalt-All product supplied is assigned to the industrial sector.

- Distillate Fuel-Product supplied is assigned to electric utilities and non-electric utilities as follows:

\section{Electric Utilities, All Periods.}

Monthly and annual consumption for 1973-1979 is assumed to be the amount of oil (minus small amounts of kerosene and kerosene-type jet fuel deliveries) reported as consumed in internal combustion and gas turbine engine plants. From January 1980, electric utility consumption of distillate fuel is assumed to be the petroleum products reported as "light oil" (minus small amounts of kerosene deliveries through 1982) consumed at electric utilities.

Sources: 1973-September 1977: FPC, Form FPC4, "Monthly Power Plant Report"; October 19771981: FERC, Form FPC-4, "Monthly Power Plant Report"; 1982 forward: EIA, Form EIA-759, "Monthly Power Plant Report."

\section{Sectors Other Than Electric Utilities, Annual Es- timates Through 1991.}

The aggregate non-electric utility use of distillate fuel is total distillate fuel supplied minus the electric utility consumption. The non-electric utility annual consumption totals are allocated to the individual non-electric utility sectors (residential, commercial, industrial, and transportation) in proportion to the share of "adjusted sales" of each end-use sector, as reported in EIA's Fuel Oil and Kerosene Sales report series (DOE/EIA-0535), which is based primarily on data collected by Form EIA-821, previously Form EIA-172. "Adjusted sales" are sales that have been adjusted at the PAD district level to equal EIA volume estimates of petroleum products supplied in the U.S. market. Following are notes on the individual sector groupings:

- Since 1979, the residential sector adjusted sales total is directly from the Sales reports. Prior to 1979 , each year's sales subtotal of the heating plus industrial category is split into residential, commercial, and industrial (including farm) in proportion to the 1979 shares.

- Since 1979, the commercial sector adjusted sales total is directly from the Sales reports. Prior to 1979 , each year's sales subtotal of the heating plus industrial category is split into residential, commer- 
cial, and industrial (including farm) in proportion to the 1979 shares.

- Since 1979, the industrial sector adjusted sales total is the sum of the adjusted sales for industrial, farm, oil company, off-highway, diesel, and all other uses. Prior to 1979, each year's sales subtotal of the heating plus industrial category is split into residential, commercial, and industrial (including farm) in proportion to the 1979 shares, and this estimated industrial portion is added to oil company, off-highway diesel, and all other uses.

- The transportation sector adjusted sales total is the sum of the adjusted sales for railroad, vessel bunkering, on-highway diesel, and military uses for all years.

\section{Sectors Other Than Electric Utilities, Monthly Es- timates Through 1991.}

- Residential and commercial monthly consumption is estimated by allocating the annual estimates described above into months in proportion to each month's share of the year's sales of No. 2 heating oil as reported in the "Monthly Report of Heating Oil Sales" by the Ethyl Corporation from 1973. 1980 and the American Petroleum Institute for 1981 and 1982, and the EIA, Form EIA-782A, "Refiners'/Gas Plant Operators' Monthly Petroleum Product Sales Report," No. 2 Fuel Oil Sales to End Users and for Resale, since 1983.

- The transportation highway use portion is allocated into the months in proportion to each month's share of the year's total sales for highway use as reported by the Federal Highway Administration's Table MF-25, "Private and Commercial Highway Use of Special Fuels by Months." The remaining transportation use of distillate fuel (i.e., for railroads, vessel bunkering, and military use) is evenly distributed over the months, adjusted for the number of days per month.

- Industrial monthly estimates are made by subtracting the residential and commercial, transportation, and electric utility sector estimates from each month's total distillate fuel supplied.

\section{Sectors Other Than Electric Utilities, 1992 and 1993}

Each month's non-electric utility consumption subtotal is disaggregated into the major end-use sectors in proportion to the shares each sector held of the non-electric utility subtotal in the same month in 1991.

- Jet Fuel-Through 1982, small amounts of kerosene-type jet fuel were consumed by electric utilities. Kerosene-type jet fuel deliveries to electric utilities as reported on the Form FERC423 (formerly Form FPC-423) were used as estimates of this consumption. All remaining jet fuel (kerosene-type and naphtha-type) is consumed by the transportation sector.

- Kerosene-Total product supplied monthly is allocated to the major end-use sectors in proportion to annual sales grouped into end-use sectors from EIA's Fuel Oil and Kerosene Sales (Sales) reports (based primarily on data collected by Form EIA-821, previously Form EIA-172), as follows:

- Residential deliveries are directly from the Sales reports for 1979-1991. Sales for 1991 are used as estimates for succeeding periods. Prior to 1979 , each year's sales category called "heating" is split into residential, commercial, and industrial in proportion to the 1979 shares.

- Commercial sales are directly from the Sales reports for 1979-1991. Sales for 1991 are used as estimates for succeeding periods. Prior to 1979. each year's sales category called "heating" is split into residential, commercial, and industrial in proportion to the 1979 shares.

- Industrial sales are directly from the Sales reports for 1979-1991. Sales for 1991 are used as estimates for succeeding periods. Prior to 1979, each year's sales category called "heating" is split into residential, commercial and industrial in proportion to the 1979 shares, and this estimated industrial (including farm) portion is added to all other uses.

- Liquefied Petroleum Gases (LPG)-The annual shares of LPG's total consumption that are estimated to be consumed by each end-use sector are applied to each month's total LPG consumption (i.e., product supplied) to create monthly end-use consumption estimates. The annual enduse shares are calculated in the following manner:

- Sales of LPG to the residential and commercial sector are converted from thousand gallons per year to thousand barrels per year and are assumed to be the annual consumption of LPG by the sector.

- The quantity of LPG sold each year for consumption in internal combustion engines is allocated between the transportation and industrial sectors on the basis of data for special fuels used on highways published by the U.S. Department of Transportation, Federal Highway Administration, in Highway Statistics. The allocations of LPG sold for internal combustion engine use to the transportation sector range from a high of 67 percent in 1981 to a low of 37 percent in 1987.

- LPG consumed annually by the industrial sector is estimated as the difference between LPG total supplied and the estimated consumption of LPG by the sum of the residential and commercial sector and the transportation sector. The industrial sector includes LPG used by chemical plants as raw materials or solvents and used in the production of 
synthetic rubber; refinery fuel use; use as synthetic natural gas feedstock and use in secondary recovery projects; all farm use; LPG sold to gas utility companies for distribution through the mains; and a portion of the use of LPG as an internal combustion engine fuel.

The sources of the annual sales data for creating annual end-use shares are:

- 1973-1982: ElA's "Sales of Liquefied Petroleum Gases and Ethane" reports, based primarily on data collected by Form EIA-174.

- 1983: End-use consumption estimates for 1983 are based on 1982 end-use consumption because the collection of data under Fonn EIA-174 was discontinued after data year 1982.

- 1984-1991: American Petroleum Institute (API), "Sales of Natural Gas Liquids and Liquefied Refinery Gases," which is based on an LPG sales survey jointly sponsored by API, the Gas Processors Association, and the National Liquefied Petrolcum Gas Association.

- 1992 and 1993: The 1991 source is used to estimate succeeding periods.

- Lubricants-Total product supplied is allocated to the industrial and transportation sectors for all months according to proportions developed from annual sales of lubricants to the two sectors from U.S. Department of Commerce, Bureau of the Census, Current Industrial Reports, "Sales of Lubricating and Industrial Oils and Greases." The 1973 shares are applied to 1973 and 1974; the 1975 shares are applied to 1975 and 1976; and the 1977 shares are applied to 1977 forward.

- Motor Gasoline-Total product supplied monthly is allocated to the major end-use sectors in proportion to aggregations of annual sales categories created on the basis of the U.S. Department of Transportation, Federal Highway Administration, Highway Statistics, Tables MF-21, MF-24, and $\mathrm{MF}-25$, as follows:

- Commercial sales are the sum of sales for public non-highway use and miscellaneous and unclassified uses.

- Industrial sales are the sum of sales for agriculture, construction, and industrial and commercial use as classified in the Highway Statistics.

- Transportation sales are the sum of sales for highway use (minus the sales of special fuels, which are primarily diesel fuel and are accounted for in the transportation sector of distillate fuel) and sales for marine use.

- Petroleum Coke-The portion consumed by electric utilities is from Form EIA-759, "Monthly
Power Plant Report" (formerly Form FPC-4). The remaining petroleum coke is assigned to the industrial sector.

- Residual Fuel-Product supplied is assigned to electric utilities and non-electric utilities as follows:

\section{Electric Utilities, All Periods.}

Monthly and annual consumpticn for 1973.1979 is assumed to be the amount of oil reported as consumed in steam-electric power plants. From January 1980 forward, electric utility consumption of residual fucl is assumed to be the petroleum products reported as heavy oil consumed at clectric utilities.

Sources: 1973-September 1977: Form FPC-4, "Monthly Power Plant Report"; October 1977. 1981: FERC, Form FPC-4, "Monthly Power Plant Report"; 1982 forw: rd: EIA, Form EIA-75\%, "Monthly Power Plant Report."

Sectors Other Than Electric Utilties, Annual Estimates Through 1991.

The aggregate non-electric utility use of residual fuel is total residual fuel supplied minus the electric utility consumption. The non-electric utility annual totals are allocated into the individual non-electric utility sectors in proportion to the amount of residual fuel sold to end users, grouped into sectors from EIA's Fuel Oil and Kerosene Sales (Sales) reports (based primarily on data collected by Form EIA-821, previously Form EIA-172), as follows:

- Since 1979, commercial sales data are directly from the Sales reports. Prior to 1979, each year's sales subtotal of the heating plus industrial category is split into commercial and industrial in proportion to the 1979 shares.

- Since 1979, industrial sales data are the sum of sales for industrial, oil company, and all other uses. Prior to 1979, each year's sales subtotal of the heating plus industrial category is split into commercial and industrial in proportion to the 1979 shares, and this estimated industrial portion is added to oil company and all other uses.

- Transportation sales are the sum of sales for railroad, vessel bunkering, and military uses for all years.

Sectors Other Than Electric Utilities, Monthly Estimates Through 1991.

- Commercial sector monthly consumption is estimated by allocating the annual commercial sector estimates described above into months in proportion to each month's share of the year's sales of No. 2 fuel oil as reported in the "Monthly Report 
of Heating Oll Sales" by the Ethyl Corporation for 1973-1980 and the American Petroleum Institute for 1981 and 1982, and the EIA, Form EIA-782A, "Refiners'/Gas Plant Operators' Monthly Petroleum Product Sales Report," No. 2 Fuel Oil Sales to End Users and for Resale, since 1983.

- Transportation monthly estimates are made by evenly distributing the annual sector estimate over the months, adjusting for the number of days per month.

- Industrial monthly estimates are made by subtracting the commercial, transportation, and electric utility sector estimates from each month's lotal residual fuel supplied.

\section{Sectors Other Than Electric Utilutes, 1992 and 1993}

Each month's non-electric utility consumption subtotal is disaggregated into the major end-use sectors in proportion to the shares each sector held of the non-electric utility subtotal in the same month in 1991.

- Road Oll-All product supplied is assigned to the industrial sector.

- All Other Petroleum Products-The product supplied of all remaining petroleum products is assigned to the industrial sector.

7. Nuclear Electric Power and Wood, Waste, Geothermal, Wind, Photovoltalc, and Solar Thermal Energy Sources Connected to Electric Utility Distribution Systems: Sources:

- 1973-1976: FPC, Form FPC-4, "Monthly Power Plant Report."

- 1977-1981: FERC, Form FPC-4, "Monthly Power Plant Report."

- 1982 forward: EIA, Form EIA-759, "Monthly Power Plant Report."

8. Hydroelectric Power: Includes electricity generated by hydroelectric power at electric utilities, small amounts in the industrial sector, and net imports of electricity, which are assumed to be generated by hydroelectric power and are included in the electric utilities sector.

Sources for electric utilities sector:

- 1973-1976: FPC, Form FPC-4, "Monthly Power Plant Report."

- 1977-1981: FERC, Form FPC-4, "Monthly Power Plant Report."

- 1982 forward: EIA, Form EIA-759, "Monthly Power Plant Report."

Sources for industrial sector:
- 1973-1978: FPC, Form FPC-4, "Monthly Power Plant Report," for plants with generating capacity exceeding 10 megawatts, ens FPC. Form FPC. 12C. "Industrial Electric Generating Capacity," for all other plants.

- 1979: FPC, Form FPC-4, "Monthly Power Plant Report," for plants with generating capacity exceeding 10 megawatts and EIA estimates for all other plants.

- 1980 forward: Annual generation estimated by EIA as the average generation over the 6-year period of 1974.1979; monthly generation estimated to be in proportion to each month's hydroelectricity generation in the electric utility industry in 1980.

\section{Sources for imports and exports of electricity:}

- 1973-September 1977: Unpublished Federal Power Commission data.

- October 1977-1980: Unpublished Economic Regulatory Administration (ERA) data.

- 1981: DOE, Office of Energy Emergency Operations. "Report on Electric Energy Exchanges with Canada and Mexico for Calendar Year 1981," April 1982 (revised June 1982).

- 1982 and 1983: DOE, ERA, Electricity Exchanges Across International Borders.

- 1984-1986: DOE, ERA, Electricity Transactions Across International Borders.

- 1987 and 1988: DOE, ERA, Form ERA-781R, "Annual Report of International Electrical Export/lmport Data."

- 1989-1991: DOE, Assistant Secretary for Fossil Energy, Form FE-781-R, "Annual Report of International Elcctrical ExporU/mport Data."

- 1992 forward: EIA estimates based on preliminary data from the National Energy Board of Canada and DOE, Assistant Secretary for Fossil Energy.

9. Net Imports of Coal Coke: Net imports means imports minus exports, and a minus sign indicates that exports are greater than imports. Sources:

- 1973-1975: DOI, BOM, Minerals Yearbook, "Coke and Coal Chemicals" chapter.

- 1976-1980: EIA, Energy Data Report, "Coke and Coal Chemicals" annual.

- 1981: EIA, Energy Data Report, "Coke Plant Report," quarterly.

- 1982 forward: EIA, Quarterly Coal Report.

10. Electricity: End-use consumption of electricity is based on Table 7.2 sales data. "Other," which is primarily for use in government buildings, is added to the commercial sector, except for approximately 4 percent used by railroads and railways and attributed to the 
transportation sector. For 1973-1983 and 1992 forward, "Monthly Serles" data are used directly. For 1984. 1991. morithly estimates are created by dividing each month's "Monthly Series" value by the "Monthly Series" total for the year and multiplying by the "Annual Serics" value for the year. Kllowatthours are converted to Btu at the rate of 3,412 Btu per kilowatthour. See Table 7.2 for sources of the electricity sales data.

11. Electrical System Energy Losseas Electrical system energy Iosses are calculated as the difference between total energy input at electric utilities and the total energy content of electricity sold to end-use consumers. Most of those losses occur at steam-electric power plants (conventional and nuclear) in the conversion of heat energy into mechanical energy to turn electric generators. The loss is a thermodynanically necessary feature of the steam-electric cycle. Part of the energy input-10-output losses is a result of imputing fossil energy equivalent inputs for hydroclectric and other energy sources, since there is no generally accepted practice for measuring those thermal conver. sion rates. In addition to conversion losses, other losses include power plant use of electricity, transmission and distribution of electricity from power plants to end-use consumers (also called "line losses"), and unaccounted for electricity. Total losses are allocated to the end-use sectors in proportion to each sector's share of total electricity sales. Overall, approximately 67 percent of total energy input is lost in conversion; of electrictiy generated, approximately 5 percent is lost in plant use and 9 percent is lost in transmission and distribution. Calculated electrical system energy losses thay be less than actual losses, because primary consumption does not include the energy equivalent of utility purchases of electricity from non-electric utilities and from Canada and Mexico, although they are included in electricity sales. 


\section{Section 3. Petroleum}

Total petroleum imports ${ }^{2}$ averaged 8.7 million harrels per day in October 1993, 3 percent ${ }^{3}$ higher than both the previous month's rate and the October 1992 rate.

In October 1993, 17.5 million barrels per day of petroleum products were supplied for domestic use. slightly higher than the October 1992 rate. Motor gasoline accounted for $\mathbf{4 3}$ percent of the total; distillate fuel oil, 19 percent; and residual fuel oil, 5 percent.

Motor gasoline supplied during October 1993 averaged 7.6 million barrels per day, slightly lower than the previous month's rate but 4 percent higher than the October 1992 rate. Total motor gasoline stocks were 209 million barrels at the end of October 1993, 2 million harrels above the stock level in the previous month and 5 million barrels above the level 1 year carlicr.
Distillate fuel oll supplled during October 1993 averaged 3.3 million barels per day, 12 pereent higher than the previous month's rate and 10 percent higher than the October 1992 rate. Disullate fuel oil ending stocks for October 1993 were 138 million barrels, 8 million barrels above the stock level in the previous month and 1 million barrels above the stock level I year carlicr.

Residual fuel oil supplied in October 1993 averaged 0.8 million barrels per day, 34 percent lower than the previous month's rate and 28 percent lower than the October 1992 ratc. Residual fuel oil stocks measured 45 million barrels at the end of October 1993, 3 million barrels above the stock level in the previous month and the same as the stock level I year earlier.

Estimates (except of crude production) for the most current month are based on Energy Information Administration (EIA) weekly data and will be revised $10 \mathrm{conform}$ with data from the EIA Petroleum Reporting System as available. For the most recent month, crude production is an EIA estimate based on historical and provisional data through July 1993.

\footnotetext{
${ }^{2}$ Total import data Include imports Into the strategle Petroleum Reserve.

${ }^{3}$ Percentage changes are based on numbers shown in the lollowing lables.
} 
Table 3.1. Petroloum Overvlow: Flold Production, stock Change, Potroloum Producte Supplied, and Ending Stocke

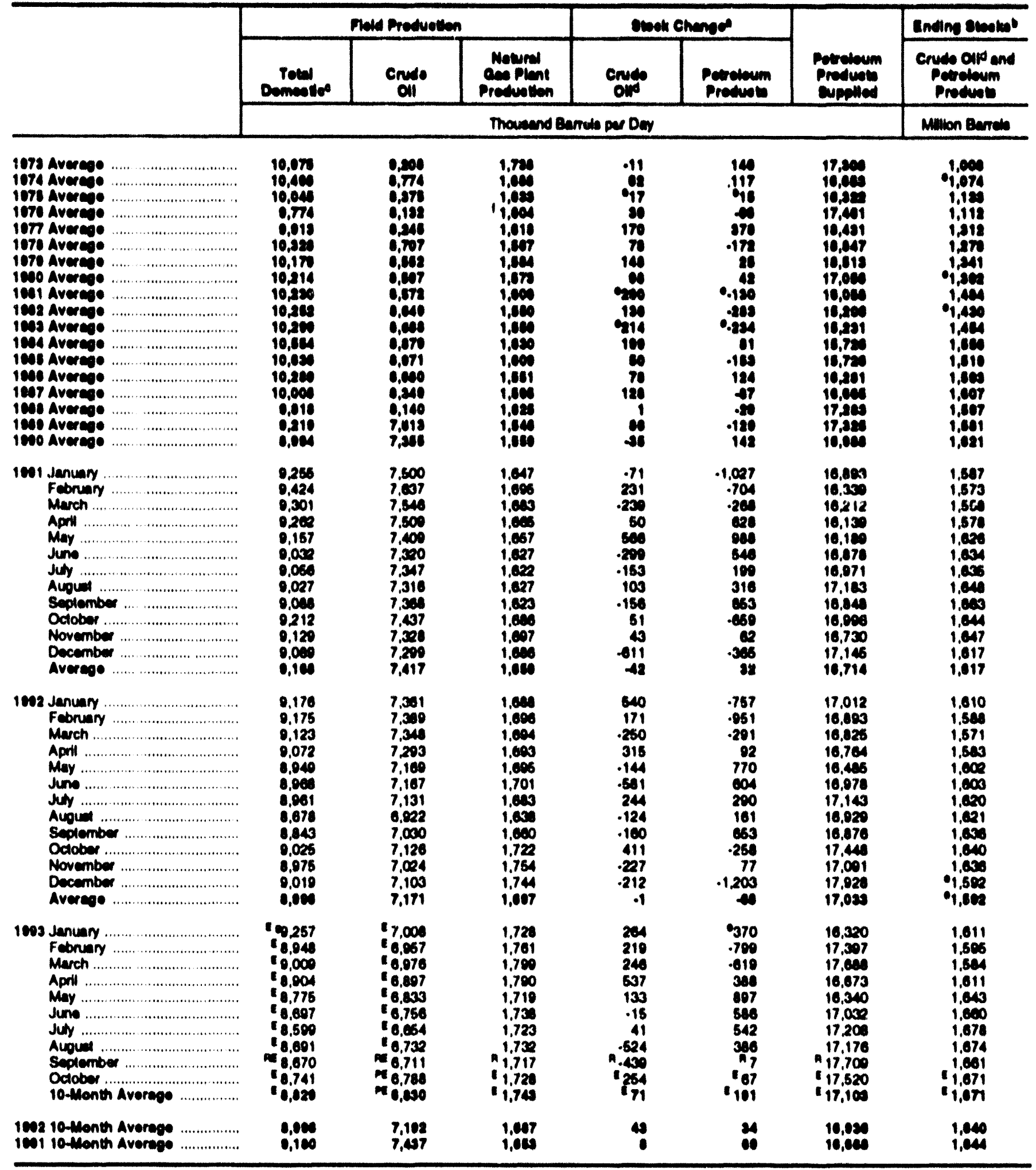

- A negetwe number indlcates a decreses in stocks and a posilve number indlcales en increase.

- stocke ar latuls es of end ol pertod.

- Inctudes crude oll, nalural gas plart liquide, and other liquide.

- Includes stocks located in the Siratecic Petroleum Reeerve.

- Ses Note 4 at end al cuction.

I seo Note 6 at and at section.

- Becining in 1003, includes luel ethand blanded into inished molor gesoline and oxyoenate produdion irom merchant MTBE (mothyl ientary

\footnotetext{
buyt cther) plents.

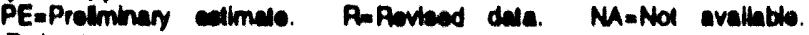
EoElmato.

Notes: - Crude ol includes bese condensale. - Geographic coverage ib the 60 States and the Distict of Columble.

Sources: - 1075-1020: Energy Intormation Adminituration (EL). Petroleum Suppy Monthy, February 1803, Tablo S1. • 1001 forwerd: EU, Potroleum Suppy Monthy, November 1903, Table 81.
} 
Table 3.1b Potroloum Overvlow: Imports, Exports, and Not Imports

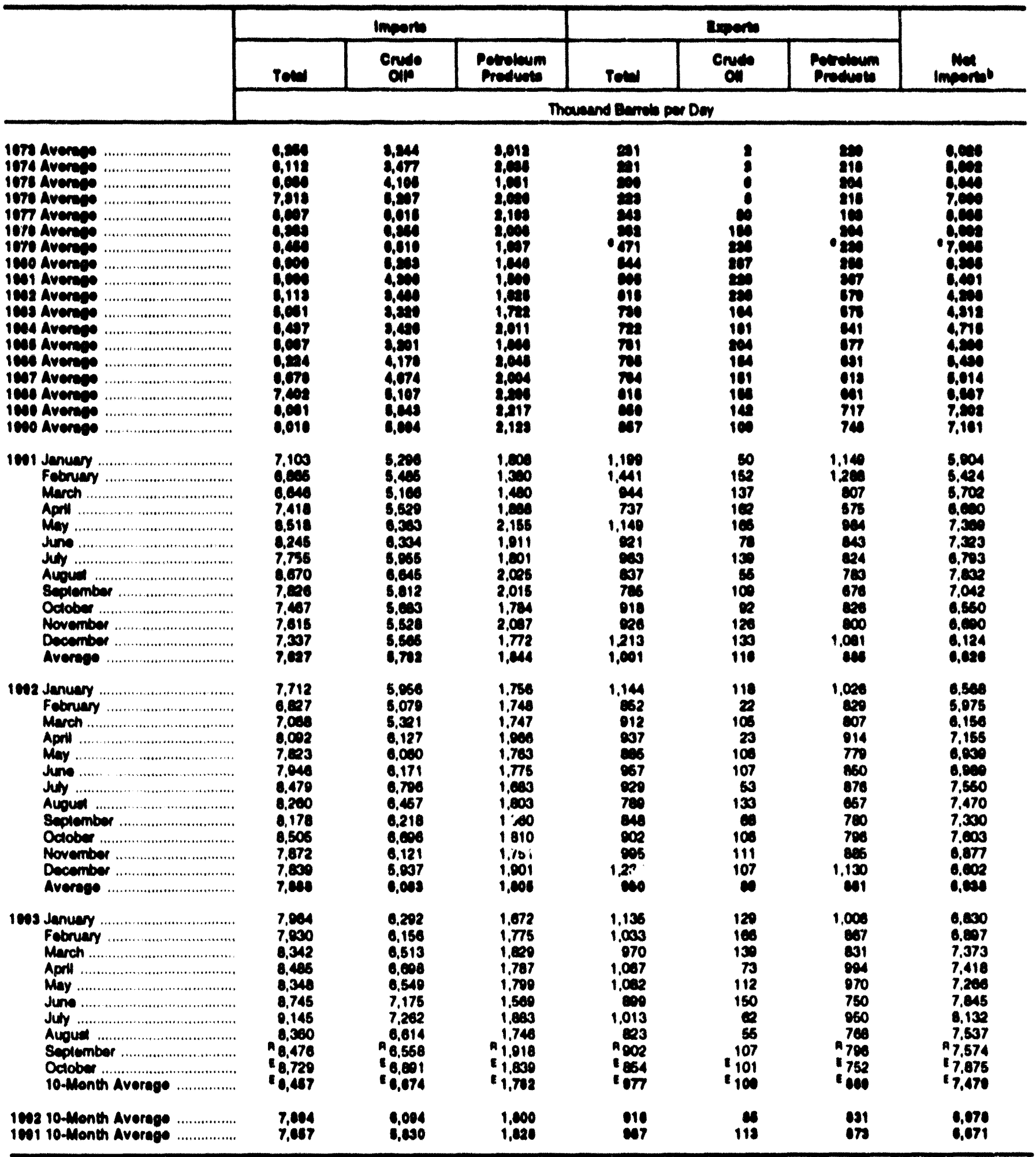

- Includes cnde oll lor storsoe in the Stratecic Petroleum Reserve.

- Nel imports equal imports minus exports.

- So Nole 8 at and of section.

AnReviend data. EnEstimale.

Noles: - Ceographic coverage is the 50 sutea and the Diatrict of Columbia.
- Totels may not equal sum of components due to independent tounding Sources: - 1075-1000: Energy Intormation Administration (EU). Puratum Supoy Moniny, February 1003, Tabie 81. - 1081 lonwerd: EU, Puroteum Supply Monthy. November 1093. Table 81 . 
Figure 3.1 Potroloum Overviow

(Million Barrole por Day)

Overvlow, January-October

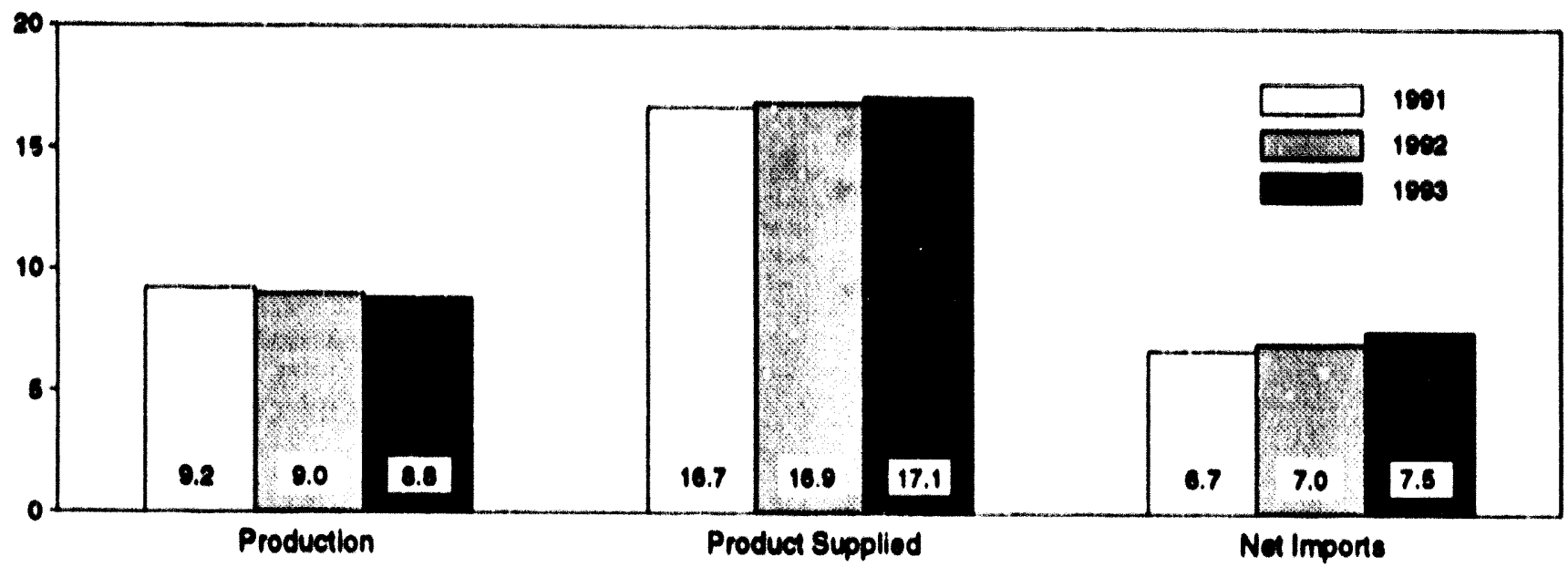

Overview, 1973-1992

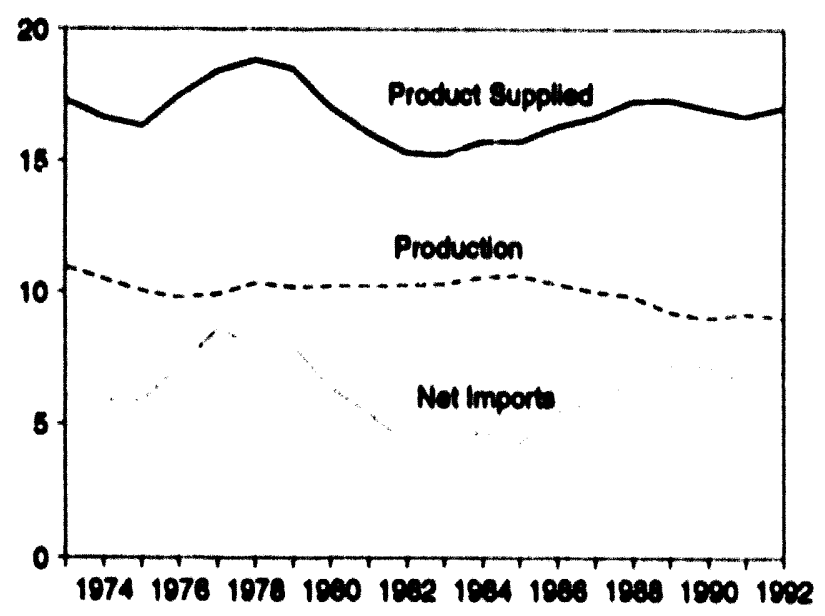

Crude Oll Production, 1973-1992

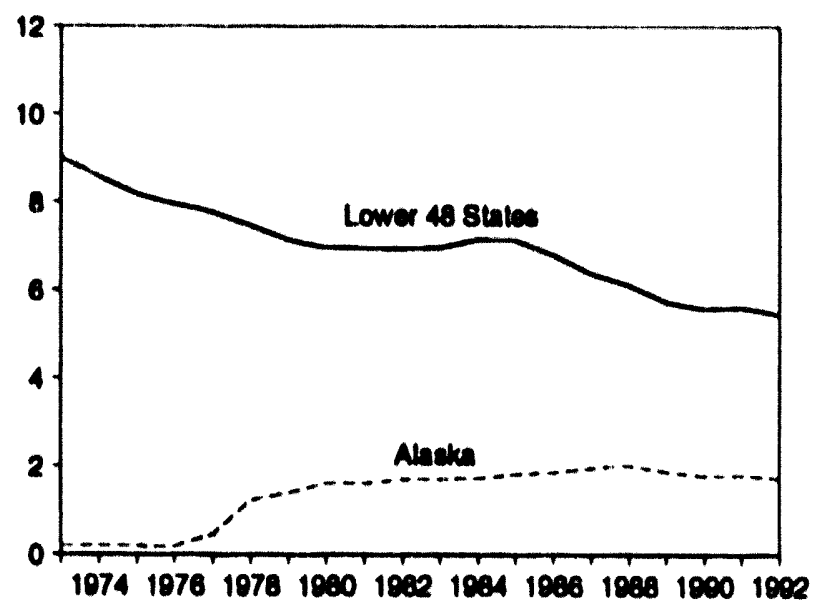

Production, 1973-1992

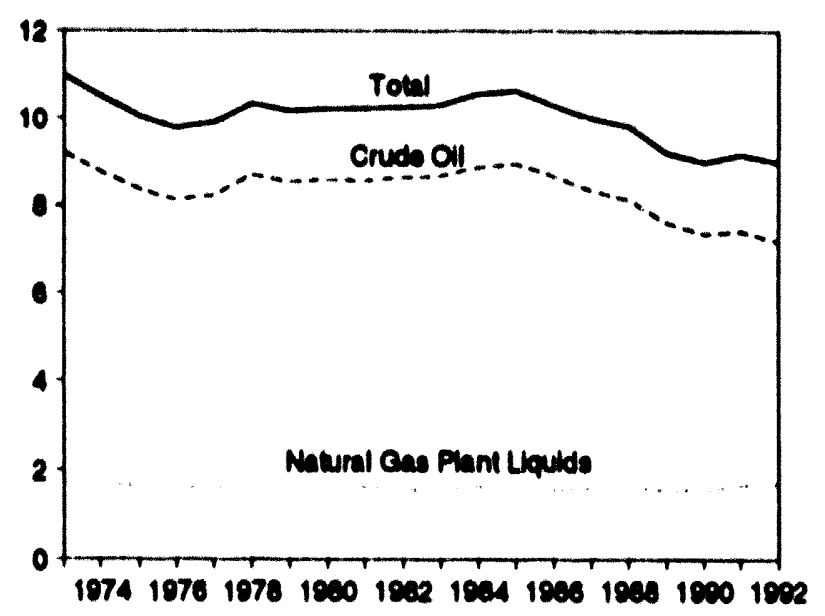

Total Production, Monthly

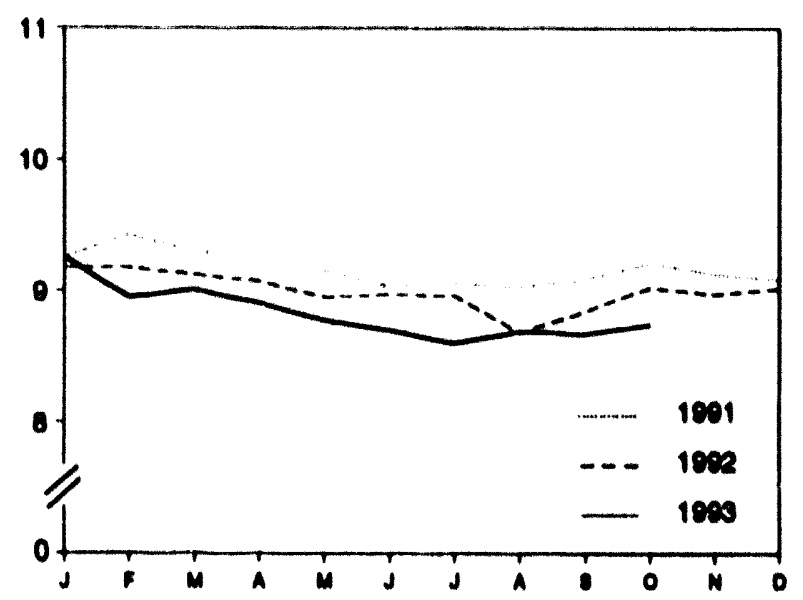

Nolo: Because verticel ecelos diler, eraphe should not be compered. sourom: Trbise 3.1a, 3.1b, and $3.2 a$. 
Flgure 3.1 Potroloum Overviow (Continued)

(Milition Barrels per Day, Except as Noted)

Product Supplied, 1973-1992

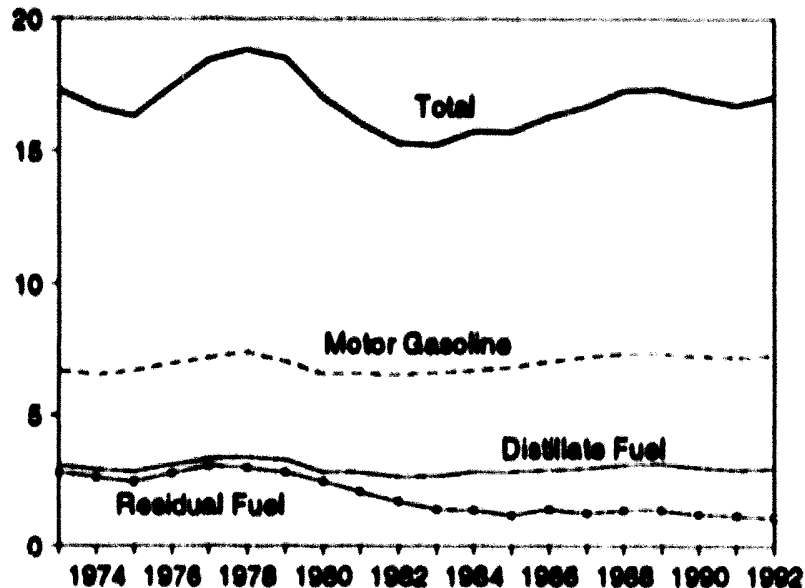

Total Product Supplied, Monthly

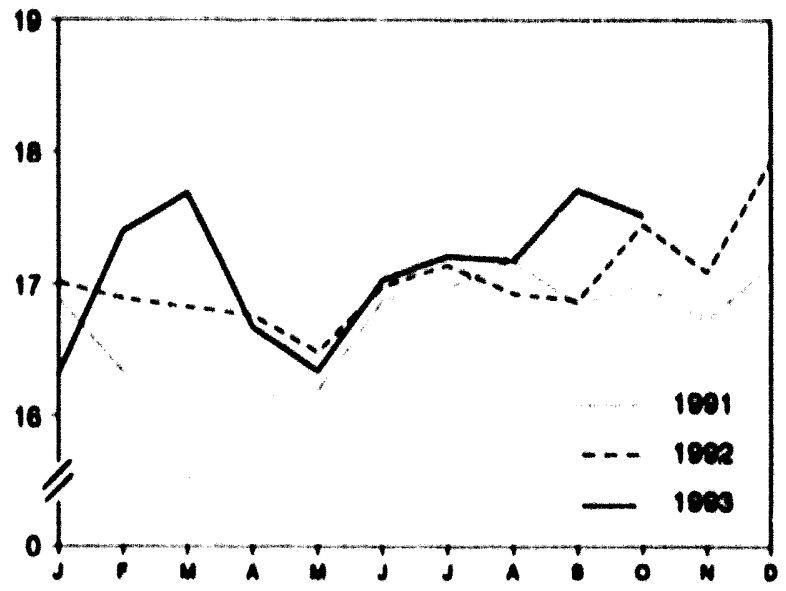

Imports from Selected Countries, September 1993

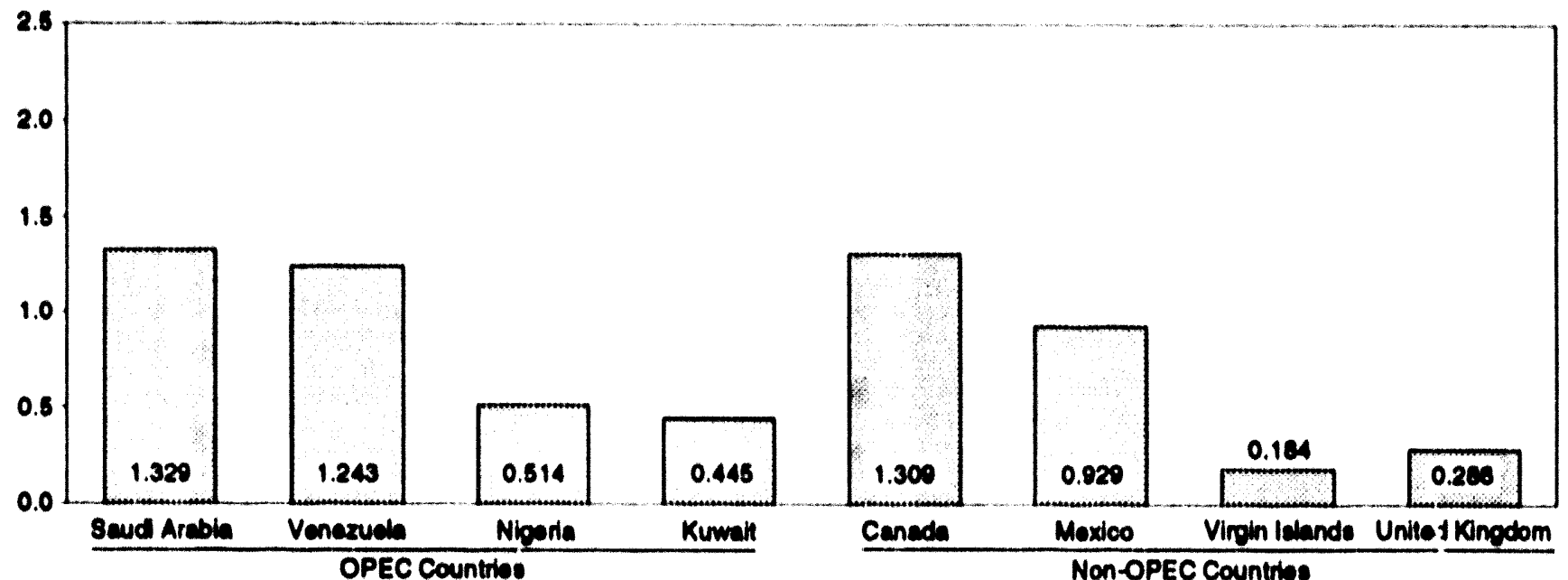

Stocks, End of Year, 1973-1992

Total Petroleum Stocks, End of Month

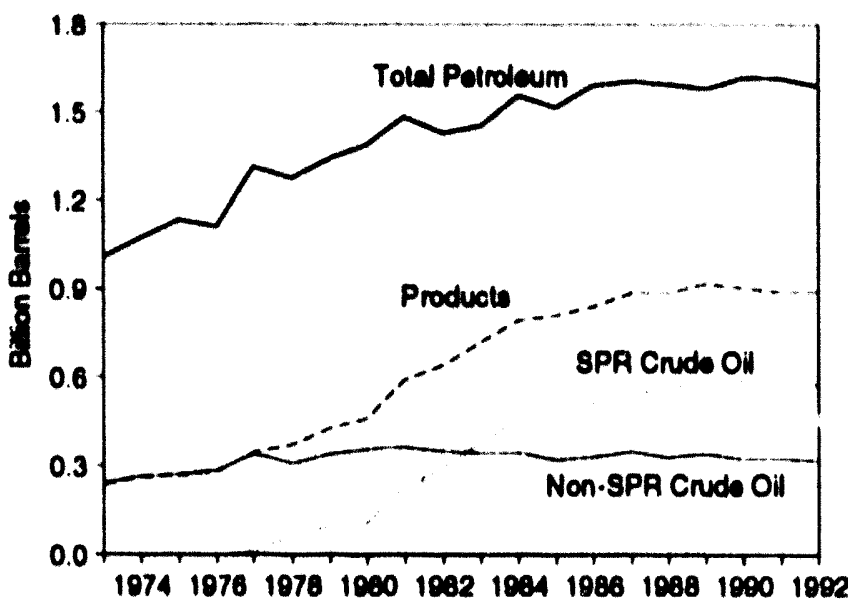

Nole: OfEC - Organtzallon of Pelrobum Exponting Countites. Note: Because ventical scales oner, greph should not be compared. Sources: Tebles 3.1e, 3.2b, 3.3a, 3.3b, 3.3d-3.3h, 3.4, 3.5, and 3.6.

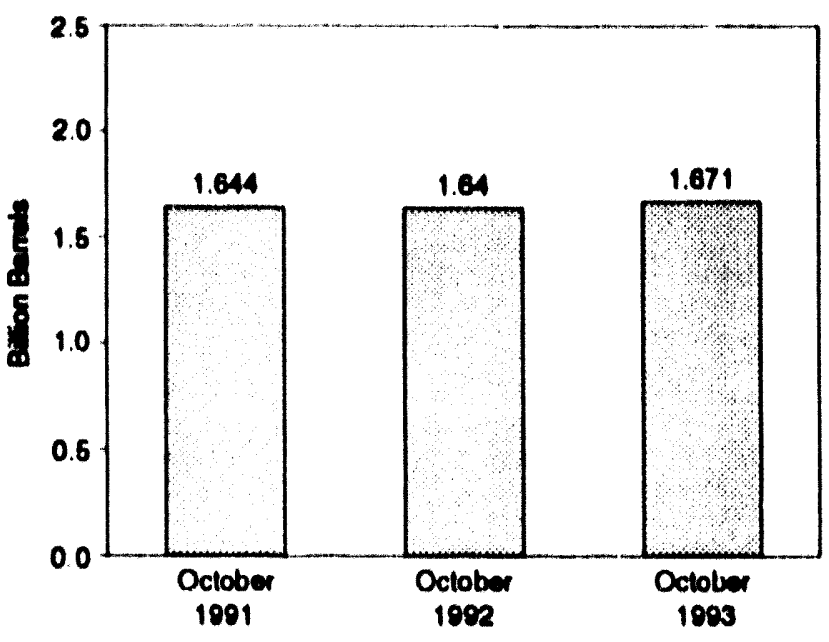

Note: SPR $=$ Strategis Potrobum Reservo. 
Tablo 3.2a Crudo OII Supply and Dlapostiton: Supply

\begin{tabular}{|c|c|c|c|c|c|c|c|}
\hline & \multicolumn{7}{|c|}{ supply } \\
\hline & \multicolumn{2}{|c|}{ Fide Preduetion } & \multicolumn{3}{|c|}{ Imperts } & \multirow{2}{*}{$\begin{array}{l}\text { Uncecounied } \\
\text { rer Crude } \\
\text { oflo }\end{array}$} & \multirow{2}{*}{$\begin{array}{l}\text { cruce oll } \\
\text { uned } \\
\text { orretye }\end{array}$} \\
\hline & $\begin{array}{l}\text { Tetal } \\
\text { Dements }\end{array}$ & Alacken & Totyl & $\cos ^{\circ}$ & One & & \\
\hline & \multicolumn{7}{|c|}{ Thounand Barnis per Day } \\
\hline 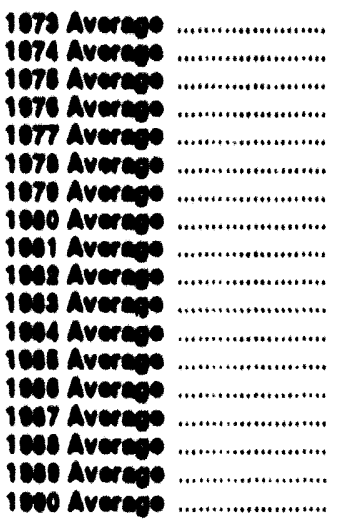 & 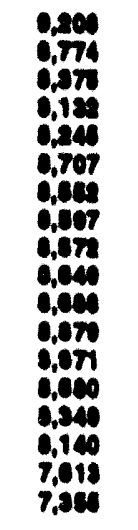 & 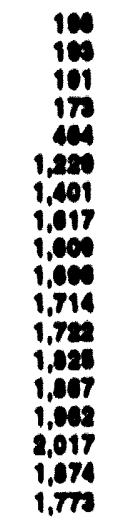 & 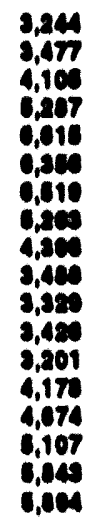 & $\begin{array}{r}= \\
= \\
81 \\
01 \\
101 \\
14 \\
106 \\
104 \\
107 \\
110 \\
4 \\
71 \\
61 \\
67 \\
87\end{array}$ & 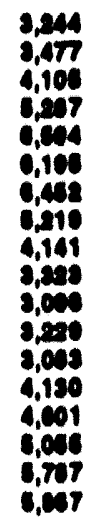 & 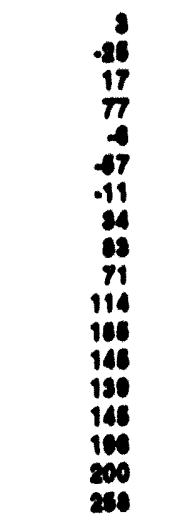 & $\begin{array}{r}.10 \\
.10 \\
.17 \\
0.10 \\
.14 \\
0.10 \\
0.14 \\
0.14 \\
=0 \\
-10 \\
= \\
= \\
= \\
= \\
=\end{array}$ \\
\hline 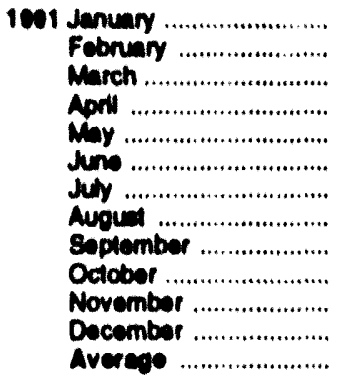 & $\begin{array}{l}7,500 \\
7,007 \\
7,540 \\
7,600 \\
7,400 \\
7,020 \\
7,347 \\
7,310 \\
7,300 \\
7,437 \\
7,320 \\
7,290 \\
7,017\end{array}$ & $\begin{array}{l}1,640 \\
1,900 \\
1,007 \\
1,700 \\
1,771 \\
1,767 \\
1,776 \\
1,731 \\
1,787 \\
1,643 \\
1,706 \\
1,718 \\
1,700\end{array}$ & $\begin{array}{l}5,296 \\
5,486 \\
5,160 \\
5,520 \\
6,306 \\
6,334 \\
5,065 \\
6,645 \\
5,012 \\
5,603 \\
5,528 \\
5,506 \\
5,762\end{array}$ & $\begin{array}{l}0 \\
0 \\
0 \\
0 \\
0 \\
0 \\
0 \\
0 \\
0 \\
0 \\
0 \\
0 \\
0\end{array}$ & 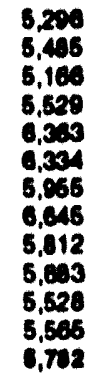 & $\begin{array}{r}.59 \\
324 \\
43 \\
230 \\
613 \\
50 \\
403 \\
11 \\
404 \\
.50 \\
203 \\
146 \\
106\end{array}$ & $\begin{array}{l}= \\
= \\
= \\
= \\
= \\
=\end{array}$ \\
\hline 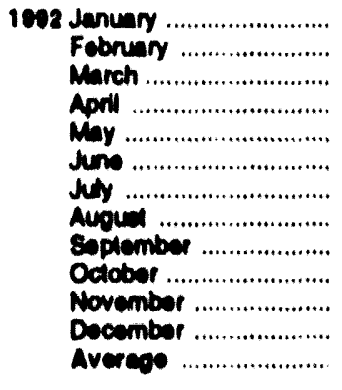 & $\begin{array}{l}7.361 \\
7,300 \\
7,340 \\
7,200 \\
7.100 \\
7.167 \\
7.131 \\
0,020 \\
7,020 \\
7.120 \\
7,024 \\
7,100 \\
7,17\end{array}$ & $\begin{array}{l}1,760 \\
1,800 \\
1,706 \\
1,741 \\
1,802 \\
1.700 \\
1,865 \\
1,036 \\
1,700 \\
1,000 \\
1,074 \\
1,706 \\
1,714\end{array}$ & $\begin{array}{l}5,050 \\
5,079 \\
5,321 \\
8,127 \\
8,000 \\
6,171 \\
8,700 \\
8,467 \\
8.216 \\
8,000 \\
0,121 \\
8,037 \\
6,009\end{array}$ & $\begin{array}{c}0 \\
0 \\
0 \\
0 \\
0 \\
34 \\
0 \\
18 \\
10 \\
40 \\
0 \\
0 \\
10\end{array}$ & $\begin{array}{l}5,060 \\
5,070 \\
5,321 \\
6,127 \\
0,000 \\
6,130 \\
6,706 \\
6,430 \\
6,202 \\
8,047 \\
6,121 \\
5,037 \\
0,070\end{array}$ & $\begin{array}{c}200 \\
220 \\
287 \\
100 \\
201 \\
250 \\
330 \\
06 \\
305 \\
200 \\
200 \\
61 \\
200\end{array}$ & $\begin{array}{l}= \\
\overline{-} \\
\overline{-} \\
\overline{-} \\
\overline{-} \\
\overline{-} \\
=\end{array}$ \\
\hline 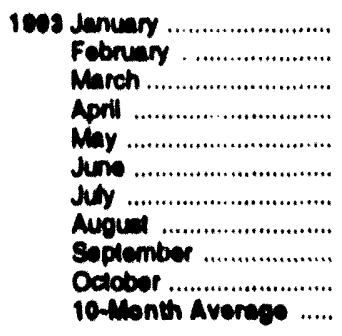 & 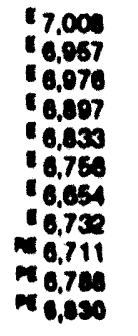 & 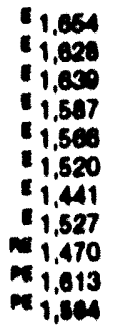 & 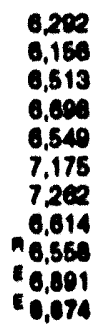 & $\begin{array}{r}0 \\
0 \\
32 \\
112 \\
0 \\
0 \\
0 \\
0 \\
34 \\
10 \\
10\end{array}$ & 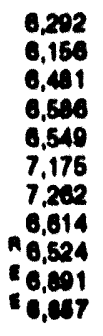 & $\begin{array}{r}12 \\
206 \\
150 \\
536 \\
576 \\
336 \\
311 \\
32 \\
253 \\
253 \\
442 \\
203\end{array}$ & $\begin{array}{l}\overline{-} \\
\bar{z} \\
\bar{z} \\
\bar{z} \\
\overline{-}\end{array}$ \\
\hline 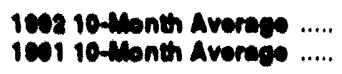 & $\begin{array}{l}7,102 \\
7,497\end{array}$ & $\begin{array}{l}1,710 \\
1,010\end{array}$ & $\begin{array}{l}0,044 \\
8,080\end{array}$ & $\begin{array}{r}12 \\
0\end{array}$ & $\begin{array}{l}0,002 \\
0,010\end{array}$ & $\begin{array}{l}278 \\
104\end{array}$ & $=$ \\
\hline
\end{tabular}

- Stralegle Polroloum Roserve.

- A belancing liom.

- Boginning in January 1923, conde oll ueed drectly an luel bs shown as produat aupolibed.

S see Note 6 al and of section.

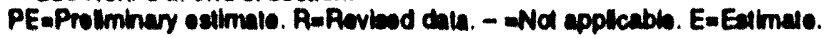

Notes: - Crude oll include beas condoneato. - Gooprephic coverege it ine 50 Siales and the Dlatict of Columbla. - Talate may nol equal sum of components dus 10 independent rounding.

Sourcs: - 16r21600: Energy Intormation Admintatration (EL), Petrobum Suppy Monthy, Fobruary 1003, Tabb S2. - 1901 forward: EU, Potroleum supply Monthy, November 1903, Tablo 82. 
Table 3.2b Crude Oll Supply and Dloposition: Disposttion and Ending Stocks

\begin{tabular}{|c|c|c|c|c|c|c|c|c|c|}
\hline & \multicolumn{6}{|c|}{ Diepectilen } & \multicolumn{3}{|c|}{ Ending steake" } \\
\hline & \multirow{2}{*}{$\begin{array}{l}\text { Crude } \\
\text { Leseses }\end{array}$} & \multicolumn{2}{|c|}{ seok Chenged } & \multirow{2}{*}{$\begin{array}{l}\text { Actinery } \\
\text { Inpute }\end{array}$} & \multirow[b]{2}{*}{ Inpere } & \multirow{2}{*}{ edvet } & \multirow[b]{2}{*}{ Toty } & \multirow[b]{2}{*}{ apno } & \multirow{2}{*}{ Onimer } \\
\hline & & ene & Orner & & & & & & \\
\hline & \multicolumn{6}{|c|}{ Mounand Barmels per Day } & \multicolumn{3}{|c|}{ Mulon Barris } \\
\hline 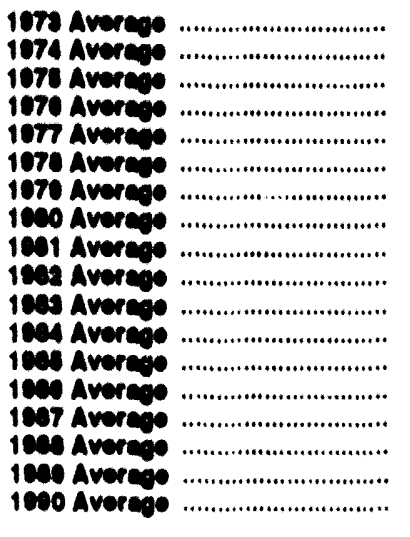 & $\begin{array}{r}18 \\
18 \\
18 \\
014 \\
10 \\
10 \\
10 \\
014 \\
5 \\
3 \\
2 \\
2 \\
1 \\
(0) \\
(0) \\
(0) \\
(0) \\
(1)\end{array}$ & 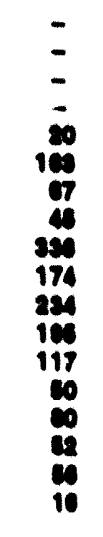 & 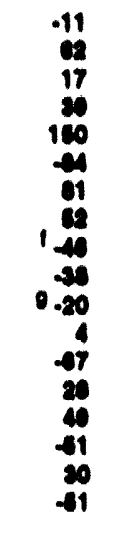 & $\begin{array}{l}12,491 \\
12,128 \\
12,442 \\
19,410 \\
14,001 \\
14,790 \\
19,040 \\
19,401 \\
12,470 \\
11,714 \\
11,04 \\
12,044 \\
12,00 \\
12,710 \\
12,044 \\
12,240 \\
12,401 \\
13,400\end{array}$ & $\begin{array}{l}2 \\
0 \\
60 \\
180 \\
201 \\
207 \\
200 \\
200 \\
104 \\
101 \\
204 \\
114 \\
181 \\
168 \\
142 \\
100\end{array}$ & $\begin{array}{l}= \\
= \\
= \\
= \\
= \\
= \\
= \\
0 \\
4 \\
40 \\
44 \\
40 \\
24\end{array}$ & 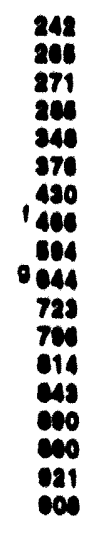 & $\begin{array}{c}- \\
- \\
- \\
- \\
7 \\
07 \\
01 \\
109 \\
290 \\
204 \\
970 \\
491 \\
190 \\
612 \\
641 \\
600 \\
600 \\
600\end{array}$ & 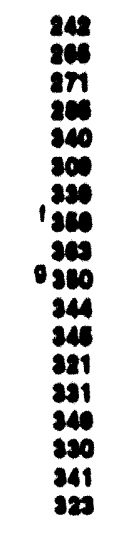 \\
\hline 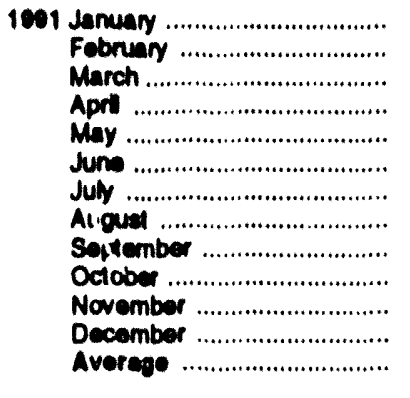 & $\begin{array}{l}0 \\
0 \\
(0) \\
(0) \\
(0) \\
(0) \\
0 \\
0 \\
0 \\
(0) \\
(0) \\
0 \\
(0)\end{array}$ & $\begin{array}{r}0 \\
.147 \\
.422 \\
0 \\
0 \\
(1) \\
(0) \\
(0) \\
0 \\
(0) \\
(0) \\
(a) \\
-17\end{array}$ & $\begin{array}{r}.71 \\
370 \\
103 \\
60 \\
660 \\
.200 \\
.163 \\
103 \\
.156 \\
51 \\
43 \\
.611 \\
.\end{array}$ & $\begin{array}{l}12,736 \\
13,046 \\
12,839 \\
13,042 \\
13,530 \\
13,016 \\
13,703 \\
13,800 \\
13,694 \\
12,896 \\
12,020 \\
13,165 \\
13,201\end{array}$ & $\begin{array}{r}60 \\
152 \\
137 \\
162 \\
166 \\
78 \\
130 \\
56 \\
100 \\
92 \\
126 \\
133 \\
110\end{array}$ & $\begin{array}{l}23 \\
17 \\
18 \\
21 \\
15 \\
16 \\
15 \\
13 \\
16 \\
22 \\
22 \\
23 \\
10\end{array}$ & $\begin{array}{l}906 \\
913 \\
906 \\
907 \\
924 \\
915 \\
911 \\
914 \\
900 \\
911 \\
912 \\
693 \\
009\end{array}$ & $\begin{array}{l}568 \\
580 \\
568 \\
568 \\
569 \\
569 \\
560 \\
560 \\
569 \\
560 \\
560 \\
569 \\
500\end{array}$ & $\begin{array}{l}320 \\
331 \\
337 \\
338 \\
368 \\
347 \\
342 \\
346 \\
341 \\
342 \\
344 \\
325 \\
325\end{array}$ \\
\hline 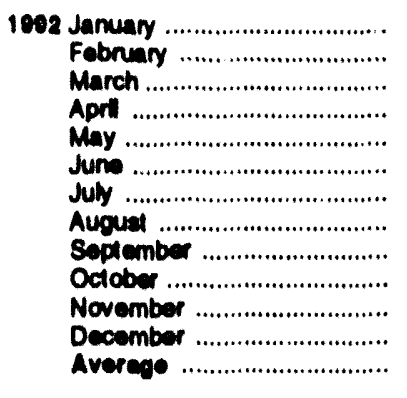 & $\begin{array}{r}0 \\
(0) \\
(0) \\
0 \\
0 \\
(0) \\
0 \\
(0) \\
0 \\
(0) \\
(0) \\
(0) \\
0\end{array}$ & $\begin{array}{l}\text { (a) } \\
0 \\
(0) \\
0 \\
(0) \\
34 \\
(0) \\
20 \\
40 \\
60 \\
15 \\
22 \\
17\end{array}$ & $\begin{array}{r}540 \\
171 \\
.250 \\
315 \\
-145 \\
.616 \\
244 \\
.144 \\
.204 \\
342 \\
.243 \\
.234 \\
.10\end{array}$ & $\begin{array}{l}12,023 \\
12,480 \\
13,063 \\
13,260 \\
13,679 \\
14,060 \\
13,063 \\
13,426 \\
13,714 \\
13,684 \\
13,647 \\
13,104 \\
13,411\end{array}$ & $\begin{array}{r}118 \\
22 \\
106 \\
23 \\
106 \\
107 \\
53 \\
133 \\
68 \\
106 \\
111 \\
107 \\
60\end{array}$ & $\begin{array}{r}26 \\
17 \\
18 \\
11 \\
10 \\
12 \\
9 \\
8 \\
11 \\
10 \\
10 \\
12 \\
10\end{array}$ & $\begin{array}{l}910 \\
915 \\
907 \\
017 \\
912 \\
895 \\
902 \\
898 \\
893 \\
900 \\
890 \\
893 \\
003\end{array}$ & $\begin{array}{l}560 \\
560 \\
569 \\
560 \\
569 \\
570 \\
570 \\
570 \\
571 \\
574 \\
574 \\
575 \\
571\end{array}$ & $\begin{array}{l}341 \\
346 \\
330 \\
340 \\
344 \\
325 \\
333 \\
328 \\
322 \\
333 \\
326 \\
318 \\
310\end{array}$ \\
\hline 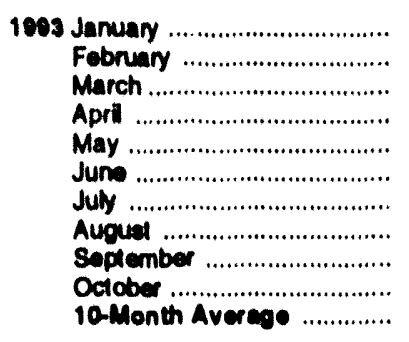 & $\begin{array}{c}(s) \\
(s) \\
0 \\
(s) \\
0 \\
0 \\
0 \\
0 \\
50 \\
0 \\
0 \\
0\end{array}$ & $\begin{array}{r}10 \\
18 \\
58 \\
130 \\
13 \\
21 \\
10 \\
24 \\
52 \\
16 \\
16 \\
130\end{array}$ & $\begin{array}{r}245 \\
202 \\
188 \\
401 \\
120 \\
.37 \\
22 \\
.548 \\
.491 \\
238 \\
E_{29}\end{array}$ & $\begin{array}{r}12,080 \\
12,023 \\
13,249 \\
13,512 \\
13,701 \\
14,125 \\
14,114 \\
13,830 \\
A 13,846 \\
13,760 \\
13,000\end{array}$ & $\begin{array}{r}120 \\
168 \\
139 \\
73 \\
112 \\
150 \\
62 \\
55 \\
107 \\
101 \\
100\end{array}$ & $\begin{array}{r}10 \\
10 \\
11 \\
8 \\
10 \\
8 \\
9 \\
8 \\
9 \\
8 \\
8 \\
0\end{array}$ & $\begin{array}{r}901 \\
907 \\
916 \\
931 \\
935 \\
936 \\
936 \\
920 \\
900 \\
908 \\
921 \\
921\end{array}$ & $\begin{array}{l}575 \\
576 \\
578 \\
582 \\
580 \\
583 \\
583 \\
584 \\
586 \\
5860 \\
580\end{array}$ & 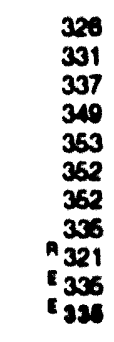 \\
\hline $\begin{array}{l}1002 \text { 10-Month Averege ............ } \\
1001 \text { 10-Month Averege ............. }\end{array}$ & (0) & $\begin{array}{r}17 \\
+7\end{array}$ & 29 & $\begin{array}{l}19,420 \\
13,222\end{array}$ & $\mathbf{1 1 3}$ & $\begin{array}{l}12 \\
10\end{array}$ & $\operatorname{los}_{11}$ & $\begin{array}{l}874 \\
660\end{array}$ & 299 \\
\hline
\end{tabular}

- Slock are lotals as of end of period.

- A nogative number indicates a decrease in slocks and a positive number indicales en increase.

- Siralegic Pelroloum Reserve.

- Bepinning In January 1083, crude oll used dhectly as full is shown as product suppliad.

- Seo Note 6 al end of section.

1 Slocks of Alaskan crude oll in Iranal aro Included Irom January 1981 lonward. See Nole 5 at end of section.
- See Nole 4 al erra of section.

ReRevised data. - wot applicable. EeEatimale. (s)=leas than +500 barrele per day and grealer than .600 berrele per day.

Notes: - Crude oll includes lease condensate. - Geographic covernge is the 60 states and the Ditita of Columbia. Tate may not equal sum of componente due to independent rounding.

Sources: - 1072-1000: Energy InI smation Adminiatration (EIA). Potroloum Supply Monthy. Febnuary 1093, Tablo S2. - 1081 fonwerd: EIA. Petroleum Supply Monthly, November 1093, Table S2. 
Table 3.3a Petroleum Imports: Algeria, Iraq, Kuwait, and Libya (Thousand Barrels per Day)

\begin{tabular}{|c|c|c|c|c|c|c|c|c|}
\hline & \multicolumn{8}{|c|}{ Arab OPEC } \\
\hline & \multicolumn{2}{|c|}{ Noerie } & \multicolumn{2}{|c|}{ Irea } & \multicolumn{2}{|c|}{ Kunalp } & \multicolumn{2}{|c|}{ Ubya } \\
\hline & Total & Crude Oll & Totel & Crude Oil & Totd & Crude OII & Totel & Crude oll \\
\hline 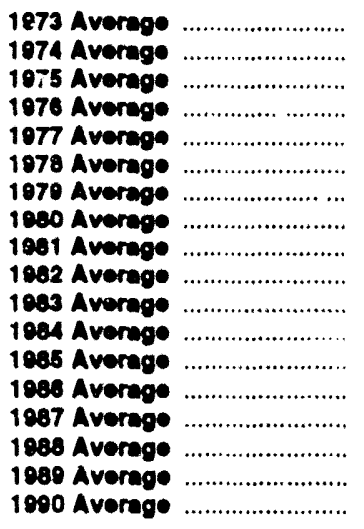 & $\begin{array}{l}130 \\
100 \\
262 \\
432 \\
550 \\
640 \\
630 \\
406 \\
911 \\
170 \\
240 \\
323 \\
187 \\
271 \\
295 \\
300 \\
280 \\
280\end{array}$ & $\begin{array}{r}120 \\
180 \\
264 \\
400 \\
544 \\
634 \\
600 \\
450 \\
261 \\
60 \\
170 \\
104 \\
84 \\
78 \\
115 \\
58 \\
60 \\
63\end{array}$ & $\begin{array}{r}4 \\
0 \\
2 \\
28 \\
74 \\
62 \\
6 \\
28 \\
(4) \\
3 \\
10 \\
12 \\
40 \\
81 \\
83 \\
345 \\
440 \\
518\end{array}$ & $\begin{array}{r}4 \\
0 \\
2 \\
26 \\
74 \\
62 \\
60 \\
20 \\
0 \\
3 \\
10 \\
12 \\
46 \\
81 \\
82 \\
343 \\
41 \\
514\end{array}$ & $\begin{array}{r}47 \\
5 \\
16 \\
5 \\
40 \\
6 \\
27 \\
0 \\
5 \\
14 \\
36 \\
21 \\
68 \\
04 \\
92 \\
157 \\
86\end{array}$ & $\begin{array}{r}12 \\
5 \\
4 \\
1 \\
12 \\
5 \\
5 \\
27 \\
0 \\
2 \\
7 \\
24 \\
4 \\
28 \\
70 \\
80 \\
155 \\
70\end{array}$ & $\begin{array}{r}164 \\
4 \\
232 \\
453 \\
723 \\
654 \\
658 \\
854 \\
310 \\
28 \\
0 \\
1 \\
4 \\
0 \\
0 \\
0 \\
0 \\
0\end{array}$ & $\begin{array}{r}133 \\
223 \\
424 \\
704 \\
630 \\
642 \\
540 \\
317 \\
23 \\
0 \\
0 \\
0 \\
0 \\
0 \\
0 \\
0 \\
0\end{array}$ \\
\hline 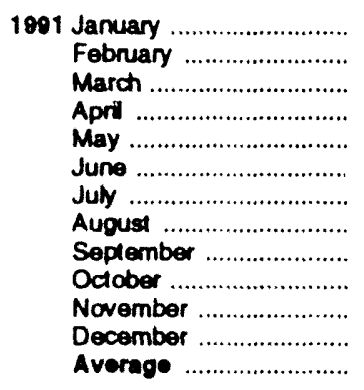 & $\begin{array}{l}327 \\
246 \\
222 \\
282 \\
308 \\
304 \\
202 \\
182 \\
206 \\
236 \\
278 \\
247 \\
253\end{array}$ & $\begin{array}{l}48 \\
20 \\
45 \\
74 \\
72 \\
37 \\
28 \\
16 \\
19 \\
53 \\
58 \\
54 \\
44\end{array}$ & $\begin{array}{l}0 \\
0 \\
0 \\
0 \\
0 \\
0 \\
0 \\
0 \\
0 \\
0 \\
0 \\
0 \\
0\end{array}$ & $\begin{array}{l}0 \\
0 \\
0 \\
0 \\
0 \\
0 \\
0 \\
0 \\
0 \\
0 \\
0 \\
0 \\
0\end{array}$ & $\begin{array}{r}0 \\
0 \\
0 \\
0 \\
0 \\
0 \\
0 \\
0 \\
34 \\
33 \\
0 \\
0 \\
6\end{array}$ & $\begin{array}{r}0 \\
0 \\
0 \\
0 \\
0 \\
0 \\
0 \\
0 \\
34 \\
33 \\
0 \\
0 \\
6\end{array}$ & $\begin{array}{l}0 \\
0 \\
0 \\
0 \\
0 \\
0 \\
0 \\
0 \\
0 \\
0 \\
0 \\
0 \\
0\end{array}$ & $\begin{array}{l}0 \\
0 \\
0 \\
0 \\
0 \\
0 \\
0 \\
0 \\
0 \\
0 \\
0 \\
0 \\
0\end{array}$ \\
\hline 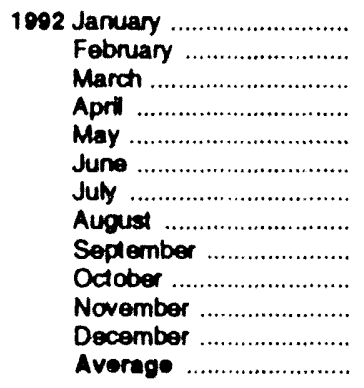 & $\begin{array}{l}206 \\
218 \\
215 \\
182 \\
208 \\
144 \\
179 \\
261 \\
184 \\
186 \\
171 \\
203 \\
106\end{array}$ & $\begin{array}{r}37 \\
57 \\
37 \\
19 \\
7 \\
12 \\
37 \\
45 \\
19 \\
8 \\
0 \\
9 \\
24\end{array}$ & $\begin{array}{l}0 \\
0 \\
0 \\
0 \\
0 \\
0 \\
0 \\
0 \\
0 \\
0 \\
0 \\
0 \\
0\end{array}$ & $\begin{array}{l}0 \\
0 \\
0 \\
0 \\
0 \\
0 \\
0 \\
0 \\
0 \\
0 \\
0 \\
0 \\
0\end{array}$ & $\begin{array}{r}0 \\
0 \\
0 \\
0 \\
0 \\
0 \\
58 \\
66 \\
70 \\
137 \\
117 \\
165 \\
51\end{array}$ & $\begin{array}{r}0 \\
0 \\
0 \\
0 \\
0 \\
0 \\
23 \\
33 \\
33 \\
109 \\
117 \\
149 \\
30\end{array}$ & $\begin{array}{l}0 \\
0 \\
0 \\
0 \\
0 \\
0 \\
0 \\
0 \\
0 \\
0 \\
0 \\
0 \\
0\end{array}$ & $\begin{array}{l}0 \\
0 \\
0 \\
0 \\
0 \\
0 \\
0 \\
0 \\
0 \\
0 \\
0 \\
0 \\
0\end{array}$ \\
\hline 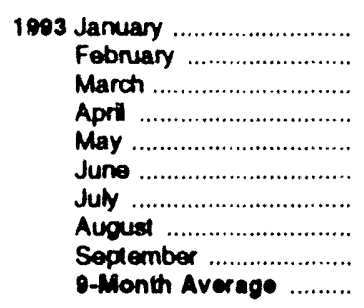 & $\begin{array}{l}153 \\
256 \\
186 \\
274 \\
228 \\
169 \\
246 \\
241 \\
192 \\
216\end{array}$ & $\begin{array}{r}28 \\
0 \\
7 \\
26 \\
3 \\
32 \\
6 \\
28 \\
0 \\
14\end{array}$ & $\begin{array}{l}0 \\
0 \\
0 \\
0 \\
0 \\
0 \\
0 \\
0 \\
0 \\
0\end{array}$ & $\begin{array}{l}0 \\
0 \\
0 \\
0 \\
0 \\
0 \\
0 \\
0 \\
0 \\
0\end{array}$ & $\begin{array}{l}144 \\
251 \\
316 \\
262 \\
222 \\
235 \\
368 \\
467 \\
445 \\
302\end{array}$ & $\begin{array}{l}129 \\
229 \\
300 \\
262 \\
222 \\
235 \\
362 \\
451 \\
431 \\
202\end{array}$ & $\begin{array}{l}0 \\
0 \\
0 \\
0 \\
0 \\
0 \\
0 \\
0 \\
0 \\
0\end{array}$ & $\begin{array}{l}0 \\
0 \\
0 \\
0 \\
0 \\
0 \\
0 \\
0 \\
0 \\
0\end{array}$ \\
\hline $\begin{array}{l}1002 \text { 9-Month Average .......... } \\
1001 \text { 9-Month Average ......... }\end{array}$ & $\begin{array}{l}100 \\
253\end{array}$ & $\begin{array}{l}30 \\
40\end{array}$ & $\begin{array}{l}0 \\
0\end{array}$ & 0 & 22 & $\begin{array}{r}10 \\
4\end{array}$ & $\begin{array}{l}0 \\
0\end{array}$ & 0 \\
\hline
\end{tabular}

axchudes petroleum imported into the Uniled States indirectly from members of the Organization of Petroleum Exporting Countries (OPEC), primarily trom Caribbean and West European areas, as petroloum products that were refined from crude oll produced by OPEC.

b Imports from the Neutral Zone between Kuwait and Saudi Arabia are included in Saud Arabla.

(s) = Less than 500 barrels per day.
Notes: - Beglnning in October 1977, Strategic Petroleum Reserve imports are included. - Geographic coverage ts the 50 Stales and the Diatrict of Columbia.

Sources: - 1073-1080: Energy Intormation Admintatration (EIA). Potroloum Supply Monthly, February 1993, Table S3. - 1001 forwerd: EIA, Petroleum Supply Monthly, November 1993, Table S3. 
Table 3.3b Petroleum Imports: Qatar, Saudi Arabia, U.A.E., and Total Arab OPEC (Thousand Barrels per Day)

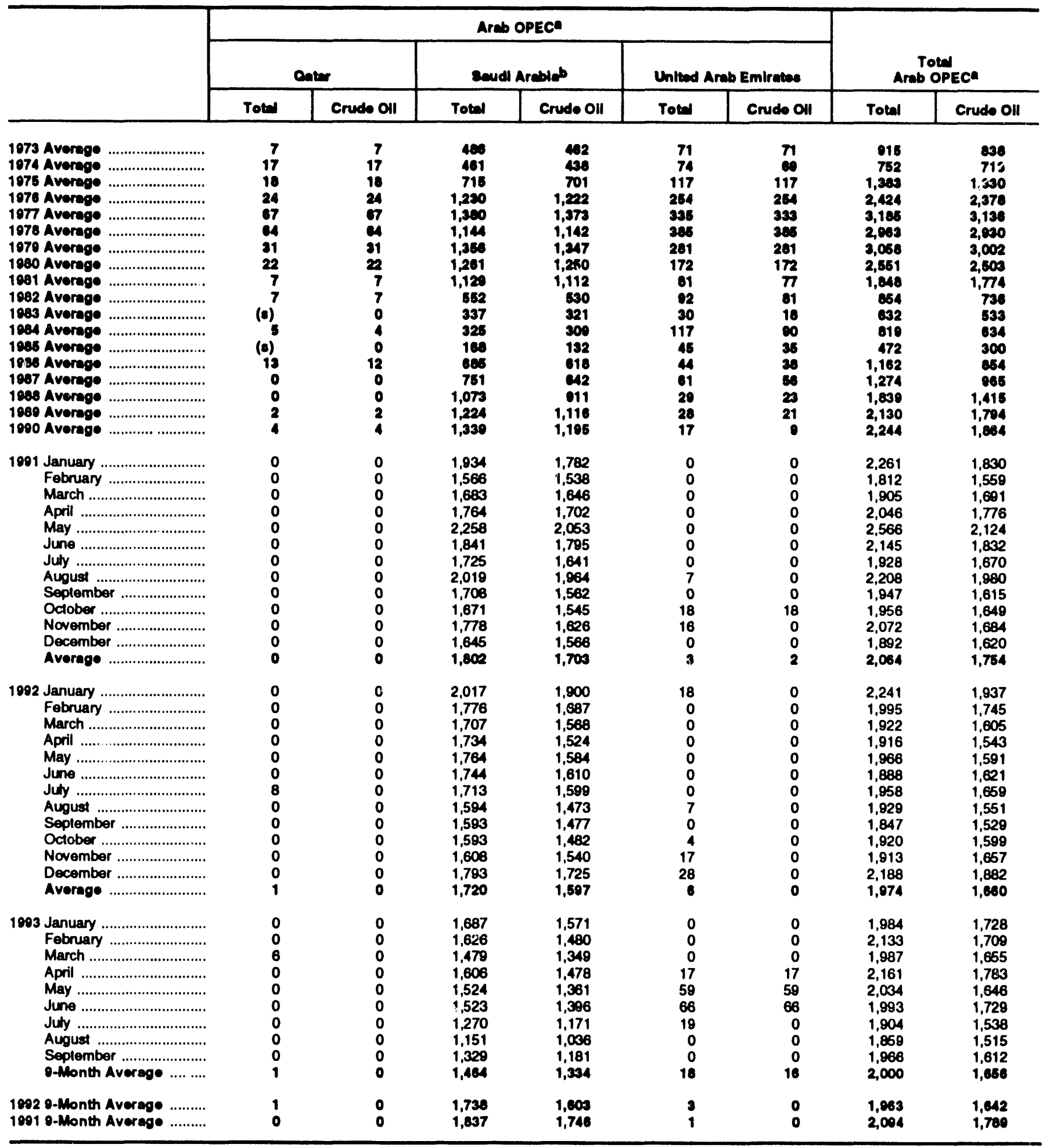

a Excludes petroleum imported into the United States indirectly from members of the Organization of Petroleum Exporting Counties (OPEC). primarily from Caribbean and West European areas, as petroleum products that were refined from crude oll produced by OPEC.

Imports from the Neutral Zone between Kuwall and Saudi Arabia are included in Saudi Arabla.

(s)=Less than 500 barrels per day.
Notes: - Beginning in October 1977, Stratogic Petroleum Reserve imports are included. - Geographic coverage to the 50 Stales and the District of Columbia. - Totals may not equal sum of components due to independent rounding.

Sources: - 1873-1080: Energy Information Administration (EIA), Patroleum Supply Monthy, February 1993, Table S3. • 1981 forwerd: EIA, Petroleum Supply Monthly, November 1993, Table S3. 
Table 3.3c Petroleum Imports: Ecuador, Gabon, Indonesia, and Iran (Thousand Barrels per Day)

\begin{tabular}{|c|c|c|c|c|c|c|c|c|}
\hline & \multicolumn{8}{|c|}{ Nen-Arab OPEca } \\
\hline & \multicolumn{2}{|c|}{ Eeundorb } & \multicolumn{2}{|c|}{ Caben } & \multicolumn{2}{|c|}{ Indoneole } & \multicolumn{2}{|c|}{ Iren } \\
\hline & Total & Crude Oll & Total & Crude Oll & Total & Crude Oll & Total & Crude Oll \\
\hline 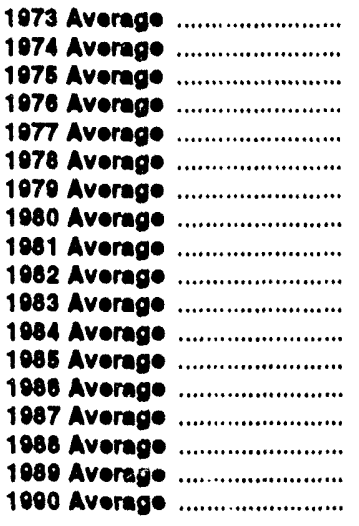 & $\begin{array}{l}48 \\
42 \\
67 \\
81 \\
67 \\
4 \\
42 \\
27 \\
48 \\
42 \\
61 \\
85 \\
67 \\
77 \\
20 \\
47 \\
80 \\
40\end{array}$ & $\begin{array}{l}47 \\
42 \\
67 \\
81 \\
85 \\
38 \\
30 \\
17 \\
38 \\
32 \\
58 \\
47 \\
86 \\
64 \\
23 \\
33 \\
80 \\
38\end{array}$ & $\begin{array}{l}0 \\
23 \\
27 \\
20 \\
42 \\
41 \\
42 \\
20 \\
28 \\
40 \\
80 \\
68 \\
32 \\
20 \\
30 \\
10 \\
60 \\
4\end{array}$ & 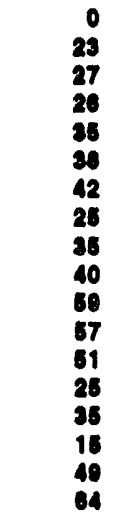 & $\begin{array}{l}213 \\
300 \\
300 \\
830 \\
541 \\
573 \\
420 \\
345 \\
349 \\
240 \\
338 \\
343 \\
314 \\
310 \\
285 \\
205 \\
109 \\
114\end{array}$ & $\begin{array}{r}200 \\
284 \\
370 \\
897 \\
807 \\
538 \\
390 \\
314 \\
318 \\
228 \\
318 \\
304 \\
292 \\
297 \\
202 \\
108 \\
138 \\
90\end{array}$ & $\begin{array}{r}203 \\
460 \\
200 \\
200 \\
528 \\
585 \\
304 \\
0 \\
0 \\
26 \\
40 \\
10 \\
27 \\
10 \\
08 \\
010) \\
0 \\
0\end{array}$ & $\begin{array}{r}218 \\
493 \\
278 \\
208 \\
090 \\
684 \\
207 \\
8 \\
0 \\
28 \\
18 \\
10 \\
27 \\
10 \\
08 \\
0 \\
(5) \\
0 \\
0\end{array}$ \\
\hline 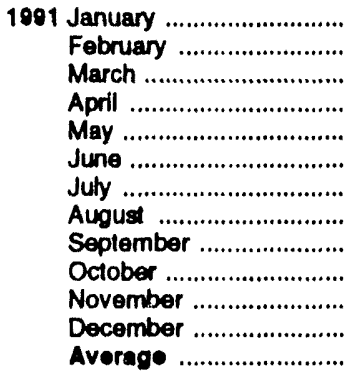 & $\begin{array}{r}18 \\
68 \\
67 \\
36 \\
109 \\
129 \\
62 \\
112 \\
31 \\
30 \\
55 \\
41 \\
63\end{array}$ & $\begin{array}{r}6 \\
55 \\
58 \\
24 \\
103 \\
126 \\
47 \\
93 \\
25 \\
24 \\
48 \\
23 \\
83\end{array}$ & $\begin{array}{r}41 \\
85 \\
28 \\
72 \\
96 \\
70 \\
137 \\
56 \\
91 \\
137 \\
91 \\
91 \\
94\end{array}$ & $\begin{array}{r}41 \\
95 \\
29 \\
72 \\
96 \\
70 \\
137 \\
56 \\
91 \\
137 \\
91 \\
81 \\
94\end{array}$ & $\begin{array}{r}70 \\
162 \\
83 \\
69 \\
97 \\
187 \\
88 \\
83 \\
83 \\
118 \\
120 \\
163 \\
111\end{array}$ & $\begin{array}{r}70 \\
163 \\
93 \\
69 \\
97 \\
187 \\
88 \\
87 \\
64 \\
91 \\
96 \\
134 \\
102\end{array}$ & $\begin{array}{r}0 \\
0 \\
0 \\
0 \\
0 \\
0 \\
81 \\
48 \\
152 \\
43 \\
64 \\
0 \\
32\end{array}$ & $\begin{array}{r}0 \\
0 \\
0 \\
0 \\
0 \\
0 \\
81 \\
48 \\
152 \\
43 \\
64 \\
0 \\
32\end{array}$ \\
\hline 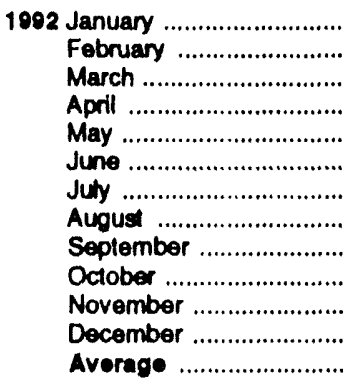 & $\begin{array}{r}56 \\
61 \\
28 \\
53 \\
51 \\
105 \\
111 \\
99 \\
97 \\
42 \\
53 \\
24 \\
88\end{array}$ & $\begin{array}{r}56 \\
48 \\
26 \\
46 \\
51 \\
101 \\
111 \\
83 \\
97 \\
36 \\
53 \\
24 \\
62\end{array}$ & $\begin{array}{r}91 \\
105 \\
25 \\
183 \\
135 \\
129 \\
143 \\
108 \\
165 \\
167 \\
114 \\
120 \\
124\end{array}$ & $\begin{array}{r}91 \\
105 \\
25 \\
188 \\
135 \\
129 \\
143 \\
108 \\
158 \\
167 \\
114 \\
120 \\
123\end{array}$ & $\begin{array}{r}125 \\
30 \\
85 \\
54 \\
155 \\
100 \\
65 \\
91 \\
57 \\
54 \\
36 \\
60 \\
70\end{array}$ & $\begin{array}{r}117 \\
30 \\
83 \\
49 \\
133 \\
102 \\
65 \\
85 \\
38 \\
43 \\
23 \\
60 \\
70\end{array}$ & $\begin{array}{l}0 \\
0 \\
0 \\
0 \\
0 \\
0 \\
0 \\
0 \\
0 \\
0 \\
0 \\
0 \\
0\end{array}$ & $\begin{array}{l}0 \\
0 \\
0 \\
0 \\
0 \\
0 \\
0 \\
0 \\
0 \\
0 \\
0 \\
0 \\
0\end{array}$ \\
\hline 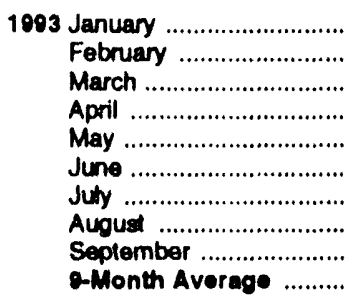 & 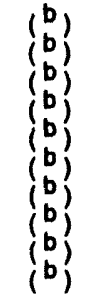 & $\begin{array}{l}\left(\begin{array}{l}b \\
b \\
b\end{array}\right) \\
\left(\begin{array}{l}b \\
b\end{array}\right) \\
\left(\begin{array}{l}b \\
b \\
b\end{array}\right) \\
\left(\begin{array}{l}b \\
b\end{array}\right)\end{array}$ & $\begin{array}{r}90 \\
88 \\
126 \\
127 \\
169 \\
107 \\
168 \\
152 \\
211 \\
139\end{array}$ & $\begin{array}{r}89 \\
88 \\
123 \\
127 \\
169 \\
107 \\
166 \\
152 \\
211 \\
137\end{array}$ & $\begin{array}{l}37 \\
52 \\
67 \\
76 \\
82 \\
97 \\
55 \\
85 \\
51 \\
68\end{array}$ & $\begin{array}{l}37 \\
51 \\
64 \\
76 \\
82 \\
67 \\
55 \\
80 \\
40 \\
61\end{array}$ & $\begin{array}{l}0 \\
0 \\
0 \\
0 \\
0 \\
0 \\
0 \\
0 \\
0 \\
0\end{array}$ & $\begin{array}{l}0 \\
0 \\
0 \\
0 \\
0 \\
0 \\
0 \\
0 \\
0 \\
0\end{array}$ \\
\hline $\begin{array}{l}1002 \text { 9-Month Average ......... } \\
1001 \text { 9-Month Average ......... }\end{array}$ & 70 & $\begin{array}{l}70 \\
60\end{array}$ & $\begin{array}{r}120 \\
70\end{array}$ & $\begin{array}{r}120 \\
76\end{array}$ & $\begin{array}{r}97 \\
104\end{array}$ & $\begin{array}{r}80 \\
100\end{array}$ & $\begin{array}{r}0 \\
31\end{array}$ & $\begin{array}{r}0 \\
31\end{array}$ \\
\hline
\end{tabular}

a Excludes petroleum imported into the United States indirectly from members of the Organization of Petroloum Exporting Countries (OPEC). primarty from Caribbean and West European areas, as petroleum products that were relined from crude oll produced by OPEC.

Ecuador whithdrew from OPEC on December 31, 1982. As of January 1993, imports from Ecuador appear on Table 3.31 under "Nor-OPEC."

c A small amount of Iranian crude oll entered the United States in January 1888 from the Virgin blands. The ol orloinated in Iran and was exported to the Virgin lalands prior to the signing of Executive Order 12813 on October
29,1987

(s)=Less than 500 barrebs per day.

Notes: - Beginning in October 1877, Strategic Petroleum Resenve imports are included. - Gecoraphic coverage is the $\mathbf{5 0}$ States and the District of Columbia.

Sources: - 1973-1080: Energy Intormation Administration (EIA), Petroleum Supply Monthly, February 1993, Table S3. - 1881 forwerd: EIA, Petroleum Supply Monthly, November 1893, Table S3. 
Table 3.3d Petroleum Imports: Nigeria, Venezuela, Total Non-Arab OPEC, and Total OPEC

(Thousand Barrels per Day)

\begin{tabular}{|c|c|c|c|c|c|c|c|c|}
\hline & \multicolumn{4}{|c|}{ Non-Arab OPECa } & \multirow{2}{*}{\multicolumn{2}{|c|}{$\begin{array}{l}\text { Total } \\
\text { Non-Arab Opeca,b }\end{array}$}} & \multirow{2}{*}{\multicolumn{2}{|c|}{$\begin{array}{l}\text { Total } \\
\text { OPECab }\end{array}$}} \\
\hline & \multicolumn{2}{|c|}{ Nigerla } & \multicolumn{2}{|c|}{ Vencezuda } & & & & \\
\hline & Total & Crudo oll & Total & Crude Oll & Totel & Crude oll & Total & Crude Oll \\
\hline 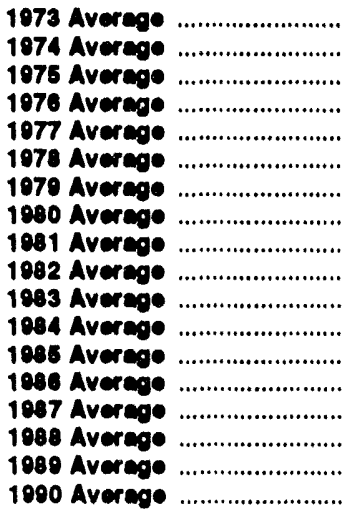 & $\begin{array}{r}450 \\
713 \\
702 \\
1,025 \\
1,143 \\
810 \\
1,080 \\
857 \\
620 \\
814 \\
302 \\
216 \\
203 \\
440 \\
636 \\
618 \\
1015 \\
100\end{array}$ & $\begin{array}{r}440 \\
607 \\
740 \\
1,014 \\
1,130 \\
010 \\
1,000 \\
641 \\
611 \\
510 \\
301 \\
207 \\
200 \\
437 \\
820 \\
807 \\
800 \\
784\end{array}$ & $\begin{array}{r}1,130 \\
970 \\
702 \\
700 \\
600 \\
640 \\
600 \\
401 \\
400 \\
412 \\
422 \\
840 \\
608 \\
703 \\
604 \\
704 \\
873 \\
1,025\end{array}$ & $\begin{array}{l}344 \\
210 \\
305 \\
241 \\
250 \\
181 \\
203 \\
186 \\
147 \\
155 \\
104 \\
283 \\
206 \\
416 \\
496 \\
430 \\
405 \\
608\end{array}$ & $\begin{array}{l}2,070 \\
2,627 \\
2,210 \\
2,042 \\
3,000 \\
2,706 \\
2,570 \\
1,740 \\
1,476 \\
1,201 \\
1,231 \\
1,230 \\
1,360 \\
1,074 \\
1,707 \\
1,001 \\
2,010 \\
2,052\end{array}$ & $\begin{array}{r}1,257 \\
1,027 \\
1,002 \\
2,167 \\
2,507 \\
2,254 \\
2,110 \\
1,361 \\
1,140 \\
000 \\
944 \\
1,010 \\
1,280 \\
1,436 \\
1,281 \\
1,582 \\
1,650\end{array}$ & $\begin{array}{l}2,000 \\
3,280 \\
3,001 \\
5,006 \\
6,193 \\
8,751 \\
8,037 \\
4,300 \\
3,323 \\
2,146 \\
1,002 \\
2,040 \\
1,030 \\
2,037 \\
3,060 \\
3,520 \\
4,140 \\
4,296\end{array}$ & $\begin{array}{l}2,006 \\
2,640 \\
3,211 \\
4,546 \\
6,849 \\
5,144 \\
5,112 \\
3,064 \\
2,022 \\
1,734 \\
1,477 \\
1,612 \\
1,312 \\
2,112 \\
2,400 \\
2,000 \\
3,376 \\
3,514\end{array}$ \\
\hline 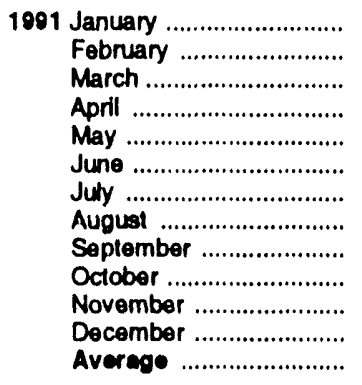 & $\begin{array}{r}504 \\
721 \\
531 \\
677 \\
860 \\
832 \\
833 \\
1,016 \\
489 \\
651 \\
704 \\
617 \\
703\end{array}$ & $\begin{array}{l}481 \\
717 \\
531 \\
649 \\
838 \\
827 \\
817 \\
983 \\
467 \\
623 \\
674 \\
593 \\
683\end{array}$ & $\begin{array}{r}1,005 \\
959 \\
998 \\
845 \\
997 \\
1,136 \\
1,102 \\
1,070 \\
1,163 \\
1,087 \\
1,065 \\
1,987 \\
1,036\end{array}$ & $\begin{array}{l}673 \\
686 \\
631 \\
470 \\
581 \\
705 \\
683 \\
701 \\
790 \\
777 \\
671 \\
655 \\
608\end{array}$ & $\begin{array}{l}1,637 \\
2,003 \\
1,718 \\
1,698 \\
2,158 \\
2,354 \\
2,304 \\
2,304 \\
2,009 \\
2,067 \\
2,099 \\
1,898 \\
2,028\end{array}$ & $\begin{array}{l}1,271 \\
1,705 \\
1,342 \\
1,283 \\
1,715 \\
1,915 \\
1,855 \\
1,966 \\
1,589 \\
1,694 \\
1,644 \\
1,496 \\
1,622\end{array}$ & $\begin{array}{l}3,898 \\
3,815 \\
3,623 \\
3,744 \\
4,724 \\
4,498 \\
4,232 \\
4,602 \\
3,956 \\
4,023 \\
4,171 \\
3,791 \\
4,002\end{array}$ & $\begin{array}{l}3,101 \\
3,264 \\
3,033 \\
3,059 \\
3,839 \\
3,747 \\
3,525 \\
3,946 \\
3,204 \\
3,343 \\
3,328 \\
3,116 \\
3,37\end{array}$ \\
\hline 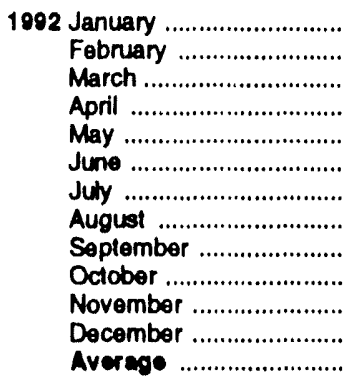 & $\begin{array}{l}593 \\
322 \\
441 \\
798 \\
773 \\
740 \\
900 \\
815 \\
774 \\
827 \\
626 \\
549 \\
881\end{array}$ & $\begin{array}{l}568 \\
303 \\
409 \\
788 \\
773 \\
740 \\
883 \\
795 \\
754 \\
813 \\
608 \\
532 \\
868\end{array}$ & $\begin{array}{l}1,119 \\
1,028 \\
1,106 \\
1,079 \\
1,038 \\
1,059 \\
1,163 \\
1,102 \\
1,333 \\
1,497 \\
1,343 \\
1,164 \\
1,170\end{array}$ & $\begin{array}{r}787 \\
655 \\
793 \\
722 \\
745 \\
738 \\
912 \\
841 \\
853 \\
1,073 \\
921 \\
763 \\
826\end{array}$ & $\begin{array}{l}1,984 \\
1,555 \\
1,684 \\
2,169 \\
2,152 \\
2,141 \\
2,382 \\
2,215 \\
2,426 \\
2,587 \\
2,173 \\
1,917 \\
2,117\end{array}$ & $\begin{array}{l}1,8: 7 \\
1,150 \\
1,336 \\
1,791 \\
1,837 \\
1,800 \\
2,114 \\
1,822 \\
2,001 \\
2,133 \\
1,719 \\
1,499 \\
1,746\end{array}$ & $\begin{array}{l}4,224 \\
3,549 \\
3,606 \\
4,085 \\
4,118 \\
4,029 \\
4,339 \\
4,144 \\
4,274 \\
4,507 \\
4,086 \\
4,105 \\
4,002\end{array}$ & $\begin{array}{l}3,554 \\
2,895 \\
2,941 \\
3,334 \\
3,428 \\
3,430 \\
3,772 \\
3,473 \\
3,531 \\
3,732 \\
3,376 \\
3,381 \\
3,406\end{array}$ \\
\hline 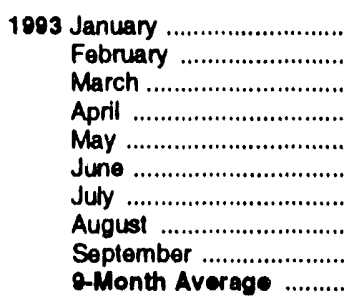 & $\begin{array}{l}729 \\
827 \\
928 \\
892 \\
741 \\
848 \\
893 \\
562 \\
514 \\
780\end{array}$ & $\begin{array}{l}729 \\
813 \\
892 \\
871 \\
723 \\
827 \\
888 \\
549 \\
496 \\
784\end{array}$ & $\begin{array}{l}1,385 \\
1,280 \\
1,200 \\
1,297 \\
1,226 \\
1,277 \\
1,384 \\
1,375 \\
1,243 \\
1,290\end{array}$ & $\begin{array}{r}1,038 \\
925 \\
817 \\
1,006 \\
954 \\
992 \\
1,068 \\
1,135 \\
1,033 \\
907\end{array}$ & $\begin{array}{r}{ }^{\circ} 2,241 \\
2,368 \\
2,330 \\
2,392 \\
2,219 \\
2,329 \\
2,500 \\
2,183 \\
2,018 \\
2,285\end{array}$ & $\begin{array}{r}b 1,892 \\
1,976 \\
1,897 \\
2,080 \\
1,929 \\
1,992 \\
2,177 \\
1,915 \\
1,779 \\
1,060\end{array}$ & $\begin{array}{l}4,225 \\
4,491 \\
4,317 \\
4,553 \\
4,253 \\
4,321 \\
4,404 \\
4,043 \\
3,984 \\
4,286\end{array}$ & $\begin{array}{r}\mathrm{b} 3,620 \\
3,685 \\
3,552 \\
3,863 \\
3,574 \\
3,721 \\
3,715 \\
3,431 \\
3,391 \\
3,616\end{array}$ \\
\hline $\begin{array}{l}1002 \text { 9-Month Average ......... } \\
1001 \text { g-Month Average ......... }\end{array}$ & $\begin{array}{l}680 \\
710\end{array}$ & $\begin{array}{l}869 \\
701\end{array}$ & $\begin{array}{l}1,114 \\
1,031\end{array}$ & $\begin{array}{l}795 \\
657\end{array}$ & $\begin{array}{l}2,081 \\
2,031\end{array}$ & $\begin{array}{l}1,733 \\
1,626\end{array}$ & $\begin{array}{l}4,044 \\
4,125\end{array}$ & $\begin{array}{l}3,376 \\
3,416\end{array}$ \\
\hline
\end{tabular}

a Excludes petroleum imported into the United States indireaty from members of the Organization of Petroleum Exporting Countries (OPEC), primarity Irom Caribbean and West European areas, as petroleum products that were refined from crude oil produced by OPEC.

As of January 1993, excludes petroleum imported trom Ecuador, which whithrew from OPEC on December 31, 1992.

Notes: - Beginning in October 1977, Strategic Petroleum Reserve imports are included. - Geographic coverage is the 50 States and the District of Columbia. - Totals may not equal sum of components due to independent rounding.

Sources: - 1973-1900: Energy Information Administration (EIA), Petroloum Supply Monthly, February 1993, Table S3. - 1081 forward: ElA, Petroloum Supply Monthly, November 1993, Table S3. 
Table 3.3e Petroleum Imports: Angola, Australia, Bahama Islands, Brazil, Canada, and China

(Thousand Barrels per Day)

\begin{tabular}{|c|c|c|c|c|c|c|c|c|c|c|c|c|}
\hline & \multicolumn{12}{|c|}{ Non-OPECA } \\
\hline & \multicolumn{2}{|c|}{ Angola } & \multicolumn{2}{|c|}{ Australla } & \multicolumn{2}{|c|}{$\begin{array}{l}\text { Bahama } \\
\text { blands }\end{array}$} & \multicolumn{2}{|c|}{ Brazll } & \multicolumn{2}{|c|}{ Ceneda } & \multicolumn{2}{|c|}{ Chine } \\
\hline & Total & Crude Oll & Total & Crude oll & Total & Cruds OII & Total & Crude oll & Total & Crude oll & Total & Crude Oll \\
\hline 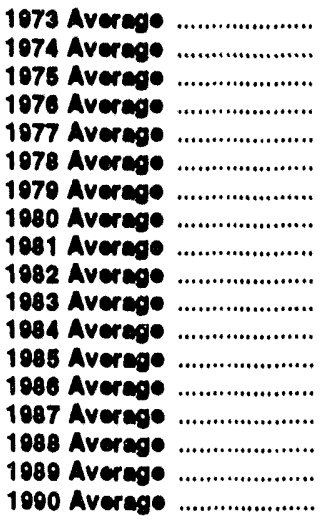 & $\begin{array}{l}49 \\
49 \\
75 \\
12 \\
24 \\
20 \\
43 \\
42 \\
40 \\
44 \\
70 \\
00 \\
110 \\
112 \\
192 \\
212 \\
284 \\
237\end{array}$ & $\begin{array}{r}40 \\
46 \\
71 \\
7 \\
17 \\
6 \\
30 \\
37 \\
45 \\
42 \\
71 \\
86 \\
104 \\
102 \\
180 \\
203 \\
270 \\
236\end{array}$ & $\begin{array}{l}2 \\
1 \\
5 \\
2 \\
3 \\
5 \\
1 \\
6 \\
5 \\
4 \\
36 \\
41 \\
50 \\
64 \\
30 \\
63\end{array}$ & $\begin{array}{l}0 \\
0 \\
0 \\
0 \\
0 \\
0 \\
0 \\
0 \\
0 \\
(0) \\
0 \\
25 \\
21 \\
30 \\
40 \\
50 \\
31 \\
47\end{array}$ & $\begin{array}{r}174 \\
164 \\
182 \\
110 \\
171 \\
160 \\
147 \\
70 \\
74 \\
66 \\
128 \\
69 \\
40 \\
37 \\
37 \\
32 \\
34 \\
37\end{array}$ & $\begin{array}{l}0 \\
0 \\
0 \\
0 \\
0 \\
0 \\
0 \\
0 \\
0 \\
0 \\
0 \\
0 \\
0 \\
0 \\
0 \\
0 \\
0 \\
0\end{array}$ & $\begin{array}{l}0 \\
2 \\
8 \\
0 \\
0 \\
0 \\
1 \\
3 \\
23 \\
47 \\
11 \\
60 \\
01 \\
50 \\
14 \\
00 \\
12 \\
40\end{array}$ & $\begin{array}{c}0 \\
0 \\
0 \\
0 \\
0 \\
0 \\
0 \\
1 \\
14 \\
10 \\
2 \\
(0) \\
0 \\
0 \\
0 \\
0 \\
0 \\
0\end{array}$ & $\begin{array}{r}1,325 \\
1,070 \\
040 \\
600 \\
517 \\
407 \\
839 \\
486 \\
447 \\
402 \\
847 \\
030 \\
770 \\
007 \\
040 \\
000 \\
031 \\
034\end{array}$ & $\begin{array}{r}1,001 \\
701 \\
600 \\
371 \\
270 \\
240 \\
271 \\
100 \\
104 \\
214 \\
274 \\
341 \\
400 \\
570 \\
600 \\
601 \\
630 \\
043\end{array}$ & $\begin{array}{c}(8) \\
0 \\
0 \\
0 \\
0 \\
0 \\
13 \\
(0) \\
10 \\
40 \\
34 \\
40 \\
80 \\
80 \\
82 \\
80 \\
80 \\
80\end{array}$ & $\begin{array}{r}0 \\
0 \\
0 \\
0 \\
0 \\
0 \\
13 \\
0 \\
0 \\
\vdots \\
18 \\
36 \\
60 \\
63 \\
12 \\
76 \\
77\end{array}$ \\
\hline 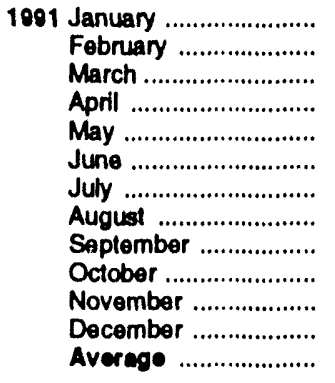 & $\begin{array}{l}232 \\
202 \\
186 \\
337 \\
220 \\
205 \\
264 \\
298 \\
230 \\
300 \\
213 \\
359 \\
254\end{array}$ & $\begin{array}{l}232 \\
202 \\
186 \\
337 \\
220 \\
205 \\
264 \\
298 \\
230 \\
300 \\
213 \\
359 \\
254\end{array}$ & $\begin{array}{r}21 \\
0 \\
0 \\
55 \\
64 \\
43 \\
20 \\
37 \\
24 \\
13 \\
25 \\
13 \\
26\end{array}$ & $\begin{array}{r}21 \\
0 \\
0 \\
55 \\
57 \\
31 \\
20 \\
22 \\
24 \\
0 \\
13 \\
13 \\
21\end{array}$ & $\begin{array}{r}25 \\
14 \\
0 \\
36 \\
42 \\
30 \\
19 \\
78 \\
29 \\
51 \\
46 \\
53 \\
35\end{array}$ & $\begin{array}{l}0 \\
0 \\
0 \\
0 \\
0 \\
0 \\
0 \\
0 \\
0 \\
0 \\
0 \\
0 \\
0\end{array}$ & $\begin{array}{r}31 \\
13 \\
0 \\
17 \\
31 \\
41 \\
21 \\
27 \\
19 \\
16 \\
45 \\
8 \\
22\end{array}$ & $\begin{array}{l}0 \\
0 \\
0 \\
0 \\
0 \\
0 \\
0 \\
0 \\
0 \\
0 \\
0 \\
0 \\
0\end{array}$ & $\begin{array}{r}978 \\
1,135 \\
1,058 \\
1,103 \\
1,027 \\
986 \\
848 \\
1,011 \\
1,137 \\
936 \\
1,107 \\
1,083 \\
1,033\end{array}$ & $\begin{array}{l}718 \\
881 \\
764 \\
768 \\
752 \\
705 \\
615 \\
694 \\
849 \\
639 \\
796 \\
759 \\
743\end{array}$ & $\begin{array}{r}68 \\
102 \\
96 \\
113 \\
119 \\
144 \\
88 \\
85 \\
91 \\
29 \\
96 \\
65 \\
91\end{array}$ & $\begin{array}{r}63 \\
96 \\
96 \\
113 \\
113 \\
139 \\
88 \\
75 \\
86 \\
24 \\
96 \\
65 \\
97\end{array}$ \\
\hline 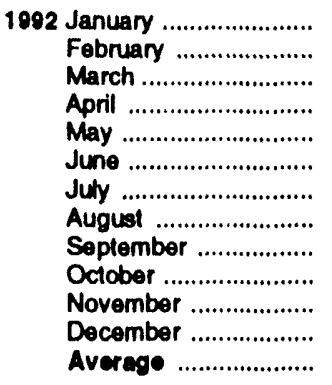 & $\begin{array}{l}360 \\
246 \\
339 \\
381 \\
264 \\
286 \\
443 \\
335 \\
248 \\
395 \\
458 \\
279 \\
336\end{array}$ & $\begin{array}{l}360 \\
246 \\
339 \\
381 \\
264 \\
286 \\
143 \\
323 \\
248 \\
395 \\
458 \\
279 \\
336\end{array}$ & $\begin{array}{r}11 \\
10 \\
0 \\
39 \\
0 \\
21 \\
20 \\
21 \\
0 \\
11 \\
53 \\
38 \\
10\end{array}$ & $\begin{array}{r}11 \\
10 \\
0 \\
22 \\
0 \\
21 \\
20 \\
21 \\
0 \\
11 \\
49 \\
38 \\
17\end{array}$ & $\begin{array}{l}63 \\
47 \\
76 \\
67 \\
46 \\
57 \\
22 \\
8 \\
8 \\
1 \\
20 \\
19 \\
36\end{array}$ & $\begin{array}{l}0 \\
0 \\
0 \\
0 \\
0 \\
0 \\
0 \\
0 \\
0 \\
0 \\
0 \\
0 \\
0\end{array}$ & $\begin{array}{l}18 \\
12 \\
(s) \\
17 \\
18 \\
28 \\
25 \\
10 \\
21 \\
10 \\
32 \\
50 \\
20\end{array}$ & $\begin{array}{l}0 \\
0 \\
0 \\
0 \\
0 \\
0 \\
0 \\
0 \\
0 \\
0 \\
0 \\
0 \\
0\end{array}$ & $\begin{array}{r}1,045 \\
1,147 \\
1,100 \\
1,121 \\
1,013 \\
970 \\
1,044 \\
1,038 \\
1,131 \\
1,063 \\
1,037 \\
1,122 \\
1,060\end{array}$ & $\begin{array}{l}786 \\
834 \\
632 \\
635 \\
779 \\
736 \\
798 \\
762 \\
839 \\
761 \\
784 \\
816 \\
707\end{array}$ & $\begin{array}{r}144 \\
80 \\
75 \\
86 \\
129 \\
110 \\
68 \\
66 \\
80 \\
61 \\
86 \\
87 \\
\infty 0\end{array}$ & $\begin{array}{r}144 \\
69 \\
75 \\
69 \\
114 \\
95 \\
64 \\
66 \\
75 \\
61 \\
86 \\
90 \\
84\end{array}$ \\
\hline 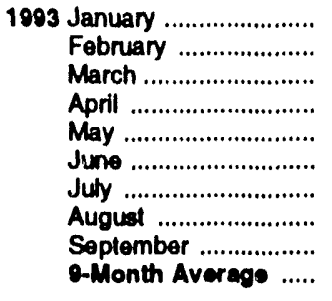 & $\begin{array}{l}354 \\
348 \\
408 \\
322 \\
287 \\
209 \\
386 \\
258 \\
282 \\
317\end{array}$ & $\begin{array}{l}354 \\
348 \\
408 \\
322 \\
287 \\
209 \\
386 \\
258 \\
282 \\
317\end{array}$ & $\begin{array}{r}0 \\
0 \\
0 \\
0 \\
13 \\
34 \\
40 \\
33 \\
0 \\
14\end{array}$ & $\begin{array}{r}0 \\
0 \\
0 \\
0 \\
13 \\
34 \\
40 \\
27 \\
0 \\
13\end{array}$ & $\begin{array}{r}18 \\
19 \\
30 \\
16 \\
8 \\
7 \\
31 \\
37 \\
27 \\
22\end{array}$ & $\begin{array}{l}0 \\
0 \\
0 \\
0 \\
0 \\
0 \\
0 \\
0 \\
0 \\
0\end{array}$ & $\begin{array}{r}3 \\
22 \\
27 \\
56 \\
41 \\
19 \\
48 \\
32 \\
59 \\
34\end{array}$ & $\begin{array}{l}0 \\
0 \\
0 \\
0 \\
0 \\
0 \\
0 \\
0 \\
0 \\
0\end{array}$ & $\begin{array}{l}1,034 \\
1,084 \\
1,065 \\
1,032 \\
1,119 \\
1,111 \\
1,247 \\
1,237 \\
1,300 \\
1,138\end{array}$ & $\begin{array}{r}778 \\
782 \\
814 \\
783 \\
874 \\
910 \\
991 \\
986 \\
1,018 \\
890\end{array}$ & $\begin{array}{r}60 \\
44 \\
79 \\
0 \\
40 \\
48 \\
24 \\
38 \\
91 \\
47\end{array}$ & $\begin{array}{r}60 \\
44 \\
73 \\
0 \\
40 \\
46 \\
24 \\
38 \\
89 \\
46\end{array}$ \\
\hline $\begin{array}{l}1992 \text { 9-Month Average .... } \\
1901 \text { g-Month Averago .... }\end{array}$ & $\begin{array}{l}323 \\
242\end{array}$ & $\begin{array}{l}322 \\
242\end{array}$ & $\begin{array}{l}13 \\
30\end{array}$ & $\begin{array}{l}12 \\
26\end{array}$ & $\begin{array}{l}44 \\
30\end{array}$ & $\frac{0}{v}$ & $\begin{array}{l}10 \\
22\end{array}$ & $\begin{array}{l}0 \\
0\end{array}$ & $\begin{array}{l}1,067 \\
1,030\end{array}$ & $\begin{array}{l}200 \\
748\end{array}$ & $\begin{array}{r}93 \\
100\end{array}$ & $\begin{array}{l}26 \\
06\end{array}$ \\
\hline
\end{tabular}

a Includes petroleum imported into the Uniled Stales indirectly from members of the Organization of Petroloum Exporting Countries (OPEC), primantly from Caribbean and West European areas, as potroloum products that were relined from crude oll produced by OPEC.

(s) =Less than 500 barrels per day.

Noles: - Beginning in October 1977, Strategic Petroloum Reserve imports are included. - Geographic coverage is the 50 States and the Diatrict of Columbia.

Sources: - 1073-1900: Energy Information Administration (EIA), Potroloum Supply Monthy, Fobruary 1993, Tablo S3. - 1981 forward: ELA, Petroleum Supply Monthy, November 1993, Table S3. 
Table 3.3f Petroleum Imports: Colombla, Ecuador, Haly, Malaysia, Mexico, and Netherlands

(Thousand Barrels per Day)

\begin{tabular}{|c|c|c|c|c|c|c|c|c|c|c|c|c|}
\hline & \multicolumn{12}{|c|}{ Non-Optea } \\
\hline & \multicolumn{2}{|c|}{ Colomble } & \multicolumn{2}{|c|}{ Ecuedorb } & \multicolumn{2}{|c|}{ tedy } & \multicolumn{2}{|c|}{ Meloyele } & \multicolumn{2}{|c|}{ Moxion } & \multicolumn{2}{|c|}{ Netherlends } \\
\hline & Total & Crude OII & Totel & Crude OII & Totul & Crude oll & Total & Crude OII & Total & Crude OII & Total & Crude oll \\
\hline 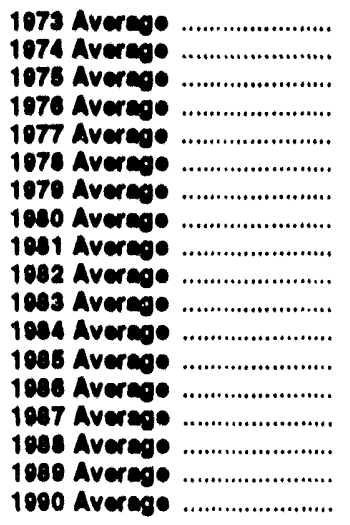 & $\begin{array}{r}0 \\
21 \\
17 \\
20 \\
18 \\
1 \\
1 \\
10 \\
23 \\
14 \\
148 \\
134 \\
172 \\
182\end{array}$ & $\begin{array}{r}2 \\
0 \\
0 \\
0 \\
0 \\
0 \\
0 \\
0 \\
0 \\
0 \\
0 \\
0 \\
0 \\
87 \\
116 \\
108 \\
136 \\
140\end{array}$ & $\begin{array}{l}= \\
= \\
= \\
= \\
= \\
= \\
= \\
= \\
=\end{array}$ & $\begin{array}{l}= \\
\bar{z} \\
\bar{z} \\
= \\
\bar{z} \\
\bar{z} \\
\bar{z} \\
-\end{array}$ & $\begin{array}{r}126 \\
74 \\
27 \\
30 \\
61 \\
10 \\
30 \\
4 \\
11 \\
10 \\
10 \\
46 \\
60 \\
70 \\
84 \\
65 \\
34 \\
80\end{array}$ & $\begin{array}{l}0 \\
0 \\
0 \\
0 \\
0 \\
0 \\
0 \\
0 \\
0 \\
(0) \\
(0) \\
(0) \\
(0) \\
0 \\
1 \\
5 \\
3 \\
2\end{array}$ & $\begin{array}{r}12 \\
12 \\
1 \\
10 \\
00 \\
42 \\
60 \\
70 \\
20 \\
20 \\
4 \\
1 \\
3 \\
12 \\
13 \\
10 \\
30 \\
41\end{array}$ & $\begin{array}{r}1 \\
1 \\
8 \\
16 \\
6 \\
37 \\
82 \\
01 \\
29 \\
10 \\
3 \\
0 \\
1 \\
11 \\
12 \\
10 \\
30 \\
40\end{array}$ & 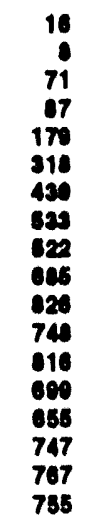 & $\begin{array}{r}1 \\
2 \\
70 \\
67 \\
177 \\
216 \\
497 \\
607 \\
400 \\
646 \\
700 \\
660 \\
718 \\
621 \\
602 \\
674 \\
716 \\
600\end{array}$ & 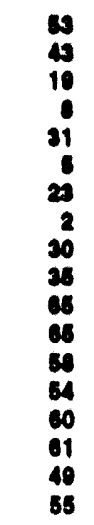 & $\begin{array}{r}0 \\
0 \\
4 \\
0 \\
1 \\
2 \\
7 \\
(0) \\
(0) \\
(0) \\
3 \\
3 \\
0 \\
0 \\
0 \\
0 \\
0 \\
0\end{array}$ \\
\hline 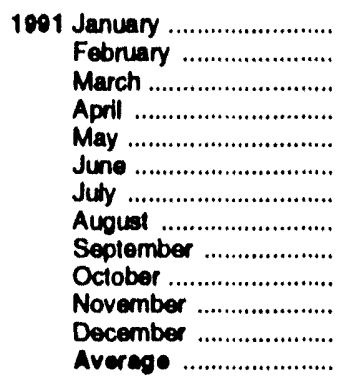 & $\begin{array}{l}194 \\
151 \\
157 \\
163 \\
163 \\
169 \\
163 \\
219 \\
168 \\
128 \\
145 \\
138 \\
163\end{array}$ & $\begin{array}{r}174 \\
98 \\
127 \\
131 \\
112 \\
124 \\
111 \\
162 \\
103 \\
80 \\
135 \\
117 \\
123\end{array}$ & $\begin{array}{l}\overline{-} \\
\overline{-} \\
\bar{z} \\
\bar{z} \\
\overline{-} \\
\overline{-}\end{array}$ & $\begin{array}{l}\overline{-} \\
\overline{-} \\
\bar{z} \\
\overline{-} \\
\overline{-} \\
\overline{-}\end{array}$ & $\begin{array}{l}25 \\
42 \\
29 \\
41 \\
60 \\
46 \\
54 \\
57 \\
89 \\
41 \\
15 \\
61 \\
47\end{array}$ & $\begin{array}{r}0 \\
13 \\
0 \\
12 \\
0 \\
0 \\
0 \\
11 \\
0 \\
0 \\
0 \\
0 \\
3\end{array}$ & $\begin{array}{r}0 \\
9 \\
21 \\
0 \\
66 \\
63 \\
9 \\
14 \\
10 \\
64 \\
10 \\
14 \\
24\end{array}$ & $\begin{array}{r}0 \\
9 \\
21 \\
0 \\
66 \\
63 \\
9 \\
14 \\
10 \\
64 \\
10 \\
14 \\
24\end{array}$ & $\begin{array}{l}798 \\
742 \\
785 \\
891 \\
757 \\
919 \\
836 \\
878 \\
805 \\
811 \\
716 \\
732 \\
807\end{array}$ & $\begin{array}{l}778 \\
603 \\
772 \\
810 \\
736 \\
872 \\
748 \\
797 \\
768 \\
754 \\
666 \\
708 \\
750\end{array}$ & $\begin{array}{r}6 \\
17 \\
33 \\
36 \\
46 \\
49 \\
47 \\
30 \\
44 \\
16 \\
24 \\
4\end{array}$ & $\begin{array}{l}0 \\
0 \\
0 \\
0 \\
0 \\
0 \\
0 \\
0 \\
0 \\
0 \\
0 \\
0 \\
0\end{array}$ \\
\hline 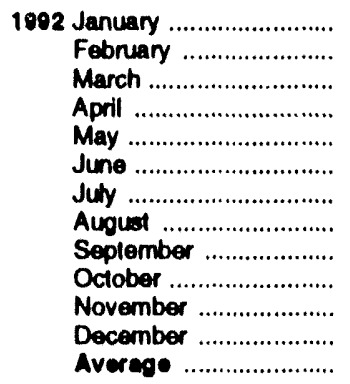 & $\begin{array}{r}158 \\
114 \\
101 \\
150 \\
57 \\
135 \\
103 \\
156 \\
190 \\
153 \\
127 \\
66 \\
126\end{array}$ & $\begin{array}{r}111 \\
92 \\
74 \\
129 \\
46 \\
114 \\
93 \\
142 \\
178 \\
132 \\
84 \\
34 \\
102\end{array}$ & $\begin{array}{l}= \\
= \\
= \\
= \\
\bar{z} \\
\overline{-} \\
=\end{array}$ & $\begin{array}{l}\overline{-} \\
= \\
= \\
z \\
z \\
z \\
z\end{array}$ & $\begin{array}{l}51 \\
48 \\
44 \\
75 \\
57 \\
69 \\
36 \\
94 \\
81 \\
37 \\
33 \\
37 \\
56\end{array}$ & $\begin{array}{l}0 \\
0 \\
0 \\
0 \\
0 \\
0 \\
0 \\
0 \\
0 \\
0 \\
0 \\
0 \\
0\end{array}$ & $\begin{array}{r}0 \\
0 \\
0 \\
0 \\
5 \\
8 \\
40 \\
22 \\
17 \\
17 \\
8 \\
4 \\
10\end{array}$ & $\begin{array}{r}0 \\
0 \\
0 \\
0 \\
5 \\
8 \\
40 \\
22 \\
17 \\
17 \\
8 \\
4 \\
10\end{array}$ & $\begin{array}{l}764 \\
838 \\
846 \\
857 \\
788 \\
906 \\
830 \\
857 \\
755 \\
829 \\
762 \\
930 \\
830\end{array}$ & $\begin{array}{l}721 \\
807 \\
809 \\
785 \\
764 \\
883 \\
788 \\
790 \\
720 \\
783 \\
700 \\
888 \\
787\end{array}$ & $\begin{array}{r}31 \\
9 \\
34 \\
8 \\
27 \\
25 \\
21 \\
45 \\
39 \\
18 \\
26 \\
33 \\
26\end{array}$ & $\begin{array}{l}0 \\
0 \\
0 \\
0 \\
0 \\
0 \\
0 \\
0 \\
0 \\
0 \\
0 \\
0 \\
0\end{array}$ \\
\hline 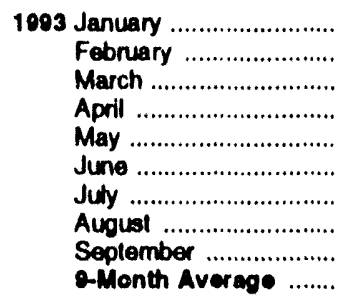 & $\begin{array}{l}188 \\
148 \\
161 \\
152 \\
147 \\
176 \\
204 \\
124 \\
224 \\
160\end{array}$ & $\begin{array}{r}167 \\
137 \\
129 \\
138 \\
90 \\
143 \\
184 \\
101 \\
170 \\
140\end{array}$ & $\begin{array}{r}76 \\
14 \\
59 \\
74 \\
56 \\
75 \\
85 \\
121 \\
49 \\
68\end{array}$ & $\begin{array}{r}70 \\
14 \\
59 \\
62 \\
56 \\
75 \\
85 \\
121 \\
49 \\
66\end{array}$ & $\begin{array}{l}48 \\
34 \\
43 \\
14 \\
18 \\
22 \\
25 \\
50 \\
32 \\
32\end{array}$ & $\begin{array}{l}0 \\
0 \\
0 \\
0 \\
0 \\
0 \\
0 \\
0 \\
0 \\
0\end{array}$ & $\begin{array}{r}0 \\
0 \\
11 \\
8 \\
21 \\
0 \\
11 \\
14 \\
28 \\
10\end{array}$ & $\begin{array}{r}0 \\
0 \\
10 \\
8 \\
10 \\
0 \\
11 \\
14 \\
28 \\
0\end{array}$ & $\begin{array}{l}858 \\
807 \\
861 \\
844 \\
907 \\
995 \\
943 \\
862 \\
829 \\
800\end{array}$ & $\begin{array}{l}820 \\
748 \\
815 \\
818 \\
846 \\
977 \\
878 \\
809 \\
867 \\
643\end{array}$ & $\begin{array}{r}11 \\
18 \\
11 \\
0 \\
10 \\
10 \\
20 \\
17 \\
22 \\
13\end{array}$ & $\begin{array}{l}0 \\
0 \\
0 \\
0 \\
0 \\
0 \\
0 \\
0 \\
0 \\
0\end{array}$ \\
\hline $\begin{array}{l}1002 \text { Q-Month Averege ........ } \\
1001 \text { Q-Month Averege ....... }\end{array}$ & $\begin{array}{l}120 \\
172\end{array}$ & $\begin{array}{l}100 \\
127\end{array}$ & - & - & $\begin{array}{l}62 \\
40\end{array}$ & : & $\begin{array}{l}10 \\
22\end{array}$ & $\begin{array}{l}10 \\
22\end{array}$ & $\begin{array}{l}827 \\
825\end{array}$ & $\begin{array}{l}788 \\
776\end{array}$ & $\begin{array}{l}27 \\
34\end{array}$ & : \\
\hline
\end{tabular}

- Includes petroleum Imported into the United States indirectly trom members of the Organization of Petroleum Exporting Countries (OPEC). primarily Irom Caribbean and West European areas, as petroleum products that were relined from crude oll produced by OPEC.

b Through 1992, Ecuador was a member of OPEC. See Table 3.3c.

- =Nol applicable. (s)=Less than 500 barrels per day.
Notes: - Beginning in October 1977 . Stralegic Pelroleum Reserve inponts are included. - Geographic coverage is the 50 States and the District of Columbia.

Sources: - 1073-1080: Energy Inlormation Administration (EIA), Petroleum Supply Monthly, February 1993, Tablo S3. - 1901 forward: EIA. Perroleum Supply Monthy, November 1993, Table S3. 
Table 3.3g Petroleum Imports: Netherlands Antilles, Norway, Puerto Rico, Russia, Spain, and Trinidad and Tobago

(Thousand Barrels per Day)

\begin{tabular}{|c|c|c|c|c|c|c|c|c|c|c|c|c|}
\hline & \multicolumn{12}{|c|}{ Non-Opece } \\
\hline & \multicolumn{2}{|c|}{$\begin{array}{l}\text { Notherlands } \\
\text { Antilles }\end{array}$} & \multicolumn{2}{|c|}{ Nonway } & \multicolumn{2}{|c|}{ Pucrto Ako } & \multicolumn{2}{|c|}{ Aceelab } & \multicolumn{2}{|c|}{ epeln } & \multicolumn{2}{|c|}{$\begin{array}{l}\text { Trinided } \\
\text { and Tobage }\end{array}$} \\
\hline & Totel & Cruds oll & Totel & Crude Oil & Total & Crude OII & Totel & Crude oll & Total & crude oll & Total & Crude OII \\
\hline 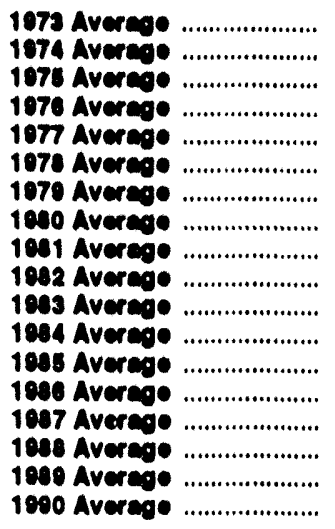 & $\begin{array}{l}648 \\
611 \\
932 \\
27 \\
211 \\
220 \\
231 \\
228 \\
107 \\
176 \\
180 \\
180 \\
40 \\
28 \\
20 \\
30 \\
42 \\
31\end{array}$ & $\begin{array}{l}0 \\
0 \\
0 \\
0 \\
0 \\
0 \\
0 \\
0 \\
0 \\
0 \\
0 \\
0 \\
0 \\
0 \\
0 \\
0 \\
0 \\
0\end{array}$ & $\begin{array}{r}1 \\
1 \\
17 \\
20 \\
60 \\
104 \\
75 \\
144 \\
110 \\
102 \\
60 \\
114 \\
32 \\
60 \\
10 \\
67 \\
130 \\
102\end{array}$ & $\begin{array}{r}0 \\
1 \\
12 \\
31 \\
40 \\
104 \\
75 \\
144 \\
114 \\
102 \\
06 \\
112 \\
31 \\
69 \\
70 \\
62 \\
127 \\
\infty 6\end{array}$ & 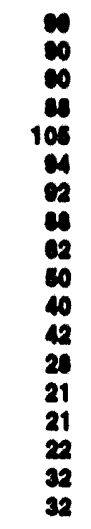 & $\begin{array}{l}0 \\
0 \\
0 \\
0 \\
0 \\
0 \\
0 \\
0 \\
0 \\
0 \\
0 \\
0 \\
0 \\
0 \\
0 \\
0 \\
0 \\
0\end{array}$ & $\begin{array}{r}20 \\
20 \\
14 \\
11 \\
12 \\
\vdots \\
1 \\
1 \\
1 \\
1 \\
13 \\
18 \\
11 \\
20 \\
16 \\
46\end{array}$ & $\begin{array}{l}0 \\
0 \\
0 \\
2 \\
2 \\
1 \\
0 \\
0 \\
(0) \\
0 \\
(0) \\
(0) \\
(0) \\
(0) \\
0 \\
0 \\
0 \\
1\end{array}$ & $\begin{array}{r}20 \\
12 \\
1 \\
1 \\
10 \\
3 \\
1 \\
1 \\
1 \\
3 \\
2 \\
11 \\
20 \\
83 \\
85 \\
69 \\
07 \\
47\end{array}$ & $\begin{array}{r}0 \\
0 \\
0 \\
0 \\
0 \\
0 \\
0 \\
0 \\
(0) \\
(0) \\
(0) \\
0 \\
1 \\
0 \\
0 \\
0 \\
0 \\
0\end{array}$ & $\begin{array}{c}200 \\
281 \\
240 \\
274 \\
200 \\
209 \\
100 \\
170 \\
139 \\
112 \\
06 \\
94 \\
113 \\
126 \\
100 \\
97 \\
94 \\
90\end{array}$ & $\begin{array}{c}60 \\
09 \\
118 \\
104 \\
184 \\
142 \\
128 \\
118 \\
102 \\
92 \\
03 \\
07 \\
08 \\
93 \\
75 \\
71 \\
73 \\
70\end{array}$ \\
\hline 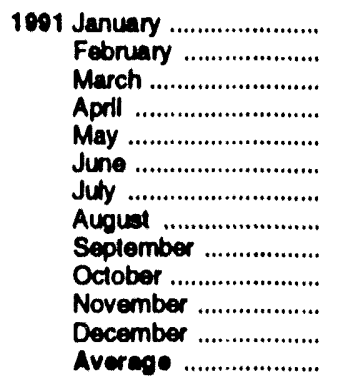 & $\begin{array}{r}103 \\
23 \\
56 \\
61 \\
113 \\
84 \\
86 \\
100 \\
67 \\
80 \\
100 \\
88 \\
01\end{array}$ & $\begin{array}{l}0 \\
0 \\
0 \\
0 \\
0 \\
0 \\
0 \\
0 \\
0 \\
0 \\
0 \\
0 \\
0\end{array}$ & $\begin{array}{r}45 \\
37 \\
25 \\
51 \\
165 \\
99 \\
69 \\
142 \\
79 \\
98 \\
73 \\
94 \\
92\end{array}$ & $\begin{array}{r}34 \\
37 \\
16 \\
35 \\
156 \\
84 \\
63 \\
136 \\
72 \\
98 \\
66 \\
88 \\
74\end{array}$ & $\begin{array}{l}22 \\
20 \\
14 \\
23 \\
42 \\
19 \\
25 \\
42 \\
34 \\
12 \\
36 \\
36 \\
27\end{array}$ & $\begin{array}{l}0 \\
0 \\
0 \\
0 \\
0 \\
0 \\
0 \\
0 \\
0 \\
0 \\
0 \\
0 \\
0\end{array}$ & $\begin{array}{l}28 \\
17 \\
13 \\
39 \\
12 \\
0 \\
58 \\
80 \\
23 \\
13 \\
16 \\
16 \\
20\end{array}$ & $\begin{array}{r}0 \\
0 \\
0 \\
0 \\
0 \\
0 \\
0 \\
11 \\
0 \\
0 \\
0 \\
0 \\
1\end{array}$ & $\begin{array}{l}28 \\
18 \\
13 \\
66 \\
53 \\
11 \\
22 \\
48 \\
42 \\
24 \\
19 \\
28 \\
39\end{array}$ & $\begin{array}{l}0 \\
0 \\
0 \\
0 \\
0 \\
0 \\
0 \\
0 \\
0 \\
0 \\
0 \\
0 \\
0\end{array}$ & $\begin{array}{r}76 \\
78 \\
86 \\
84 \\
61 \\
118 \\
81 \\
91 \\
119 \\
88 \\
77 \\
87 \\
08\end{array}$ & $\begin{array}{r}64 \\
76 \\
73 \\
64 \\
61 \\
104 \\
72 \\
68 \\
75 \\
76 \\
69 \\
71 \\
72\end{array}$ \\
\hline 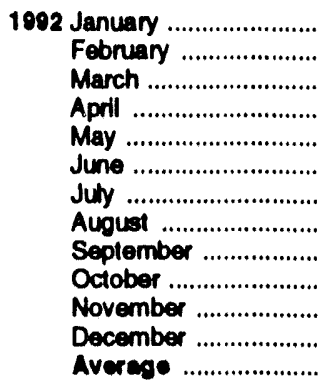 & $\begin{array}{l}40 \\
82 \\
49 \\
73 \\
59 \\
83 \\
49 \\
65 \\
60 \\
90 \\
56 \\
80 \\
68\end{array}$ & $\begin{array}{l}0 \\
0 \\
0 \\
0 \\
0 \\
0 \\
0 \\
0 \\
0 \\
0 \\
0 \\
0 \\
0\end{array}$ & $\begin{array}{r}25 \\
11 \\
11 \\
155 \\
210 \\
234 \\
186 \\
142 \\
103 \\
190 \\
111 \\
140 \\
127\end{array}$ & $\begin{array}{r}17 \\
0 \\
0 \\
147 \\
200 \\
225 \\
179 \\
134 \\
102 \\
177 \\
104 \\
133 \\
110\end{array}$ & $\begin{array}{l}32 \\
23 \\
18 \\
14 \\
22 \\
36 \\
11 \\
38 \\
37 \\
29 \\
26 \\
28 \\
26\end{array}$ & $\begin{array}{l}0 \\
0 \\
0 \\
0 \\
0 \\
0 \\
0 \\
0 \\
0 \\
0 \\
0 \\
0 \\
0\end{array}$ & $\begin{array}{r}17 \\
3 \\
0 \\
0 \\
0 \\
0 \\
72 \\
62 \\
53 \\
9 \\
0 \\
0 \\
18\end{array}$ & $\begin{array}{r}0 \\
0 \\
0 \\
0 \\
0 \\
0 \\
32 \\
31 \\
0 \\
0 \\
0 \\
0 \\
0\end{array}$ & $\begin{array}{l}36 \\
16 \\
37 \\
36 \\
30 \\
46 \\
18 \\
29 \\
56 \\
32 \\
36 \\
17 \\
32\end{array}$ & $\begin{array}{l}0 \\
0 \\
0 \\
0 \\
0 \\
0 \\
0 \\
0 \\
0 \\
0 \\
0 \\
0 \\
0\end{array}$ & $\begin{array}{r}108 \\
109 \\
105 \\
79 \\
69 \\
94 \\
103 \\
106 \\
84 \\
108 \\
85 \\
81 \\
08\end{array}$ & $\begin{array}{l}70 \\
76 \\
85 \\
75 \\
54 \\
74 \\
78 \\
54 \\
56 \\
71 \\
62 \\
71 \\
70\end{array}$ \\
\hline 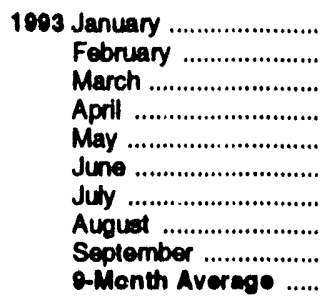 & $\begin{array}{l}73 \\
80 \\
61 \\
86 \\
77 \\
55 \\
52 \\
52 \\
97 \\
70\end{array}$ & $\begin{array}{l}0 \\
0 \\
0 \\
0 \\
0 \\
0 \\
0 \\
0 \\
0 \\
0\end{array}$ & $\begin{array}{r}70 \\
62 \\
122 \\
109 \\
65 \\
160 \\
215 \\
180 \\
113 \\
122\end{array}$ & $\begin{array}{r}70 \\
61 \\
115 \\
100 \\
65 \\
160 \\
215 \\
161 \\
113 \\
110\end{array}$ & $\begin{array}{l}37 \\
21 \\
26 \\
18 \\
38 \\
29 \\
49 \\
30 \\
28 \\
31\end{array}$ & $\begin{array}{l}0 \\
0 \\
0 \\
0 \\
0 \\
0 \\
0 \\
0 \\
0 \\
0\end{array}$ & $\begin{array}{r}0 \\
0 \\
0 \\
16 \\
32 \\
59 \\
157 \\
26 \\
57 \\
30\end{array}$ & $\begin{array}{r}0 \\
0 \\
0 \\
16 \\
32 \\
34 \\
134 \\
0 \\
29 \\
27\end{array}$ & $\begin{array}{l}44 \\
26 \\
21 \\
61 \\
34 \\
20 \\
41 \\
37 \\
54 \\
36\end{array}$ & $\begin{array}{l}0 \\
0 \\
0 \\
0 \\
0 \\
0 \\
0 \\
0 \\
0 \\
0\end{array}$ & $\begin{array}{l}59 \\
72 \\
92 \\
78 \\
61 \\
77 \\
82 \\
50 \\
70 \\
71\end{array}$ & $\begin{array}{l}48 \\
58 \\
71 \\
55 \\
51 \\
55 \\
53 \\
37 \\
56 \\
54\end{array}$ \\
\hline 1002 g-Month Average ..... & $\frac{62}{70}$ & $\begin{array}{l}0 \\
0\end{array}$ & $\begin{array}{r}120 \\
80\end{array}$ & $\begin{array}{r}112 \\
n\end{array}$ & $\begin{array}{l}20 \\
27\end{array}$ & $\begin{array}{l}0 \\
0\end{array}$ & 23 & $\begin{array}{l}7 \\
1\end{array}$ & $\begin{array}{l}33 \\
37\end{array}$ & : & $\begin{array}{l}96 \\
80\end{array}$ & $\begin{array}{l}70 \\
73\end{array}$ \\
\hline
\end{tabular}

- Includes petroleum imported into the United States indirectly from members of the Omanization of Petroleum Exporting Countiles (OPEC), primarity from Caribbean and West European areas, as petroleum products that were rethed from crude oll produced by OPEC.

Imports from other States in the lormer U.S.S.R. may be hcluded in imports from Russia for the years 1973 through 1892.

(s) =Lees than 500 barrels per day.
Notes: - Beginning in Odaber 1977, Strategic Petroleum Reserve imponts are included. - Geographic coverage bo the 50 Stales and the District of Columbia.

Sources: - 1973-1060: Energy Information Administration (ELA), Petroloum Supply Monthy, February 1993, Table S3. - 1081 forward: EIA Petroleum Supply Monthy, November 1993, Table S3. 
Table 3.3h Potroleum Imports: Unltod KIngdom, Virgin lelands, Other Non-OPEC, Total Non-OPEC, and Total Imports

(Thousand Barrels per Day)

\begin{tabular}{|c|c|c|c|c|c|c|c|c|c|c|}
\hline & \multicolumn{6}{|c|}{ Non-Opece } & \multirow{2}{*}{\multicolumn{2}{|c|}{$\begin{array}{c}\text { Taty } \\
\text { Mon-opecab } \\
\end{array}$}} & \multirow{2}{*}{\multicolumn{2}{|c|}{$\begin{array}{c}\text { Total } \\
\text { Importe }\end{array}$}} \\
\hline & \multicolumn{2}{|c|}{$\begin{array}{c}\text { Unned } \\
\text { Klnodom } \\
\end{array}$} & \multicolumn{2}{|c|}{ Viroin belande } & \multicolumn{2}{|c|}{$\begin{array}{l}\text { Oine } \\
\text { Non-opec }\end{array}$} & & & & \\
\hline & Total & Crude oll & Totel & Crude oll & Tetal & Crude of & Tord & Crude of & Toud & Crude oll \\
\hline 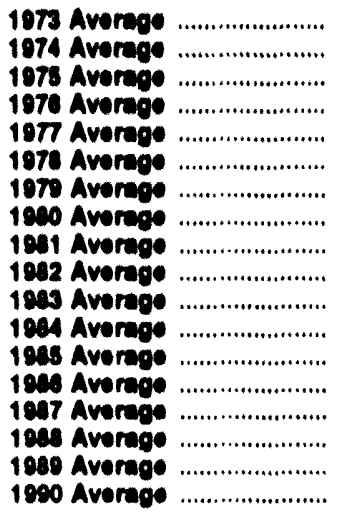 & $\begin{array}{r}18 \\
11 \\
31 \\
120 \\
180 \\
202 \\
170 \\
378 \\
480 \\
392 \\
402 \\
310 \\
350 \\
382 \\
318 \\
218 \\
180\end{array}$ & $\begin{array}{l}0 \\
0 \\
(0) \\
13 \\
97 \\
100 \\
107 \\
173 \\
309 \\
411 \\
396 \\
374 \\
270 \\
317 \\
304 \\
284 \\
160 \\
185\end{array}$ & $\begin{array}{l}320 \\
391 \\
400 \\
420 \\
404 \\
420 \\
431 \\
340 \\
327 \\
310 \\
202 \\
294 \\
247 \\
244 \\
217 \\
242 \\
321 \\
202\end{array}$ & $\begin{array}{l}0 \\
0 \\
0 \\
0 \\
0 \\
0 \\
0 \\
0 \\
0 \\
0 \\
0 \\
0 \\
0 \\
0 \\
0 \\
0 \\
0 \\
0\end{array}$ & 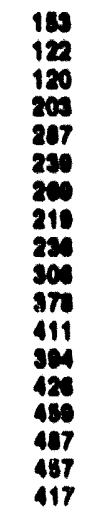 & $\begin{array}{c}31 \\
30 \\
14 \\
101 \\
187 \\
140 \\
192 \\
102 \\
109 \\
174 \\
218 \\
210 \\
197 \\
144 \\
100 \\
190 \\
197 \\
1100\end{array}$ & 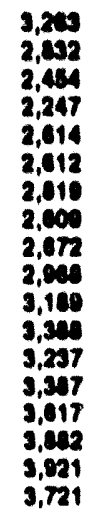 & $\begin{array}{r}1,140 \\
937 \\
993 \\
742 \\
071 \\
1,172 \\
1,407 \\
1,309 \\
1,474 \\
1,784 \\
1,084 \\
1,014 \\
1,016 \\
2,006 \\
2,274 \\
2,411 \\
2,467 \\
2,301\end{array}$ & $\begin{array}{l}6,286 \\
6,112 \\
6,056 \\
7,313 \\
6,007 \\
6,303 \\
6,480 \\
6,000 \\
6,000 \\
6,113 \\
8,051 \\
6,437 \\
8,067 \\
6,204 \\
6,670 \\
7,402 \\
6,061 \\
6,010\end{array}$ & $\begin{array}{l}3,244 \\
3,47 \\
4,106 \\
5,207 \\
6,615 \\
6,364 \\
0,310 \\
5,203 \\
4,300 \\
3,404 \\
3,340 \\
3,424 \\
3,201 \\
4,170 \\
4,074 \\
5,107 \\
5,043 \\
5,004\end{array}$ \\
\hline 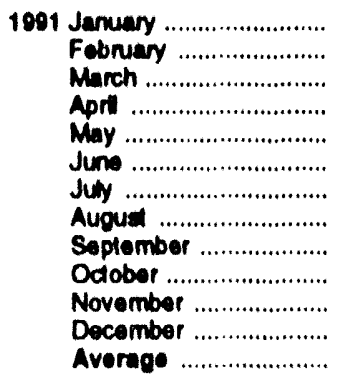 & $\begin{array}{r}32 \\
34 \\
46 \\
61 \\
222 \\
105 \\
228 \\
254 \\
218 \\
201 \\
84 \\
154 \\
138\end{array}$ & $\begin{array}{r}10 \\
21 \\
10 \\
37 \\
188 \\
70 \\
164 \\
217 \\
194 \\
166 \\
18 \\
151 \\
106\end{array}$ & $\begin{array}{l}261 \\
222 \\
214 \\
246 \\
264 \\
234 \\
101 \\
206 \\
269 \\
262 \\
264 \\
286 \\
243\end{array}$ & $\begin{array}{l}0 \\
0 \\
0 \\
0 \\
0 \\
0 \\
0 \\
0 \\
0 \\
0 \\
0 \\
0 \\
0\end{array}$ & $\begin{array}{l}236 \\
180 \\
170 \\
256 \\
230 \\
340 \\
384 \\
360 \\
374 \\
252 \\
335 \\
220 \\
2112\end{array}$ & $\begin{array}{l}91 \\
96 \\
60 \\
90 \\
63 \\
189 \\
275 \\
197 \\
197 \\
139 \\
130 \\
104 \\
137\end{array}$ & $\begin{array}{l}3,206 \\
3,061 \\
3,023 \\
3,074 \\
3,794 \\
3,747 \\
3,524 \\
4,067 \\
3,871 \\
3,444 \\
3,444 \\
3,546 \\
3,535\end{array}$ & $\begin{array}{l}2,195 \\
2,221 \\
2,133 \\
2,470 \\
2,524 \\
2,587 \\
2,430 \\
2,400 \\
2,600 \\
2,340 \\
2,200 \\
2,446 \\
2,405\end{array}$ & $\begin{array}{l}7,103 \\
6,865 \\
6,646 \\
7,418 \\
8,518 \\
6,245 \\
7,755 \\
6,670 \\
7,826 \\
7,467 \\
7,615 \\
7,337 \\
7,627\end{array}$ & $\begin{array}{l}5,290 \\
5.486 \\
5,166 \\
5,529 \\
6,363 \\
6,334 \\
5,955 \\
6.645 \\
5,812 \\
5,683 \\
5,528 \\
5.565 \\
5,782\end{array}$ \\
\hline 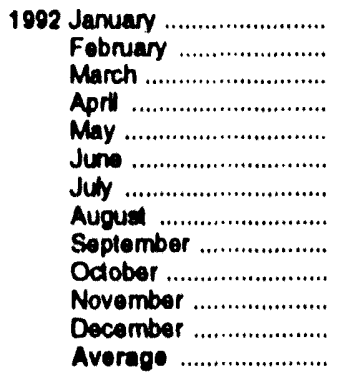 & $\begin{array}{r}129 \\
63 \\
70 \\
157 \\
198 \\
248 \\
354 \\
295 \\
341 \\
411 \\
336 \\
146 \\
230\end{array}$ & $\begin{array}{r}115 \\
0 \\
52 \\
128 \\
180 \\
206 \\
337 \\
282 \\
291 \\
111 \\
285 \\
110 \\
200\end{array}$ & $\begin{array}{l}250 \\
222 \\
202 \\
234 \\
240 \\
266 \\
280 \\
263 \\
217 \\
254 \\
274 \\
273 \\
249\end{array}$ & $\begin{array}{l}0 \\
0 \\
0 \\
0 \\
0 \\
0 \\
0 \\
0 \\
0 \\
0 \\
0 \\
0 \\
0\end{array}$ & $\begin{array}{l}208 \\
106 \\
346 \\
458 \\
467 \\
297 \\
415 \\
464 \\
382 \\
279 \\
218 \\
283 \\
336\end{array}$ & $\begin{array}{r}50 \\
50 \\
114 \\
212 \\
225 \\
95 \\
152 \\
357 \\
160 \\
144 \\
124 \\
92 \\
149\end{array}$ & $\begin{array}{l}3,468 \\
3,278 \\
3,462 \\
4,007 \\
3,705 \\
3,917 \\
4,140 \\
4,116 \\
3,904 \\
3,998 \\
3,786 \\
3,734 \\
3,706\end{array}$ & $\begin{array}{l}2,402 \\
2.184 \\
2,380 \\
2.793 \\
2.633 \\
2.741 \\
3.024 \\
2,984 \\
2.687 \\
2.964 \\
2.745 \\
2,556 \\
2,676\end{array}$ & $\begin{array}{l}7,712 \\
6,827 \\
7,068 \\
8,092 \\
7,823 \\
7,946 \\
8,478 \\
8,260 \\
8,178 \\
8,505 \\
7,872 \\
7,839 \\
7,888\end{array}$ & $\begin{array}{l}5,056 \\
5,070 \\
5.321 \\
6.127 \\
6,060 \\
6,171 \\
6.706 \\
6,467 \\
6,218 \\
6,686 \\
6.121 \\
5,237 \\
6,003\end{array}$ \\
\hline 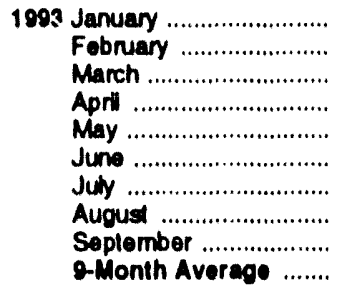 & $\begin{array}{l}228 \\
173 \\
315 \\
348 \\
486 \\
458 \\
292 \\
343 \\
286 \\
327\end{array}$ & $\begin{array}{l}201 \\
127 \\
281 \\
281 \\
458 \\
408 \\
247 \\
323 \\
217 \\
284\end{array}$ & $\begin{array}{l}252 \\
244 \\
244 \\
245 \\
279 \\
290 \\
202 \\
256 \\
184 \\
244\end{array}$ & $\begin{array}{l}0 \\
0 \\
0 \\
0 \\
0 \\
0 \\
0 \\
0 \\
0 \\
0\end{array}$ & $\begin{array}{l}325 \\
223 \\
390 \\
456 \\
356 \\
570 \\
585 \\
520 \\
551 \\
143\end{array}$ & $\begin{array}{l}104 \\
151 \\
186 \\
243 \\
152 \\
405 \\
299 \\
329 \\
251 \\
236\end{array}$ & $\begin{array}{r}3,730 \\
3,439 \\
4,026 \\
3,933 \\
4,006 \\
4,423 \\
4,741 \\
4,318 \\
4,493 \\
4,140\end{array}$ & $\begin{array}{r}0,672 \\
2,471 \\
2,961 \\
2,836 \\
2,974 \\
3,454 \\
3,546 \\
3,184 \\
3,167 \\
3,034\end{array}$ & $\begin{array}{r}7,964 \\
7,930 \\
8,342 \\
8,495 \\
8,348 \\
8,745 \\
9,145 \\
8,360 \\
A 8,476 \\
8,426\end{array}$ & $\begin{array}{r}0,282 \\
6,156 \\
6,513 \\
6,698 \\
6,540 \\
7,175 \\
7,262 \\
6,614 \\
{ }^{R} 6,558 \\
6,650\end{array}$ \\
\hline $\begin{array}{l}1992 \text { 9-Month Avorage ........ } \\
1091 \text { 9-Month Average ........ }\end{array}$ & $\begin{array}{l}208 \\
135\end{array}$ & $\begin{array}{l}178 \\
104\end{array}$ & $\begin{array}{l}242 \\
234\end{array}$ & $\begin{array}{l}0 \\
0\end{array}$ & $\begin{array}{l}360 \\
286\end{array}$ & $\begin{array}{l}159 \\
141\end{array}$ & $\begin{array}{l}3,781 \\
3,354\end{array}$ & $\begin{array}{l}2,650 \\
2,431\end{array}$ & $\begin{array}{l}7,825 \\
7,670\end{array}$ & $\begin{array}{l}6,026 \\
5,846\end{array}$ \\
\hline
\end{tabular}

a Includes petroleum imported into the United Stales indirectly Irom members of the Organization of Petroloum Exporting Counitios (OPEC). pitmarity Irom Caribbean and West Eumopean areas, as petroloum products that were refined from crude oll produced by OPEC.

is of January 1993, includes petrotoum imported from Ecuador, which withdrew from OPEC on Decomber 31, 1982.

$R=$ Revised data. (s)=Less than 500 barrels per day
Notes: - Begining in October 1977, Stralegic Polroloum Reserve imports are included. - Geographic coverage is the 50 States and the Diatina of Columbla. - Totals may not equal sum of components due to independent rounding.

Sources: - 1973-1980: Energy Intormation Administration (EIA). Petroloum Supply Monthy, February 1993, Table S3. - 1981 torward: ElA Petroleum Supply Monthly, November 1993, Table S3. 
Figure 3.2 Finished Motor Casollno

(Million Barrole per Day, Except as Noted)

Overviow, 1973-1992

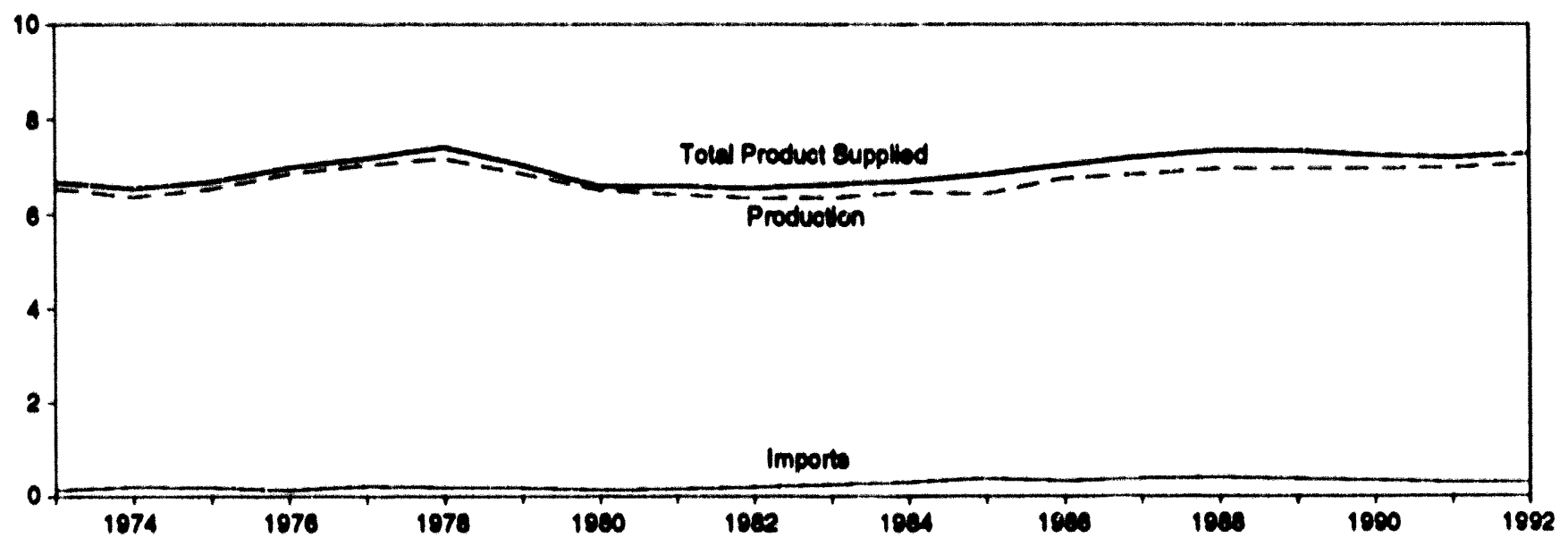

Overview, Monthly

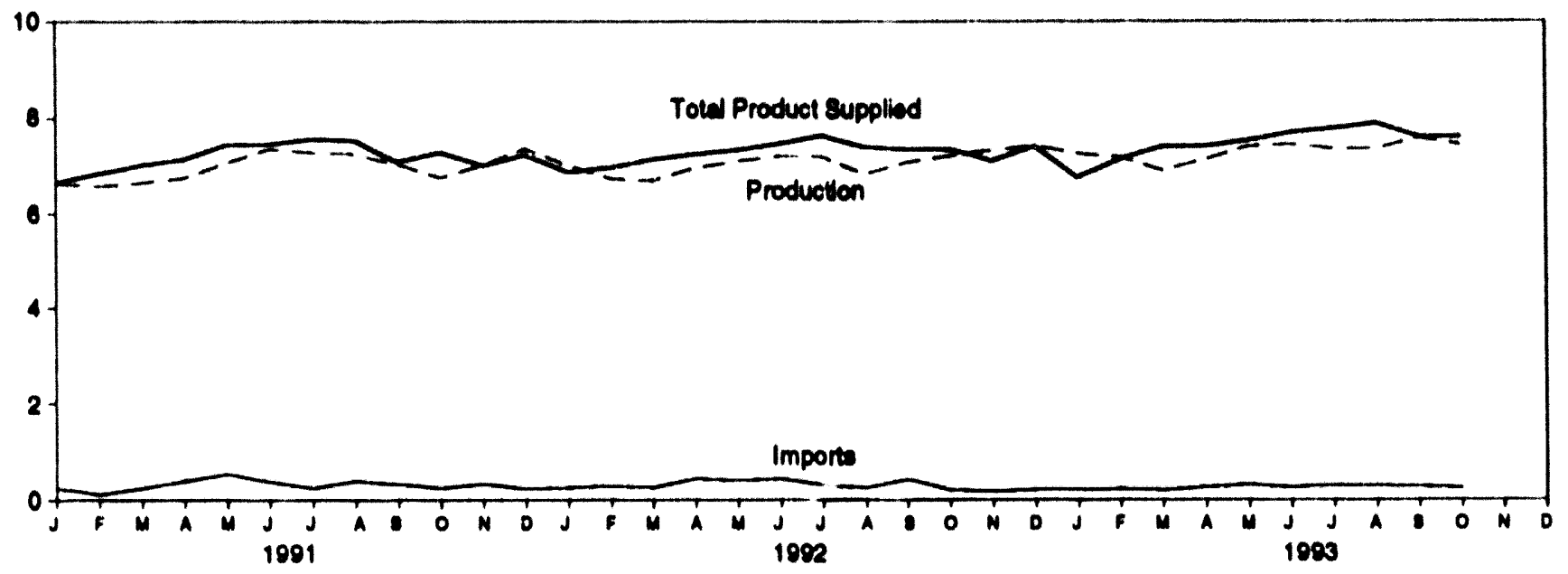

Total Product Supplied, January-October

Total Stocks, End of Month
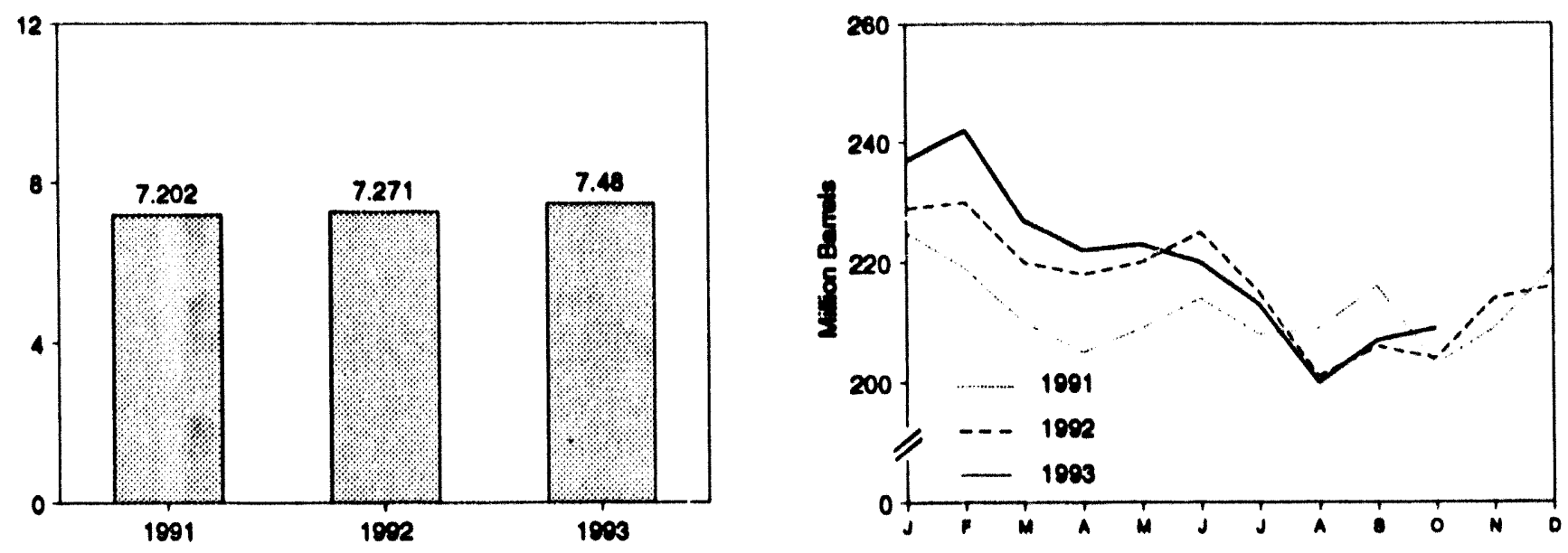

Note: Because ventical ecalse differ, orephe should not be compared.

Source: Trble 3.4. 
Table 3.4 Finished Motor Casoline Supply and Dleposttion

\begin{tabular}{|c|c|c|c|c|c|c|c|c|}
\hline & \multicolumn{2}{|c|}{ supow } & \multicolumn{3}{|c|}{ Dipreseition } & \multicolumn{2}{|c|}{$\begin{array}{l}\text { Meter enoling } \\
\text { Ending Eveolve }\end{array}$} & \multirow{2}{*}{$\begin{array}{c}\text { oryente } \\
\text { Eneoling }\end{array}$} \\
\hline & Production & lonpertat & chenest.o & Exate & Supplied & Tand & Flablen & \\
\hline & \multicolumn{5}{|c|}{ Thousand Bamis per Dey } & \multicolumn{3}{|c|}{ Mimon Barrels } \\
\hline 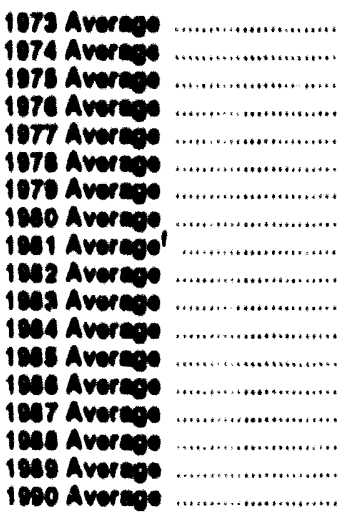 & 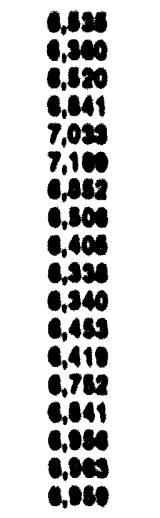 & 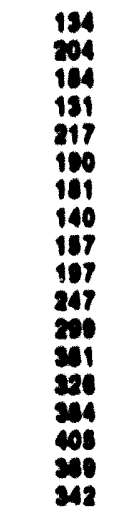 & 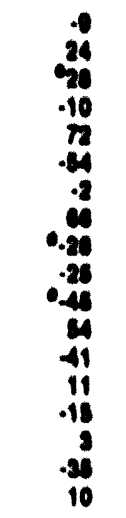 & 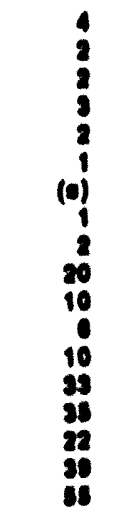 & 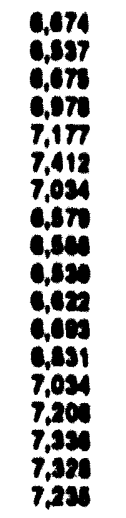 & 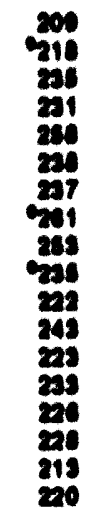 & 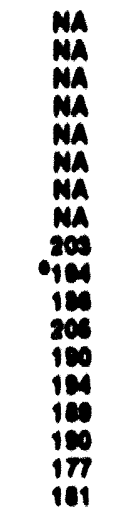 & $\begin{array}{l}M A \\
M A \\
M A \\
M A \\
M A \\
M A \\
M A \\
M A \\
M A \\
M A \\
M A \\
M A \\
M A \\
M A \\
M A \\
M A \\
M A\end{array}$ \\
\hline 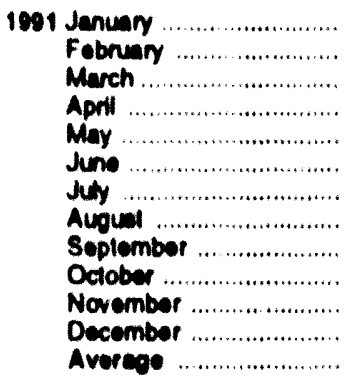 & $\begin{array}{l}6.020 \\
6.573 \\
6.043 \\
6,740 \\
7.043 \\
7.351 \\
7.274 \\
7.247 \\
7.030 \\
6.740 \\
7.010 \\
7.364 \\
0.075\end{array}$ & $\begin{array}{l}220 \\
116 \\
235 \\
301 \\
528 \\
394 \\
232 \\
305 \\
312 \\
230 \\
322 \\
216 \\
297\end{array}$ & $\begin{array}{r}102 \\
.252 \\
.236 \\
.67 \\
85 \\
160 \\
.177 \\
7 \\
105 \\
.364 \\
220 \\
267 \\
3\end{array}$ & $\begin{array}{r}50 \\
102 \\
97 \\
63 \\
50 \\
90 \\
122 \\
90 \\
63 \\
60 \\
104 \\
70 \\
12\end{array}$ & 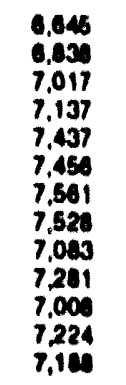 & $\begin{array}{l}226 \\
210 \\
210 \\
205 \\
200 \\
214 \\
200 \\
200 \\
210 \\
203 \\
200 \\
210 \\
210\end{array}$ & $\begin{array}{l}180 \\
170 \\
171 \\
160 \\
172 \\
177 \\
172 \\
172 \\
178 \\
197 \\
173 \\
189 \\
182\end{array}$ & $M_{M M}^{M}$ \\
\hline 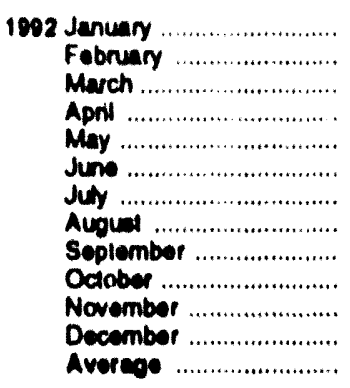 & $\begin{array}{l}7.013 \\
6.720 \\
0.603 \\
6.051 \\
7.092 \\
7.100 \\
7.195 \\
6.017 \\
7.071 \\
7.100 \\
7.323 \\
7.411 \\
7.050\end{array}$ & $\begin{array}{l}240 \\
275 \\
217 \\
428 \\
302 \\
424 \\
303 \\
240 \\
410 \\
103 \\
170 \\
202 \\
204\end{array}$ & $\begin{array}{r}304 \\
.22 \\
.276 \\
54 \\
74 \\
76 \\
.240 \\
.440 \\
60 \\
.41 \\
316 \\
32 \\
.11\end{array}$ & $\begin{array}{r}87 \\
59 \\
71 \\
00 \\
82 \\
80 \\
100 \\
123 \\
85 \\
04 \\
74 \\
184 \\
04\end{array}$ & 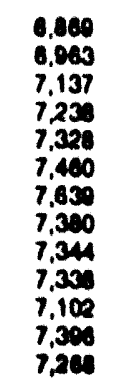 & $\begin{array}{l}220 \\
230 \\
220 \\
216 \\
220 \\
225 \\
216 \\
201 \\
206 \\
204 \\
214 \\
216 \\
216\end{array}$ & $\begin{array}{l}101 \\
191 \\
182 \\
180 \\
180 \\
180 \\
180 \\
167 \\
160 \\
167 \\
177 \\
170 \\
170\end{array}$ & $M_{M}^{M}$ \\
\hline 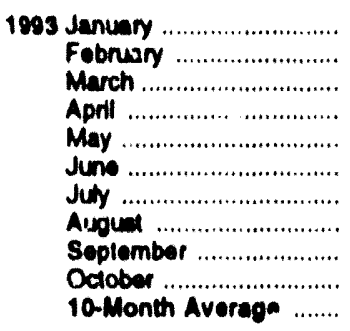 & 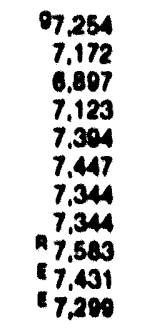 & $\begin{array}{l}204 \\
216 \\
190 \\
263 \\
308 \\
251 \\
202 \\
283 \\
263 \\
230 \\
230 \\
262\end{array}$ & $\begin{array}{r}571 \\
160 \\
.411 \\
.137 \\
80 \\
.75 \\
.242 \\
.330 \\
154 \\
.7 \\
.28\end{array}$ & $\begin{array}{r}142 \\
90 \\
100 \\
111 \\
90 \\
61 \\
100 \\
77 \\
905 \\
178 \\
197\end{array}$ & $\begin{array}{l}00,740 \\
7,129 \\
7,307 \\
7,401 \\
7,531 \\
7,002 \\
7,777 \\
7,806 \\
7,612 \\
7,800 \\
7,460\end{array}$ & $\begin{array}{l}237 \\
242 \\
227 \\
222 \\
223 \\
220 \\
213 \\
200 \\
207 \\
200 \\
200\end{array}$ & $\begin{array}{l}105 \\
200 \\
187 \\
183 \\
186 \\
180 \\
176 \\
186 \\
170 \\
171 \\
171\end{array}$ & $\begin{array}{l}n 14 \\
13 \\
14 \\
15 \\
17 \\
10 \\
20 \\
21 \\
20 \\
N \\
\mathrm{NA}\end{array}$ \\
\hline $\begin{array}{l}1992 \text { 10-Month Averseg ....... } \\
1901 \text { 10-Month Averseg ....... }\end{array}$ & $\begin{array}{l}6,090 \\
6,032\end{array}$ & $\begin{array}{l}316 \\
303\end{array}$ & -4 & 19 & $\begin{array}{l}7,271 \\
7,202\end{array}$ & $\begin{array}{l}204 \\
203\end{array}$ & $\begin{array}{l}167 \\
167\end{array}$ & MA \\
\hline
\end{tabular}

- Stocks ere tolals as of end of period.

- From 1981 lonward, blending compononis are excluded.

- A negative number indicales a cocrease in stocks and a positve mumber indicates an increase.

I Indudes motor gasoline blanding components, but excludes axygenates, which ere reported seperately.

- See Nole 4 al end of section.

I See Note 2 al end of rection.

- Beginning in 1993. molor gasoline production and product suppliod

imbalance of motor eneoline blending componerts. Ses Note 2 at end of section.

hee Note 1 al end of eaction.

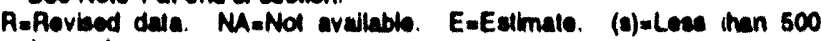
barrels per day.

Note: Cecoraphic coverage is the 50 States and the Diatrict of Columbla.

Sources: - 1075-1090: Enoroy Intormalion Admintatration (EU), Patroleum Supply Monthy. Fobnuary 1003, Table S4. - 181 Iorward: EU, Patroloum Supphy Monthy, November 1993, Table S4. 
Overview, 1973-1992

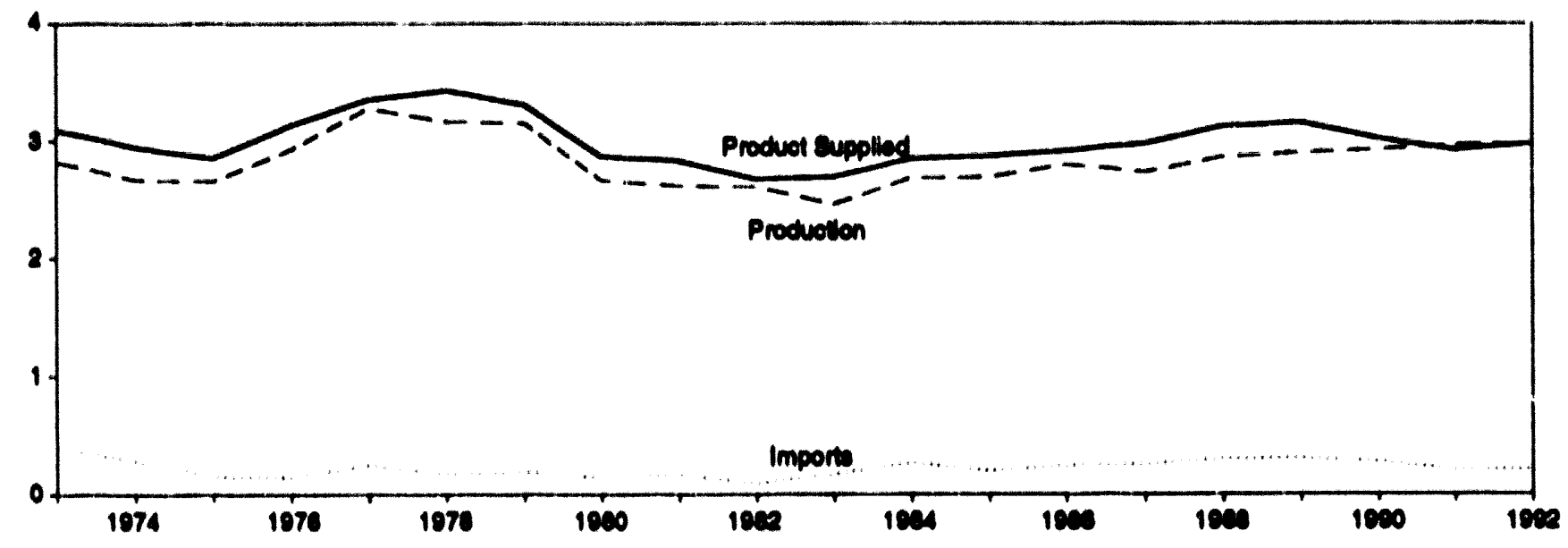

\section{Overview, Monthly}

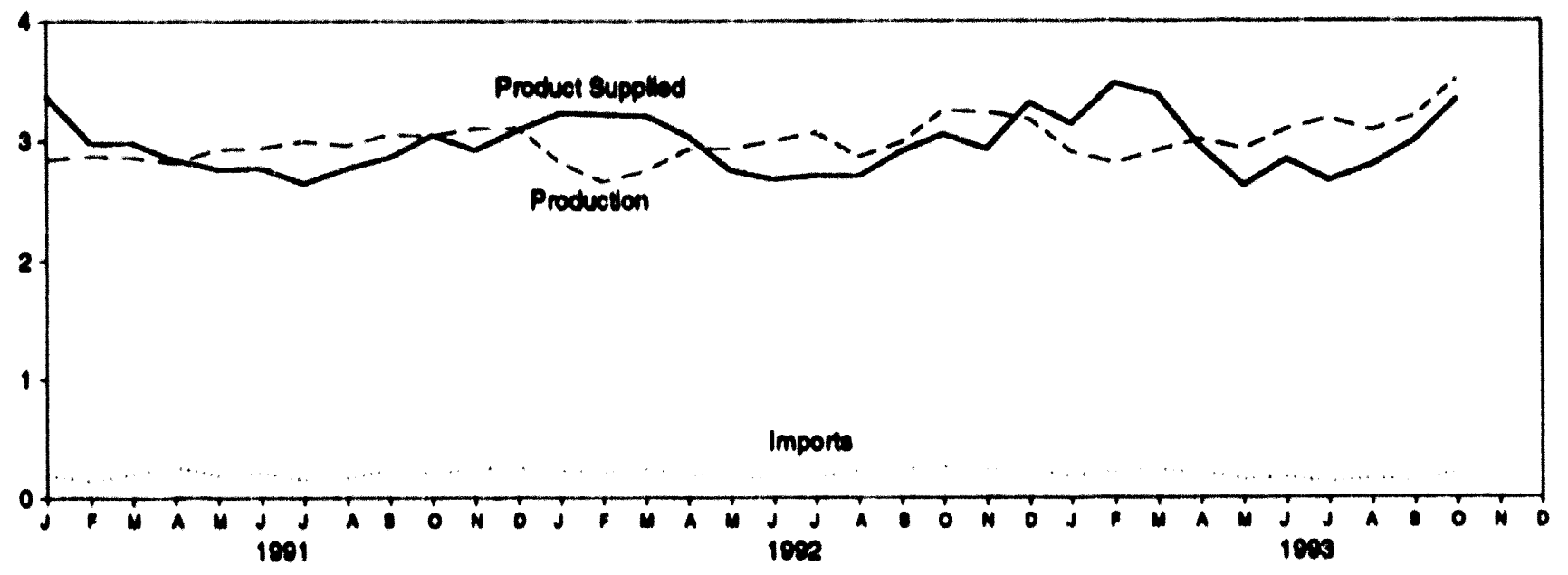

Product Supplied, January-October

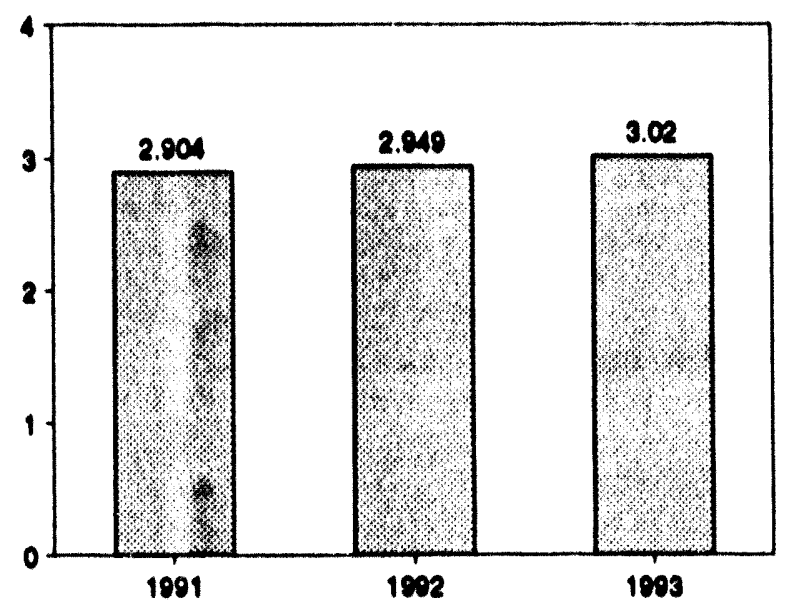

Stocks, End of Month

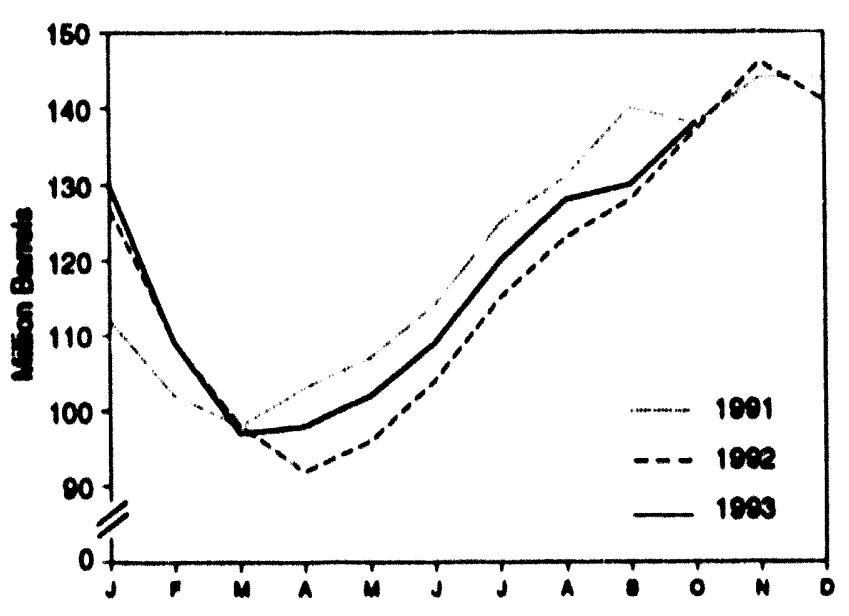

Bouros: Thble 3.5 . 


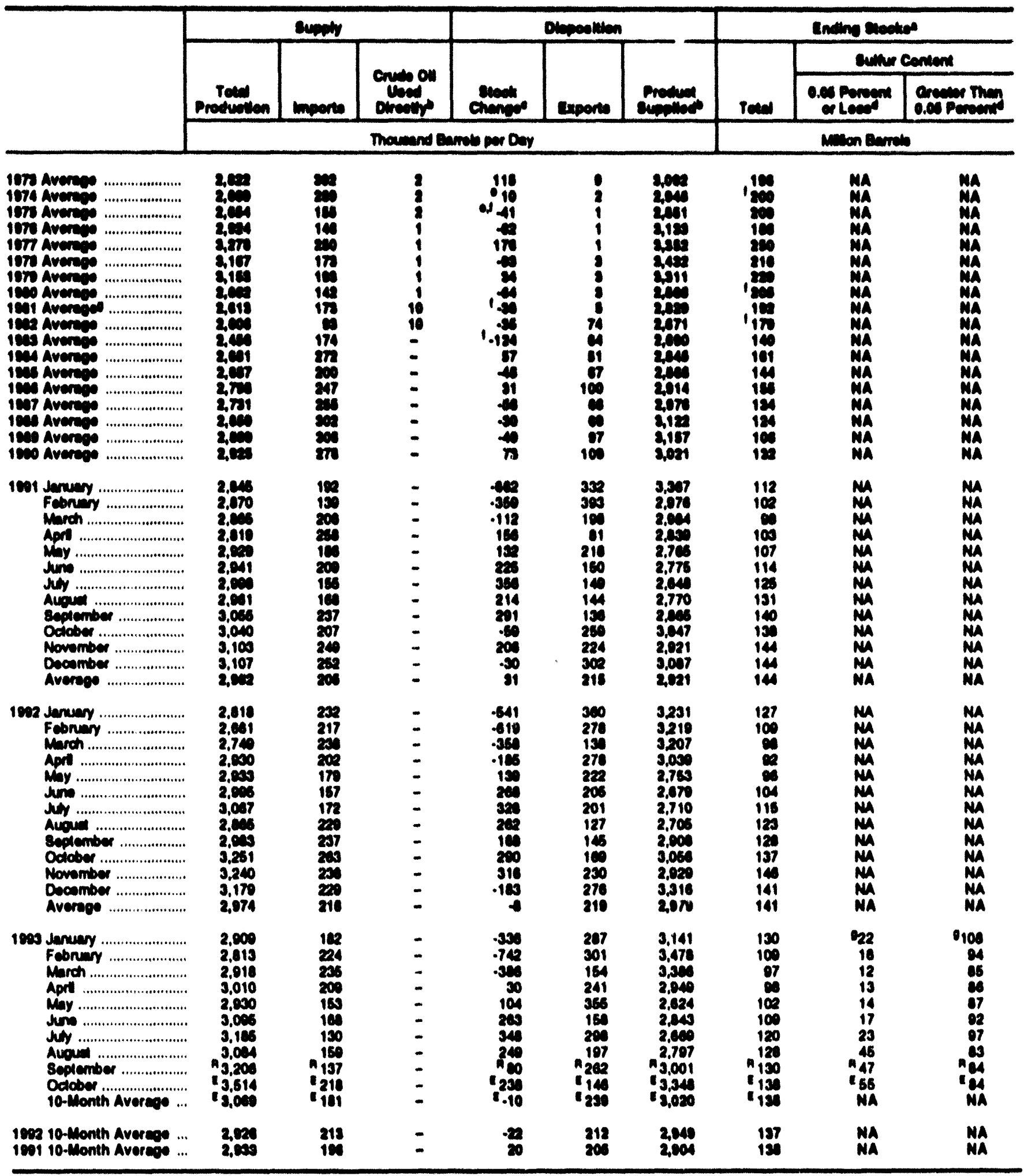

- Brock are lotats es of end of potod.

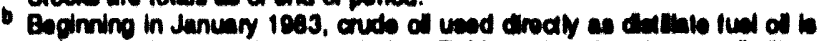
reponted es encls of product supplied on Tabl 3.26 rether than es dellinte luel oll product supplied.

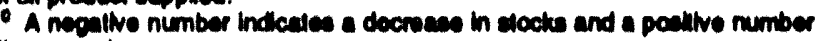
indlates en horease.

B wioh.

- See Nole 6 al end of section.

I see Nolo 4 al end of section.
- sen Noes 3 at end of endion.

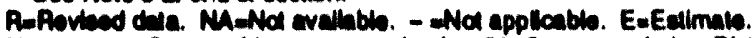

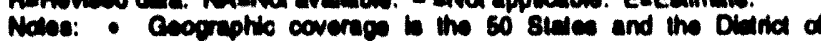

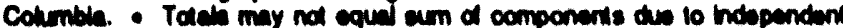
rounding.

Borros: - 107s-1eso: Enory Intomation Adminituation (EW). Peraleum suppy Monthy, Fobruny 1003, Table 85. - Ies1 forwerd: EIA Potrotum Suppy Monthy, November 1903, Table 86. 


\section{Figure 3.4 Rooldual Fual}

(Million Barrole per Day, Except as Noled)

\section{Overview, 1973-1992}

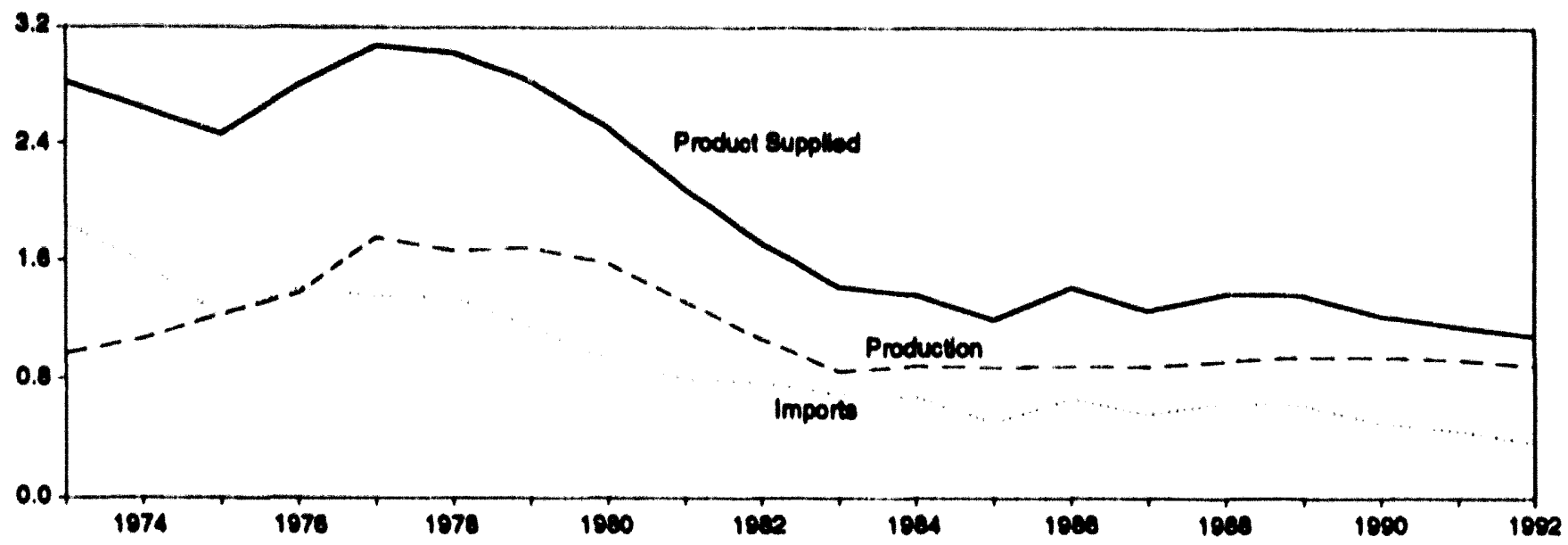

\section{Overview, Monthly}

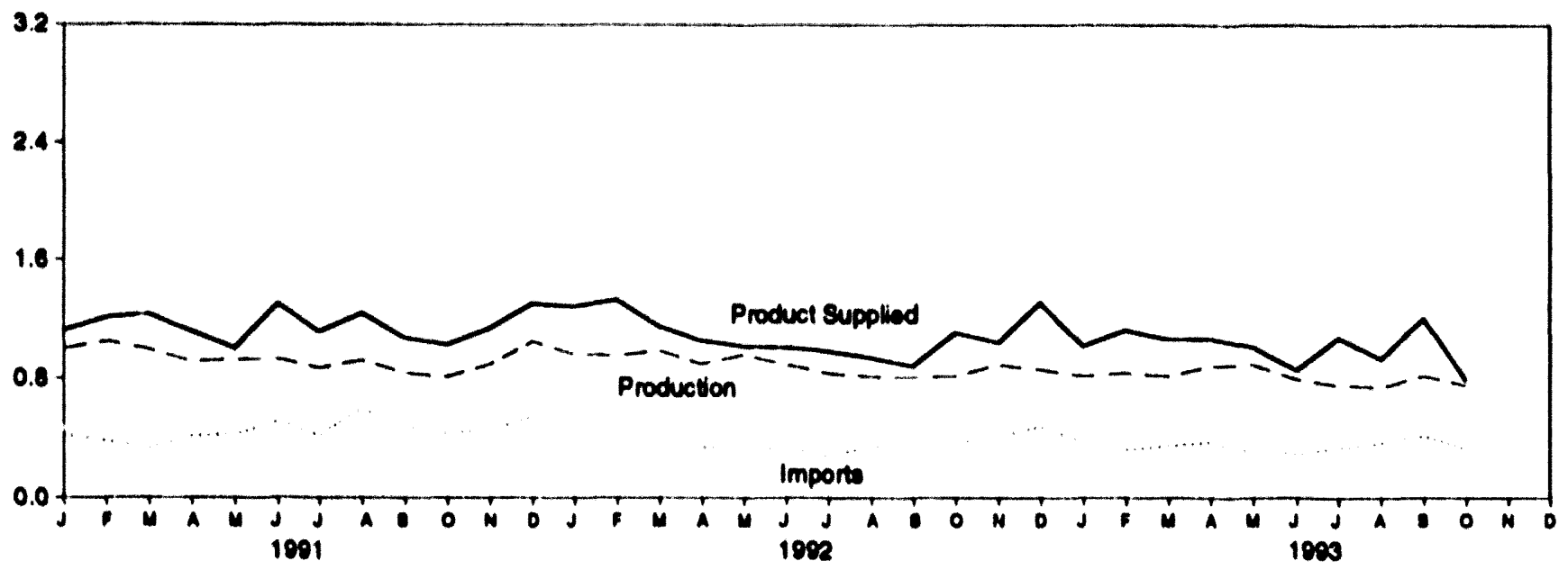

Product Supplied, January-October

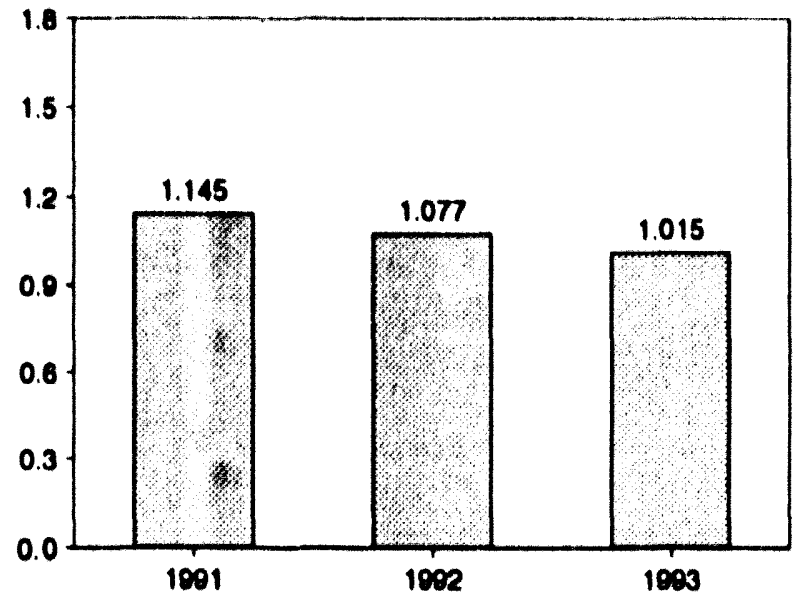

Stocks, End of Month

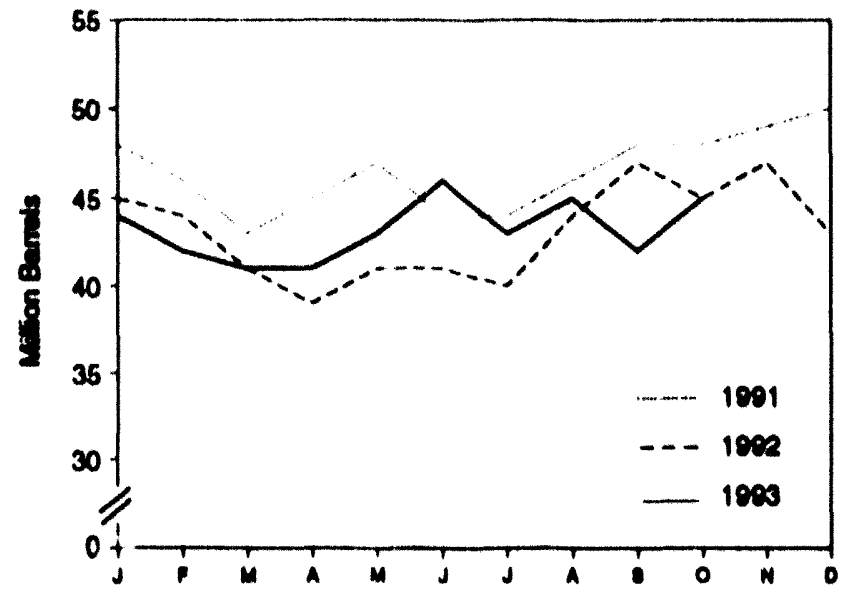

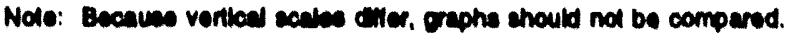
Source: Teble 3.6 . 


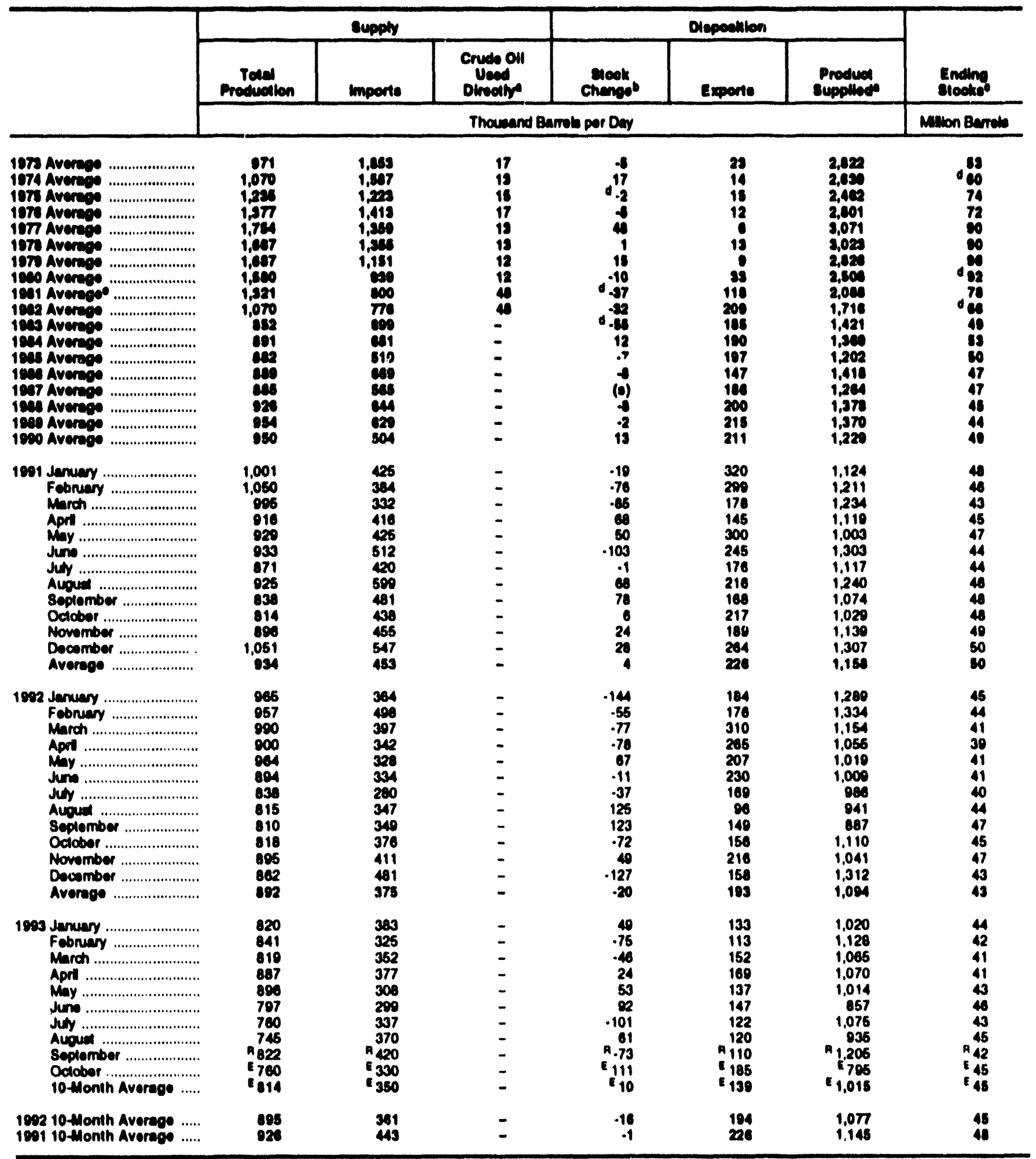

- Becinino in January 1893, crude ofl uned directly as residual lual ofl b reported as crude oll product supplied on Table $3.2 \mathrm{~b}$ ralher than es resicual luel oll product supplied.

b a mogitive number indicates a decreses h socke and a postive number indicalos en increase.

- Slocle are lolals as of and of period.

a See Nole 4 at end of section.
- See Nole 3 al ind of section.

Ranovied date. - -Not applicable. EaEatimate. (a)=Lese than $\$ 500$ barreta per day and greater than -500 berrale par day.

Note: Geocraphic coverag is the 50 States and the Ditrict of Coumbla.

Sources: - 1072-10c0: Enomy Intormation Adminidiration (EIA). Petrobum Supply Morthy, February 1003, Table So. - 1981 fonwerd: EIA. Potrobum Supply Monthy. November 1003, Table $\mathbf{s e}$ 
Figure 3.5 Jet Fuel

(Million Barrels per Day, Except as Noted)

Total Jet Fuel Overview, 1973-1992

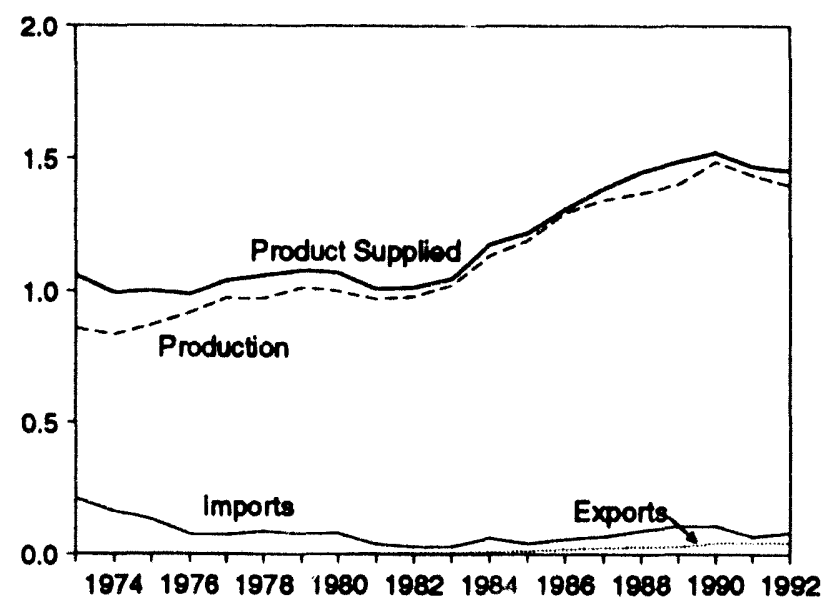

Product Supplied by Type, 1973-1992

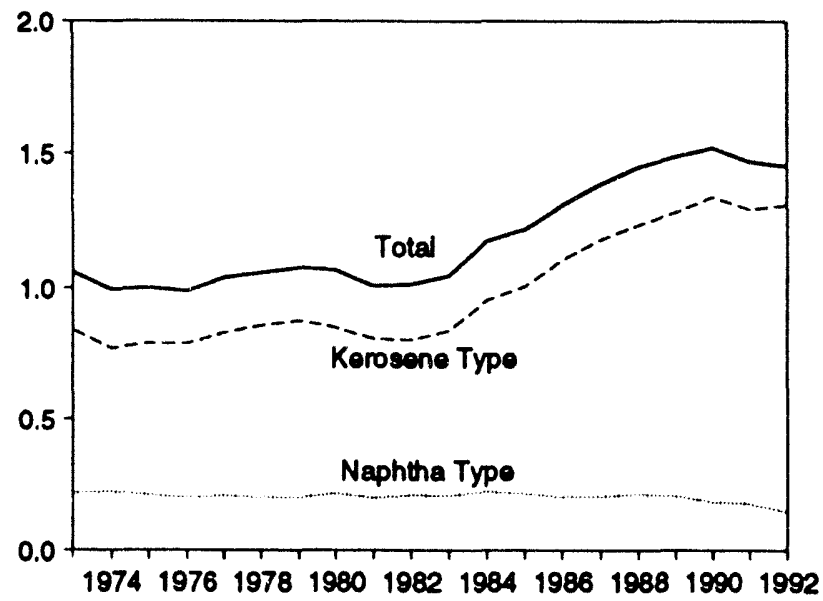

Total Product Supplied, January-October

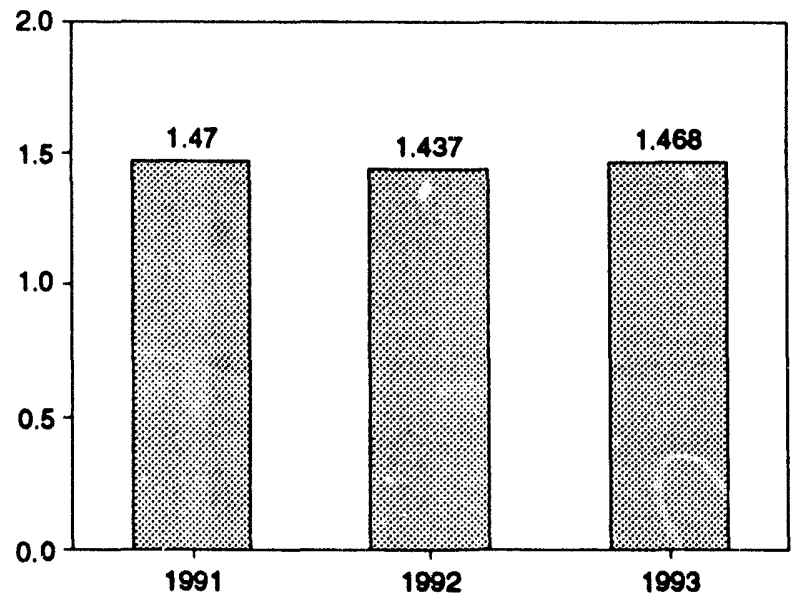

Total Jet Fuel Overview, Monthly

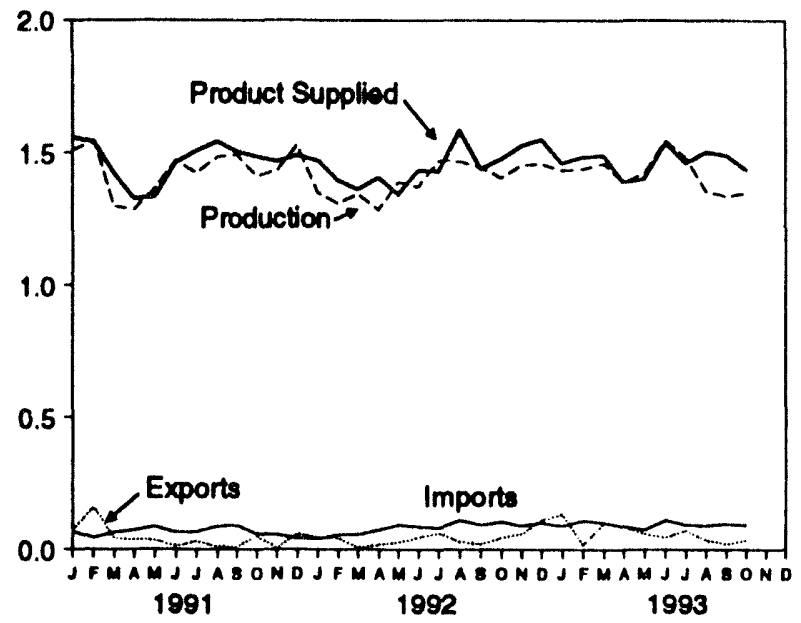

Product Supplied by Type, Monthly

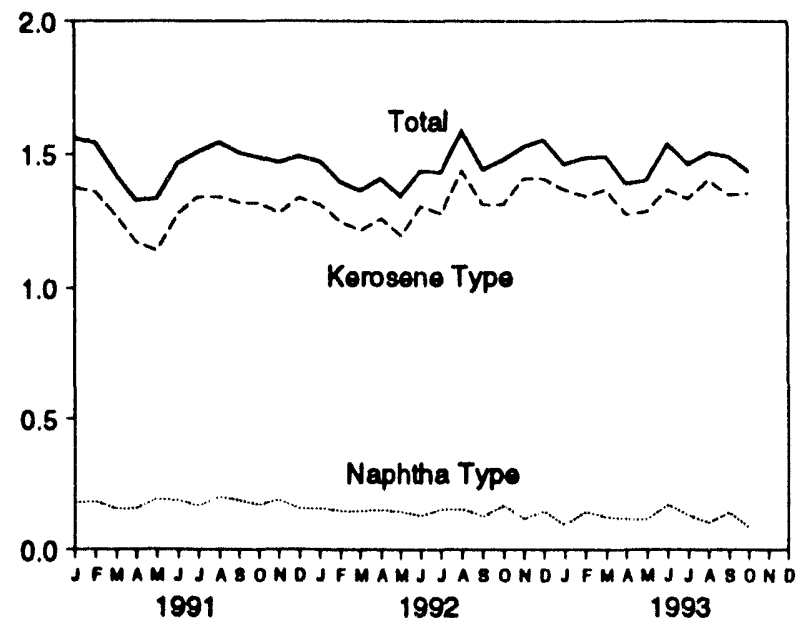

Total Stocks, End of Month

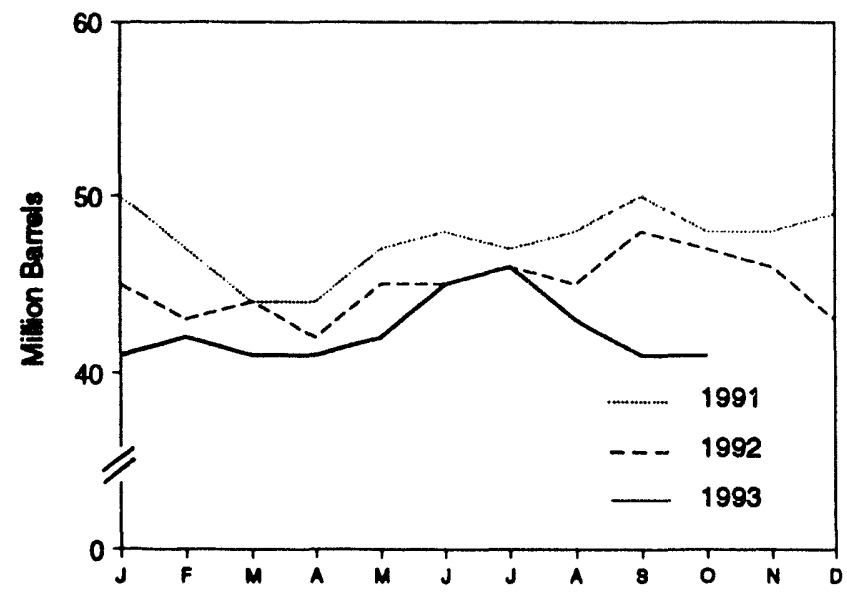

Source: Table 3.7. 


\begin{tabular}{|c|c|c|c|c|c|c|c|c|c|}
\hline & \multicolumn{3}{|c|}{ supply } & \multicolumn{4}{|c|}{ Diepostion } & & \\
\hline & \multicolumn{2}{|c|}{ Production } & \multirow[b]{2}{*}{ Imports } & \multirow{2}{*}{$\begin{array}{l}\text { Stock } \\
\text { Chengeb }\end{array}$} & \multirow[b]{2}{*}{ Exporte } & \multicolumn{2}{|c|}{ Product supplied } & \multicolumn{2}{|c|}{ Ending Stocke } \\
\hline & Totel & Kerosene Type & & & & Total & Kerosene Type & Total & Kerosene Type \\
\hline & \multicolumn{7}{|c|}{ Thousand Barrole per Day } & \multicolumn{2}{|c|}{ Million Barrels } \\
\hline 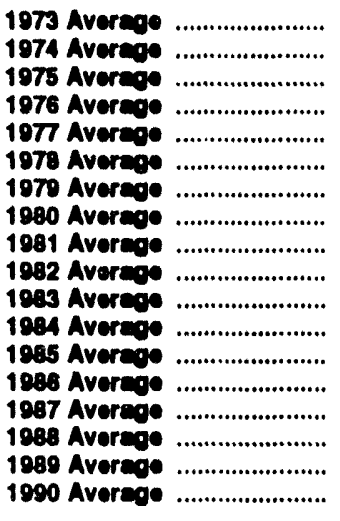 & $\begin{array}{r}859 \\
836 \\
871 \\
910 \\
973 \\
970 \\
1,012 \\
009 \\
968 \\
978 \\
1,022 \\
1,132 \\
1,189 \\
1,293 \\
1,343 \\
1,370 \\
1,403 \\
1,488\end{array}$ & $\begin{array}{l}670 \\
641 \\
601 \\
731 \\
787 \\
701 \\
895 \\
811 \\
775 \\
778 \\
817 \\
919 \\
893 \\
1,097 \\
1,138 \\
1,164 \\
1,197 \\
1,311\end{array}$ & $\begin{array}{r}212 \\
163 \\
133 \\
76 \\
75 \\
86 \\
78 \\
80 \\
38 \\
29 \\
29 \\
62 \\
39 \\
57 \\
67 \\
90 \\
106 \\
108\end{array}$ & $\begin{array}{r}8 \\
2 \\
c 2 \\
5 \\
7 \\
-2 \\
13 \\
10 \\
c-4 \\
-12 \\
(0) \\
0 \\
-4 \\
25 \\
(0) \\
-17 \\
-8 \\
31\end{array}$ & $\begin{array}{r}4 \\
3 \\
2 \\
2 \\
2 \\
1 \\
1 \\
1 \\
2 \\
6 \\
6 \\
9 \\
13 \\
18 \\
24 \\
28 \\
27 \\
43\end{array}$ & $\begin{array}{r}1,050 \\
903 \\
1,001 \\
987 \\
1,030 \\
1,057 \\
1,076 \\
1,068 \\
1,007 \\
1,013 \\
1,048 \\
1,175 \\
1,218 \\
1,307 \\
1,385 \\
1,449 \\
1,489 \\
1,522\end{array}$ & $\begin{array}{r}842 \\
771 \\
781 \\
780 \\
831 \\
858 \\
878 \\
851 \\
809 \\
804 \\
830 \\
953 \\
1,005 \\
1,105 \\
1,181 \\
1,238 \\
1,284 \\
1,340\end{array}$ & $\begin{array}{r}20 \\
\mathbf{2 0} \\
30 \\
32 \\
35 \\
34 \\
30 \\
\times \mathbf{4 2} \\
\mathbf{4 1} \\
\mathbf{3 1} \\
30 \\
42 \\
40 \\
50 \\
50 \\
44 \\
41 \\
52\end{array}$ & $\begin{array}{r}23 \\
\mathbf{r}^{24} \\
25 \\
28 \\
28 \\
28 \\
33 \\
\mathrm{c} 38 \\
\mathbf{3 6} \\
\mathbf{3 4} \\
\mathbf{3 1} \\
32 \\
35 \\
34 \\
43 \\
42 \\
38 \\
34 \\
46\end{array}$ \\
\hline 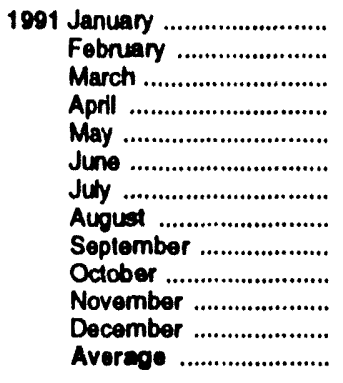 & $\begin{array}{l}1,509 \\
1,548 \\
1,299 \\
1,286 \\
1,367 \\
1,473 \\
1,426 \\
1,486 \\
1,495 \\
1,416 \\
1,433 \\
1,530 \\
1,438\end{array}$ & $\begin{array}{l}1,354 \\
1,384 \\
1,157 \\
1,135 \\
1,191 \\
1,300 \\
1,255 \\
1,316 \\
1,322 \\
1,253 \\
1,276 \\
1,357 \\
1,274\end{array}$ & $\begin{array}{l}67 \\
44 \\
65 \\
73 \\
87 \\
64 \\
67 \\
88 \\
92 \\
59 \\
56 \\
42 \\
67\end{array}$ & $\begin{array}{r}-55 \\
-108 \\
-99 \\
-8 \\
85 \\
58 \\
-47 \\
21 \\
71 \\
-66 \\
15 \\
22 \\
-0\end{array}$ & $\begin{array}{r}73 \\
159 \\
40 \\
38 \\
35 \\
13 \\
31 \\
11 \\
10 \\
50 \\
5 \\
59 \\
43\end{array}$ & $\begin{array}{l}1,559 \\
1,541 \\
1,423 \\
1,329 \\
1,334 \\
1,465 \\
1,509 \\
1,543 \\
1,506 \\
1,489 \\
1,469 \\
1,492 \\
1,471\end{array}$ & $\begin{array}{l}1,378 \\
1,360 \\
1,270 \\
1,173 \\
1,143 \\
1,280 \\
1,343 \\
1,343 \\
1,321 \\
1,319 \\
1,282 \\
1,338 \\
1,296\end{array}$ & $\begin{array}{l}50 \\
47 \\
44 \\
44 \\
47 \\
48 \\
47 \\
48 \\
50 \\
48 \\
48 \\
49 \\
49\end{array}$ & $\begin{array}{l}44 \\
41 \\
38 \\
38 \\
41 \\
43 \\
41 \\
42 \\
45 \\
43 \\
44 \\
44 \\
44\end{array}$ \\
\hline 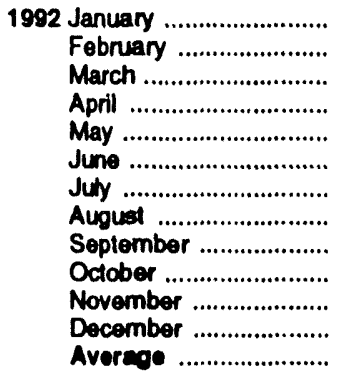 & $\begin{array}{l}1,352 \\
1,311 \\
1,347 \\
1,286 \\
1,393 \\
1,374 \\
1,473 \\
1,471 \\
1,448 \\
1,408 \\
1,456 \\
1,462 \\
1,399\end{array}$ & $\begin{array}{l}1,200 \\
1,164 \\
1,215 \\
1,131 \\
1,214 \\
1,234 \\
1,328 \\
1,339 \\
1,296 \\
1,265 \\
1,319 \\
1,336 \\
1,254\end{array}$ & $\begin{array}{r}39 \\
56 \\
56 \\
74 \\
93 \\
86 \\
81 \\
111 \\
93 \\
105 \\
90 \\
102 \\
82\end{array}$ & $\begin{array}{r}-127 \\
-73 \\
31 \\
-68 \\
114 \\
-21 \\
59 \\
-32 \\
78 \\
-12 \\
-41 \\
-101 \\
-16\end{array}$ & $\begin{array}{r}44 \\
42 \\
7 \\
18 \\
26 \\
45 \\
62 \\
28 \\
20 \\
44 \\
58 \\
112 \\
43\end{array}$ & $\begin{array}{l}1,473 \\
1,398 \\
1,365 \\
1,409 \\
1,346 \\
1,436 \\
1,433 \\
1,585 \\
1,442 \\
1,480 \\
1,528 \\
1,553 \\
1,454\end{array}$ & $\begin{array}{l}1,314 \\
1,250 \\
1,218 \\
1,262 \\
1,198 \\
1,308 \\
1,260 \\
1,438 \\
1,313 \\
1,315 \\
1,411 \\
1,410 \\
1,310\end{array}$ & $\begin{array}{l}45 \\
43 \\
44 \\
42 \\
45 \\
45 \\
46 \\
45 \\
48 \\
47 \\
46 \\
43 \\
43\end{array}$ & $\begin{array}{l}40 \\
38 \\
39 \\
37 \\
40 \\
39 \\
42 \\
41 \\
43 \\
43 \\
41 \\
39 \\
39\end{array}$ \\
\hline 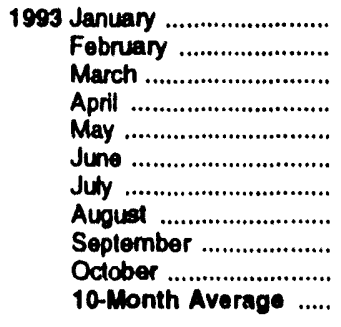 & 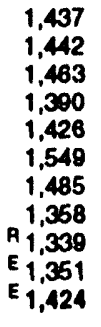 & $\begin{array}{l}1,306 \\
1,318 \\
1,332 \\
1,262 \\
1,300 \\
1,409 \\
1,359 \\
1,257 \\
\text { A }_{1,242} \\
\text { E }_{1,270} \\
\text { E }_{1,305}\end{array}$ & $\begin{array}{r}89 \\
110 \\
102 \\
88 \\
75 \\
111 \\
94 \\
91 \\
A_{97} \\
E_{93} \\
E_{95}\end{array}$ & $\begin{array}{r}-73 \\
46 \\
-29 \\
-4 \\
37 \\
78 \\
41 \\
-91 \\
-78 \\
-78 \\
E-30 \\
-11\end{array}$ & $\begin{array}{r}134 \\
17 \\
101 \\
88 \\
60 \\
45 \\
73 \\
34 \\
\mathrm{~A}_{21} \\
E_{36} \\
E_{61}\end{array}$ & $\begin{array}{l}1,464 \\
1,488 \\
1,483 \\
1,393 \\
1,404 \\
1,538 \\
1,465 \\
1,506 \\
\text { P }_{1,483} \\
\text { E } 1,438 \text { E } 1,468^{1,4}\end{array}$ & $\begin{array}{r}1,371 \\
1,346 \\
1,371 \\
1,278 \\
1,289 \\
1,370 \\
1,337 \\
1,405 \\
P_{1,352} \\
E_{1,357} \\
E_{1,348}\end{array}$ & $\begin{array}{l}41 \\
42 \\
41 \\
41 \\
42 \\
45 \\
46 \\
43 \\
\mathrm{P}_{41} \\
\mathrm{E}_{41} \\
\mathrm{E}_{41}\end{array}$ & $\begin{array}{l}\mathbf{3 6} \\
\mathbf{3 8} \\
\mathbf{3 7} \\
\mathbf{3 7} \\
\mathbf{3 8} \\
\mathbf{4 1} \\
42 \\
\mathbf{3 9} \\
\text { R } \mathbf{3 8} \\
\mathrm{E}_{\mathbf{3 8}} \\
\mathrm{E}_{\mathbf{3 8}}\end{array}$ \\
\hline $\begin{array}{l}190210 \text {-Month Average ..... } \\
1991 \text { 10-Month Average ..... }\end{array}$ & $\begin{array}{l}1,387 \\
1,429\end{array}$ & $\begin{array}{l}1,239 \\
1,266\end{array}$ & $\begin{array}{l}80 \\
71\end{array}$ & $\begin{array}{r}-5 \\
-14\end{array}$ & $\begin{array}{l}34 \\
45\end{array}$ & $\begin{array}{l}1,437 \\
1,470\end{array}$ & $\begin{array}{l}1,280 \\
1,293\end{array}$ & $\begin{array}{l}47 \\
48\end{array}$ & $\begin{array}{l}43 \\
43\end{array}$ \\
\hline
\end{tabular}

- Stocks are totals as of end of period. grealer than -500 barrels per day.
Note: Geographic coverage bo the 50 States and the District of Columbia.

b A negative number indicates a decrease in stocks and a positive number indicates an increase.

c See Note 4 al end of section.

$R=$ Revised data. E=Estimate. (s)=Less than +500 berrels per day and

Sources: - 1973-1980: Energy intormation Administration (ELA), Petroleum Supply Monthly, February 1983, Table S7. - 1981 fonward: EIA, Petroleum Supply Monthly, Novembor 1993, Table S7. 
Figure 3.6 Liquefled Petroleum Gases

(Million Barrels per Day, Except as Noted)

Overview, 1973-1992

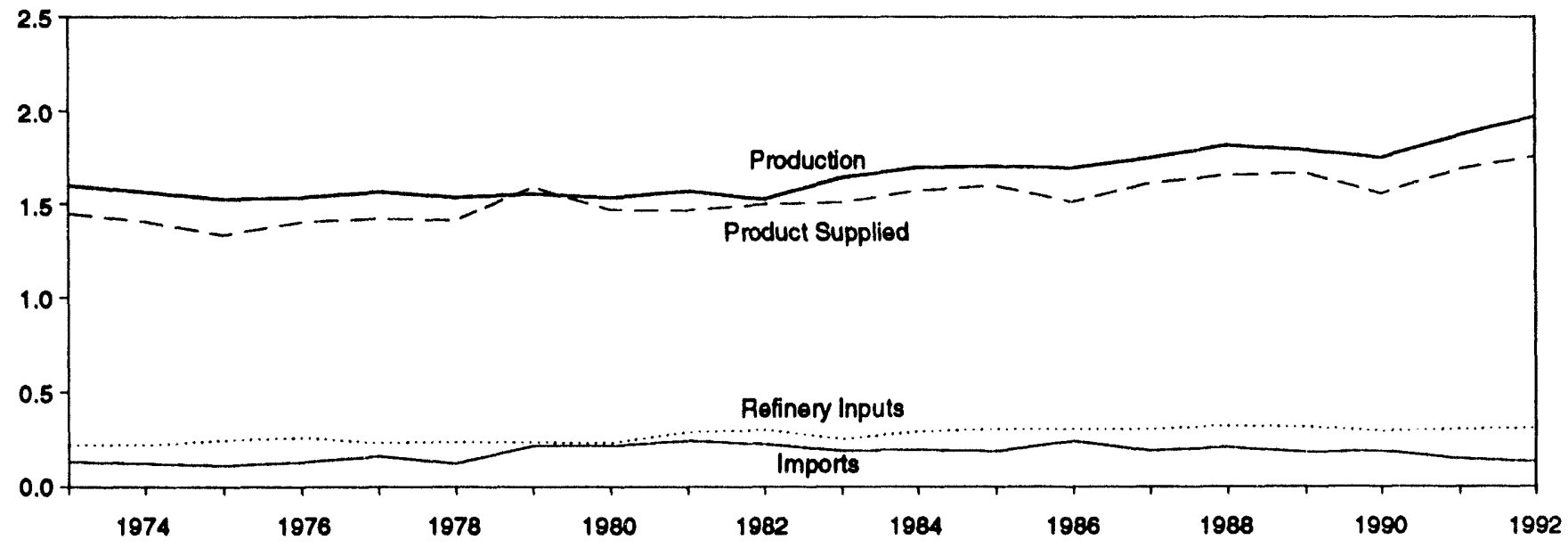

Overview, Monthly

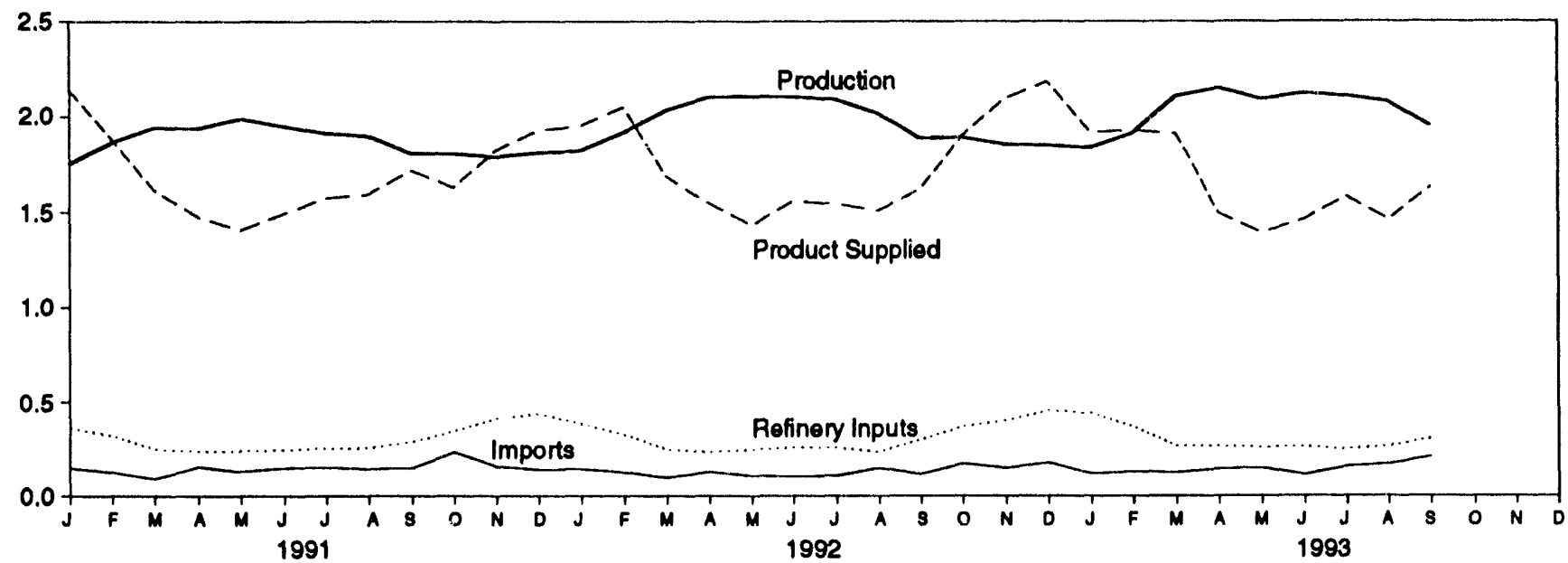

Product Supplied, January-September

Stocks, End of Month
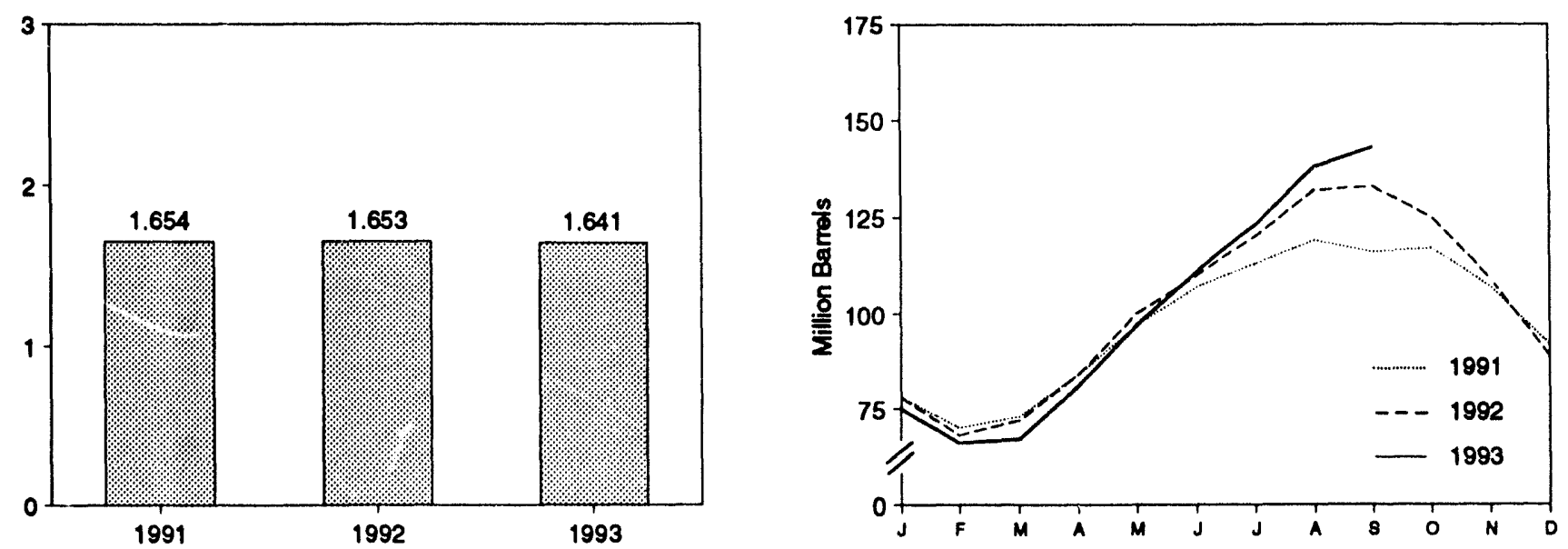

Note: Because vertical scales differ, graphs should not be compared. Source: Table 3.8. 
Table 3.8 Llquefled Petroleum Gases Supply and Disposition

\begin{tabular}{|c|c|c|c|c|c|c|c|}
\hline & \multicolumn{2}{|c|}{ Supply } & \multicolumn{4}{|c|}{ Dispostition } & \multirow{3}{*}{$\frac{\begin{array}{c}\text { Ending } \\
\text { Stocks }\end{array}}{\text { Million Barrels }}$} \\
\hline & $\begin{array}{c}\text { Total } \\
\text { Production }\end{array}$ & Imports & $\begin{array}{c}\text { Stook } \\
\text { Chango" }\end{array}$ & $\begin{array}{c}\text { Rofinory } \\
\text { Inpute }\end{array}$ & Exports & $\begin{array}{l}\text { Product } \\
\text { Supplied }\end{array}$ & \\
\hline & \multicolumn{6}{|c|}{ Thousand Barreb per Day } & \\
\hline 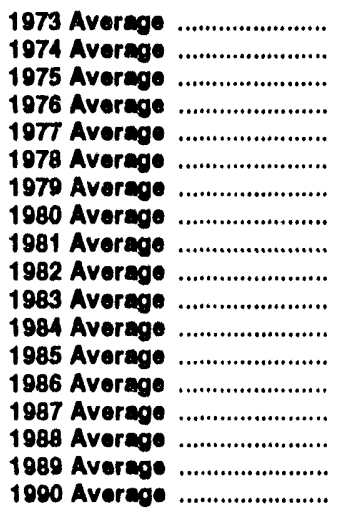 & $\begin{array}{r}1,600 \\
1,565 \\
1,527 \\
1,535 \\
1,566 \\
1,537 \\
1,556 \\
1,535 \\
1,571 \\
d 1,527 \\
1,642 \\
1,697 \\
1,704 \\
1,695 \\
1,748 \\
1,817 \\
1,791 \\
1,740\end{array}$ & $\begin{array}{l}132 \\
123 \\
112 \\
130 \\
161 \\
123 \\
217 \\
216 \\
214 \\
226 \\
190 \\
195 \\
187 \\
242 \\
190 \\
209 \\
181 \\
188\end{array}$ & $\begin{array}{r}35 \\
38 \\
c 35 \\
-24 \\
55 \\
-12 \\
c-70 \\
27 \\
c 18 \\
-111 \\
c-4 \\
c .19 \\
-75 \\
80 \\
-15 \\
1 \\
-47 \\
48\end{array}$ & $\begin{array}{l}220 \\
220 \\
246 \\
260 \\
233 \\
239 \\
236 \\
233 \\
289 \\
300 \\
253 \\
291 \\
304 \\
302 \\
304 \\
321 \\
315 \\
203\end{array}$ & $\begin{array}{l}27 \\
25 \\
26 \\
25 \\
18 \\
20 \\
15 \\
21 \\
42 \\
65 \\
73 \\
48 \\
62 \\
42 \\
38 \\
49 \\
35 \\
40\end{array}$ & $\begin{array}{l}1,449 \\
1,406 \\
1,333 \\
1,404 \\
1,422 \\
1,413 \\
1,592 \\
1,469 \\
1,466 \\
1,499 \\
1,509 \\
1,572 \\
1,599 \\
1,512 \\
1,612 \\
1,656 \\
1,668 \\
1,556\end{array}$ & $\begin{array}{r}99 \\
c 113 \\
125 \\
116 \\
136 \\
c^{132} \\
111 \\
111 \\
c^{120} \\
135 \\
c 94 \\
c \quad 101 \\
101 \\
74 \\
103 \\
97 \\
97 \\
80 \\
90\end{array}$ \\
\hline 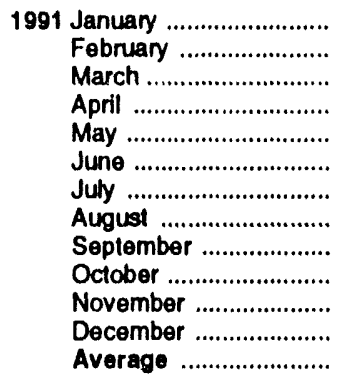 & $\begin{array}{l}1,753 \\
1,865 \\
1,942 \\
1,937 \\
1,989 \\
1,949 \\
1,913 \\
1,899 \\
1,806 \\
1,805 \\
1,789 \\
1,810 \\
1,871\end{array}$ & $\begin{array}{r}148 \\
126 \\
91 \\
154 \\
129 \\
148 \\
151 \\
143 \\
147 \\
233 \\
156 \\
139 \\
147\end{array}$ & $\begin{array}{r}-658 \\
-271 \\
113 \\
346 \\
428 \\
328 \\
211 \\
175 \\
-84 \\
33 \\
-330 \\
-488 \\
-15\end{array}$ & $\begin{array}{l}364 \\
322 \\
249 \\
237 \\
239 \\
245 \\
253 \\
255 \\
288 \\
345 \\
413 \\
437 \\
304\end{array}$ & $\begin{array}{l}56 \\
60 \\
56 \\
31 \\
45 \\
32 \\
24 \\
18 \\
31 \\
31 \\
40 \\
73 \\
41\end{array}$ & $\begin{array}{l}2,139 \\
1,880 \\
1,615 \\
1,477 \\
1,407 \\
1,492 \\
1,575 \\
1,594 \\
1,718 \\
1,629 \\
1,821 \\
1,927 \\
1,689\end{array}$ & $\begin{array}{r}78 \\
70 \\
73 \\
84 \\
97 \\
107 \\
113 \\
119 \\
116 \\
117 \\
107 \\
92 \\
92\end{array}$ \\
\hline 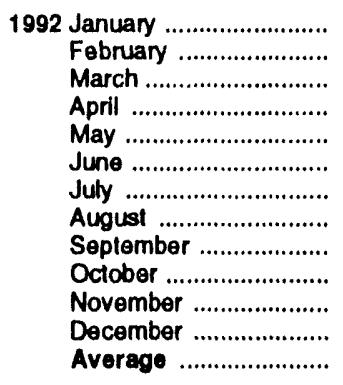 & $\begin{array}{l}1,820 \\
1,917 \\
2,033 \\
2,102 \\
2,106 \\
2,102 \\
2,090 \\
2,016 \\
1,886 \\
1,892 \\
1,854 \\
1,849 \\
1,972\end{array}$ & $\begin{array}{r}142 \\
126 \\
97 \\
127 \\
106 \\
104 \\
106 \\
148 \\
114 \\
171 \\
148 \\
176 \\
131\end{array}$ & $\begin{array}{r}-452 \\
-365 \\
153 \\
401 \\
489 \\
334 \\
345 \\
369 \\
37 \\
-242 \\
-541 \\
-660 \\
-10\end{array}$ & $\begin{array}{l}384 \\
326 \\
247 \\
233 \\
245 \\
257 \\
255 \\
233 \\
299 \\
369 \\
403 \\
453 \\
309\end{array}$ & $\begin{array}{l}80 \\
33 \\
43 \\
45 \\
44 \\
59 \\
52 \\
55 \\
45 \\
39 \\
43 \\
49 \\
49\end{array}$ & $\begin{array}{l}1,950 \\
2,051 \\
1,687 \\
1,549 \\
1,433 \\
1,556 \\
1,544 \\
1,507 \\
1,620 \\
1,898 \\
2,097 \\
2,184 \\
1,755\end{array}$ & $\begin{array}{r}78 \\
68 \\
72 \\
84 \\
100 \\
110 \\
120 \\
132 \\
133 \\
125 \\
109 \\
89 \\
89\end{array}$ \\
\hline 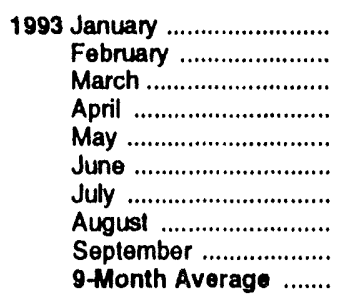 & $\begin{array}{l}1,837 \\
1,912 \\
2,106 \\
2,151 \\
2,091 \\
2,122 \\
2,108 \\
2,078 \\
1,952 \\
2,041\end{array}$ & $\begin{array}{l}117 \\
128 \\
123 \\
142 \\
148 \\
111 \\
155 \\
167 \\
206 \\
144\end{array}$ & $\begin{array}{r}-441 \\
-310 \\
9 \\
466 \\
538 \\
469 \\
380 \\
475 \\
188 \\
201\end{array}$ & $\begin{array}{l}440 \\
367 \\
263 \\
263 \\
258 \\
260 \\
246 \\
263 \\
304 \\
296\end{array}$ & $\begin{array}{l}30 \\
55 \\
47 \\
68 \\
50 \\
41 \\
54 \\
45 \\
35 \\
46\end{array}$ & $\begin{array}{l}1,917 \\
1,928 \\
1,910 \\
1,495 \\
1,393 \\
1,463 \\
1,583 \\
1,462 \\
1,632 \\
1,641\end{array}$ & $\begin{array}{r}75 \\
66 \\
67 \\
81 \\
97 \\
111 \\
123 \\
138 \\
143 \\
143\end{array}$ \\
\hline $\begin{array}{l}1992 \text { 9-Month Average ....... } \\
1991 \text { 9-Month Average ....... }\end{array}$ & $\begin{array}{l}2,008 \\
1,895\end{array}$ & $\begin{array}{l}119 \\
137\end{array}$ & $\begin{array}{r}148 \\
68\end{array}$ & $\begin{array}{l}275 \\
272\end{array}$ & $\begin{array}{l}51 \\
39\end{array}$ & $\begin{array}{l}1,653 \\
1,654\end{array}$ & $\begin{array}{l}133 \\
116\end{array}$ \\
\hline
\end{tabular}

- A negative number indicates a decrease in stocks and a postive number indicates an increase.

Stocks are totals as of end of period.

c Seo Note 4 at end of section.

d See Note 6 at end of section.

Notes: - Liquelied petroleum gases include ethane, ethylene, propane, propylene, nomal butane, butylone, bobutane and bobutylene. - Geographic coverage is the 50 States and the District of Columbia.

Sources: - 1973-1980: Energy Information Administration (EIA), Petroleum Supply Monthly, February 1993, Table S8. • 1981 forward: EIA, Petroleum Supply Monthly, November 1993, Table S9. 
Figure 3.7 Propane and Propylene

(Million Barrels per Day, Except as Noted)

Overview, 1973-1992

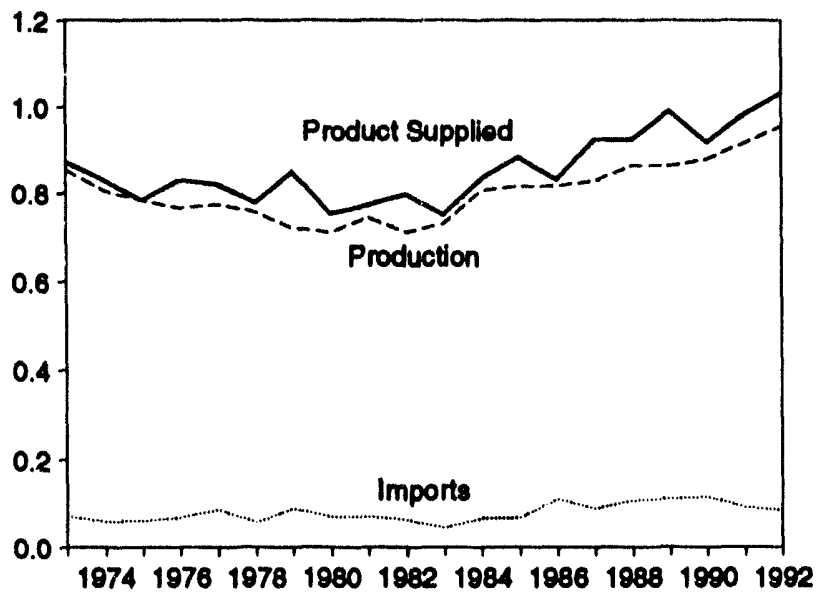

Product Supplied, Monthly

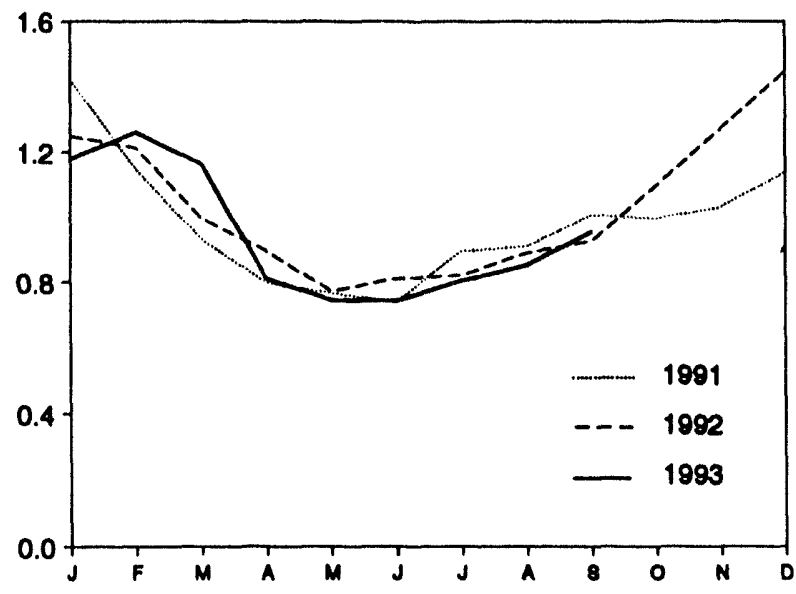

Stocks, End of Month

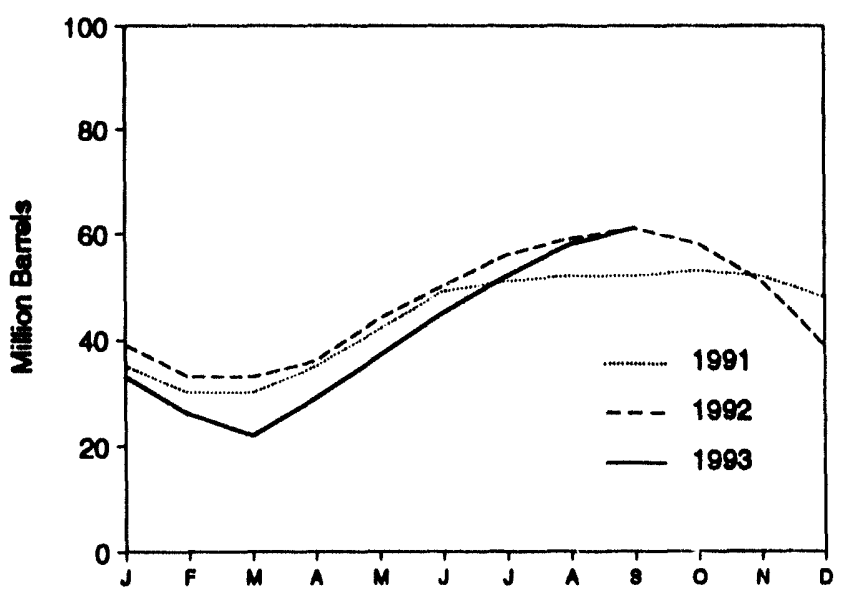

Product Supplied, January-September

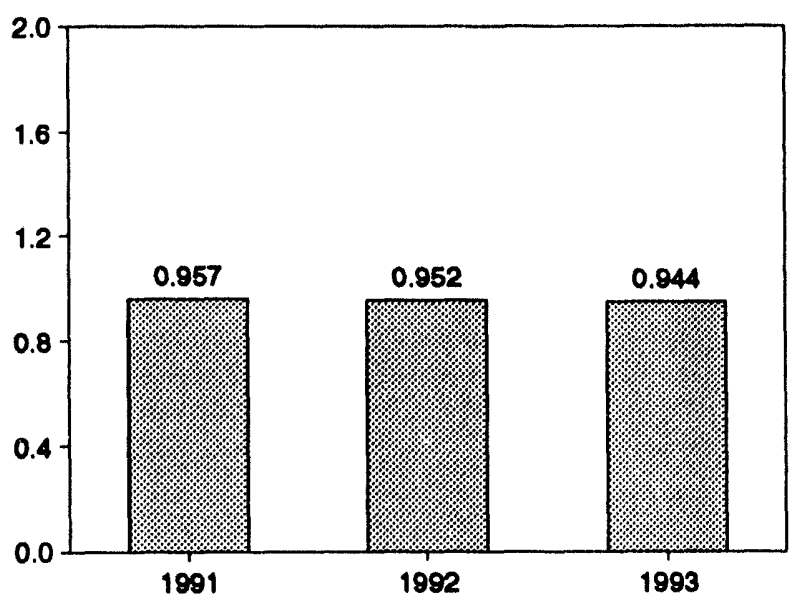

\section{Share of Liquefied Petroleum Gases, September}

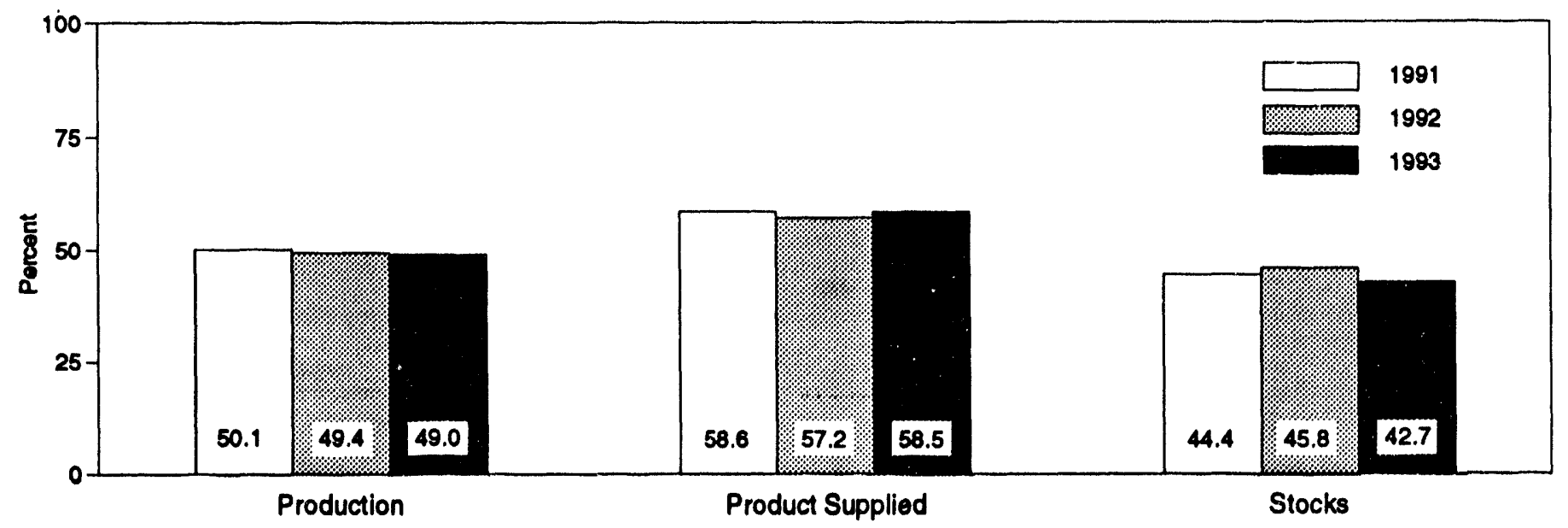

Note: Because vertical scales ciffer, graphs should not be compared.

Sources: Table 3.8 and, for calculation of shares, data prior to rounding for publlcallon in Tables 3.8 and 3.8 . 
Table 3.9 Propane and Propylene Supply and Dlsposition (A Subset of Table 3.8)

\begin{tabular}{|c|c|c|c|c|c|c|c|}
\hline & \multicolumn{2}{|c|}{ Supply } & \multicolumn{4}{|c|}{ Diepostition } & \multirow{3}{*}{$\begin{array}{c}\begin{array}{c}\text { Ending } \\
\text { Stooks }\end{array} \\
\text { Milion Barrele }\end{array}$} \\
\hline & $\begin{array}{c}\text { Total } \\
\text { Production }\end{array}$ & Importe & $\begin{array}{c}\text { Stook } \\
\text { Chengen }\end{array}$ & $\begin{array}{l}\text { Reflinery } \\
\text { Inpute }\end{array}$ & Exporte & $\begin{array}{l}\text { Products } \\
\text { Supplled }\end{array}$ & \\
\hline & \multicolumn{6}{|c|}{ Thousand Barreb per Day } & \\
\hline 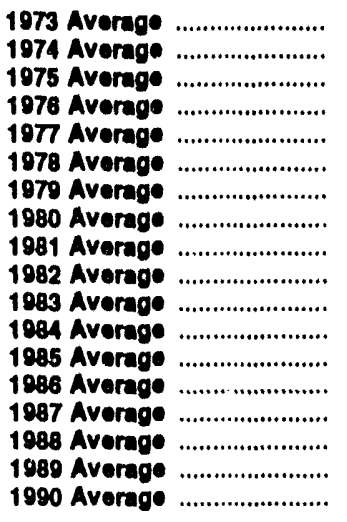 & $\begin{array}{l}854 \\
805 \\
783 \\
766 \\
775 \\
758 \\
721 \\
711 \\
745 \\
711 \\
730 \\
806 \\
816 \\
817 \\
828 \\
863 \\
662 \\
878\end{array}$ & $\begin{array}{r}71 \\
50 \\
60 \\
66 \\
86 \\
57 \\
86 \\
69 \\
70 \\
68 \\
44 \\
67 \\
67 \\
110 \\
89 \\
108 \\
111 \\
115\end{array}$ & $\begin{array}{r}30 \\
11 \\
36 \\
-22 \\
21 \\
15 \\
c-61 \\
4 \\
618 \\
-69 \\
6.24 \\
67 \\
-50 \\
64 \\
41 \\
7 \\
52 \\
48\end{array}$ & $\begin{array}{r}8 \\
9 \\
11 \\
12 \\
10 \\
13 \\
14 \\
12 \\
5 \\
4 \\
4 \\
4 \\
3 \\
4 \\
8 \\
8 \\
11 \\
(0)\end{array}$ & $\begin{array}{r}15 \\
14 \\
13 \\
13 \\
10 \\
9 \\
8 \\
10 \\
18 \\
31 \\
43 \\
30 \\
48 \\
28 \\
24 \\
31 \\
24 \\
28\end{array}$ & $\begin{array}{l}872 \\
830 \\
783 \\
830 \\
821 \\
778 \\
810 \\
754 \\
773 \\
790 \\
751 \\
833 \\
883 \\
831 \\
924 \\
923 \\
990 \\
917\end{array}$ & $\begin{array}{r}65 \\
69 \\
62 \\
74 \\
81 \\
687 \\
64 \\
64 \\
065 \\
76 \\
084 \\
640 \\
80 \\
30 \\
63 \\
40 \\
50 \\
32 \\
40\end{array}$ \\
\hline 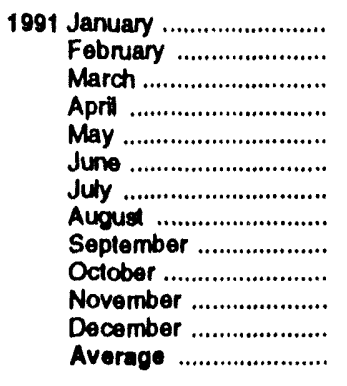 & $\begin{array}{l}920 \\
923 \\
912 \\
900 \\
922 \\
906 \\
901 \\
891 \\
905 \\
902 \\
930 \\
964 \\
915\end{array}$ & $\begin{array}{r}105 \\
90 \\
56 \\
101 \\
90 \\
81 \\
91 \\
73 \\
92 \\
146 \\
82 \\
86 \\
91\end{array}$ & $\begin{array}{r}.449 \\
-174 \\
-10 \\
179 \\
214 \\
223 \\
81 \\
40 \\
-22 \\
35 \\
-37 \\
-128 \\
-3\end{array}$ & $\begin{array}{l}0 \\
0 \\
0 \\
0 \\
0 \\
0 \\
0 \\
0 \\
0 \\
0 \\
0 \\
(\mathbf{s}) \\
(\mathbf{a})\end{array}$ & $\begin{array}{l}51 \\
40 \\
45 \\
25 \\
31 \\
22 \\
15 \\
13 \\
14 \\
18 \\
20 \\
38 \\
28\end{array}$ & $\begin{array}{r}1,422 \\
1,147 \\
933 \\
798 \\
767 \\
741 \\
895 \\
910 \\
1,006 \\
995 \\
1,030 \\
1,139 \\
982\end{array}$ & $\begin{array}{l}35 \\
30 \\
30 \\
35 \\
42 \\
49 \\
51 \\
52 \\
52 \\
53 \\
52 \\
48 \\
48\end{array}$ \\
\hline 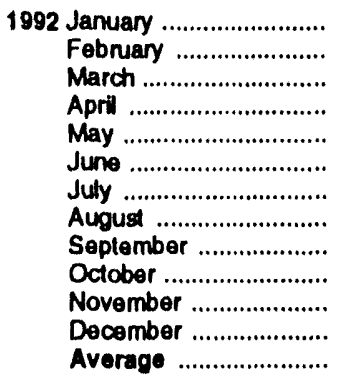 & $\begin{array}{l}949 \\
955 \\
840 \\
961 \\
977 \\
978 \\
964 \\
946 \\
931 \\
933 \\
964 \\
977 \\
956\end{array}$ & $\begin{array}{r}90 \\
86 \\
68 \\
80 \\
72 \\
66 \\
68 \\
85 \\
71 \\
104 \\
99 \\
131 \\
85\end{array}$ & $\begin{array}{r}-282 \\
-200 \\
-15 \\
120 \\
253 \\
206 \\
176 \\
117 \\
51 \\
-88 \\
-243 \\
-385 \\
-24\end{array}$ & $\begin{array}{l}\text { (s) } \\
\text { (s) } \\
(\mathrm{s}) \\
0 \\
(\mathrm{~s}) \\
(\mathbf{s}) \\
(\mathrm{s}) \\
(\mathrm{s}) \\
(\mathrm{s}) \\
(\mathrm{s}) \\
0 \\
0 \\
(\mathbf{s})\end{array}$ & $\begin{array}{l}72 \\
27 \\
26 \\
24 \\
23 \\
27 \\
35 \\
25 \\
25 \\
30 \\
33 \\
45 \\
33\end{array}$ & $\begin{array}{r}1,249 \\
1,214 \\
997 \\
896 \\
773 \\
811 \\
821 \\
889 \\
927 \\
1,095 \\
1,273 \\
1,448 \\
1,032\end{array}$ & $\begin{array}{l}39 \\
33 \\
33 \\
36 \\
44 \\
50 \\
56 \\
59 \\
61 \\
58 \\
51 \\
39 \\
39\end{array}$ \\
\hline 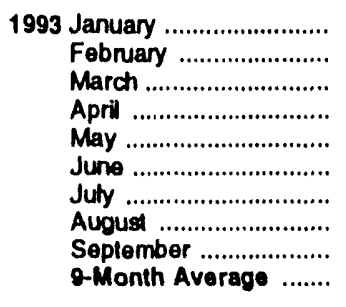 & $\begin{array}{l}965 \\
959 \\
971 \\
973 \\
942 \\
958 \\
956 \\
945 \\
956 \\
958\end{array}$ & $\begin{array}{r}72 \\
78 \\
85 \\
112 \\
96 \\
75 \\
105 \\
116 \\
132 \\
97\end{array}$ & $\begin{array}{r}-173 \\
-261 \\
-140 \\
233 \\
262 \\
266 \\
232 \\
184 \\
116 \\
82\end{array}$ & $\begin{array}{r}1 \\
(\mathbf{s}) \\
(\mathbf{s}) \\
(\mathbf{s}) \\
0 \\
0 \\
0 \\
0 \\
0 \\
(\mathbf{8})\end{array}$ & $\begin{array}{l}31 \\
37 \\
32 \\
40 \\
30 \\
23 \\
26 \\
27 \\
17 \\
29\end{array}$ & $\begin{array}{r}1,179 \\
1,261 \\
1,165 \\
812 \\
746 \\
744 \\
804 \\
851 \\
955 \\
944\end{array}$ & $\begin{array}{l}33 \\
26 \\
22 \\
29 \\
37 \\
45 \\
52 \\
58 \\
61 \\
61\end{array}$ \\
\hline $\begin{array}{l}1992 \text { g-Month Average ....... } \\
1991 \text { 9-Month Average ....... }\end{array}$ & $\begin{array}{l}956 \\
909\end{array}$ & $\begin{array}{l}76 \\
86\end{array}$ & $\begin{array}{l}48 \\
10\end{array}$ & (s) & $\begin{array}{l}32 \\
28\end{array}$ & $\begin{array}{l}952 \\
957\end{array}$ & $\begin{array}{l}61 \\
52\end{array}$ \\
\hline
\end{tabular}

- A negative number indicates a decrease in stocks and a positive number indicales an increase.

Stocks are totals as of end of period.

c See Note 4 at end of section.

(s)=Less than 500 barrels per day.

Nole: Geographic coverage is the 50 States and the District of Columbla.

Sources: - 1973 through 1975: U.S. Department of the Interior, Burean of Mines, Mineral Industry Surveys, "Pelroloum Stalement, Annual." • 1976 through 1980: Energy Information Administration (EIA). Energy Date Reports, Petroleum Statement, Annual." - 1981 fonward: ElA, Petroleum Supply Monthly, November 1993, Table S8. 
Table 3.10 Other Petroleum Products Supply and Disposition

\begin{tabular}{|c|c|c|c|c|c|c|c|}
\hline & \multicolumn{2}{|c|}{ Supply } & \multicolumn{4}{|c|}{ Diepostilon } & \multirow{3}{*}{$\begin{array}{c}\begin{array}{c}\text { Ending } \\
\text { Stooks }\end{array} \\
\text { Million Barrels }\end{array}$} \\
\hline & $\begin{array}{c}\text { Total } \\
\text { Production }\end{array}$ & Imports & $\begin{array}{c}\text { 8took } \\
\text { Chengo" }\end{array}$ & $\begin{array}{l}\text { Rofinery } \\
\text { Inpute }\end{array}$ & Exporte & $\begin{array}{l}\text { Producte } \\
\text { Supplled }\end{array}$ & \\
\hline & \multicolumn{6}{|c|}{ Thousand Barrels per Day } & \\
\hline 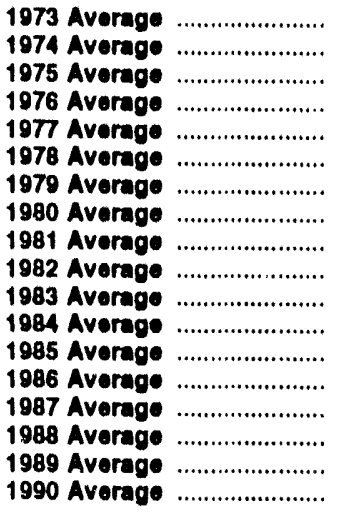 & $\begin{array}{l}2,833 \\
2,722 \\
2,547 \\
2,725 \\
2,939 \\
3,076 \\
3,141 \\
2,957 \\
2,771 \\
2,475 \\
2,437 \\
2,500 \\
2,532 \\
2,704 \\
2,737 \\
2,773 \\
2,771 \\
2,842\end{array}$ & $\begin{array}{r}290 \\
269 \\
144 \\
129 \\
130 \\
80 \\
116 \\
130 \\
188 \\
305 \\
382 \\
503 \\
550 \\
504 \\
543 \\
645 \\
627 \\
705\end{array}$ & $\begin{array}{c}1 \\
25 \\
c-6 \\
(0) \\
20 \\
-12 \\
24 \\
15 \\
c-42 \\
-68 \\
c-6 \\
c-32 \\
22 \\
-15 \\
-1 \\
22 \\
12 \\
-32\end{array}$ & $\begin{array}{l}750 \\
685 \\
537 \\
524 \\
514 \\
402 \\
352 \\
310 \\
723 \\
787 \\
712 \\
701 \\
886 \\
888 \\
829 \\
790 \\
707 \\
887\end{array}$ & $\begin{array}{l}162 \\
172 \\
158 \\
172 \\
164 \\
165 \\
208 \\
107 \\
197 \\
205 \\
236 \\
238 \\
227 \\
291 \\
264 \\
294 \\
305 \\
289\end{array}$ & $\begin{array}{r}2,211 \\
2,129 \\
2,001 \\
2,156 \\
2,371 \\
2,511 \\
2,673 \\
2,568 \\
2,081 \\
d 1,867 \\
1,877 \\
2,007 \\
1,947 \\
2,045 \\
2,187 \\
2,303 \\
2,285 \\
2,402\end{array}$ & $\begin{array}{r}179 \\
c 188 \\
188 \\
188 \\
195 \\
191 \\
200 \\
c 205 \\
241 \\
c 218 \\
c 217 \\
198 \\
206 \\
201 \\
200 \\
208 \\
213 \\
201\end{array}$ \\
\hline 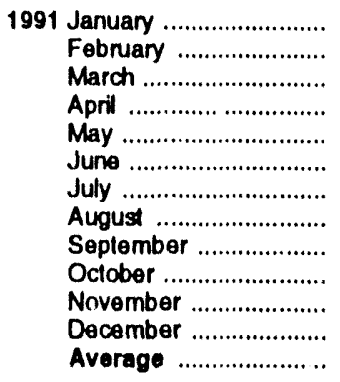 & $\begin{array}{l}2,653 \\
2,668 \\
2,576 \\
2,724 \\
2,853 \\
3,030 \\
3,029 \\
2,993 \\
3,010 \\
2,824 \\
2,750 \\
2,787 \\
2,826\end{array}$ & $\begin{array}{l}748 \\
573 \\
551 \\
607 \\
800 \\
615 \\
776 \\
642 \\
746 \\
611 \\
850 \\
577 \\
675\end{array}$ & $\begin{array}{r}204 \\
363 \\
151 \\
133 \\
198 \\
-123 \\
-143 \\
-169 \\
101 \\
-218 \\
-81 \\
-163 \\
18\end{array}$ & $\begin{array}{r}844 \\
726 \\
819 \\
753 \\
900 \\
1,092 \\
1,081 \\
1,013 \\
802 \\
944 \\
1,093 \\
1,147 \\
936\end{array}$ & $\begin{array}{l}317 \\
275 \\
239 \\
228 \\
327 \\
304 \\
321 \\
296 \\
267 \\
211 \\
238 \\
304 \\
277\end{array}$ & $\begin{array}{l}2,036 \\
1,876 \\
1,919 \\
2,217 \\
2,228 \\
2,372 \\
2,545 \\
2,496 \\
2,586 \\
2,498 \\
2,349 \\
2,085 \\
2,269\end{array}$ & $\begin{array}{l}207 \\
217 \\
222 \\
226 \\
232 \\
228 \\
224 \\
219 \\
222 \\
215 \\
213 \\
208 \\
208\end{array}$ \\
\hline 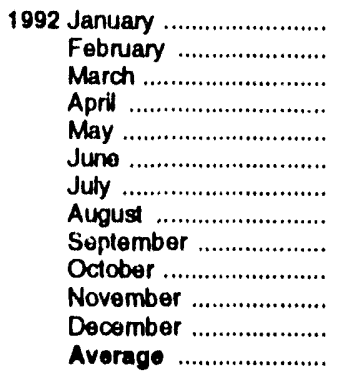 & $\begin{array}{l}2,702 \\
2,642 \\
2,752 \\
2,900 \\
2,929 \\
3,126 \\
3,207 \\
3,068 \\
3,114 \\
2,923 \\
2,915 \\
2,853 \\
2,928\end{array}$ & $\begin{array}{l}734 \\
575 \\
713 \\
793 \\
665 \\
669 \\
740 \\
729 \\
748 \\
701 \\
697 \\
711 \\
707\end{array}$ & $\begin{array}{r}203 \\
183 \\
238 \\
-31 \\
-113 \\
-42 \\
.156 \\
-116 \\
188 \\
-182 \\
-24 \\
-165 \\
-3\end{array}$ & $\begin{array}{r}787 \\
883 \\
730 \\
1,043 \\
910 \\
787 \\
986 \\
884 \\
675 \\
954 \\
889 \\
1,223 \\
806\end{array}$ & $\begin{array}{l}272 \\
240 \\
239 \\
217 \\
199 \\
225 \\
284 \\
227 \\
336 \\
295 \\
264 \\
352 \\
263\end{array}$ & $\begin{array}{l}2,175 \\
1,911 \\
2,258 \\
2,464 \\
2,588 \\
2,826 \\
2,822 \\
2,802 \\
2,663 \\
2,557 \\
2,383 \\
2,154 \\
2,470\end{array}$ & $\begin{array}{l}214 \\
219 \\
227 \\
226 \\
222 \\
221 \\
216 \\
212 \\
218 \\
212 \\
212 \\
c_{207} \\
c_{207}\end{array}$ \\
\hline 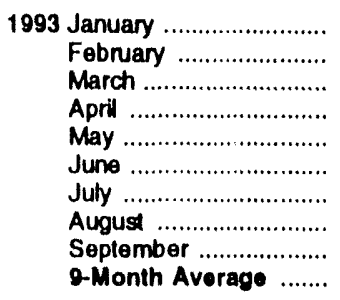 & $\begin{array}{l}0,026 \\
2,815 \\
2,866 \\
2,862 \\
2,899 \\
3,022 \\
3,116 \\
3,094 \\
3,016 \\
2,070\end{array}$ & $\begin{array}{l}698 \\
773 \\
818 \\
719 \\
808 \\
630 \\
875 \\
676 \\
789 \\
754\end{array}$ & $\begin{array}{r}{ }^{c} 600 \\
122 \\
243 \\
9 \\
85 \\
-240 \\
116 \\
27 \\
-265 \\
80\end{array}$ & $\begin{array}{r}829 \\
949 \\
747 \\
800 \\
979 \\
881 \\
945 \\
865 \\
1,031 \\
913\end{array}$ & $\begin{array}{l}271 \\
282 \\
269 \\
315 \\
278 \\
278 \\
302 \\
295 \\
282 \\
286\end{array}$ & $\begin{array}{l}2,023 \\
2,235 \\
2,425 \\
2,357 \\
2,364 \\
2,632 \\
2,628 \\
2,583 \\
2,757 \\
2,446\end{array}$ & $\begin{array}{l}225 \\
228 \\
236 \\
236 \\
239 \\
231 \\
235 \\
236 \\
228 \\
228\end{array}$ \\
\hline $\begin{array}{l}1992 \text { 9-Month Average ....... } \\
1991 \text { 9-Wonth Average ....... }\end{array}$ & $\begin{array}{l}2,939 \\
2,838\end{array}$ & $\begin{array}{l}708 \\
674\end{array}$ & $\frac{38}{77}$ & $\begin{array}{l}855 \\
894\end{array}$ & $\begin{array}{l}249 \\
286\end{array}$ & $\begin{array}{l}2,505 \\
2,255\end{array}$ & $\begin{array}{l}218 \\
222\end{array}$ \\
\hline
\end{tabular}

- A negative number indicales a decrease in stocks and a positive number indicates an increase.

Stocks are totals as of end of period.

c See Note 4 at end of section.

d See Nole 6 at end of section.

- Beginning in 1993, other petroleum products production, expons, and products supplied include an adjustment to oxygenates and motor gasoline blending components.

(s)=Less than +500 barrels per day and greater than -500 barrels per day.

Notes: - Other petroloum products include pentanes plus, other hydrocarbons and oxygenates, unlinished olls, gasoline blending components, and all finished petroleum products except finished motor gasoline, distillate fuel oll, residual fuel oll, jet fuel, and liquefled potroleum gases. - Geographic coverage is the 50 States and the District of Columbia.

Sources: - 1973-1080: Energy Intormation Administration (ELA), Petroloum Supply Monthly, Fobruary 1993, Table S8. - 1081 forwerd: EIA Petroleum Supply Monthly, November 1993, Table $\$ 10$. 


\section{Petroleum Notes}

1. The Energy Information Administration (BIA) uses a number of sources and methods to maintain the survey respondent lists. On a regular basis, survey managers review such industry publications as the Oil and Gas Journal and Oil Daily for information on facilities or companies starting up or closing down opcrations. Those sources are augmented by articles in newspapers, letters from respondents indicating changes in status, and information received from survey systems.

To supplement routine frames maintenance and to provide more thorough coverage, a comprehensive frames investigation is conducted every 3 years. This investigation results in the reassessment and recompilation of the complete frame for each survey. The effort also includes the evaluation of the impact of potential frame changes on the historical time series of data published from these respondents. The results of this frame study are usually implemented in January to provide a full year under the same frame.

In 1991, the EIA conducted a frame identifier survey of companies that produce, blend, store, or import oxygenates. A summary of the results from the identification survey was published in the Weekly Petroleum Status Report dated February 12, 1992, and in the February 1992 issue of the Petroleum Supply Monthly. In order to continue to provide relevant information about U.S. and regional gasoline supply, the EIA conducted a second frame identifier survey of those companies during 1992. As a result, numerous respondents were added to the monthly surveys effective in January 1993. See Explanatory Note 7 in the Petroleum Supply Monthly.

2. Motor Gasoline: Beginning in January 1981, the EIA expanded its universe to include non-refinery blenders and sepaated blending components from finished motor gasoline as a reporting category. Also, survey forms were modified to describe refinery operations more accurately.

Beginning with the reporting of January 1993 data, the EIA made adjustments to the product supplied series for finished motor gasoline. It was recognized that motor gasoline statistics published by the EIA through 1992 were underreported because the reporting system was (1) not collecting all fuel ethanol blending, and (2) there was a misreporting of motor gasoline blending components that were blended into finished gasoline. The adjustments are incorporated into EIA's data beginning in January 1993. To facilitate data analysis across the 1992-1993 period, EIA has prepared a table of 1992 data adjusted according to the 1993 basis. See Petroleum Supply Monthly, March 1993, Table H3.

3. Distillate and Residual Fuel Oils: The requirement to report crude oil in pipelines or burned on leases as either distillate or residual fuel oil has been eliminated.
Prior to January 1981, the refinery input of unfinished olls typically exceeded the available supply of un. finished oils. That discrepancy was assumed to be duc to the redesignation of distillate and residual fuel oils received as such but used as unfinished oil inputs by the receiving refinery. The imbalance hetween supply and disposition of unfinished olls would then be sub. tracted from the production of distillate and residual fucl oils. Two-thirds of that difference was subtracted from distillate and onc-third from residual. Beginning in January 1981, the EIA modified its survey forms to account for redesignated product and discontinued the above-mentioned adjustment.

Beginning in January 1993, the end-of-month stocks of distillate fuel oil are split into two sulfur categories ( 0.05 percent sulfur or less and greater than 0.05 percent sulfur) to meet Environmental Protection Agency requirements effective in October 1992. For further details, see the EIA, Petroleum Supply Monthly.

4. New Stock Basis: In January 1975, 1979, 1981 , and 1983, numerous respondents were added to bulk terminal and pipeline surveys, affecting subsequent stocks reported and stock change calculations. Using the expanded coverage (new basis), the end-of-year stocks, in million barrels, would have been:

- Crude Oil: 1982-645 (Total) and 351 (Other Primary).

- Crude Oil and Petroleum Products: 1974-1,121; 1980-1,425; and 1982-1,461.

- Motor Gasoline: 1974-225; 1980-263; 1982244 (Total) and 202 (Finished).

- Distillate Fuel Oil: 1974-224; 1980-205; and 1982-186.

- Residual Fuel Oil: 1974-75; 1980-91; and 1982-69.

- Jet Fuel: 1974-30 (Total) and 24 (Kerosene Type); $1980-42$ (Total) and 36 (Kerosene Type); and 1982-39 (Total) and 32 (Kerosene Type).

- Liquefied Petroleum Gases: 1974-113; 1978136; 1980-128; and 1982-102.

- Propane and Propylene: 1978-86; 1980-69; and $1982-57$.

- Other Petroleum Products: 1974-190; 1980 207; and 1982-219.

Stock change calculations beginning in 1975,1979 , 1981, and 1983 were made by using new basis stock levels.

In January 1984, changes were made in the reporting of natural gas liquids. As a result, unfractionated stream, which was formerly included in the "Other Petroleum Products Supply and Disposition" table, is now reponed on a component basis (ethane, propane, normal butane, isobutane, and pentanes plus). Most of these stocks now appear in the "Liquefied Petroleum Gases Supply 
and Disposition" table. This change affects stocks reported and stock change calculations in each table. Under the new basis, end-of-year 1983 stocks, in million barrels, would have been:

- Liquefled Petroleum Gases: 1983-108.

- Propane and Propylene: 1983-55.

- Other Petroleum Products: 1983-210.

In January 1993, changes were made in the monthly surveys to begin collecting bulk terminal and pipeline stocks of oxygenates. This change affected stocks reported and stock change calculations. However, a new basis stock level was not calculated for 1992 end-of-year stocks.
5. Stocks of Alankan Crude Olls Stocks of Alaskan Crude oil in transit were Included for the first time in January 1981. The major impact of this change is on the reporting of stock change calculations. Using the expanded coverage (new basis), 1980 end-of-year stocks, in million barrels, would have been 488 (Total) and 380 (Other Primary).

6. Datu Discrepancles: Due to differences intemal to EIA data processing systems, some small discrepancies exist between data in the Monthly Energy Review (MER) and the Petroleum Supply Annual (PSA) and Petroleum Supply Monthly (PSM). The data that have discrepan. cies are footnoted in Section 3 tables and summarized here.

\begin{tabular}{lllrr} 
Table & \multicolumn{1}{c}{ Data Series } & $\begin{array}{c}\text { Year } \\
\text { Average }\end{array}$ & $\begin{array}{c}\text { MER } \\
\text { Data }\end{array}$ & $\begin{array}{c}\text { PSA and PSM } \\
\text { Data }\end{array}$ \\
\hline 3.1a & Natural Gas Plant Production & 1976 & 1,604 & 1,603 \\
$3.1 \mathrm{~b}$ & Exports, Total & 1979 & 471 & 472 \\
$3.1 \mathrm{~b}$ & Exports, Petroleum Products & 1979 & 236 & 237 \\
$3.1 \mathrm{~b}$ & Net Imports & 1979 & 7,985 & 7,984 \\
$3.2 \mathrm{a}$ & Crude Used Directly & 1976 & -19 & -18 \\
$3.2 \mathrm{a}$ & Imports, SPR & 1978 & 161 & 162 \\
$3.2 \mathrm{a}$ & Crude Used Directly & 1978 & -15 & -14 \\
$3.2 \mathrm{a}$ & Crude Used Directly & 1979 & -14 & -13 \\
$3.2 \mathrm{a}$ & Crude Used Directly & 1980 & -14 & -13 \\
$3.2 \mathrm{~b}$ & Crude Losses & 1976 & 14 & 15 \\
$3.2 \mathrm{~b}$ & Crude Losses & 1980 & 14 & 15 \\
3.5 & Stock Change & 1974 & 10 & 9 \\
3.5 & Stock Change & 1975 & -41 & -40 \\
3.8 & Total Production & 1982 & 1,527 & 1,525 \\
3.10 & Products Supplied & 1982 & 1,857 & 1,856 \\
\hline
\end{tabular}




\section{Section 4. Natural Gas}

Total dry natural gas production in the United States during September 1993 was an estimated 1.5 trillion cubic ieet, 2 percent 4 higher than production during the previous September. Dry natural gas production during the rirst 3 quarters of 1993 was 13.5 trillion cublc feet, 3 percent higher than during the first 3 quarters of 1992.

Consumption of natural and supplemental gas in September 1993 was 1.3 trillion cubic feet, 5 percent above the level in September 1992. Consumption of natural and supplemental gas during the first 3 quarters of 1993 was an estimated 14.9 trillion cubic fect, 3 percent higher than the consumption level during the first 3 quarters of 1992.

Deliveries to residential consumers in August 1993 (latest date for which data are available) were 120 billion cubic feet. 5 percent lower than the previous
August's deliveries. Total deliveries to industrial con. sumers during August 1993 were 612 billion cubic fech, 5 percent more than the previous Augusi's level.

Imports of natural gas in Seplember 1993 were 188 billion cubic feet, 13 percent higher than imports in the previous Sepiember. Imports of natural gas during the first 3 quarters of 1993 were 1.7 billion cubic feet. 8 percent higher than imports during the first 3 quarters of 1992.

Stocks of working $\mathrm{gas}^{3}$ in underground natural gas storage reservoirs at the end of September 1993 totaled 2.9 trillion cubic feet, 5 percent below the level of stocks available 1 year earlier. Net injections into storage during September 1993 were 352 billion cubic feet, 18 percent above the amount injected during the previous September.

\footnotetext{
"Percentage changes are based on unrounded data.
}

"Cas avallable lor whithawal. 
Overviow, 1973-1992

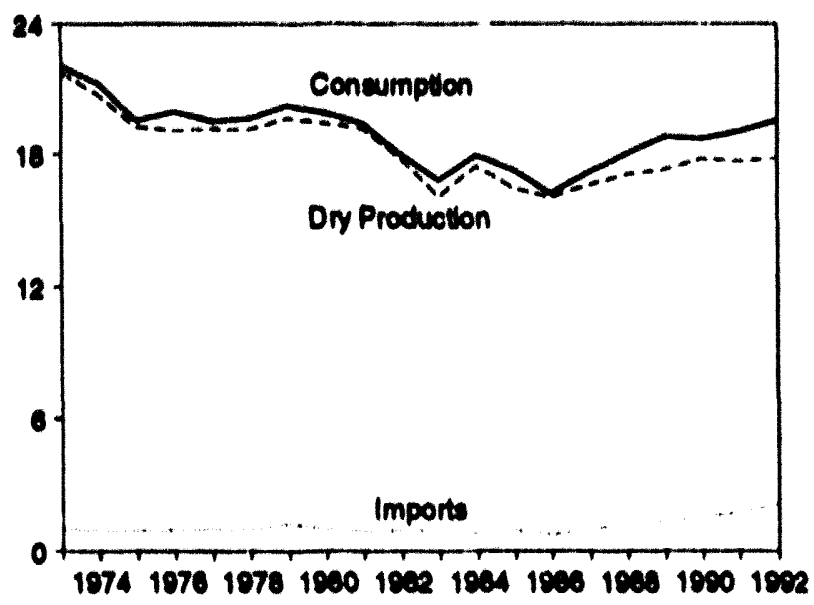

Consumption by Sector, 1973-1992

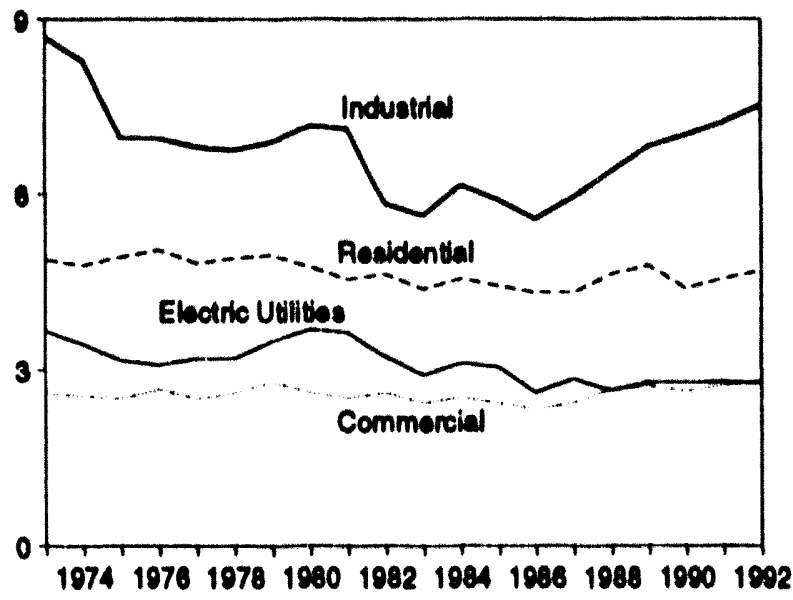

Underground Storage, End of Year, 1973-1992

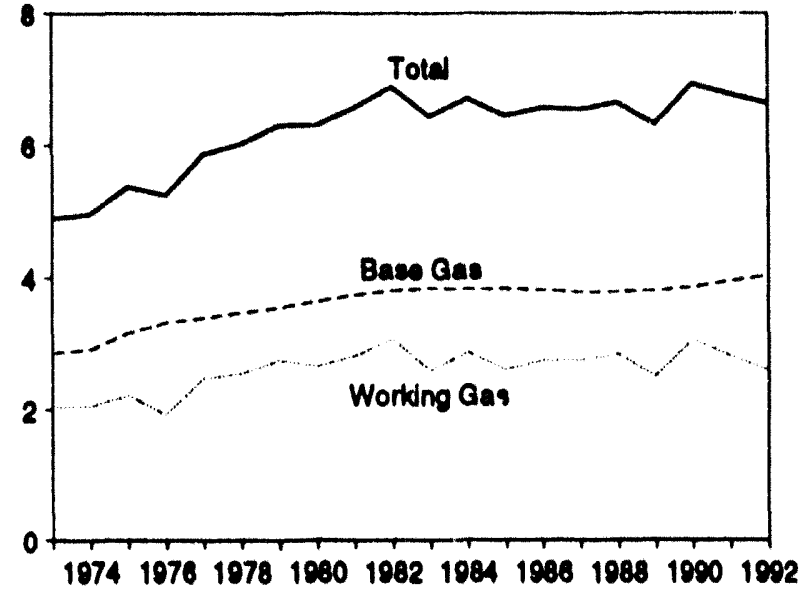

Overview, Monthly

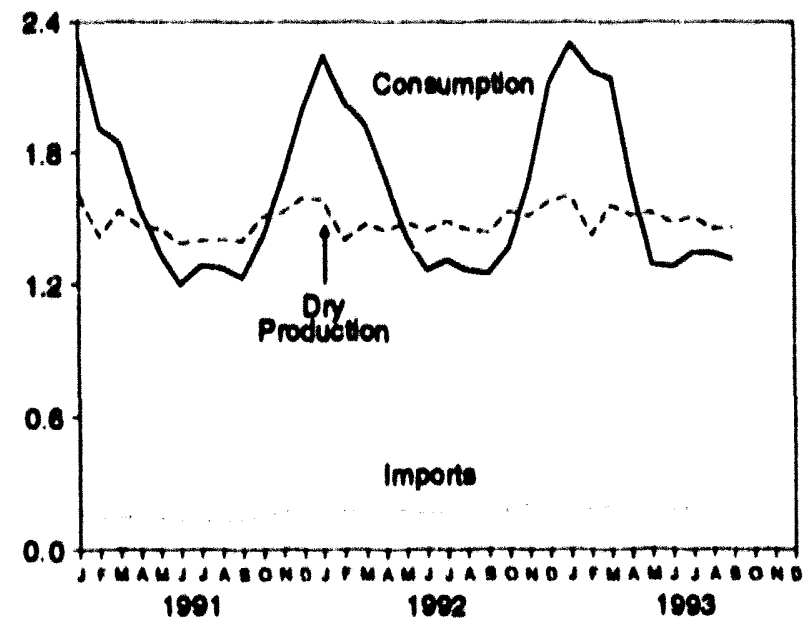

Consumption by Sector, Monthly

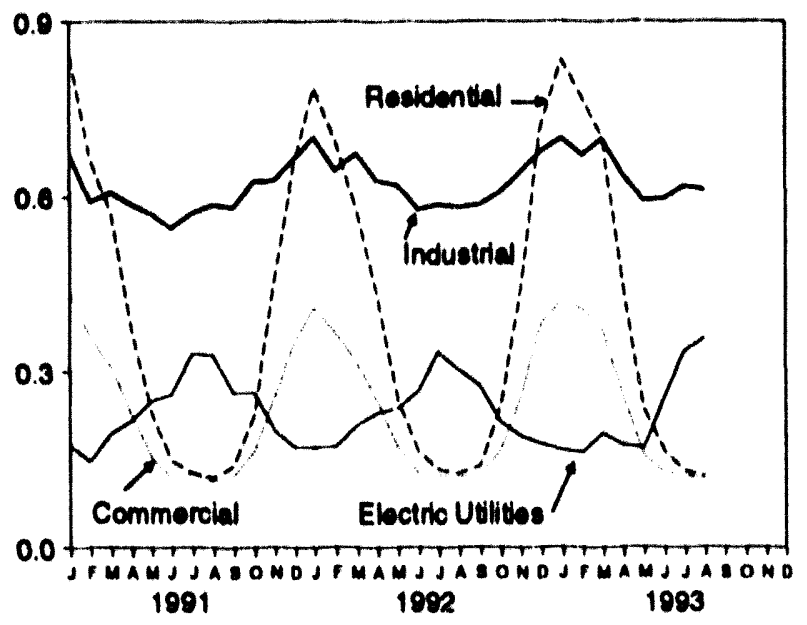

Underground Storage, End of Month

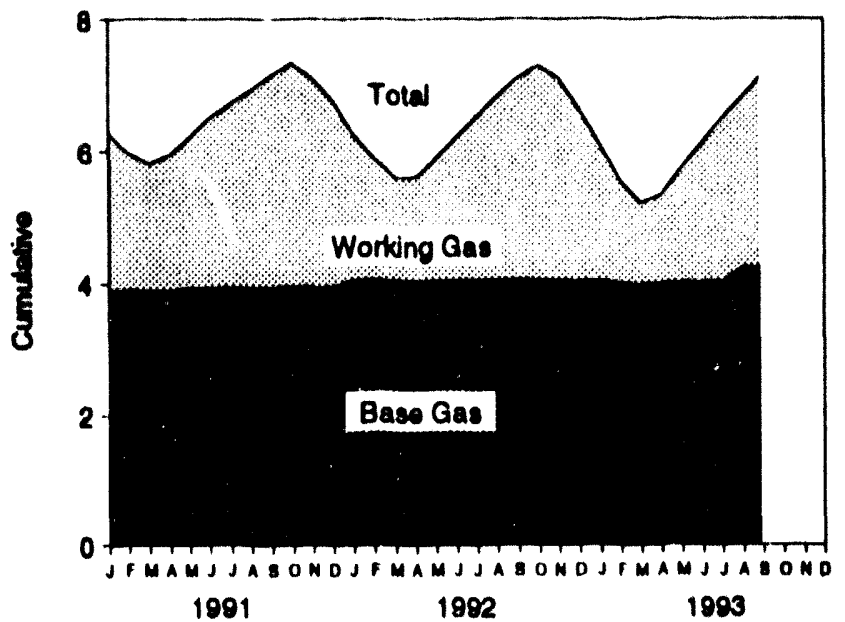

Nols: Because vertical scales oller, eraphe should not be compered. Sources: Tables $4.2,4.4$, and 4.5 . 
Table 4.1 Natural Cas Production (Billon Cublo Foet)

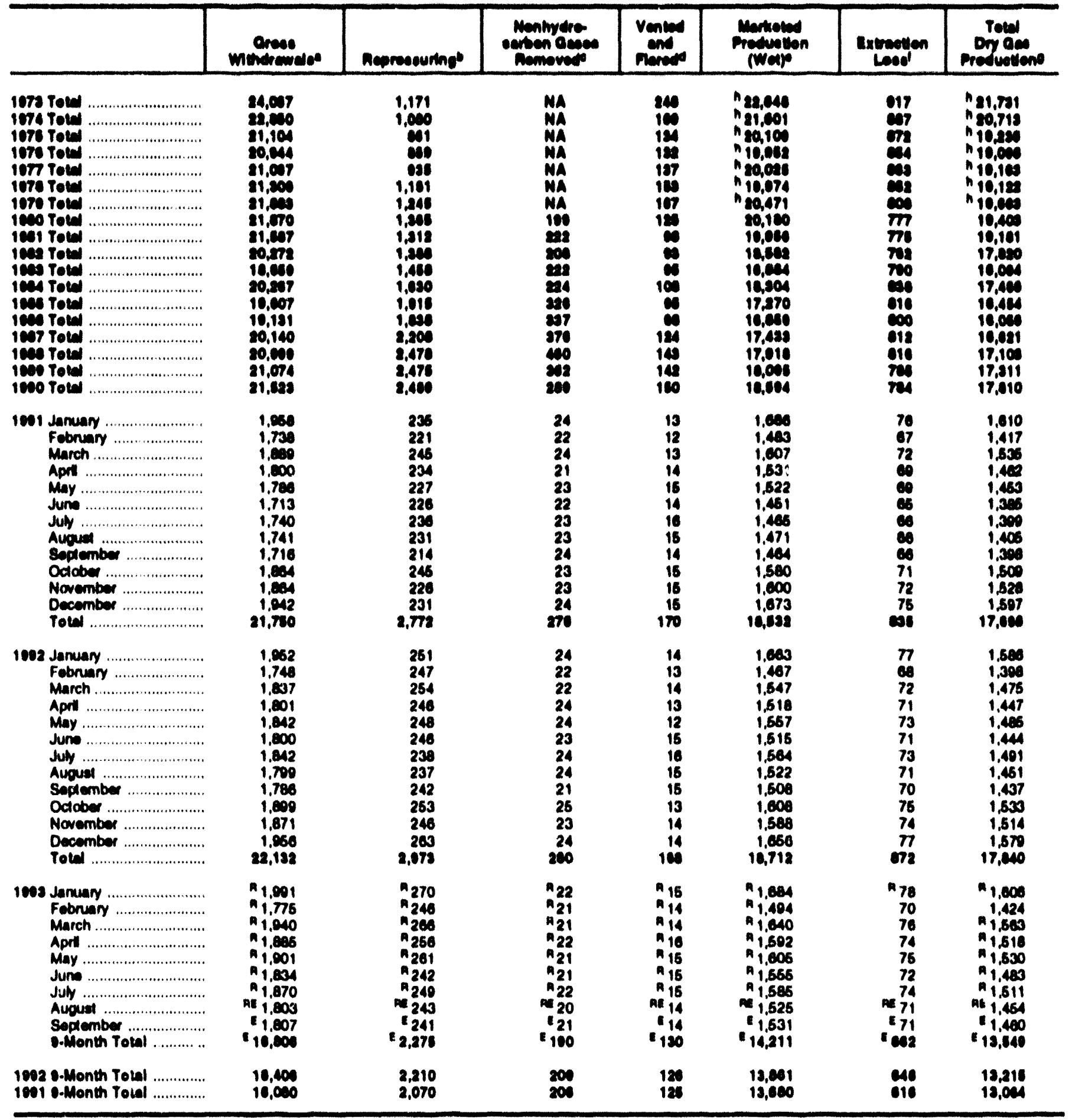

- Cas whindrawn trom oses and oll wolb.

- The injection of naturd gas into oll and gas lormations lor preasure maintenunce and cycling purposes.

- See Note 1 al end of section.

d Vented: Natural oas released into the alf on the base sile of at processing plants. Flared: Nalurd gas bumed in flares on the base athe or al gas processing plante.

- "Oross Withdrawals" minus 'Repressuring. "Nonhydrocarton Geses Removed," and 'Vented and Flared." see Note 2 al end of sectlon.

\footnotetext{
Isen Note 3 al and of section.

- "Marketed Production (Wel)" minus "Extraction Lose."

" May include uniknown quantilibe of nonhydrocarbon gases.

ReRovised dala. NA=Nol aveliable. E=Ealimale.

Notes: • Geopraphic coverage to the 50 States and the Diatrict of Columbla.

- Total may not equal sum of componenta due lo independent rounding

Sources: - 1073-10ea: Eneroy Iniormation Adminibiration (EL). Naturas

Gas Amnual 1901, Table 95. - 1097 fonwerd: EU, Notural Gas Monthy. November 1003, Table 1.
} 


\begin{tabular}{|c|c|c|c|c|c|c|c|c|c|}
\hline & \multicolumn{5}{|c|}{ supply } & $\begin{array}{l}\text { Toul } \\
\text { supply/ } \\
\text { Olepoeltiond }\end{array}$ & \multicolumn{3}{|c|}{ Dlepealiten } \\
\hline 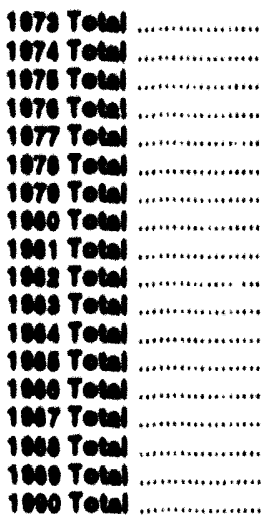 & 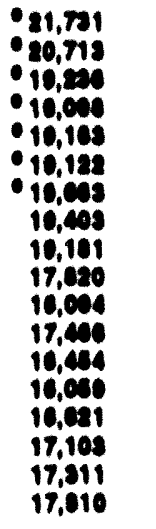 & 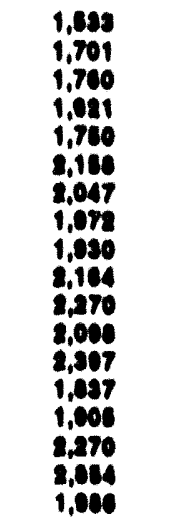 & $\begin{array}{l}\text { NA } \\
\text { NA } \\
\text { NA } \\
\text { NA } \\
\text { NA } \\
\text { MA } \\
100 \\
100 \\
100 \\
198 \\
110 \\
180 \\
118 \\
101 \\
101 \\
107 \\
120\end{array}$ & 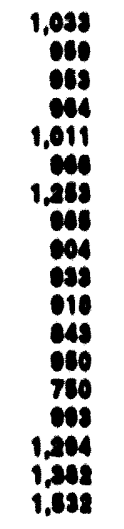 & 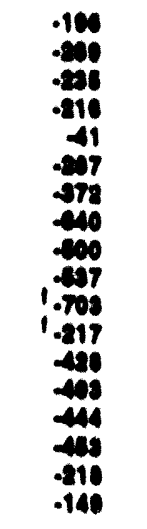 & 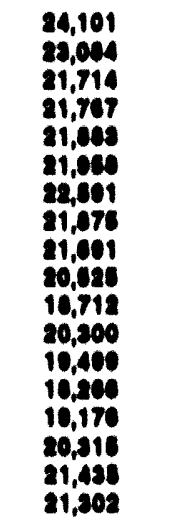 & $\begin{array}{l}1,074 \\
1,704 \\
2,104 \\
1,740 \\
2,207 \\
2,270 \\
2,240 \\
1,040 \\
2,204 \\
2,47 \\
1,04 \\
2,201 \\
2,104 \\
1,044 \\
1,011 \\
2,211 \\
2,080 \\
2,401\end{array}$ & $\begin{array}{r}77 \\
7 \\
78 \\
68 \\
60 \\
69 \\
60 \\
40 \\
60 \\
68 \\
68 \\
68 \\
61 \\
64 \\
74 \\
107 \\
00\end{array}$ & 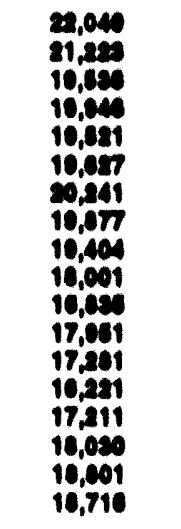 \\
\hline 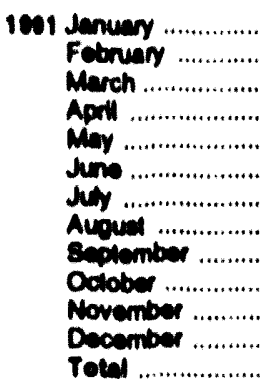 & $\begin{array}{l}1,610 \\
1,417 \\
1,535 \\
1,402 \\
1,463 \\
1,306 \\
1,300 \\
1,406 \\
1,300 \\
1,500 \\
1,520 \\
1,507 \\
17,006\end{array}$ & $\begin{array}{r}602 \\
400 \\
207 \\
104 \\
50 \\
42 \\
75 \\
62 \\
70 \\
103 \\
300 \\
461 \\
2,762\end{array}$ & $\begin{array}{r}12 \\
10 \\
11 \\
8 \\
6 \\
8 \\
8 \\
8 \\
10 \\
11 \\
11 \\
118\end{array}$ & $\begin{array}{l}163 \\
138 \\
151 \\
144 \\
141 \\
133 \\
135 \\
127 \\
134 \\
157 \\
160 \\
181 \\
1,773\end{array}$ & 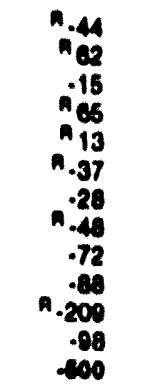 & 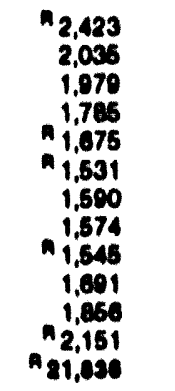 & $\begin{array}{l}115 \\
112 \\
120 \\
234 \\
331 \\
320 \\
290 \\
290 \\
304 \\
260 \\
150 \\
125 \\
2.078\end{array}$ & $\begin{array}{r}10 \\
11 \\
10 \\
9 \\
8 \\
7 \\
8 \\
10 \\
11 \\
14 \\
15 \\
18 \\
120\end{array}$ & $\begin{array}{l}2,200 \\
1,012 \\
1,640 \\
1,540 \\
1,337 \\
1,100 \\
1,208 \\
1,274 \\
1,231 \\
1,410 \\
1,001 \\
2,000 \\
10,030\end{array}$ \\
\hline 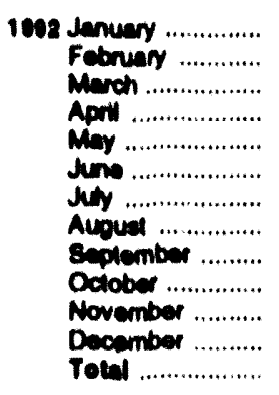 & $\begin{array}{l}1.580 \\
1.308 \\
1.475 \\
1,447 \\
1.486 \\
1.444 \\
1.401 \\
1.461 \\
1.437 \\
1.533 \\
1.514 \\
1.570 \\
17,040\end{array}$ & 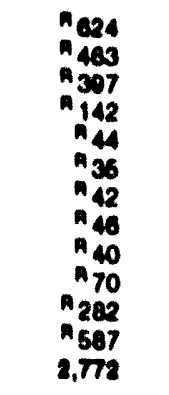 & $\begin{array}{r}12 \\
11 \\
11 \\
10 \\
0 \\
8 \\
8 \\
98 \\
98 \\
10 \\
11 \\
12 \\
110\end{array}$ & $\begin{array}{r}166 \\
175 \\
180 \\
176 \\
174 \\
162 \\
167 \\
175 \\
160 \\
176 \\
210 \\
200 \\
2,190\end{array}$ & 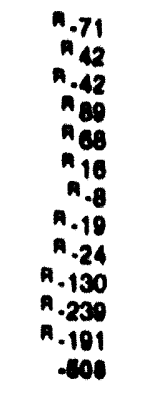 & $\begin{array}{l}A_{2,315} \\
A_{2,000} \\
A_{2,022} \\
A_{1,804} \\
A_{1,780} \\
A_{1,606} \\
A_{1,700} \\
A_{1,602} \\
A_{1,020} \\
A_{1,060} \\
A_{1,770} \\
A_{2,106} \\
22,300\end{array}$ & 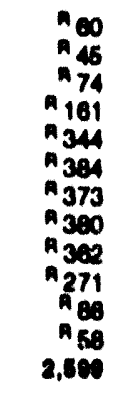 & $\begin{array}{l}16 \\
14 \\
23 \\
18 \\
19 \\
18 \\
16 \\
18 \\
18 \\
10 \\
10 \\
10 \\
216\end{array}$ & $\begin{array}{r}2,280 \\
2,031 \\
1,020 \\
1,606 \\
1,416 \\
1,264 \\
1,311 \\
1,264 \\
1,240 \\
1,360 \\
1,672 \\
2,110 \\
10,644\end{array}$ \\
\hline 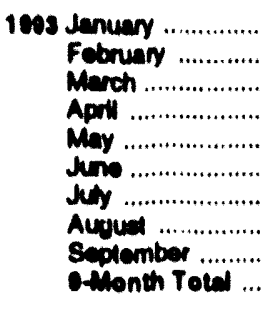 & 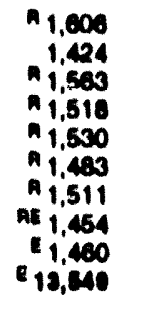 & $\begin{array}{r}605 \\
578 \\
381 \\
111 \\
25 \\
43 \\
46 \\
08 \\
25 \\
1,010\end{array}$ & $\begin{array}{r}13 \\
12 \\
12 \\
10 \\
8 \\
10 \\
9 \\
9 \\
9 \\
0\end{array}$ & $\begin{array}{l}190 \\
183 \\
190 \\
180 \\
100 \\
160 \\
178 \\
190 \\
184 \\
180 \\
1,060\end{array}$ & 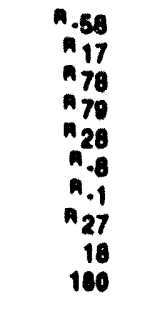 & $\begin{array}{l}n_{2,304} \\
n_{2,214} \\
n_{2,234} \\
n_{1,004} \\
n_{1,751} \\
n_{1,700} \\
n_{1,758} \\
n_{1,772} \\
1,600 \\
17,402\end{array}$ & $\begin{array}{r}60 \\
27 \\
78 \\
210 \\
447 \\
416 \\
308 \\
410 \\
378 \\
2.480\end{array}$ & $\begin{array}{r}18 \\
13 \\
17 \\
12 \\
12 \\
11 \\
114 \\
111 \\
11 \\
110\end{array}$ & $\begin{array}{l}n_{2,207} \\
n_{2,174} \\
n_{2,140} \\
n_{1,073} \\
n_{1}, 201 \\
A_{1}, 200 \\
A_{1}, 340 \\
n_{1,342} \\
1,311 \\
14,014\end{array}$ \\
\hline 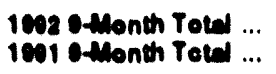 & $\begin{array}{l}13,216 \\
13,044\end{array}$ & $\begin{array}{l}1,093 \\
1,027\end{array}$ & $\mathbf{6}$ & $\begin{array}{l}1,641 \\
1,207\end{array}$ & $\begin{array}{r}89 \\
-104\end{array}$ & $\begin{array}{l}10,720 \\
10,120\end{array}$ & $\begin{array}{l}2,199 \\
2,120\end{array}$ & $\begin{array}{r}160 \\
82\end{array}$ & $\begin{array}{l}14,948 \\
13,017\end{array}$ \\
\hline
\end{tabular}

- Dala for 1080-1002 include underground storage and laquelled natural gae storage. All other datu include underground storage only. Computation procentures are diecuesed in Nole 8 at end of section.

- ses Notes al end of section.

- See Table 4.3.

- Data lor 1978 tonward do nat halude in-lranill recelple and dellvaries.

- May include unkenown quartilles of nonhydrocartion gasea.

1 geo Nole 7 al end of eaction.

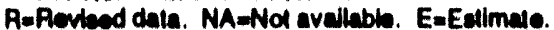

Notes: - Geographic coverage is the 50 statea and the Diturict of Columbia. - Todals may nol equal sum d components dive to independent rounding.
Sourcas: - 1972-1600: Totel Dry Cas Produetton-Energy Inlomualion Administration (EIA), Natural Gas Annual 1891, Tabis Q5. Whathervale from Storage, 107s-1075 and 1090-1064-EU, Nalural Gas Annual 1991. Table co. Withdrawele from storme, 1076-1070-EIA, Natural Ges Production and Conoumption 1970. Table 1. Supplomental Cascous Fuds. 1080-1004-EU, Natural Ges Amual 1000, Volums 2, Tabis 12. Imports, Additions to Btorage, Exporte, and Conoumption-ElA. Natural Gas

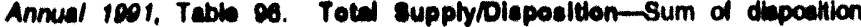
Mems. Delenoing Hem-Total supply/dispoettion minus all other supply Hems. - 1907 formard: ElA, Natural Gas Monthy, November 1903, Table 2. 


\begin{tabular}{|c|c|c|c|c|c|c|c|c|}
\hline & \multicolumn{4}{|c|}{ Imperts } & \multicolumn{4}{|c|}{ Experts } \\
\hline & Cenede & Alectat & Otron & Total & Canede: & Moxdoo" & Jepen' & Teen \\
\hline 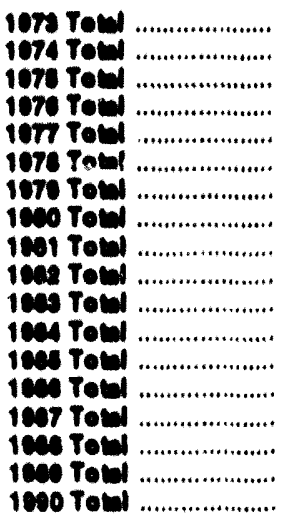 & 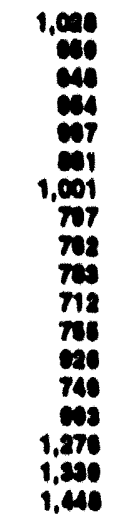 & 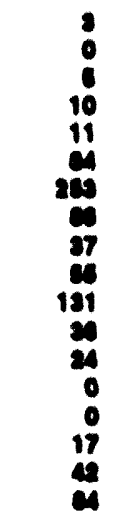 & $\begin{array}{r}(0) \\
0 \\
0 \\
2 \\
0 \\
0 \\
102 \\
100 \\
08 \\
70 \\
0 \\
0 \\
0 \\
0 \\
0 \\
0 \\
0\end{array}$ & 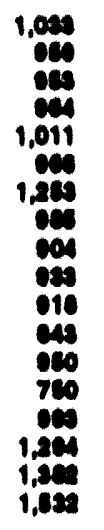 & $\begin{array}{l}18 \\
18 \\
10 \\
(0) \\
(0) \\
(0) \\
(0) \\
(0) \\
(0) \\
(0) \\
(0) \\
80 \\
09 \\
17\end{array}$ & $\begin{array}{r}14 \\
18 \\
7 \\
4 \\
4 \\
1 \\
2 \\
2 \\
2 \\
2 \\
2 \\
2 \\
2 \\
17 \\
10\end{array}$ & 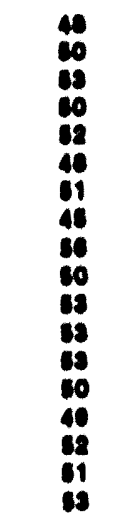 & 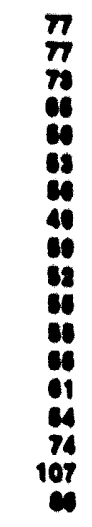 \\
\hline 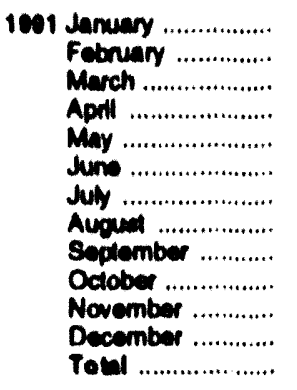 & $\begin{array}{l}156 \\
133 \\
140 \\
130 \\
136 \\
131 \\
130 \\
127 \\
131 \\
146 \\
164 \\
170 \\
1,710\end{array}$ & $\begin{array}{r}8 \\
5 \\
5 \\
5 \\
5 \\
3 \\
5 \\
0 \\
3 \\
10 \\
5 \\
10 \\
4\end{array}$ & $\begin{array}{l}0 \\
0 \\
0 \\
0 \\
0 \\
0 \\
0 \\
0 \\
0 \\
0 \\
0 \\
0 \\
0\end{array}$ & $\begin{array}{l}160 \\
130 \\
151 \\
144 \\
141 \\
130 \\
135 \\
127 \\
134 \\
157 \\
169 \\
181 \\
1,77\end{array}$ & $\begin{array}{r}2 \\
3 \\
1 \\
(0) \\
(1) \\
(0) \\
(0) \\
1 \\
(0) \\
2 \\
2 \\
3 \\
16\end{array}$ & $\begin{array}{r}3 \\
3 \\
4 \\
3 \\
5 \\
4 \\
3 \\
3 \\
6 \\
8 \\
8 \\
10 \\
10\end{array}$ & $\begin{array}{l}4 \\
4 \\
4 \\
6 \\
3 \\
3 \\
4 \\
6 \\
4 \\
4 \\
4 \\
14\end{array}$ & $\begin{array}{r}10 \\
11 \\
10 \\
9 \\
8 \\
7 \\
8 \\
10 \\
11 \\
14 \\
15 \\
18 \\
120\end{array}$ \\
\hline 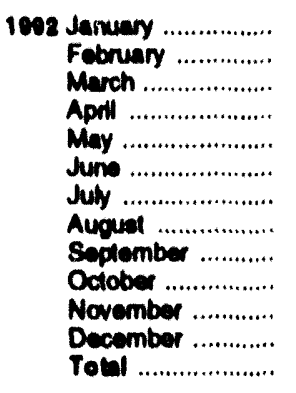 & $\begin{array}{l}157 \\
170 \\
178 \\
174 \\
174 \\
160 \\
167 \\
172 \\
164 \\
174 \\
203 \\
202 \\
2,004\end{array}$ & $\begin{array}{l}8 \\
5 \\
3 \\
3 \\
0 \\
3 \\
0 \\
2 \\
3 \\
3 \\
8 \\
8 \\
4\end{array}$ & $\begin{array}{l}0 \\
0 \\
0 \\
0 \\
0 \\
0 \\
0 \\
0 \\
0 \\
0 \\
0 \\
0 \\
0\end{array}$ & $\begin{array}{r}166 \\
176 \\
180 \\
176 \\
174 \\
160 \\
167 \\
176 \\
168 \\
176 \\
210 \\
200 \\
2,120\end{array}$ & $\begin{array}{r}2 \\
1 \\
11 \\
6 \\
6 \\
6 \\
5 \\
5 \\
6 \\
6 \\
3 \\
7 \\
6\end{array}$ & $\begin{array}{r}10 \\
6 \\
7 \\
7 \\
7 \\
7 \\
6 \\
0 \\
8 \\
10 \\
11 \\
8 \\
96\end{array}$ & $\begin{array}{l}4 \\
4 \\
4 \\
6 \\
4 \\
4 \\
4 \\
3 \\
4 \\
4\end{array}$ & $\begin{array}{l}16 \\
14 \\
23 \\
18 \\
10 \\
18 \\
16 \\
18 \\
18 \\
10 \\
10 \\
19 \\
210\end{array}$ \\
\hline 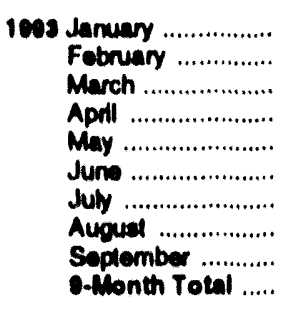 & $\begin{array}{l}193 \\
176 \\
194 \\
178 \\
155 \\
171 \\
183 \\
170 \\
177 \\
1,105\end{array}$ & $\begin{array}{r}5 \\
8 \\
5 \\
6 \\
5 \\
8 \\
8 \\
6 \\
10 \\
01\end{array}$ & $\begin{array}{l}0 \\
0 \\
0 \\
0 \\
0 \\
0 \\
0 \\
0 \\
0 \\
0\end{array}$ & $\begin{array}{l}109 \\
180 \\
100 \\
186 \\
160 \\
178 \\
100 \\
184 \\
189 \\
1,069\end{array}$ & $\begin{array}{l}6 \\
6 \\
8 \\
5 \\
4 \\
4 \\
5 \\
4 \\
4 \\
4\end{array}$ & $\begin{array}{r}8 \\
2 \\
3 \\
3 \\
3 \\
4 \\
4 \\
3 \\
2 \\
32\end{array}$ & $\begin{array}{r}4 \\
4 \\
9 \\
4 \\
4 \\
3 \\
5 \\
5 \\
5 \\
41\end{array}$ & $\begin{array}{r}18 \\
13 \\
17 \\
12 \\
12 \\
11 \\
14 \\
11 \\
11 \\
118\end{array}$ \\
\hline $\begin{array}{l}1002 \text { - Month Total .... } \\
1001 \text { - Month Total ..... }\end{array}$ & $\begin{array}{l}1,510 \\
1,220\end{array}$ & $\underset{30}{20}$ & $\begin{array}{l}0 \\
0\end{array}$ & $\begin{array}{l}1,641 \\
1,207\end{array}$ & s! & $\begin{array}{l}97 \\
36\end{array}$ & 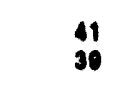 & $\begin{array}{r}100 \\
82\end{array}$ \\
\hline
\end{tabular}

- By pipoline, excepl lor very emall amounis of liquelied natural gas Imported Irom Canada in 1073, 1077 and 1981. See Note 5 at end of section.

- As liquetled natural gas.

- For 1973-1984, impons are from Maxico; for 1986, importa are from Indonesia.

(s) = Leas than 500 million cublc led.
Notes: - See Note 5 at end of section. - Geopraphic coverage is the 50 States and the District of Columbia. - Totale may not equal sum of components dus 10 independent rounding.

Sourca: Eneroy Information Administration, Natural Gas Monthis. November 1993, Tables 5 and 6. 
Table 4.4 Natural Gas Consumption by End-Use Sector (Billion Cubic Feet)

\begin{tabular}{|c|c|c|c|c|c|c|c|c|}
\hline & \multirow[b]{2}{*}{$\begin{array}{l}\text { Lease and } \\
\text { Plant Fud }\end{array}$} & \multirow[b]{2}{*}{$\begin{array}{l}\text { Plpelline } \\
\text { Fuete }\end{array}$} & \multicolumn{5}{|c|}{ Dellvered to Consumers } & \multirow[b]{2}{*}{$\begin{array}{c}\text { Toted } \\
\text { Consumption }\end{array}$} \\
\hline & & & Aesldental & Commerelal & Induetrial & $\begin{array}{l}\text { Electric } \\
\text { Utilities }\end{array}$ & Total & \\
\hline 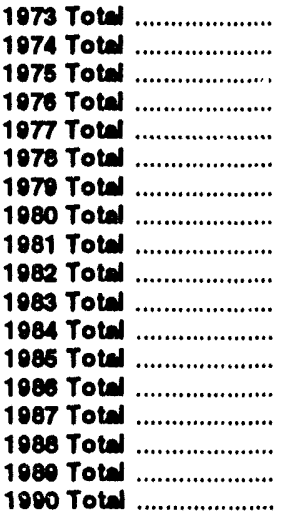 & $\begin{array}{r}1,496 \\
1,477 \\
1,306 \\
1,634 \\
1,659 \\
1,648 \\
1,400 \\
1,028 \\
928 \\
1,100 \\
978 \\
1,077 \\
086 \\
1,140 \\
1,023 \\
1,006 \\
1,236 \\
\end{array}$ & $\begin{array}{l}728 \\
680 \\
583 \\
548 \\
533 \\
530 \\
601 \\
635 \\
642 \\
506 \\
490 \\
520 \\
504 \\
485 \\
510 \\
614 \\
620 \\
660\end{array}$ & $\begin{array}{l}4,870 \\
4,788 \\
4,024 \\
5,051 \\
4,621 \\
4,003 \\
4,965 \\
4,752 \\
4,546 \\
4,633 \\
4,381 \\
4,555 \\
4,433 \\
4,314 \\
4,315 \\
4,630 \\
4,781 \\
4,391\end{array}$ & $\begin{array}{l}2,507 \\
2,556 \\
2,500 \\
2,688 \\
2,501 \\
2,601 \\
2,786 \\
2,611 \\
2,520 \\
2,606 \\
2,433 \\
2,524 \\
2,432 \\
2,318 \\
2,430 \\
2,670 \\
2.718 \\
2,623\end{array}$ & $\begin{array}{l}8,680 \\
8,202 \\
6,988 \\
6,964 \\
6,815 \\
6,767 \\
6,800 \\
7,172 \\
7,128 \\
5,831 \\
5,643 \\
6,154 \\
5,001 \\
5,579 \\
5,053 \\
6,383 \\
6,816 \\
7,018\end{array}$ & $\begin{array}{l}3,660 \\
3,443 \\
3,158 \\
3,081 \\
3,191 \\
3,188 \\
3,491 \\
3,682 \\
3,640 \\
3,226 \\
2,911 \\
3,111 \\
3,044 \\
2,602 \\
2,844 \\
2,636 \\
2,787 \\
2,787\end{array}$ & $\begin{array}{l}19,825 \\
19,077 \\
17,558 \\
17,764 \\
17,320 \\
17,440 \\
18,141 \\
18,216 \\
17,834 \\
16,295 \\
15,367 \\
16,345 \\
15,811 \\
14,814 \\
15,542 \\
16,320 \\
17,102 \\
16,820\end{array}$ & $\begin{array}{l}22,040 \\
21,223 \\
18,838 \\
19,046 \\
19,521 \\
19,027 \\
20,241 \\
19,877 \\
10,404 \\
18,001 \\
18,835 \\
17,851 \\
17,281 \\
18,221 \\
17,211 \\
18,030 \\
18,801 \\
18,716\end{array}$ \\
\hline 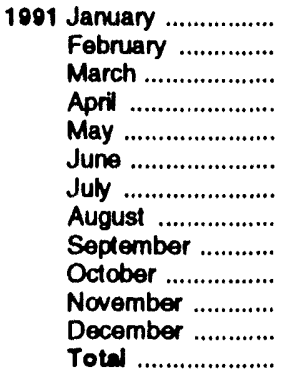 & $\begin{array}{r}102 \\
90 \\
98 \\
93 \\
93 \\
89 \\
90 \\
90 \\
89 \\
97 \\
97 \\
101 \\
1,129\end{array}$ & $\begin{array}{l}74 \\
61 \\
58 \\
49 \\
42 \\
37 \\
40 \\
40 \\
38 \\
44 \\
54 \\
64 \\
601\end{array}$ & $\begin{array}{r}844 \\
664 \\
573 \\
373 \\
229 \\
148 \\
126 \\
118 \\
138 \\
225 \\
459 \\
658 \\
4,556 \\
\end{array}$ & $\begin{array}{r}434 \\
359 \\
\times 310 \\
225 \\
154 \\
119 \\
125 \\
113 \\
121 \\
163 \\
256 \\
350 \\
2,720\end{array}$ & $\begin{array}{r}672 \\
591 \\
607 \\
586 \\
571 \\
546 \\
572 \\
586 \\
582 \\
626 \\
627 \\
665 \\
7,231\end{array}$ & $\begin{array}{r}173 \\
146 \\
193 \\
216 \\
249 \\
260 \\
330 \\
328 \\
263 \\
263 \\
198 \\
170 \\
2,780\end{array}$ & $\begin{array}{r}2,123 \\
1,761 \\
1,683 \\
1,400 \\
1,202 \\
1,073 \\
1,153 \\
1,144 \\
1,103 \\
1,278 \\
1,540 \\
1,843 \\
17,305\end{array}$ & $\begin{array}{r}2,299 \\
1,812 \\
1,840 \\
1,542 \\
1,337 \\
1,199 \\
1,283 \\
1,274 \\
1,231 \\
1,419 \\
1,691 \\
2,009 \\
19,005\end{array}$ \\
\hline 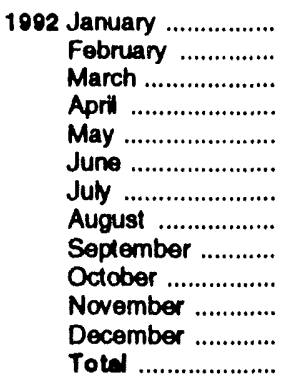 & $\begin{array}{r}104 \\
92 \\
97 \\
95 \\
97 \\
95 \\
98 \\
95 \\
94 \\
101 \\
99 \\
104 \\
1,171\end{array}$ & $\begin{array}{r}68 \\
62 \\
58 \\
51 \\
42 \\
37 \\
39 \\
37 \\
37 \\
41 \\
50 \\
64 \\
588\end{array}$ & $\begin{array}{l}786 \\
696 \\
574 \\
431 \\
251 \\
162 \\
132 \\
126 \\
137 \\
241 \\
437 \\
717 \\
4,690\end{array}$ & $\begin{array}{r}P_{410} \\
366 \\
315 \\
250 \\
170 \\
125 \\
122 \\
121 \\
121 \\
166 \\
256 \\
381 \\
2,803 \\
\end{array}$ & $\begin{array}{r}701 \\
644 \\
674 \\
628 \\
620 \\
578 \\
587 \\
582 \\
586 \\
608 \\
641 \\
677 \\
7,527\end{array}$ & $\begin{array}{r}169 \\
170 \\
208 \\
229 \\
236 \\
266 \\
334 \\
303 \\
274 \\
213 \\
189 \\
176 \\
2,766\end{array}$ & $\begin{array}{r}2,067 \\
1,876 \\
1,770 \\
1,539 \\
1,278 \\
1,132 \\
1,175 \\
1,131 \\
1,117 \\
1,227 \\
1,523 \\
1,951 \\
17,786\end{array}$ & $\begin{array}{r}2,239 \\
2,031 \\
1,926 \\
1,685 \\
1,418 \\
1,264 \\
1,311 \\
1,264 \\
1,249 \\
1,368 \\
1,672 \\
2,119 \\
19,544\end{array}$ \\
\hline 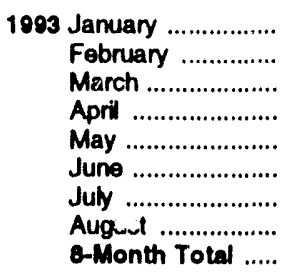 & $\begin{array}{r}R_{105} \\
R_{94} \\
103 \\
100 \\
100 \\
97 \\
99 \\
95 \\
704\end{array}$ & $\begin{array}{l}R_{73} \\
R_{69} \\
R_{68} \\
R_{53} \\
R_{41} \\
R_{40} \\
R_{43} \\
42 \\
428\end{array}$ & $\begin{array}{r}834 \\
770 \\
703 \\
450 \\
234 \\
164 \\
R \quad 130 \\
120 \\
3,405\end{array}$ & $\begin{array}{r}R \\
R_{421} \\
R_{408} \\
R_{374} \\
257 \\
156 \\
R_{127} \\
123 \\
115 \\
1,082\end{array}$ & 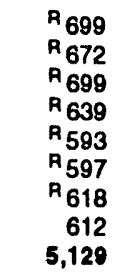 & $\begin{array}{r}164 \\
162 \\
194 \\
174 \\
167 \\
255 \\
333 \\
357 \\
1,806\end{array}$ & $\begin{array}{l}A_{2,119} \\
R_{2,012} \\
R_{1,969} \\
R_{1,521} \\
R_{1}, 150 \\
R_{1,142} \\
R_{1,204} \\
1,204 \\
12,322\end{array}$ & $\begin{array}{r}A_{2,297} \\
R_{2,174} \\
R_{2,140} \\
R_{1,673} \\
R_{1,291} \\
R_{1,280} \\
R_{1,346} \\
1,342 \\
13,543\end{array}$ \\
\hline $\begin{array}{l}1092 \text { a-Month Total ..... } \\
1091 \text { Q-Month Total ..... }\end{array}$ & $\begin{array}{l}773 \\
745\end{array}$ & $\begin{array}{l}305 \\
401\end{array}$ & $\begin{array}{l}3,158 \\
3,075\end{array}$ & $\begin{array}{l}1,879 \\
1,838\end{array}$ & $\begin{array}{l}5,015 \\
4,731\end{array}$ & $\begin{array}{l}1,014 \\
1,805\end{array}$ & $\begin{array}{l}11,087 \\
11,530\end{array}$ & $\begin{array}{l}13,138 \\
12,686\end{array}$ \\
\hline
\end{tabular}

a Natural gas consumed in the operation of pipelines, primarity in compressors.

$R=$ Revised data.

Notes: - Natural gas includes supplemental gaseous fuels. - Geographic coverage is the 50 States and the District of Columbia. - Totals may not equal sum of components due to independent rounding.

Sources: - 1073-1086: Energy Information Administration (EIA), Natural Gas Arnual 1991, Table 97. - 1987 forward: ElA, Natural Gas Monthly, November 1993, Table 3. 


\begin{tabular}{|c|c|c|c|c|c|c|c|c|}
\hline & \multicolumn{3}{|c|}{$\begin{array}{l}\text { Natural Gas in } \\
\text { Underground Storage, } \\
\text { End of Porlod }\end{array}$} & \multicolumn{2}{|c|}{$\begin{array}{c}\text { Change In Working ane } \\
\text { from Seme Perlod } \\
\text { Previous Year }\end{array}$} & \multicolumn{3}{|c|}{ Storege Activity } \\
\hline & Bace Oas & Working Gas & Totala & Volume & Pereent & Injectlons & Withdrawals $b$ & Notc \\
\hline 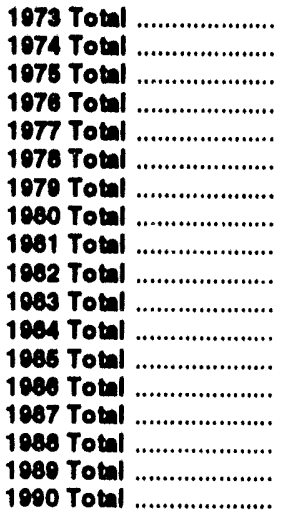 & $\begin{array}{l}2,804 \\
2,012 \\
3,102 \\
3,323 \\
3,301 \\
3,473 \\
3,553 \\
3,642 \\
3,752 \\
3,808 \\
3,847 \\
3,830 \\
3,842 \\
3,810 \\
3,702 \\
3,800 \\
3,812 \\
3,868\end{array}$ & $\begin{array}{l}2,034 \\
2,050 \\
2,212 \\
1,026 \\
2,475 \\
2,547 \\
2,753 \\
2,855 \\
2,817 \\
3,071 \\
2,895 \\
2,876 \\
2,807 \\
2,740 \\
2,756 \\
2,850 \\
2,513 \\
3,068\end{array}$ & $\begin{array}{l}4,898 \\
4,962 \\
5,374 \\
5,250 \\
5,866 \\
6,020 \\
6,306 \\
6,297 \\
6,560 \\
6,870 \\
6,442 \\
6,708 \\
6,448 \\
6,567 \\
6,548 \\
6,650 \\
6,325 \\
6,936\end{array}$ & $\begin{array}{r}305 \\
16 \\
182 \\
-280 \\
540 \\
72 \\
207 \\
-90 \\
162 \\
255 \\
-476 \\
281 \\
-270 \\
142 \\
7 \\
94 \\
-337 \\
555\end{array}$ & $\begin{array}{r}17.0 \\
.8 \\
7.0 \\
-12.0 \\
28.5 \\
2.0 \\
6.1 \\
-3.6 \\
6.1 \\
0.0 \\
-15.5 \\
10.8 \\
-0.4 \\
5.5 \\
.3 \\
3.4 \\
-11.8 \\
22.1\end{array}$ & $\begin{array}{l}1,074 \\
1,784 \\
2,104 \\
1,756 \\
2,307 \\
2,278 \\
2,295 \\
1,806 \\
2,180 \\
2,309 \\
1,700 \\
2,252 \\
2,128 \\
1,052 \\
1,887 \\
2,174 \\
2,491 \\
2,433\end{array}$ & $\begin{array}{l}1,533 \\
1,701 \\
1,760 \\
1,021 \\
1,750 \\
2,158 \\
2,047 \\
1,010 \\
1,887 \\
2,094 \\
2,142 \\
2,084 \\
2,359 \\
1,812 \\
1,881 \\
2,244 \\
2,804 \\
1,034\end{array}$ & $\begin{array}{r}442 \\
84 \\
344 \\
-165 \\
657 \\
120 \\
248 \\
-14 \\
293 \\
308 \\
-442 \\
188 \\
-231 \\
140 \\
6 \\
-89 \\
-313 \\
490\end{array}$ \\
\hline 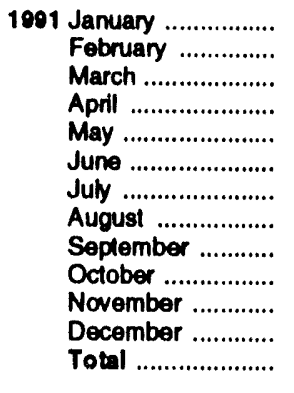 & $\begin{array}{l}3,911 \\
3,908 \\
3,895 \\
3,898 \\
3,931 \\
3,939 \\
3,942 \\
3,949 \\
3,950 \\
3,961 \\
3,952 \\
3,954 \\
3,054\end{array}$ & $\begin{array}{l}2,362 \\
2,063 \\
1,912 \\
2,037 \\
2,273 \\
2,553 \\
2,771 \\
2,978 \\
3,201 \\
3,369 \\
3,148 \\
2,824 \\
2,824\end{array}$ & $\begin{array}{l}6,273 \\
5,972 \\
5,806 \\
5,935 \\
6,204 \\
6,492 \\
6,713 \\
6,927 \\
7,151 \\
7,330 \\
7,100 \\
6,778 \\
6,778\end{array}$ & $\begin{array}{r}92 \\
59 \\
37 \\
91 \\
93 \\
68 \\
-20 \\
.93 \\
.120 \\
.98 \\
.324 \\
.244 \\
-244\end{array}$ & $\begin{array}{r}4.1 \\
\text { A.0 } \\
2.0 \\
4.7 \\
4.3 \\
2.7 \\
-.7 \\
-3.0 \\
-3.6 \\
-2.8 \\
-9.3 \\
-8.0 \\
-8.0\end{array}$ & $\begin{array}{r}115 \\
112 \\
129 \\
228 \\
319 \\
314 \\
\mathrm{~A}_{290} \\
282 \\
294 \\
251 \\
150 \\
125 \\
2,608\end{array}$ & $\begin{array}{r}{ }^{A} 660 \\
397 \\
291 \\
104 \\
58 \\
42 \\
75 \\
82 \\
78 \\
103 \\
352 \\
448 \\
2,680\end{array}$ & $\begin{array}{r}-545 \\
-285 \\
-162 \\
124 \\
261 \\
272 \\
214 \\
200 \\
216 \\
148 \\
-202 \\
-323 \\
-80\end{array}$ \\
\hline 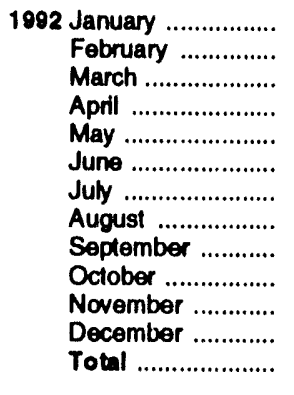 & $\begin{array}{l}4,061 \\
4,057 \\
4,046 \\
4,038 \\
4,044 \\
4,050 \\
4,064 \\
4,062 \\
4,061 \\
4,065 \\
4,061 \\
4,044 \\
4,044\end{array}$ & $\begin{array}{l}2,216 \\
1,837 \\
1,545 \\
1,573 \\
1,848 \\
2,153 \\
2,460 \\
2,761 \\
3,044 \\
3,223 \\
3,054 \\
2,597 \\
2,597\end{array}$ & $\begin{array}{l}6,277 \\
5,894 \\
5,591 \\
5,611 \\
5,892 \\
6,203 \\
6,524 \\
6,823 \\
7,105 \\
7,288 \\
7,115 \\
6,641 \\
6,641\end{array}$ & $\begin{array}{l}-146 \\
-226 \\
.367 \\
-463 \\
-425 \\
-400 \\
-311 \\
-217 \\
-157 \\
-146 \\
-94 \\
-227 \\
-227\end{array}$ & $\begin{array}{r}-6.2 \\
-10.9 \\
-19.2 \\
-22.8 \\
-18.7 \\
-15.7 \\
-11.2 \\
-7.3 \\
-4.9 \\
-4.3 \\
-3.0 \\
-8.0 \\
-8.0\end{array}$ & $\begin{array}{r}68 \\
52 \\
81 \\
167 \\
330 \\
366 \\
357 \\
364 \\
346 \\
264 \\
95 \\
65 \\
2,555\end{array}$ & $\begin{array}{r}591 \\
441 \\
381 \\
150 \\
53 \\
43 \\
50 \\
54 \\
48 \\
78 \\
276 \\
557 \\
2,724\end{array}$ & $\begin{array}{r}-524 \\
-389 \\
-301 \\
18 \\
277 \\
323 \\
307 \\
309 \\
298 \\
186 \\
-181 \\
-491 \\
-168\end{array}$ \\
\hline 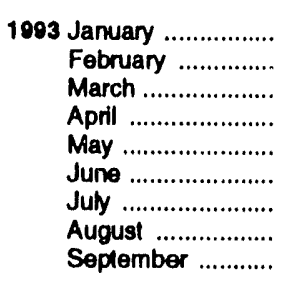 & $\begin{array}{l}4,040 \\
4,014 \\
3,993 \\
3,999 \\
4,017 \\
4,029 \\
4,030 \\
4,254 \\
4,254\end{array}$ & $\begin{array}{l}2,045 \\
1,519 \\
1,237 \\
1,335 \\
1,738 \\
2,100 \\
2,465 \\
2,566 \\
2,901\end{array}$ & $\begin{array}{l}6,086 \\
5,532 \\
5,230 \\
5,334 \\
5,755 \\
6,128 \\
6,495 \\
6,820 \\
7,155\end{array}$ & $\begin{array}{r}-170 \\
-319 \\
-308 \\
-238 \\
-111 \\
-53 \\
5 \\
-195 \\
-143\end{array}$ & $\begin{array}{r}-7.7 \\
-17.3 \\
-19.9 \\
-15.1 \\
-6.0 \\
-2.5 \\
.2 \\
-7.1 \\
-4.7\end{array}$ & $\begin{array}{r}50 \\
27 \\
78 \\
219 \\
447 \\
416 \\
398 \\
419 \\
378\end{array}$ & $\begin{array}{r}605 \\
578 \\
381 \\
111 \\
25 \\
43 \\
48 \\
98 \\
25\end{array}$ & $\begin{array}{r}-556 \\
-552 \\
-304 \\
108 \\
423 \\
372 \\
350 \\
321 \\
352\end{array}$ \\
\hline
\end{tabular}

- Tolal underground storage capacity at the end of each calendar year (in billon cubic feet): 1975--6,280 (irst year for which data are available); 1976-6,544; 1977-6,678; 1978-6,890; 1979-6,929; 1980-7,434; 1981--7,805; 1982-7,915; 1983-7,985; 1984-8,043; 1985-8,087; 1986--8,145; 1987, 1988, and 1989--8,124; 1990-8,125; 1991-.7,993; and 1992--7,932. Current capacity remains at 7,932.

b For 1980-1991, data differ from those shown on Table 4.2, which includes liquetied natural gas storage for that period.

c Positive numbers indicate injections are greater than withdrawals. Negative numbers indicate withdrawals are greater than injections. Net injections or withdrawals may not equal the difference between applicable ending stocks. See Note 8 at end of section.

$\mathrm{R}=$ Revised data.

Notes: - Geographic coverage is the 50 States and the District of Columbia.

- Totals may not equal sum of components due to independent rounding.

Sources: - Storage Activity: 1973-1975-Energy Information
Administration (ElA), Natural Gas Annual 1990, Volume 2, Table 9. 1976-1970-EIA, Natural Gas Production and Consumption 1979, Table 1 1080-1986-EIA, Natural Gas Annual 1990, Volume 2, Table 11. 1987 forward-EIA, Natural Gas Monthy, November 1993, Table 13. - Other Data: 1973 and 1974-American Gas Association (AGA), Gas Facts, 1972 Data, Table 57, Gas Facts, 1973 Data, Table 57, and Gas Facts, 1974 Data, Table 40. 1075 and 1976-Federal Energy Administration (FEA), Form FEA-G318-M-0, "Underground Gas Storage Report," and Federal Power Commission (FPC), Form FPC-8, "Underground Gas Storage Report." 1977 and 1978-EIA, Form FEA-G318-M-0, "Underground Gas Storage Report," and Federal Energy Regulatory Commission (FERC), Form FERC-8, "Underground Gas Storage Repon." 1979-1986-ElA, Form ElA-191, "Underground Gas Storage Report," and FERC, Form FERC-8, "Underground Gas Storage Report." 1987 forward-EIA, Natural Gas Monthly, November 1993, Table 13. 


\section{Natural Gas Notes}

1. Nonhydrocarbon Gases Removed: Annual data on nonhydrocarbon gases removed from marketed production-carbon dioxide, helium, hydrogen sulfide, and nitrogen-are from the Energy Information Administration (EIA) Natural Gas Annual (NGA) 1991. Data are not available for periods prior to 1980 . Monthly data are reported by three States and computed for six States. Monthly data are preliminary until after publication of the EIA NGA. Differences between annual data published in the EIA $N G A$ and the sum of the preliminary monthly data (January-December) are allocated proportionally to the months to create final monthly data. For further information on methods of estimating preliminary monthly data, see the EIA Natural Gas Monthly (NGM).

\section{Production.}

- Annual data: Final annual data are from the EIA $N G A$.

- Estimated monthly data: Data for the two most recent months presented are estimated. Some of the data for earlier months are also estimated or computed. For a discussion of computation and estimation procedures, see the EIA NGM.

- Preliminary monthly data: Monthly data are considered preliminary until after publication of the EIA NGA. Preliminary monthly data are gathered from reports to the Interstate Oil Compact Commission and the U.S. Minerals Management Service. Volumetric data are converted, as necessary, to a standard 14.73 psi pressure base. Unless there are major changes, data are not revised until after publication of the EIA $N G A$.

- Final monthly data: Differences between annual data in the EIA $N G A$ and the sum of preliminary monthly data (January-December) are allocated proportionally to the months to create final monthly data.

3. Extraction Loss: Extraction loss is the reduction in volume of natural gas resulting from the removal of natural gas liquid constituents at natural gas processing plants.

Annual data for extraction loss are from the EIA $N G A$, where they are estimated on the basis of the type and quantity of liquid products extracted from the gas stream and the calculated volume of such products at standard conditions. For a detailed explanation of the calculations used to derive estimated extraction losses, see the EIA $N G A$.

Preliminary monthly data are estimated on the basis of extraction loss as an annual percentage of marketed production. This percentage is applied to each month's marketed production to estimate monthly extraction loss.
Monthly data are revised and considered final after the publication of the EIA NGA. Final monthly data are estimated by allocating annual extraction loss data to the months on the basis of total natural gas marketed production data from the EIA $N G A$.

4. Supplemental Gaseous Fuels: Any gaseous substance that, introduced into or commingled with natural gas, increases the volume available for disposition. Such substances include, but are not limited to, propane-air, refinery gas, coke oven gas, still gas, manufactured gas, biomass gas, or air or inert gases added for Btu stabilization.

Annual data beginning with 1980 are from the EIA $N G A$. Unknown quantities of supplemental gaseous fuels are included in consumption data for 1979 and earlier years.

Monthly data are considered preliminary until after the publication of the EIA NGA. Monthly estimates are based on the annual ratio of supplemental gaseous fuels to the sum of dry gas production, net imports, and net withdrawals from storage. The ratio is applied to the monthly sum of the three elements to compute a monthly supplemental gaseous fuels figure.

5. Imports and Exports: The United States imports natural gas via pipeline from Canada. Prior to 1985, it also imported natural gas via pipeline from Mexico. Liquefied natural gas (LNG) arrives via tanker from Algeria. One shipment of $L N G$ was received from Indonesia in December 1986. Very small amounts of LNG arrived from Canada in 1973 (667 million cubic feet), 1977 (572 million cubic feet), and 1981 (6 million cubic feet). The United States exports natural gas via pipeline to Canada and Mexico and LNG via tanker to Japan.

Annual and final monthly data are from the annual Form FPC-14, "Annual Report for Importers and Exporters of Natural Gas," which requires data to be reported by month for the calendar year.

Preliminary monthly data are EIA estimates. For a discussion of estimation procedures, see the EIA NGM. Preliminary data are revised after the publication of the EIA U.S. Imports and Exports of Natural Gas.

6. Consumption: Consumption includes pipeline fuel use, lease and plant fuel use, and deliveries to consuming sectors.

Final data are from the EIA NGA. Monthly data are considered preliminary until after publication of the EIA $N G A$. For more detailed information on the methods of estimating preliminary and final monthly data, see the EIA $N G M$.

7. Balancing Item: The balancing item for natural gas represents the difference between the sum of the com- 
ponents of natural gas supply and the sum of components of natural gas disposition. The differences may be due to quantities lost or to the effects of data reporting problems. Reporting problems include differences due to the net result of conversions of flow data metered at varying temperature and pressure bases and converted to a standard temperature and pressure base; the effect of variations in company accounting and billing practices; differences between billing cycle and calendar period time frames; and imbalances resulting from the merger of data reporting systems which vary in scope, format, definitions, and type of respondents.

The increase of 0.2 trillion cubic feet (Tcf) in the "Balancing Item" category in 1983, followed by a decline of $0.5 \mathrm{Tcf}$ in 1984, reflected unusually large differences resulting from the use of the annual billing cycle (essentially December 15 through the following December 14) consumption data in conjunction with calendar year supply data. Record cold temperatures during the last half of December 1983 resulted in a reported 0.3 Tcf increase in net withdrawals from underground storage for peak shaving as compared with the same period in 1982, but the effect of this cold weather was reflected primarily in 1984 consumption data. For underground storage data, see Table $F 2$ in the May 1985 NGM, which was published in July 1985.
8. Natural Gas Storage: Gas in storage at the end of a reporting period may not equal the quantity derived by adding or subtracting net injections or withdrawals from the quantity in storage at the end of the previous period. The difference is due to changes in the quantity of native gas included in the base gas and/or losses in base gas due to migration from storage reservoirs.

Monthly underground storage data are collected from the Forms FERC-8 (interstate data) and EIA-191 (intrastate data). Beginning in January 1991, all data are collected on the revised Form EIA-191. Injection and withdrawal data from the FERC-8/EIA-191 survey are adjusted to correspond to data from Form EIA-176 following publication of the EIA NGA.

The final monthly and annual storage and withdrawal data for 1980-1989 include both underground and liquefied natural gas (LNG) storage. Annual data on LNG additions and withdrawals are from Form ElA176. Monthly data are estimated by computing the ratio of each month's underground storage additions and withdrawals $h$ annual underground storage additions and withdrawals and applying the ratio to the annual LNG data. 


\section{Section 5. Oil and Gas Resource Development}

A total of 81 seismic exploration crews were active in October 1993, 5 more crews than were active a year earlier. Of the total, 66 were land crews and 15 were aboard marine vessels. The number of land crews was unchanged but the number of operating marine vessels increased by 5 vessels from the October 1992 count.

The October 1993 rotary rig count of 860 was 1 percent higher than the count in the previous month and 7 percent higher than the count in October 1992. Of the total number of rigs in operation, 767 were onshore and 93 were offshore. The number of onshore rigs was up 2 percent from the number in October 1992, and the number of offshore rigs was up 75 percent.

Total footage drilled in October 1993 was 12.11 million feet, up 5 percent from footage drilled in September
1993 and up 2 percent from that drilled in October 1992.

The estimated number of exploratory and development gas and oil wells drilled during October 1993 was $1,726,2$ percent higher than the number drilled in September 1993 but 2 percent lower than the number drilled in October 1992. The estimated number of oil wells drilled was 850 and the estimated number of gas wells was 876, 9 percent higher but 11 percent lower, respectively, from the October 1992 levels. The estimated number of dry holes drilled in October 1993 was 789,15 percent higher than the number drilled in September 1993 and 40 percent higher than the number drilled in October 1992.

Figure 5.1 Oil and Gas Resource Development Indicators

\section{Crews Engaged in Exploration}

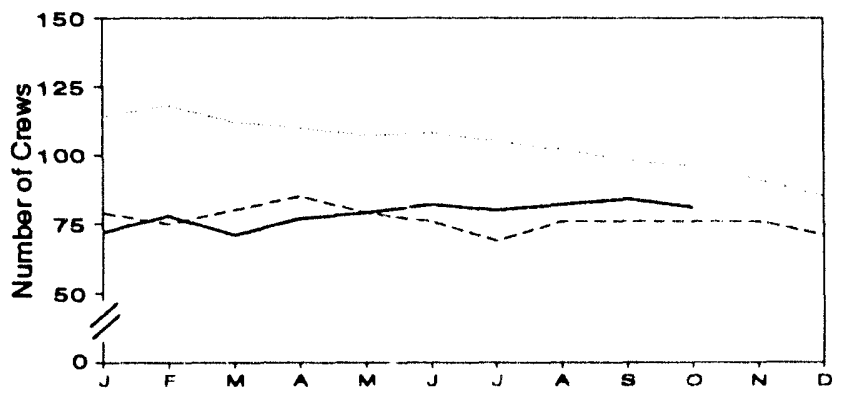

Wells Drilled

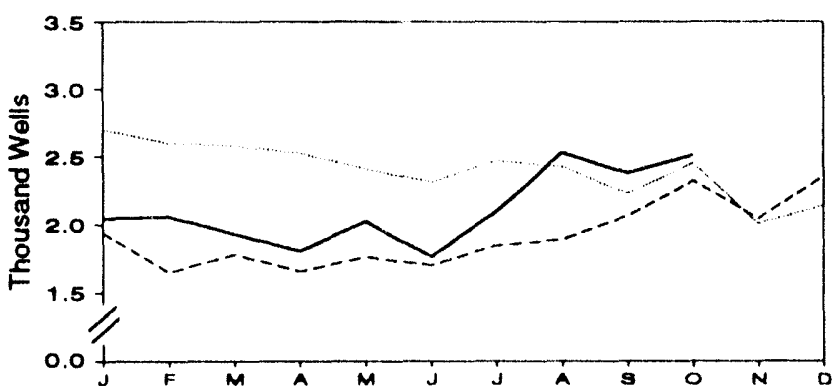
Rotary Rigs in Operation $\quad-1993$

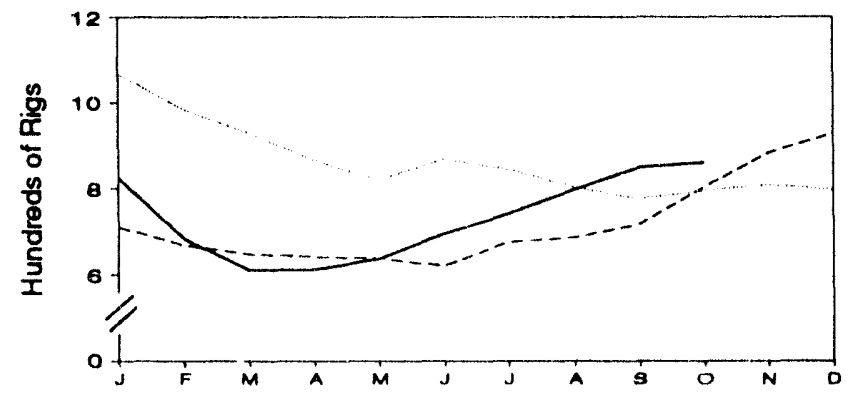

Footage Drilled

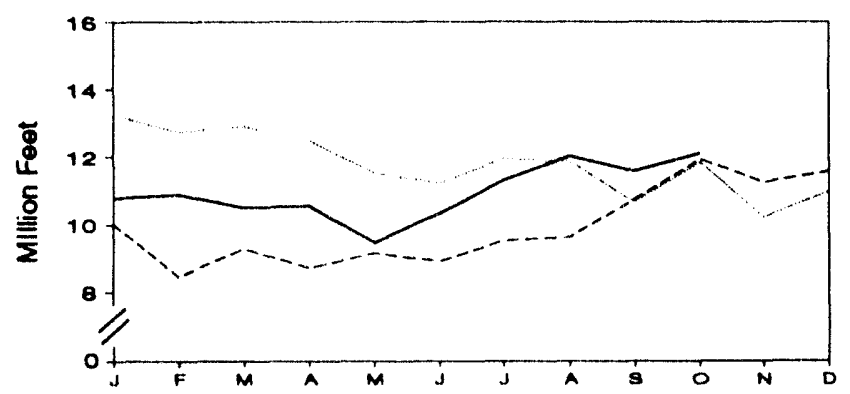

Sources: Tables 5.1 and 5.2 . 
Table 5.1 Oll and Gas Drilling Activity Measurements

\begin{tabular}{|c|c|c|c|c|c|c|c|c|c|c|}
\hline & \multicolumn{3}{|c|}{$\begin{array}{l}\text { Crowe Engaged in } \\
\text { selemle Exploration }\end{array}$} & \multicolumn{5}{|c|}{ Rotary Alge in Operatton } & \multirow{3}{*}{$\begin{array}{l}\text { Total } \\
\text { Footnge } \\
\text { Drillede }\end{array}$} & \multirow{3}{*}{$\begin{array}{l}\text { Actve } \\
\text { Well Eorvicing } \\
\text { Unitod }\end{array}$} \\
\hline & \multirow[b]{2}{*}{ Otishore } & \multirow[b]{2}{*}{ Onshore } & \multirow[b]{2}{*}{ Total } & \multicolumn{2}{|c|}{ By site } & \multicolumn{2}{|c|}{ Ву Туре } & \multirow[b]{2}{*}{ Totalb } & & \\
\hline & & & & Olishore & Onshore & Oll & Ges & & & \\
\hline & \multicolumn{3}{|c|}{ Monthly Average } & \multicolumn{4}{|c|}{ Weekly Avorage } & & Thousand Feot & Number \\
\hline 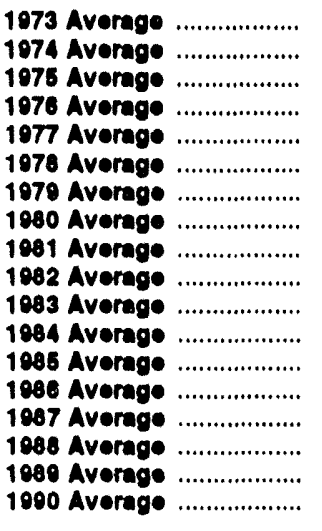 & $\begin{array}{l}23 \\
31 \\
30 \\
26 \\
27 \\
25 \\
30 \\
37 \\
44 \\
57 \\
17 \\
19 \\
45 \\
24 \\
24 \\
29 \\
23 \\
23\end{array}$ & $\begin{array}{l}227 \\
274 \\
284 \\
237 \\
281 \\
327 \\
370 \\
493 \\
637 \\
531 \\
426 \\
446 \\
333 \\
176 \\
153 \\
153 \\
109 \\
102\end{array}$ & $\begin{array}{l}260 \\
305 \\
284 \\
282 \\
308 \\
352 \\
400 \\
630 \\
681 \\
888 \\
173 \\
494 \\
378 \\
200 \\
177 \\
182 \\
132 \\
125\end{array}$ & $\begin{array}{r}84 \\
04 \\
108 \\
120 \\
187 \\
185 \\
207 \\
231 \\
286 \\
243 \\
190 \\
213 \\
208 \\
90 \\
05 \\
123 \\
105 \\
108\end{array}$ & $\begin{array}{r}1,110 \\
1,370 \\
1,564 \\
1,520 \\
1,834 \\
2,074 \\
1,070 \\
2,670 \\
3,714 \\
2,862 \\
2,033 \\
2,218 \\
1,774 \\
865 \\
241 \\
813 \\
704 \\
002\end{array}$ & $\begin{array}{l}\text { NA } \\
\text { NA } \\
\text { NA } \\
\text { NA } \\
\text { NA } \\
\text { NA } \\
N A \\
N A \\
N A \\
N A \\
N A \\
N A \\
N A \\
N A \\
N A \\
554 \\
453 \\
532\end{array}$ & $\begin{array}{l}\text { NA } \\
\text { NA } \\
\text { NA } \\
\text { NA } \\
\text { NA } \\
\text { NA } \\
\text { NA } \\
\text { NA } \\
\text { NA } \\
\text { NA } \\
\text { NA } \\
\text { NA } \\
\text { NA } \\
\text { NA } \\
\text { NA } \\
354 \\
401 \\
464\end{array}$ & $\begin{array}{r}1,194 \\
1,472 \\
1,660 \\
1,650 \\
2,001 \\
2,250 \\
2,177 \\
2,900 \\
3,070 \\
3,105 \\
2,232 \\
2,428 \\
1,080 \\
964 \\
936 \\
938 \\
860 \\
1,010\end{array}$ & $\begin{array}{l}139,427 \\
163,701 \\
181,046 \\
187,291 \\
216,686 \\
238,388 \\
243,686 \\
312,303 \\
408,842 \\
378,437 \\
318,585 \\
370,730 \\
312,569 \\
177,486 \\
161,226 \\
153,340 \\
133,383 \\
149,378\end{array}$ & $\begin{array}{c}N A \\
N A \\
N A \\
2,601 \\
2,828 \\
2,088 \\
3,300 \\
4,080 \\
4,850 \\
4,248 \\
3,732 \\
4,863 \\
4,718 \\
3,038 \\
3,060 \\
3,341 \\
3,391 \\
3,858\end{array}$ \\
\hline 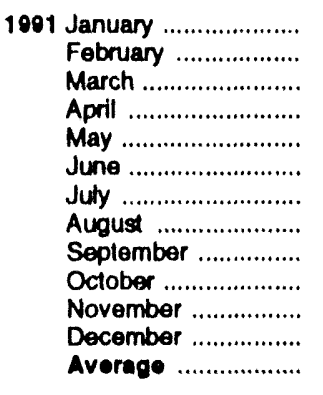 & $\begin{array}{l}22 \\
21 \\
24 \\
23 \\
22 \\
21 \\
16 \\
15 \\
14 \\
15 \\
18 \\
18 \\
10\end{array}$ & $\begin{array}{l}92 \\
97 \\
88 \\
87 \\
85 \\
87 \\
89 \\
87 \\
84 \\
81 \\
73 \\
66 \\
86\end{array}$ & $\begin{array}{r}114 \\
118 \\
112 \\
110 \\
107 \\
108 \\
105 \\
102 \\
88 \\
96 \\
91 \\
85 \\
104\end{array}$ & $\begin{array}{l}91 \\
88 \\
81 \\
95 \\
98 \\
93 \\
80 \\
68 \\
71 \\
68 \\
72 \\
65 \\
11\end{array}$ & $\begin{array}{l}977 \\
896 \\
848 \\
770 \\
721 \\
774 \\
764 \\
735 \\
704 \\
727 \\
736 \\
731 \\
770\end{array}$ & $\begin{array}{l}633 \\
564 \\
520 \\
469 \\
430 \\
483 \\
472 \\
451 \\
433 \\
433 \\
457 \\
469 \\
482\end{array}$ & $\begin{array}{l}413 \\
405 \\
389 \\
374 \\
354 \\
342 \\
332 \\
326 \\
314 \\
330 \\
328 \\
308 \\
351\end{array}$ & $\begin{array}{r}1,068 \\
984 \\
929 \\
865 \\
819 \\
867 \\
844 \\
803 \\
775 \\
795 \\
808 \\
786 \\
860\end{array}$ & $\begin{array}{r}13,243 \\
12,738 \\
12,905 \\
12,490 \\
11,514 \\
11,214 \\
11,940 \\
11,861 \\
10,669 \\
A_{11,830} \\
10,215 \\
10,980 \\
141,500\end{array}$ & $\begin{array}{l}3,579 \\
3,512 \\
3,444 \\
3,416 \\
3,394 \\
3,363 \\
3,369 \\
3,257 \\
3,208 \\
3,138 \\
3,113 \\
3,183 \\
3,331\end{array}$ \\
\hline 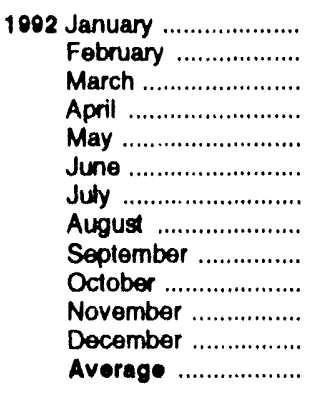 & $\begin{array}{r}18 \\
13 \\
13 \\
13 \\
13 \\
12 \\
9 \\
9 \\
10 \\
10 \\
15 \\
13 \\
12\end{array}$ & $\begin{array}{l}61 \\
62 \\
67 \\
72 \\
66 \\
64 \\
60 \\
67 \\
66 \\
66 \\
61 \\
58 \\
64\end{array}$ & $\begin{array}{l}79 \\
75 \\
60 \\
85 \\
79 \\
76 \\
69 \\
76 \\
76 \\
76 \\
76 \\
71 \\
76\end{array}$ & $\begin{array}{l}56 \\
51 \\
54 \\
55 \\
47 \\
44 \\
48 \\
51 \\
45 \\
53 \\
60 \\
59 \\
52\end{array}$ & $\begin{array}{l}654 \\
618 \\
594 \\
587 \\
591 \\
577 \\
628 \\
635 \\
672 \\
750 \\
822 \\
867 \\
660\end{array}$ & $\begin{array}{l}400 \\
378 \\
381 \\
370 \\
358 \\
343 \\
349 \\
334 \\
345 \\
392 \\
418 \\
397 \\
373\end{array}$ & $\begin{array}{l}294 \\
277 \\
250 \\
251 \\
260 \\
260 \\
310 \\
331 \\
356 \\
399 \\
451 \\
509 \\
331\end{array}$ & $\begin{array}{l}710 \\
669 \\
648 \\
642 \\
638 \\
621 \\
676 \\
686 \\
717 \\
803 \\
882 \\
926 \\
721\end{array}$ & $\begin{array}{r}10,017 \\
8,456 \\
9,289 \\
8,726 \\
9,158 \\
8,915 \\
9,529 \\
9,635 \\
10,748 \\
\mathrm{~A}^{11,925} \\
11,250 \\
11,570 \\
\mathrm{~A}_{119,218}\end{array}$ & $\begin{array}{l}2,912 \\
2,704 \\
2,592 \\
2,727 \\
2,264 \\
2,369 \\
2,492 \\
2,630 \\
2,825 \\
3,076 \\
2,977 \\
3,218 \\
2,732\end{array}$ \\
\hline 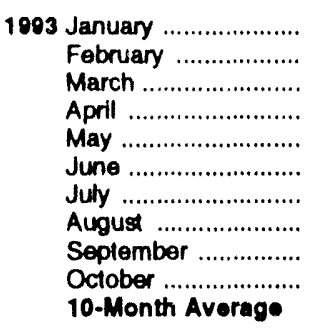 & $\begin{array}{l}17 \\
15 \\
16 \\
14 \\
15 \\
17 \\
15 \\
16 \\
18 \\
15 \\
16\end{array}$ & $\begin{array}{l}55 \\
63 \\
55 \\
63 \\
64 \\
65 \\
65 \\
66 \\
66 \\
66 \\
63\end{array}$ & $\begin{array}{l}72 \\
78 \\
71 \\
77 \\
79 \\
82 \\
80 \\
82 \\
84 \\
81 \\
70\end{array}$ & $\begin{array}{l}72 \\
69 \\
62 \\
69 \\
73 \\
83 \\
85 \\
87 \\
89 \\
93 \\
70\end{array}$ & $\begin{array}{l}752 \\
615 \\
549 \\
543 \\
564 \\
612 \\
656 \\
710 \\
759 \\
767 \\
653\end{array}$ & $\begin{array}{l}335 \\
311 \\
315 \\
320 \\
323 \\
350 \\
368 \\
397 \\
418 \\
441 \\
350\end{array}$ & $\begin{array}{l}454 \\
334 \\
268 \\
270 \\
294 \\
327 \\
360 \\
390 \\
421 \\
411 \\
353\end{array}$ & $\begin{array}{l}824 \\
684 \\
611 \\
612 \\
637 \\
695 \\
741 \\
797 \\
848 \\
860 \\
732\end{array}$ & $\begin{array}{r}10,784 \\
10,891 \\
10,501 \\
10,553 \\
9,469 \\
10,321 \\
11,308 \\
12,023 \\
11,575 \\
12,110 \\
109,535\end{array}$ & $\begin{array}{r}2,807 \\
2,899 \\
2,829 \\
2,703 \\
2,848 \\
3,087 \\
3,178 \\
3,423 \\
\text { P }_{3,341} \\
E_{3,400} \\
E_{3,052}\end{array}$ \\
\hline $\begin{array}{l}1002 \text { 10-Month Average } \\
1001 \text { 10-Month Average }\end{array}$ & $\begin{array}{l}12 \\
10\end{array}$ & $\begin{array}{l}65 \\
88\end{array}$ & $\underset{107}{7}$ & $\begin{array}{l}50 \\
83\end{array}$ & $\begin{array}{l}633 \\
780\end{array}$ & $\begin{array}{l}388 \\
485\end{array}$ & $\begin{array}{l}300 \\
357\end{array}$ & $\begin{array}{l}683 \\
872\end{array}$ & $\begin{array}{r}96,398 \\
120,404\end{array}$ & $\begin{array}{l}2,850 \\
3,368\end{array}$ \\
\hline
\end{tabular}

Monthly data are averages of 4- or 5-week reporting periods, not calendar months. Annual data are averages of 52. or 53-weok reporting periods, not calendar years.

Sum of oll, gas, and miscellaneous other rigs, which is not shown.

c Values shown are totals.

d See Glossary.

$R=$ Revised data. $N A=N o t$ available. $E=E s t i m a t e$.

Note: Geographic coverage is the 50 States and the District of Columbia.
Sources: - Crewe Engaged in Solsmlc Exploration: Sociely of Exploration Geophysicists, Tulsa, Oklahorna, Monthly Seismic Crew Count. - Rotary Rlge in Operation: Baker Hughes, Inc. Houston, Texas, Rotary Rigs Running--by State. - Total Footage Drilled: Energy Inlormation Administration computations, which are based on well reports submitted to the American Petroleum Institute by the Pelroleum Intormation Corporation Denver, Colorado. - Active Well Sorvicing Units: American Association of Oiwell Servicing Contractors, Dallas, Texas, Well Servicing. 


\begin{tabular}{|c|c|c|c|c|c|c|c|c|c|c|c|c|}
\hline & \multicolumn{4}{|c|}{ Exploratory } & \multicolumn{4}{|c|}{ Development } & \multicolumn{4}{|c|}{ Total } \\
\hline & Oll & Gas & Dry & Total & Oll & Gas & Dry & Total & oll & Gas & Dry & Total \\
\hline 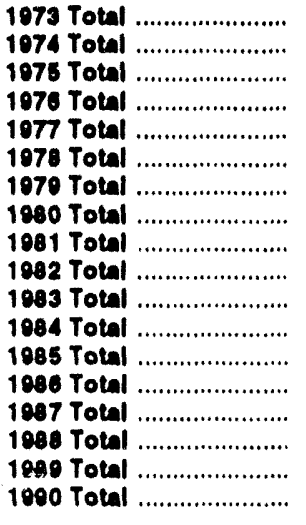 & $\begin{array}{r}654 \\
870 \\
091 \\
1,100 \\
1,183 \\
1,191 \\
1,335 \\
1,781 \\
2,667 \\
2,470 \\
2,113 \\
2,335 \\
1,870 \\
988 \\
880 \\
702 \\
580 \\
617\end{array}$ & $\begin{array}{r}1,070 \\
1,206 \\
1,263 \\
1,362 \\
1,562 \\
1,792 \\
1,020 \\
2,094 \\
2,533 \\
2,168 \\
1,660 \\
1,599 \\
1,282 \\
733 \\
673 \\
663 \\
1,652 \\
578\end{array}$ & $\begin{array}{r}6,038 \\
6,804 \\
7,207 \\
6,854 \\
7,402 \\
8,054 \\
7,478 \\
0,035 \\
12,297 \\
11,346 \\
10,271 \\
11,482 \\
0,445 \\
8,511 \\
5,170 \\
4,766 \\
4,001 \\
3,782\end{array}$ & $\begin{array}{r}7,771 \\
8,069 \\
9,481 \\
9,316 \\
10,147 \\
11,037 \\
10,733 \\
12,910 \\
17,497 \\
18,084 \\
14,044 \\
15,416 \\
12,006 \\
7,232 \\
6,711 \\
8,221 \\
A, 5,233 \\
4,977\end{array}$ & $\begin{array}{r}9,597 \\
12,704 \\
15,988 \\
16,597 \\
17,517 \\
17,874 \\
10,368 \\
30,497 \\
40,176 \\
36,672 \\
35,086 \\
40,250 \\
33,142 \\
17,713 \\
15,327 \\
12,530 \\
9,750 \\
11,533\end{array}$ & $\begin{array}{r}8,896 \\
5,065 \\
8,007 \\
8,076 \\
10,557 \\
12,613 \\
13,250 \\
15,120 \\
17,374 \\
16,776 \\
12,896 \\
15,413 \\
12,070 \\
7,402 \\
7,084 \\
7,575 \\
R, 8,573 \\
0,862\end{array}$ & $\begin{array}{r}\mathbf{4 , 4 2 8} \\
\mathbf{5 , 3 1 1} \\
\mathbf{6 , 5 2 0} \\
\mathbf{6 , 9 5 1} \\
\mathbf{7 , 6 3 4} \\
\mathbf{8 , 5 3 7} \\
\mathbf{9 , 5 6 0} \\
11,302 \\
14,987 \\
15,036 \\
14,065 \\
14,315 \\
11,763 \\
7,255 \\
\mathbf{6 , 3 0 2} \\
\mathbf{5 , 4 7 6} \\
\mathbf{4 , 4 9 0} \\
A, 793\end{array}$ & $\begin{array}{r}19,021 \\
24,070 \\
29,424 \\
31,624 \\
36,708 \\
39,024 \\
41,178 \\
50,028 \\
72,537 \\
69,484 \\
62,047 \\
69,078 \\
57,875 \\
32,370 \\
28,713 \\
25,581 \\
A_{22,822} \\
A_{26,188}\end{array}$ & $\begin{array}{l}10,251 \\
13,684 \\
16,070 \\
17,697 \\
18,700 \\
10,065 \\
20,703 \\
32,278 \\
42,843 \\
30,142 \\
37,199 \\
42,585 \\
35,021 \\
18,701 \\
16,186 \\
13,322 \\
10,330 \\
12,150\end{array}$ & $\begin{array}{r}6,975 \\
7,170 \\
8,170 \\
9,438 \\
12,119 \\
14,405 \\
15,170 \\
17,223 \\
19,907 \\
19,044 \\
14,556 \\
17,012 \\
14,252 \\
8,135 \\
7,757 \\
8,238 \\
0,225 \\
10,440\end{array}$ & $\begin{array}{r}10,486 \\
12,205 \\
13,736 \\
13,805 \\
15,036 \\
16,501 \\
16,038 \\
20,337 \\
27,284 \\
26,382 \\
24,336 \\
25,707 \\
21,206 \\
12,766 \\
11,481 \\
10,242 \\
8,401 \\
{ }^{8}, 576\end{array}$ & $\begin{array}{r}27,002 \\
33,030 \\
38,085 \\
40,040 \\
46,855 \\
50,081 \\
51,011 \\
60,838 \\
00,034 \\
84,468 \\
78,001 \\
85,394 \\
70,481 \\
39,002 \\
36,424 \\
31,802 \\
28,058 \\
31,168\end{array}$ \\
\hline 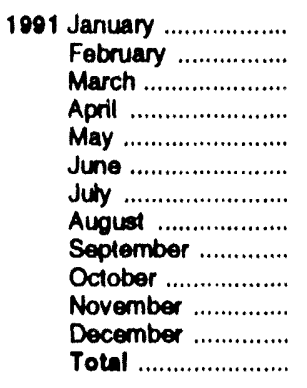 & $\begin{array}{l}56 \\
47 \\
53 \\
55 \\
39 \\
51 \\
56 \\
48 \\
39 \\
32 \\
25 \\
43 \\
544\end{array}$ & $\begin{array}{r}46 \\
47 \\
32 \\
35 \\
34 \\
42 \\
A_{36} \\
\text { ค } 37 \\
\text { ค }_{30} \\
\text { ค } 45 \\
35 \\
42 \\
\text { P }_{461}\end{array}$ & $\begin{array}{l}247 \\
271 \\
267 \\
279 \\
263 \\
251 \\
301 \\
309 \\
255 \\
286 \\
302 \\
271 \\
3,302\end{array}$ & $\begin{array}{r}349 \\
365 \\
352 \\
369 \\
336 \\
344 \\
\text { R } 393 \\
\text { A } 394 \\
\text { A } 324 \\
\text { R } 363 \\
362 \\
356 \\
4,307\end{array}$ & $\begin{array}{r}1,166 \\
1,173 \\
1,098 \\
1,063 \\
996 \\
878 \\
903 \\
921 \\
816 \\
911 \\
726 \\
718 \\
11,360\end{array}$ & $\begin{array}{r}834 \\
681 \\
753 \\
705 \\
692 \\
727 \\
\text { ค } 775 \\
\text { P }_{755} \\
\text { ค } 716 \\
\text { ค } 767 \\
571 \\
693 \\
8,660\end{array}$ & $\begin{array}{r}352 \\
382 \\
379 \\
392 \\
387 \\
365 \\
401 \\
357 \\
374 \\
\text { R } 413 \\
347 \\
375 \\
4,524\end{array}$ & $\begin{array}{r}2,352 \\
2,236 \\
2,230 \\
2,160 \\
2,075 \\
1,970 \\
A_{2,079} \\
A_{2,033} \\
A_{1,906} \\
A_{2,091} \\
1,644 \\
1,786 \\
A_{24,562}\end{array}$ & $\begin{array}{r}1,222 \\
1,220 \\
1,151 \\
1,118 \\
1,035 \\
929 \\
959 \\
969 \\
855 \\
943 \\
751 \\
761 \\
11,913\end{array}$ & $\begin{array}{r}880 \\
728 \\
785 \\
740 \\
726 \\
769 \\
811 \\
792 \\
746 \\
P_{812} \\
606 \\
735 \\
P, 130\end{array}$ & $\begin{array}{r}599 \\
653 \\
646 \\
671 \\
650 \\
616 \\
702 \\
666 \\
629 \\
\text { P } 699 \\
649 \\
646 \\
7,826\end{array}$ & $\begin{array}{r}2,701 \\
2,601 \\
2,582 \\
2,529 \\
2,411 \\
2,314 \\
2,472 \\
2,427 \\
2,230 \\
R 2,454 \\
2,006 \\
2,142 \\
R 28,860\end{array}$ \\
\hline 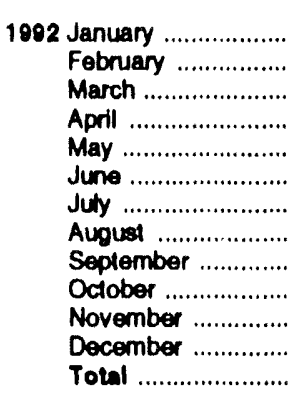 & $\begin{array}{r}46 \\
34 \\
38 \\
32 \\
35 \\
41 \\
43 \\
42 \\
36 \\
28 \\
38 \\
43 \\
458\end{array}$ & $\begin{array}{r}{ }^{A} 32 \\
{ }^{A} 30 \\
30 \\
22 \\
22 \\
33 \\
31 \\
A_{32} \\
19 \\
31 \\
30 \\
33 \\
A_{345}\end{array}$ & $\begin{array}{r}218 \\
167 \\
205 \\
233 \\
225 \\
209 \\
256 \\
241 \\
222 \\
\text { R } 205 \\
165 \\
225 \\
R_{2,571}\end{array}$ & $\begin{array}{r}A_{296} \\
A_{231} \\
273 \\
287 \\
282 \\
283 \\
330 \\
R_{315} \\
277 \\
A_{264} \\
233 \\
301 \\
A_{3,372}\end{array}$ & $\begin{array}{r}740 \\
590 \\
721 \\
656 \\
636 \\
626 \\
664 \\
R_{617} \\
785 \\
R_{750} \\
686 \\
751 \\
R_{8,222}\end{array}$ & $\begin{array}{r}P_{586} \\
R_{553} \\
465 \\
415 \\
470 \\
461 \\
542 \\
R_{600} \\
663 \\
R_{952} \\
795 \\
915 \\
R_{7,417}\end{array}$ & $\begin{array}{r}317 \\
273 \\
320 \\
297 \\
374 \\
330 \\
312 \\
357 \\
339 \\
\text { ค } 358 \\
331 \\
391 \\
R_{3,000}\end{array}$ & $\begin{array}{r}A_{1,643} \\
A_{1,416} \\
1,506 \\
1,368 \\
1,480 \\
1,417 \\
1,518 \\
A_{1,574} \\
1,787 \\
A_{2,060} \\
1,812 \\
2,057 \\
A_{19,638}\end{array}$ & $\begin{array}{r}786 \\
624 \\
759 \\
688 \\
671 \\
667 \\
707 \\
659 \\
821 \\
\text { R } 778 \\
724 \\
794 \\
\mathrm{~A}_{8,678}\end{array}$ & $\begin{array}{r}618 \\
583 \\
495 \\
437 \\
492 \\
494 \\
573 \\
632 \\
682 \\
\text { ค } 983 \\
825 \\
948 \\
\text { R }_{7,762}\end{array}$ & $\begin{array}{r}535 \\
440 \\
525 \\
530 \\
599 \\
539 \\
568 \\
598 \\
561 \\
\text { P } 563 \\
496 \\
616 \\
\mathrm{~A}_{6,570}\end{array}$ & $\begin{array}{r}1,939 \\
1,647 \\
1,779 \\
1,655 \\
1,762 \\
1,700 \\
1,848 \\
1,880 \\
2,064 \\
\text { म } 2,324 \\
2,045 \\
2,358 \\
\text { R } 23,010\end{array}$ \\
\hline 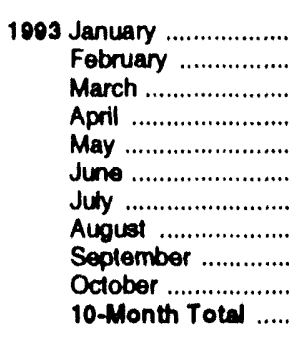 & $\begin{array}{r}41 \\
32 \\
23 \\
41 \\
36 \\
36 \\
\text { R } \\
48 \\
48 \\
39 \\
377\end{array}$ & $\begin{array}{r}35 \\
42 \\
28 \\
28 \\
33 \\
28 \\
P_{26} \\
36 \\
29 \\
36 \\
321\end{array}$ & $\begin{array}{r}162 \\
171 \\
175 \\
A_{205} \\
176 \\
193 \\
254 \\
254 \\
253 \\
278 \\
2,121\end{array}$ & $\begin{array}{r}238 \\
245 \\
226 \\
R_{274} \\
245 \\
256 \\
R^{2514} \\
338 \\
330 \\
353 \\
2,810\end{array}$ & $\begin{array}{r}614 \\
551 \\
593 \\
R 562 \\
537 \\
617 \\
\text { ค } 706 \\
780 \\
777 \\
811 \\
6,546\end{array}$ & $\begin{array}{r}902 \\
917 \\
875 \\
P_{614} \\
785 \\
574 \\
R_{549} \\
937 \\
838 \\
840 \\
7,831\end{array}$ & $\begin{array}{r}290 \\
346 \\
236 \\
{ }^{2} 355 \\
456 \\
318 \\
A_{527} \\
477 \\
435 \\
511 \\
3,951\end{array}$ & $\begin{array}{r}1,806 \\
1,814 \\
1,704 \\
R 1,539 \\
1,778 \\
1,509 \\
A 1,782 \\
2,194 \\
2,050 \\
2,162 \\
18,330\end{array}$ & $\begin{array}{r}655 \\
583 \\
616 \\
\text { ค } 603 \\
573 \\
652 \\
740 \\
828 \\
825 \\
850 \\
6,925\end{array}$ & $\begin{array}{r}937 \\
959 \\
903 \\
{ }^{\circ} 642 \\
818 \\
602 \\
A^{575} \\
973 \\
867 \\
876 \\
8,152\end{array}$ & $\begin{array}{r}452 \\
517 \\
411 \\
A^{4} 560 \\
632 \\
511 \\
P^{2} 781 \\
731 \\
688 \\
789 \\
6,072\end{array}$ & $\begin{array}{r}2,044 \\
2,059 \\
1,830 \\
A 1,805 \\
2,023 \\
1,765 \\
A_{2}, 096 \\
2,532 \\
2,380 \\
2,515 \\
21,140\end{array}$ \\
\hline $\begin{array}{l}100210 \text {-Month Total ..... } \\
1001 \text { 10-Month Total ..... }\end{array}$ & $\begin{array}{l}375 \\
476\end{array}$ & $\begin{array}{l}282 \\
384\end{array}$ & $\begin{array}{l}2,181 \\
2,720\end{array}$ & $\begin{array}{l}2,838 \\
3,580\end{array}$ & $\begin{array}{l}6,785 \\
9,925\end{array}$ & $\begin{array}{l}5,707 \\
7,406\end{array}$ & $\begin{array}{l}3,27 \\
3,802\end{array}$ & $\begin{array}{l}15,760 \\
21,132\end{array}$ & $\begin{array}{r}7,160 \\
10,401\end{array}$ & $\begin{array}{l}5,980 \\
7,780\end{array}$ & $\begin{array}{l}5,458 \\
6,531\end{array}$ & $\begin{array}{l}18,607 \\
24,721\end{array}$ \\
\hline
\end{tabular}

$\mathrm{R}=$ Revised data.

Notes: - Service wells, stratigraphic tests, and core lests are excluded. - Geographic coverage is the 50 States and the District of Columbia. Due to the method of estimation, data shown on this page are frequently revised.
See end of section.

Sources: Energy Intormation Administration computations, which are based on well reports subrnitted to the Amorican Petroleum Institute by the Petrobum Intormation Corporation, Denver, Colorado. 


\section{Oil and Gas Resource Development Notes}

Three well types are considered in the Monthly Energy Review (MER) drilling statistics: "completed for oil," "completed for gas," and "dry hole." Wells that productively encounter both crude oil and natural gas are categorized as "completed for oil." Both development wells and exploratory wells (new field wildcats, new pool tests, and extension tests) are included in the statistics. All other classes of wells drilled in connection with the search for producible hydrocarbons are excluded.

Prior to the March $1985 M E R$, drilling statistics consisted of completion data for the above types and classes of wells as reported to the American Petroleum Institute (API) during a given month. Due to time lags between the date of well completion and the date of completion reporting to the API, as-reported well completions proved to be an inaccurate indicator of drilling activity.
During 1982, for example, as-reported well completions rose, while the number of actual completions fell. Consequently, the drilling statistics published since the March 1985 MER are Energy Information Administration-generated (EIA) estimates produced by statistically imputing well counts and footage based on the partial data available from the API.

Estimates for a given month are first published in the MER for that month. Revisions of the "oil," "gas," and "dry" components are made in the 6th, 12th, and 24th subsequent months, as newly reported data allow refinement of the estimates. Unscheduled revisions may also occur when the latest estimate differs by more than 15 percent during the first 5 months, more than 10 percent during the next 6 months, or more than 2 percent thereafter through 5 years. After 5 years, the reported API data are published in lieu of EIA-generated estimates. Additional information about the EIA estimation methodology may be found in "Estimating Well Completions," the feature article published in the March 1985 MER. 


\section{Section 6. Coal}

Coal production in September 1993 totaled 83 million short tons, 1 percent ${ }^{6}$ lower than coal production in September 1992. Coal production for the first 9 months of 1993 amounted to 713 million short tons, 35 million short tons lower than the comparable period of 1992 .

Electric utility coal consumption in August 1993 totaled 78 million short tons, 10 percent higher than the consumption level in August 1992.
Electric utility coal stocks were 114 million short tons at the end of August 1993, down from 153 million short tons at the end of August 1992.

Coal exports in August 1993 totaled 6 million short tons, 23 percent lower than exports in August 1992. Coal imports in August 1993 totaled 747 thousand short tons, 550 thousand short tons higher than imports in August 1992.

'Percertage changes are based on unrounded dala. 
Figure 6.1 Coal

(Million Short Tons)

Overview, 1973-1992

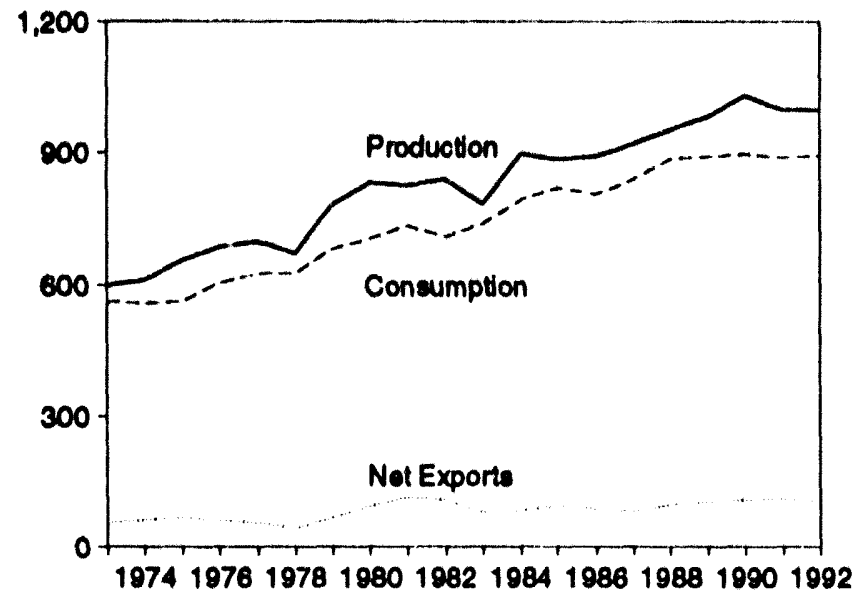

Consumption by Sector, $1973-1992$

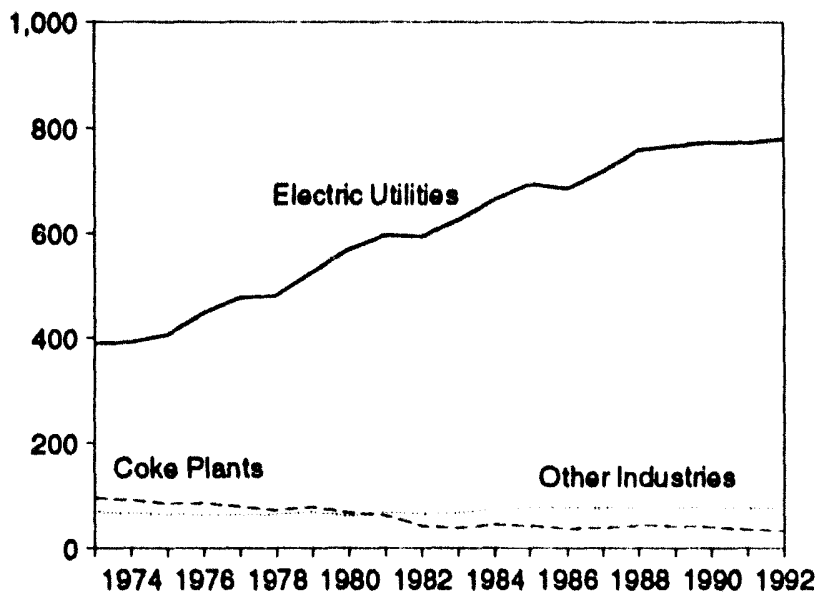

Stocks, End of Year, 1973-1992

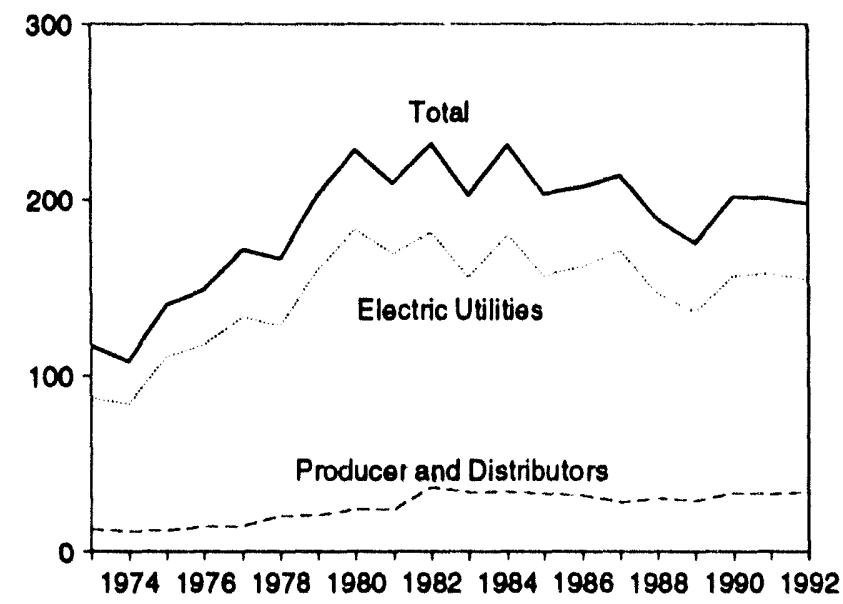

Overview, Monthly

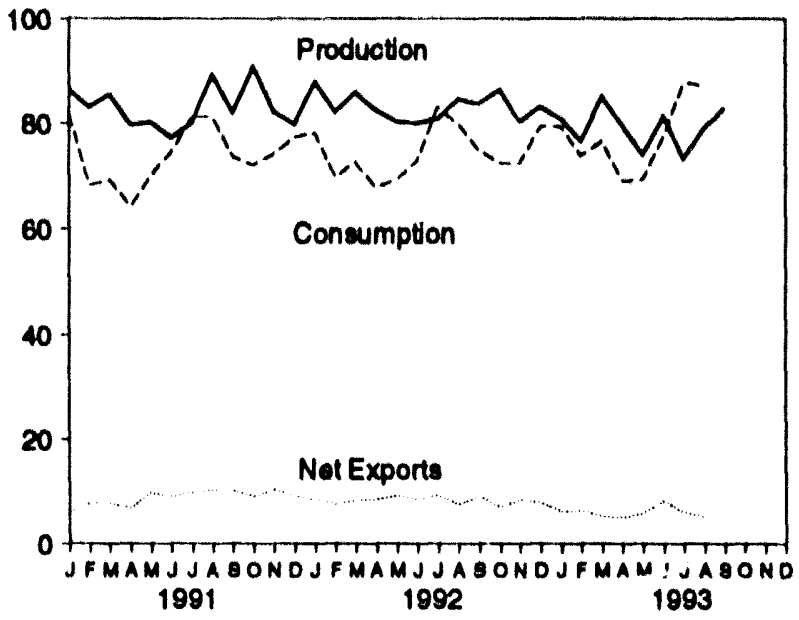

Consumption by Electric Utilities, Monthly

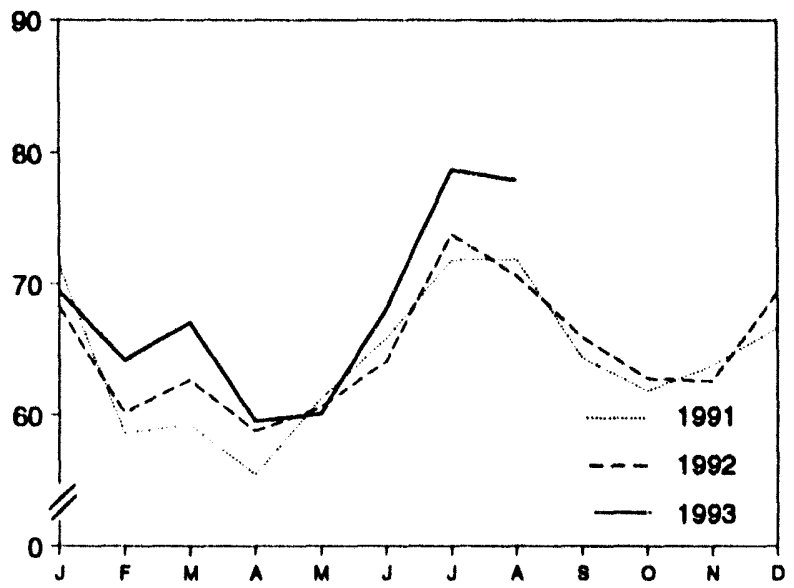

Stocks at Electric Utilities, End of Month

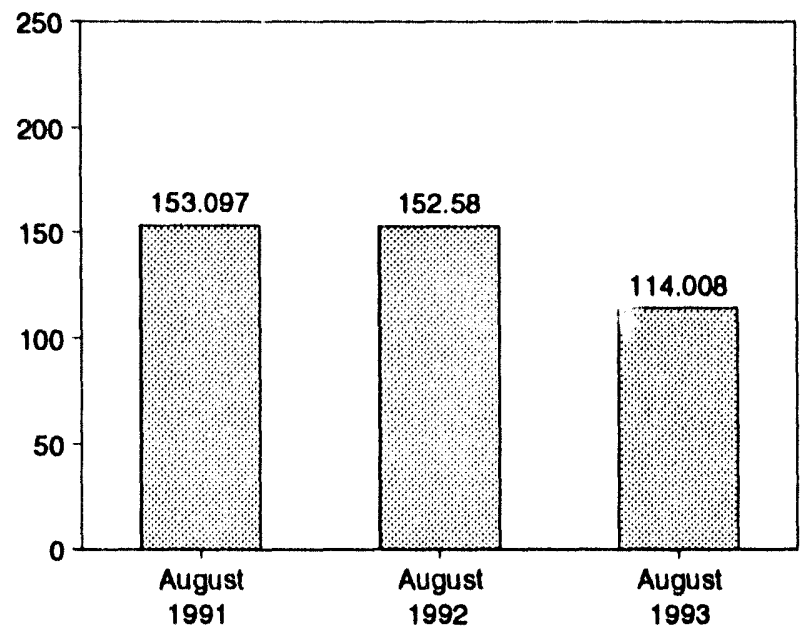

Note: Because vertical scales difer, graphs should not be compared. Sources: Tables 6.1, 6.2, and 6.3. 
Table 6.1 Coal Overview

(Thousand Short Tons)

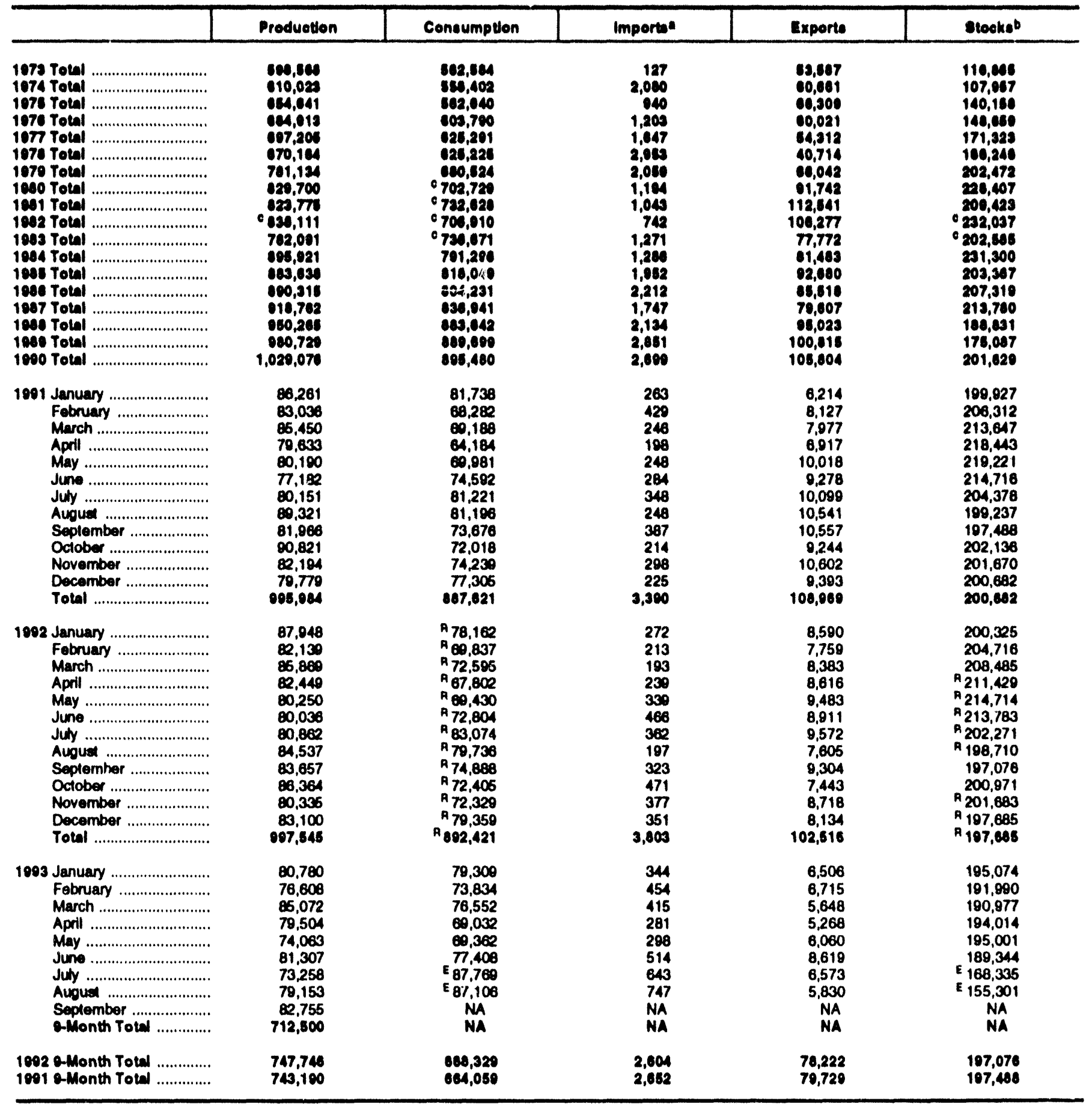

- Includas Puerto Rico.

- Stocks held by electric utilties, coke plants, general indusiry, and coal producers and distributors at end of period. Stocks held at retail dealers for consumption by the residential and commercial sector are excluded.

c See Note 6 al end of section.

R=Revised data. NA=Not avaliable. Ex=Estimale.

Notes: - Geographic coverage is the 50 Stales and the Districa of Columbla. - Data through 1991 are thal. Subsequent data are preliminary. - Totats may not equal sum of components due to independent rounding.
- For methodology used to calculate production, consumption, and stocks, $s 00$ Notes 1, 2, and 3 at end of section.

Sources: - Production: 1973-Soptember 1077-U.S. Department of the Interior, Bureau of Mines, Minerals Yearbook and Minerals Industry Surveys. Oetober 1077 forward-Energy Inlormation Administration. Woeky Coal Production. - Consumption: Table 6.2. - Imports and Exports: U.S Department of Commerce, Bureau of the Census, Morthly Reports IM-145 (Imports) and EM-522 (Exports). - stocks: Table 6.3. 


\begin{tabular}{|c|c|c|c|c|c|}
\hline & \multirow[b]{2}{*}{ 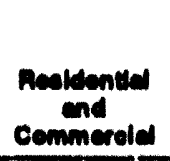 } & \multicolumn{2}{|c|}{ Induatriel } & \multirow[b]{2}{*}{ 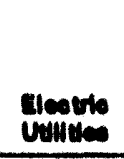 } & \multirow[b]{2}{*}{ Total } \\
\hline & & $\begin{array}{l}\text { Colve } \\
\text { Plente }\end{array}$ & 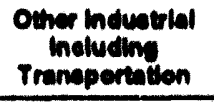 & & \\
\hline 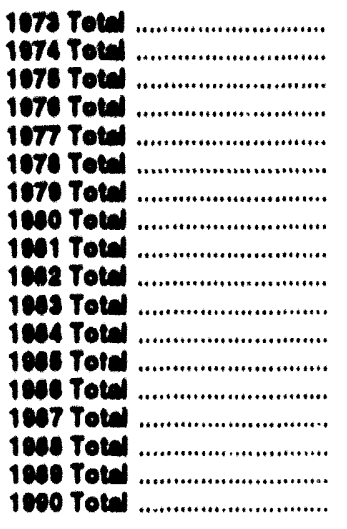 & 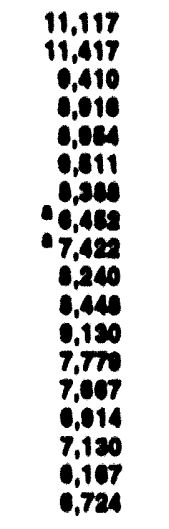 & 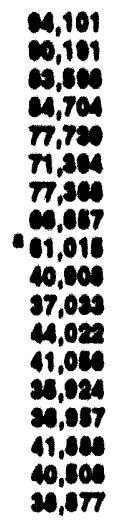 & 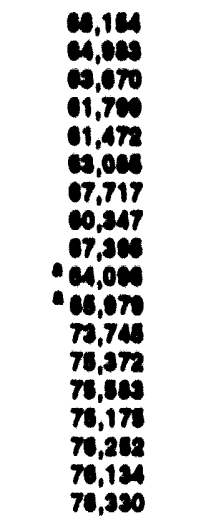 & 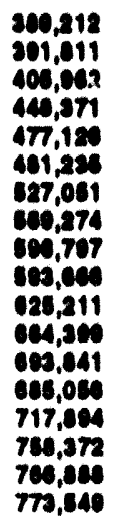 & 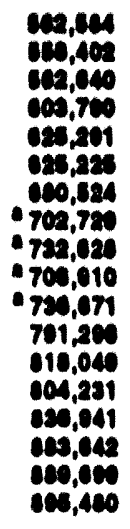 \\
\hline 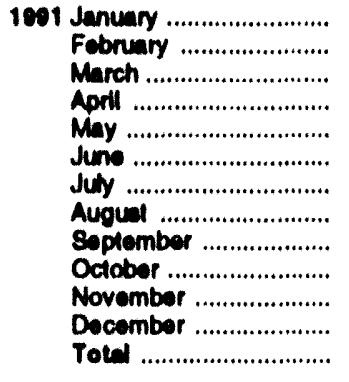 & $\begin{array}{l}862 \\
606 \\
541 \\
100 \\
330 \\
322 \\
427 \\
386 \\
319 \\
363 \\
677 \\
869 \\
6,084\end{array}$ & $\begin{array}{r}2,028 \\
2,470 \\
2,803 \\
2,675 \\
2,710 \\
2,600 \\
2,020 \\
2,916 \\
2,930 \\
2,900 \\
2,890 \\
2,913 \\
29,064\end{array}$ & $\begin{array}{l}6,541 \\
6,584 \\
6,402 \\
6,683 \\
5,613 \\
5,763 \\
6,014 \\
6,011 \\
6,028 \\
6,880 \\
6,852 \\
6,865 \\
78,406\end{array}$ & $\begin{array}{l}71,406 \\
68,614 \\
59,272 \\
55,443 \\
61,228 \\
66,817 \\
71,852 \\
71,864 \\
64,397 \\
61,883 \\
63,814 \\
66,659 \\
772,260\end{array}$ & $\begin{array}{l}81,730 \\
69,289 \\
60,180 \\
64,184 \\
60,081 \\
74,692 \\
61,221 \\
81,186 \\
73,678 \\
72,018 \\
74,239 \\
77,306 \\
007,021\end{array}$ \\
\hline 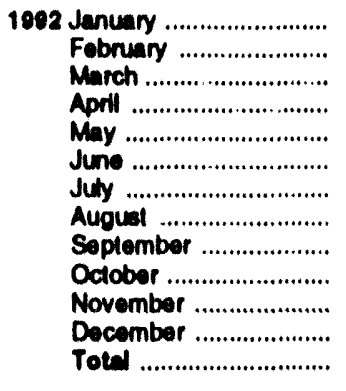 & $\begin{array}{r}736 \\
582 \\
526 \\
532 \\
321 \\
296 \\
474 \\
393 \\
368 \\
367 \\
642 \\
916 \\
0,163\end{array}$ & $\begin{array}{r}2,783 \\
2,660 \\
2,801 \\
2,723 \\
2,757 \\
2,617 \\
2,800 \\
2,773 \\
2,625 \\
2,586 \\
2,562 \\
2,581 \\
32,366\end{array}$ & $\begin{array}{r}A_{6,379} \\
A_{6,376} \\
A_{6,416} \\
A_{6,464} \\
A_{5,754} \\
A_{5,762} \\
A_{5,760} \\
A_{5,983} \\
A_{5,933} \\
A_{5,927} \\
A_{6,646} \\
A_{6,613} \\
A_{6,513} \\
A_{6,497} \\
A_{74,042}\end{array}$ & $\begin{array}{c}68,264 \\
60,183 \\
62,706 \\
58,794 \\
60,591 \\
64,122 \\
73,815 \\
70,637 \\
66,967 \\
62,806 \\
62,612 \\
69,366 \\
770,060\end{array}$ & $\begin{array}{r}A_{78,160} \\
A_{60,837} \\
A_{72,506} \\
A_{67,802} \\
A_{60,430} \\
A_{72,804} \\
A_{83,074} \\
A_{79,736} \\
A_{74,886} \\
A_{72,406} \\
A_{72,320} \\
A_{79,360} \\
A_{002,421}\end{array}$ \\
\hline 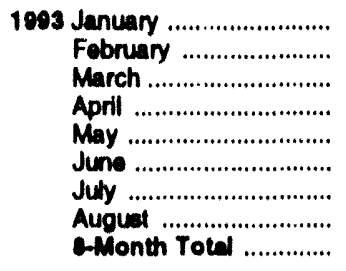 & $\begin{array}{r}747 \\
725 \\
580 \\
721 \\
380 \\
482 \\
449 \\
4420 \\
E \\
4,514\end{array}$ & $\begin{aligned} 2,674 \\
2,468 \\
2,640 \\
2,578 \\
2,710 \\
2,588 \\
E_{2,734} \\
E_{2,790} \\
E_{21,201}\end{aligned}$ & $\begin{array}{r}\mathbf{6 , 3 9 7} \\
\mathbf{6 , 4 4 0} \\
\mathbf{6 , 2 5 9} \\
\mathbf{6 , 1 6 8} \\
\mathbf{6 , 1 6 2} \\
\mathbf{6 , 2 1 6} \\
\mathbf{E}^{\mathbf{5}, 878} \\
\mathbf{E}_{\mathbf{5}} \mathbf{5 , 9 5 6} \\
\mathbf{E}_{\mathbf{4 0 , 4 7 8}}\end{array}$ & $\begin{array}{l}69,490 \\
64,201 \\
67,073 \\
59,563 \\
60,102 \\
68,113 \\
78,706 \\
77,932 \\
848,182\end{array}$ & $\begin{array}{r}79,300 \\
73,834 \\
76,552 \\
69,032 \\
69,362 \\
77,400 \\
E_{87,760} \\
687,100 \\
E_{820,372}\end{array}$ \\
\hline $\begin{array}{l}1002 \text { \&-Month Totel .............. } \\
1001 \text { \&-Month Total ................ }\end{array}$ & $\begin{array}{l}3,880 \\
3,870\end{array}$ & $\begin{array}{l}22,012 \\
22,210\end{array}$ & $\begin{array}{l}48,460 \\
49,702\end{array}$ & $\begin{array}{l}510,111 \\
515,515\end{array}$ & $\begin{array}{l}803,441 \\
600,3103\end{array}$ \\
\hline
\end{tabular}

- See Nute 6 al end of section.

$R=$ Rovised data. Ex=Estimate.

Notes: - For sector-specific reporting and estimating intormation, see Note 2 at end of section. - Geographic coverage is the 50 States and the District of Columbla. - Data through 1891 are final. Subsequent data are prellminary. - Totals may not equal sum of components due to independent rounding.

Sources: - Reoldentlel and Commercle: 1973-1076-U.S. Department of the Interior (DOI). Bureau of Mines (BOM). Minerals Yearbook. Januery-8optember 1977-DOI, BOM, Form 6-1400, "Monthly Coal Peport, Relall Dealers-Upper I.ake Docks." October 1077-1070-Energy Intormation Administration (EIA), Form ELA-2, "Monthly Coal Report, Relail Dealers-Upper Lake Docks." 1080 forward-EIA, Form EIA-6, "Coal Distribution Report." "Coke Plantw: 1973-September 1077-DOI,
BOM, Minerals Yearbook and Minerals Industry Surveys. Octobor 1077.1000-ELA, Form EIA.5/5A, "Coke and Coal Chemicals. Monthly/Annual.' 1081-1084-EIA,' Form EIA-5/5A, "Coke Plant Report-Quarterty/Annual Supplement." 1085 forward-EIA. Form ElA-5, "Coke Plant Repont: quarterly. - Other Industrlal: 1073-80ptember 1977-DOI, BOM, Minerals Yearbook and Minerale Industry Survoys. October 1977.1970-EIA, Form EIA.3, "Monthly Coal Consumption Heport-Manufacturing Plants." 1080 forward-EIA, Form ELA-3, 'Quarterty Coal Consumption Report-Manulacturing Plants," and Form EIA-6. "Coal Distribution Report." Electric Utilities: 1073-Septomber 1977-DOI, BOM, Minerals Yearbook and Minerals Indusiny Sunveys. Oetobor 1977 forward-EIA, Form EIA-759 (formerty Form FPC-4), Monthly Power Plant Report." 
Table 6.3 Coal Stocks, End of Perlod

(Thousand Short Tons)

\begin{tabular}{|c|c|c|c|c|c|c|}
\hline & \multicolumn{4}{|c|}{ Cenoumer } & \multirow{2}{*}{$\begin{array}{l}\text { Producere } \\
\text { and } \\
\text { Distrlbuters }\end{array}$} & \multirow[b]{2}{*}{ Torde } \\
\hline & $\begin{array}{l}\text { Coth } \\
\text { Pente }\end{array}$ & $\begin{array}{c}\text { Other } \\
\text { Industelel }\end{array}$ & $\begin{array}{l}\text { Eleotils } \\
\text { Utilltes }\end{array}$ & Totof & & \\
\hline 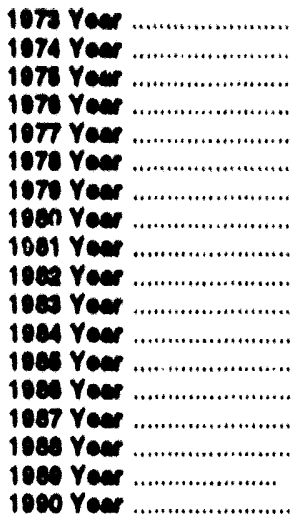 & 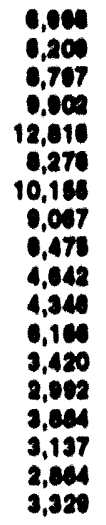 & 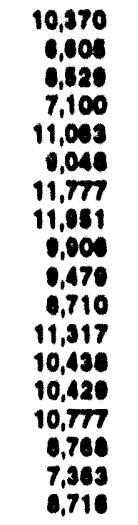 & 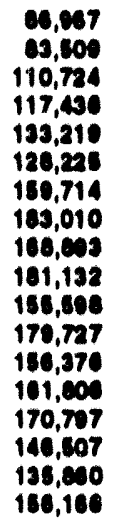 & 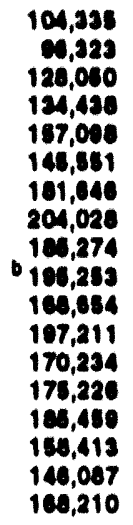 & $\begin{array}{l}12,590 \\
11,034 \\
12,100 \\
14,221 \\
14,228 \\
20,085 \\
20,820 \\
24,370 \\
24,140 \\
30,784 \\
33,931 \\
34,090 \\
33,133 \\
32,093 \\
20,321 \\
30,418 \\
20,000 \\
33,418\end{array}$ & 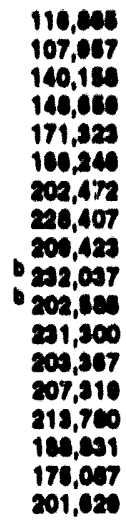 \\
\hline 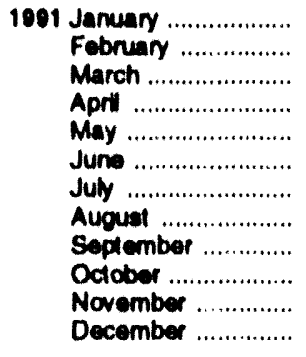 & $\begin{array}{l}3,262 \\
3,196 \\
3,130 \\
3,181 \\
3,232 \\
3,283 \\
3,097 \\
2,891 \\
2,696 \\
2,721 \\
2,747 \\
2,773\end{array}$ & $\begin{array}{l}8,234 \\
7,753 \\
7,271 \\
7,154 \\
7,038 \\
6,921 \\
7,033 \\
7,145 \\
7,258 \\
7,102 \\
7,127 \\
7,081\end{array}$ & $\begin{array}{l}152,007 \\
156,116 \\
: 9:, 084 \\
166,315 \\
167,528 \\
163,450 \\
155,680 \\
153,007 \\
153,007 \\
158,813 \\
158,605 \\
157,070\end{array}$ & $\begin{array}{l}163,594 \\
167,065 \\
171,485 \\
176,650 \\
177,707 \\
173,663 \\
165,800 \\
163,133 \\
163,660 \\
168,726 \\
168,479 \\
167,711\end{array}$ & $\begin{array}{l}36,333 \\
39,248 \\
42,162 \\
41,793 \\
41,423 \\
11,064 \\
38,578 \\
36,103 \\
33,628 \\
33,409 \\
33,190 \\
32,071\end{array}$ & $\begin{array}{l}100,927 \\
208,312 \\
213,647 \\
218,443 \\
219,221 \\
214,716 \\
204,378 \\
100,237 \\
107,480 \\
202,130 \\
201,670 \\
200,162\end{array}$ \\
\hline 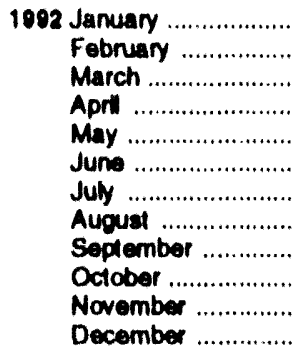 & $\begin{array}{l}2,807 \\
2,841 \\
2,875 \\
2,842 \\
2,800 \\
2,776 \\
2,589 \\
2,402 \\
2,215 \\
2,342 \\
2,470 \\
2,547\end{array}$ & $\begin{array}{r}6,616 \\
A_{6,171} \\
5,725 \\
A_{5,923} \\
A_{6,100} \\
A_{6,317} \\
A_{6,538} \\
A_{6,758} \\
6,979 \\
6,974 \\
6,969 \\
A_{6,965}\end{array}$ & $\begin{array}{l}155,637 \\
158,145 \\
160,032 \\
162,591 \\
165,512 \\
164,176 \\
154,403 \\
152,580 \\
152,685 \\
156,859 \\
157,849 \\
184,130\end{array}$ & $\begin{array}{r}166,060 \\
167,157 \\
168,632 \\
A_{171,366} \\
A_{174,421} \\
A_{173,270} \\
A_{163,530} \\
A_{161,740} \\
161,878 \\
168,175 \\
A_{167,288} \\
A_{103,092}\end{array}$ & $\begin{array}{l}35,265 \\
37,559 \\
39,853 \\
40,073 \\
40,293 \\
40,513 \\
38,741 \\
36,970 \\
35,198 \\
34,796 \\
34,395 \\
33,903\end{array}$ & $\begin{array}{r}200,326 \\
204,716 \\
208,485 \\
A_{211}, 420 \\
A_{214,714} \\
A_{213,783} \\
A_{202,271} \\
A_{198,710} \\
107,076 \\
200,071 \\
A_{201,680} \\
A_{107,085}\end{array}$ \\
\hline 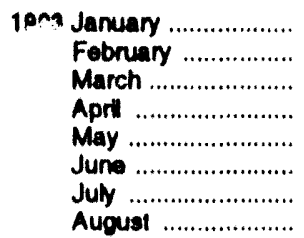 & $\begin{array}{l}2,668 \\
2,739 \\
2,800 \\
2,879 \\
2,949 \\
3,020 \\
2,656 \\
2,560\end{array}$ & $\begin{array}{l}6,600 \\
6,236 \\
6,872 \\
5,931 \\
6,090 \\
6,049 \\
7,044 \\
6,733\end{array}$ & $\begin{array}{l}150,371 \\
148,139 \\
143,978 \\
148,049 \\
150,070 \\
146,448 \\
126,635 \\
114,008\end{array}$ & $\begin{array}{l}159,639 \\
155,113 \\
152,659 \\
156,859 \\
159,010 \\
154,517 \\
E 136,336 \\
E 123,301\end{array}$ & $\begin{array}{l}35,435 \\
36,877 \\
38,319 \\
37,155 \\
35,991 \\
34,827 \\
\text { E2,000 } \\
\text { E2,000 }\end{array}$ & $\begin{array}{l}105,074 \\
101,000 \\
100,977 \\
104,014 \\
105,001 \\
180,344 \\
169,335 \\
165,301\end{array}$ \\
\hline
\end{tabular}

- Excludes stocks hold at retall deaters for consumption by the residential and commercial sector.

b See Note 6 at end of section.

$\mathrm{R}=$ Poviaed data. Exatimate.

Notes: - For sector-specific reporting and estimating intormation, see Note 3 at end of section. Geocraphic coverage ts the 50 Stales and the District of Columbia. - Dala through 1891 are final. Subsequent data are preliminary. - Totals may not equal sum of components dus to independent rounding.

Source: - Coke Plants: 1973-8optember 1077-U.S. Deparment of the Intertor (DOI), Bureau of Mines (BOM), Minerals Yeartook and Minerals Industry Surveys. October 1077.1080-Energy Inlormation Adminiatration
(EIA), Form EIA.5/5A, "Coke and Coal Chemicals-Monthy/Annual." 1081-1084-EIA, Form EIA-5/5A, "Coke Plant Report-Qunrterty/Annual Supplement." 1098 forward-EIA, Form EIA.5, "Coke Plan Repon. quartery. - Othe Industrlal: 1073-soptember 1077-DOI, BOM, Minerals Yearbook and Minerab Industry Sunveys. October 1077-1079-ElA, Form EIA-3, "Monthly Coal Consumplion Peport-Manutacturing Piants." 1080 forwerd-EIA, Form EIA-3, "Quarierty Coal Consumption Report. Manulacturing Plants," and Form ElA.6, "Coal Distribution Repon." - Electric Udilites: 1073-september 1077-DOI, BOM, Minerals Yearbook and Minerale Indusin Surveys. Ootober 1077 forward-EL, Fom EU.750 (lormerty Form FPC-4). "Monthly Power Plant Pepon." - Preducere end Dietrlbutors: EIA, Form ElA.6, "Coal Distribution Repont. 


\section{Coal Notes}

1. Production: Preliminary monthly estimates of national coal production are the sum of weekly estimates developed by the linergy Information Administration. (EIA) and published in the Weekly Coal Production report. When a weck extends into a new mon:h. production is allocated on a daily hasis and added to the appropriate month. Weckly estimates are based on Association of American Railroads data showing the number of railcars loaded with coal during the week by Class $I$ and certain other railroads. This number is converted into cons of coal by BIA by using the average number of tons of coal per railcar loaded reported in the most recent "Quarterly Freight Commodity Statistics" from the Interstate Commerce Commission. If an average coal tonnage per railcar loaded is not available for a specific railroad, the national average is used. To derive the estimate of total weekly produstion, the total rail tonnage for the week is divided by the ratio of quarterly production shipped by rail and total quarterly production. Data for the corresponding quarter of previous years are used to derive this ratio. This method insures that the seasonal variations are preserved in the production estimates.

When preliminary quarterly data become available, the monthly and weekly estimates are adjusted to conform to the quarterly figure. The adjustment procedure uses State-level production data and is explained in EIA's Quarterly Coal Report. Initial estimates of annual production published in January of the following year are based on preliminary production data covering the first 9 months (three quarters) and weekly/monthly estimates for the fourth quarter. The fourth quarter estimates may or may not be revised when preliminary data hecome available in March of the following year. depending on the magnitude of the difference hetween the estimates and the preliminary data. In any event, all quarterly, monthly, and weekly production figures are adjusted to conform to the final annual production data published in the Monthly Energy Review in the fall of the following year.

2. Consumption: Coal consumption data are reported by major end-use sector. Estimated data for the most recent months (designated by an "l:") are derived from forecasted values shown in the EIA Short-Term Energy Outlook (DOL:LIA-0202) table titled "Supply and Disposition of Coal: Mid World Oil Price Case." The monthly estimates are one-third of the quarterly values shown in the then current issue of the publication, regularly released in February, May, Augusi, and November. The estimates are revised quarterly as collected data become available from the data sources. Sector-specific information follows.

- Residential and Commercial-Prior 101980. monthly consumption estimales for the residential and commercial sector were derived by using reported data to modify baseline figures developed by the Bureau of Mines. From 1980-1987, month- ly estimates were derived hy proportioning reported quarterly data by using the ratios of monthly-10-quarterly consumption data in 1979, the last year in which monthly data were reported on Form EIA-2. During 1981 and 1982, the estimales were also modified lo reflect air temperature degree-days. Quarterly consumption data were directly from reported data and were defined as distribution to the residential and commercial sector as reported hy coal producers and distributors on form lilA-6. Beginning in January 1988 , monthly residential and commercial consumption estimates are derived from reported quarterly data by using monthly national average population weighted heating/cooling degree-days obtained from the National Oceanic and Aumospheric Administration. The monthly ratios are the monthly national sum of heating and cooling degree-days as a proportion of the quarterly national sum. Quarterly consumption data are direclly from reported data.

- Coke Plants--Prior to 1980, monthly coke plant consumplion data were laken directly from reported data. From 1980.1987, coke plant consumption estimates were derived hy proportioning reported quarterly data hy using the ratios of monthly-10-quarterly consumption data in 1979, the last year in which monthly data were reported. Beginning in January 1988, monthly coke plant consumption estimates are derived froun the reported quarterly data hy using monthly ratios of raw stecl production data from the American Iron and Steel Institute. The ratios are the monthly raw steel production from open hearth and hasic oxygen process furnaces as a proportion of the quarterly production from those kinds of furnaces.

- Other Industrial-Prior to 1978, monthly consumption data for the other industrial sector (i.e., all industrial users minus coke plants) were derived hy using reported data to modify baseline consumption figures from the most recent Bureau of the Census Annual Survey of Manufactures or Census of Manufactures. For 1978 and 1979. monthly estimates were derived from data reported on Forms EIA-3 and EIA-6. From 1980-1987, monthly figures were estimated by proportioning quarterly data by using the ratios of monthly-10quarterly consumption data in 1979, the last year in which monthly data were reported on form EIA-3. Quarterly consumption data were derived by adding heginning stocks al manufacturing plants to current receipts and subtracting ending stocks at manufacturing plants. In this calculation, current receipts uere the greater of either reported receipts from manufacturing plants (1\%orm EIA-3) or reported shipments to the other industrial sector (form EIA-6), thereby ensuring that agriculture, forestry, fishing, mining, and construction consumption data were included where appropriale. Starting in January 1988, monthly consumption for the other industrial sector is estimated from reported quarterly data by using 


\section{Coal Notes}

1. Production: Preliminary monthly estimates of national coal production are the sum of weekly estimates developed by the Energy Information Administration(EIA) and published in the Weekly Coal Production report. When a week extends into a new month, production is allocated on a daily basis and added to the appropriate month. Weekly estimates are based on Association of American Railroads data showing the number of railcars loaded with coal during the week by Class I and certain other railroads. This number is converted into tons of coal by EIA by using the average number of tons of coal per railcar loaded reported in the most recent "Quarterly Freight Commodity Statistics" from the Interstate Commerce Commission. If an average coal tonnage per railcar loaded is not available for a specific railroad, the national average is used. To derive the estimate of total weekly production, the total rail tonnage for the week is divided by the ratio of quarterly production shipped by rail and total quarterly production. Data for the corresponding quarter of previous years are used to derive this ratio. This method insures that the seasonal variations are preserved in the production estimates.

When preliminary quarterly data become available, the monthly and weekly estimates are adjusted to conform to the quarterly figure. The adjustment procedure uses State-level production data and is explained in EIA's Quarterly Coal Report. Initial estimates of annual production published in January of the following year are based on preliminary production data covering the first 9 months (three quarters) and weekly/monthly estimates for the fourth quarter. The fourth quarter estimates may or may not be revised when preliminary data become available in March of the following year, depending on the magnitude of the difference between the estimates and the preliminary data. In any event, all quarterly, monthly, and weekly production figures are adjusted to conform to the final annual production data published in the Monthly Energy Review in the fall of the following year.

2. Consumption: Coal consumption data are reported by major end-use sector. Estimated data for the most recent months (designated by an "E") are derived from forecasted values shown in the EIA Short-Term Energy Outlook (DOE/EIA-0202) table titled "Supply and Disposition of Coal: Mid World Oil Price Case." The monthly estimates are one-third of the quarterly values shown in the then current issue of the publication, regularly released in February, May, August, and November. The estimates are revised quarterly as collected data become available from the data sources. Sector-specific information follows.

- Residential and Commercial-Prior to 1980, monthly consumption estimates for the residential and commercial sector were derived by using reported data to modify baseline figures developed by the Bureau of Mines. From 1980-1987, month- ly estimates were derived by proportioning reported quarterly data by using the ratios of monthly-to-quarterly consumption data in 1979, the last year in which monthly data were reported on Form EIA-2. During 1981 and 1982, the estimates were also modified to reflect air temperature degree-days. Quarterly consumption data were directly from reported data and were defined as distribution to the residential and commercial sector as reported by coal producers and distributors on Form EIA-6. Beginning in January 1988 , monthly residential and commercial consumption estimates are derived from reported quarterly data by using monthly national average population weighted heating/cooling degree-days obtained from the National Oceanic and Atmospheric Administration. The monthly ratios are the monthly national sum of heating and cooling degree-days as a proportion of the quarterly national sum. Quarterly consumption data are directly from reported data.

- Coke Plants-Prior to 1980 , monthly coke plant consumption data were taken directly from reported data. From 1980-1987, coke plant consumption estimates were derived by proportioning reported quarterly data by using the ratios of monthly-to-quarterly consumption data in 1979, the last year in which monthly data were reported. Beginning in January 1988, monthly coke plant consumption estimates are derived from the reported quarterly data by using monthly ratios of raw steel production data from the American Iron and Steel Institute. The ratios are the monthly raw steel production from open hearth and basic oxygen process furnaces as a proportion of the quarterly production from those kinds of furnaces.

- Other Industrial-Prior to 1978, monthly consumption data for the other industrial sector (i.e., all industrial users minus coke plants) were derived by using reported data to modify baseline consumption figures from the most recent Bureau of the Census Annual Survey of Manufactures or Census of Manufactures. For 1978 and 1979, monthly estiniates were derived from data reported on Forms EIA-3 and EIA-6. From 1980-1987, monthly figures were estimated by proportioning quarterly data by using the ratios of monthly-toquarterly consumption data in 1979 , the last year in which monthly data were reported on Form EIA-3. Quarterly consumption data were derived by adding beginning stocks at manufacturing plants to current receipts and subtracting ending stocks at manufacturing plants. In this calculation, current receipts were the greater of either reported receipts from manufacturing plants (Form EIA-3) or reported shipments to the other industrial sector (Form EIA-6), thereby ensuring that agriculture, forestry, fishing, mining, and construction consumption data were included where appropriate. Starting in January 1988, monthly consumption for the other industrial sector is estimated from reported quarterly data by using 
ratios derived from industrial production indices published by the Board of Governors of the Federal Reserve System. Indices for six major industry groups are used as the basis for calculating the ratios: foods, Standard Industrial Classification (SIC) 20; paper and products, SIC 26; chemicals and products, SIC 28; petroleum products, SIC 29; clay, glass, and stone products, SIC 32; and primary metals, SIC 33. The monthly ratios are computed as the monthly sum of the weighted indices as a proportion of the quarterly sum of the weighted indices by using the 1977 proportion as the weights.

- Electric Utilities-Monthly consumption data for electric utility plants are directly from reported data.

3. Stocks: Coal stocks data are reported by major enduse sector. Estimated data for the most recent months (designated by an "E") are derived from forecasted values shown in the EIA Short-Term Energy Outlook (DOE/EIA-0202) table titled "Supply and Disposition of Coal: Mid World Oil Price Case." The monthly estimates are one-third of the quarterly values shown in the then current issue of the publication, regularly released in February, May, August, and November. The estimates are revised quarterly as collected data become available from the data sources. Sector-specific information follows.

- Coke Plants-Prior to 1980 , monthly stocks at coke plants were taken directly from reported data. From 1980 forward, coke plant stocks are estimated by using one-third of the current quarterly change to indicate the monthly change in stocks. Quarterly stocks are directly from data reported on Form EIA-5.
- Other Industrial-Prior to 1978 , stocks for the other industrial sector were derived by using reported data to modify baseline figures from a one-time Bureau of Mines survey of consumers. For 1978-1982, monthly estimates were derived by judgmentally proportioning reported quarterly data based on representative seasonal patterns of supply and demand. From 1983 forward, other industrial coal stocks are estimated as indicated above for coke plants. Quarterly stocks are taken directly from data reported on Form EIA-3 and therefore include only manufacturing industries; data for agriculture, forestry, fishing, miring, and construction stocks are not available.

- Electric Utilities-Monthly stocks data at electric utility plants are taken directly from reported data.

- Producers and Distributors-Quarterly stocks at producers and distributors are taken directly from reported data. Monthly data are estimated by using one-third of the current quarterly change to indicate the monthly change in stocks.

4. Imports and Exports: All coal import and export figures are taken directly from data reported monthly by the Bureau of the Census.

5. Additional Information: EIA's Quarterly Coal Report provides additional information about coal data and estimation procedures.

6. Data Discrepancies: Due to differences internal to EIA data processing systems, some small discrepancies exist between data in the Monthly Energy Review (MER) and the Quarterly Coal Report (QCR). The data that have discrepancies are footnoted in Section 6 tables and summarized here.

\begin{tabular}{llrrr} 
Table & \multicolumn{1}{c}{ Data Series } & Year & $\begin{array}{c}M E R \\
\text { Data }\end{array}$ & \multicolumn{1}{c}{$\begin{array}{c}\text { QCR } \\
\text { Data }\end{array}$} \\
\hline 6.1 & Consumption & 1980 & 702,729 & 702,730 \\
6.1 & Consumption & 1981 & 732,628 & 732,627 \\
6.1 & Production & 1982 & 838,111 & 838,112 \\
6.1 & Consumption & 1982 & 706,910 & 706,911 \\
6.1 & Stocks & 1982 & 232,037 & 232,038 \\
6.1 & Consumption & 1983 & 736,671 & 736,672 \\
6.1 & Stocks & 1983 & 202,585 & 202,584 \\
6.2 & Residential and Commercial & 1980 & 6,452 & 6,451 \\
6.2 & Total & 1980 & 702,729 & 702,730 \\
6.2 & Residential and Commercial & 1981 & 7,422 & 7,421 \\
6.2 & Coke Plants & 1981 & 61,015 & 61,014 \\
6.2 & Total & 1981 & 732,628 & 732,627 \\
6.2 & Other Industrial & 1982 & 64,096 & 64,097 \\
6.2 & Total & 1982 & 706,910 & 706,911 \\
6.2 & Other Industrial & 1983 & 65,979 & 65,980 \\
6.2 & Total & 1983 & 736,671 & 736,672 \\
6.3 & Consumer, Total & 1982 & 195,253 & 195,254 \\
6.3 & Total & 1982 & 232,037 & 232,038 \\
6.3 & Total & 1983 & 202,585 & 202,584 \\
\hline
\end{tabular}




\section{Section 7. Electricity}

During August 1993, electric utilities generated 279 billion kilowatthours of electricity, 9 percent ${ }^{7}$ more than in August 1992. Coal-fired generation totaled 156 billion kilowatthours, 10 percent more than in August 1992. Nuclear generation totaled 56 billion kilowatthours, 4 percent below the level 1 year earlier. Natural gas-fired generation was 34 billion kilowatthours, 19 percent more the August 1992 level. Hydroelectric generation totaled 20 billion kilowatthours, 9 percent above the August 1992 level. Petroleum-fired generation totaled 12 billion kilowatthours, 73 percent above the level 1 year earlier.

Sales of electricity to all ultimate consumers in the United States in August were 274 billion kilowatthours, 9 percent more than sales during August 1992. Sales to residential consumers during August 1993 were 102 billion kilowatthours, 16 percent above the level of sales during the previous year. Sales to industrial consumers totaled 87 billion kilowatthours in August 1993, 2 percent above the level a year ago. Commer- cial sales were 77 billion kilowatthours, 8 percent above the level of commercial sales 1 year earlier. In August 1993, other sales totaled 9 billion kilowatthours, 3 percent abeve the August 1992 level.

Electric utility consumption of coal during August 1993 was 78 million short tons, 10 percent above consumption in August 1992. Petroleum consumption (excluding petroleum coke) during August 1993 was 20 million barrels, 69 percent above the August 1992 level. During August 1993, electric utilities consumed 357 billion cubic feet of natural gas, 18 percent above the August 1992 consumption level.

On August 31, 1993, electric utility stocks of all types of coal totaled 114 million stort tons, 25 percent below the level on August 31, 1992. Stocks of petroleum (excluding petroleum coke) on August 31, 1993, totaled 59 million barrels, 16 percent below the level on August 31, 1992.

${ }^{7}$ Percentage changes are based on numbers shown in the following tables. 
Figure 7.1 Electric Utility Net Generation of Electricity

(Billion Kilowatthours)

Net Generation by Source, 1973-1992

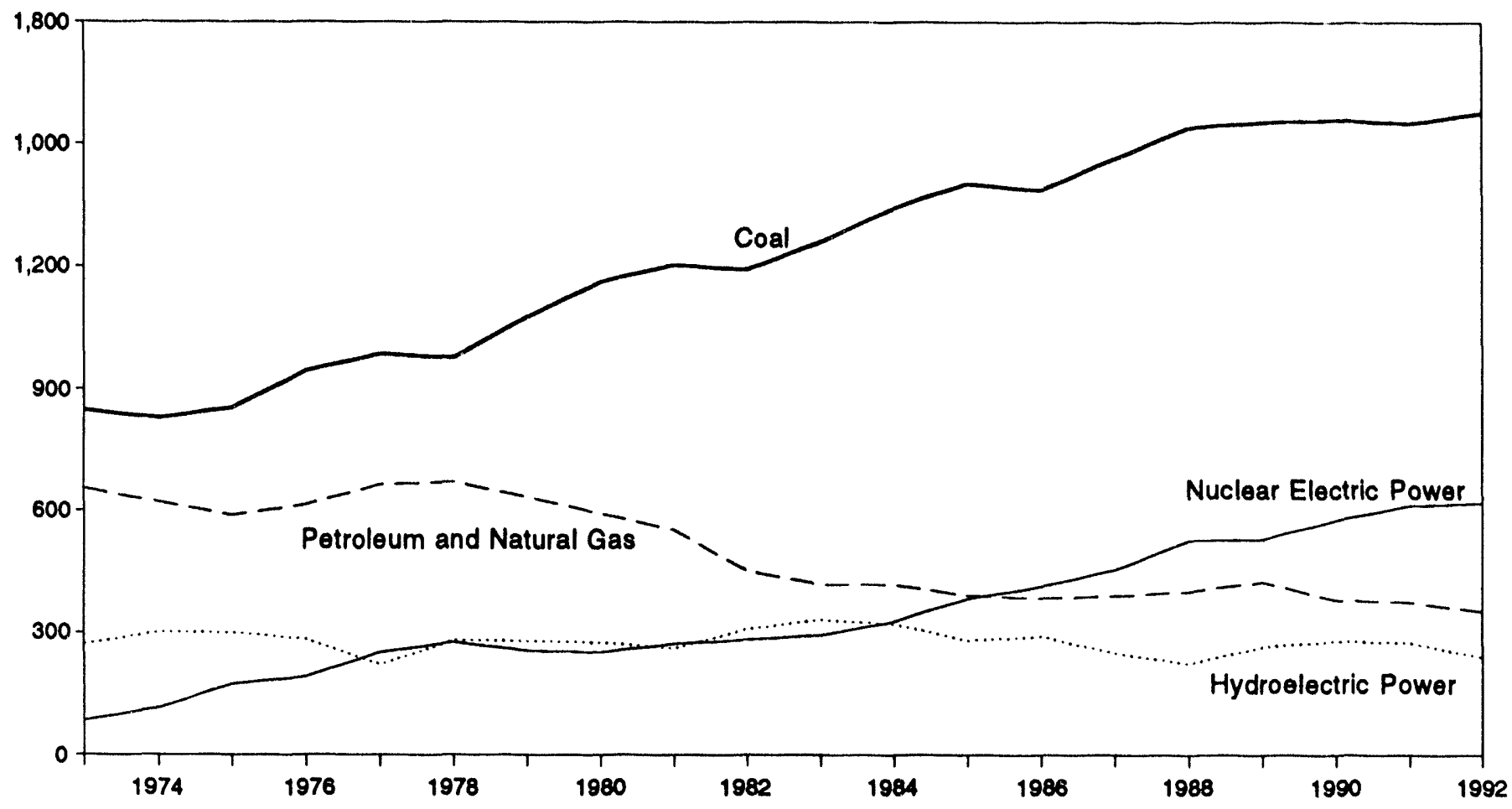

Net Generation, January-August

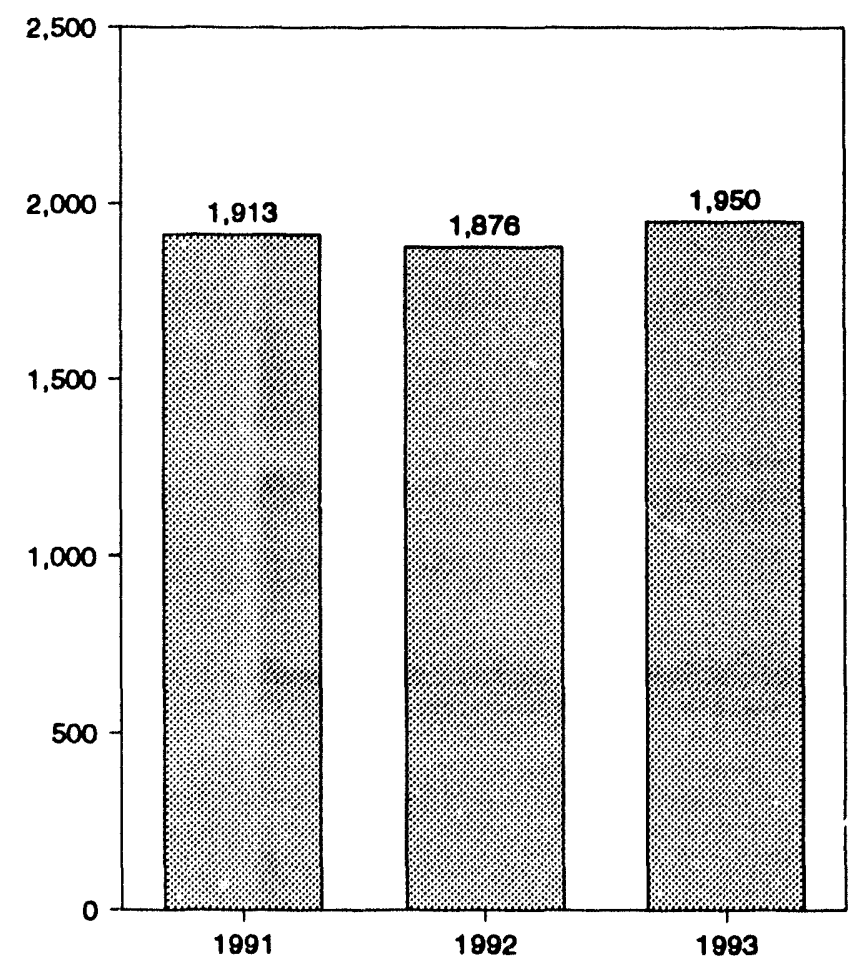

Net Generation by Source, August 1993

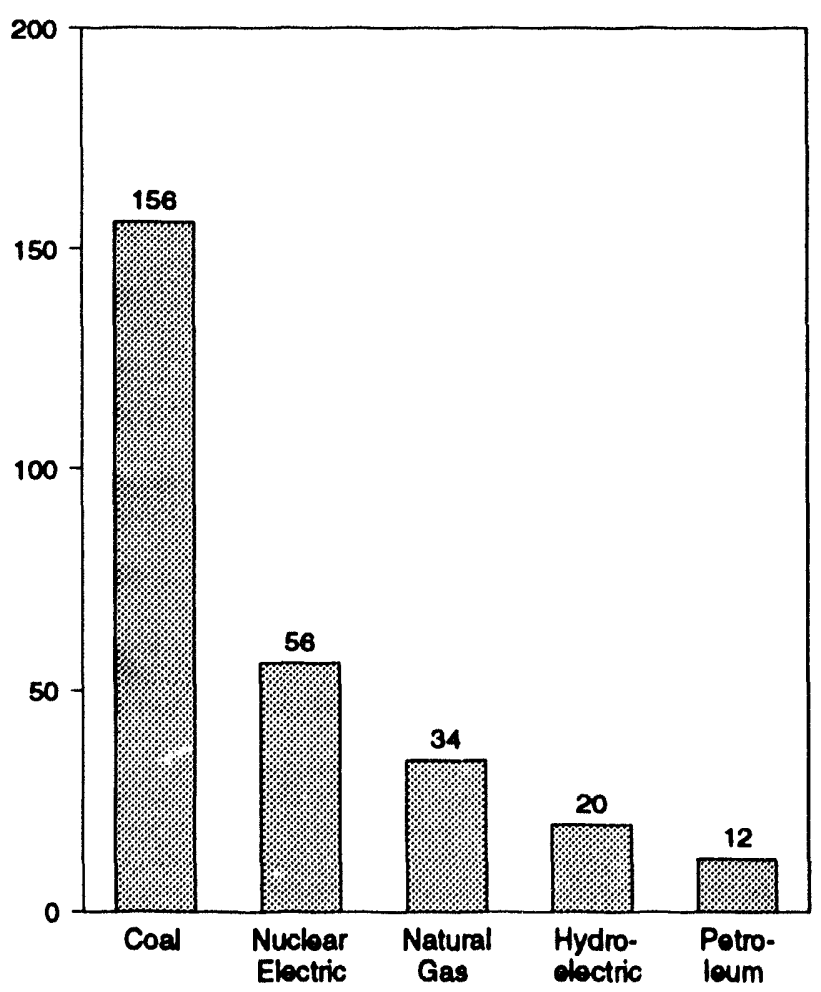

Note: Because vertical scales difier, graphs should not be compared.

Source: Table 7.1. 
Table 7.1 Electric Utility Net Generation of Electricity

(Million Kilowatthours)

\begin{tabular}{|c|c|c|c|c|c|c|c|}
\hline & Coal & $\begin{array}{l}\text { Natural } \\
\text { Gesa }\end{array}$ & Potroleumb & $\begin{array}{l}\text { Nuclew } \\
\text { Electule } \\
\text { Power }\end{array}$ & $\begin{array}{l}\text { Hydro- } \\
\text { Electric } \\
\text { Power }\end{array}$ & Othere & Total \\
\hline 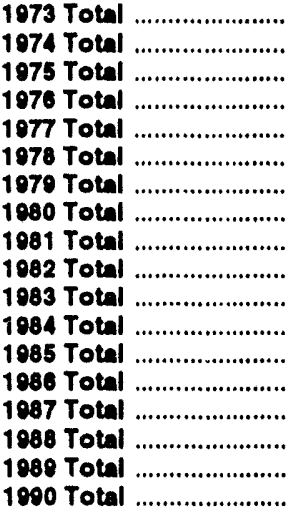 & $\begin{array}{r}847,051 \\
828,433 \\
852,786 \\
944,301 \\
985,210 \\
975,742 \\
1,075,097 \\
1,161,582 \\
1,203,203 \\
1,192,004 \\
1,259,424 \\
1,341,081 \\
1,402,128 \\
1,385,831 \\
1,463,781 \\
1,540,653 \\
1,553,681 \\
1,559,608\end{array}$ & $\begin{array}{l}340,858 \\
320,065 \\
209,778 \\
204,024 \\
305,505 \\
305,301 \\
329,485 \\
346,240 \\
345,777 \\
305,260 \\
274,008 \\
207,304 \\
201,046 \\
248,508 \\
272,621 \\
252,801 \\
266,598 \\
264,080\end{array}$ & $\begin{array}{l}314,343 \\
300,931 \\
289,005 \\
319,088 \\
268,170 \\
385,080 \\
303,525 \\
245,004 \\
206,421 \\
146,707 \\
144,409 \\
110,008 \\
100,202 \\
136,585 \\
118,403 \\
148,000 \\
168,318 \\
117,017\end{array}$ & $\begin{array}{r}83,470 \\
113,076 \\
172,508 \\
191,104 \\
250,083 \\
276,103 \\
255,158 \\
251,116 \\
272,874 \\
282,773 \\
293,077 \\
327,034 \\
383,091 \\
414,038 \\
455,270 \\
526,073 \\
529,356 \\
576,062\end{array}$ & $\begin{array}{l}272,083 \\
301,032 \\
300,047 \\
283,707 \\
220,475 \\
280,410 \\
270,783 \\
276,021 \\
260,684 \\
300,213 \\
332,130 \\
321,150 \\
281,140 \\
290,844 \\
249,695 \\
222,040 \\
208,063 \\
270,026\end{array}$ & $\begin{array}{r}2,204 \\
2,703 \\
3,437 \\
3,083 \\
4,063 \\
3,316 \\
4,387 \\
5,506 \\
6,054 \\
5,164 \\
6,456 \\
8,038 \\
10,724 \\
11,503 \\
12,267 \\
11,084 \\
11,300 \\
10,651\end{array}$ & $\begin{array}{l}1,860,710 \\
1,867,140 \\
1,917,640 \\
2,037,806 \\
2,124,323 \\
2,206,331 \\
2,247,372 \\
2,286,430 \\
2,204,812 \\
2,241,211 \\
2,310,285 \\
2,416,304 \\
2,460,841 \\
2,487,310 \\
2,572,127 \\
2,704,250 \\
2,784,304 \\
2,808,151\end{array}$ \\
\hline 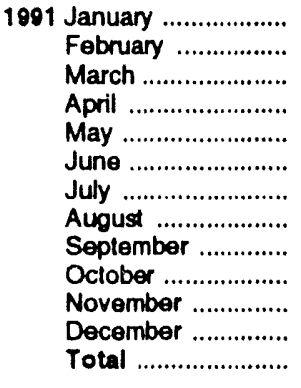 & $\begin{array}{r}141,945 \\
117,867 \\
118,366 \\
112,418 \\
123,906 \\
131,964 \\
143,997 \\
144,194 \\
129,141 \\
125,523 \\
129,125 \\
132,721 \\
1,551,167\end{array}$ & $\begin{array}{r}16,348 \\
13,723 \\
18,446 \\
20,504 \\
23,455 \\
24,417 \\
31,145 \\
30,970 \\
24,966 \\
25,390 \\
18,990 \\
15,819 \\
264,172\end{array}$ & $\begin{array}{r}9,222 \\
8,689 \\
8,785 \\
7,984 \\
10,995 \\
11,159 \\
11,010 \\
11,866 \\
8,646 \\
6,483 \\
7,784 \\
8,841 \\
111,463\end{array}$ & $\begin{array}{l}54,369 \\
47,863 \\
49,121 \\
41,631 \\
46,755 \\
54,208 \\
60,735 \\
58,473 \\
51,874 \\
47,653 \\
46,295 \\
53,589 \\
612,565\end{array}$ & $\begin{array}{r}25,676 \\
21,915 \\
25,820 \\
25,687 \\
28,455 \\
25,830 \\
24,250 \\
21,747 \\
18,428 \\
17,538 \\
18,300 \\
21,873 \\
275,519\end{array}$ & $\begin{array}{r}897 \\
764 \\
863 \\
780 \\
808 \\
848 \\
839 \\
865 \\
830 \\
843 \\
883 \\
916 \\
10,137\end{array}$ & $\begin{array}{r}248,455 \\
210,821 \\
221,400 \\
209,004 \\
234,373 \\
248,427 \\
271,976 \\
268,115 \\
233,885 \\
223,430 \\
221,377 \\
233,760 \\
2,825,023\end{array}$ \\
\hline 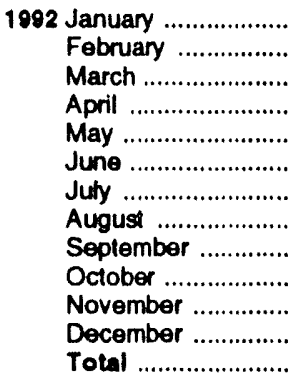 & $\begin{array}{r}137,327 \\
121,732 \\
127,678 \\
119,909 \\
123,768 \\
129,607 \\
149,028 \\
141,900 \\
133,239 \\
127,940 \\
125,535 \\
138,234 \\
1,575,895\end{array}$ & $\begin{array}{r}16,178 \\
16,165 \\
19,906 \\
21,913 \\
22,689 \\
24,997 \\
31,950 \\
28,778 \\
26,099 \\
20,420 \\
18,031 \\
16,744 \\
263,872\end{array}$ & $\begin{array}{r}10,202 \\
8,296 \\
8,809 \\
6,505 \\
5,156 \\
7,508 \\
8,540 \\
6,923 \\
6,841 \\
6,908 \\
6,838 \\
6,390 \\
88,016\end{array}$ & $\begin{array}{r}57,849 \\
52,804 \\
45,835 \\
42,268 \\
45,627 \\
51,185 \\
56,049 \\
58,656 \\
50,919 \\
48,784 \\
50,726 \\
58,075 \\
618,776\end{array}$ & $\begin{array}{r}21,502 \\
17,966 \\
21,566 \\
19,454 \\
22,285 \\
22,698 \\
19,711 \\
18,062 \\
16,838 \\
16,375 \\
19,294 \\
23,808 \\
230,550\end{array}$ & $\begin{array}{r}912 \\
798 \\
871 \\
788 \\
830 \\
846 \\
869 \\
885 \\
825 \\
862 \\
840 \\
874 \\
10,200\end{array}$ & $\begin{array}{r}243,970 \\
217,761 \\
224,665 \\
210,837 \\
220,355 \\
236,842 \\
266,148 \\
255,203 \\
234,760 \\
221,289 \\
221,263 \\
244,126 \\
2,707,210\end{array}$ \\
\hline 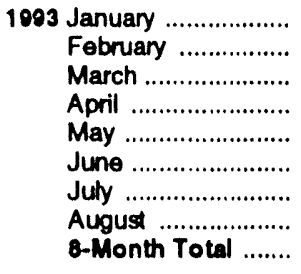 & $\begin{array}{r}138,357 \\
130,078 \\
136,280 \\
120,325 \\
120,878 \\
137,464 \\
158,380 \\
156,193 \\
1,097,054\end{array}$ & $\begin{array}{r}15,811 \\
15,773 \\
18,740 \\
16,591 \\
15,843 \\
24,391 \\
31,684 \\
34,262 \\
173,096\end{array}$ & $\begin{array}{r}7,226 \\
6,950 \\
8,569 \\
5,205 \\
5,268 \\
7,819 \\
11,341 \\
11,978 \\
84,357\end{array}$ & $\begin{array}{r}59,076 \\
51,319 \\
46,603 \\
43,199 \\
50,367 \\
52,620 \\
56,502 \\
56,209 \\
415,809\end{array}$ & $\begin{array}{r}24,474 \\
19,743 \\
23,583 \\
25,171 \\
29,323 \\
26,606 \\
23,575 \\
19,685 \\
102,161\end{array}$ & $\begin{array}{l}853 \\
800 \\
852 \\
802 \\
716 \\
725 \\
788 \\
820 \\
6,356\end{array}$ & $\begin{array}{r}245,797 \\
224,663 \\
234,630 \\
211,292 \\
222,396 \\
249,625 \\
282,270 \\
279,147 \\
1,049,821\end{array}$ \\
\hline $\begin{array}{l}1092 \text { 8-Month Total ....... } \\
1901 \text { 8-Month Total ....... }\end{array}$ & $\begin{array}{l}1,050,048 \\
1,034,657\end{array}$ & $\begin{array}{l}182,577 \\
179,008\end{array}$ & $\begin{array}{l}81,939 \\
79,708\end{array}$ & $\begin{array}{l}410,272 \\
413,154\end{array}$ & $\begin{array}{l}163,245 \\
109,380\end{array}$ & $\begin{array}{l}6,800 \\
6,664\end{array}$ & $\begin{array}{l}1,875,780 \\
1,012,571\end{array}$ \\
\hline
\end{tabular}

Includes supplemental gaseous fuel.

b Includes fuel cil nos. 1, 2, 4, 5, and 6, crude oil, kercsene, and petroleum coke.

c "Other" is electricity produced from geothermal, wood, waste, wind, photovoltaic, and solar thermal energy sources connected to electric unility distribution systems.

Notes: - Geographic coverage is the 50 States and the District of Columbia. - Totals may not equal sum of components due to independent rounding.

Sources: - 1973-Septomber 1977: Federal Power Commission, Form FPC-4, "Monthly Power Plant Report." - October 1977-1979: Federal Energy Regulatory Commission, Form FPC.4, "Monthly Power Plant Report." - 1080: Energy Information Administration (EIA), Eloctric Power Monthly. March 1991, Table 4. - 1981: ElA, Electric Power Monthly, March 1992, Table 4. - 1982 and 1901 monthly data: ElA. Electric Power Monthly, March 1993, Table 4. - 1083 forwerd (oxcopt 1091 monthly data): EIA, Electric Power Monthly, November 1993, Table 4. 
Flgure 7.2 Electricity Sales

(Billion Kilowatthours)

Total Sales, January-August

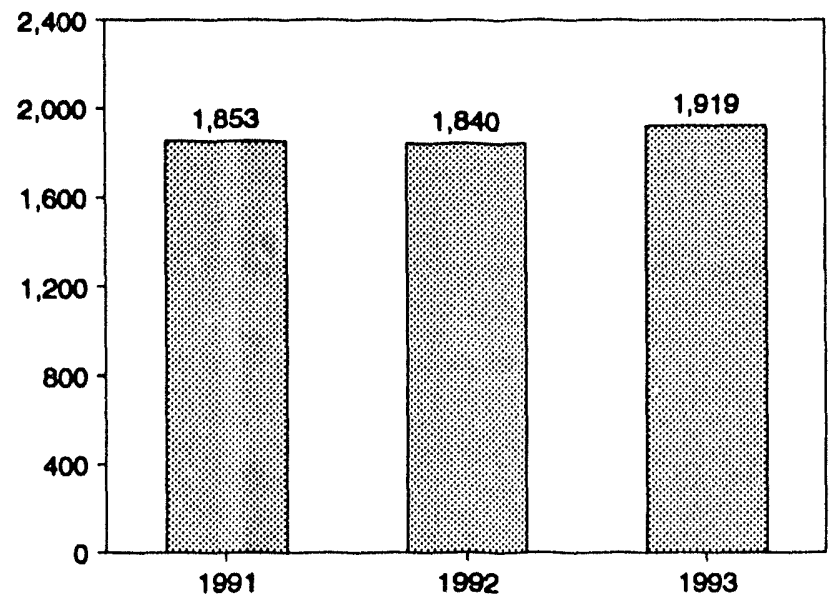

Total Sales, Monthly

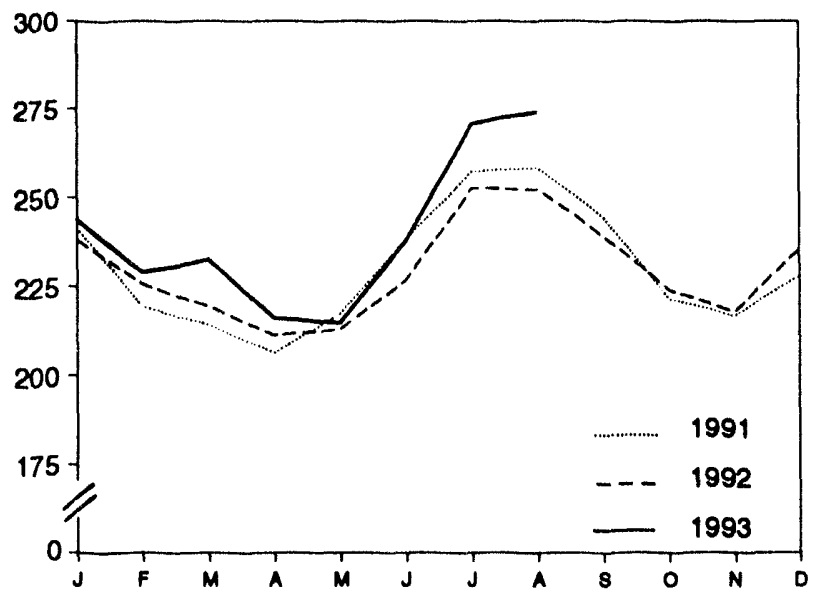

Sales by Sector, Monthly

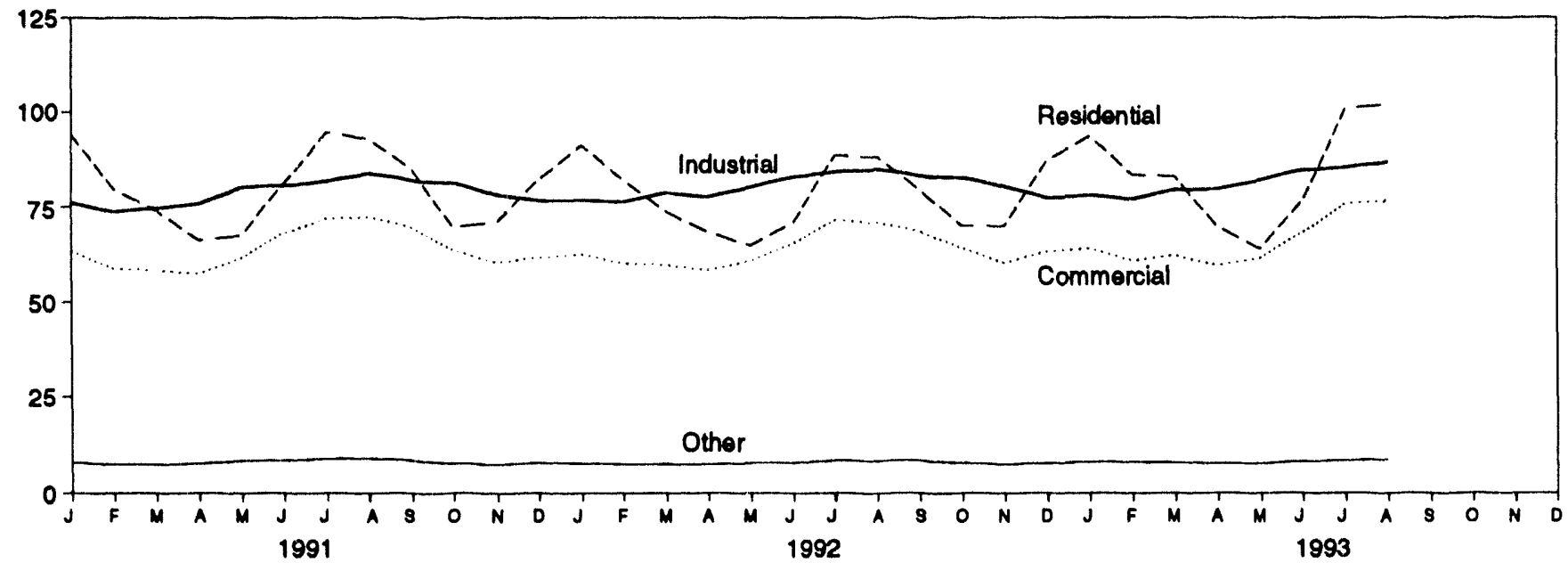

Sales by Sector, 1973-1992

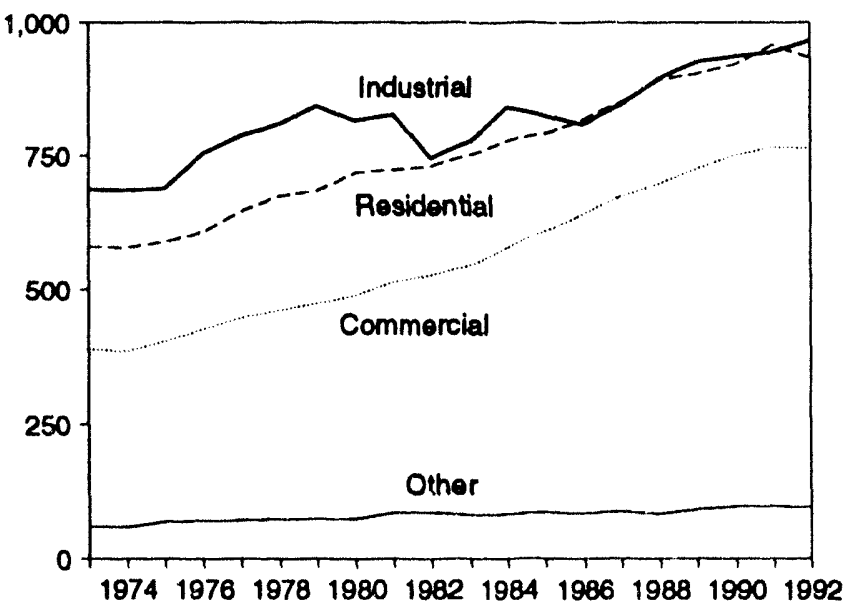

Sales by Sector, August 1993

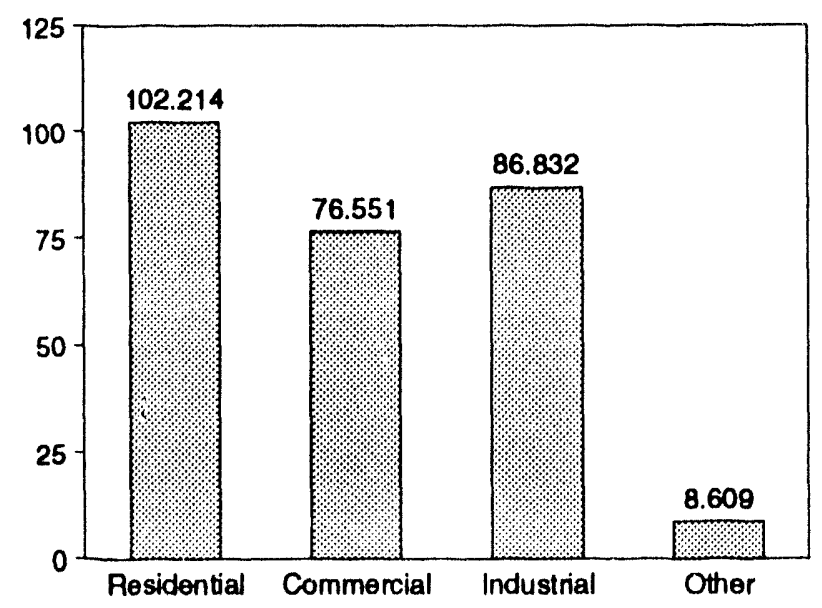

Note: Because vertical scales difter, oraphs should not be compared. Source: Teble 7.2, Morthly Serles. 
Table 7.2 Electricity Sales by End-Use Sector

(Million Kilowatthours)

\begin{tabular}{|c|c|c|c|c|c|c|c|c|c|c|}
\hline & \multicolumn{2}{|c|}{ Realdential } & \multicolumn{2}{|c|}{ Commercial } & \multicolumn{2}{|c|}{ Industrial } & \multicolumn{2}{|c|}{ Other" } & \multicolumn{2}{|c|}{ Total } \\
\hline & $\begin{array}{l}\text { Monthly } \\
\text { Series }\end{array}$ & $\begin{array}{l}\text { Annue } \\
\text { Series }\end{array}$ & $\begin{array}{l}\text { Monthly } \\
\text { Series }\end{array}$ & $\begin{array}{l}\text { Annual } \\
\text { Series }\end{array}$ & $\begin{array}{l}\text { Monthly } \\
\text { Serieeb }\end{array}$ & $\begin{array}{l}\text { Annual } \\
\text { Series }\end{array}$ & $\begin{array}{l}\text { Monthly } \\
\text { Seriese }\end{array}$ & $\begin{array}{l}\text { Annual } \\
\text { Series }\end{array}$ & $\begin{array}{l}\text { Monthly } \\
\text { Series }\end{array}$ & $\begin{array}{l}\text { Annual } \\
\text { Series }\end{array}$ \\
\hline 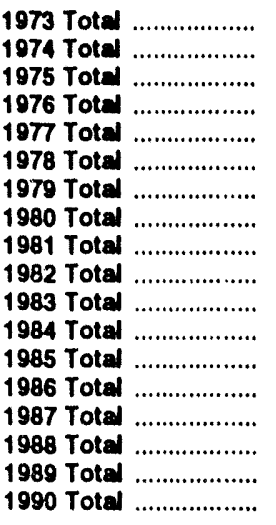 & $\begin{array}{l}579,231 \\
578,184 \\
588,140 \\
606,452 \\
645,239 \\
674,466 \\
682,819 \\
717,495 \\
722,265 \\
729,520 \\
750,948 \\
777,654 \\
790,977 \\
817,663 \\
849,613 \\
892,125 \\
903,979 \\
921,473\end{array}$ & $\begin{array}{l}\text { NA } \\
\text { NA } \\
\text { NA } \\
\text { NA } \\
\text { NA } \\
\text { NA } \\
\text { NA } \\
\text { NA } \\
\text { NA } \\
\text { NA } \\
\text { NA } \\
780,092 \\
793,934 \\
819,088 \\
850,410 \\
892,866 \\
905,525 \\
924,010\end{array}$ & $\begin{array}{l}388,266 \\
384,826 \\
403,049 \\
425,094 \\
446,514 \\
461,163 \\
473,307 \\
488,155 \\
514,338 \\
526,397 \\
543,788 \\
578,281 \\
608,968 \\
641,469 \\
673,707 \\
697,711 \\
725,229 \\
750,835\end{array}$ & $\begin{array}{l}\text { NA } \\
\text { NA } \\
\text { NA } \\
\text { NA } \\
\text { NA } \\
\text { NA } \\
\text { NA } \\
\text { NA } \\
\text { NA } \\
\text { NA } \\
\text { NA } \\
582,621 \\
605,989 \\
630,520 \\
660,433 \\
699,100 \\
725,861 \\
751,027\end{array}$ & $\begin{array}{l}686,085 \\
684,875 \\
687,680 \\
754,060 \\
786,037 \\
809,078 \\
841,903 \\
815,067 \\
825,743 \\
744,949 \\
775,999 \\
840,588 \\
824,523 \\
808,292 \\
845,266 \\
895,751 \\
926,376 \\
936,428\end{array}$ & $\begin{array}{c}\text { NA } \\
N A \\
N A \\
N A \\
N A \\
N A \\
N A \\
N A \\
N A \\
N A \\
N A \\
837,836 \\
836,772 \\
830,531 \\
858,233 \\
896,498 \\
925,659 \\
945,522\end{array}$ & $\begin{array}{l}59,326 \\
58,030 \\
68,222 \\
69,631 \\
70,571 \\
73,215 \\
73,070 \\
73,732 \\
84,756 \\
85,575 \\
80,219 \\
81,849 \\
85,075 \\
83,409 \\
86,854 \\
82,362 \\
91,066 \\
95,936\end{array}$ & $\begin{array}{c}\text { NA } \\
\text { NA } \\
\text { NA } \\
\text { NA } \\
\text { NA } \\
\text { NA } \\
\text { NA } \\
\text { NA } \\
\text { NA } \\
\text { NA } \\
\text { NA } \\
85,248 \\
87,279 \\
88,615 \\
88,196 \\
89,598 \\
89,765 \\
91,988\end{array}$ & $\begin{array}{l}1,712,909 \\
1,705,924 \\
1,747,091 \\
1,855,246 \\
1,948,361 \\
2,017,922 \\
2,071,099 \\
2,094,449 \\
2,147,103 \\
2,086,441 \\
2,150,955 \\
2,278,372 \\
2,309,543 \\
2,350,835 \\
2,455,440 \\
2,567,949 \\
2,646,651 \\
2,704,672\end{array}$ & $\begin{array}{c}\text { NA } \\
\text { NA } \\
\text { NA } \\
\text { NA } \\
\text { NA } \\
\text { NA } \\
\text { NA } \\
\text { NA } \\
\text { NA } \\
\text { NA } \\
\text { NA } \\
2,285,796 \\
2,323,974 \\
2,368,753 \\
2,457,272 \\
2,578,062 \\
2,646,809 \\
2,712,555\end{array}$ \\
\hline 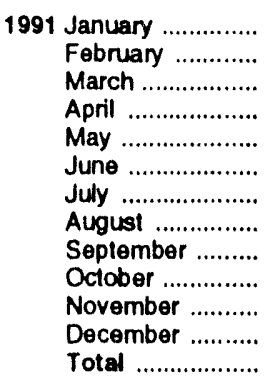 & $\begin{array}{l}94,144 \\
79,676 \\
74,078 \\
66,079 \\
67,450 \\
81,116 \\
94,738 \\
93,127 \\
84,696 \\
69,422 \\
71,114 \\
82,160 \\
957,801\end{array}$ & $\begin{array}{l}- \\
- \\
- \\
- \\
- \\
- \\
- \\
- \\
- \\
955,417\end{array}$ & $\begin{array}{r}63,336 \\
58,582 \\
58,157 \\
57,155 \\
61,434 \\
67,991 \\
71,872 \\
72,360 \\
69,501 \\
63,439 \\
60,133 \\
61,516 \\
765,476\end{array}$ & $\begin{array}{l}- \\
- \\
- \\
- \\
- \\
- \\
- \\
- \\
- \\
- \\
765,664\end{array}$ & $\begin{array}{l}76,111 \\
73,715 \\
74,720 \\
75,706 \\
80,236 \\
80,569 \\
81,700 \\
83,974 \\
81,967 \\
81,209 \\
78,176 \\
76,601 \\
944,684\end{array}$ & $\begin{array}{l}- \\
- \\
- \\
- \\
- \\
- \\
- \\
- \\
- \\
- \\
246,583\end{array}$ & $\begin{array}{r}7,905 \\
7,424 \\
7,459 \\
7,600 \\
8,378 \\
8,502 \\
8,877 \\
8,986 \\
8,476 \\
7,654 \\
7,463 \\
7,790 \\
96,513\end{array}$ & $\begin{array}{l}- \\
- \\
- \\
- \\
- \\
- \\
- \\
- \\
- \\
-\overline{94,339}\end{array}$ & $\begin{array}{r}241,497 \\
219,397 \\
214,414 \\
206,541 \\
217,499 \\
238,177 \\
257,187 \\
258,447 \\
244,639 \\
221,723 \\
216,886 \\
228,068 \\
2,764,474\end{array}$ & $\begin{array}{c}- \\
- \\
- \\
- \\
- \\
- \\
- \\
- \\
- \\
2,762,003\end{array}$ \\
\hline 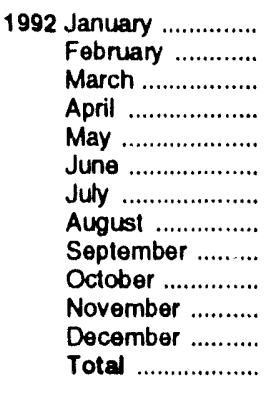 & $\begin{array}{r}91,310 \\
82,022 \\
73,635 \\
68,322 \\
64,662 \\
70,745 \\
88,510 \\
88,251 \\
79,400 \\
69,838 \\
69,970 \\
87,378 \\
934,044\end{array}$ & $\begin{array}{l}- \\
- \\
- \\
- \\
- \\
- \\
- \\
- \\
\overline{-} \\
\text { NA }\end{array}$ & $\begin{array}{r}62,441 \\
59,876 \\
59,574 \\
58,081 \\
60,559 \\
65,209 \\
71,445 \\
70,844 \\
68,437 \\
63,985 \\
60,131 \\
63,082 \\
763,664\end{array}$ & $\begin{array}{l}- \\
- \\
- \\
- \\
- \\
- \\
- \\
- \\
- \\
\overline{-} \\
\overline{N A}\end{array}$ & $\begin{array}{r}76,760 \\
76,312 \\
78,741 \\
77,607 \\
80,191 \\
82,900 \\
84,195 \\
85,013 \\
83,182 \\
82,678 \\
80,421 \\
77,358 \\
965,356\end{array}$ & $\begin{array}{l}- \\
- \\
- \\
- \\
- \\
- \\
- \\
- \\
- \\
- \\
\text { NA }\end{array}$ & $\begin{array}{r}7,725 \\
7,507 \\
7,542 \\
7,448 \\
7,767 \\
7,901 \\
8,392 \\
8,327 \\
8,441 \\
7,766 \\
7,462 \\
7,725 \\
94,003\end{array}$ & $\begin{array}{l}- \\
- \\
- \\
- \\
- \\
- \\
- \\
- \\
- \\
\overline{-} \\
\text { NA }\end{array}$ & $\begin{array}{r}238,235 \\
225,717 \\
219,491 \\
211,458 \\
213,179 \\
226,755 \\
252,541 \\
252,435 \\
239,460 \\
224,267 \\
217,984 \\
235,543 \\
2,757,067\end{array}$ & $\begin{array}{l}- \\
- \\
- \\
- \\
- \\
- \\
- \\
- \\
- \\
\text { NA }\end{array}$ \\
\hline 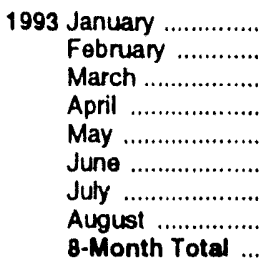 & $\begin{array}{r}93,739 \\
83,416 \\
83,023 \\
69,668 \\
63,852 \\
76,584 \\
101,023 \\
102,214 \\
673,520\end{array}$ & $\begin{array}{l}- \\
\overline{-} \\
- \\
- \\
- \\
-\end{array}$ & $\begin{array}{r}63,930 \\
60,624 \\
62,169 \\
59,389 \\
61,420 \\
68,171 \\
75,704 \\
76,551 \\
527,958\end{array}$ & $\begin{array}{l}- \\
\overline{-} \\
- \\
- \\
- \\
-\end{array}$ & $\begin{array}{r}78,074 \\
77,017 \\
79,504 \\
79,593 \\
82,100 \\
84,768 \\
85,370 \\
86,832 \\
653,260\end{array}$ & $\begin{array}{l}- \\
\overline{-} \\
- \\
- \\
- \\
-\end{array}$ & $\begin{array}{r}8,113 \\
7,940 \\
7,919 \\
7,588 \\
7,602 \\
8,138 \\
8,457 \\
8,609 \\
64,365\end{array}$ & $\begin{array}{l}- \\
- \\
- \\
- \\
- \\
-\end{array}$ & $\begin{array}{r}243,856 \\
228,997 \\
232,615 \\
216,238 \\
214,975 \\
237,662 \\
270,555 \\
274,206 \\
1,919,104\end{array}$ & $\begin{array}{l}- \\
- \\
- \\
- \\
- \\
-\end{array}$ \\
\hline $\begin{array}{l}1992 \text { 8-Month Total ... } \\
1991 \text { 8-Month Total ... }\end{array}$ & $\begin{array}{l}627,458 \\
650,408\end{array}$ & $\overline{-}$ & $\begin{array}{l}508,029 \\
510,888\end{array}$ & $\overline{-}$ & $\begin{array}{l}641,717 \\
626,731\end{array}$ & $\overline{-}$ & $\begin{array}{l}62,609 \\
65,131\end{array}$ & $\overline{-}$ & $\begin{array}{l}1,839,812 \\
1,853,158\end{array}$ & - \\
\hline
\end{tabular}

a "Other" is public street and highway lighting, other sales 10 public authorities, sales to rallroads and railways, and interdepartmental sales. b Annual totals are the sums of the monthly values.

NA $=$ Not available. $-=$ Not applicable.

Notes: - Geographic coverage is the $\mathbf{5 0}$ States and the District of Columbia

- Totals may nol equal sum ol components dure to independent rounding.

Sources: - 1973-September 1977: Federal Power Commission, Form

FPC-5, "Monthly Statement of Electric Operating Revenue and Income."
October 1977-1979: Federal Energy Regulatory Commission, Form FERC-5, "Elociric Operating Revenue and Income." 1980: Energy Infomation Administration (ELA), Electric Power Monthy, March 1991, Table 51. - 1981: ElA, Electric Power Monthly, March 1992, Table 51. - 1982 and 1991 monthly data: ElA, Eloctric Power Monthly, March 1993. Table 51. 1983 forward (except 1991 monthly data): ElA, Electric Power Monthly, November 1993, Table 51. 
Figure 7.3 Electric Utility Consumption and Stocks of Fossil Fuels

Fuels Consumed, 1973-1992

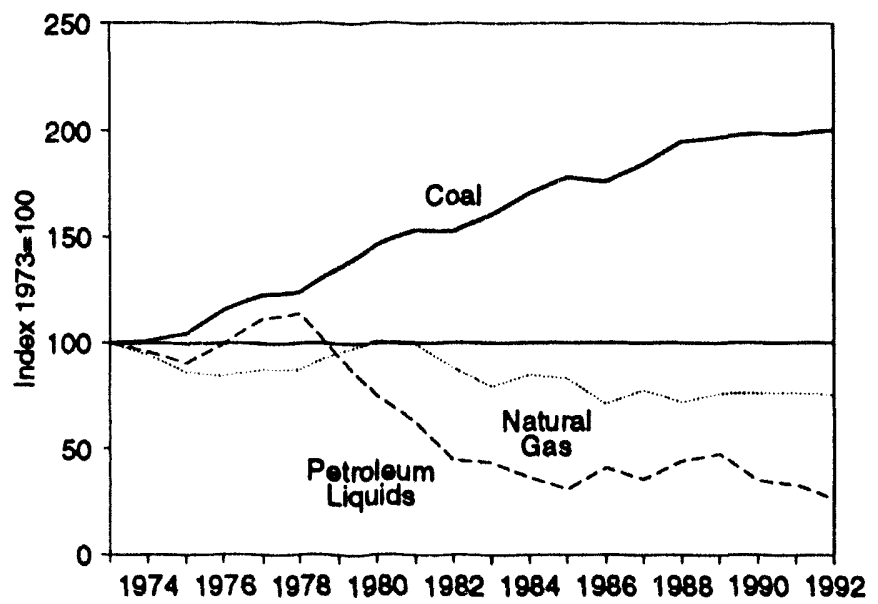

Petroleum Liquids Consumed, Monthly

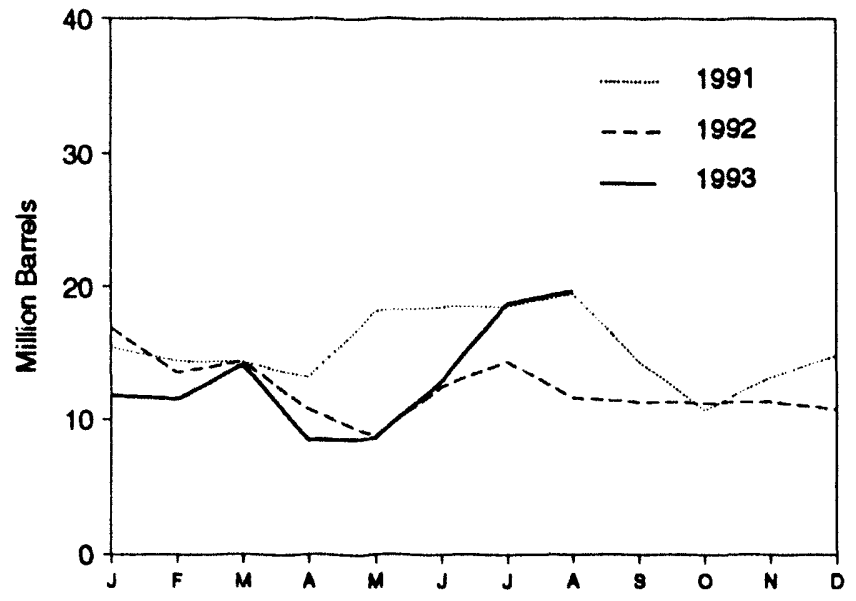

Coal Stocks, End of Month

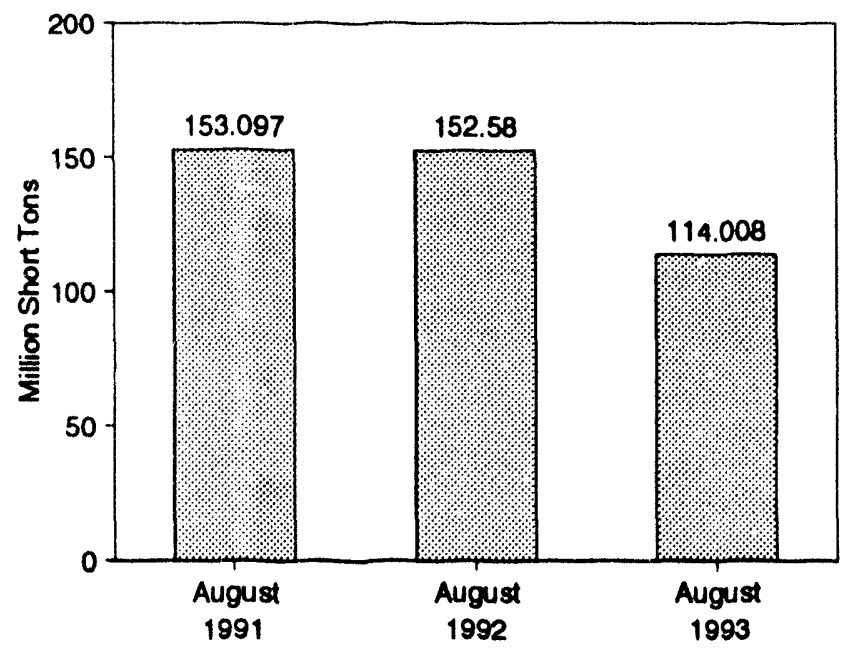

Coal Consumed, Monthly

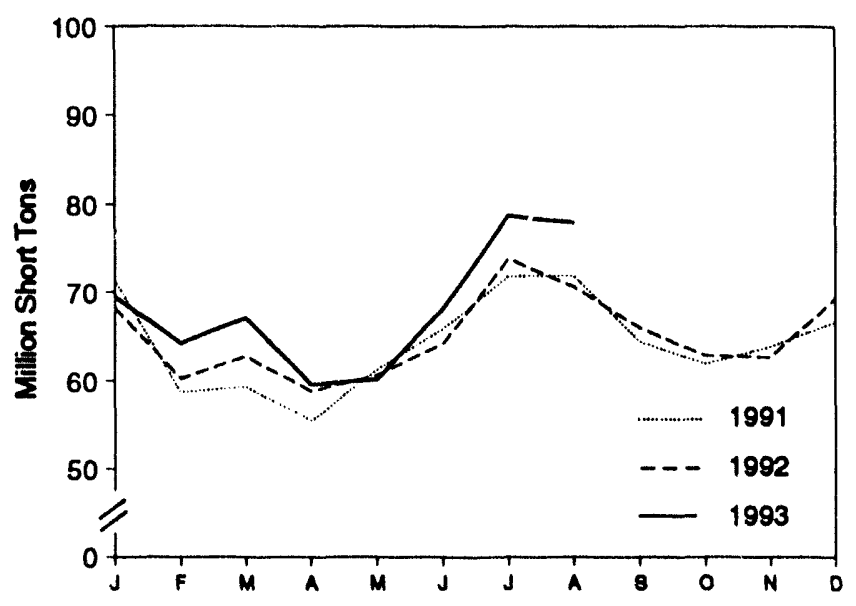

Natural Gas Consumed, Monthly

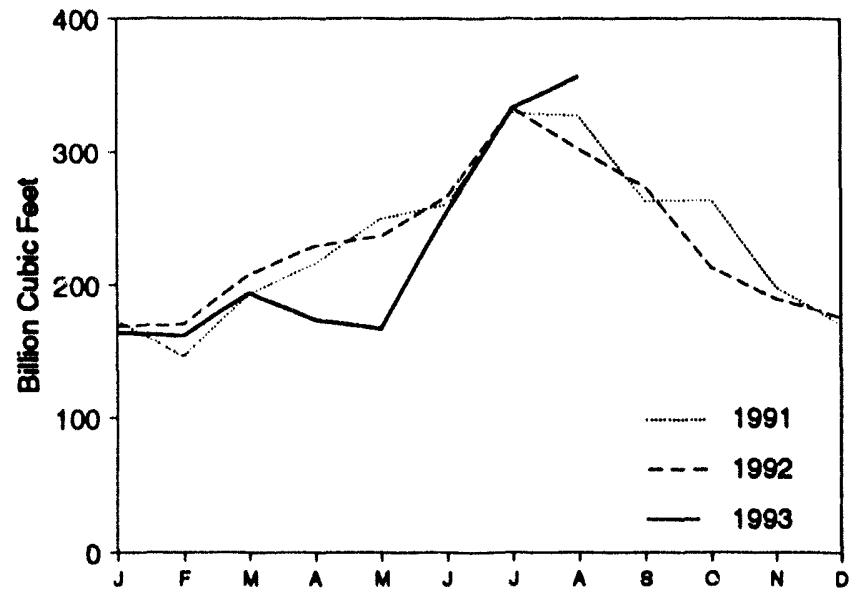

Petroleum Liquids Stocks, End of Month

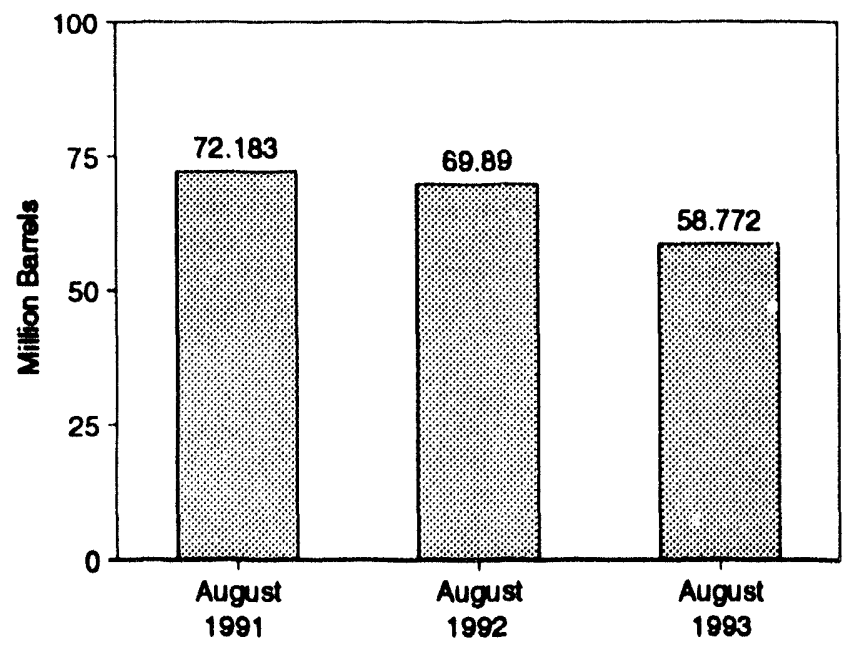

Note: Because vertical scales difler. graphs should not be compared. Sources: Tables 7.3 and 7.4. 


\begin{tabular}{|c|c|c|c|c|c|c|c|c|c|c|c|}
\hline & \multicolumn{4}{|c|}{ Cod } & \multicolumn{6}{|c|}{ Petroleum } & \multirow[b]{3}{*}{$\begin{array}{l}\text { Naturel } \\
\text { Coned }\end{array}$} \\
\hline & \multirow[b]{2}{*}{$\begin{array}{l}\text { Anthre- } \\
\text { clte }\end{array}$} & \multirow[b]{2}{*}{$\underset{\text { Coel }}{\text { Bltuminous }}$} & \multirow[b]{2}{*}{ Uenite } & \multirow[b]{2}{*}{ Total } & \multicolumn{2}{|c|}{$\begin{array}{l}\text { By Type } \\
\text { of Potroleum }\end{array}$} & \multicolumn{2}{|c|}{$\begin{array}{l}\text { By Prime } \\
\text { Mover Typo }\end{array}$} & \multirow[b]{2}{*}{$\begin{array}{l}\text { Totel } \\
\text { Uquide }\end{array}$} & \multirow[b]{2}{*}{$\begin{array}{c}\text { Potroloum } \\
\text { Coks }\end{array}$} & \\
\hline & & & & & $\begin{array}{l}\text { Heavy } \\
\text { Olpa }\end{array}$ & $\begin{array}{l}\text { Wght } \\
\text { OIf }\end{array}$ & $\begin{array}{l}\text { 8tiomm } \\
\text { Plante }\end{array}$ & GTnce & & & \\
\hline & \multicolumn{4}{|c|}{ Thousand Shont Tons } & \multicolumn{5}{|c|}{ Thousand Barrels } & $\begin{array}{l}\text { Thousand } \\
\text { Shont Tons }\end{array}$ & $\begin{array}{l}\text { Million } \\
\text { Cuble Feet }\end{array}$ \\
\hline 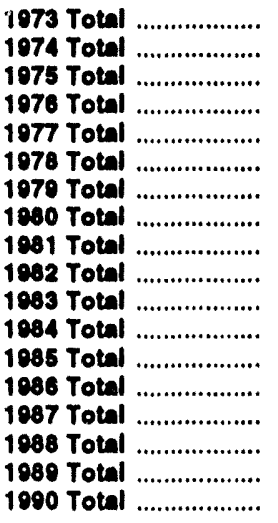 & $\begin{array}{r}1,443 \\
1,400 \\
1,400 \\
1,350 \\
1,426 \\
1,034 \\
1,046 \\
081 \\
1,221 \\
1,078 \\
1,036 \\
1,070 \\
1,033 \\
820 \\
972 \\
1,033 \\
1,040 \\
1,031\end{array}$ & 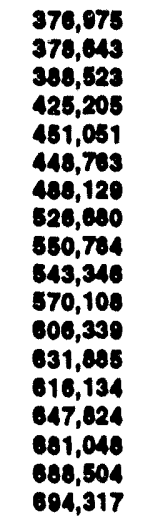 & $\begin{array}{l}10,704 \\
11,070 \\
18,060 \\
21,017 \\
24,080 \\
31,407 \\
37,076 \\
41,042 \\
44,702 \\
49,246 \\
54,067 \\
68,090 \\
60,023 \\
68,093 \\
89,098 \\
76,260 \\
77,338 \\
78,201\end{array}$ & $\begin{array}{l}389,212 \\
301,811 \\
408,082 \\
448,371 \\
477,126 \\
481,238 \\
627,081 \\
600,274 \\
608,707 \\
603,068 \\
625,211 \\
694,300 \\
693,841 \\
685,058 \\
717,804 \\
758,372 \\
766,868 \\
773,540\end{array}$ & $\begin{array}{c}\text { NA } \\
\text { NA } \\
\text { NA } \\
\text { NA } \\
\text { NA } \\
\text { NA } \\
\text { NA } \\
301,183 \\
320,798 \\
234,434 \\
228,084 \\
180,280 \\
159,770 \\
216,156 \\
184,011 \\
220,327 \\
241,080 \\
181,231\end{array}$ & $\begin{array}{c}\text { NA } \\
\text { NA } \\
\text { NA } \\
\text { NA } \\
\text { NA } \\
\text { NA } \\
\text { NA } \\
29,051 \\
21,313 \\
15,337 \\
16,512 \\
15,190 \\
14,636 \\
14,328 \\
15,367 \\
18,789 \\
25,491 \\
14,823\end{array}$ & $\begin{array}{l}513,160 \\
403,140 \\
407,221 \\
514,077 \\
574,860 \\
588,310 \\
402,800 \\
401,863 \\
330,860 \\
243,637 \\
237,846 \\
197,080 \\
168,842 \\
222,500 \\
190,818 \\
235,817 \\
260,315 \\
187,531\end{array}$ & $\begin{array}{r}47,058 \\
53,128 \\
38,007 \\
41,043 \\
48,037 \\
47,820 \\
30,001 \\
18,351 \\
11,431 \\
6,234 \\
7,052 \\
7,420 \\
8,572 \\
7,093 \\
8,830 \\
12,270 \\
17,136 \\
8,523\end{array}$ & $\begin{array}{l}560,248 \\
639,274 \\
806,128 \\
856,020 \\
623,708 \\
936,030 \\
820,207 \\
420,214 \\
381,111 \\
240,771 \\
246,407 \\
204,470 \\
173,414 \\
230,482 \\
190,370 \\
240,096 \\
207,461 \\
196,054\end{array}$ & $\begin{array}{r}607 \\
628 \\
70 \\
98 \\
98 \\
208 \\
208 \\
170 \\
130 \\
140 \\
281 \\
262 \\
231 \\
313 \\
348 \\
400 \\
517 \\
810\end{array}$ & 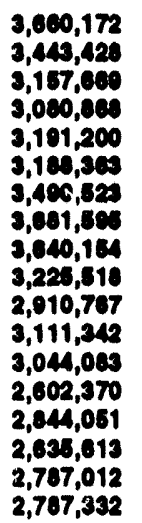 \\
\hline 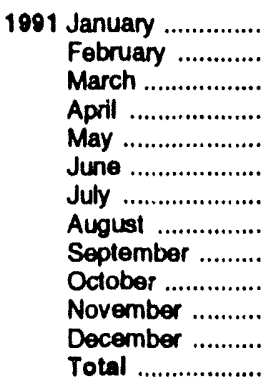 & $\begin{array}{r}74 \\
68 \\
93 \\
92 \\
73 \\
72 \\
101 \\
90 \\
90 \\
86 \\
79 \\
77 \\
904\end{array}$ & $\begin{array}{l}63,779 \\
52,090 \\
52,924 \\
50,131 \\
55,229 \\
58,455 \\
64,202 \\
64,280 \\
57,474 \\
55,586 \\
57,662 \\
59,462 \\
691,275\end{array}$ & $\begin{array}{l}7,553 \\
6,456 \\
6,255 \\
5,219 \\
5,926 \\
7,290 \\
7,548 \\
7,514 \\
6,833 \\
6,212 \\
6,073 \\
7,120 \\
79,090\end{array}$ & $\begin{array}{l}71,406 \\
58,614 \\
59,272 \\
55,443 \\
61,228 \\
65,817 \\
71,852 \\
71,884 \\
64,397 \\
61,883 \\
63,814 \\
66,659 \\
772,268\end{array}$ & $\begin{array}{r}14,264 \\
13,595 \\
13,513 \\
12,142 \\
16,312 \\
17,325 \\
17,289 \\
18,041 \\
13,209 \\
9,791 \\
12,020 \\
13,656 \\
171,157\end{array}$ & $\begin{array}{r}1,187 \\
804 \\
828 \\
1,019 \\
1,814 \\
1,122 \\
1,218 \\
1,380 \\
1,165 \\
902 \\
1,146 \\
1,143 \\
13,720\end{array}$ & $\begin{array}{r}14,911 \\
14,021 \\
13,999 \\
12,641 \\
16,919 \\
17,845 \\
17,737 \\
18,500 \\
13,634 \\
10,269 \\
12,575 \\
14,214 \\
177,286\end{array}$ & $\begin{array}{r}541 \\
377 \\
341 \\
510 \\
1,208 \\
602 \\
770 \\
921 \\
740 \\
403 \\
591 \\
586 \\
7,600\end{array}$ & $\begin{array}{r}15,452 \\
14,398 \\
14,340 \\
13,161 \\
18,128 \\
18,447 \\
18,507 \\
19,421 \\
14,374 \\
10,693 \\
13,166 \\
14,800 \\
184,886\end{array}$ & $\begin{array}{l}74 \\
57 \\
73 \\
72 \\
66 \\
50 \\
61 \\
56 \\
52 \\
50 \\
52 \\
59 \\
722\end{array}$ & $\begin{array}{r}173,138 \\
146,266 \\
192,809 \\
215,850 \\
249,454 \\
260,153 \\
329,861 \\
327,621 \\
262,825 \\
263,376 \\
197,831 \\
160,931 \\
2,789,014\end{array}$ \\
\hline 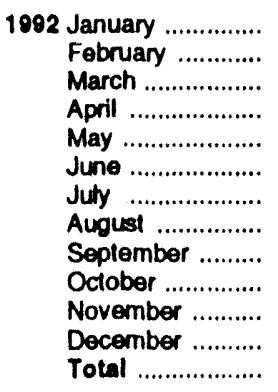 & $\begin{array}{l}80 \\
80 \\
93 \\
73 \\
69 \\
84 \\
90 \\
84 \\
83 \\
85 \\
74 \\
93 \\
986\end{array}$ & $\begin{array}{l}60,881 \\
53,687 \\
56,243 \\
53,314 \\
54,664 \\
57,179 \\
66,318 \\
62,937 \\
58,899 \\
56,368 \\
56,186 \\
61,951 \\
698,626\end{array}$ & $\begin{array}{r}7,304 \\
6,415 \\
6,368 \\
5,407 \\
5,858 \\
6,859 \\
7,407 \\
7,616 \\
6,885 \\
6,356 \\
6,352 \\
7,321 \\
00,246\end{array}$ & $\begin{array}{l}68,264 \\
60,183 \\
62,705 \\
58,794 \\
60,591 \\
64,122 \\
73,815 \\
70,637 \\
65,867 \\
62,606 \\
62,612 \\
69,365 \\
770,860\end{array}$ & $\begin{array}{r}15,811 \\
12,730 \\
13,492 \\
9,929 \\
7,910 \\
11,372 \\
12,939 \\
10,607 \\
10,456 \\
10,454 \\
10,330 \\
9,749 \\
135,770\end{array}$ & $\begin{array}{r}1,103 \\
806 \\
843 \\
811 \\
843 \\
1,077 \\
1,428 \\
1,011 \\
849 \\
792 \\
1,004 \\
889 \\
11,556\end{array}$ & $\begin{array}{r}16,332 \\
13,093 \\
13,932 \\
10,335 \\
8,385 \\
11,881 \\
13,392 \\
11,067 \\
10,820 \\
10,867 \\
10,803 \\
10,256 \\
141,163\end{array}$ & $\begin{array}{l}582 \\
444 \\
404 \\
404 \\
387 \\
568 \\
974 \\
551 \\
485 \\
379 \\
531 \\
482 \\
, 172 \\
\end{array}$ & $\begin{array}{r}16,915 \\
13,536 \\
14,338 \\
10,740 \\
8,752 \\
12,440 \\
14,367 \\
11,610 \\
11,305 \\
11,246 \\
11,333 \\
10,737 \\
147,335\end{array}$ & $\begin{array}{r}71 \\
76 \\
83 \\
66 \\
50 \\
66 \\
72 \\
116 \\
98 \\
103 \\
93 \\
105 \\
09\end{array}$ & $\begin{array}{r}169,125 \\
170,293 \\
207,656 \\
220,012 \\
238,316 \\
265,882 \\
333,567 \\
300,544 \\
273,670 \\
212,640 \\
189,296 \\
175,608 \\
2,768,608\end{array}$ \\
\hline $\begin{array}{l}1903 \text { January ............... } \\
\text { February ........... } \\
\text { March .................. } \\
\text { April ................. } \\
\text { May .................... } \\
\text { June ................. } \\
\text { July .................... } \\
\text { August ............ } \\
\text { 8-Month Total ... }\end{array}$ & $\begin{array}{r}79 \\
88 \\
101 \\
84 \\
81 \\
80 \\
73 \\
67 \\
653\end{array}$ & $\begin{array}{r}61,793 \\
57,682 \\
60,969 \\
53,722 \\
53,450 \\
61,085 \\
71,124 \\
70,241 \\
400,068\end{array}$ & $\begin{array}{r}7,617 \\
6,431 \\
6,002 \\
5,757 \\
6,570 \\
6,948 \\
7,511 \\
7,624 \\
54,461\end{array}$ & $\begin{array}{r}69,490 \\
64,201 \\
67,073 \\
59,563 \\
60,102 \\
68,113 \\
78,708 \\
77,932 \\
545,182\end{array}$ & $\begin{array}{r}10,804 \\
10,591 \\
12,784 \\
7,629 \\
7,722 \\
11,758 \\
16,896 \\
18,044 \\
96,227\end{array}$ & $\begin{array}{r}1,011 \\
934 \\
1,277 \\
819 \\
867 \\
1,113 \\
1,815 \\
1,570 \\
0,407\end{array}$ & $\begin{array}{r}11,265 \\
11,023 \\
13,313 \\
8,094 \\
8,198 \\
12,249 \\
17,406 \\
18,515 \\
100,063\end{array}$ & $\begin{array}{r}550 \\
502 \\
748 \\
354 \\
392 \\
621 \\
1,305 \\
1,099 \\
8,571\end{array}$ & $\begin{array}{r}11,815 \\
11,525 \\
14,062 \\
8,448 \\
8,580 \\
12,870 \\
18,711 \\
19,614 \\
105,634\end{array}$ & $\begin{array}{r}92 \\
81 \\
87 \\
70 \\
86 \\
98 \\
125 \\
112 \\
750\end{array}$ & $\begin{array}{r}164,400 \\
161,778 \\
193,795 \\
173,700 \\
167,146 \\
254,601 \\
333,406 \\
356,695 \\
1,806,528\end{array}$ \\
\hline $\begin{array}{l}1992 \text { 8-Month Total ... } \\
1991 \text { 8-Month Total ... }\end{array}$ & $\begin{array}{l}852 \\
683\end{array}$ & $\begin{array}{l}465,224 \\
461,001\end{array}$ & $\begin{array}{l}53,235 \\
53,760\end{array}$ & $\begin{array}{l}510,111 \\
515,515\end{array}$ & $\begin{array}{r}94,790 \\
122,481\end{array}$ & $\begin{array}{l}7,923 \\
9,372\end{array}$ & $\begin{array}{r}98,418 \\
128,574\end{array}$ & $\begin{array}{l}4,285 \\
5,280\end{array}$ & $\begin{array}{l}102,713 \\
131,858\end{array}$ & $\begin{array}{l}600 \\
509\end{array}$ & $\begin{array}{l}1,914,304 \\
1,898,081\end{array}$ \\
\hline
\end{tabular}

- Heavy oil includes fuel oil nos. 4, 5, and 6, and resichual fuel oils.

b Light oil includes fuel oil nos. 1 and 2, kerosene, and jot fuel.

c $\mathrm{GT} / \mathrm{KC}=$ Gas turbine and intermal combustion plants.

Includes supplemental gaseous fuels.

NA $=$ Not available.

Notes: - Geographic coverage is the 50 States and the District of Columbia.

- Tolals may not equal sum of components due to independent rounding.

Sources: - Prime Mover Type Data: 1073-Soptember 1077-Federal

Power Commission (FPC), Form FPC.4, "Monthly Power Plant Repon."

October 1077-1981-Federal Energy Regulatory Commission (FERC). Form
FPC-4, "Monthly Power Plant Report." 1082 forward-Energy Inlormation Administration (ELA), Form ElA.759, "Monthly Power Plant Repont." - All Other Data: 1073-September 1077-FPC. Form FPC-4, "Monthly Power Plant Report." Oetober 1077.1970-FERC. Form FPC.4, "Monithy Power Plant Report." 1980_EIA, Eloctric Power Monthly, March 1991, Table 17. - 1001: ElA, Eloctric Power Monthly, March 1982, Table 17. - 1002 and 1091 monthly data: EIA, Eloctric Power Monthy, March 1983, Tablo 17. - 1983 forward (oxcept 1001 monthly data): ElA, Eloctric Power Monthis, November 1993, Table 17. 


\begin{tabular}{|c|c|c|c|c|c|c|c|c|c|c|}
\hline & \multicolumn{4}{|c|}{ Cod } & \multicolumn{6}{|c|}{ Potroleum } \\
\hline & \multirow[b]{2}{*}{ Anthreolto } & \multirow[b]{2}{*}{$\begin{array}{c}\text { Bituminous } \\
\text { Col }\end{array}$} & \multirow[b]{2}{*}{ Uonite } & \multirow[b]{2}{*}{ Total } & \multicolumn{2}{|c|}{$\begin{array}{c}\text { By Typo } \\
\text { of Potroloum }\end{array}$} & \multicolumn{2}{|c|}{$\begin{array}{l}\text { By Prime } \\
\text { Mover Typo }\end{array}$} & \multirow[b]{2}{*}{$\begin{array}{l}\text { Total } \\
\text { Uquide }\end{array}$} & \multirow{3}{*}{$\begin{array}{c}\begin{array}{c}\text { Potroleum } \\
\text { Colks }\end{array} \\
\begin{array}{c}\text { Thounand } \\
\text { Shont Tons }\end{array}\end{array}$} \\
\hline & & & & & $\begin{array}{l}\text { Heany } \\
\text { Olla }\end{array}$ & $\underset{\text { Olf }}{\text { Lloht }}$ & $\begin{array}{l}\text { Steam } \\
\text { Plents }\end{array}$ & OTACo & & \\
\hline & \multicolumn{4}{|c|}{ Thousand Shont Tons } & \multicolumn{5}{|c|}{ Thousand Barreb } & \\
\hline 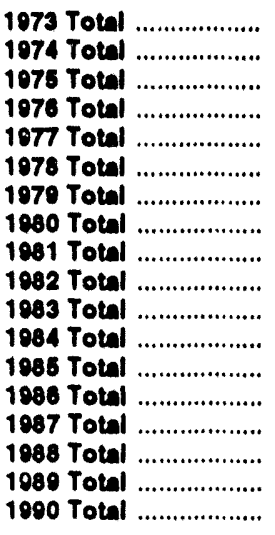 & $\begin{array}{r}1,080 \\
930 \\
982 \\
1,000 \\
2,321 \\
2,170 \\
3,274 \\
4,741 \\
5,537 \\
6,080 \\
6,507 \\
6,710 \\
7,180 \\
7,000 \\
6,040 \\
6,581 \\
6,403 \\
6,409\end{array}$ & $\begin{array}{r}84,041 \\
81,712 \\
107,027 \\
114,130 \\
128,210 \\
123,080 \\
162,081 \\
174,154 \\
188,258 \\
170,480 \\
145,250 \\
167,118 \\
142,144 \\
148,865 \\
168,070 \\
133,434 \\
122,067 \\
142,050\end{array}$ & $\begin{array}{r}081 \\
867 \\
1,815 \\
2,308 \\
2,688 \\
3,027 \\
3,489 \\
4,115 \\
8,008 \\
4,673 \\
3,041 \\
5,009 \\
7,043 \\
8,042 \\
7,187 \\
6,812 \\
6,490 \\
7,010\end{array}$ & 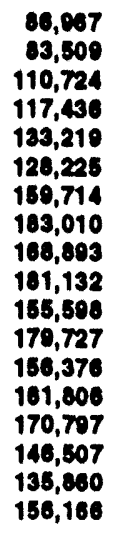 & $\begin{array}{c}N A \\
N A \\
N A \\
N A \\
N A \\
N A \\
N A \\
108,381 \\
102,042 \\
95,515 \\
70,573 \\
68,503 \\
57,304 \\
84,841 \\
58,000 \\
84,187 \\
\mathbf{4 7 , 4 4 0} \\
67,030\end{array}$ & $\begin{array}{c}N A \\
N A \\
N A \\
N A \\
N A \\
N A \\
N A \\
30,023 \\
26,004 \\
23,390 \\
18,801 \\
10,116 \\
18,398 \\
16,280 \\
15,780 \\
15,000 \\
13,024 \\
18,471\end{array}$ & 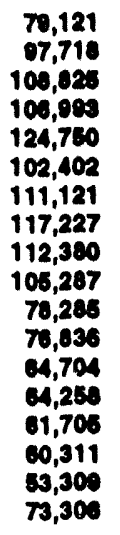 & 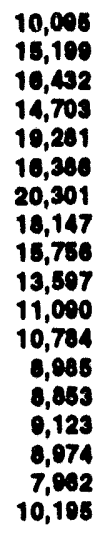 & $\begin{array}{r}89,216 \\
112,017 \\
128,267 \\
121,060 \\
144,031 \\
118,789 \\
131,420 \\
136,374 \\
128,136 \\
118,884 \\
80,378 \\
87,810 \\
73,689 \\
73,111 \\
70,027 \\
80,288 \\
81,270 \\
83,501\end{array}$ & $\begin{array}{r}312 \\
38 \\
31 \\
32 \\
44 \\
108 \\
103 \\
52 \\
42 \\
41 \\
56 \\
80 \\
40 \\
40 \\
51 \\
86 \\
108 \\
94\end{array}$ \\
\hline $\begin{array}{l}1001 \text { January } \\
\text { February .................. } \\
\text { March ................. } \\
\text { April .................... } \\
\text { May ................... } \\
\text { June ................... } \\
\text { Juty .................... } \\
\text { August ............... } \\
\text { September ........ } \\
\text { October .............. } \\
\text { November .......... } \\
\text { December .......... }\end{array}$ & $\begin{array}{l}6,470 \\
6,442 \\
\mathbf{6 , 3 8 4} \\
\mathbf{6 , 3 4 7} \\
\mathbf{6 , 3 8 7} \\
\mathbf{6 , 4 4 1} \\
\mathbf{6 , 4 8 4} \\
\mathbf{6 , 5 0 8} \\
\mathbf{6 , 5 1 4} \\
\mathbf{6 , 5 4 4} \\
\mathbf{6 , 5 3 3} \\
\mathbf{6 , 5 1 3}\end{array}$ & $\begin{array}{l}138,220 \\
142,454 \\
147,460 \\
152,830 \\
154,172 \\
150,554 \\
142,804 \\
140,320 \\
141,463 \\
146,178 \\
145,775 \\
148,367\end{array}$ & $\begin{array}{l}7,407 \\
7,220 \\
7,231 \\
7,135 \\
6,968 \\
6,463 \\
6,392 \\
6,272 \\
5,930 \\
6,090 \\
6,298 \\
8,008\end{array}$ & $\begin{array}{l}152,097 \\
156,116 \\
161,084 \\
166,315 \\
167,528 \\
163,459 \\
155,680 \\
153,097 \\
153,907 \\
158,813 \\
158,605 \\
187,076\end{array}$ & $\begin{array}{l}\mathbf{6 4 , 3 4 4} \\
\mathbf{6 0 , 4 9 0} \\
\mathbf{5 8 , 1 7 2} \\
\mathbf{5 8 , 8 3 5} \\
\mathbf{5 7 , 2 4 7} \\
\mathbf{5 8 , 3 4 5} \\
\mathbf{5 7 , 9 3 2} \\
\mathbf{5 8 , 5 8 8} \\
\mathbf{5 9 , 0 3 5} \\
\mathbf{6 0 , 2 2 5} \\
\mathbf{5 8 , 8 1 4} \\
\mathbf{6 8 , 6 3 0}\end{array}$ & $\begin{array}{l}16,601 \\
16,892 \\
16,376 \\
16,175 \\
15,574 \\
15,680 \\
15,654 \\
15,596 \\
15,514 \\
15,790 \\
15,780 \\
16,367\end{array}$ & $\begin{array}{l}70,744 \\
67,367 \\
64,699 \\
65,393 \\
63,531 \\
64,604 \\
64,119 \\
62,813 \\
65,168 \\
66,257 \\
64,963 \\
68,032\end{array}$ & $\begin{array}{r}10,201 \\
10,014 \\
9,848 \\
9,618 \\
9,290 \\
9,421 \\
9,467 \\
9,370 \\
9,363 \\
9,758 \\
9,631 \\
9,461\end{array}$ & $\begin{array}{l}80,945 \\
77,382 \\
74,547 \\
75,011 \\
72,822 \\
74,025 \\
73,586 \\
72,183 \\
74,550 \\
76,015 \\
74,594 \\
74,093\end{array}$ & $\begin{array}{r}103 \\
111 \\
101 \\
80 \\
81 \\
89 \\
86 \\
79 \\
73 \\
64 \\
75 \\
70\end{array}$ \\
\hline 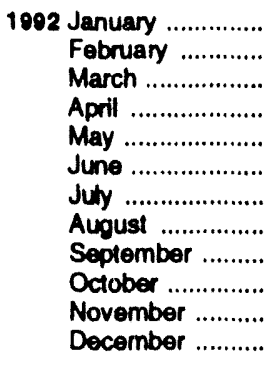 & $\begin{array}{l}6,488 \\
6,455 \\
6,398 \\
6,379 \\
6,370 \\
6,355 \\
6,341 \\
6,343 \\
6,329 \\
6,304 \\
6,273 \\
6,215\end{array}$ & $\begin{array}{l}143,468 \\
146,338 \\
147,978 \\
149,824 \\
152,275 \\
151,224 \\
141,613 \\
140,168 \\
140,409 \\
144,068 \\
145,408 \\
142,156\end{array}$ & $\begin{array}{l}\mathbf{5 , 6 8 3} \\
\mathbf{5 , 3 5 2} \\
\mathbf{5 , 6 5 6} \\
\mathbf{6 , 3 8 7} \\
\mathbf{6 , 8 6 7} \\
\mathbf{6 , 5 8 6} \\
\mathbf{6 , 4 4 9} \\
\mathbf{6 , 0 7 1} \\
\mathbf{5 , 9 4 6} \\
\mathbf{6 , 4 8 7} \\
\mathbf{6 , 1 6 9} \\
\mathbf{6 , 7 5 0}\end{array}$ & $\begin{array}{l}155,637 \\
158,145 \\
160,032 \\
162,591 \\
165,512 \\
164,176 \\
154,403 \\
152,580 \\
152,685 \\
156,869 \\
157,849 \\
154,130\end{array}$ & $\begin{array}{l}53,136 \\
54,750 \\
54,513 \\
52,815 \\
55,144 \\
53,794 \\
53,445 \\
54,434 \\
52,731 \\
52,819 \\
53,632 \\
56,136\end{array}$ & $\begin{array}{l}15,712 \\
15,655 \\
15,589 \\
15,371 \\
15,214 \\
15,117 \\
14,995 \\
15,456 \\
15,251 \\
15,351 \\
15,302 \\
15,714\end{array}$ & $\begin{array}{l}59,340 \\
61,086 \\
60,840 \\
59,044 \\
61,145 \\
59,648 \\
59,273 \\
60,644 \\
58,646 \\
58,869 \\
59,536 \\
62,374\end{array}$ & $\begin{array}{l}9,500 \\
9,321 \\
9,262 \\
9,143 \\
9,214 \\
9,263 \\
9,167 \\
9,246 \\
9,336 \\
9,400 \\
9,398 \\
0,475\end{array}$ & $\begin{array}{l}68,849 \\
70,408 \\
70,103 \\
68,188 \\
70,358 \\
68,910 \\
68,440 \\
69,890 \\
67,980 \\
68,260 \\
68,934 \\
71,840\end{array}$ & $\begin{array}{l}75 \\
62 \\
56 \\
47 \\
63 \\
67 \\
56 \\
46 \\
51 \\
55 \\
59 \\
67\end{array}$ \\
\hline 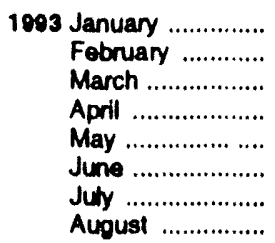 & $\begin{array}{l}6,166 \\
6,107 \\
6,036 \\
5,802 \\
5,773 \\
5,766 \\
5,755 \\
5,745\end{array}$ & $\begin{array}{l}138,685 \\
134,674 \\
132,183 \\
136,159 \\
138,165 \\
133,673 \\
115,184 \\
102,612\end{array}$ & $\begin{array}{l}5,521 \\
5,357 \\
5,758 \\
6,088 \\
\mathbf{6 , 1 3 2} \\
6,009 \\
5,686 \\
5,651\end{array}$ & $\begin{array}{l}150,371 \\
146,139 \\
143,978 \\
148,049 \\
150,070 \\
145,448 \\
126,635 \\
114,008\end{array}$ & $\begin{array}{l}53,781 \\
50,009 \\
45,313 \\
47,858 \\
50,422 \\
40,294 \\
47,401 \\
43,943\end{array}$ & $\begin{array}{l}15,058 \\
15,205 \\
15,001 \\
14,805 \\
14,682 \\
14,923 \\
14,605 \\
14,830\end{array}$ & $\begin{array}{l}60,200 \\
56,306 \\
51,528 \\
54,069 \\
56,512 \\
55,595 \\
53,631 \\
50,223\end{array}$ & $\begin{array}{l}9,527 \\
8,907 \\
8,785 \\
8,724 \\
8,591 \\
8,621 \\
8,376 \\
8,550\end{array}$ & $\begin{array}{l}69,738 \\
65,213 \\
60,314 \\
62,793 \\
65,103 \\
64,217 \\
62,007 \\
58,772\end{array}$ & $\begin{array}{l}65 \\
60 \\
66 \\
77 \\
82 \\
92 \\
73 \\
99\end{array}$ \\
\hline
\end{tabular}

- Heavy oll includes fuel oil nos. 4,5, and 6, and residual fuel olls.

- Ught oil includes fuel oll nos. 1 and 2, kerosene, and let luel.

- $\mathrm{GT} / \mathrm{C}=$ Gas turbine and intemal combustion plants.

NA=Not available.

Notes: - Geographic coverage is the 50 States and the Distrlct of Columbia.

- Totals may not equal sum of components due to independent rounding.

Sources: - Prime Mover Typo Datu: 1973-Septomber 1077-Federal

Power Commission (FPC), Form FPC-4, "Monthly Power Plant Report."

Oetober 1077.1061 Federal Energy Regulatory Commiseion (FERC), Form
FPC.4, "Monthly Power Plant Repont" 1022 fonward-Eneroy Inlormation Administration (EIA), Form EL-759, "Monthly Power Plant Report." - All Other Data: 1073-september 1077-FPC, Form FPC-4, Monthly Power Plant Report." Oetober 1077.1970-FERC, Form FPC.4. "Monthy Power Plant Report: 1080-EIA, Electric Power Monthly, March 1901, Table 28. 1081-ElA, Electric Power Monthy, March 1092, Table 28, 1082 and 1091 menthly dats-ELA, Electric Power Monthy, March 1993, Table 28. 1083 Ionward (cxcept 1001 monthly data)-EIA, Eloctric Power Monthly. November 1993, Table 28. 


\section{Section 8. Nuclear Energy}

In August 1993, U.S. nuclear generating units produced a total of 56 net terawatthours (billion kilowatthours) of electricity, 4 percent $^{8}$ less than in August 1992. Nuclear units generated at an average capacity factor of 76.3 percent, 3 percentage points lower than in August 1992. Nuclear power supplied 20.1 percent of the total electric utility-generated electricity in August 1993, compared with 23.0 percent in August 1992.

No low- or full power licenses for nuclear power plants were issued by Nuclear Regulatory Commission during August 1993.

On August 31, 1993, there were 109 operable nuclear generating units in the United States, with a collective net summer capability of 99.0 million kilowatts of electricity. Of the 109 operable units, 18 units generated at less than 25 percent of capacity because of maintenance, refueling, or repair outage, and 14 of the 18 units generated no electricity during the month.

Two operable units, Browns Ferry 1 and 3, have been shut down since March 1985. Each unit had a capacity of 1.065 megawatts electric.

As of August 31, there were 116 domestic nuclear generating units in all stages of construction and operation. The aggregate net design capacity of operable units was 101.1 million kilowatts, and the design capacity of units under construction was $\mathbf{8 . 5}$ million kilowatts, for a total design capacity of 109.6 million kilowatts. 
Flgure 8.1 Nuclear Power Plant Operations

Operable Units, End of Year, 1973-1992

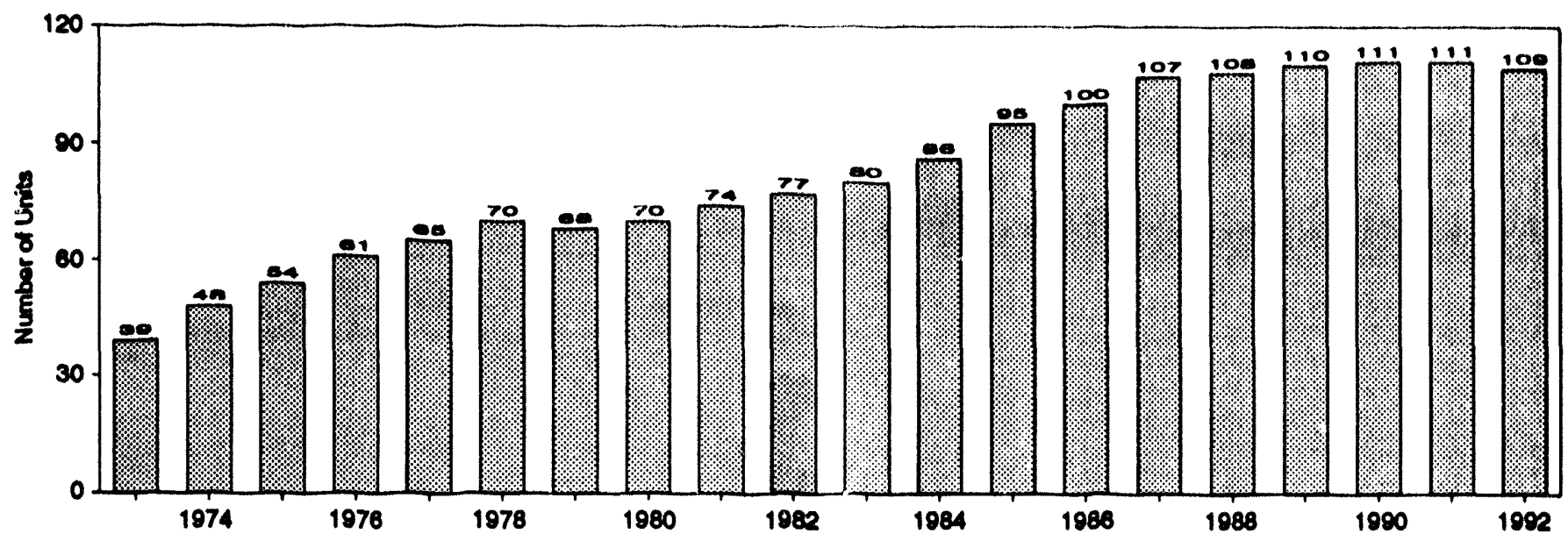

Net Generation of Electricity, 1973-1992

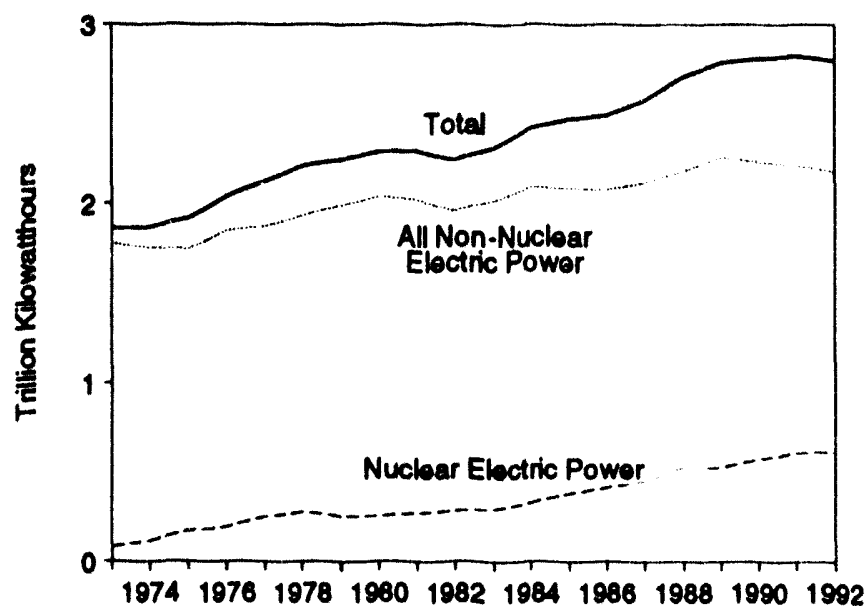

Nuclear Electricity Net Generation

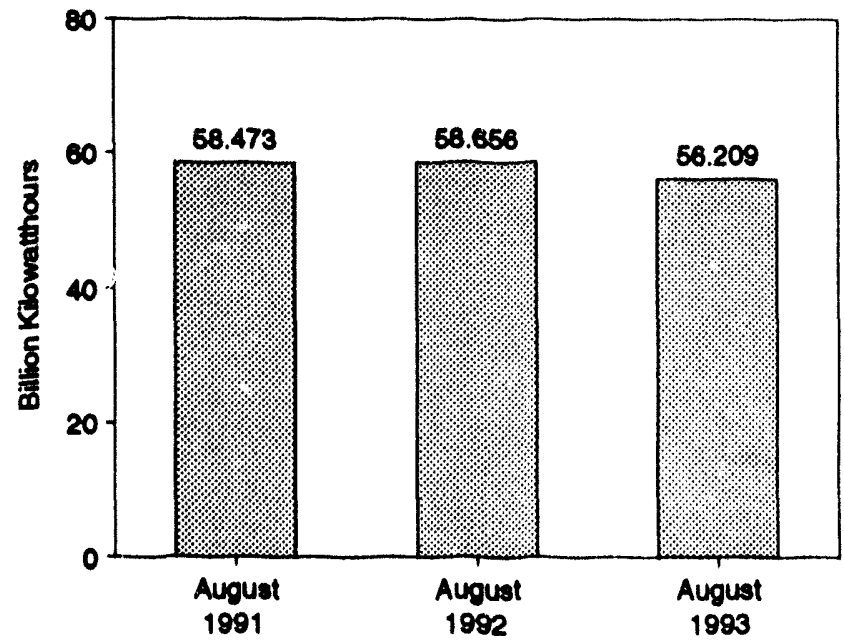

Nuclear Portion of Domestic Electricity

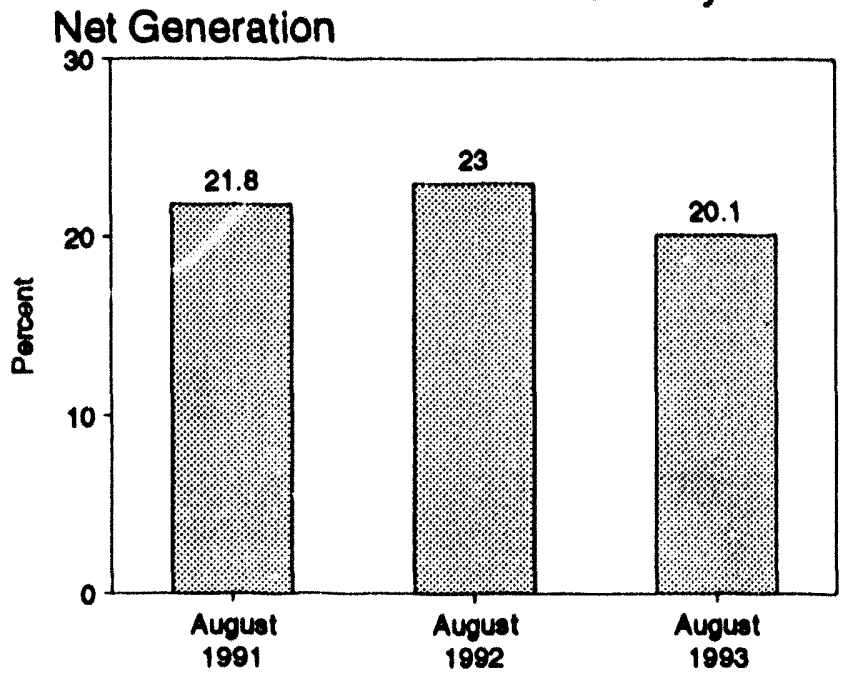

Capacity Factor, Monthly

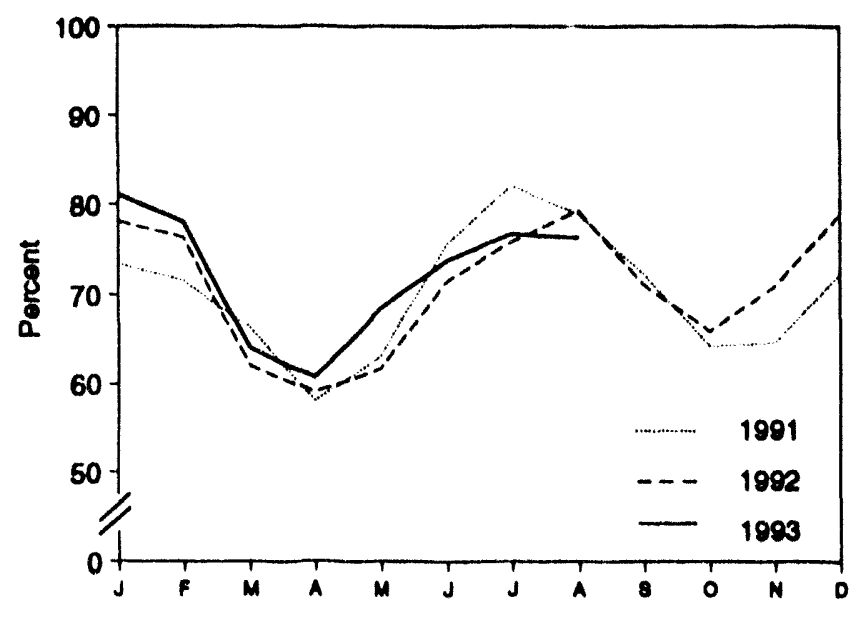

Note: Because vertical scales after, graphs should not be compared. Sources: Tables 7.1 and 8.1 . 


\begin{tabular}{|c|c|c|c|c|c|}
\hline & $\begin{array}{l}\text { Operable } \\
\text { Units a,b }\end{array}$ & $\begin{array}{c}\text { Nuclear } \\
\text { Electricity } \\
\text { Net } \\
\text { Generation }\end{array}$ & $\begin{array}{l}\text { Nuciear } \\
\text { Portion of } \\
\text { Domentie } \\
\text { Electrielty } \\
\text { Net Generation }\end{array}$ & $\begin{array}{c}\text { Mot } \\
\text { Summer } \\
\text { Capabillty } \\
\text { of Operable } \\
\text { Unitea,c }\end{array}$ & $\begin{array}{l}\text { Capacity } \\
\text { Factor }\end{array}$ \\
\hline & Number & $\begin{array}{c}\text { Million } \\
\text { Kllowatthours }\end{array}$ & Percent & $\begin{array}{l}\text { Million } \\
\text { Kilowatts }\end{array}$ & Percent \\
\hline 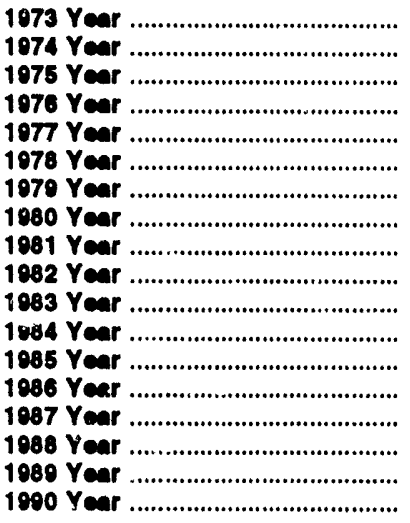 & $\begin{array}{r}30 \\
68 \\
54 \\
61 \\
65 \\
70 \\
68 \\
70 \\
74 \\
77 \\
80 \\
86 \\
85 \\
100 \\
107 \\
108 \\
110 \\
111\end{array}$ & $\begin{array}{r}83,470 \\
113,076 \\
172,505 \\
101,104 \\
250,863 \\
276,403 \\
255,155 \\
251,116 \\
272,674 \\
282,773 \\
203,677 \\
327,634 \\
383,601 \\
414,038 \\
455,270 \\
526,073 \\
529,355 \\
576,862\end{array}$ & $\begin{array}{r}4.5 \\
6.1 \\
0.0 \\
9.4 \\
11.8 \\
12.5 \\
11.4 \\
11.0 \\
11.9 \\
12.6 \\
12.7 \\
13.6 \\
15.5 \\
16.6 \\
17.7 \\
10.5 \\
10.0 \\
20.5\end{array}$ & $\begin{array}{l}22.683 \\
31.867 \\
37.267 \\
43.822 \\
\mathbf{4 6 . 3 0 3} \\
\mathbf{5 0 . 8 2 4} \\
49.747 \\
\mathbf{5 1 . 8 1 0} \\
\mathbf{5 6 . 0 4 2} \\
\mathbf{6 0 . 0 3 5} \\
\mathbf{6 3 . 0 0 0} \\
\mathbf{6 9 . 8 5 2} \\
\mathbf{7 9 . 3 9 7} \\
\mathbf{8 5 . 2 4 1} \\
\mathbf{9 3 . 5 8 3} \\
94.605 \\
98.161 \\
09.624\end{array}$ & $\begin{array}{l}53.5 \\
47.8 \\
55.0 \\
54.7 \\
63.3 \\
64.5 \\
58.4 \\
56.3 \\
58.2 \\
56.6 \\
54.4 \\
56.3 \\
58.0 \\
56.0 \\
57.4 \\
63.5 \\
62.2 \\
66.0\end{array}$ \\
\hline 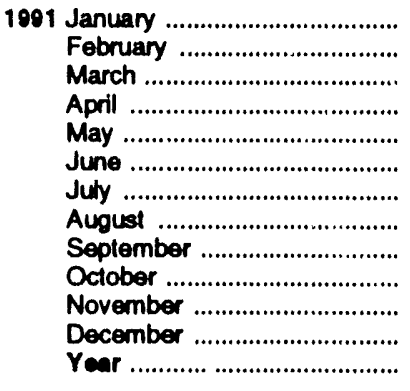 & $\begin{array}{l}111 \\
111 \\
111 \\
111 \\
111 \\
111 \\
111 \\
111 \\
111 \\
111 \\
111 \\
111 \\
111\end{array}$ & $\begin{array}{l}54,369 \\
47,863 \\
49,121 \\
41,631 \\
46,755 \\
54,208 \\
60,735 \\
58,473 \\
51,874 \\
47,653 \\
46,295 \\
53,589 \\
612,565\end{array}$ & $\begin{array}{l}21.9 \\
22.7 \\
22.2 \\
19.9 \\
19.9 \\
21.8 \\
22.3 \\
21.8 \\
22.2 \\
21.3 \\
20.9 \\
22.9 \\
21.7\end{array}$ & $\begin{array}{l}99.624 \\
99.624 \\
99.624 \\
99.624 \\
99.624 \\
99.624 \\
99.589 \\
99.589 \\
99.589 \\
99.589 \\
99.589 \\
99.589 \\
99.589\end{array}$ & $\begin{array}{l}73.4 \\
71.5 \\
66.3 \\
58.1 \\
63.1 \\
75.6 \\
82.0 \\
78.9 \\
72.3 \\
64.2 \\
64.6 \\
72.3 \\
70.2\end{array}$ \\
\hline 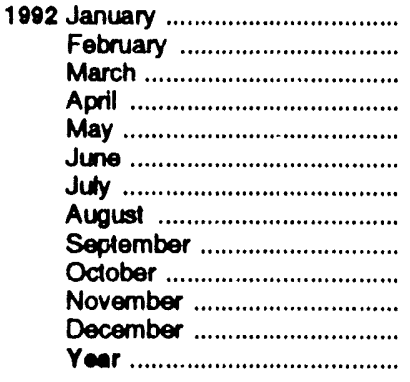 & $\begin{array}{l}111 \\
110 \\
110 \\
110 \\
110 \\
110 \\
110 \\
110 \\
110 \\
110 \\
110 \\
109 \\
100\end{array}$ & $\begin{array}{l}57,849 \\
52,804 \\
45,835 \\
42,268 \\
45,627 \\
51,185 \\
56,049 \\
58,656 \\
50,919 \\
48,784 \\
50,726 \\
58,075 \\
618,776\end{array}$ & $\begin{array}{l}23.7 \\
24.2 \\
20.4 \\
20.0 \\
20.7 \\
21.6 \\
21.1 \\
23.0 \\
21.7 \\
22.0 \\
22.9 \\
23.8 \\
22.1\end{array}$ & $\begin{array}{l}99.589 \\
99.422 \\
99.422 \\
99.422 \\
99.422 \\
99.422 \\
99.422 \\
99.422 \\
99.422 \\
99.422 \\
99.422 \\
98.986 \\
98.086\end{array}$ & $\begin{array}{l}78.1 \\
76.3 \\
62.0 \\
59.1 \\
61.7 \\
71.5 \\
75.8 \\
79.3 \\
71.1 \\
65.9 \\
70.9 \\
78.9 \\
70.9\end{array}$ \\
\hline 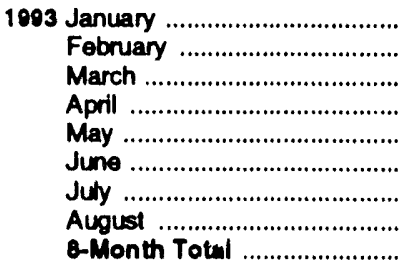 & $\begin{array}{l}108 \\
108 \\
108 \\
109 \\
109 \\
109 \\
109 \\
109 \\
109\end{array}$ & $\begin{array}{r}59,076 \\
51,319 \\
46,606 \\
43,199 \\
50,367 \\
52,620 \\
56,502 \\
56,209 \\
415,808\end{array}$ & $\begin{array}{l}24.0 \\
22.8 \\
19.9 \\
20.4 \\
22.6 \\
21.1 \\
20.0 \\
20.1 \\
21.3\end{array}$ & $\begin{array}{l}97.882 \\
97.882 \\
97.882 \\
99.032 \\
99.032 \\
99.032 \\
99.031 \\
99.031 \\
99.031\end{array}$ & $\begin{array}{l}81.1 \\
78.0 \\
64.0 \\
60.7 \\
68.4 \\
73.8 \\
76.7 \\
76.3 \\
72.3\end{array}$ \\
\hline 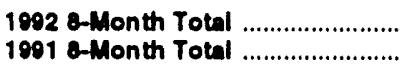 & $\begin{array}{l}110 \\
111\end{array}$ & $\begin{array}{l}410,272 \\
413,154\end{array}$ & $\begin{array}{l}21.0 \\
21.6\end{array}$ & $\begin{array}{l}09.422 \\
00.589\end{array}$ & $\begin{array}{l}70.5 \\
71.1\end{array}$ \\
\hline
\end{tabular}

At end of period.

- See Nole 1 al end of section.

c For the definition of "Net Summer Capability." see Note 3 at end of section.

For an explanation of the method of calculating the capacity factor, see Note 4 at end of section.

Notes: - Geographic coverage is the 50 States and the District of Columbia.

- Nuclear electricity nel generation totals may not equal sum of components due to independent rounding.

Sources: - Operable Units: 1073-1082-U.S. Department of Energy (DOE), Otice of Nuclear Programs, U.S. Central Siation Nuclear Eloctric

Generating Units: Signilicant Milestones." 1083 forward-Nuclear Regulatory Commission (NRC), "Licensed Operating Reactors" (NUREG-0020). - Nuclear Electricity Not Generation: Table 7.1. - Nuclear Portion of Domestic Electrlelty Not Generation: Calculated from data in Table 7.1. - Net Summer Capability of Operable Units: 1973-1082-Complled from various sources, primarily DOE, Otfice of Nuclear Reactor Programs, "U.S. Central Station Nuclear Electric Generating Units: Signilicant Milestones." 1983 forward-Energy Information Administration (EIA), Form ElA-860, "Annual Electric Generation Repont" and monthly updales as appropriate. - Cepacity Factor: EIA, Otfice of Coal, Nuclear, Eloctric and Altemate Fuels. 
Table 8.2 Nuclear Generating Units, End of Period

\begin{tabular}{|c|c|c|c|c|c|c|c|c|}
\hline & \multicolumn{2}{|c|}{$\begin{array}{l}\text { Lleensed } \\
\text { for Operation }\end{array}$} & \multicolumn{2}{|c|}{$\begin{array}{l}\text { Construction } \\
\text { Pormits }\end{array}$} & \multirow[b]{2}{*}{ On Order } & \multirow[b]{2}{*}{ Announced } & \multirow[b]{2}{*}{ Total } & \multirow{2}{*}{$\frac{\begin{array}{c}\text { Total } \\
\text { Deslgn } \\
\text { Capacityc }\end{array}}{\begin{array}{c}\text { Million } \\
\text { Kilowatts }\end{array}}$} \\
\hline & Oporablea & In Startupb & Granted & Pending & & & & \\
\hline 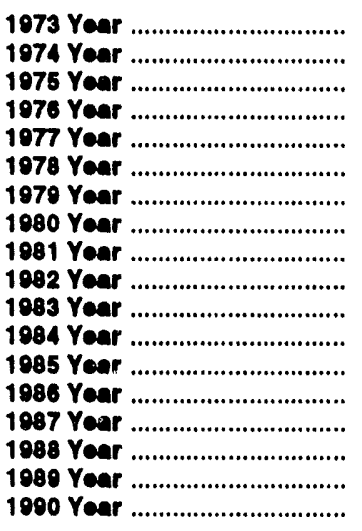 & $\begin{array}{l}30 \\
48 \\
54 \\
61 \\
65 \\
70 \\
68 \\
70 \\
74 \\
77 \\
80 \\
86 \\
95 \\
100 \\
107 \\
108 \\
110 \\
111\end{array}$ & $\begin{array}{l}2 \\
5 \\
2 \\
1 \\
2 \\
0 \\
0 \\
1 \\
0 \\
2 \\
3 \\
6 \\
3 \\
7 \\
4 \\
3 \\
1 \\
0\end{array}$ & $\begin{array}{l}57 \\
62 \\
89 \\
71 \\
76 \\
80 \\
90 \\
82 \\
76 \\
60 \\
53 \\
38 \\
30 \\
10 \\
14 \\
12 \\
10 \\
8\end{array}$ & $\begin{array}{l}52 \\
75 \\
60 \\
63 \\
40 \\
32 \\
21 \\
12 \\
11 \\
3 \\
0 \\
0 \\
0 \\
0 \\
0 \\
0 \\
0 \\
0\end{array}$ & $\begin{array}{r}49 \\
30 \\
14 \\
16 \\
13 \\
5 \\
3 \\
3 \\
2 \\
2 \\
2 \\
2 \\
2 \\
2 \\
2 \\
0 \\
0 \\
0\end{array}$ & $\begin{array}{l}0 \\
0 \\
5 \\
2 \\
2 \\
0 \\
0 \\
0 \\
0 \\
0 \\
0 \\
0 \\
0 \\
0 \\
0 \\
0 \\
0 \\
0\end{array}$ & $\begin{array}{l}208 \\
226 \\
213 \\
214 \\
200 \\
105 \\
185 \\
168 \\
183 \\
144 \\
138 \\
132 \\
130 \\
128 \\
127 \\
123 \\
121 \\
110\end{array}$ & $\begin{array}{l}198 \\
223 \\
212 \\
211 \\
203 \\
191 \\
180 \\
162 \\
157 \\
134 \\
120 \\
123 \\
121 \\
110 \\
110 \\
115 \\
113 \\
111\end{array}$ \\
\hline 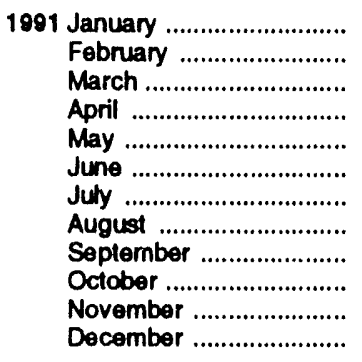 & $\begin{array}{l}111 \\
111 \\
111 \\
111 \\
111 \\
111 \\
111 \\
111 \\
111 \\
111 \\
111 \\
111\end{array}$ & $\begin{array}{l}0 \\
0 \\
0 \\
0 \\
0 \\
0 \\
0 \\
0 \\
0 \\
0 \\
0 \\
0\end{array}$ & $\begin{array}{l}8 \\
8 \\
8 \\
8 \\
8 \\
8 \\
8 \\
8 \\
8 \\
8 \\
8 \\
8\end{array}$ & $\begin{array}{l}0 \\
0 \\
0 \\
0 \\
0 \\
0 \\
0 \\
0 \\
0 \\
0 \\
0 \\
0\end{array}$ & $\begin{array}{l}0 \\
0 \\
0 \\
0 \\
0 \\
0 \\
0 \\
0 \\
0 \\
0 \\
0 \\
0\end{array}$ & $\begin{array}{l}0 \\
0 \\
0 \\
0 \\
0 \\
0 \\
0 \\
0 \\
0 \\
0 \\
0 \\
0\end{array}$ & $\begin{array}{l}119 \\
119 \\
119 \\
119 \\
119 \\
119 \\
119 \\
119 \\
119 \\
119 \\
119 \\
119\end{array}$ & $\begin{array}{l}111 \\
111 \\
111 \\
111 \\
111 \\
111 \\
111 \\
111 \\
111 \\
111 \\
111 \\
111\end{array}$ \\
\hline 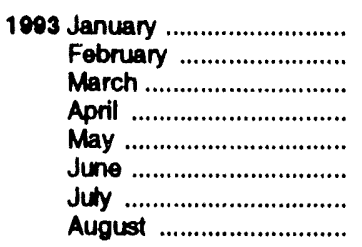 & $\begin{array}{l}108 \\
108 \\
108 \\
109 \\
109 \\
109 \\
109 \\
109\end{array}$ & $\begin{array}{l}0 \\
1 \\
1 \\
0 \\
0 \\
0 \\
0 \\
0\end{array}$ & $\begin{array}{l}8 \\
7 \\
7 \\
7 \\
7 \\
7 \\
7 \\
7\end{array}$ & $\begin{array}{l}0 \\
0 \\
0 \\
0 \\
0 \\
0 \\
0 \\
0\end{array}$ & $\begin{array}{l}0 \\
0 \\
0 \\
0 \\
0 \\
0 \\
0 \\
0\end{array}$ & $\begin{array}{l}0 \\
0 \\
0 \\
0 \\
0 \\
0 \\
0 \\
0\end{array}$ & $\begin{array}{l}116 \\
116 \\
116 \\
116 \\
116 \\
116 \\
116 \\
116\end{array}$ & $\begin{array}{l}110 \\
110 \\
110 \\
110 \\
110 \\
110 \\
110 \\
110\end{array}$ \\
\hline
\end{tabular}

See Note 1 at end of section.

bee Note 2 at end of section.

c Net design electrical rating (DER) is used because many of the units were canceled prior to being assigned a net summer capability. See Note 3 at end of section.

Note: Geographic coverage is the $\mathbf{5 0}$ States and the District of Columbla.

Sources: - Liceneed for Operation: 1973-1982-U.S. Department of Energy (DOE), Office of Nuclear Programs, "U.S. Central Station Nuclear Electric Generating Units: Significant Milestones." 1083 forward-Nuclear Regulatory Commission (NRC), "Licensed Operating Reactors" (NUREG-0020). Construction Permits, On Order, and Announced:

1973-1982-Complled from vartous sources, primarily DOE, Otfice of Nuclear Reactor Programs, "U.S. Central Station Nudear Electric Generating Units: Significart Milestones"; Energy Intormation Administration (ELA), Office of Coal, Nuclear, Electric and Altemale Fuels (CNEAF), "Nuclear Steam-Eleciric
Units That Have Been in Operation as of 1957-1989"; EIA, CNEAF, "Nuchar Plant Cancellations: Causes, Costs, and Consequences'; and Utilify Dala Institute, Inc., "U.S. Nuclear Plant Statistics, 1987." 1983 torward-NRC, "Summary Information Report" (NUREG-0871); NRC, "Licensed Operating Peactors" (NUREG-0020); and various joumals. - Total Design Capacity: 1973-1062-Complled from various sources, primarily DOE, Otfice of Nuclear Reactor Programs, "U.S. Central Station Nuclear Eloctric Generating Units: Signilicant Milestones"; EIA, CNEAF. "Nuclear Steam-Electric Units That Have Been in Operation as of 1957-1987"; EIA, CNEAF, "Monthly Report for Electric Utillies-Power Generation"; EIA, CNEAF, "Nuclear Plant Cancellations: Causes, Costs, and Consequences"; and Uility Data Institute, Inc., "U.S. Nuclear Plant Statistics, 1987:" 1083 forward-NRC, "Summary Information Report" (NUREG-0871); NRC, "Licensed Operating Reactors" (NUREG-0020); and EIA, Form EIA-860, "Annual Electric Generalor Repont." 


\section{Nuclear Energy Notes}

1. Operable Units: Nuclear generating units that have been issued a full-power license by the Nuclear Regulatory Commission (NRC).

Exceptions: The Shippingport (60 megawatts (MW)) and the Hanford-N (840 MW) nuclear units were included in the operable units until 1982 and 1988, respectively. The Shippingport unit was excluded from the operable category during March 1974-August 1977 due to a major core modification outage. Hanford-N, an unlicensed unit used for defense material production, was included in the operable category because power was produced as by-product and sold commercially. Three Mile Island 2 ( $880 \mathrm{MW}$ ) experienced a major accident in 1979 and, although that unit still retains its operating license and site cleanup continues, there is no plan to restart it. Therefore, it has not been included in the operable category since March 1979. Although Shoreham received a full-power license in April 1989, the unit is not currently scheduled to operate and, therefore, has not been included in the operable category. Rancho Seco (873 MW) was shut down by the Sacramento Municipal Utility District (SMUD) in June 1989 following a referendum on its continued operation. Because there are currently no plans to operate it as a nuclear unit, it is no longer included as an operable unit but is identified as a unit shut down for an extended period. As soon as SMUD and the NRC formalize the plant's official retirement, it will be noted as such in this report. The Department of Energy-operated Experimental Breeder Reactor 2 unit is not a commercial reactor and is therefore not included in the operable category.

In addition, nine units have been retired and therefore removed from the operable category. Those units are: Peach Bottom 1 (40 MW) and Indian Point 1 (265 MW), both retired in 1974; Humboldt Bay (65 MW), officially retired in 1976; Dresden 1 (200 MW), retired in August 1979; LaCrosse (51 MW), retired in May 1987; Fort Saint Vrain (217 MW), retired in August 1989; Yankee Rowe 1 (185 MW), retired in February 1992; San Onofre 1 (436 MW), retired in December 1992; and Trojan (1,104 MW), retired in January 1993.

2. In Startup: The period of time between a nuclear generating unit's initial fuel loading date and the issuance of its full-power license. During that period, the unit is undergoing low-power testing and the maximum level of operation is 5 percent of the unit's design thermal rating.

3. Capacity: Nuclear generating units may have more than one type of net capacity rating, including the following:

(a) Net Summer Capability-The steady hourly output that generating equipment is expected to supply to system load, exclusive of auxiliary power, as demonstrated by test at the time of summer peak demand. Auxiliary power of a typical nuclear power plant is about 5 percent of gross generation.

(b) Net Design Capacity or Net Design Electrical Rating (DER)-The nominal net electrical output of a unit, specified by the utility and used for plant design.

4. Monthly Capacity Factors: The monthly capacity factors are computed as the actual monthly generation divided by the maximum possible generation for that month. The maximum possible generation is the number of hours in the month multiplied by the net summer capability at the end of the month. That fraction is then multiplied by 100 to obtain a percentage. Annual capacity factors are averages of the monthly values for that year. 


\section{Section 9. Energy Prices}

Crude Oil. The average price of domestic crude oil purchased at the wellhead was $\$ 13.71$ per barrel in August 1993, 20 percent below the level in August 1992. The refiner acquisition cost of imported crude oil in August 1993 was $\$ 15.62$ per barrel, 19 percent below the August 1992 level. The average cost of domestic crude oil in August 1993 was \$16.03, 19 percent less than the August 1992 average.

Motor Gasoline. The national city average retail price of ur.leaded regular gasoline at all types of stations was $\$ 1.09$ per gallon in September 1993, 6 percent lower than the price in September 1992. The price of unleaded premium gasoline averaged $\$ 1.28$ per gallon in September 1993, 5 percent lower than the price in September 1992.

Residual Fuel Oil. The average price, excluding taxes, of residual fuel oil sold to end users in August 1993 was 32 cents per gallon, 5 percent lower than the previous month's price and 18 percent below the August 1992 average. The average resale price, excluding taxes, of residual fuel oil in August 1993 was 27 cents per gallon, slightly lower than the July 1993 average and 21 percent below the price 1 year earlier.

Aviation Fuel. The average price, excluding taxes, of aviation gasoline sold to end users in August 1993 was 99 cents per gallon, 1 percent lower than the previous month's price and 7 percent lower than the August 1992 price. The average price, excluding taxes, of kerosene-type jet fuel sold to end users in August 1993 was 55 cents per gallon, 1 percent lower than the previous month's average price and 15 percent lower than the August 1992 average price.

No. 2 Distillate Fuel Oil. The August 1993 national average price, excluding taxes, of heating oil sold to residential customers was 84 cents per gallon, 2 percent lower than the July 1993 price and 5 percent lower than the August 1992 price. The average price of No. 2 fuel oil sold to all end users was 55 cents per gallon in August 1993, 1 percent higher than the July 1993 price but 12 percent lower than the August 1992 price.

Electricity. The average price of electricity sold to all ultimate consumers in the United States in August 1993 was 7.3 cents per kilowatthour, 1 percent above the August 1992 mean price. The price of electricity sold to residential consumers in August 1993 averaged 8.7 cents per kilowatthour, 1 percent above the August 1992 price. The price of electricity sold to commercial consumers averaged 8.0 cents per kilowatthour in August 1993, 1 percent above the August 1992 price. The price of electricity sold to other consumers was 7.0 cents per kilowatthour, 1 percent above the August 1992 price. The price of electricity sold to industrial users in August 1993 averaged 5.2 cents per kilowatthour, 2 percent higher than the price 1 year earlier.

Beginning with January 1986, there were new series of national average price estimates based on a statistically derived sample of both publicly and privately owned electric utilities. Previously, average price estimates were derived from selected privately owned electric utilities and were not national averages.

Natural Gas. The estimated average wellhead price of natural gas for August 1993 was $\$ 2.07$ per thousand cubic feet, 13 percent above the August 1992 price.

The average price of natural gas delivered to electric utility plants was $\$ 2.46$ per thousand cubic feet in July 1993 (latest date for which data are available), 15 percent above the July 1992 price. The average price of natural gas used by residential consumers in August 1993 was $\$ 8.10$ per thousand cubic feet, 9 percent above the August 1992 price. The average price of natural gas used by commercial consumers in August 1993 was $\$ 5.26$ per thousand cubic feet, 11 percent higher than the August 1992 price. The average price of natural gas used by industrial consumers in August 1993 was $\$ 2.86$ per thousand cubic feet, 6 percent above the August 1992 price. 
'L'6 pur ' $S$ '6 ' I'6 selqe :sesunos

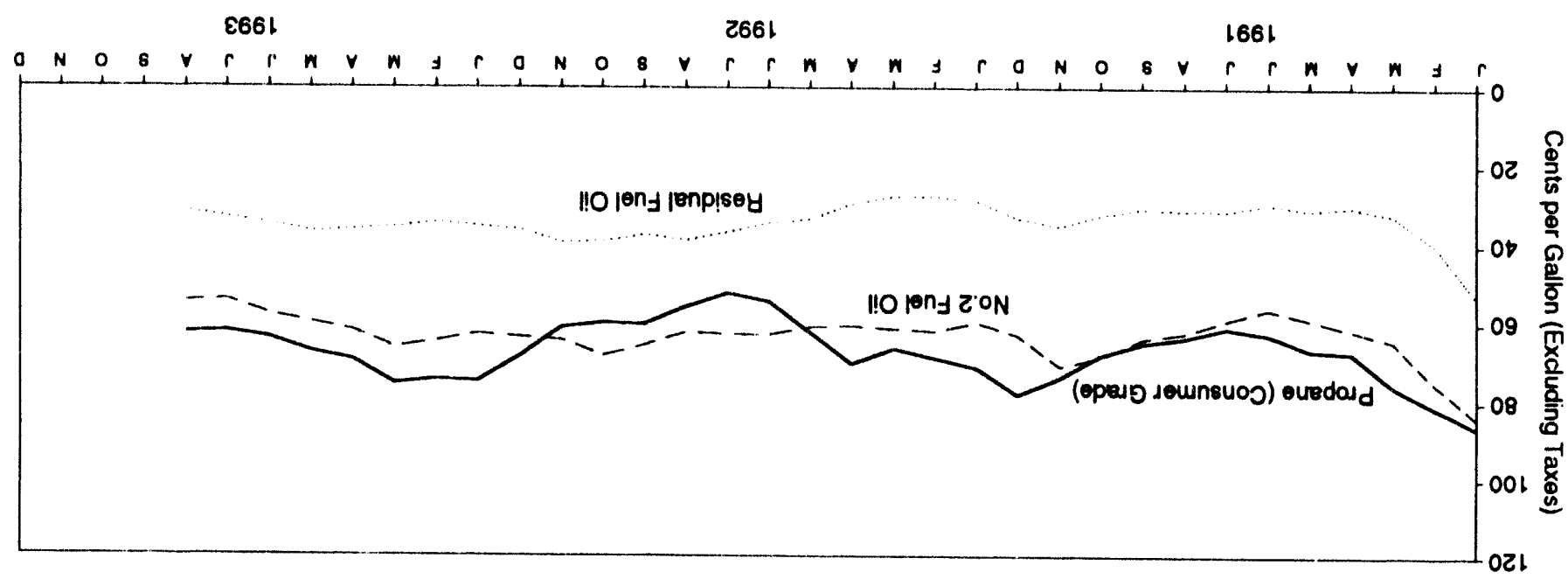

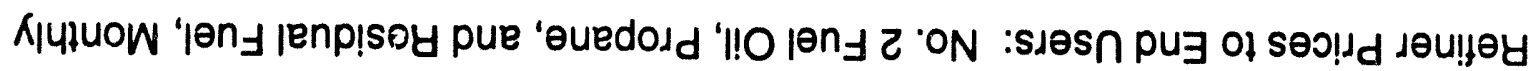

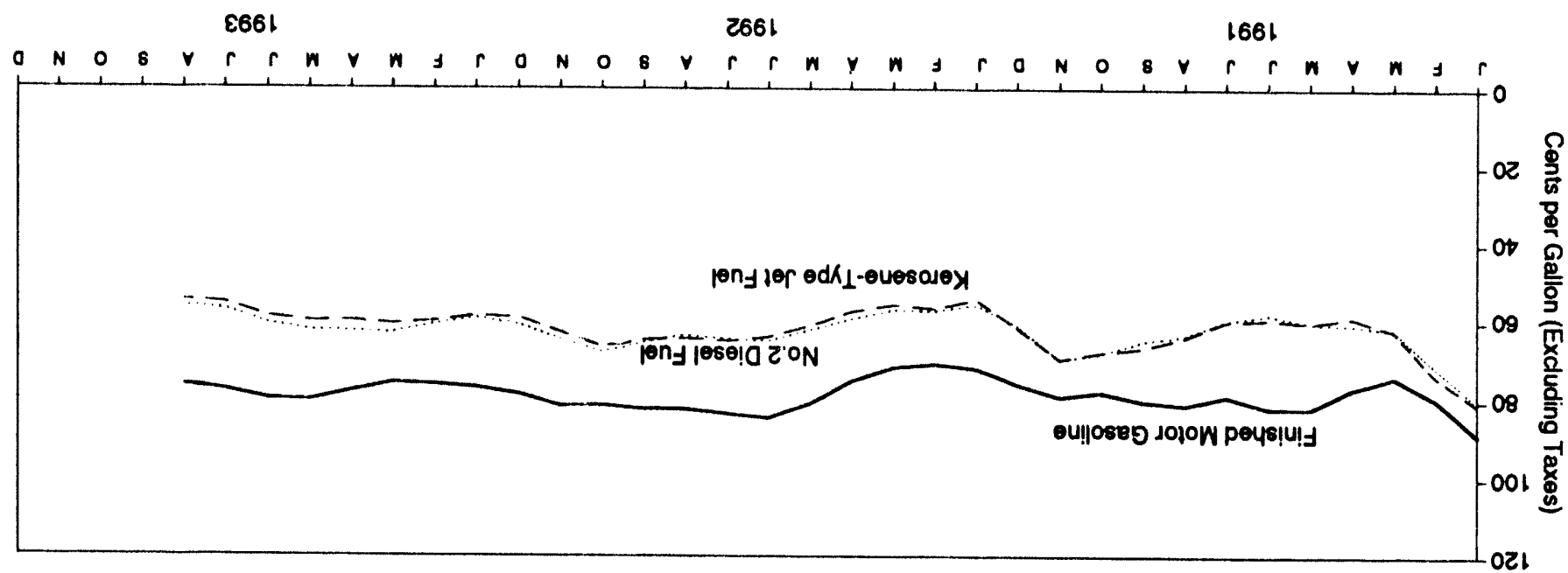

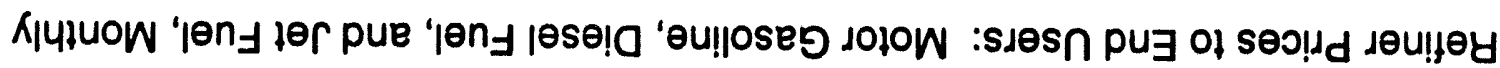

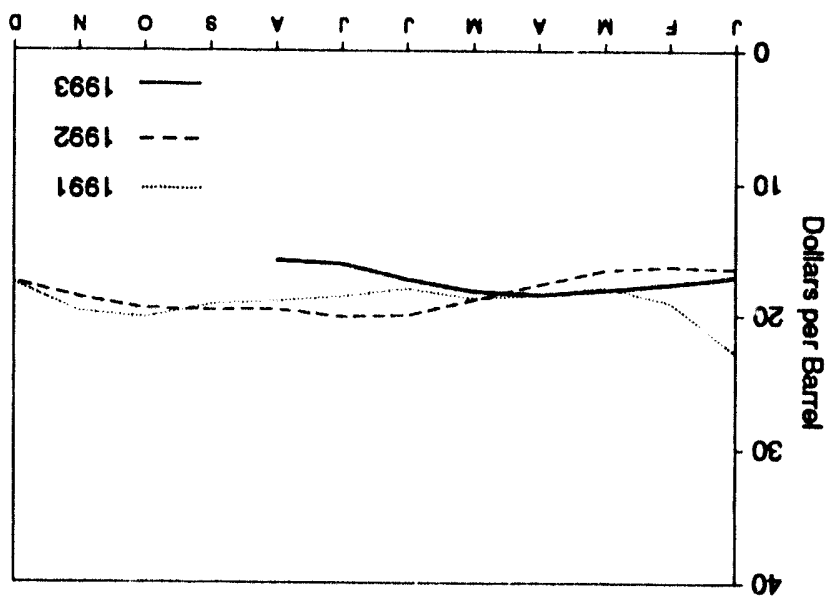

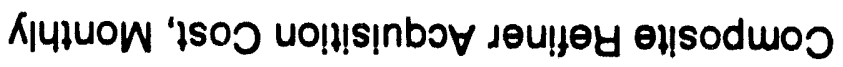

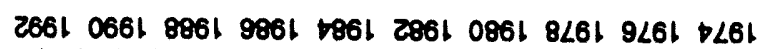

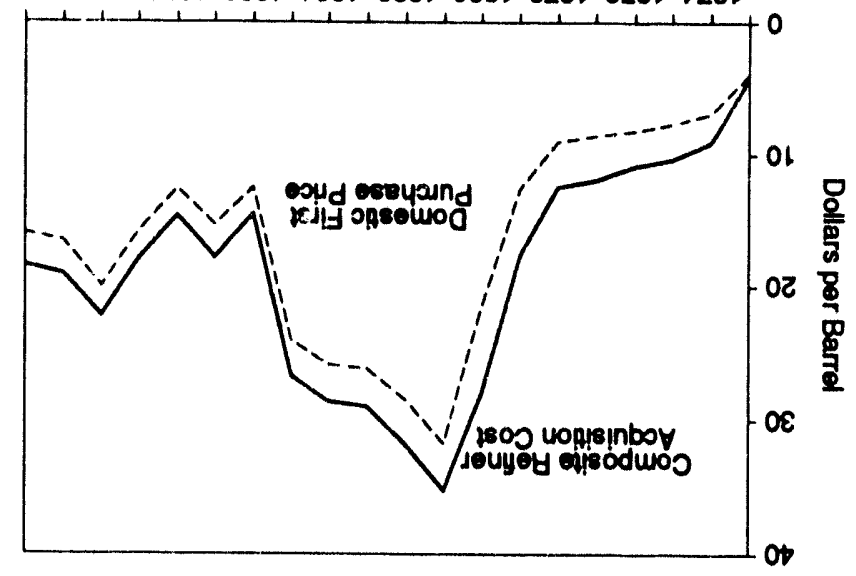

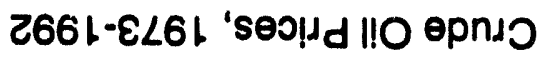


Table 9.1 Crude Oil Price Summary

(Dollars per Barrel)

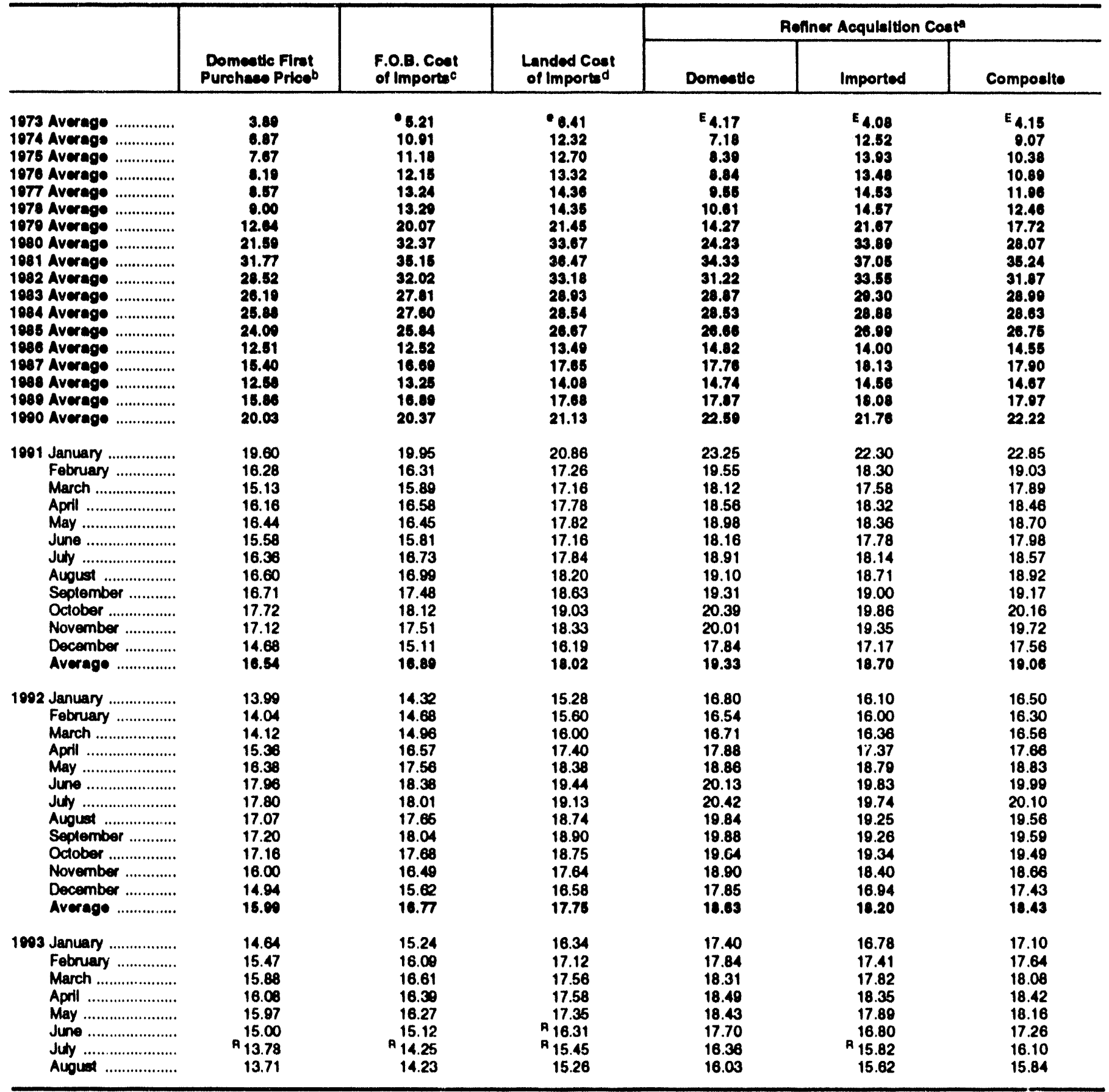

- See Note 4 at and of section.

b See Note 1 at end of section.

c See Note 2 at end of section.

- See Note 3 at end a section.

- Based on October, November, and December data only.

$R=$ Revlsed data. ExEstimate.

Notes: - Geographic coverage is the 50 States, the District of Columbia, Puerto Rico, the Virgin lalands, and all U.S. Tertitorles and Possessions. - Vakues lor Domestic Fira Purchase Price and Reliner Acquisition Cost lor the current month and for F.O.B. and Landed Cost of Imports for the current 2 monthe are preiminary. - F.O.B. and landed costs through 1980 reflect the period of reponting; prices after 1880 rellect the perlod of kading - Annual averapes are the averages of the monthy prices, weichled by volume.

Sources: - Domestle Firet Purchene Price: 1973-1070-U.S. Department of the Interior (DOI). Bureau of Mines (BOM). Minerals Yeartook, 'Crude Petroleum and Petroleum Products' chapler. 1977-Federal Energy Adminiatration (FEA), based on Form FEA.P124, "Domeatic Cnude Oil
Purchaser's Monthly Report: 1978 fonwerd-Energy Informalion Adrnintatration (ELA), Potroloum Marketing Monthly, November 1993, Table 1. - F.0.8. and Landed Cost of Imports: October 1973-Soptember 1977-FEA, Form FEA.F701-M-0, "Transler Pricing Report." October. December 1077-ELA, Form FEA-F701-M-0, "Transler Pricing Pepont." 1978 forwerd-ELA, Potroloum Marketing Monthly, November 1993, Table 1. - Refiner Acquieltion Cost: 1973-ElA estimates. The domestic price was derived by adding est'mated iraneportation costs to the reported dornestic first purchase price. The imported price was derived by adding an estimaled ocean transport cost to the average "Free Alongside Ship" value published by the U.S. Bureau of the Consus. 1074-1976-DOI, BOM, Minerals Yearbook, "Crude Pelroloum and Petroleum Products' chapter. 1077-Jenuery. 8eptember-FEA, based on Form FEA.P110-M-1, "Retiners' Monthly Cost Allocation Repont." October-Decomber-EIA, based on Form FEA.P110-M-1, 'Reliners' Monthly Cos Allocation Report." 1078 forwerd-ELA, Petrobum Marketing Monthy, Nwember 1993, Table 1. 
Table 9.2 F.O.B. Costs of Crude Oll Imports from Selected Countries (Dollars per Barrel)

\begin{tabular}{|c|c|c|c|c|c|c|c|c|c|c|c|}
\hline & Agerla & Indoncela & Iran & Moxico & Nigerla & $\begin{array}{l}\text { seudl } \\
\text { Arabla }\end{array}$ & $\begin{array}{l}\text { United } \\
\text { Kingdem }\end{array}$ & Venorucio & $\begin{array}{c}\text { Other } \\
\text { Countrie }\end{array}$ & $\begin{array}{c}\text { Arab } \\
\text { OPECa }\end{array}$ & $\begin{array}{l}\text { Totel } \\
\text { OPECb }\end{array}$ \\
\hline 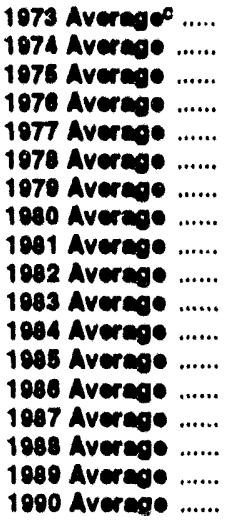 & $\begin{array}{l}7.29 \\
13.24 \\
11.09 \\
13.03 \\
14.36 \\
14.12 \\
20.09 \\
30.67 \\
30.09 \\
34.20 \\
30.09 \\
20.34 \\
28.00 \\
13.62 \\
18.70 \\
W \\
W \\
W\end{array}$ & $\begin{array}{r}8.67 \\
11.00 \\
12.88 \\
12.76 \\
13.57 \\
13.61 \\
10.09 \\
22.17 \\
35.02 \\
34.11 \\
20.02 \\
29.13 \\
27.12 \\
13.10 \\
17.40 \\
13.01 \\
17.01 \\
21.20\end{array}$ & $\begin{array}{l}4.24 \\
10.05 \\
10.01 \\
11.01 \\
12.05 \\
12.05 \\
22.09 \\
N A \\
(d) \\
30.07 \\
24.30 \\
27.42 \\
W \\
W \\
W \\
\text { (d) } \\
\text { d) } \\
\text { (d) }\end{array}$ & $\begin{array}{l}N A \\
W \\
11.44 \\
12.22 \\
13.42 \\
13.24 \\
20.27 \\
31.00 \\
33.01 \\
28.01 \\
28.20 \\
29.30 \\
28.33 \\
11.04 \\
16.38 \\
12.10 \\
18.90 \\
10.28\end{array}$ & $\begin{array}{r}7.41 \\
12.44 \\
11.02 \\
13.08 \\
14.44 \\
14.08 \\
21.00 \\
38.03 \\
30.31 \\
38.18 \\
29.81 \\
29.81 \\
29.04 \\
14.35 \\
18.47 \\
18.16 \\
18.31 \\
22.46\end{array}$ & $\begin{array}{r}3.24 \\
10.17 \\
10.07 \\
11.02 \\
12.30 \\
12.70 \\
17.20 \\
26.17 \\
32.00 \\
33.79 \\
27.89 \\
27.07 \\
22.04 \\
11.36 \\
16.12 \\
12.16 \\
16.29 \\
20.39\end{array}$ & $\begin{array}{c}N A \\
N A \\
N A \\
W \\
14.11 \\
13.02 \\
21.70 \\
24.30 \\
34.04 \\
33.42 \\
20.01 \\
20.07 \\
27.04 \\
13.04 \\
10.21 \\
14.00 \\
17.06 \\
23.43\end{array}$ & $\begin{array}{r}5.30 \\
10.71 \\
11.04 \\
11.30 \\
12.68 \\
12.34 \\
16.00 \\
24.01 \\
28.06 \\
23.74 \\
21.49 \\
24.25 \\
23.04 \\
10.02 \\
15.00 \\
12.06 \\
18.09 \\
18.55\end{array}$ & $\begin{array}{r}4.04 \\
10.02 \\
10.86 \\
11.02 \\
13.19 \\
13.35 \\
21.10 \\
34.34 \\
36.89 \\
31.08 \\
27.08 \\
27.79 \\
26.12 \\
13.32 \\
17.11 \\
13.45 \\
17.12 \\
19.88\end{array}$ & $\begin{array}{r}4.06 \\
10.06 \\
11.18 \\
12.08 \\
13.13 \\
13.20 \\
10.27 \\
31.57 \\
34.78 \\
33.84 \\
28.28 \\
37.70 \\
24.34 \\
11.50 \\
15.00 \\
12.87 \\
18.72 \\
18.84\end{array}$ & $\begin{array}{r}8.43 \\
11.33 \\
11.34 \\
12.23 \\
13.20 \\
13.31 \\
10.01 \\
32.21 \\
38.17 \\
33.46 \\
28.46 \\
27.70 \\
25.67 \\
12.21 \\
16.49 \\
13.43 \\
17.06 \\
20.40\end{array}$ \\
\hline $\begin{array}{l}1801 \text { January ......... } \\
\text { February ...... } \\
\text { March ........... } \\
\text { Aprll ............. } \\
\text { May ............. } \\
\text { June ............ } \\
\text { Juty .............. } \\
\text { Auguet ......... } \\
\text { September ... } \\
\text { October ........ } \\
\text { November .... } \\
\text { December .... } \\
\text { Averege ...... }\end{array}$ & $\begin{array}{l}w \\
w \\
w \\
w \\
w \\
w \\
w \\
w \\
w \\
w \\
w \\
w \\
w\end{array}$ & $\begin{array}{c}W \\
20.82 \\
W \\
18.85 \\
W \\
16.77 \\
W \\
W \\
W \\
18.50 \\
W \\
W \\
18.60\end{array}$ & $\begin{array}{l}\left(\begin{array}{l}d \\
d \\
d\end{array}\right) \\
(d) \\
w \\
(d) \\
w \\
w \\
w \\
w \\
w \\
(d) \\
(d) \\
15.58\end{array}$ & $\begin{array}{l}19.39 \\
13.62 \\
13.50 \\
15.34 \\
15.24 \\
14.68 \\
15.24 \\
15.34 \\
15.40 \\
16.91 \\
16.30 \\
13.47 \\
15.37\end{array}$ & $\begin{array}{l}24.68 \\
20.49 \\
19.44 \\
19.12 \\
19.35 \\
18.38 \\
19.44 \\
20.20 \\
21.10 \\
22.55 \\
21.63 \\
18.99 \\
20.29\end{array}$ & $\begin{array}{c}12.69 \\
14.08 \\
w \\
16.14 \\
15.15 \\
14.54 \\
w \\
16.35 \\
15.86 \\
14.61 \\
13.33 \\
12.72 \\
14.62\end{array}$ & $\begin{array}{c}W \\
W \\
24.50 \\
W \\
W \\
W \\
19.45 \\
W \\
20.24 \\
W \\
21.67 \\
W \\
20.81\end{array}$ & $\begin{array}{l}17.04 \\
14.50 \\
14.00 \\
15.38 \\
14.68 \\
13.62 \\
14.85 \\
14.64 \\
15.53 \\
16.44 \\
14.77 \\
12.62 \\
14.01\end{array}$ & $\begin{array}{l}21.24 \\
17.12 \\
16.18 \\
16.90 \\
16.95 \\
16.33 \\
17.41 \\
17.82 \\
18.79 \\
19.42 \\
18.97 \\
16.57 \\
17.70\end{array}$ & $\begin{array}{l}16.04 \\
14.56 \\
15.24 \\
15.72 \\
15.71 \\
15.29 \\
15.86 \\
16.81 \\
16.76 \\
15.76 \\
15.02 \\
14.32 \\
15.50\end{array}$ & $\begin{array}{l}19.45 \\
16.73 \\
16.48 \\
16.88 \\
16.71 \\
16.04 \\
16.86 \\
17.23 \\
17.57 \\
18.12 \\
17.43 \\
15.03 \\
16.09\end{array}$ \\
\hline $\begin{array}{l}1902 \text { January ........ } \\
\text { February ...... } \\
\text { March ........... } \\
\text { Aprll ............. } \\
\text { May .............. } \\
\text { June ............ } \\
\text { Juty .............. } \\
\text { Aupuet ........ } \\
\text { Seplember ... } \\
\text { October ........ } \\
\text { November .... } \\
\text { December .... } \\
\text { Averege ...... }\end{array}$ & $\begin{array}{l}w \\
w \\
(d \\
w \\
w \\
w \\
w \\
w \\
(d)\end{array}$ & $\begin{array}{c}W \\
W \\
W \\
16.23 \\
W \\
W \\
W \\
W \\
W \\
W \\
W \\
W \\
17.00\end{array}$ & $\begin{array}{l}\left(\begin{array}{l}d \\
d \\
d\end{array}\right) \\
\left(\begin{array}{l}0 \\
d \\
d\end{array}\right) \\
\left(\begin{array}{l}d \\
d\end{array}\right) \\
\left(\begin{array}{l}d \\
d\end{array}\right) \\
\left(\begin{array}{l}d \\
d\end{array}\right) \\
\left(\begin{array}{l}d \\
d\end{array}\right) \\
\left(\begin{array}{l}d \\
\text { f }\end{array}\right.\end{array}$ & $\begin{array}{l}12.45 \\
12.40 \\
12.68 \\
14.11 \\
16.05 \\
17.09 \\
16.88 \\
16.38 \\
16.88 \\
16.90 \\
15.78 \\
14.79 \\
18.28\end{array}$ & $\begin{array}{l}18.58 \\
18.28 \\
18.10 \\
10.59 \\
20.47 \\
21.42 \\
20.83 \\
20.33 \\
20.84 \\
20.76 \\
20.00 \\
18.42 \\
10.00\end{array}$ & $\begin{array}{c}W \\
14.61 \\
14.87 \\
W \\
17.61 \\
W \\
17.60 \\
W \\
16.60 \\
W \\
14.62 \\
15.62 \\
16.65\end{array}$ & 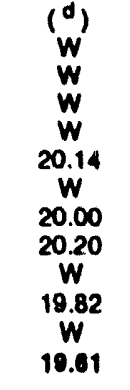 & $\begin{array}{l}12.32 \\
12.53 \\
12.46 \\
14.38 \\
15.03 \\
15.33 \\
15.10 \\
15.38 \\
16.21 \\
15.40 \\
13.82 \\
13.38 \\
14.30\end{array}$ & $\begin{array}{l}15.44 \\
16.04 \\
16.01 \\
17.10 \\
18.35 \\
10.20 \\
18.74 \\
18.43 \\
18.65 \\
18.70 \\
17.57 \\
16.13 \\
17.65\end{array}$ & $\begin{array}{l}14.07 \\
15.35 \\
15.20 \\
17.26 \\
18.13 \\
17.95 \\
18.20 \\
17.89 \\
17.11 \\
15.89 \\
15.12 \\
15.91 \\
16.50\end{array}$ & $\begin{array}{l}14.50 \\
15.04 \\
15.28 \\
17.25 \\
17.83 \\
18.44 \\
18.00 \\
17.69 \\
18.01 \\
17.42 \\
15.97 \\
15.60 \\
18.07\end{array}$ \\
\hline $\begin{array}{l}1003 \text { January ........ } \\
\text { February ...... } \\
\text { March ........... } \\
\text { April ............. } \\
\text { May ............. } \\
\text { June ............ } \\
\text { July .............. } \\
\text { Auguist ......... }\end{array}$ & $\begin{array}{l}\left(\begin{array}{l}d \\
w\end{array}\right) \\
\left(\begin{array}{l}d \\
d\end{array}\right) \\
\left(\begin{array}{l}d \\
\text { d }\end{array}\right) \\
\text { (d) } \\
(d)\end{array}$ & $\begin{array}{c}W \\
W \\
W \\
W \\
19.14 \\
W \\
A_{16.48} \\
18.11\end{array}$ & $\begin{array}{l}\left(\begin{array}{l}d \\
d \\
d\end{array}\right) \\
\left(\begin{array}{l}d \\
d\end{array}\right) \\
\left(\begin{array}{l}d \\
d\end{array}\right) \\
\left(\begin{array}{l}d \\
d\end{array}\right)\end{array}$ & $\begin{array}{r}14.14 \\
14.64 \\
15.17 \\
15.04 \\
15.15 \\
14.08 \\
13.09 \\
13.38\end{array}$ & $\begin{array}{r}17.95 \\
19.08 \\
19.33 \\
19.10 \\
18.92 \\
18.01 \\
A 17.45 \\
17.33\end{array}$ & $\begin{array}{l}16.55 \\
16.17 \\
16.45 \\
16.03 \\
14.54 \\
W \\
W \\
W\end{array}$ & $\begin{array}{r}18.29 \\
18.13 \\
18.51 \\
18.36 \\
18.20 \\
17.15 \\
A 16.07 \\
17.03\end{array}$ & $\begin{array}{r}12.99 \\
13.68 \\
14.22 \\
14.52 \\
13.89 \\
12.47 \\
\text { ค } 11.95 \\
12.76\end{array}$ & $\begin{array}{r}15.17 \\
18.51 \\
16.85 \\
16.90 \\
16.73 \\
15.89 \\
R_{14.96} \\
14.62\end{array}$ & $\begin{array}{l}15.60 \\
16.39 \\
16.83 \\
16.24 \\
15.03 \\
14.29 \\
13.95 \\
14.68\end{array}$ & $\begin{array}{r}15.62 \\
16.49 \\
16.82 \\
16.59 \\
16.32 \\
A_{14.94} \\
A_{14.25} \\
14.32\end{array}$ \\
\hline
\end{tabular}

- The Arab members of OPEC are Aberia, Iraq, Kuwall, Libya, Qatar, Saudl Arabla, and the Uniled Arab Emirates.

- Current members of OPEC are Gabon, indonesia, Iran, Nigoria, and Venezuola, as well as the Arab members. Pritor to 1993. Ecuador was also a member. The cost of imports from the Neutral Zone between Kuwah and Saudl Arabla is included in the coet of imports from "Total OPEC."

- Based on October, November, and December data onty.

d No data reported.

$R=R e v i s e d$ data. NA=Not avallabla. W=Value witheld to avold dieclosure of individual company data.

Noles: - The Free on Board (F.O.B.) coet at the courtry of ortoin exchudes all costs related to insurance and tranaportation. See Nole 2 at end of section. - Values for the current 2 monthe are preliminary. - Prices through
1980 reflect the period of reporting: prices after 1980 reflect the pertod of bading. - Annual averages are averages of the monthly prices, including prices not publlahed, weighted by volume. - Cargoes that are purchased on a "netback" basia, or under similar contractual arrangements whereby the ectual purchase price is not establiahed at the time the crude oll is acquired for importation into the Uniled States, are not included in the published date untll the actual prices have been determined and reported.

Sourcea: - Oetober 1073-September 1077: Federal Energy Administralion, Form FEA-F701-M0. "Trander Pricing Report." - Detober 1077-December 1977: Energy Information Administration (ElA). Fom FEA.F701-M-0, "Trander Pricing Repon." - 1070 forwerd: ElA, Potroleum Marketing Monthy. November 1993, Table 21. 
Table 9.3 Landed Costs of Crude Oll Imports from Selected Countries (Dollars per Barrel)

\begin{tabular}{|c|c|c|c|c|c|c|c|c|c|c|c|c|}
\hline & Algerte & Cenada & Indonesla & Iran & Mexleo & Noerte & $\begin{array}{l}\text { Saudl } \\
\text { Arcbla }\end{array}$ & $\begin{array}{l}\text { United } \\
\text { KIngdem }\end{array}$ & Veneruela & $\begin{array}{c}\text { Other } \\
\text { Countries }\end{array}$ & $\underset{\text { APECa }}{\text { Anb }}$ & $\begin{array}{c}\text { Tolal } \\
\text { OPECb }\end{array}$ \\
\hline 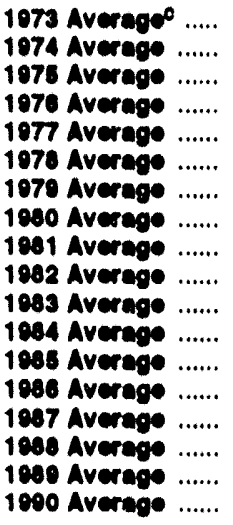 & 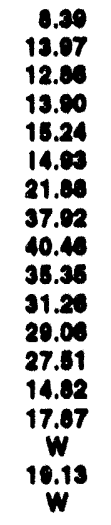 & 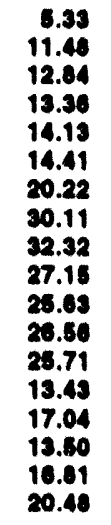 & $\begin{array}{r}7.22 \\
13.20 \\
13.83 \\
13.88 \\
14.88 \\
14.68 \\
20.83 \\
33.02 \\
37.31 \\
34.70 \\
31.87 \\
30.87 \\
28.67 \\
14.63 \\
18.40 \\
18.18 \\
18.38 \\
22.50\end{array}$ & 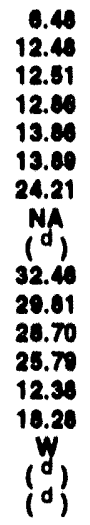 & 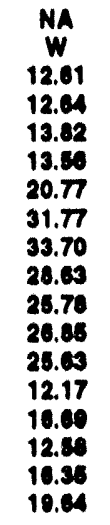 & 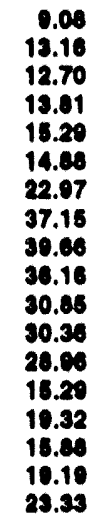 & $\begin{array}{r}8.37 \\
11.03 \\
12.50 \\
13.06 \\
13.60 \\
13.04 \\
18.08 \\
29.00 \\
34.20 \\
34.09 \\
29.27 \\
29.20 \\
24.72 \\
12.04 \\
16.81 \\
13.37 \\
17.34 \\
21.02\end{array}$ & $\begin{array}{c}N A \\
N A \\
N A \\
W \\
14.03 \\
14.63 \\
22.07 \\
38.68 \\
37.20 \\
34.25 \\
30.87 \\
20.48 \\
28.36 \\
14.63 \\
18.76 \\
18.02 \\
18.74 \\
22.06\end{array}$ & $\begin{array}{r}8.00 \\
11.28 \\
12.38 \\
11.80 \\
13.11 \\
12.84 \\
17.68 \\
28.02 \\
29.01 \\
24.03 \\
22.04 \\
25.10 \\
24.43 \\
11.52 \\
18.70 \\
13.09 \\
10.79 \\
20.31\end{array}$ & $\begin{array}{r}6.00 \\
12.03 \\
12.80 \\
13.38 \\
14.86 \\
14.88 \\
22.86 \\
34.18 \\
38.54 \\
34.03 \\
29.68 \\
29.21 \\
27.33 \\
14.26 \\
18.30 \\
14.46 \\
18.08 \\
20.82\end{array}$ & $\begin{array}{r}6.02 \\
12.30 \\
12.71 \\
13.31 \\
14.30 \\
14.30 \\
20.79 \\
32.07 \\
30.22 \\
36.18 \\
20.07 \\
20.10 \\
26.00 \\
13.14 \\
17.32 \\
13.00 \\
17.41 \\
20.04\end{array}$ & 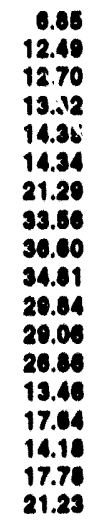 \\
\hline $\begin{array}{l}1001 \text { January ........ } \\
\text { February ...... } \\
\text { March ........... } \\
\text { April ............ } \\
\text { May .............. } \\
\text { June ............ } \\
\text { July ............. } \\
\text { Augus ........ } \\
\text { Seplember ... } \\
\text { October ........ } \\
\text { Nevember .... } \\
\text { Dicember .... } \\
\text { Average ...... }\end{array}$ & $\begin{array}{l}w \\
w \\
w \\
w \\
w \\
W \\
W \\
w \\
w \\
w \\
w \\
w \\
w\end{array}$ & $\begin{array}{l}20.81 \\
17.05 \\
15.20 \\
16.26 \\
16.28 \\
16.19 \\
17.14 \\
17.61 \\
17.84 \\
18.38 \\
17.53 \\
15.87 \\
17.16\end{array}$ & $\begin{array}{c}W \\
22.61 \\
20.03 \\
18.85 \\
W \\
18.25 \\
17.76 \\
W \\
W \\
19.85 \\
21.05 \\
W \\
20.20\end{array}$ & 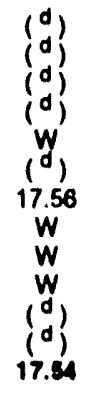 & $\begin{array}{l}19.88 \\
14.23 \\
14.15 \\
15.85 \\
15.81 \\
15.20 \\
15.89 \\
15.78 \\
15.80 \\
17.34 \\
16.53 \\
13.89 \\
18.89\end{array}$ & $\begin{array}{l}26.00 \\
21.68 \\
20.60 \\
20.31 \\
20.50 \\
19.79 \\
20.73 \\
21.29 \\
22.13 \\
23.68 \\
22.71 \\
19.86 \\
21.30\end{array}$ & $\begin{array}{l}18.53 \\
16.18 \\
17.08 \\
17.54 \\
17.34 \\
16.85 \\
17.48 \\
18.04 \\
18.19 \\
17.62 \\
16.46 \\
15.03 \\
17.22\end{array}$ & $\begin{array}{l}W \\
W \\
25.77 \\
20.56 \\
20.21 \\
19.36 \\
20.47 \\
20.71 \\
21.16 \\
22.07 \\
22.71 \\
20.29 \\
21.37\end{array}$ & $\begin{array}{l}18.35 \\
15.76 \\
16.18 \\
16.35 \\
15.74 \\
14.61 \\
15.92 \\
15.64 \\
16.44 \\
17.28 \\
15.66 \\
13.46 \\
15.02\end{array}$ & $\begin{array}{l}24.08 \\
19.42 \\
18.59 \\
18.77 \\
19.53 \\
18.38 \\
18.82 \\
19.30 \\
20.35 \\
20.81 \\
21.04 \\
18.67 \\
10.73\end{array}$ & $\begin{array}{l}18.94 \\
16.29 \\
17.23 \\
17.65 \\
17.49 \\
17.01 \\
17.01 \\
18.17 \\
18.42 \\
17.97 \\
16.90 \\
15.49 \\
17.48\end{array}$ & $\begin{array}{l}20.16 \\
17.43 \\
17.88 \\
18.17 \\
17.98 \\
17.32 \\
17.98 \\
18.40 \\
18.70 \\
19.03 \\
17.95 \\
16.94 \\
18.08\end{array}$ \\
\hline $\begin{array}{l}1002 \text { Janua.y ........ } \\
\text { February ...... } \\
\text { March .......... } \\
\text { Aprl ........... } \\
\text { May .............. } \\
\text { June ............ } \\
\text { Juty ............. } \\
\text { Augued ........ } \\
\text { September .... } \\
\text { October ........ } \\
\text { November .... } \\
\text { December .... } \\
\text { Averege ...... }\end{array}$ & $\begin{array}{l}w \\
w \\
(d) \\
w \\
w \\
w \\
w \\
w \\
\left(\begin{array}{c}w \\
w\end{array}\right) \\
\left(\begin{array}{c}w \\
w\end{array}\right) \\
w\end{array}$ & $\begin{array}{l}14.83 \\
15.57 \\
15.68 \\
16.42 \\
17.35 \\
18.40 \\
18.50 \\
18.28 \\
18.35 \\
18.35 \\
17.26 \\
15.85 \\
17.04\end{array}$ & $\begin{array}{c}W \\
W \\
W \\
17.76 \\
17.66 \\
19.60 \\
21.06 \\
21.26 \\
W \\
W \\
W \\
W \\
10.70\end{array}$ & $\left(\begin{array}{l}d \\
d\end{array}\right)$ & $\begin{array}{l}13.02 \\
12.78 \\
13.08 \\
14.40 \\
16.39 \\
17.41 \\
17.20 \\
16.74 \\
17.34 \\
17.26 \\
16.18 \\
15.12 \\
18.60\end{array}$ & $\begin{array}{l}19.34 \\
19.10 \\
19.06 \\
20.32 \\
21.25 \\
22.11 \\
21.49 \\
21.05 \\
21.57 \\
21.60 \\
20.79 \\
19.32 \\
20.76\end{array}$ & $\begin{array}{l}14.81 \\
15.61 \\
16.05 \\
18.01 \\
18.62 \\
18.49 \\
18.00 \\
18.45 \\
18.45 \\
17.96 \\
17.02 \\
16.64 \\
17.46\end{array}$ & $\begin{array}{c}W \\
W \\
18.63 \\
18.97 \\
10.09 \\
20.85 \\
21.46 \\
21.37 \\
20.72 \\
21.17 \\
21.00 \\
19.40 \\
20.65\end{array}$ & $\begin{array}{l}13.20 \\
13.47 \\
13.41 \\
15.08 \\
15.73 \\
16.01 \\
15.78 \\
16.10 \\
16.89 \\
16.14 \\
14.51 \\
14.07 \\
16.13\end{array}$ & $\begin{array}{l}17.46 \\
17.64 \\
17.44 \\
18.10 \\
19.58 \\
20.83 \\
20.49 \\
20.10 \\
20.12 \\
20.09 \\
19.26 \\
17.80 \\
19.26\end{array}$ & $\begin{array}{l}15.16 \\
15.85 \\
18.14 \\
18.11 \\
18.80 \\
19.60 \\
19.15 \\
18.79 \\
18.51 \\
18.08 \\
17.05 \\
18.69 \\
17.03\end{array}$ & $\begin{array}{l}15.38 \\
15.87 \\
16.20 \\
18.07 \\
18.66 \\
10.57 \\
19.08 \\
18.70 \\
18.83 \\
18.58 \\
17.28 \\
18.62 \\
17.01\end{array}$ \\
\hline 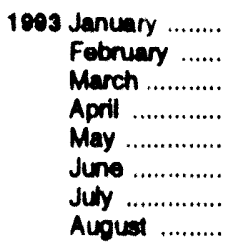 & $\begin{array}{l}\left(\begin{array}{l}d \\
d\end{array}\right) \\
w \\
w \\
w \\
\left(\begin{array}{l}d \\
w\end{array}\right) \\
\left(\begin{array}{l}d \\
w\end{array}\right)\end{array}$ & $\begin{array}{l}15.27 \\
15.84 \\
16.48 \\
16.79 \\
16.82 \\
16.25 \\
15.29 \\
14.93\end{array}$ & $\begin{array}{c}W \\
W \\
W \\
19.89 \\
20.57 \\
W \\
17.87 \\
19.67\end{array}$ & $\left.\begin{array}{l}\text { (d) } \\
\text { (d) } \\
\text { d } \\
\text { d } \\
\text { d }\end{array}\right)$ & $\begin{array}{r}14.50 \\
14.88 \\
15.50 \\
15.55 \\
15.57 \\
14.50 \\
13.44 \\
13.85\end{array}$ & $\begin{array}{r}18.98 \\
19.92 \\
20.25 \\
20.18 \\
19.79 \\
18.23 \\
\text { A } 18.25 \\
18.08\end{array}$ & $\begin{array}{r}16.38 \\
17.29 \\
17.56 \\
17.56 \\
16.64 \\
A \quad 15.72 \\
A 14.99 \\
14.94\end{array}$ & $\begin{array}{r}19.12 \\
19.28 \\
19.43 \\
19.32 \\
19.33 \\
18.67 \\
A 17.51 \\
17.59\end{array}$ & $\begin{array}{r}14.07 \\
14.60 \\
15.14 \\
15.54 \\
14.81 \\
A_{13.53} \\
A_{12.81} \\
13.52\end{array}$ & $\begin{array}{r}17.21 \\
18.17 \\
18.43 \\
18.48 \\
18.41 \\
17.44 \\
\text { ค } 16.46 \\
15.92\end{array}$ & $\begin{array}{r}16.39 \\
17.29 \\
17.63 \\
17.55 \\
16.79 \\
A 15.86 \\
A 14.98 \\
14.05\end{array}$ & $\begin{array}{r}18.64 \\
17.43 \\
17.83 \\
17.77 \\
17.30 \\
\text { A } 16.03 \\
\text { A } 15.32 \\
15.28\end{array}$ \\
\hline
\end{tabular}

- The Arab members of OPEC are Aberia, Iraq, Kuwall, Llbya, Qatar, Saudi Arabia, and the United Arab Emirates.

- Cument members of OPEC are Gabon, Indoneala, Iran. Nigeria, and Venezuela, so well as the Arab members. Prior to 1993, Ecundor was alao member. The coet at imports trom the Noural Zone between Kuwak and Saudl Arabla be included in the coet of imports from "Tolal OPEC."

Based on October, November, and December data only.

No dala reported.

RaRevised dala. $\mathrm{NA}=\mathrm{No}$ available. W=Vake whtheld to avoid disclosure of individual company data.

Notes: - See Note 3 at end of section. - Vakes for the current 2 monthe are proliminary. - Pricee through 1980 reflect the pertod of reporting; prices since then refled the pertod of loading. - Annual averages are averages of the monthy prices, including prices nol published, weighted by volume. - Carpoes that are purchased on a "notuack' basis, or under sinilar contractual arranoements whereby the actual purchise price is not cetablished at the time the crude oll be acquired for Importation into the Uniled states, are not included in the publlahed data uniti the actual prices have been determined and reported.

Sourcen: - Oetober 1073-Soptember 1077: Federal Energy Adminteatration, Form FEA.F701-Mt0, "Traneler Pricing Repurt:" Oobber 1077-December 1077: Energy Intormalion Adminiatration (EUA), Form FEA-F701-M-0, "Trander Pricing Roport:" 1978 forward: ELA, Petrodeum Marketing Monithy, November 1983, Table 22. 
Table 9.4 Motor Gasoline Retail Prices, U.S. City Average

(Cents per Gallon, Including Taxes)

\begin{tabular}{|c|c|c|c|c|}
\hline & $\begin{array}{l}\text { Leaded } \\
\text { Reouler }\end{array}$ & $\begin{array}{l}\text { Unleaded } \\
\text { Acoular }\end{array}$ & $\begin{array}{l}\text { Unicaded } \\
\text { Premlum }\end{array}$ & All Types" \\
\hline 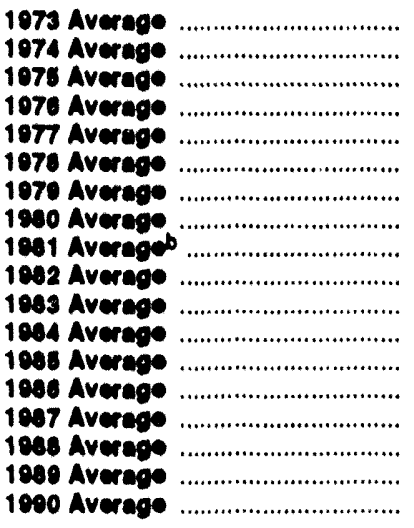 & 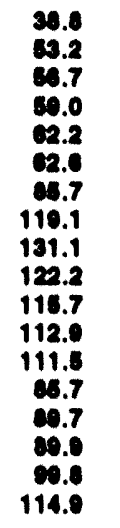 & 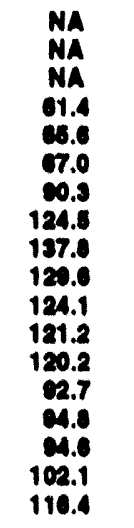 & $\begin{array}{l}\text { NA } \\
\text { NA } \\
\text { NA } \\
\text { NA } \\
\text { NA } \\
\text { NA } \\
\text { NA } \\
\text { c147.0 } \\
141.8 \\
134.3 \\
130.8 \\
134.0 \\
109.5 \\
109.3 \\
110.7 \\
119.7 \\
134.0\end{array}$ & 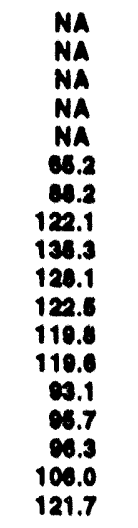 \\
\hline 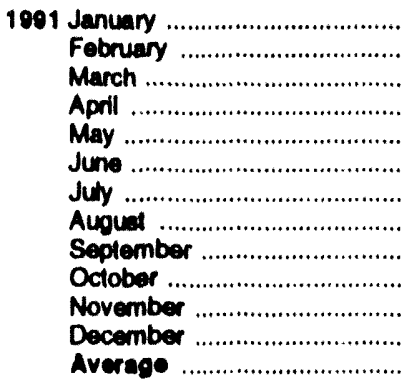 & $\begin{array}{l}124.6 \\
113.7 \\
104.7 \\
108.2 \\
\text { NA } \\
\text { NA } \\
\text { NA } \\
\text { NA } \\
\text { NA } \\
\text { NA } \\
\text { NA } \\
\text { NA } \\
\text { NA }\end{array}$ & $\begin{array}{l}124.7 \\
114.3 \\
108.2 \\
110.4 \\
115.6 \\
116.0 \\
112.7 \\
114.0 \\
114.3 \\
112.2 \\
113.4 \\
112.3 \\
114.0\end{array}$ & $\begin{array}{l}143.1 \\
132.1 \\
126.4 \\
128.1 \\
133.1 \\
133.8 \\
131.3 \\
131.8 \\
132.4 \\
130.7 \\
131.8 \\
130.8 \\
132.1\end{array}$ & $\begin{array}{l}130.4 \\
118.8 \\
113.8 \\
115.8 \\
120.8 \\
121.4 \\
118.5 \\
119.6 \\
119.9 \\
118.0 \\
119.3 \\
118.2 \\
118.6\end{array}$ \\
\hline 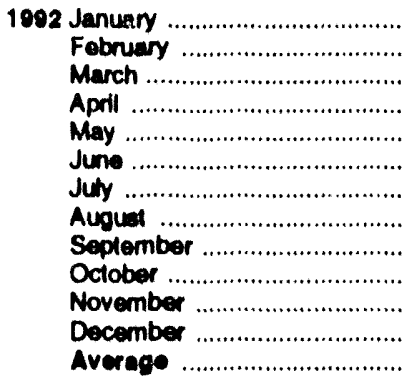 & $\begin{array}{l}\text { NA } \\
\text { NA } \\
\text { NA } \\
\text { NA } \\
\text { MA } \\
\text { NA } \\
\text { NA } \\
\text { NA } \\
\text { NA } \\
\text { NA } \\
\text { NA }\end{array}$ & $\begin{array}{l}107.3 \\
105.4 \\
105.8 \\
107.8 \\
113.8 \\
117.8 \\
117.5 \\
115.8 \\
115.8 \\
115.4 \\
115.9 \\
113.6 \\
112.7\end{array}$ & $\begin{array}{l}126.7 \\
124.8 \\
125.0 \\
126.8 \\
131.7 \\
136.9 \\
136.3 \\
134.8 \\
134.6 \\
134.5 \\
136.1 \\
133.0 \\
131.6\end{array}$ & $\begin{array}{l}113.5 \\
111.7 \\
112.2 \\
114.3 \\
119.7 \\
123.9 \\
123.8 \\
122.1 \\
122.2 \\
121.9 \\
122.3 \\
120.1 \\
110.0\end{array}$ \\
\hline 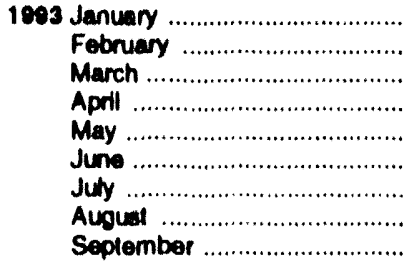 & $\begin{array}{l}\text { NA } \\
\text { NA } \\
\text { NA } \\
\text { NA } \\
\text { NA } \\
\text { NA } \\
\text { NA }\end{array}$ & $\begin{array}{l}111.7 \\
110.8 \\
109.8 \\
111.2 \\
112.8 \\
113.0 \\
110.8 \\
109.7 \\
108.5\end{array}$ & $\begin{array}{l}131.3 \\
130.1 \\
120.4 \\
130.4 \\
131.9 \\
132.1 \\
130.5 \\
129.4 \\
128.2\end{array}$ & $\begin{array}{l}118.2 \\
117.2 \\
118.3 \\
117.5 \\
118.3 \\
118.4 \\
117.4 \\
116.3 \\
115.1\end{array}$ \\
\hline
\end{tabular}

- Also includes types of motor gasoline not shown separately.

- In September 1981, the Bureau of Labor Statistics changed the weights used in the calculation of average motor gasoline prices. From September 1881 lonward, gasohol is included in the average for all types, and unleaded premium to weighted more heavlly.

c September through Decomber data onty.

NAmNol avallable.

Noles: - See Note 5 al and of section. - Geographic coverage for
1973-1977 is 56 urtan areas. Geographic coverage for 1978 forward to 85 urban areas.

Sources: - Monthly Data: U.S. Department of Labor, Bureaul of Labor Statbitica, Consumer Prices: Energy. - Annual Deta: 1973-Platt's Oil Price Handbook and Oilmanac, 1974, 51st Edition. 1974 forwerd-calculated by the Energy Inlormation Administration as the simple averages of monthily data. 
Table 9.5 Refiner Prices of Residual Fuel Oil (Cents per Gallon, Excluding Taxes)

\begin{tabular}{|c|c|c|c|c|c|c|}
\hline & \multicolumn{2}{|c|}{$\begin{array}{l}\text { Realdual Fuel Oll } \\
\text { gulthr Content Loes } \\
\text { Then or Equal to } 1 \text { Percent }\end{array}$} & \multicolumn{2}{|c|}{$\begin{array}{l}\text { Realduel Fuel Oll } \\
\text { Sultur Content } \\
\text { Ornmtor Then } 1 \text { Pereent }\end{array}$} & \multicolumn{2}{|c|}{ Averege } \\
\hline & $\begin{array}{l}\text { seles for } \\
\text { Reacle }\end{array}$ & $\begin{array}{l}\text { ede to } \\
\text { End Ueere }\end{array}$ & $\begin{array}{l}\text { Eleles for } \\
\text { Resede }\end{array}$ & $\begin{array}{l}\text { Eles to } \\
\text { Bnd Uenre }\end{array}$ & $\begin{array}{l}\text { 8ales for } \\
\text { Posele }\end{array}$ & $\begin{array}{l}\text { sales to } \\
\text { End Uere }\end{array}$ \\
\hline 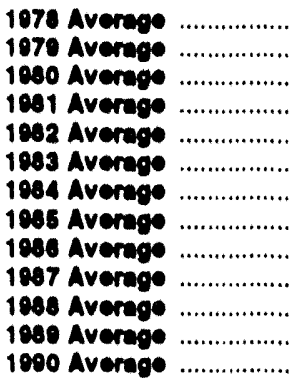 & $\begin{array}{l}20.3 \\
4.0 \\
60.8 \\
74.0 \\
00.5 \\
4.3 \\
4.8 \\
61.0 \\
32.8 \\
41.2 \\
33.3 \\
40.7 \\
47.2\end{array}$ & $\begin{array}{l}31.4 \\
46.8 \\
67.5 \\
02.0 \\
74.7 \\
69.8 \\
72.0 \\
64.4 \\
37.2 \\
44.7 \\
37.2 \\
43.6 \\
50.6\end{array}$ & $\begin{array}{l}24.0 \\
39.0 \\
47.0 \\
62.2 \\
67.2 \\
69.1 \\
0.0 \\
69.0 \\
20.0 \\
39.2 \\
27.1 \\
33.1 \\
37.2\end{array}$ & $\begin{array}{l}27.8 \\
38.0 \\
52.3 \\
67.3 \\
61.1 \\
61.1 \\
68.0 \\
68.2 \\
31.7 \\
30.0 \\
30.0 \\
34.4 \\
40.0\end{array}$ & $\begin{array}{l}26.3 \\
30.0 \\
52.0 \\
88.3 \\
61.2 \\
00.0 \\
68.4 \\
37.7 \\
30.5 \\
38.6 \\
30.0 \\
38.0 \\
41.3\end{array}$ & $\begin{array}{l}29.0 \\
43.6 \\
60.7 \\
75.6 \\
67.6 \\
85.1 \\
68.7 \\
61.0 \\
34.3 \\
12.3 \\
33.4 \\
\mathbf{3 4 . 5} \\
44.4\end{array}$ \\
\hline 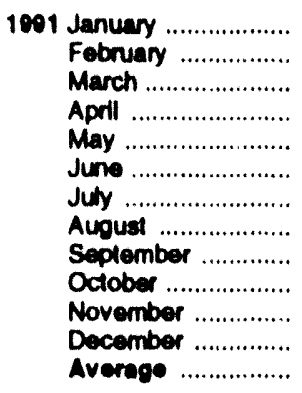 & $\begin{array}{l}52.1 \\
36.5 \\
38.0 \\
33.6 \\
36.6 \\
32.1 \\
32.6 \\
33.4 \\
33.7 \\
34.1 \\
38.6 \\
34.8 \\
\mathbf{3 0 . 4}\end{array}$ & $\begin{array}{l}59.8 \\
44.6 \\
38.3 \\
37.8 \\
36.6 \\
35.3 \\
36.4 \\
36.8 \\
36.8 \\
38.5 \\
40.8 \\
40.0 \\
40.2\end{array}$ & $\begin{array}{l}49.2 \\
32.0 \\
24.2 \\
25.8 \\
27.7 \\
28.6 \\
27.4 \\
25.9 \\
25.4 \\
27.6 \\
27.9 \\
26.1 \\
20.2\end{array}$ & $\begin{array}{l}49.7 \\
37.1 \\
28.2 \\
27.0 \\
27.6 \\
26.9 \\
28.2 \\
27.7 \\
27.3 \\
29.7 \\
31.8 \\
28.8 \\
30.6\end{array}$ & $\begin{array}{l}50.2 \\
33.4 \\
28.2 \\
28.7 \\
30.3 \\
29.7 \\
28.8 \\
27.9 \\
27.9 \\
28.5 \\
30.7 \\
28.9 \\
31.4\end{array}$ & $\begin{array}{l}53.4 \\
30.8 \\
32.3 \\
30.2 \\
31.0 \\
29.5 \\
31.2 \\
31.1 \\
30.6 \\
32.3 \\
36.1 \\
33.1 \\
34.0\end{array}$ \\
\hline 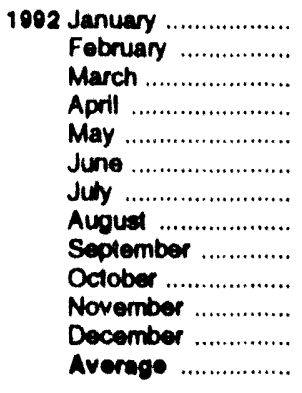 & $\begin{array}{l}30.3 \\
32.7 \\
30.8 \\
31.6 \\
33.1 \\
35.9 \\
38.0 \\
37.7 \\
37.9 \\
41.4 \\
39.2 \\
35.9 \\
38.4\end{array}$ & $\begin{array}{l}35.7 \\
36.2 \\
34.8 \\
35.3 \\
37.2 \\
38.8 \\
41.4 \\
42.1 \\
42.0 \\
44.7 \\
42.8 \\
40.2 \\
34.0\end{array}$ & $\begin{array}{l}21.1 \\
20.9 \\
21.1 \\
25.2 \\
29.1 \\
30.7 \\
33.3 \\
33.2 \\
32.9 \\
36.5 \\
33.8 \\
28.1 \\
20.4\end{array}$ & $\begin{array}{l}24.7 \\
23.6 \\
24.4 \\
27.5 \\
32.0 \\
33.1 \\
34.9 \\
37.0 \\
35.3 \\
37.3 \\
37.6 \\
33.4 \\
31.3\end{array}$ & $\begin{array}{l}24.4 \\
25.6 \\
24.6 \\
27.4 \\
30.2 \\
32.5 \\
34.7 \\
34.7 \\
34.8 \\
37.4 \\
35.9 \\
30.6 \\
30.7\end{array}$ & $\begin{array}{l}28.8 \\
27.7 \\
27.7 \\
29.6 \\
33.4 \\
34.5 \\
36.7 \\
38.8 \\
37.5 \\
39.2 \\
39.4 \\
36.2 \\
33.8\end{array}$ \\
\hline 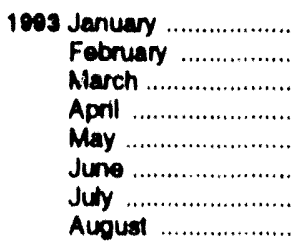 & $\begin{array}{l}38.6 \\
35.5 \\
39.0 \\
38.4 \\
34.7 \\
33.7 \\
32.7 \\
32.1\end{array}$ & $\begin{array}{r}40.8 \\
40.8 \\
42.6 \\
43.6 \\
41.9 \\
40.6 \\
41.9 \\
37.2\end{array}$ & $\begin{array}{r}27.2 \\
27.1 \\
27.5 \\
29.2 \\
27.8 \\
28.4 \\
24.6 \\
24.6 \\
24.0\end{array}$ & $\begin{array}{l}32.4 \\
30.8 \\
31.6 \\
32.2 \\
34.1 \\
31.5 \\
28.5 \\
28.4\end{array}$ & $\begin{array}{l}31.2 \\
31.1 \\
32.9 \\
33.6 \\
31.0 \\
30.0 \\
27.4 \\
27.3\end{array}$ & $\begin{array}{r}36.3 \\
34.4 \\
36.6 \\
36.3 \\
36.8 \\
34.7 \\
\text { ค } \\
33.2 \\
31.7\end{array}$ \\
\hline
\end{tabular}

R=Revised dala.

Notes: - Sales for reasale are those made to purchasers other than ultimate consumers. Sales to end users are those made directly to the ullimate consumer, including bulk customers, such as acriculture, indusiry, and obctric utilities, as woll as commercial cuetomers. - Geographic coverage is the 50 States and the District of Columbia. - Vakues for the current month are preliminary. - Prices prior to 1883 are Energy Inlormation Administration (EL) eotimates. See Note 6 at and of section.

Source: ElA, Pardeum Marketing Monthly, November 1993, Table 17. 
Table 9.6 Refiner Prices of Petroleum Products for Resale (Cents per Gallon, Excluding Taxes)

\begin{tabular}{|c|c|c|c|c|c|c|c|}
\hline & 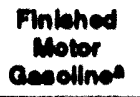 & $\begin{array}{l}\text { Finibhed } \\
\text { Avleden } \\
\text { Cesedine }\end{array}$ & $\begin{array}{c}\text { Kereone } \\
\text { Type } \\
\text { Jef Fuel }\end{array}$ & Keresen & $\begin{array}{l}\text { No. } 2 \\
\text { Fuel } \\
\text { Oll }\end{array}$ & $\begin{array}{l}\text { No. } 2 \\
\text { Dlesel } \\
\text { Fuel }\end{array}$ & $\begin{array}{l}\text { Propano } \\
\text { (Consumer } \\
\text { Crede) }\end{array}$ \\
\hline 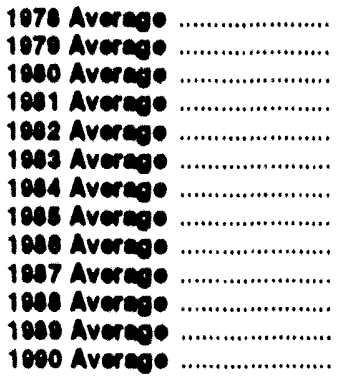 & $\begin{array}{r}43.4 \\
03.7 \\
04.1 \\
109.4 \\
07.3 \\
01.2 \\
03.2 \\
03.1 \\
63.1 \\
61.0 \\
67.7 \\
05.4 \\
70.0\end{array}$ & $\begin{array}{r}03.7 \\
72.1 \\
112.0 \\
128.0 \\
122.8 \\
117.0 \\
110.0 \\
113.0 \\
01.2 \\
08.0 \\
05.0 \\
10.0 \\
105.0\end{array}$ & $\begin{array}{r}38.4 \\
64.0 \\
08.0 \\
101.2 \\
08.3 \\
08.4 \\
63.0 \\
70.4 \\
40.5 \\
63.8 \\
40.8 \\
60.3 \\
7.3\end{array}$ & 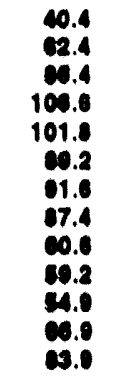 & 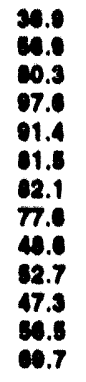 & 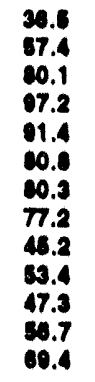 & $\begin{array}{l}23.7 \\
21.1 \\
41.8 \\
4 . .0 \\
42.7 \\
4.4 \\
4.0 \\
29.0 \\
20.0 \\
25.2 \\
24.0 \\
24.7 \\
39.6\end{array}$ \\
\hline 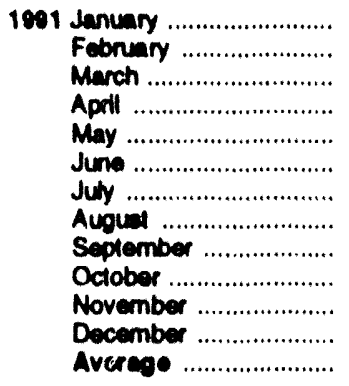 & $\begin{array}{l}76.2 \\
68.0 \\
67.3 \\
70.7 \\
74.2 \\
70.5 \\
69.1 \\
72.7 \\
69.1 \\
68.8 \\
69.9 \\
62.9 \\
60.0\end{array}$ & $\begin{array}{r}111.2 \\
104.2 \\
97.4 \\
97.8 \\
100.3 \\
99.5 \\
98.9 \\
100.2 \\
99.9 \\
98.8 \\
99.5 \\
97.3 \\
100.1\end{array}$ & $\begin{array}{l}82.0 \\
74.0 \\
62.4 \\
58.9 \\
60.8 \\
58.8 \\
59.4 \\
63.3 \\
65.0 \\
67.1 \\
68.2 \\
60.1 \\
65.0\end{array}$ & $\begin{array}{l}68.0 \\
76.1 \\
68.2 \\
63.0 \\
61.4 \\
59.0 \\
62.6 \\
67.1 \\
68.9 \\
73.5 \\
74.6 \\
62.6 \\
\mathbf{7 2 . 2}\end{array}$ & $\begin{array}{l}76.6 \\
67.0 \\
58.6 \\
57.2 \\
58.0 \\
54.0 \\
56.7 \\
60.6 \\
62.1 \\
68.3 \\
66.6 \\
55.9 \\
62.2\end{array}$ & $\begin{array}{l}75.5 \\
67.4 \\
57.7 \\
57.4 \\
57.2 \\
54.5 \\
57.1 \\
61.9 \\
62.0 \\
65.6 \\
66.5 \\
55.6 \\
61.6\end{array}$ & $\begin{array}{l}42.2 \\
31.6 \\
31.3 \\
31.8 \\
31.0 \\
29.3 \\
27.6 \\
29.6 \\
34.9 \\
40.2 \\
43.0 \\
37.7 \\
34.0\end{array}$ \\
\hline 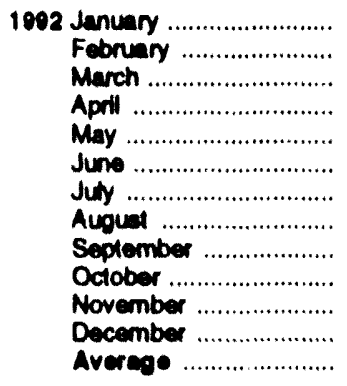 & $\begin{array}{l}60.0 \\
61.7 \\
62.7 \\
68.6 \\
71.5 \\
74.2 \\
71.0 \\
70.6 \\
71.0 \\
70.4 \\
68.1 \\
63.8 \\
67.7\end{array}$ & $\begin{array}{r}94.9 \\
93.1 \\
92.5 \\
96.4 \\
100.5 \\
101.5 \\
102.0 \\
102.6 \\
102.3 \\
100.5 \\
99.7 \\
97.6 \\
99.1\end{array}$ & $\begin{array}{l}53.9 \\
55.2 \\
54.6 \\
58.9 \\
60.8 \\
63.3 \\
64.8 \\
63.9 \\
64.3 \\
66.0 \\
61.5 \\
58.9 \\
60.4\end{array}$ & $\begin{array}{l}59.9 \\
62.0 \\
59.1 \\
61.6 \\
62.1 \\
63.7 \\
66.7 \\
64.2 \\
68.8 \\
70.1 \\
64.5 \\
62.8 \\
63.2\end{array}$ & $\begin{array}{l}51.0 \\
54.0 \\
53.7 \\
56.5 \\
58.8 \\
61.7 \\
61.3 \\
60.1 \\
62.7 \\
64.6 \\
58.8 \\
55.7 \\
57.0\end{array}$ & $\begin{array}{l}51.4 \\
54.1 \\
54.0 \\
57.0 \\
60.1 \\
62.7 \\
61.8 \\
60.4 \\
63.3 \\
65.5 \\
60.4 \\
56.4 \\
59.0\end{array}$ & $\begin{array}{l}30.9 \\
30.2 \\
29.5 \\
29.0 \\
29.4 \\
31.6 \\
31.5 \\
32.9 \\
36.4 \\
36.6 \\
36.2 \\
36.3 \\
32.8\end{array}$ \\
\hline 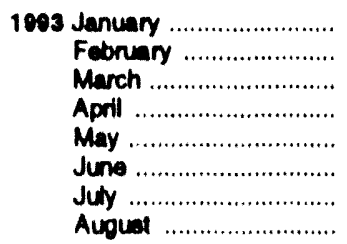 & $\begin{array}{r}63.8 \\
63.8 \\
65.2 \\
67.7 \\
69.2 \\
66.2 \\
\text { A } 62.7 \\
62.9\end{array}$ & $\begin{array}{l}96.0 \\
98.5 \\
97.4 \\
97.7 \\
90.4 \\
99.1 \\
97.9 \\
98.9\end{array}$ & $\begin{array}{r}57.7 \\
60.5 \\
60.3 \\
50.0 \\
60.1 \\
58.4 \\
55.1 \\
55.2\end{array}$ & $\begin{array}{l}61.4 \\
63.7 \\
65.4 \\
60.8 \\
58.3 \\
58.9 \\
53.6 \\
55.6\end{array}$ & $\begin{array}{l}54.4 \\
56.9 \\
50.0 \\
57.5 \\
58.0 \\
54.9 \\
51.0 \\
50.9\end{array}$ & $\begin{array}{l}54.9 \\
57.4 \\
60.0 \\
59.9 \\
59.6 \\
57.2 \\
53.1 \\
53.3\end{array}$ & $\begin{array}{l}\mathbf{4 0 . 2} \\
\mathbf{3 9 . 7} \\
\mathbf{3 9 . 2} \\
\mathbf{3 8 . 2} \\
\mathbf{3 4 . 0} \\
\mathbf{3 3 . 8} \\
\mathbf{3 3 . 3} \\
\mathbf{3 3 . 3}\end{array}$ \\
\hline
\end{tabular}

- See Note 5 at end of section.

A=Revised data.

Notes: - Sales for resab are those made to purchnasers other than ultimate consumers. Sales to end users are shown in Table 9.7; thoy are sabes made directly to the ulimate consumer, including bulk custorivers, such as agicutiure, industry, and electric viltibs, as woll as residential and commercial customers. Geographic coverage the 50 States and the District of Columbla. - Values for the current month are preliminary. Prices prior to 1983 are Energy Intormation Administration (EIA) estimates. See Note 6 at end of section.

Source: ElA, Petroleum Marketing Monthy, November 1993, Table 4. 
Table 9.7 Refiner Prices of Petroleum Products to End Users (Cents per Gallon, Excluding Taxes)

\begin{tabular}{|c|c|c|c|c|c|c|c|}
\hline & $\begin{array}{l}\text { Pinlahed } \\
\text { Motor } \\
\text { exwellnea }\end{array}$ & $\begin{array}{l}\text { Findehed } \\
\text { Aviation } \\
\text { Cacollno }\end{array}$ & $\begin{array}{l}\text { Koresene } \\
\text { Type } \\
\text { Jelfuld }\end{array}$ & Keresene & $\begin{array}{l}\text { No. } 2 \\
\text { Fual } \\
\text { Oll }\end{array}$ & $\begin{array}{l}\text { No. } 2 \\
\text { Olocel } \\
\text { Fuel }\end{array}$ & $\begin{array}{l}\text { Propene } \\
\text { (Concumer } \\
\text { Orado) }\end{array}$ \\
\hline 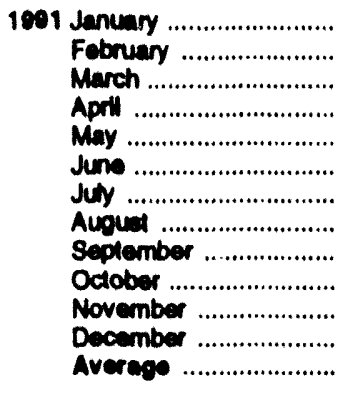 & $\begin{array}{l}89.8 \\
79.5 \\
74.0 \\
77.0 \\
82.0 \\
81.9 \\
78.9 \\
81.1 \\
80.2 \\
77.9 \\
79.1 \\
76.0 \\
70.7\end{array}$ & $\begin{array}{l}112.1 \\
108.4 \\
101.3 \\
101.2 \\
106.3 \\
106.2 \\
103.6 \\
106.8 \\
105.7 \\
104.6 \\
104.3 \\
102.0 \\
104.7\end{array}$ & $\begin{array}{l}81.1 \\
73.7 \\
62.1 \\
58.7 \\
60.1 \\
59.2 \\
50.7 \\
63.8 \\
66.6 \\
67.8 \\
69.6 \\
61.5 \\
65.2\end{array}$ & $\begin{array}{l}106.0 \\
68.0 \\
88.8 \\
73.8 \\
60.3 \\
62.3 \\
64.7 \\
68.7 \\
73.6 \\
81.6 \\
04.3 \\
86.8 \\
61.0\end{array}$ & $\begin{array}{l}84.3 \\
75.2 \\
64.5 \\
61.6 \\
58.0 \\
56.3 \\
59.1 \\
62.3 \\
63.0 \\
66.5 \\
70.0 \\
63.0 \\
66.8\end{array}$ & $\begin{array}{l}80.5 \\
71.4 \\
61.8 \\
60.6 \\
60.1 \\
57.9 \\
59.5 \\
63.3 \\
64.8 \\
68.0 \\
60.7 \\
60.9 \\
64.8\end{array}$ & $\begin{array}{l}83.7 \\
81.4 \\
76.0 \\
67.4 \\
66.7 \\
62.8 \\
61.1 \\
63.6 \\
65.0 \\
68.0 \\
73.7 \\
78.2 \\
73.0\end{array}$ \\
\hline 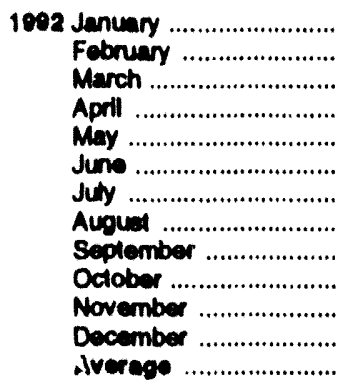 & $\begin{array}{l}71.0 \\
70.8 \\
71.6 \\
76.2 \\
80.8 \\
81.5 \\
83.5 \\
82.3 \\
82.3 \\
81.3 \\
81.5 \\
78.5 \\
71.4\end{array}$ & $\begin{array}{r}98.5 \\
98.5 \\
98.0 \\
99.1 \\
102.4 \\
108.4 \\
108.8 \\
105.7 \\
104.9 \\
104.3 \\
103.4 \\
101.3 \\
102.7\end{array}$ & $\begin{array}{l}54.2 \\
56.5 \\
55.5 \\
57.3 \\
61.0 \\
63.0 \\
64.0 \\
64.2 \\
64.0 \\
66.4 \\
62.7 \\
58.9 \\
61.0\end{array}$ & $\begin{array}{l}83.3 \\
78.3 \\
80.2 \\
78.3 \\
73.3 \\
68.7 \\
70.5 \\
60.0 \\
70.5 \\
87.2 \\
83.3 \\
84.0 \\
70.0\end{array}$ & $\begin{array}{l}59.7 \\
62.0 \\
61.4 \\
60.6 \\
60.0 \\
62.9 \\
62.8 \\
62.3 \\
66.6 \\
68.2 \\
64.3 \\
63.6 \\
62.7\end{array}$ & $\begin{array}{l}65.6 \\
57.1 \\
56.8 \\
69.2 \\
62.1 \\
64.0 \\
64.5 \\
63.4 \\
66.3 \\
67.8 \\
64.5 \\
60.8 \\
61.0\end{array}$ & $\begin{array}{c}71.3 \\
\mathrm{NM} \\
66.4 \\
70.3 \\
62.5 \\
54.5 \\
52.3 \\
56.8 \\
60.3 \\
50.0 \\
61.1 \\
68.4 \\
6.2\end{array}$ \\
\hline
\end{tabular}

- See Note 5 at end of section.

RaRevised dale. NA=Not avellable.

Notes: - Sales to end ween are those made drectly to the vitimale coneumer, including bulk customers, such as agriculture, industry, and

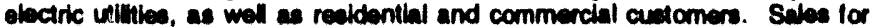
realb are shown in Table 9.6; they are sales made to purchasers other than ulimate coneumers. - Geographic covernge b the 50 States and the Ditilat of Columbia. - Value for the current month ere preliminary. Prices prior to 1883 are Energy Iniormation Adminiatraton (EIA) estmates. Ses Nole 6 at and of section.

Source: EM, Petrobum Marketing Monthy, November 1993, Table 2. 
Table 9.8a No. 2 Distillate Prices to Residences: Northeastern States (Cents per Gallon, Excluding Taxes)

\begin{tabular}{|c|c|c|c|c|c|c|c|c|c|}
\hline & Malno & $\begin{array}{c}\text { Now } \\
\text { Hampohire }\end{array}$ & Vermont & Meserehusette & $\begin{array}{l}\text { Rhode } \\
\text { lelend }\end{array}$ & Connectleut & $\begin{array}{l}\text { Now } \\
\text { Yortk }\end{array}$ & $\begin{array}{l}\text { New } \\
\text { Jereoy }\end{array}$ & Penneylvania \\
\hline 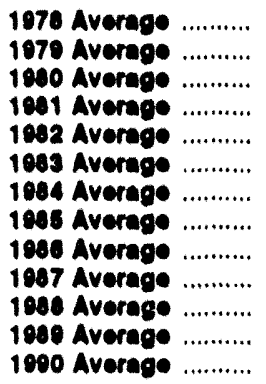 & 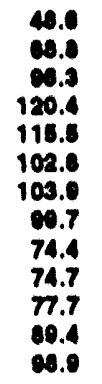 & $\begin{array}{r}60.3 \\
72.5 \\
100.4 \\
123.7 \\
117.4 \\
104.1 \\
108.4 \\
102.4 \\
78.0 \\
76.8 \\
78.2 \\
89.3 \\
102.8\end{array}$ & $\begin{array}{r}10.0 \\
7.8 \\
101 . \\
128.4 \\
120.1 \\
112.0 \\
111.0 \\
107.7 \\
64.6 \\
81.1 \\
82.6 \\
00.5 \\
107.0\end{array}$ & \begin{tabular}{r|}
48.6 \\
70.9 \\
07.0 \\
121.3 \\
117.6 \\
100.1 \\
111.6 \\
107.0 \\
82.1 \\
60.8 \\
12.1 \\
02.6 \\
108.4
\end{tabular} & $\begin{array}{r}60.7 \\
72.8 \\
101.1 \\
128.8 \\
120.1 \\
110.8 \\
111.4 \\
109.7 \\
82.8 \\
82.8 \\
83.8 \\
93.8 \\
108.6\end{array}$ & $\begin{array}{r}50.1 \\
72.0 \\
98.3 \\
121.7 \\
118.3 \\
109.1 \\
112.1 \\
108.0 \\
89.0 \\
83.4 \\
85.3 \\
02.0 \\
109.8\end{array}$ & $\begin{array}{r}80.1 \\
71.2 \\
6.2 \\
123.2 \\
120.5 \\
112.1 \\
115.5 \\
111.3 \\
01.1 \\
88.2 \\
68.3 \\
95.8 \\
112.8\end{array}$ & \begin{tabular}{r|}
49.6 \\
71.0 \\
87.0 \\
121.6 \\
117.4 \\
107.0 \\
111.0 \\
108.0 \\
00.2 \\
04.8 \\
84.8 \\
01.8 \\
108.7
\end{tabular} & \begin{tabular}{r|}
48.8 \\
09.8 \\
98.4 \\
118.1 \\
113.7 \\
108.8 \\
107.8 \\
102.3 \\
81.4 \\
76.9 \\
77.8 \\
83.1 \\
102.6
\end{tabular} \\
\hline $\begin{array}{l}1991 \text { January ............ } \\
\text { February .......... } \\
\text { March ............... } \\
\text { Aprll ................ } \\
\text { May .................. } \\
\text { June ............... } \\
\text { July .................. } \\
\text { Augus ........... } \\
\text { September ....... } \\
\text { October ............ } \\
\text { November ........ } \\
\text { December ......... } \\
\text { Averege ......... }\end{array}$ & $\begin{array}{r}114.4 \\
105.9 \\
85.4 \\
87.1 \\
81.9 \\
79.6 \\
82.3 \\
83.4 \\
87.3 \\
91.3 \\
85.1 \\
89.3 \\
86.0\end{array}$ & $\begin{array}{r}107.2 \\
100.7 \\
90.5 \\
83.9 \\
79.4 \\
77.3 \\
77.6 \\
80.6 \\
84.2 \\
87.8 \\
90.1 \\
88.8 \\
81.6\end{array}$ & $\begin{array}{r}117.7 \\
111.3 \\
104.4 \\
98.5 \\
23.5 \\
91.3 \\
88.1 \\
88.6 \\
91.9 \\
93.9 \\
95.7 \\
94.1 \\
101.0\end{array}$ & $\begin{array}{r}118.1 \\
111.3 \\
102.6 \\
96.1 \\
91.7 \\
88.9 \\
88.5 \\
88.7 \\
90.9 \\
94.9 \\
97.5 \\
95.8 \\
103.0\end{array}$ & $\begin{array}{l}113.3 \\
100.5 \\
101.8 \\
94.7 \\
89.7 \\
87.1 \\
88.8 \\
88.7 \\
90.3 \\
94.9 \\
95.8 \\
93.4 \\
00.0\end{array}$ & $\begin{array}{r}122.5 \\
116.0 \\
109.0 \\
101.4 \\
96.5 \\
92.7 \\
90.0 \\
89.7 \\
92.0 \\
96.3 \\
99.8 \\
98.3 \\
108.2\end{array}$ & $\begin{array}{r}124.6 \\
120.2 \\
112.8 \\
108.7 \\
101.2 \\
98.1 \\
93.9 \\
93.0 \\
98.7 \\
103.3 \\
108.1 \\
106.7 \\
111.3\end{array}$ & $\begin{array}{r}119.6 \\
113.2 \\
104.3 \\
98.6 \\
94.4 \\
90.3 \\
88.5 \\
89.0 \\
92.2 \\
96.9 \\
100.7 \\
96.6 \\
104.0\end{array}$ & $\begin{array}{r}117.7 \\
110.9 \\
101.8 \\
95.5 \\
89.9 \\
85.7 \\
80.8 \\
81.8 \\
83.4 \\
88.8 \\
93.6 \\
93.1 \\
90.7\end{array}$ \\
\hline 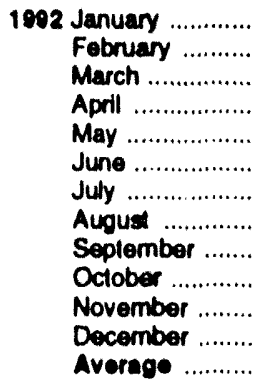 & $\begin{array}{l}87.7 \\
88.2 \\
88.4 \\
86.5 \\
86.5 \\
87.1 \\
87.7 \\
87.8 \\
86.8 \\
89.3 \\
88.3 \\
85.7 \\
87.1\end{array}$ & $\begin{array}{l}88.1 \\
86.5 \\
83.3 \\
81.8 \\
81.7 \\
82.9 \\
82.3 \\
81.8 \\
83.0 \\
87.6 \\
87.6 \\
87.7 \\
85.6\end{array}$ & $\begin{array}{l}92.4 \\
92.8 \\
92.2 \\
91.7 \\
91.5 \\
90.7 \\
89.1 \\
99.4 \\
91.6 \\
92.0 \\
92.6 \\
92.9 \\
92.2\end{array}$ & $\begin{array}{l}93.2 \\
92.5 \\
91.5 \\
91.4 \\
91.0 \\
91.3 \\
90.4 \\
89.6 \\
90.7 \\
93.5 \\
93.8 \\
93.5 \\
92.4\end{array}$ & $\begin{array}{l}90.7 \\
91.7 \\
90.9 \\
90.4 \\
90.9 \\
89.7 \\
89.9 \\
89.4 \\
89.8 \\
92.7 \\
92.5 \\
91.5 \\
91.2\end{array}$ & $\begin{array}{l}96.4 \\
95.5 \\
94.0 \\
93.3 \\
93.1 \\
91.8 \\
93.1 \\
90.5 \\
91.8 \\
94.9 \\
95.8 \\
95.2 \\
94.7\end{array}$ & $\begin{array}{r}103.4 \\
103.8 \\
102.1 \\
101.1 \\
101.1 \\
101.7 \\
100.7 \\
99.0 \\
99.7 \\
102.7 \\
104.7 \\
104.3 \\
102.8\end{array}$ & $\begin{array}{l}95.6 \\
95.1 \\
93.5 \\
92.0 \\
89.2 \\
90.4 \\
90.3 \\
88.1 \\
90.8 \\
94.0 \\
94.6 \\
95.4 \\
93.0\end{array}$ & $\begin{array}{l}91.4 \\
91.5 \\
90.1 \\
89.4 \\
88.6 \\
86.5 \\
83.0 \\
81.7 \\
84.4 \\
87.5 \\
89.6 \\
89.3 \\
88.0\end{array}$ \\
\hline 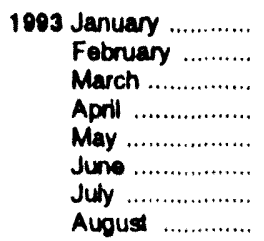 & $\begin{array}{r}85.2 \\
85.4 \\
86.5 \\
83.0 \\
81.5 \\
80.8 \\
\text { ค } 78.2 \\
77.4\end{array}$ & $\begin{array}{r}87.1 \\
87.0 \\
86.6 \\
85.0 \\
83.6 \\
82.5 \\
\text { ค } 78.0 \\
76.6\end{array}$ & $\begin{array}{l}93.4 \\
93.3 \\
93.7 \\
91.2 \\
91.2 \\
89.7 \\
85.5 \\
86.6\end{array}$ & $\begin{array}{r}94.0 \\
94.4 \\
94.8 \\
91.3 \\
90.9 \\
88.6 \\
83.9 \\
83.1\end{array}$ & $\begin{array}{r}91.7 \\
91.8 \\
92.4 \\
90.3 \\
90.6 \\
87.6 \\
985.2 \\
82.7\end{array}$ & $\begin{array}{r}94.9 \\
86.2 \\
86.7 \\
83.6 \\
91.7 \\
88.6 \\
\text { ค } 85.5 \\
83.9\end{array}$ & $\begin{array}{r}104.3 \\
104.2 \\
104.2 \\
100.1 \\
99.3 \\
97.8 \\
\text { P } 95.2 \\
93.5\end{array}$ & $\begin{array}{r}96.5 \\
96.7 \\
96.2 \\
95.1 \\
91.6 \\
88.0 \\
{ }^{8} 87.9 \\
85.8\end{array}$ & $\begin{array}{r}89.0 \\
89.1 \\
89.8 \\
89.0 \\
86.6 \\
84.0 \\
\text { ค } 78.8 \\
77.0\end{array}$ \\
\hline
\end{tabular}

$R=$ Revised data.

- Prices prior to 1983 are Energy Information Administration (EIA) estimates. Notes: - States are grouped in Tables 9.8a, 9.8b, and 9.8c by geographic region of the country. Values for the current month are preliminary. 
Table 9.8b No. 2 Distillate Prices to Residences: Selected South Atlantic and Midwestern States

(Cents per Gallon, Excluding Taxes)

\begin{tabular}{|c|c|c|c|c|c|c|c|c|c|c|c|}
\hline & Dolewere & $\begin{array}{c}\text { Distriet } \\
\text { of } \\
\text { Columbla }\end{array}$ & Marylend & Virglnie & $\begin{array}{l}\text { Weat } \\
\text { Virginla }\end{array}$ & Onlo & Michlean & Indlane & IIIInole & Meconein & Minnesote \\
\hline 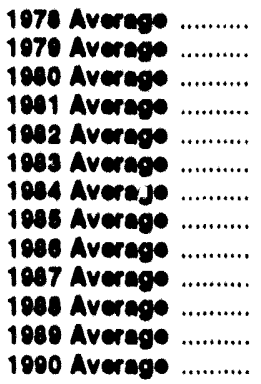 & 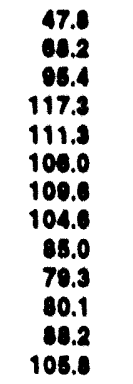 & $\begin{array}{r}60.7 \\
74.2 \\
102.0 \\
127.4 \\
124.8 \\
117.0 \\
118.7 \\
114.9 \\
98.1 \\
91.8 \\
91.0 \\
90.6 \\
107.0\end{array}$ & $\begin{array}{r}40.2 \\
70.1 \\
97.0 \\
121.4 \\
117.1 \\
110.3 \\
113.8 \\
108.0 \\
91.4 \\
80.0 \\
87.0 \\
93.0 \\
111.0\end{array}$ & $\begin{array}{r}40.1 \\
70.4 \\
00.5 \\
120.5 \\
117.7 \\
100.7 \\
110.5 \\
108.3 \\
10.8 \\
70.5 \\
00.5 \\
67.0 \\
110.8\end{array}$ & $\begin{array}{r}46.2 \\
05.1 \\
92.2 \\
116.0 \\
109.3 \\
101.0 \\
102.1 \\
08.0 \\
74.0 \\
70.4 \\
74.2 \\
93.0 \\
09.1\end{array}$ & $\begin{array}{r}47.4 \\
69.0 \\
91.0 \\
113.2 \\
110.2 \\
101.9 \\
102.1 \\
00.7 \\
77.7 \\
74.7 \\
74.7 \\
81.8 \\
08.1\end{array}$ & $\begin{array}{r}47.0 \\
70.0 \\
97.0 \\
118.3 \\
113.0 \\
105.4 \\
105.0 \\
102.1 \\
01.0 \\
77.5 \\
77.5 \\
08.9 \\
100.0\end{array}$ & $\begin{array}{r}49.1 \\
72.7 \\
09.1 \\
110.8 \\
114.3 \\
100.7 \\
103.1 \\
09.1 \\
74.8 \\
78.4 \\
78.4 \\
13.2 \\
09.3\end{array}$ & 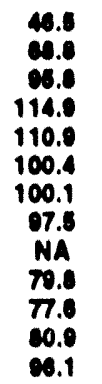 & $\begin{array}{r}44.7 \\
67.3 \\
01.8 \\
109.1 \\
107.8 \\
101.2 \\
101.0 \\
98.3 \\
75.0 \\
78.1 \\
73.0 \\
11.1 \\
04.2\end{array}$ & $\begin{array}{r}47.8 \\
72.4 \\
60.9 \\
118.4 \\
118.1 \\
109.1 \\
104.1 \\
101.0 \\
70.2 \\
74.6 \\
73.6 \\
02.4 \\
101.4\end{array}$ \\
\hline 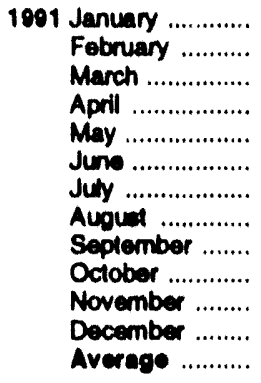 & $\begin{array}{r}113.0 \\
105.4 \\
88.4 \\
92.3 \\
91.5 \\
84.0 \\
81.5 \\
86.0 \\
87.3 \\
92.8 \\
86.8 \\
84.9 \\
00.7\end{array}$ & $\begin{array}{r}124.1 \\
118.6 \\
112.3 \\
105.6 \\
101.1 \\
85.3 \\
88.6 \\
88.6 \\
101.7 \\
104.0 \\
107.3 \\
107.7 \\
112.2\end{array}$ & $\begin{array}{r}122.0 \\
116.1 \\
107.7 \\
102.7 \\
98.7 \\
98.2 \\
93.7 \\
94.0 \\
96.8 \\
100.1 \\
103.2 \\
102.6 \\
108.4\end{array}$ & $\begin{array}{r}117.2 \\
110.3 \\
102.4 \\
88.1 \\
80.7 \\
87.8 \\
86.8 \\
87.5 \\
90.4 \\
93.6 \\
67.0 \\
95.2 \\
101.1\end{array}$ & $\begin{array}{r}110.5 \\
101.5 \\
90.8 \\
87.6 \\
85.8 \\
83.6 \\
81.7 \\
82.4 \\
84.8 \\
89.7 \\
91.8 \\
89.0 \\
93.4\end{array}$ & $\begin{array}{r}105.6 \\
94.6 \\
85.7 \\
83.2 \\
83.1 \\
60.7 \\
79.6 \\
81.1 \\
84.8 \\
88.7 \\
91.8 \\
66.0 \\
91.0\end{array}$ & $\begin{array}{r}109.8 \\
88.5 \\
91.5 \\
80.7 \\
88.1 \\
87.4 \\
83.3 \\
84.4 \\
88.8 \\
89.5 \\
92.8 \\
89.9 \\
94.2\end{array}$ & $\begin{array}{r}105.9 \\
05.4 \\
87.9 \\
86.0 \\
86.3 \\
80.3 \\
78.8 \\
85.5 \\
85.5 \\
86.7 \\
87.8 \\
83.3 \\
81.0\end{array}$ & $\begin{array}{r}102.5 \\
82.9 \\
86.5 \\
88.3 \\
88.5 \\
88.8 \\
82.2 \\
86.5 \\
87.3 \\
88.4 \\
92.4 \\
69.9 \\
92.7\end{array}$ & $\begin{array}{r}102.4 \\
92.4 \\
87.8 \\
84.0 \\
82.9 \\
80.0 \\
78.0 \\
78.8 \\
82.7 \\
85.7 \\
89.9 \\
85.4 \\
89.5\end{array}$ & $\begin{array}{r}105.4 \\
83.5 \\
87.2 \\
87.8 \\
88.1 \\
87.1 \\
84.4 \\
86.3 \\
84.0 \\
86.8 \\
89.2 \\
64.4 \\
01.1\end{array}$ \\
\hline $\begin{array}{l}1002 \text { January ............ } \\
\text { February .......... } \\
\text { March ............... } \\
\text { Aprll ................ } \\
\text { May ................. } \\
\text { June ................. } \\
\text { Juty ................. } \\
\text { Augut ............ } \\
\text { September ....... } \\
\text { October ............ } \\
\text { November ........ } \\
\text { December ........ } \\
\text { Averege ......... }\end{array}$ & $\begin{array}{l}94.4 \\
92.7 \\
92.4 \\
91.5 \\
90.2 \\
91.4 \\
90.6 \\
89.5 \\
90.3 \\
93.7 \\
92.8 \\
90.8 \\
92.4\end{array}$ & $\begin{array}{l}107.3 \\
107.3 \\
105.3 \\
104.7 \\
102.3 \\
102.7 \\
102.0 \\
101.9 \\
101.2 \\
104.0 \\
105.7 \\
105.4 \\
108.7\end{array}$ & $\begin{array}{r}101.6 \\
100.8 \\
100.3 \\
99.0 \\
97.2 \\
97.6 \\
95.7 \\
95.2 \\
95.7 \\
98.8 \\
100.4 \\
100.4 \\
90.0\end{array}$ & $\begin{array}{l}94.3 \\
93.7 \\
93.7 \\
92.6 \\
91.7 \\
89.6 \\
90.2 \\
88.4 \\
69.4 \\
91.9 \\
92.1 \\
93.3 \\
92.8\end{array}$ & $\begin{array}{l}85.5 \\
86.9 \\
86.6 \\
85.6 \\
84.2 \\
86.5 \\
82.3 \\
81.4 \\
85.4 \\
88.3 \\
88.0 \\
89.0 \\
88.4\end{array}$ & $\begin{array}{l}82.0 \\
83.0 \\
82.5 \\
82.9 \\
83.5 \\
85.3 \\
81.7 \\
82.3 \\
84.7 \\
88.4 \\
84.6 \\
84.5 \\
83.6\end{array}$ & $\begin{array}{l}86.6 \\
86.5 \\
86.6 \\
86.7 \\
86.4 \\
86.1 \\
86.0 \\
86.7 \\
88.2 \\
80.0 \\
88.2 \\
87.9 \\
87.1\end{array}$ & $\begin{array}{l}77.8 \\
78.7 \\
79.5 \\
80.2 \\
81.2 \\
79.6 \\
82.4 \\
83.1 \\
84.8 \\
85.8 \\
82.7 \\
81.8 \\
81.1\end{array}$ & $\begin{array}{l}85.2 \\
86.6 \\
88.1 \\
88.4 \\
89.0 \\
80.8 \\
87.0 \\
88.4 \\
88.0 \\
80.6 \\
80.4 \\
88.2 \\
87.6\end{array}$ & $\begin{array}{l}80.1 \\
79.8 \\
79.2 \\
80.4 \\
81.5 \\
81.9 \\
81.1 \\
80.6 \\
83.6 \\
84.1 \\
83.7 \\
84.3 \\
81.8\end{array}$ & $\begin{array}{l}79.4 \\
79.6 \\
79.7 \\
81.8 \\
83.0 \\
82.0 \\
84.5 \\
84.1 \\
85.0 \\
87.1 \\
86.0 \\
83.1 \\
82.3\end{array}$ \\
\hline 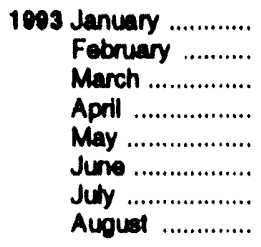 & $\begin{array}{r}80.8 \\
90.8 \\
92.4 \\
91.6 \\
89.4 \\
90.9 \\
990.2 \\
86.0\end{array}$ & $\begin{array}{r}105.2 \\
108.8 \\
108.5 \\
107.1 \\
104.3 \\
100.4 \\
\text { A } 100.2 \\
98.3\end{array}$ & $\begin{array}{r}100.5 \\
101.3 \\
101.6 \\
98.2 \\
96.2 \\
95.2 \\
992.3 \\
91.7\end{array}$ & $\begin{array}{r}92.4 \\
93.5 \\
94.2 \\
90.3 \\
88.6 \\
88.0 \\
{ }^{9} 8.7 \\
84.3\end{array}$ & $\begin{array}{l}88.3 \\
88.6 \\
89.9 \\
88.9 \\
84.8 \\
88.7 \\
81.2 \\
78.7\end{array}$ & $\begin{array}{r}84.2 \\
85.5 \\
86.6 \\
86.9 \\
86.0 \\
85.7 \\
79.3 \\
78.7\end{array}$ & $\begin{array}{l}88.3 \\
87.6 \\
90.1 \\
90.8 \\
89.8 \\
87.4 \\
83.4 \\
83.0\end{array}$ & $\begin{array}{r}81.8 \\
82.3 \\
83.1 \\
84.9 \\
83.6 \\
82.1 \\
\text { A } \\
79.0 \\
76.5\end{array}$ & $\begin{array}{r}87.2 \\
88.2 \\
90.0 \\
N A \\
84.8 \\
81.2 \\
A 79.4 \\
77.2\end{array}$ & $\begin{array}{r}82.1 \\
83.3 \\
84.0 \\
84.7 \\
84.9 \\
84.2 \\
\text { R4.1 } \\
79.1\end{array}$ & $\begin{array}{l}82.9 \\
83.0 \\
83.9 \\
83.3 \\
84.1 \\
83.4 \\
\varepsilon^{-} .0 \\
79.4\end{array}$ \\
\hline
\end{tabular}

$R=R e v i e e d$ dala. $N A=N o t$ avallable.

Notes: - Stales are grouped in Tables $9.8 \mathrm{a}, 8.8 \mathrm{~b}$, and $9.8 \mathrm{c}$ by geographic recion of the country. - Values lor the current month are preliminary.
- Prices prior to 1983 are Energy Information Administration (EIA) estimales. See Note 6 at end of section.

Source: ElA, Petroleum Marketing Monthy, November 1993, Table 16 
Table 9.8c No. 2 Distillate Prices to Residences: Selected Western States and U.S. Average

(Cents per Gallon, Excluding Taxes)

\begin{tabular}{|c|c|c|c|c|c|}
\hline & Idaho & Weshington & Oregon & Alaskn & $\begin{array}{c}\text { U.S. } \\
\text { Avorage }\end{array}$ \\
\hline 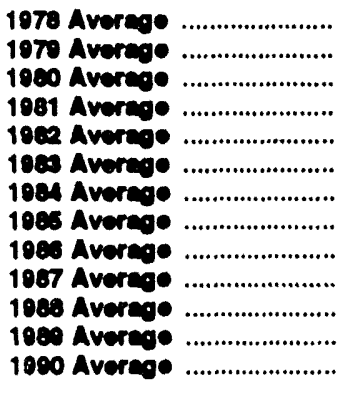 & $\begin{array}{r}43.6 \\
62.1 \\
91.6 \\
110.4 \\
110.4 \\
101.0 \\
60.6 \\
97.2 \\
73.8 \\
66.8 \\
68.0 \\
77.8 \\
97.4\end{array}$ & $\begin{array}{r}40.6 \\
60.7 \\
100.8 \\
118.5 \\
117.0 \\
100.0 \\
102.0 \\
101.1 \\
71.5 \\
70.5 \\
78.5 \\
87.4 \\
102.8\end{array}$ & $\begin{array}{r}46.8 \\
86.0 \\
97.3 \\
111.4 \\
111.0 \\
103.6 \\
09.3 \\
07.1 \\
70.4 \\
72.5 \\
70.0 \\
80.2 \\
97.0\end{array}$ & $\begin{array}{r}53.2 \\
60.2 \\
97.8 \\
116.0 \\
117.4 \\
100.8 \\
106.0 \\
108.3 \\
24.0 \\
80.5 \\
86.0 \\
86.4 \\
110.1\end{array}$ & $\begin{array}{r}49.0 \\
70.4 \\
97.4 \\
110.4 \\
116.0 \\
107.8 \\
100.1 \\
105.3 \\
83.6 \\
80.3 \\
81.3 \\
90.0 \\
106.3\end{array}$ \\
\hline 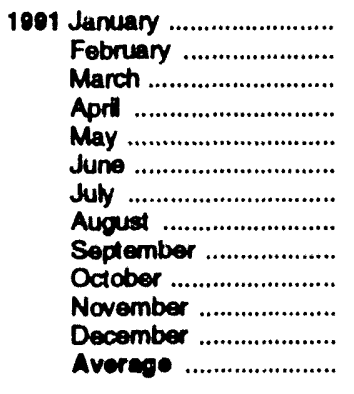 & $\begin{array}{r}110.8 \\
97.3 \\
84.0 \\
83.4 \\
84.4 \\
83.4 \\
80.0 \\
84.6 \\
87.4 \\
87.6 \\
93.3 \\
94.7 \\
95.1\end{array}$ & $\begin{array}{r}118.4 \\
112.0 \\
95.3 \\
23.5 \\
24.9 \\
91.7 \\
85.5 \\
92.6 \\
93.5 \\
85.2 \\
99.5 \\
96.2 \\
101.6\end{array}$ & $\begin{array}{r}108.4 \\
102.9 \\
88.8 \\
86.4 \\
86.5 \\
85.6 \\
83.6 \\
87.3 \\
90.8 \\
89.1 \\
90.6 \\
87.0 \\
03.3\end{array}$ & $\begin{array}{r}129.3 \\
122.8 \\
109.5 \\
101.9 \\
101.3 \\
98.2 \\
98.6 \\
96.8 \\
92.4 \\
91.3 \\
96.0 \\
96.2 \\
105.0\end{array}$ & $\begin{array}{r}117.1 \\
110.5 \\
102.6 \\
96.9 \\
92.5 \\
89.3 \\
86.6 \\
87.0 \\
89.7 \\
94.0 \\
98.0 \\
95.9 \\
101.9\end{array}$ \\
\hline 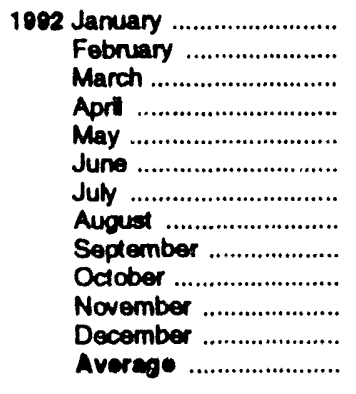 & $\begin{array}{l}86.1 \\
79.2 \\
82.2 \\
84.2 \\
86.1 \\
84.6 \\
86.1 \\
79.4 \\
86.0 \\
89.6 \\
91.7 \\
86.8 \\
85.7\end{array}$ & $\begin{array}{l}92.0 \\
90.9 \\
91.8 \\
92.0 \\
94.3 \\
90.6 \\
88.0 \\
84.0 \\
90.3 \\
94.5 \\
98.7 \\
99.7 \\
94.3\end{array}$ & $\begin{array}{l}85.3 \\
83.5 \\
82.6 \\
85.5 \\
88.9 \\
89.2 \\
87.3 \\
84.0 \\
87.6 \\
91.7 \\
92.8 \\
91.5 \\
87.0\end{array}$ & $\begin{array}{l}92.7 \\
91.1 \\
93.0 \\
92.1 \\
93.6 \\
93.9 \\
93.0 \\
96.8 \\
93.4 \\
96.8 \\
97.7 \\
95.8 \\
94.0\end{array}$ & $\begin{array}{l}94.2 \\
94.2 \\
93.2 \\
92.5 \\
92.3 \\
92.0 \\
90.4 \\
88.6 \\
90.1 \\
93.7 \\
94.8 \\
94.5 \\
93.4\end{array}$ \\
\hline
\end{tabular}

$R=$ Revised dala.

Notes: - States are grouped in Tables 9.8a, 9.8b, and 9.8c by geographic region of the country. "Values for the current month are preliminary.
- Prices prior to 1983 are Energy Information Administration (EIA) estimates. Seo Note 6 at end of section.

Source: EIA, Petroleum Marketing Monthly, November 1993, Table 16. 
Figure 9.2 Electricity Retail Prices

(Cents per Kilowatthour)

Prices by Sector, 1973-1992

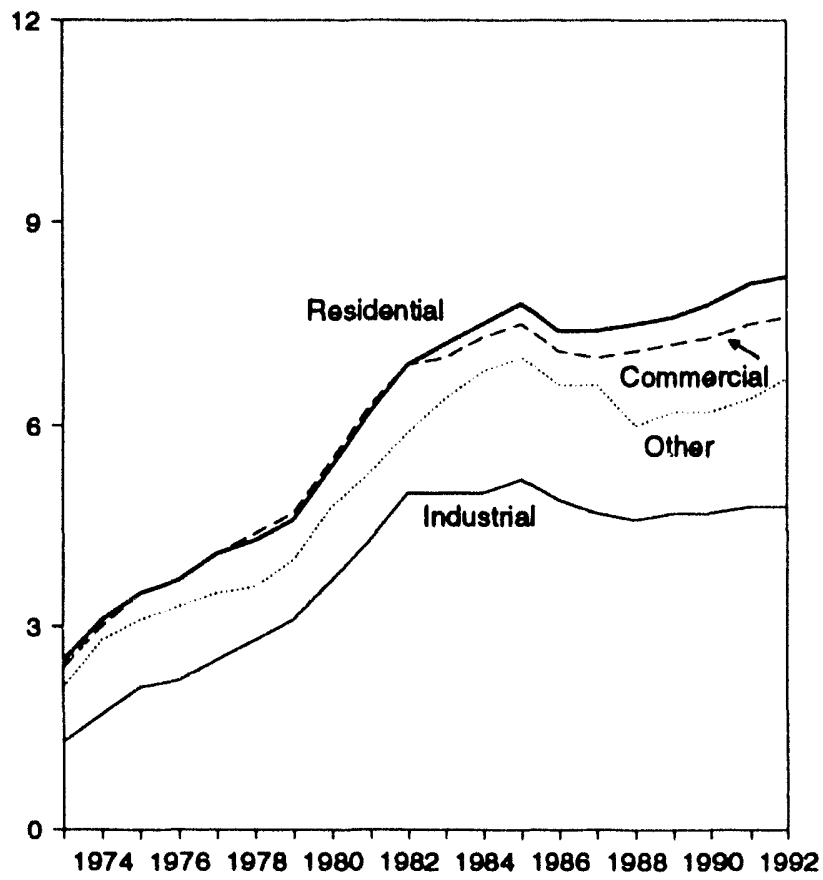

Prices by Sector, Monthly

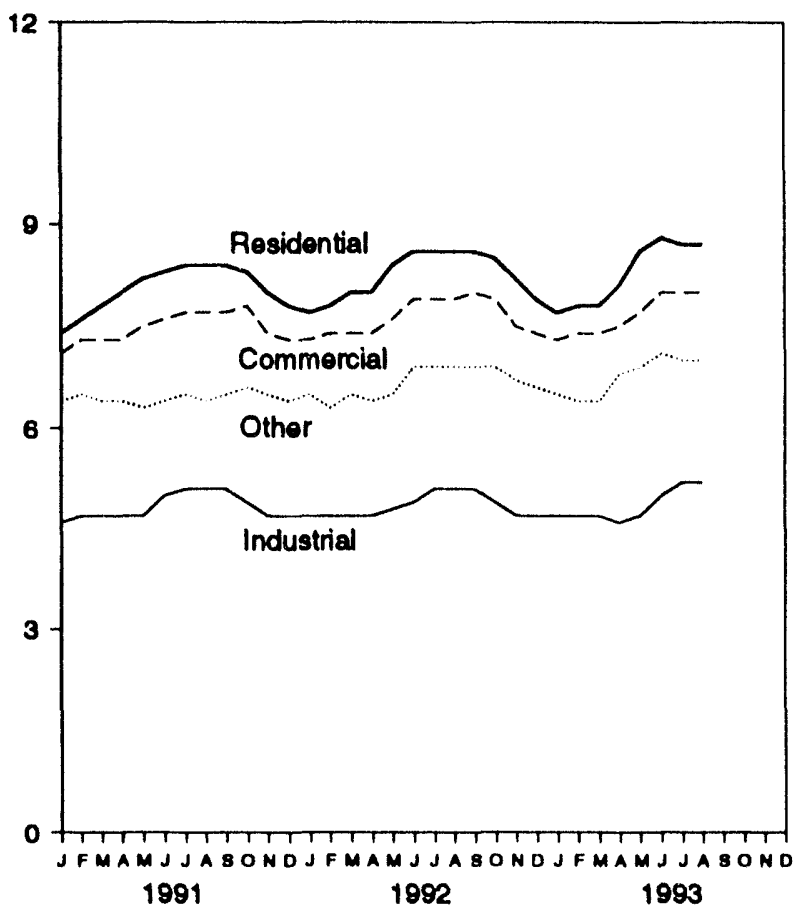

Source: Table 9.9, Monthly Series.

Figure 9.3 Cost of Fossil-Fuel Recelpts at Steam-Electric Plants (Dollars per Million Btu)

Fossil Fuels Costs, 1973-1992

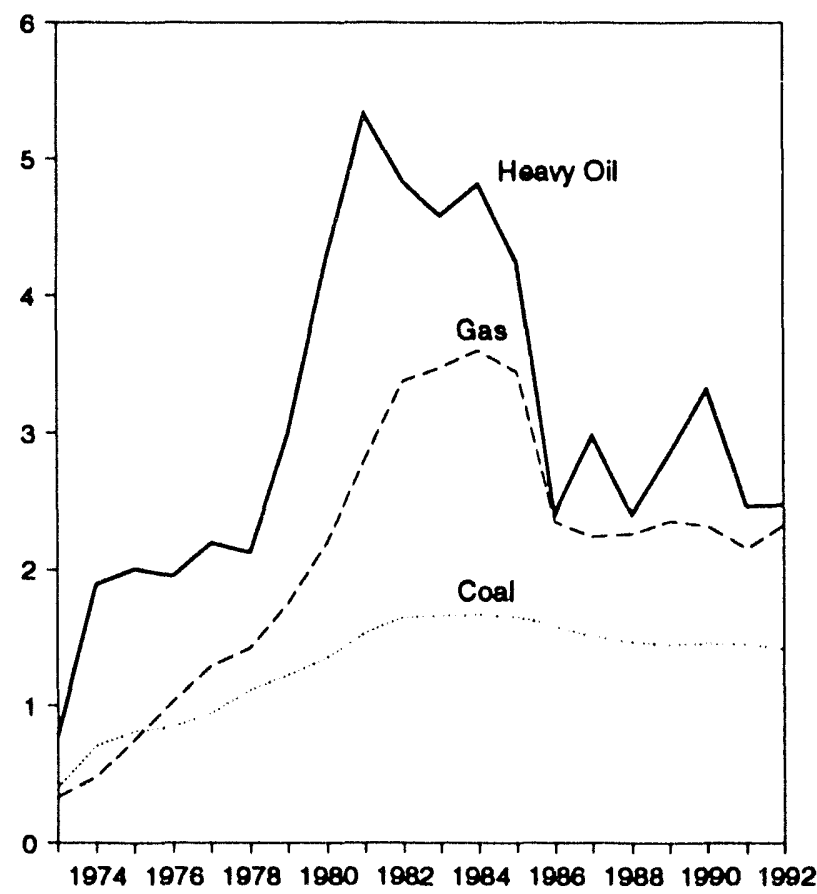

Fossil Fuel Costs, Monthly

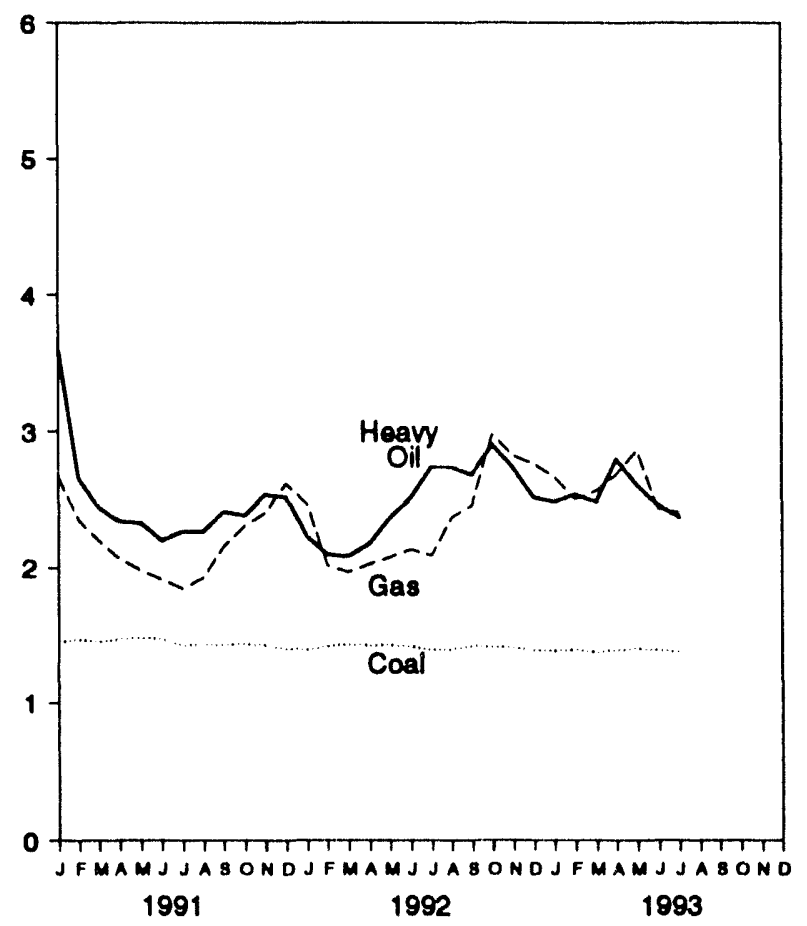




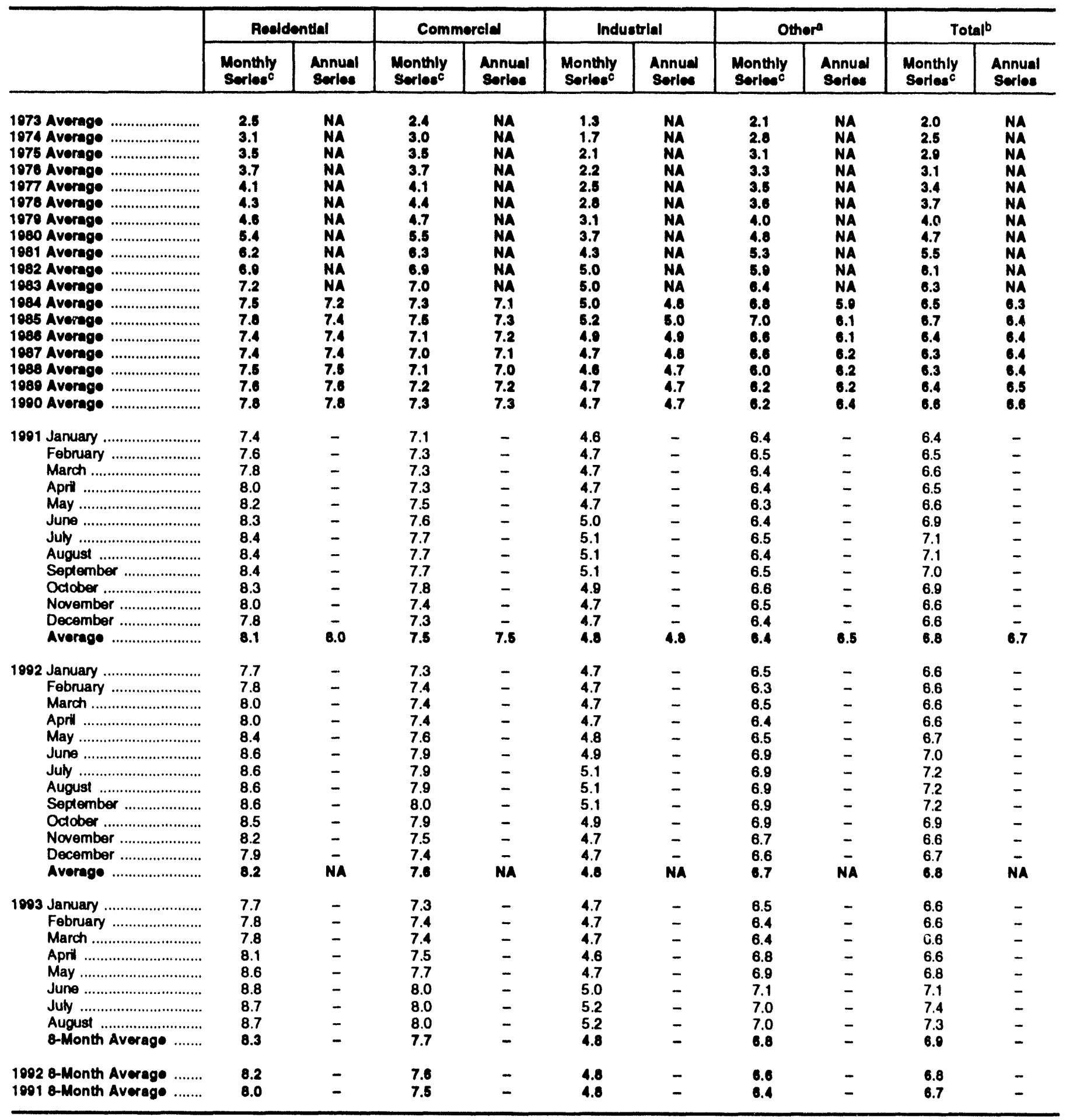

- "Other" is public street and highway lighting, oher sales to public authorities, sales to railroads and railways, and interdepartmental sales.

b Averago price for lotal sales to unimate consumers.

c Annual values are the sum of the monthly revenue divided by the sum of the monthly sales. Data through 1979 cover privately owned electric utilities in Classes A and B. Data for 1980-1985 cover selected privalely owned electric utilities in Class $A$ whose electric operating revenue was $\$ 100$ million or more during the previous year. See Nole 7 at end of section.

$\mathrm{NA}=\mathrm{Not}$ available. $-=\mathrm{Not}$ applicable.

Notes: - Prices are calculated by dividing revenue by sales. Revenue may not correspond to sales for a particular month because of electric utility billing and accounting procedures. That lack of correspondence could resul in uncharacteristic increases or decreases in the monthly prices. See Note 7 at end of section. - Geographic coverage is the 50 States and the District of Columbia.

Sources: - Monthly Sorles: 1073-September 1977-Federal Power Commission, Form FPC-5, "Monthly Statement of Eectric Operaling Revenue and Income." October 1077-February 1080-Federal Energy Regulatory Commission (FERC), Form FERC-5, "Electric Operating Revenue and Income." March 1080-December 1980-FERC, Form FERC-5, "Electric Utility Company Monthly Slatement. 1081-Energy Intormation Administration (EIA), Electric Power Monthy, March 1992, Table 59. 1982 and 1091 monthly data-EIA, Electric Power Monthly, March 1993, Table 59. 1983 fonwerd (oxcept 1091 monthly data)-ElA, Electric Powet Monthly, November 1993, Table 59. - Annual Series: ElA, Electric Power Monthly, November 1993, Table 59. 
Table 9.10 Quantity and Cost of Fossil-Fuel Receipts at Steam-Electric Utility Plants

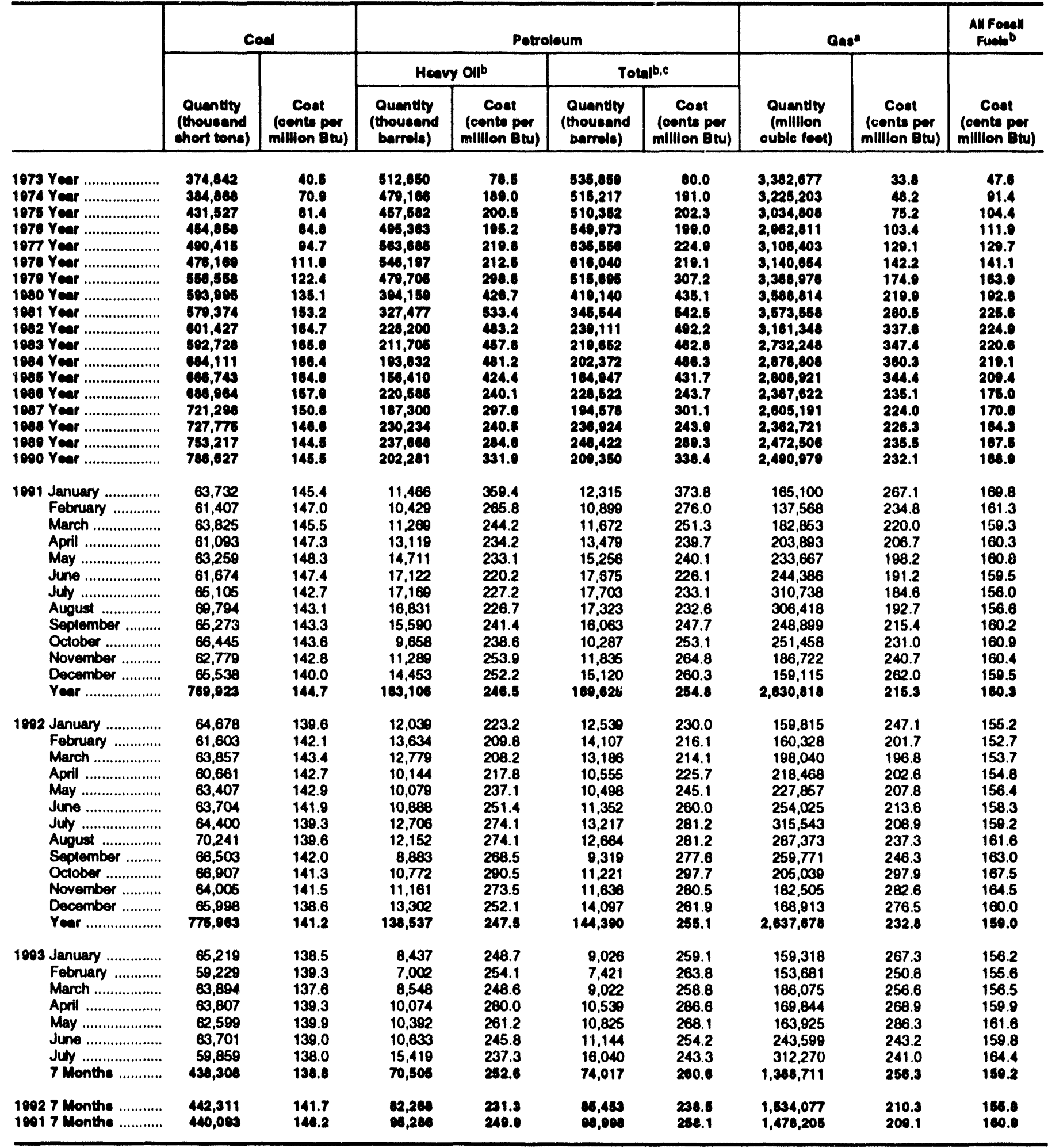

- Includes supplemental asseous fuels.

- Heavy oil includes fuel oll nos. 4, 5, and 6, and topped crude oll. The weighted averages for petrobum and all fossil fueb include both heavy and light ofl (No. 2 fuel oil, kerosene, and let fuel) prices. Data do not include petroleum coke.

c Data for 1973-1982 do not include small quantilies of rerelined molor oll. bunker oll, and liquelied petrobum gas.

Notes: - Dala lor 1973-1982 cover all electric generating plants at which the generator nameplate capacity of all steam-electric units combined lotaled
25 megawatts or greater. For 1974-1982, peaking units were included in the data and counted towards the 25-megawatt-or-greater lotal. Dath for 1983-1990 cover all electric generating plants at which the ceneralor nameplate capacily of all steam-electric units combined totaled 50 megawatts or greater. Data for 1891 forward cover all electric generating plants al which the cenerator nameplate capacity of all steam-electric units and combined-cycle unls combined lotaled 50 megawatts or greater. - Geographic coverago is the 50 Siates and the Disirict of Columbia.

Sources: See end of section. 
Figure 9.4 Natural Gas Prices

(Dollars per Thousand Cubic Feet)

Selected Prices, 1973-1992

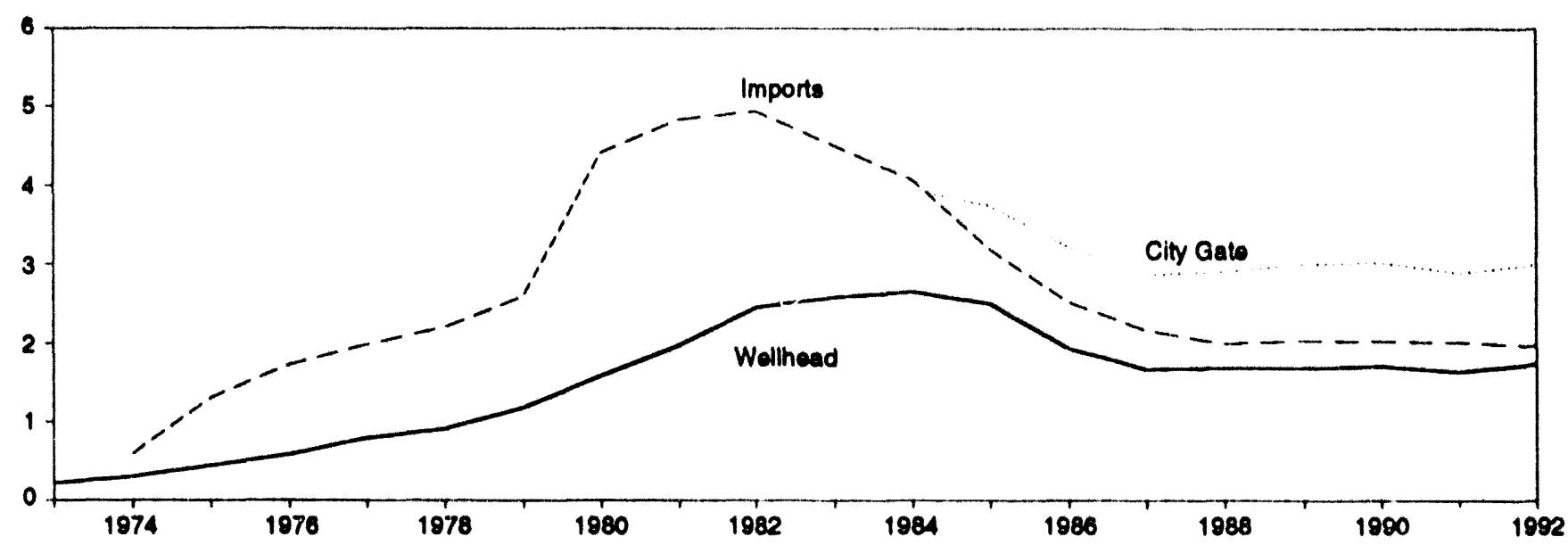

Delivered to Consumers, $1973-1992$

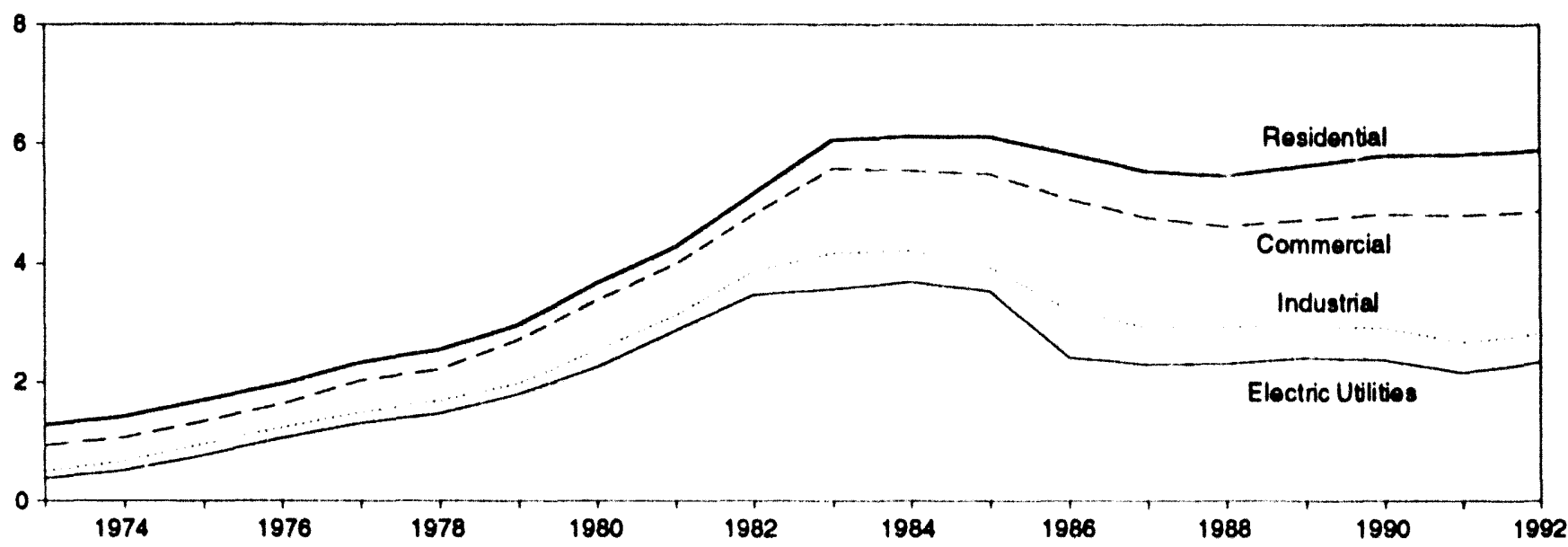

Delivered to Consumers, Monthly

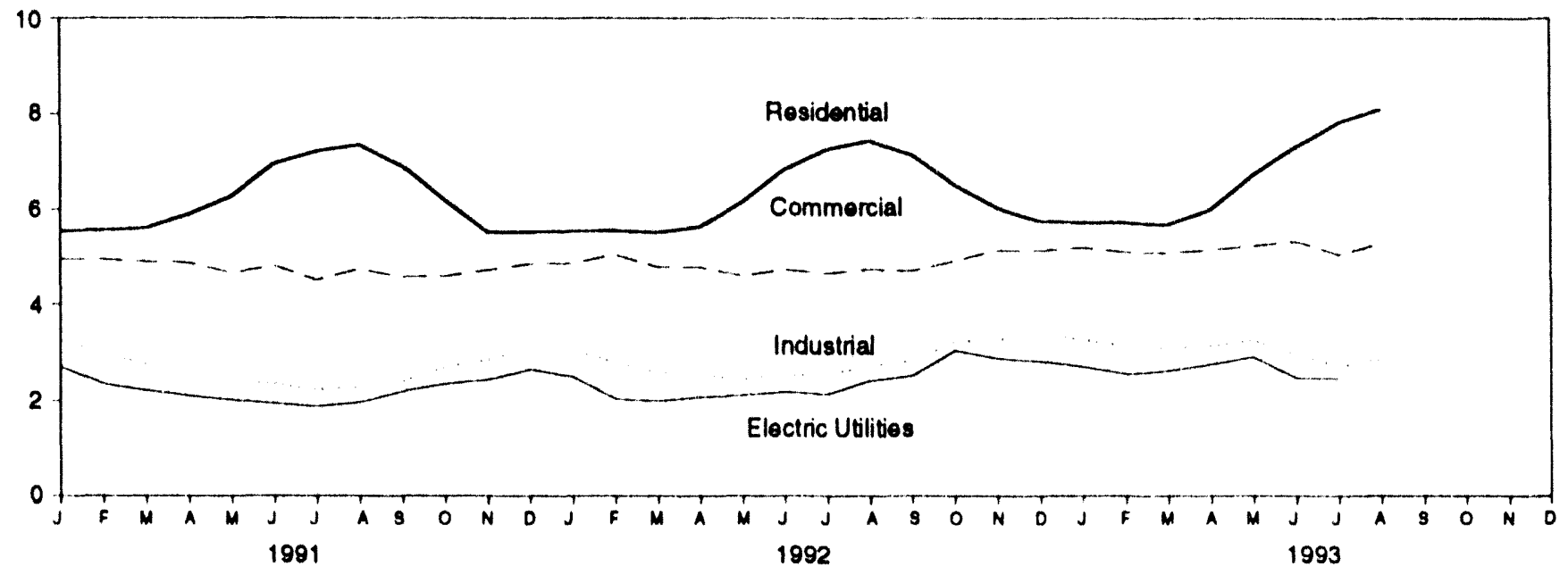

Nole: Because vertical scales dilter, graphs should nol be compared Source: Table 9.11. 


\begin{tabular}{|c|c|c|c|c|c|c|c|c|}
\hline & \multirow[b]{2}{*}{ Wellheed } & \multicolumn{2}{|c|}{$\begin{array}{l}\text { Mejor Interetate } \\
\text { Pipeline Companice }\end{array}$} & \multirow[b]{2}{*}{$\begin{array}{l}\text { Clity } \\
\text { Onto }\end{array}$} & \multicolumn{4}{|c|}{ Delluered to Coneumerse.t. } \\
\hline & & Importa & $\begin{array}{l}\text { Purcheses from } \\
\text { Producers }\end{array}$ & & Acoldential & Commerelal & Industrlal & $\begin{array}{l}\text { Electulo } \\
\text { Ualliteab }\end{array}$ \\
\hline 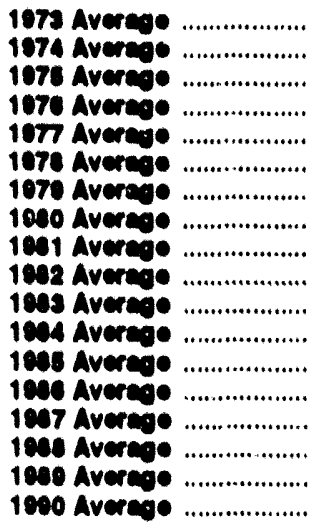 & $\begin{array}{r}0.22 \\
.20 \\
.44 \\
.60 \\
.70 \\
.01 \\
1.10 \\
1.00 \\
1.00 \\
2.46 \\
2.80 \\
2.06 \\
2.11 \\
1.04 \\
1.07 \\
1.00 \\
1.06 \\
1.71\end{array}$ & 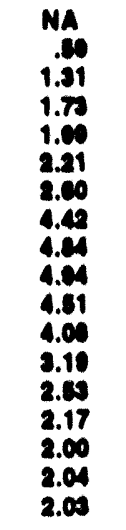 & 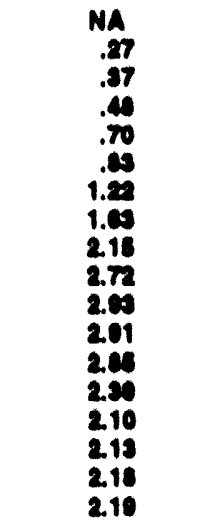 & $\begin{array}{l}\text { NA } \\
N A \\
N A \\
N A \\
N A \\
N A \\
N A \\
N A \\
N A \\
N A \\
N A \\
N A \\
3.06 \\
3.7 \\
3.20 \\
2.07 \\
2.02 \\
3.01 \\
3.05\end{array}$ & 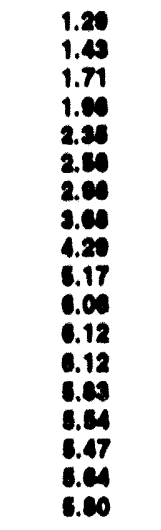 & $\begin{array}{l}0.04 \\
1.07 \\
1.36 \\
1.04 \\
2.04 \\
2.29 \\
2.73 \\
3.30 \\
1.00 \\
4.82 \\
6.80 \\
6.86 \\
6.80 \\
6.00 \\
4.77 \\
4.03 \\
4.74 \\
4.03\end{array}$ & $\begin{array}{r}0.80 \\
.07 \\
.00 \\
1.24 \\
1.80 \\
1.70 \\
1.00 \\
2.60 \\
3.14 \\
3.07 \\
4.10 \\
4.22 \\
3.09 \\
3.23 \\
2.04 \\
2.05 \\
2.06 \\
2.03\end{array}$ & $\begin{array}{r}0.30 \\
.11 \\
.77 \\
1.00 \\
1.22 \\
1.40 \\
1.01 \\
2.27 \\
2.00 \\
3.40 \\
3.40 \\
3.70 \\
3.48 \\
2.49 \\
2.29 \\
2.39 \\
2.49 \\
2.20\end{array}$ \\
\hline 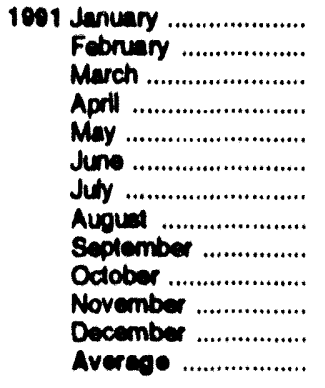 & $\begin{array}{l}1.08 \\
1.62 \\
1.40 \\
1.50 \\
1.48 \\
1.43 \\
1.34 \\
1.43 \\
1.50 \\
1.82 \\
1.89 \\
2.00 \\
1.04\end{array}$ & $\begin{array}{l}2.20 \\
2.10 \\
1.02 \\
2.03 \\
1.00 \\
2.03 \\
2.11 \\
1.71 \\
1.84 \\
2.00 \\
2.20 \\
2.00 \\
2.02\end{array}$ & $\begin{array}{l}2.19 \\
1.03 \\
2.02 \\
1.87 \\
1.06 \\
1.75 \\
1.79 \\
1.71 \\
1.76 \\
1.94 \\
2.02 \\
2.11 \\
1.02\end{array}$ & $\begin{array}{l}3.08 \\
2.04 \\
2.78 \\
2.74 \\
2.76 \\
2.86 \\
2.74 \\
2.78 \\
2.91 \\
2.92 \\
2.02 \\
3.06 \\
2.00\end{array}$ & $\begin{array}{l}5.54 \\
5.56 \\
5.60 \\
6.00 \\
6.28 \\
6.97 \\
7.23 \\
7.36 \\
6.02 \\
6.20 \\
5.51 \\
5.51 \\
1.02\end{array}$ & $\begin{array}{l}4.94 \\
4.94 \\
4.89 \\
4.87 \\
4.65 \\
4.80 \\
4.50 \\
4.73 \\
4.57 \\
4.58 \\
4.71 \\
4.84 \\
4.81\end{array}$ & $\begin{array}{l}3.25 \\
2.97 \\
2.75 \\
2.68 \\
2.40 \\
2.34 \\
2.23 \\
2.29 \\
2.40 \\
2.69 \\
2.84 \\
3.09 \\
2.00\end{array}$ & $\begin{array}{l}2.70 \\
2.36 \\
2.21 \\
2.10 \\
2.01 \\
1.94 \\
1.80 \\
1.06 \\
2.10 \\
2.36 \\
2.43 \\
2.64 \\
2.10\end{array}$ \\
\hline 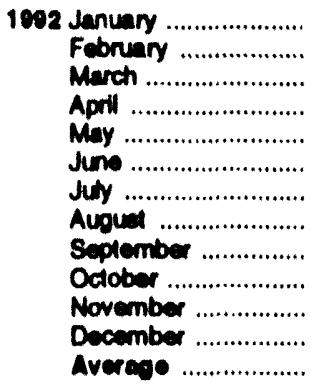 & $\begin{array}{l}A_{1} 1.74 \\
A_{1} \\
A_{1} .26 \\
A_{1.42} \\
A_{1.51} \\
A_{1.62} \\
A_{1.55} \\
A_{1.84} \\
A_{1.02} \\
A_{2.38} \\
A_{2.13} \\
A_{2.07} \\
A_{1.74}\end{array}$ & $\begin{array}{r}2.20 \\
1.08 \\
1.46 \\
2.01 \\
1.70 \\
2.03 \\
1.80 \\
n_{1.86} \\
2.06 \\
2.13 \\
2.32 \\
1.02 \\
1.07\end{array}$ & $\begin{array}{l}2.10 \\
1.70 \\
1.00 \\
1.73 \\
1.90 \\
2.16 \\
1.86 \\
2.14 \\
2.13 \\
2.60 \\
2.37 \\
2.40 \\
2.10\end{array}$ & $\begin{array}{l}2.20 \\
2.70 \\
2.61 \\
2.74 \\
2.00 \\
3.00 \\
3.01 \\
3.16 \\
3.23 \\
3.50 \\
3.33 \\
3.17 \\
3.01\end{array}$ & $\begin{array}{l}5.63 \\
5.54 \\
5.50 \\
5.62 \\
6.15 \\
6.84 \\
7.27 \\
7.45 \\
7.15 \\
6.52 \\
6.00 \\
5.74 \\
6.60\end{array}$ & $\begin{array}{l}4.85 \\
5.03 \\
4.77 \\
4.77 \\
4.59 \\
4.72 \\
4.64 \\
4.73 \\
4.60 \\
4.90 \\
5.12 \\
5.11 \\
4.40\end{array}$ & $\begin{array}{l}3.04 \\
2.78 \\
2.58 \\
2.54 \\
2.44 \\
2.53 \\
2.54 \\
2.71 \\
2.82 \\
3.21 \\
3.26 \\
3.38 \\
2.04\end{array}$ & $\begin{array}{r}2.40 \\
2.03 \\
1.90 \\
R 2.07 \\
2.11 \\
2.18 \\
2.13 \\
2.42 \\
2.51 \\
3.04 \\
2.87 \\
2.81 \\
2.20\end{array}$ \\
\hline 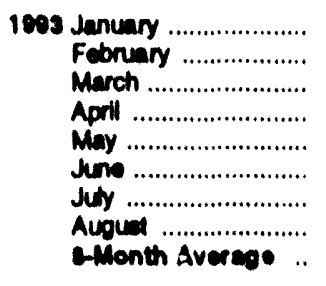 & $\begin{array}{l}A_{1} .06 \\
A_{1} 1.72 \\
A_{1} \\
A_{2.00} \\
A_{2.30} \\
A_{1.87} \\
A_{1.01} \\
C_{2.07} \\
E_{1.07}\end{array}$ & $\begin{array}{l}2.02 \\
1.91 \\
1.78 \\
2.15 \\
2.13 \\
1.96 \\
1.78 \\
2.02 \\
2.00\end{array}$ & $\begin{array}{l}2.17 \\
1.04 \\
2.20 \\
2.34 \\
2.81 \\
2.00 \\
2.02 \\
2.36 \\
2.23\end{array}$ & $\begin{array}{r}A_{3.11} \\
2.04 \\
3.06 \\
3.24 \\
P_{3.58} \\
{ }^{A} 3.44 \\
3.34 \\
3.36 \\
3.10\end{array}$ & $\begin{array}{r}5.71 \\
6.71 \\
5.66 \\
5.69 \\
6.72 \\
7.32 \\
7.83 \\
6.10 \\
0.06\end{array}$ & $\begin{array}{r}5.18 \\
5.08 \\
5.06 \\
5.13 \\
5.21 \\
5.31 \\
R 5.03 \\
5.26 \\
5.13\end{array}$ & $\begin{array}{r}3.26 \\
3.12 \\
3.08 \\
3.13 \\
3.24 \\
2.95 \\
R_{2.71} \\
2.66 \\
3.00\end{array}$ & $\begin{array}{l}2.70 \\
2.55 \\
2.81 \\
2.75 \\
2.00 \\
2.47 \\
2.40 \\
\text { NA } \\
\text { NA }\end{array}$ \\
\hline $\begin{array}{l}1002 \text { E-Month Avermos ... } \\
1901 \text { E-Month Averege ... }\end{array}$ & $\begin{array}{l}1.64 \\
1.63\end{array}$ & $\begin{array}{l}1.00 \\
2.01\end{array}$ & $\begin{array}{l}1.04 \\
1.00\end{array}$ & $\begin{array}{l}2.04 \\
2.07\end{array}$ & $\begin{array}{l}8.00 \\
6.10\end{array}$ & $\begin{array}{l}4.01 \\
4.08\end{array}$ & $\begin{array}{l}2.68 \\
2.68\end{array}$ & $\begin{array}{l}2.10 \\
2.05\end{array}$ \\
\hline
\end{tabular}

- Includes supplemental ansecous furets.

- See Nole 8 at end of section.

R=Revised dalle. NA= Nol avallable. Ese Ealmate.

Noles: - Prices shown on this page are intended to include all waxee. See Nole 8 at and of eedion. - Geographic coverage it the 50 states and the

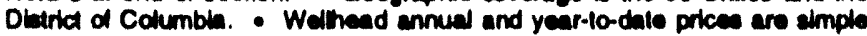
averages of the monthly prices; al other annual and year-10-date prices are volume-welohted averagee of the monthly prioes.

Sourcea: - 1073-10es: Wellihead-Energy Intormation Adminiatration (EU), Netural Gas Annual 1991, Table 95.
Compenles, 1074.1077-Catculated from revenue and salee data reponted to the Federal Power Commiaton (FPC). Form FPC-11, 'Natural Gas Pipellne Company Monthly Statement." Mejor Interstato Pipellno Compentes, 1074-10es-EIA, Natural Ges Monthy, December 1984. Tablo 10. Malor Interetats PIpelino Companies, 1064-1060-EIA, Natural Gas Montity. December 1989, Tablo 4. Clty Cato, 10s4.10en-EU, Natural Gas Monthy, Decernber 1989. Table 4. Delivered to Consumers, 107s-10e4-EK, Natural Gas Amual 1891, Table 98. - 1067 forward: EIA, Netural Gas Monthy, November 1003, Table 4. 


\section{Energy Prices Notes}

1. The average domestic first purchase price represents the average price at which all domestic crude oil is purchased. Prior to February 1976, the price represented an estimate of the average of posted prices; beginning with February 1976, the price represents an average of actual first purchase prices. The data series was previously called "Actual Domestic Wellhead Price."

2. F.O.B. literally means "Free on Board." It denotes a transaction whereby the seller makes the product avall. able with an agreement on a given port at a given price; it is the responsibility of the buyer to arrange for the transportation and insurance.

3. The landed cost of imported crude oil from selected countries does not represent the total cost of all imported crude. Prior to March 1975, imported crude costs to U.S. company-owned refineries in the Caribbean were not included in the landed cost, and costs of crude oil from countries that export only small amounts to the United States were also excluded. Beginning in March 1975, however, coverage was expanded to include U.S. company-owned refineries in the Caribbean. Landed costs do not include supplemental fees.

4. Beginning with January 1981 , refiner acquisition costs of crude oil are from data collected on Form EIA. 14, "Refiners' Monthly Cost Report." Those costs were previously published from data collected on Form ERA49. "Domestic Crude Oil Entitlements Program Refiners Monthly Report." Form ERA-49 was discontinued with the decontrol of crude oil on January 28, 1981. Crude oil purchases and costs are defined for Form EIA-14 in accordance with conventions used for Form ERA-49. Also, the respondents for the two forms are essentially the same. However, due to possible different interpretations of the filing requirements and a different method for handling prior period adjustments, care must be taken when comparing the data collected on the two forms.

The refiner acquisition cost of crude oll is the average price paid by refiners for crude oil booked into their refineries in accordance with accounting procedures generally accepted and consistently and historically applied by the refiners concerned. Domestic crude oil is that oil produced in the United States or from the outer continental shelf as defined in 43 USC Section 1331. Imported crude oil is either that oil reported on Form ERA.51, "Transfer Pricing Report," or any crude oil that is not domestic oil. The composite cost is the weighted average of domestic and imported crude oil costs.

Crude oil costs and volumes reported on Form ERA-49 excluded unfinished oils but included the Strategic Petroleum Reserve (SPR). Crude oil costs and volumes reported on Form FEA.P110-M-1, "Refiners' Monthly Cost Allocation Report," included unfinished oils but excluded SPR. Imported averages derived from Form
IERA-49 exclude oil purchased for SPR, whereas the composite averages derived from Form ERA-49 include SPR. None of the prices derived from Form EIA-14 include cither unfinished olls or SPR.

5. Several different series of motor gasoline prices are published in this section. U.S. City Average Retall Prices of Motor Gasoline are calculated monthly by the Bureau of Labor Statistics during the development of the Consumer Price Index (CPI). These prices Include all Federal, State, and local taxes pald at the time of sale. For the period 1974.1977, prices were collected in 56 urban areas. For the period 1978 forward, prices were collected from a new sample of service stations in 85 urban areas selected to represent all urban consumersabout 80 percent of the total U.S. population. The service stations are selected initially, and on a replacement basis, in such a way that they represent the purchasing habits of the CPI population. Service stations in the current sample include those providing all types of service (i.e., full-, mini-, and self-scrve).

Refiner prices of finished motor gasoline for resale and to end users ar: determined by the Energy Information Administration (EIA) in a monthly survey of refiners and gas plant operators (Form EIA-782A). The prices do not include any Federal, State, or local taxes paid at the time of sale. Estimates of prices prior to January 1983 are based on Form FEA.P302-M-1/EIA-460, "Petroleum Industry Monthly Report for Product Prices," and also exclude all Federal, State, or local taxes paid at the time of sale. Sales for resale are those made to purchasers who are other-than-ultimate consumers. Sales to end users are sales made directly to the consumer of the product, including bulk consumers, such as agriculture, industry, and utilities, as well as residential and commercial consumers.

6. Starting in January 1983, Form EIA-782, "Monthly Petrolcum Product Sales Report," replaced 10 previous surveys. Every attempt was made to continue the most important price series. However, prices published through December 1982 and those published since January 1983 do not necessarily form continuous data series due to changes in survey forms, definitions, instructions, populations, samples, processing systems, and statistical procedures. To provide historical data, continuous series were generated for annual data 1978-1982 and for monthly data 1981 and 1982 by estimating the prices that would have been published had Form EIA-782 survey and system been in operation at that time. This form of estimation was performed after detailed adjustment was made for product and sales type matching and for discontinuity ciue to other factors. An important difference between the previous and present prices is the distinction between wholesale and resale and between retail and end user. The resale category continues to include sales among resellers. However, bulk sales 10 utility, industrial, and commercial accounts previously included in the wholesale category are now counted as made to end users. The end-user category continues to include retail sales through company owned and operated 
outlets but also includes the bulk utility, industrial, and commercial sales. Additional information may be found in Estimated Historic Tine Series for the EIA-782, a feature article reprinted from the December 1983 (3) Petroleum Marketing Monthly, published by EIA.

7. National average electricity prices are shown in two data series. The "Annual Series" is based on data from more than 3,000 publicly and privately owned electric utilites that report on Form EIA-861, "Annual Electric Utility Report." The "Monthly Series" is based on data from over 400 utilities statistically chosen as a stratified sample of the utilities that report on Form EIA.861. The selected utilities report monthly on Form EIA.826, "Monthly Electric Utility Sales and Revenue Report with State Distributions," formerly the "Electric Utility Company Monthly Statement." Annual values shown for the monthly series are the sum of the monthly revenue divided by the sum of the monthly sales. Prior to January 1986, only privately owned utilities were included in the monthly survey and the sample was chosen by using cut-off, rather than stratification, techniques.

8. Natural gas prices are intended to include all taxes. Instructions on the data collection forms specifically direct that all Federa. State, and local taxes, surcharges, and/or adjustments billed to consumers are to be included. However, sales and other taxes itemized on consumers' bills are sometimes excluded by the reporting utilities.

Delivered-to-consumers prices for 1987 forward represent natural gas delivered and sold to residential, commercial, industrial, and electric utility consumers. They do not include the price of natural gas delivered to industrial and commercial consumers on behalf of third parties. Volumes of natural gas delivered on behalf of third parties are included in the consumption data shown in Table 4.3. Additional information is available in the EIA Natural Gas Monthly. Appendix C.
Electric utility data for $1973-1982$ cover all electric generating plants at which the generator nameplate capacity of all steam-electric units combined totaled 25 megawatts or greater. From 1974-1982, peaking units were included in the data and counted towards the 25. megawatt-or-greater total. Data for 1983.1990 cover all electric generating plants at which the generator nameplate capacity of all steam-electric units combined totaled 50 megawatts or greater. Data for 1991 forward cover all electric generating plants at which the generator nameplate capacity of all steam-electric units and combined-cycle units together totaled 50 megawatts or greater.

\section{Sources for Table 9.10}

- 1973-1979: Annual data for quantity are simple sums of unrounded monthly values and for cost are averages of monthly values, weighted by quantities, from the following: 1973-May 1977-Federal Power Commission, Form FPC-423, "Monthly Report on Cost and Quality of Fucls for Electric Utility Plants." June 1977. December 1977-Federal Energy Regulatory Commission, Form FERC-423, "Monthly Report on Cost and Quality of Fucls for Electric Utility Plants." 1978 and 1979-Energy Information Administration (EIA), Form FERC-423, "Monthly Report on Cost and Quality of Fuels for Electric Utility Plants."

- 1980: EIA, Electric Power Monthly, April 1991, Table 33.

- 1981: EIA, Electric Power Monthly, April 1992. Table 33.

- 1982 and 1991 monthly data: EIA, Electric Power Monthly, April 1993, Table 33.

- 1983 forward: (except 1991 monthly data): EIA, Electric Power Monihly, November 1993, Table 33. 


\section{Section 10. International Energy}

Crude Oll Production. World crude oll production during August 1993 was 60 million barrels per day, down 0.1 million barrels per day from the level in the previous month.

Organization of Petroleum Exporting Countries (OPEC) production during August 1993 averaged 26 million barrels per day, down slightly from the level during the previous month. Production by the Arab members of OPEC in August 1993 averaged 16 million barrels per day, up 0.2 million barrels per day from the July 1993 level. During August 1993, production increased in both Kuwait and Saudi Arabia by 105 thousand barrels per day and in Libya by 20 thousand barrels per day. Production remained unchanged in Algeria, Iraq, Qatar, and the United Arab Emirates. Among the nonArab members of OPEC, production during August 1993 increased in Nigeria by 50 thousand barrels per day and decreased in Iran by 300 thousand barrels per day. Production remained unchanged in Indonesia and Venezuela.

Among the non-OPEC nations, production during August 1993 increased in the United Statcs by 78 thousand barrels per day and in Mexico by 20 thousand barrels per day. Production decreased in the former U.S.S.R. by 75 thousand barrels per day, in Canada by 20 thousand barrels per day, and in the United Kingdom by 5 thousand barrels per day. Production remained unchanged in China.

Petroleum Consumption. In June 1993, consumption in all Organization for Economic Cooperation and Development (OECD) countries was 38.6 million barrels per day, 2 percent ${ }^{9}$ higher than the June 1992 rate. The consumption rate was higher than it was 1 year ago in Germany $(+13$ percent). France $(+8$ percent), United Kingdom and Canada (each +4 percent), Italy $(+3$ percent), Japan $(+1$ percent), and slightly higher in the United States.
Petroleum Stocks. For all OECD countries, petroleum stocks at the end of June 1993 totaled 3.6 billion bar. rels, 2 percent higher than the ending stock level in June 1992. Stock levels were higher than the levels 1 year ago in the United Kingdom and the United States (each +4 percent), Japan $(+3$ percent), and Germany $(+1$ percent). Stocks were lower in June 1993 than in June 1992 in France $(.6$ percent), Canada $(.4$ percent), and ltaly (-2 percent) compared with levels I year earlier.

Nuclear Electricity Generation. Based on Nucleonics Week information for August 1993, reporting countries with nuclear capacity generated 161 gross terawatthours of nuclear-generated electricity, 1 percent more than in August 1992.

Two new units began commercial operation in August. In the United States, Comanche Peak-2, which had become operable in April 1993, actually began commercial operation on August 3, 1993. It is a 1,161. gross megawall pressurized light-water reactor. In Japan, Kashiwazaki-Kariwa-3, a 1,100-gross megawall boiling-water reactor, began commercial operation on August 11, 1993.

Two units shut down permanently on July 20, 1993. In the United Kingdom, Trawsfynydd-1 and Trawsfynydd-2, both 290-gross megawall gas-cooled reactors, last generated nuclear electricily in February 1991 and had been in commercial service for 26 years.

As of August 31, 1993, there were 358 operable nuclear generating units in the reporting countrics. The units had a collective gross generating capacity of 304.6 gigawatts. The 109 U.S. units accounted for 105.3 gross gigawatts, 34.6 percent of the total reported nuclear generating capacity.

\footnotetext{
- Percentege changes ere besed on urrounded data

${ }^{10}$ One torawathour equals 1 blliton klowathour

"On megawatt equale it thousand kllowatle.

${ }^{12}$ One glowwatl equals 1 million klowatls
} 
Table 10.1a World Crude Oll Production: Algerla Through Venezuela

(Thousand Barrels per Day)

\begin{tabular}{|c|c|c|c|c|c|c|c|c|c|c|c|c|}
\hline & Arenta & Irma & Kumatr & Ubye & Coter & Arelyen & emiratu & apteb & Indencele & Iran & Nigerle & Venuezude \\
\hline 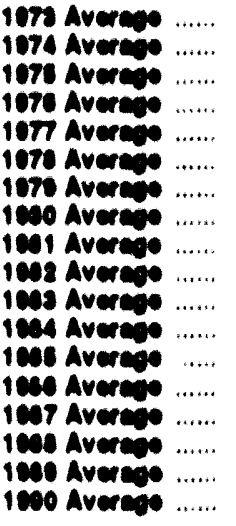 & 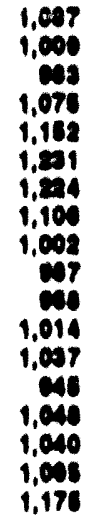 & $\begin{array}{l}2,010 \\
1,071 \\
2,202 \\
2,410 \\
8,249 \\
2,004 \\
2,477 \\
2,014 \\
1,000 \\
1,012 \\
1,000 \\
1,200 \\
1,449 \\
1,000 \\
2,07 \\
2,045 \\
2,007 \\
2,040\end{array}$ & 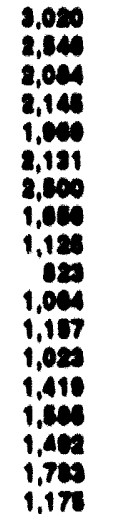 & 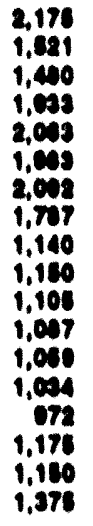 & 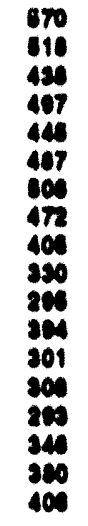 & 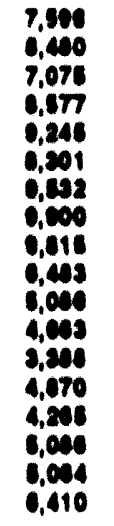 & 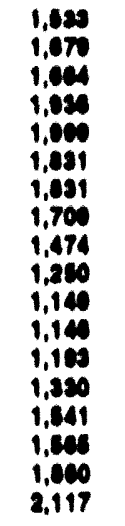 & 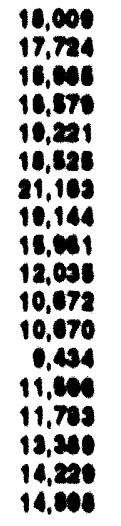 & 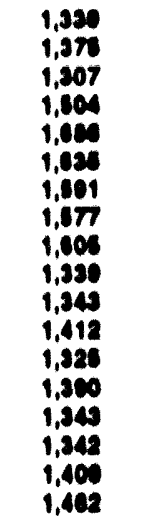 & $\begin{array}{l}6,011 \\
0,022 \\
6,940 \\
5,019 \\
6,498 \\
6,242 \\
3,160 \\
1,142 \\
1,290 \\
2,214 \\
2,440 \\
2,174 \\
2,280 \\
2,030 \\
2,200 \\
2,210 \\
2,010 \\
3,090\end{array}$ & 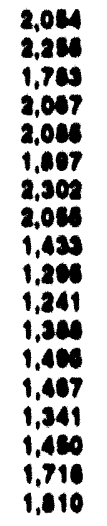 & $\begin{array}{l}3,290 \\
2,070 \\
2,210 \\
2,214 \\
2,201 \\
2,104 \\
2,290 \\
2,101 \\
2,108 \\
1,008 \\
1,601 \\
1,700 \\
1,077 \\
1,707 \\
1,798 \\
1,009 \\
1,007 \\
2,197\end{array}$ \\
\hline 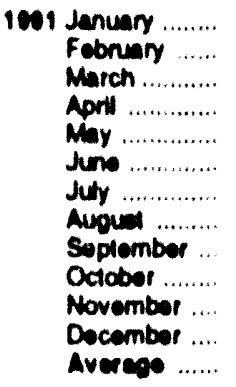 & $\begin{array}{l}1.230 \\
1.230 \\
1.230 \\
1.230 \\
1,230 \\
1.230 \\
1.230 \\
1.230 \\
1,230 \\
1.230 \\
1.230 \\
1.230 \\
1.230\end{array}$ & $\begin{array}{r}250 \\
0 \\
0 \\
200 \\
360 \\
360 \\
400 \\
400 \\
400 \\
400 \\
400 \\
400 \\
200\end{array}$ & $\begin{array}{r}50 \\
0 \\
0 \\
0 \\
0 \\
75 \\
165 \\
105 \\
299 \\
429 \\
490 \\
519 \\
197\end{array}$ & $\begin{array}{l}1.500 \\
1.500 \\
1.450 \\
1.450 \\
1.460 \\
1.450 \\
1.460 \\
1.450 \\
1.500 \\
1.500 \\
1.550 \\
1.550 \\
1.493\end{array}$ & $\begin{array}{l}381 \\
402 \\
402 \\
402 \\
402 \\
402 \\
402 \\
402 \\
402 \\
402 \\
302 \\
320 \\
300\end{array}$ & $\begin{array}{l}8.140 \\
8.200 \\
., 000 \\
7.400 \\
7.400 \\
0.150 \\
.475 \\
0.465 \\
0.400 \\
0.460 \\
8.440 \\
0.440 \\
0.101\end{array}$ & $\begin{array}{l}2,510 \\
2,535 \\
2,560 \\
2,560 \\
2,300 \\
2,390 \\
2,360 \\
2,360 \\
2,360 \\
2,440 \\
2,505 \\
2,470 \\
2,447\end{array}$ & $\begin{array}{l}14,041 \\
13,087 \\
13,642 \\
13,242 \\
13,102 \\
14,017 \\
14,462 \\
14,602 \\
14,582 \\
14,061 \\
15,005 \\
15,120 \\
14,210\end{array}$ & $\begin{array}{l}1,630 \\
1,630 \\
1,630 \\
1.630 \\
1,630 \\
1,630 \\
1,680 \\
1,630 \\
1,580 \\
1,530 \\
1,580 \\
1,580 \\
1,613\end{array}$ & $\begin{array}{l}3,200 \\
\mathbf{3 , 3 0 0} \\
\mathbf{3 , 4 0 0} \\
\mathbf{3 , 3 0 0} \\
\mathbf{3 , 3 0 0} \\
\mathbf{3 , 3 0 0} \\
\mathbf{3 , 4 0 0} \\
\mathbf{3 , 4 0 0} \\
\mathbf{3 , 3 0 0} \\
\mathbf{3 , 3 0 0} \\
\mathbf{3 , 3 0 0} \\
\mathbf{3 . 5 0 0} \\
\mathbf{3 , 3 3 4}\end{array}$ & $\begin{array}{l}1,908 \\
1,008 \\
1,008 \\
1,008 \\
1,900 \\
1,050 \\
1,850 \\
1,908 \\
1,908 \\
1,800 \\
1,908 \\
1,931 \\
1,002\end{array}$ & $\begin{array}{l}2.300 \\
2,306 \\
2,306 \\
2,346 \\
2,346 \\
2,346 \\
2,346 \\
2,346 \\
2,346 \\
2,306 \\
2,306 \\
2,446 \\
2,378\end{array}$ \\
\hline 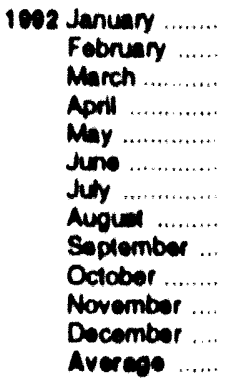 & $\begin{array}{l}1.230 \\
1.230 \\
1.230 \\
1.230 \\
1.210 \\
1.210 \\
1.210 \\
1.210 \\
1.210 \\
1.210 \\
1.210 \\
1.210 \\
1.217\end{array}$ & $\begin{array}{l}450 \\
450 \\
450 \\
450 \\
450 \\
450 \\
450 \\
450 \\
450 \\
450 \\
450 \\
450 \\
460\end{array}$ & $\begin{array}{l}565 \\
630 \\
736 \\
000 \\
015 \\
1,015 \\
1,000 \\
1,130 \\
1,200 \\
1,280 \\
1,375 \\
1,550 \\
1,020\end{array}$ & $\begin{array}{l}1,550 \\
1,550 \\
1,450 \\
1,500 \\
1,450 \\
1,450 \\
1,450 \\
1.425 \\
1,475 \\
1.500 \\
1.500 \\
1,500 \\
1,493\end{array}$ & $\begin{array}{l}360 \\
325 \\
375 \\
375 \\
375 \\
375 \\
400 \\
425 \\
425 \\
440 \\
40 \\
440 \\
34\end{array}$ & 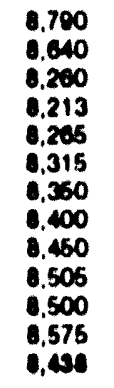 & $\begin{array}{l}2,435 \\
2,425 \\
2,300 \\
2,300 \\
2,300 \\
2,275 \\
2,300 \\
2.300 \\
2,320 \\
2,310 \\
2,306 \\
2,306 \\
2,320\end{array}$ & $\begin{array}{l}15,370 \\
15,260 \\
14,800 \\
14,030 \\
14,005 \\
15,000 \\
15,240 \\
15,370 \\
15,530 \\
15,695 \\
15,780 \\
18,030 \\
18,310\end{array}$ & $\begin{array}{l}1,580 \\
1,605 \\
1,650 \\
1.600 \\
1,530 \\
1,560 \\
1,550 \\
1,540 \\
1,550 \\
1,550 \\
1,550 \\
1,550 \\
1,540\end{array}$ & $\begin{array}{l}3.500 \\
3.500 \\
3.360 \\
3.250 \\
3.250 \\
3.250 \\
3.300 \\
3.450 \\
3.450 \\
3.650 \\
3.650 \\
3.550 \\
3.420\end{array}$ & $\begin{array}{l}1,075 \\
1,925 \\
1,000 \\
1,025 \\
1,025 \\
1,025 \\
1,075 \\
2,000 \\
2,025 \\
2,050 \\
2,050 \\
2,100 \\
1,002\end{array}$ & $\begin{array}{l}2,300 \\
2,340 \\
2,190 \\
2,100 \\
2,200 \\
2,200 \\
2,200 \\
2,340 \\
2,300 \\
2,440 \\
2,440 \\
2,415 \\
2,294\end{array}$ \\
\hline 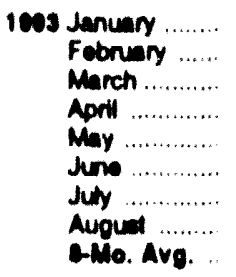 & $\begin{array}{l}1.210 \\
1,210 \\
1.200 \\
1,200 \\
1.200 \\
1,200 \\
1.180 \\
1.180 \\
1,107\end{array}$ & $\begin{array}{l}500 \\
500 \\
500 \\
500 \\
500 \\
500 \\
500 \\
500 \\
500\end{array}$ & $\begin{array}{l}1,675 \\
1,806 \\
1,660 \\
1,645 \\
1,713 \\
1,775 \\
1,040 \\
2,045 \\
1,700\end{array}$ & $\begin{array}{l}1,490 \\
1,425 \\
1,350 \\
1,350 \\
1,350 \\
1,350 \\
1,360 \\
1,370 \\
1,370\end{array}$ & $\begin{array}{l}450 \\
430 \\
400 \\
400 \\
420 \\
400 \\
410 \\
410 \\
410\end{array}$ & $\begin{array}{r}8,500 \\
8,440 \\
8,300 \\
8,000 \\
8,000 \\
8,150 \\
9,240 \\
8,345 \\
0,210\end{array}$ & $\begin{array}{l}2,205 \\
2,305 \\
2,270 \\
2,270 \\
2,230 \\
2,230 \\
2,210 \\
2,210 \\
2,292\end{array}$ & $\begin{array}{r}16,110 \\
16,175 \\
15,670 \\
15,335 \\
15,413 \\
15,605 \\
15,030 \\
16,000 \\
19,770\end{array}$ & $\begin{array}{l}1,550 \\
1,530 \\
1,500 \\
1,400 \\
1,510 \\
1.510 \\
1.510 \\
1.510 \\
1,612\end{array}$ & $\begin{array}{l}3.650 \\
3.750 \\
3.7100 \\
3.500 \\
3.650 \\
3.650 \\
3.800 \\
3.500 \\
3.640\end{array}$ & $\begin{array}{l}2,126 \\
2,105 \\
2,075 \\
2,025 \\
2.025 \\
1,005 \\
1,075 \\
2,025 \\
2,040\end{array}$ & $\begin{array}{r}2,410 \\
2,300 \\
2,340 \\
2,340 \\
2,340 \\
2,340 \\
2,300 \\
2,300 \\
2,397\end{array}$ \\
\hline $\begin{array}{l}\text { 1es AMo. Avo. } \\
\text { 101 c-Mo. Avg. }\end{array}$ & $\begin{array}{l}1.220 \\
1.230\end{array}$ & $\begin{array}{l}460 \\
249\end{array}$ & oc & $\begin{array}{l}1,470 \\
1,492\end{array}$ & 378 & $\begin{array}{l}0,403 \\
0,020\end{array}$ & $\begin{array}{l}2,313 \\
2,460\end{array}$ & $\begin{array}{l}18,127 \\
13,075\end{array}$ & $\begin{array}{l}1,676 \\
1,036\end{array}$ & $\begin{array}{l}3,368 \\
3,326\end{array}$ & $\begin{array}{l}1,044 \\
1,004\end{array}$ & $\begin{array}{l}2,200 \\
2,344\end{array}$ \\
\hline
\end{tabular}

- Includes about one-hall of the production in the Kuwell.Saud Arabla Neured Zons from 1973 through Juty 1990 and in Jum 1991. Kuwall Noural Zons oupue was discontinued lollowing lreq's invesion of Kuwall on Augut 2. 1000. but was resumed in Jume 1001. In Aloued 1003. Noutral Zone production by toah Kuwall and Saud Arabla toteled abou 300 thousand berrole per day

- The Arab members of ithe Organization a Petroloum Exponing Countries (OPEC) wo Abgeria, Iraq. Kuwath, Libya, Oale, Saudl Arabie, and the United Arab Emirates. Production in the Neutral Zon between Kiwweit and Saudi
Arabla in included in 'Arab OPEC.

R=Roviead dalle.

Notes: - Cnde oll includes base condensale but excludee naturd gas plant liquide. - U.S. ocographic covernge be the 50 Sialos and the Dietrict of Columbin. - Monthy dali are often preliminary ligures and may not everepe 10 the annual lotals becauses of rounding of because updales to the preliminary monthy dala are not avellable

Sources: See ond of section. 
Table 10.1b World Crude Oll Production: Total OPEC, Canada Through Former U.S.S.R., and World

(Thousand Barrels per Day)

\begin{tabular}{|c|c|c|c|c|c|c|c|c|c|c|}
\hline & $\begin{array}{l}\text { Tot } \\
\text { opece }\end{array}$ & Aull & Cenede & Mexles & $\begin{array}{l}\text { United } \\
\text { Kingdom }\end{array}$ & $\begin{array}{l}\text { Unlied } \\
\text { stetes }\end{array}$ & Chine & $\begin{array}{l}\text { Formes } \\
\text { U.8.8.R. }\end{array}$ & Other & World \\
\hline 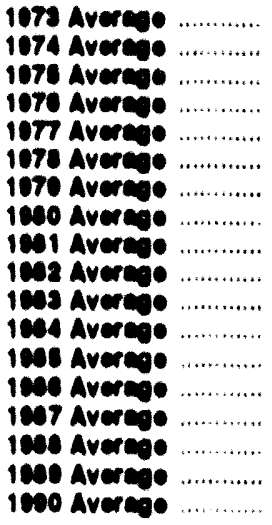 & 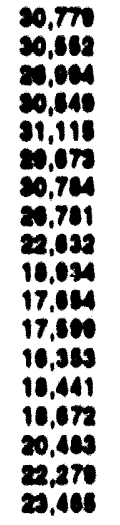 & 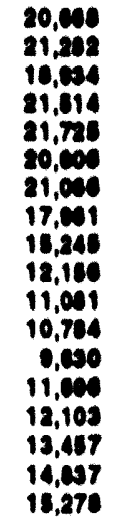 & 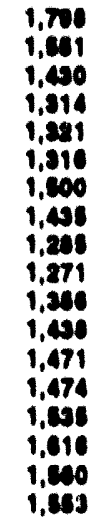 & $\begin{array}{l}101 \\
671 \\
705 \\
191 \\
001 \\
1,200 \\
1,401 \\
1,030 \\
2,819 \\
2,740 \\
2,000 \\
2,700 \\
2,740 \\
2,420 \\
2,540 \\
2,612 \\
2,520 \\
2,509\end{array}$ & 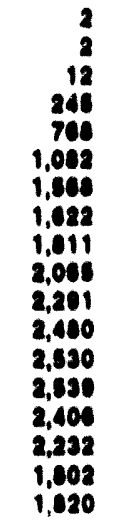 & 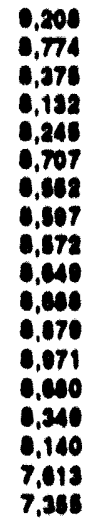 & $\begin{array}{l}1,000 \\
1,510 \\
1,400 \\
1,070 \\
1,074 \\
2,002 \\
2,120 \\
2,114 \\
2,012 \\
2,040 \\
2,180 \\
2,200 \\
2,005 \\
2,120 \\
2,060 \\
2,730 \\
2,707 \\
2,774\end{array}$ & $\begin{array}{r}1,324 \\
1,012 \\
1,623 \\
10,000 \\
10,003 \\
11,104 \\
11,304 \\
11,704 \\
11,060 \\
11,012 \\
11,072 \\
11,001 \\
11,008 \\
11,005 \\
11,008 \\
11,070 \\
11,025 \\
10,000\end{array}$ & 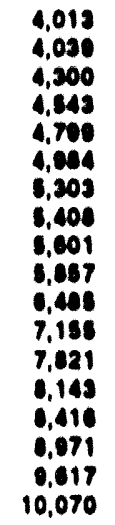 & 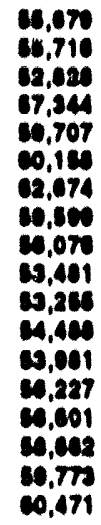 \\
\hline 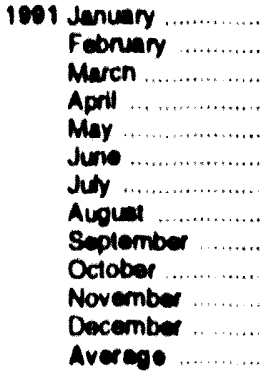 & $\begin{array}{l}23,487 \\
23,414 \\
23,263 \\
22,712 \\
22,600 \\
20,430 \\
24,053 \\
24,072 \\
24,002 \\
24,186 \\
24,486 \\
24,884 \\
23,728\end{array}$ & $\begin{array}{l}14,553 \\
14,477 \\
14,405 \\
13,003 \\
13,084 \\
14,674 \\
15,240 \\
15,200 \\
15,101 \\
15,450 \\
15,565 \\
15,889 \\
14,070\end{array}$ & $\begin{array}{l}1.501 \\
1.021 \\
1.546 \\
1.445 \\
1.505 \\
1.525 \\
1.506 \\
1.501 \\
1.581 \\
1.505 \\
1.621 \\
1.586 \\
1,640\end{array}$ & $\begin{array}{l}2,660 \\
2,674 \\
2,669 \\
2,685 \\
2,605 \\
2,720 \\
2,600 \\
2,660 \\
2,675 \\
2,680 \\
2,660 \\
2,675 \\
2,070\end{array}$ & $\begin{array}{l}1.675 \\
1,004 \\
2,008 \\
1.526 \\
1.306 \\
1,525 \\
1.005 \\
1,027 \\
1,696 \\
1.090 \\
1.975 \\
1.979 \\
1,707\end{array}$ & $\begin{array}{l}7.500 \\
7.637 \\
7.540 \\
7.509 \\
7.400 \\
7.320 \\
7.347 \\
7.316 \\
7.369 \\
7.437 \\
7.328 \\
7.299 \\
7.417\end{array}$ & $\begin{array}{l}2,792 \\
2,802 \\
2,797 \\
2.802 \\
2,802 \\
2,812 \\
2,812 \\
2,812 \\
2,807 \\
2,807 \\
2,812 \\
2,807 \\
2,008\end{array}$ & $\begin{array}{r}10,663 \\
9,943 \\
10,307 \\
10,310 \\
10,222 \\
9,800 \\
9.800 \\
9.420 \\
9,886 \\
9.192 \\
9.378 \\
9.347 \\
9,107\end{array}$ & $\begin{array}{l}10,300 \\
10,430 \\
10,432 \\
10,320 \\
10,402 \\
10,139 \\
10,230 \\
9,697 \\
10,434 \\
10,484 \\
10,570 \\
10,663 \\
10,387\end{array}$ & $\begin{array}{l}60,730 \\
60,430 \\
60,687 \\
50,270 \\
50,003 \\
60,289 \\
60,281 \\
59,584 \\
60,818 \\
60,580 \\
60,830 \\
61,230 \\
60,221\end{array}$ \\
\hline 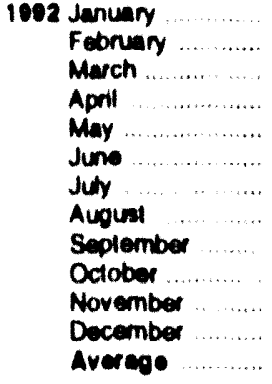 & $\begin{array}{l}25,100 \\
24,880 \\
24,170 \\
24,205 \\
24,286 \\
24,420 \\
24,600 \\
25,005 \\
25,246 \\
25.685 \\
25,770 \\
25,045 \\
24,047\end{array}$ & $\begin{array}{l}16,130 \\
18,010 \\
15,510 \\
15,187 \\
15,502 \\
15,716 \\
15,916 \\
16,220 \\
16,330 \\
16,670 \\
16,755 \\
16,905 \\
16,104\end{array}$ & 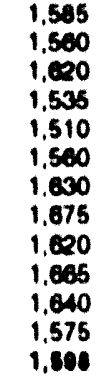 & $\begin{array}{l}2,675 \\
2,605 \\
2,680 \\
2,680 \\
2,680 \\
2,690 \\
2,600 \\
2,685 \\
2,685 \\
2,655 \\
2,640 \\
2,655 \\
2,040\end{array}$ & $\begin{array}{l}1.920 \\
1,905 \\
1,755 \\
1.835 \\
1,700 \\
1,545 \\
1,780 \\
1,025 \\
1,830 \\
1,930 \\
1.945 \\
1,935 \\
1,025\end{array}$ & $\begin{array}{l}7.361 \\
7.390 \\
7,348 \\
7.293 \\
7.160 \\
7.167 \\
7.131 \\
6.922 \\
7.000 \\
7.126 \\
7.024 \\
7.103 \\
7.171\end{array}$ & $\begin{array}{l}2,830 \\
2,806 \\
2,835 \\
2.855 \\
2,836 \\
2,830 \\
2,825 \\
2,815 \\
2,860 \\
2,875 \\
2.845 \\
2,785 \\
2,030\end{array}$ & $\begin{array}{l}9.115 \\
8,650 \\
8,760 \\
9.025 \\
0.455 \\
0.440 \\
8,365 \\
8.130 \\
7,980 \\
7,966 \\
7,910 \\
7,870 \\
0.300\end{array}$ & $\begin{array}{l}10,821 \\
10,670 \\
10,744 \\
10,838 \\
10,566 \\
10,758 \\
10,818 \\
10,802 \\
10,873 \\
11,017 \\
10,847 \\
11,074 \\
10,820\end{array}$ & $\begin{array}{l}61,407 \\
60,584 \\
50,012 \\
60,268 \\
50,160 \\
50,400 \\
50,869 \\
50,858 \\
60,123 \\
60,018 \\
60,621 \\
60,942 \\
60,248\end{array}$ \\
\hline 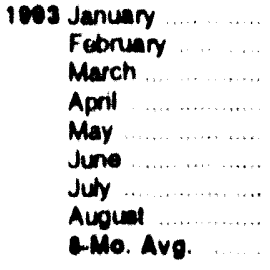 & $\begin{array}{r}26,145 \\
26,250 \\
25,585 \\
25,010 \\
25.238 \\
25,400 \\
25,706 \\
25,775 \\
28,040\end{array}$ & $\begin{array}{l}17,105 \\
17,325 \\
16,855 \\
16,350 \\
16,540 \\
16,740 \\
17,136 \\
17,045 \\
18,018\end{array}$ & $\begin{array}{l}1,570 \\
1,610 \\
1,635 \\
1,605 \\
A_{1}, 600 \\
1,725 \\
A_{1,710} \\
1,690 \\
1,081\end{array}$ & $\begin{array}{r}2,606 \\
2,610 \\
2,636 \\
2,674 \\
2,673 \\
2,675 \\
2,650 \\
2,650 \\
2,670 \\
2,640\end{array}$ & $\begin{array}{l}1,810 \\
1,930 \\
1,710 \\
1,605 \\
1,745 \\
1,675 \\
1,945 \\
1,940 \\
1,000\end{array}$ & $\begin{array}{l}7,008 \\
6,067 \\
6,076 \\
6,897 \\
6,833 \\
6,756 \\
6,664 \\
6,732 \\
6,080\end{array}$ & $\begin{array}{r}2,805 \\
2,875 \\
2,885 \\
2,005 \\
2,806 \\
2,890 \\
2,890 \\
2,800 \\
2,001\end{array}$ & $\begin{array}{r}7.800 \\
7.785 \\
7.685 \\
7.605 \\
7.495 \\
7.400 \\
7.120 \\
7.045 \\
7.406\end{array}$ & $\begin{array}{r}10,138 \\
10,877 \\
11,044 \\
11,014 \\
11,058 \\
10,744 \\
11,108 \\
10,997 \\
10,040\end{array}$ & $\begin{array}{r}60,559 \\
60,804 \\
60,145 \\
A_{50,466} \\
A_{50,607} \\
A_{50,266} \\
A_{50,872} \\
50,730 \\
50,039\end{array}$ \\
\hline $\begin{array}{l}1002 \text { \&Mo. Avg. } \\
1001 \text { \&Mo. Avg. }\end{array}$ & $\begin{array}{l}24,00 \\
20,300\end{array}$ & $\begin{array}{l}18,128 \\
14,010\end{array}$ & $\begin{array}{l}1,505 \\
1,530\end{array}$ & $\begin{array}{l}2,073 \\
2,670\end{array}$ & $\begin{array}{l}1,703 \\
1,718\end{array}$ & $\begin{array}{l}7,291 \\
7,446\end{array}$ & $\begin{array}{l}2,034 \\
2,004\end{array}$ & $\begin{array}{r}0,610 \\
10,060\end{array}$ & $\begin{array}{l}10,752 \\
10,281\end{array}$ & $\begin{array}{r}0,014 \\
50,022\end{array}$ \\
\hline
\end{tabular}

- "TOAal OPEC" conside of Algeria, Gabon, Indonesia, Iran, Iraq, Kuwar, Lbya. Nigenta, Oalar, Saud Arabia, tho United Arab Emirales, and Venezuale Production from the Neurna Zone berween Kuwell and Saudi Arabia in included in "Total OPEC."

- Thu Porsian Guil Nations aro Bahrain, Iren, Iraq, Kuwat, Oalar, Saudi Arabia, orro the Unted Arab Emirates. Production Irom the Neutral Zono between Kuwat and Saudl Arabia is included in "Porsian Gull Nations"

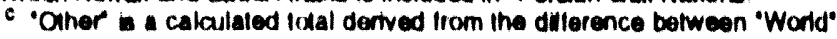
and the sum of production in "Total OPEC." Canada. Moxico, the Unined
Kingdom, the United Stales, China, and the lormer US S.A Rasevised date. Ex Estimale.

Notes: - Crude ol inchudes lases condereale but excludes naturel gus plant liquide. - U.S. geographic coverage is the 50 States and the District of Columbia. Monthy dala we dien presimuinary ligures and may not averago 10 the annual lotals because of rounding ox because updales to the prediminary monthly dali are not availatio

Sources: See end of section 
Figure 10.1 Crude Oll Production

(Million Barrole per Day)

World Production, 1973-1992

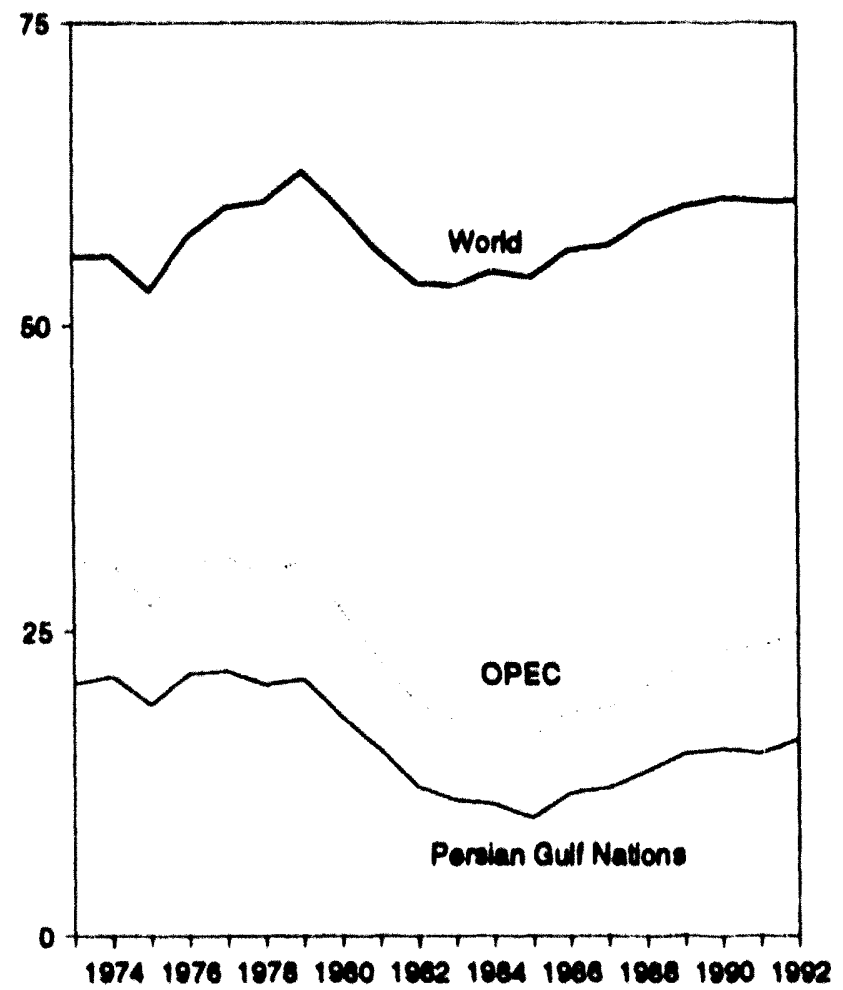

Leading Producers, 1973-1992

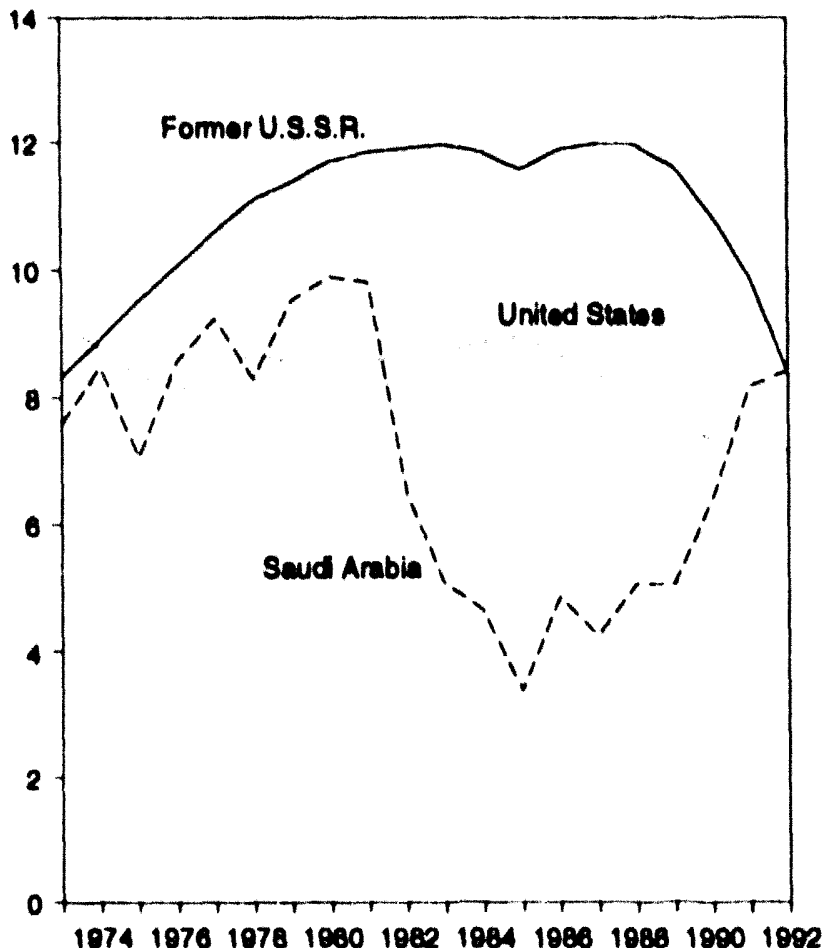

World Production, Monthly

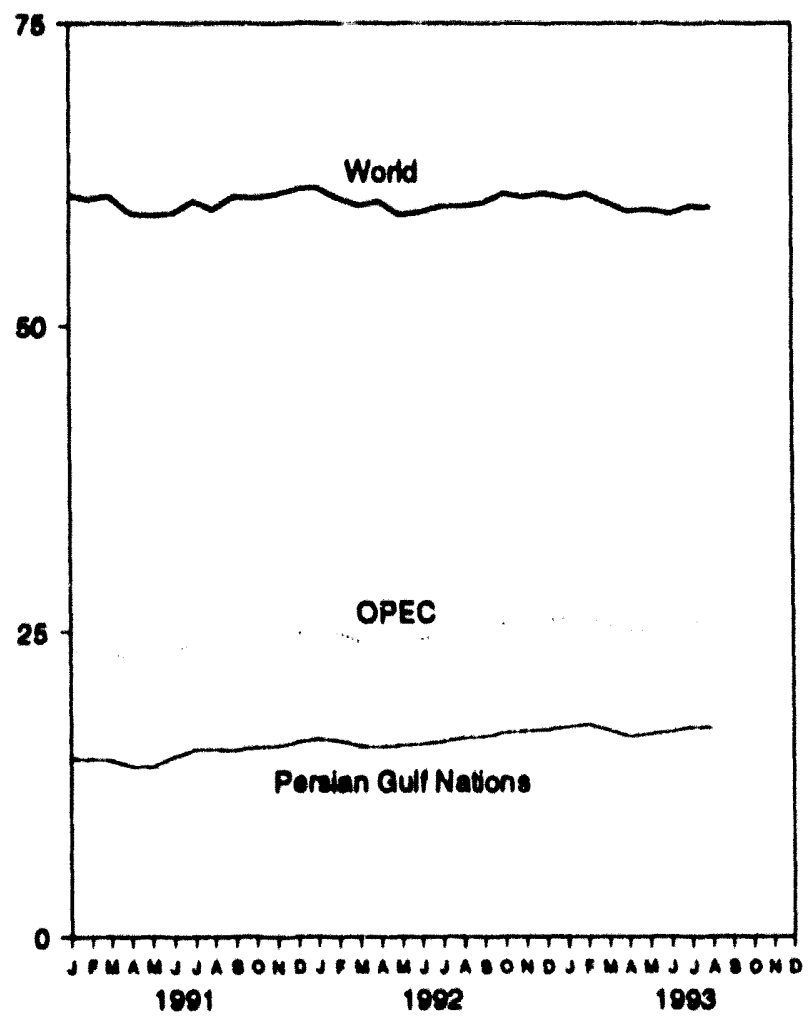

Leading Producers, Monthly

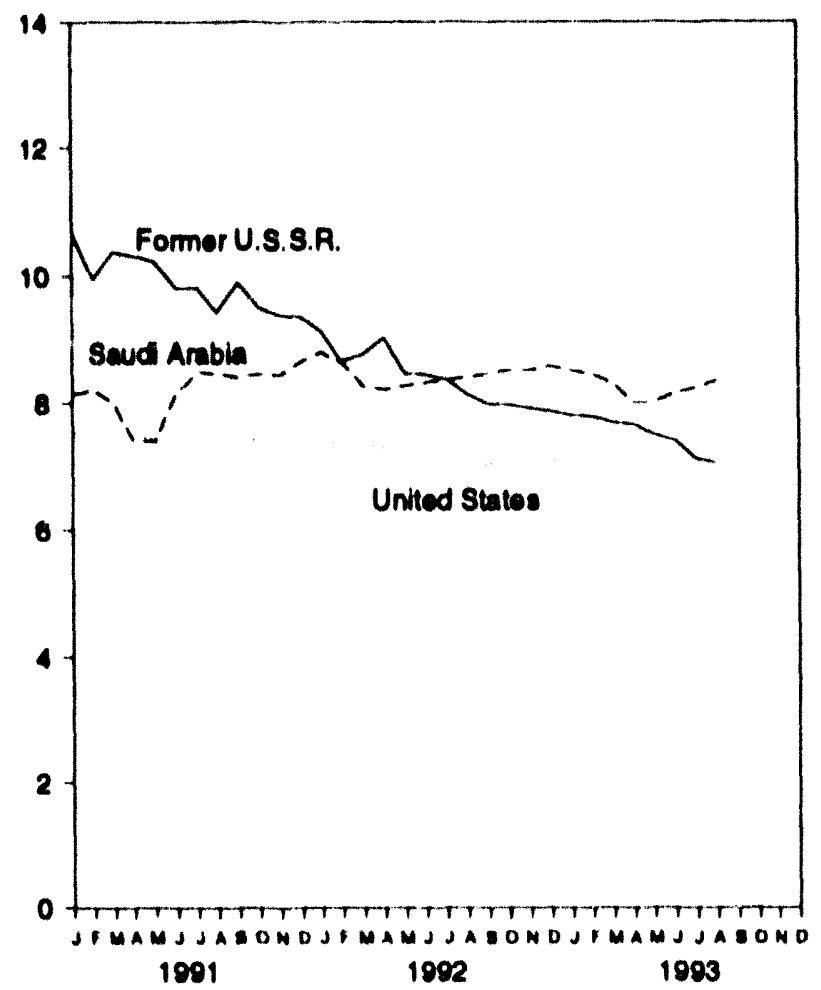

Nole: OPEC in the Oreanization of Petrobum Exporing Counitise. Sources: Trobe 10.1a and 10.16. 
Figure 10.2 Crudo Oll Production by Selected Country (Million Barrole por Day)

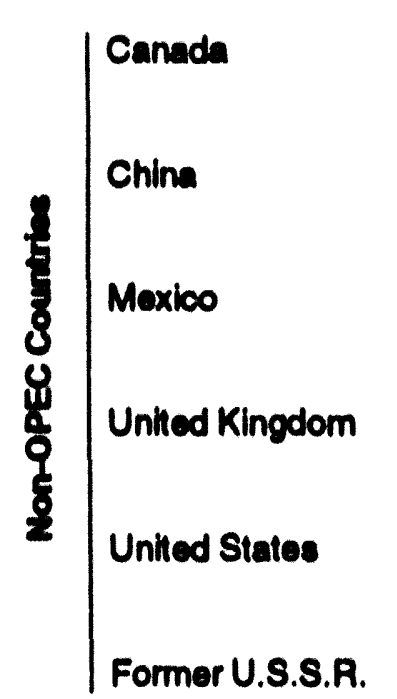

$\mid$\begin{tabular}{l|l|} 
Algeria & \\
Iran & Iraq \\
8 & Kuwait \\
$\frac{\mathrm{U}}{8}$ & Libya \\
Nigeria
\end{tabular}

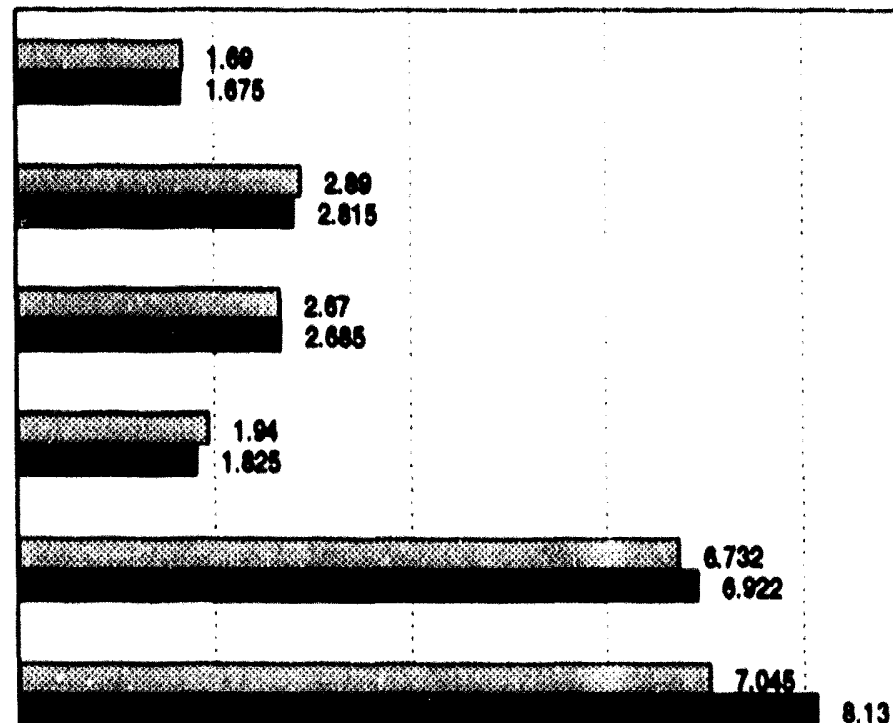

8.13

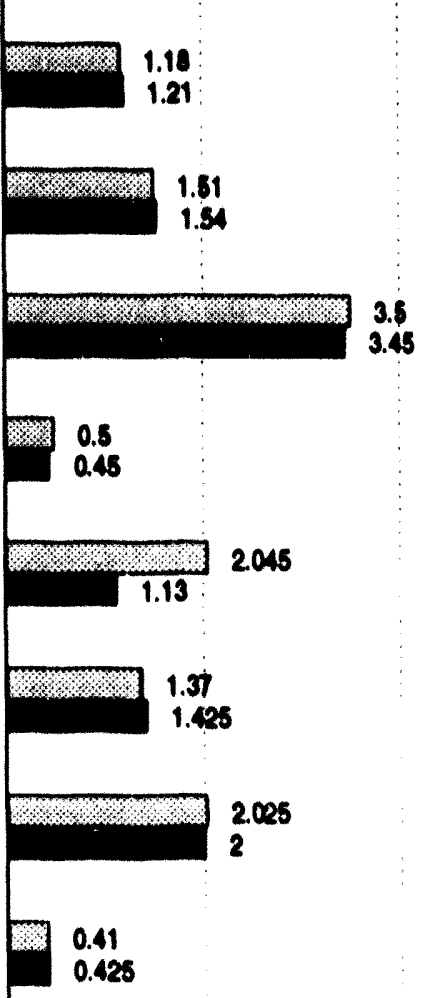

Saudi Arabia

United Arab

Emirates

Venezuela

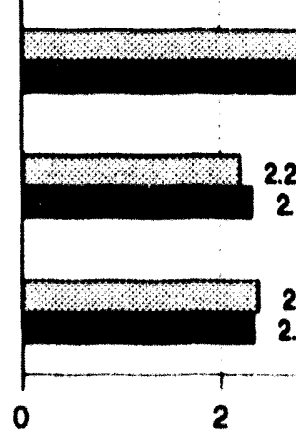

8.345

8.4
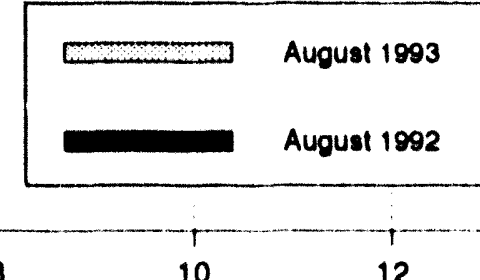

10

12

14

Noto: OPEC t the Organization of Pelroleum Exponting Countiles.

Sources: Tables 10.1a and 10.1b. 
Figure 10.3 Petroloum Consumption in OECD Countries

(Million Barrels per Day)

OECD Consumption, 1973-1992

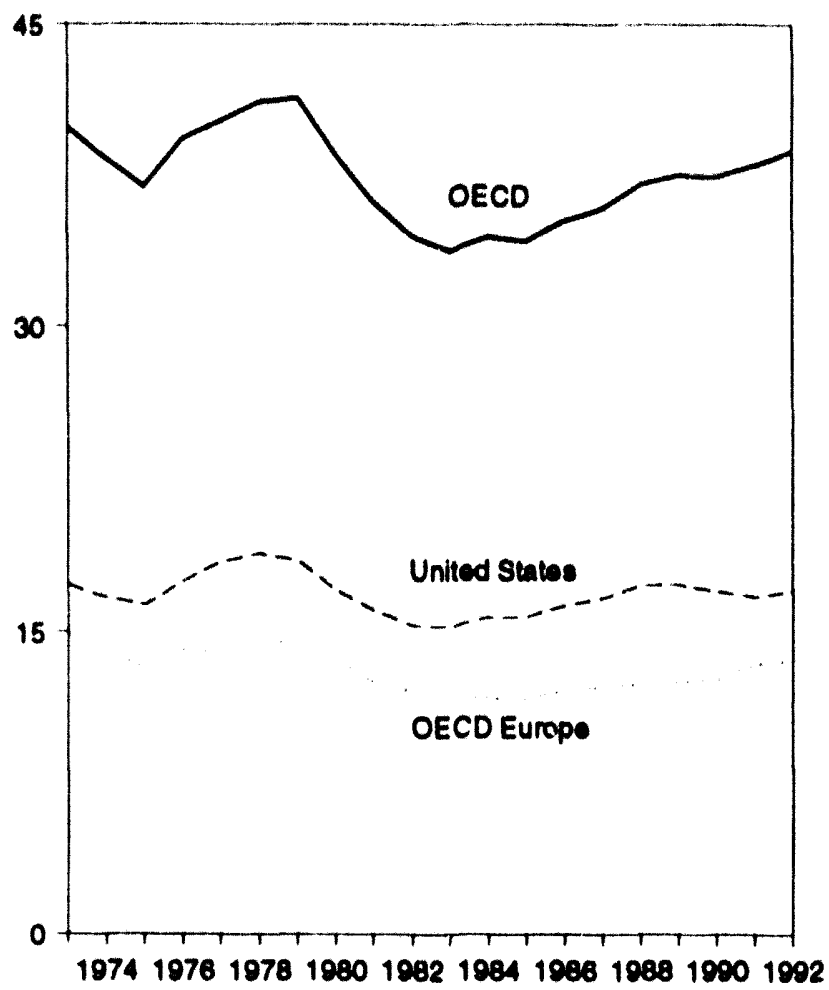

OECD Consumption

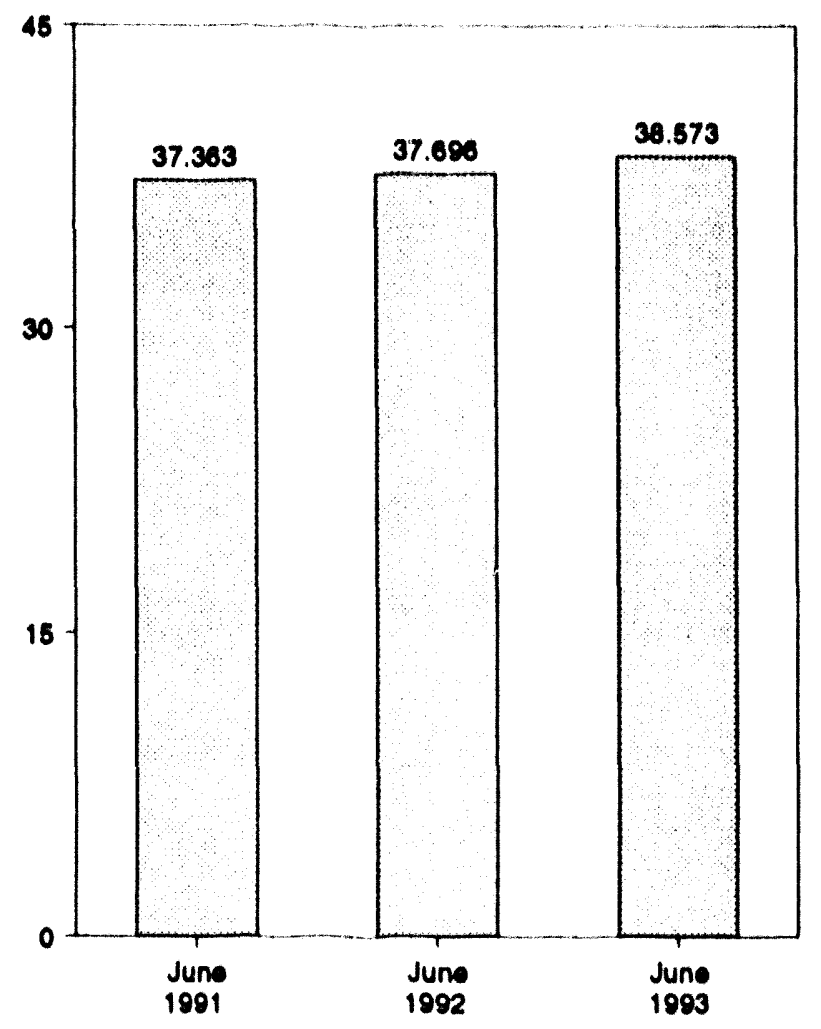

Consumption by Selected OECD Country

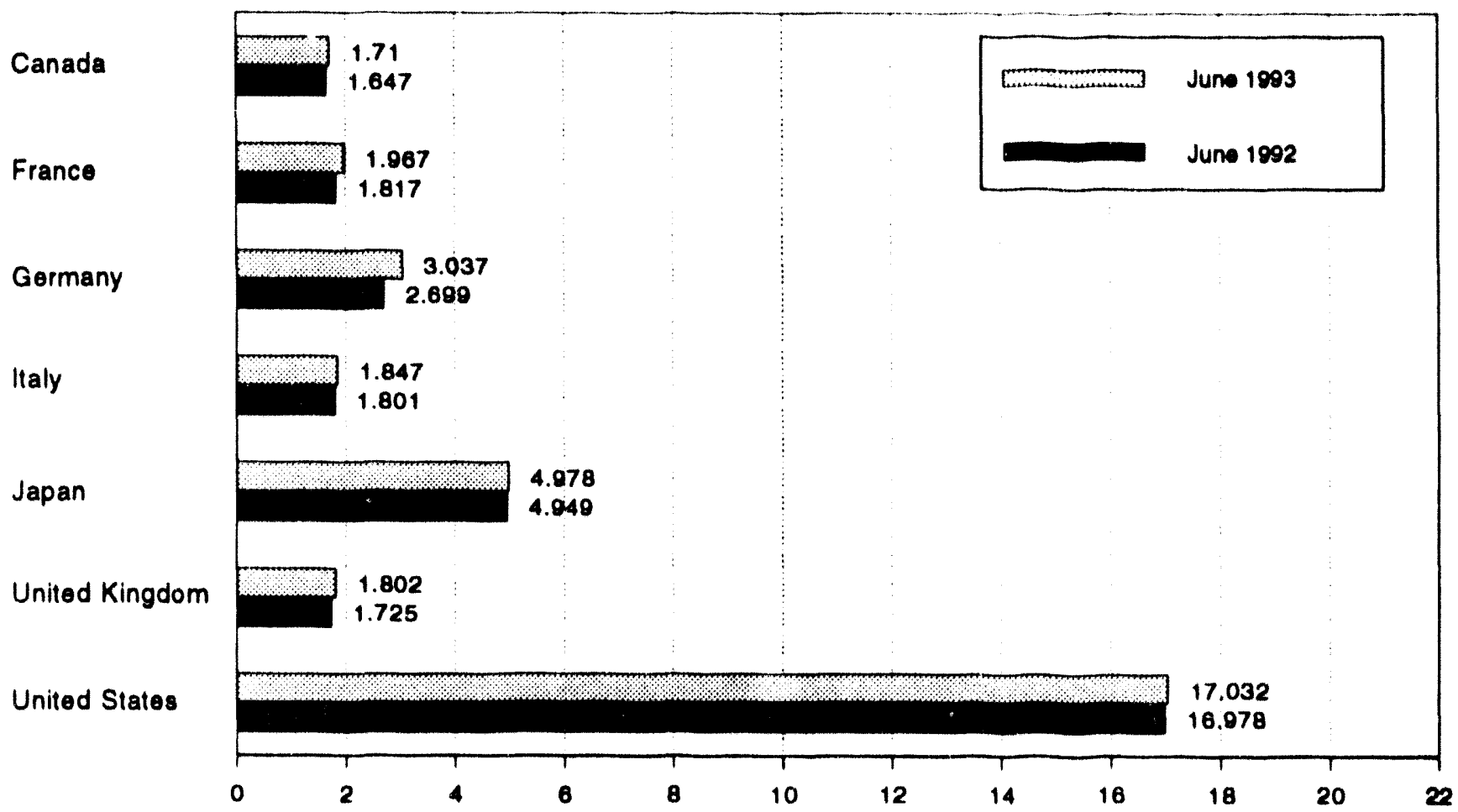

Note: OECD the Organization for Economic Cooperation and Development. Source: Table 10.2 . 
Table 10.2 Petroleum Consumption in OECD Countries

(Thousand Barrels per Day)

\begin{tabular}{|c|c|c|c|c|c|c|c|c|c|c|}
\hline & Caneda & France & Germany & Italy & Japan & $\begin{array}{l}\text { United } \\
\text { KIngdom }\end{array}$ & $\begin{array}{l}\text { United } \\
\text { Statos }\end{array}$ & $\begin{array}{l}\text { OECD } \\
\text { Europob }\end{array}$ & $\begin{array}{l}\text { Other } \\
\text { OECDC }\end{array}$ & OECD \\
\hline 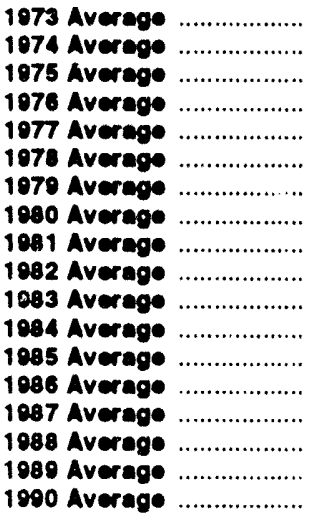 & $\begin{array}{l}1,720 \\
1,770 \\
1,770 \\
1,818 \\
1,850 \\
1,902 \\
1,071 \\
1,873 \\
1,788 \\
1,578 \\
1,448 \\
1,472 \\
1,504 \\
1,508 \\
1,548 \\
1,693 \\
1,733 \\
1,690\end{array}$ & $\begin{array}{l}2,601 \\
2,447 \\
2,252 \\
2,420 \\
2,294 \\
2,408 \\
2,463 \\
2,268 \\
2,023 \\
1,800 \\
1,838 \\
1,754 \\
1,775 \\
1,772 \\
1,789 \\
1,797 \\
1,857 \\
1,818\end{array}$ & $\begin{array}{l}3,056 \\
2,748 \\
2,650 \\
2,877 \\
2,868 \\
2,027 \\
3,003 \\
2,707 \\
2,449 \\
2,372 \\
2,324 \\
2,322 \\
2.338 \\
2,498 \\
2,424 \\
2,422 \\
2,280 \\
2,382\end{array}$ & $\begin{array}{l}2,068 \\
2,004 \\
1,855 \\
1,971 \\
1,897 \\
1,052 \\
2,030 \\
1,034 \\
1,674 \\
1,781 \\
1,760 \\
1,648 \\
1,717 \\
1,738 \\
1,856 \\
1,836 \\
1,030 \\
1,872\end{array}$ & $\begin{array}{l}4,940 \\
4,864 \\
4,621 \\
4,837 \\
4,880 \\
4,945 \\
6,050 \\
4,960 \\
4,848 \\
4,582 \\
4,395 \\
4,576 \\
4,384 \\
4,430 \\
4,484 \\
4,752 \\
4,983 \\
5,140\end{array}$ & $\begin{array}{l}2,341 \\
2,210 \\
1,011 \\
1,802 \\
1,005 \\
1,038 \\
1,071 \\
1,725 \\
1,590 \\
1,590 \\
1,681 \\
1,840 \\
1,634 \\
1,640 \\
1,603 \\
1,607 \\
1,738 \\
1,752\end{array}$ & $\begin{array}{l}17,308 \\
16,853 \\
16,322 \\
17,461 \\
18,431 \\
18,847 \\
18,513 \\
17,058 \\
16,058 \\
15,296 \\
15,231 \\
15,726 \\
15,726 \\
16,281 \\
16,865 \\
17,283 \\
17,325 \\
16,986\end{array}$ & $\begin{array}{l}14,925 \\
13,088 \\
13,217 \\
14,124 \\
13,016 \\
14,290 \\
14,687 \\
13,634 \\
12,515 \\
12,053 \\
11,765 \\
11,736 \\
11,681 \\
12,102 \\
12,255 \\
12,427 \\
12,531 \\
12,629\end{array}$ & $\begin{array}{r}988 \\
1,095 \\
1,041 \\
1,110 \\
1,160 \\
1,204 \\
1,178 \\
1,072 \\
1,080 \\
1,008 \\
954 \\
980 \\
978 \\
951 \\
958 \\
930 \\
998 \\
1,027\end{array}$ & $\begin{array}{l}30,000 \\
38,370 \\
36,980 \\
30,358 \\
40,237 \\
41,187 \\
41,370 \\
38,595 \\
36,260 \\
34,517 \\
33,793 \\
34,500 \\
34,271 \\
35,270 \\
35,011 \\
37,093 \\
37,570 \\
37,475\end{array}$ \\
\hline 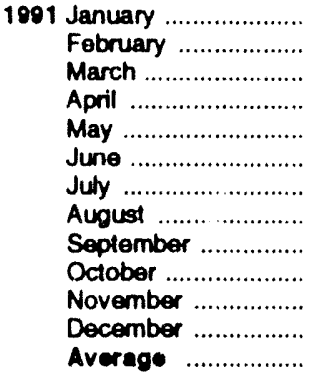 & $\begin{array}{l}1,599 \\
1,613 \\
1,484 \\
1,595 \\
1,637 \\
1,589 \\
1,707 \\
1,693 \\
1,583 \\
1,693 \\
1,602 \\
1,662 \\
1,622\end{array}$ & $\begin{array}{l}2,294 \\
2,009 \\
1,759 \\
1,808 \\
1,773 \\
1,807 \\
1,989 \\
1,795 \\
1,824 \\
2,075 \\
1,953 \\
2,132 \\
1,935\end{array}$ & $\begin{array}{l}2,998 \\
2,783 \\
2,858 \\
2,953 \\
2,912 \\
3,269 \\
2,272 \\
2,609 \\
2,679 \\
2,919 \\
2,860 \\
2,829 \\
2,828\end{array}$ & $\begin{array}{l}2,185 \\
2,025 \\
1,660 \\
1,813 \\
1,722 \\
1,535 \\
1,665 \\
1,546 \\
1,824 \\
2,126 \\
2,031 \\
2,231 \\
1,863\end{array}$ & $\begin{array}{l}5,852 \\
6,155 \\
5,789 \\
5,025 \\
4,880 \\
4,765 \\
5,000 \\
4,888 \\
4,724 \\
4,848 \\
5,581 \\
5,852 \\
5,284\end{array}$ & $\begin{array}{l}1,819 \\
1,837 \\
1,725 \\
1,793 \\
1,799 \\
1,769 \\
1,853 \\
1,812 \\
1,753 \\
1,864 \\
1,829 \\
1,765 \\
1,801\end{array}$ & $\begin{array}{l}16,893 \\
16,339 \\
16,212 \\
16,139 \\
16,189 \\
16,878 \\
16,971 \\
17,183 \\
16,848 \\
16,996 \\
16,730 \\
17,145 \\
16,714\end{array}$ & $\begin{array}{l}14,564 \\
13,804 \\
12,609 \\
13,073 \\
12,965 \\
13,184 \\
12,648 \\
12,727 \\
12,999 \\
14,178 \\
13,736 \\
14,228 \\
13,301\end{array}$ & $\begin{array}{r}1,063 \\
1,039 \\
1,091 \\
1,082 \\
1,104 \\
947 \\
1,001 \\
989 \\
1,024 \\
1,113 \\
1,128 \\
1,043 \\
1,052\end{array}$ & $\begin{array}{l}39,971 \\
38,950 \\
37,185 \\
36,914 \\
36,775 \\
37,363 \\
37,327 \\
37,480 \\
37,178 \\
38,827 \\
38,777 \\
40,029 \\
38,063\end{array}$ \\
\hline 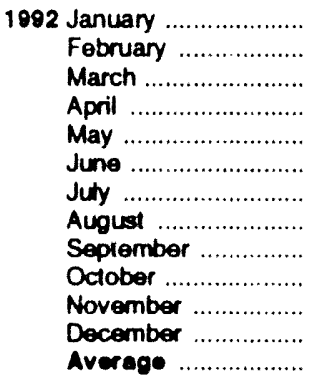 & 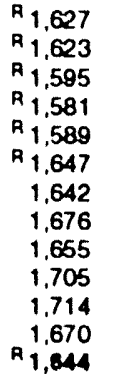 & $\begin{array}{l}{ }_{2} 2,213 \\
A_{2,108} \\
A_{1,939} \\
A_{1,993} \\
A_{1,632} \\
A_{1,817} \\
A_{1,929} \\
R_{1,735} \\
A_{1,956} \\
A_{1,942} \\
A_{1}, 890 \\
A_{2,000} \\
A_{1,920}\end{array}$ & $\begin{array}{l}2,968 \\
2,814 \\
2,809 \\
2,893 \\
2,588 \\
2,699 \\
3,029 \\
2,829 \\
3,072 \\
2,752 \\
2,823 \\
2,841 \\
2,843\end{array}$ & $\begin{array}{r}2,237 \\
R_{2,149} \\
R_{1,856} \\
1,891 \\
R_{1,671} \\
1,801 \\
1,900 \\
1,655 \\
2,003 \\
1,830 \\
2,053 \\
2,076 \\
1,036\end{array}$ & $\begin{array}{l}A_{5,776} \\
R_{6,347} \\
R_{5,873} \\
A_{5,212} \\
A_{4,845} \\
A_{4,949} \\
A_{5,124} \\
A_{4,964} \\
A_{5,147} \\
A_{5,310} \\
A_{5,644} \\
A_{6,285} \\
A_{5,454}\end{array}$ & $\begin{array}{r}1,832 \\
R 1,819 \\
1,818 \\
1,858 \\
1,694 \\
1,725 \\
1,804 \\
1,699 \\
1,870 \\
1,825 \\
1,852 \\
1,839 \\
P_{1,803}\end{array}$ & $\begin{array}{l}17,012 \\
16,893 \\
16,825 \\
16,764 \\
16,485 \\
16,978 \\
17,143 \\
16,929 \\
16,876 \\
17,448 \\
17,091 \\
17,928 \\
17,033\end{array}$ & $\begin{array}{l}R_{14,459} \\
R_{14,052} \\
R_{13,682} \\
R_{13,667} \\
A_{12,347} \\
A_{13,036} \\
A_{13,662} \\
A_{12,909} \\
A_{14,224} \\
A_{13,475} \\
R_{13,806} \\
A_{13,991} \\
R_{13,606}\end{array}$ & $\begin{array}{r}1,014 \\
1,045 \\
1,054 \\
1,042 \\
R 1,002 \\
1,086 \\
1,027 \\
946 \\
1,046 \\
1,014 \\
1,049 \\
1,103 \\
1,035\end{array}$ & 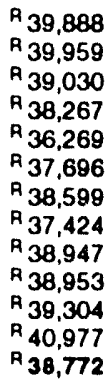 \\
\hline 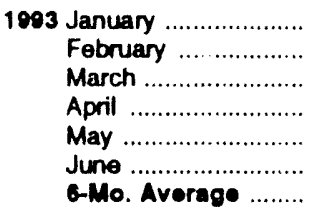 & $\begin{array}{l}\text { R } 1,581 \\
\text { A }_{1,728} \\
\text { A }_{1,696} \\
\text { A }_{1,615} \\
\text { म }_{1,624} \\
1,710 \\
1,660\end{array}$ & $\begin{array}{r}1,950 \\
2,138 \\
2,010 \\
R \quad 1,931 \\
R_{1,696} \\
1,967 \\
1,046\end{array}$ & $\begin{array}{r}A_{2,521} \\
A_{2,930} \\
{ }_{2}, 953 \\
A_{2,814} \\
2,584 \\
3,037 \\
2,803\end{array}$ & $\begin{array}{r}1,859 \\
2,106 \\
2,005 \\
R \quad 1,809 \\
\mathrm{P}_{1,706} \\
1,847 \\
1,886\end{array}$ & $\begin{array}{r}A_{5,937} \\
R_{6,286} \\
R_{6,238} \\
R_{5,447} \\
R_{4,760} \\
4,978 \\
5,601\end{array}$ & $\begin{array}{l}\text { ค } 1,721 \\
\text { ค }_{1,872} \\
\text { ค }_{1,881} \\
\text { ค }_{1,726} \\
\text { ค }_{1,671} \\
1,802 \\
1,778\end{array}$ & $\begin{array}{l}16,320 \\
17,397 \\
17,688 \\
16,673 \\
16,340 \\
17,032 \\
16,901\end{array}$ & $\begin{array}{l}R_{12,823} \\
A_{14,214} \\
R_{14,023} \\
R_{13,339} \\
R_{12,222} \\
13,763 \\
13,382\end{array}$ & $\begin{array}{r}943 \\
\text { म }_{1,104} \\
\text { म }_{1,145} \\
\text { म }_{1,099} \\
\text { म }_{1,110} \\
1,090 \\
1,082\end{array}$ & $\begin{array}{l}R_{37,614} \\
R_{40,729} \\
R_{40,790} \\
R_{38,173} \\
R_{36,055} \\
38,573 \\
38,625\end{array}$ \\
\hline $\begin{array}{l}1002 \text { \&-Mo. Avorage ........ } \\
1001 \text { G-Mo. Ayorage ........ }\end{array}$ & $\begin{array}{l}1,610 \\
1,586\end{array}$ & $\begin{array}{l}1,040 \\
1,008\end{array}$ & $\begin{array}{l}2,705 \\
2,963\end{array}$ & $\begin{array}{l}1,038 \\
1,822\end{array}$ & $\begin{array}{l}5,496 \\
5,404\end{array}$ & $\begin{array}{l}1,701 \\
1,700\end{array}$ & $\begin{array}{l}16,825 \\
16,443\end{array}$ & $\begin{array}{l}13,537 \\
13,362\end{array}$ & $\begin{array}{l}1,040 \\
1,055\end{array}$ & $\begin{array}{l}38,508 \\
37,850\end{array}$ \\
\hline
\end{tabular}

- Through December 1990, the dala for Germany are for the former West Germany only. Beginning with January 1991, the data lor Germany are for the unitied Germany, l.e., the former East Germany and West Germany.

b 'OECD Europe" consists of Austria, Belgium, Denmark, Finland, France, Germany. Greece, Iceland, Ireland, Maly, Luxemtmirg, the Netherlands, Norway, Portugal, Spain, Sweden, Swizertand, Turkey, and the United Kingdom

c 'Other OECD" consists of Australia. New Zealand, and the U.S Territories.

$R=$ Revised data

Notes: - The Organization for Economic Cooperation and Development (OECD) consists of Canada, Japan, and the United States, as well as "OECD Europe" and "Other OECD." - U.S. geographic coverage is the 50 States and the District of Columbia. - Totals may not equal sum of components due to independent rounding. - Data through 1990 are linal. Subsequent data are preliminary.

Sources: - United States: Table 3.1a. - All Other Data: 1073-1970-Intemational Energy Agency (IEA). Annual Oil and Gas STatistics of OECD Countries. 1080 forward-IEA, quarterty and monthly computer tapes supporting Quarterty Oil STatistics and Energy Balances. 
Figure 10.4 Petroleum Stocks in OECD Countries

(Billion Barrels)

OECD Stocks, End of Year, 1973-1992

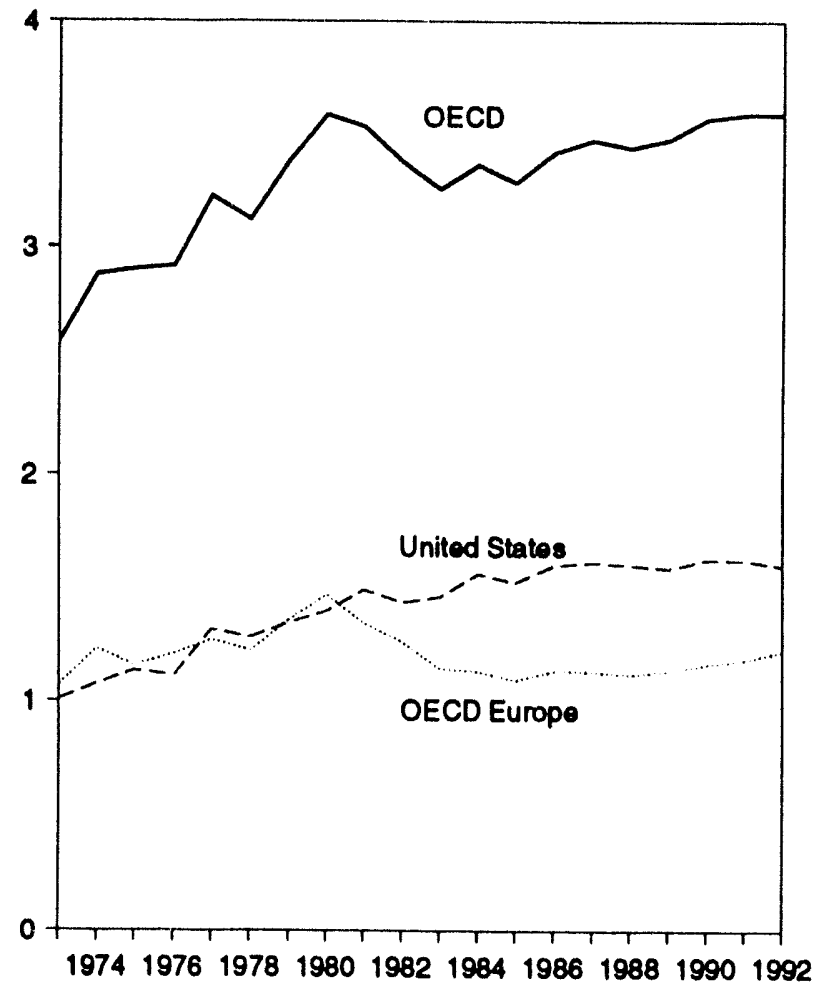

OECD Stocks, End of Month

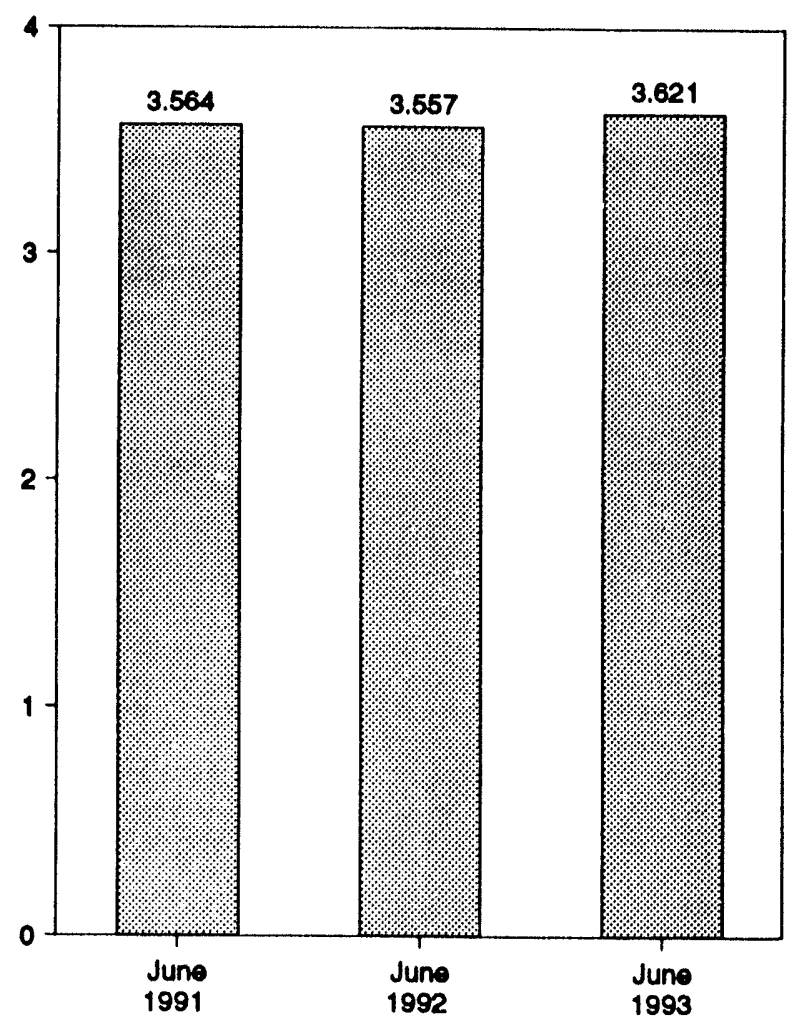

Stocks by Selected Country, End of Month

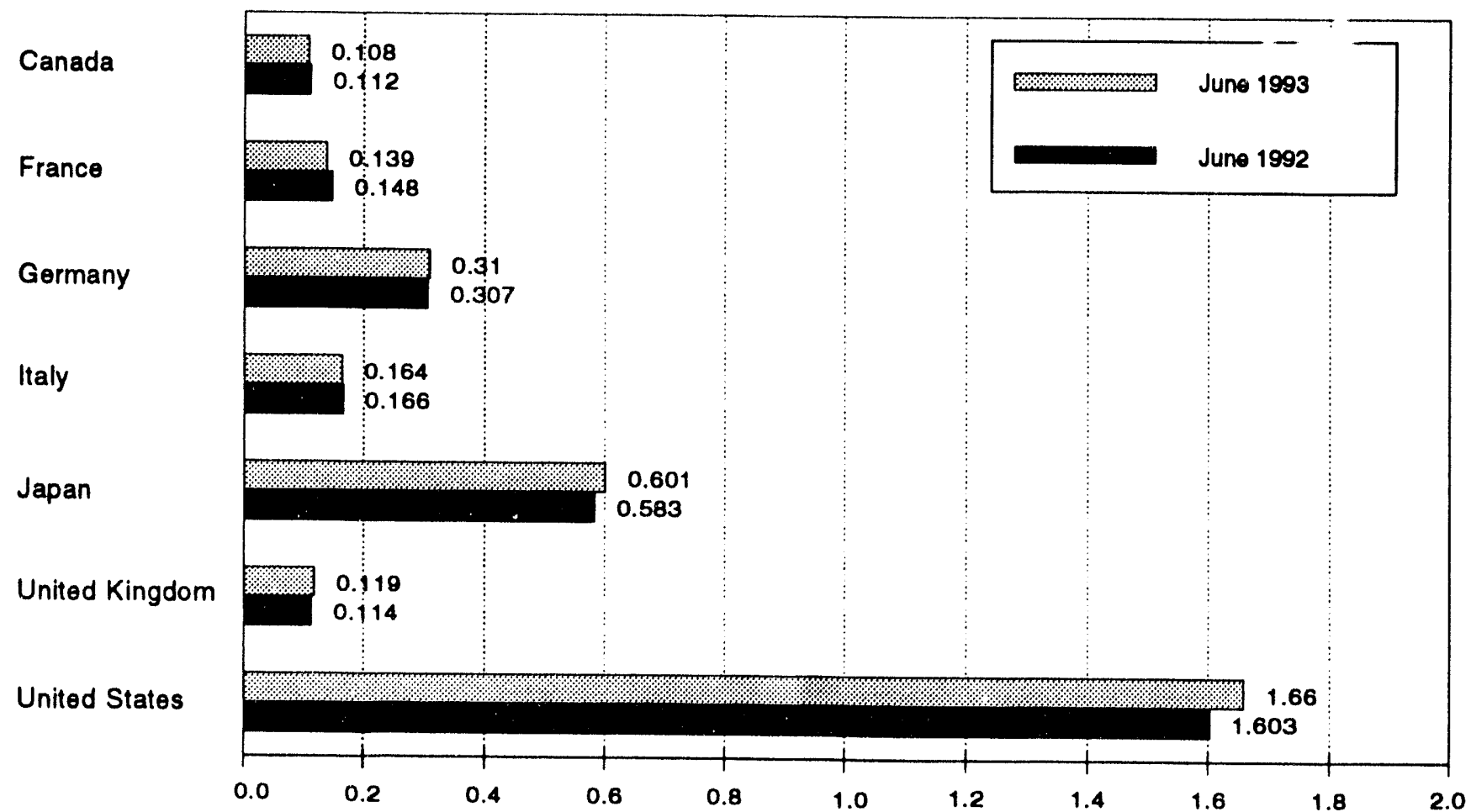

Note: OECD b the Organization for Economic Cooperation and Development.

Source: Table 10.3. 
Table 10.3 Petroleum Stocks in OECD Countries, End of Period (Million Barrels)

\begin{tabular}{|c|c|c|c|c|c|c|c|c|c|c|}
\hline & Canade & Frence & Germany & italy & Jepen & $\begin{array}{c}\text { United } \\
\text { Kingdom }\end{array}$ & $\begin{array}{l}\text { United } \\
\text { States }\end{array}$ & $\begin{array}{c}\text { OECD } \\
\text { Europeb }\end{array}$ & $\begin{array}{l}\text { Other } \\
\text { OECDC }\end{array}$ & OECD \\
\hline 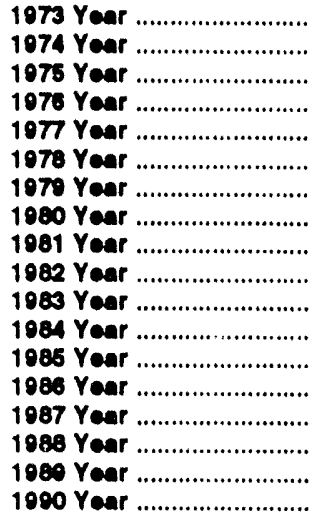 & $\begin{array}{l}140 \\
145 \\
174 \\
153 \\
167 \\
144 \\
180 \\
164 \\
181 \\
138 \\
121 \\
128 \\
113 \\
111 \\
126 \\
116 \\
114 \\
121\end{array}$ & $\begin{array}{l}201 \\
249 \\
225 \\
234 \\
230 \\
201 \\
226 \\
243 \\
214 \\
193 \\
153 \\
152 \\
130 \\
127 \\
127 \\
140 \\
138 \\
140\end{array}$ & $\begin{array}{l}181 \\
213 \\
187 \\
200 \\
225 \\
238 \\
272 \\
310 \\
207 \\
272 \\
249 \\
230 \\
233 \\
252 \\
269 \\
206 \\
271 \\
265\end{array}$ & $\begin{array}{l}152 \\
167 \\
143 \\
143 \\
161 \\
154 \\
183 \\
170 \\
167 \\
170 \\
149 \\
159 \\
157 \\
155 \\
189 \\
155 \\
184 \\
172\end{array}$ & $\begin{array}{l}303 \\
370 \\
375 \\
380 \\
400 \\
413 \\
460 \\
495 \\
482 \\
484 \\
470 \\
470 \\
494 \\
500 \\
540 \\
530 \\
577 \\
540\end{array}$ & $\begin{array}{l}186 \\
191 \\
186 \\
185 \\
148 \\
187 \\
180 \\
186 \\
143 \\
125 \\
118 \\
112 \\
123 \\
124 \\
121 \\
112 \\
118 \\
112\end{array}$ & $\begin{array}{l}1,003 \\
1,074 \\
1,133 \\
1,112 \\
1,312 \\
1,278 \\
1,341 \\
1,302 \\
1,484 \\
1,430 \\
1,454 \\
1,550 \\
1,510 \\
1,503 \\
1,607 \\
1,507 \\
1,581 \\
1,621\end{array}$ & $\begin{array}{l}1,070 \\
1,227 \\
1,154 \\
1,205 \\
1,288 \\
1,210 \\
1,353 \\
1,464 \\
1,337 \\
1,258 \\
1,142 \\
1,130 \\
1,092 \\
1,133 \\
1,130 \\
1,118 \\
1,133 \\
1,163\end{array}$ & $\begin{array}{l}67 \\
64 \\
67 \\
68 \\
68 \\
68 \\
75 \\
72 \\
67 \\
68 \\
68 \\
69 \\
66 \\
72 \\
72 \\
71 \\
71 \\
73\end{array}$ & $\begin{array}{l}2,588 \\
2,800 \\
2,403 \\
2,918 \\
3,224 \\
3,122 \\
3,370 \\
3,507 \\
3,531 \\
3,376 \\
3,255 \\
3,362 \\
3,284 \\
3,418 \\
3,474 \\
3,440 \\
3,476 \\
3,568\end{array}$ \\
\hline 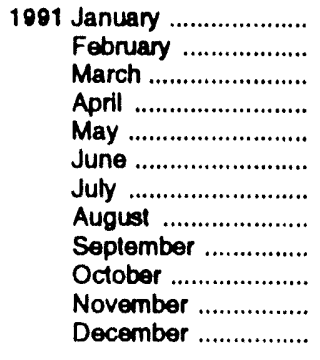 & $\begin{array}{l}116 \\
114 \\
117 \\
110 \\
107 \\
107 \\
118 \\
116 \\
117 \\
118 \\
122 \\
110\end{array}$ & $\begin{array}{l}133 \\
137 \\
142 \\
138 \\
138 \\
144 \\
145 \\
152 \\
150 \\
148 \\
152 \\
153\end{array}$ & $\begin{array}{l}278 \\
278 \\
280 \\
277 \\
279 \\
274 \\
285 \\
284 \\
287 \\
286 \\
289 \\
288\end{array}$ & $\begin{array}{l}174 \\
169 \\
178 \\
177 \\
174 \\
173 \\
169 \\
171 \\
170 \\
165 \\
163 \\
180\end{array}$ & $\begin{array}{l}591 \\
572 \\
593 \\
585 \\
586 \\
590 \\
594 \\
610 \\
622 \\
625 \\
607 \\
607\end{array}$ & $\begin{array}{l}116 \\
118 \\
124 \\
119 \\
113 \\
118 \\
113 \\
118 \\
120 \\
119 \\
120 \\
110\end{array}$ & $\begin{array}{l}1,587 \\
1,573 \\
1,558 \\
1,578 \\
1,626 \\
1,634 \\
1,635 \\
1,648 \\
1,663 \\
1,644 \\
1,647 \\
1,617\end{array}$ & $\begin{array}{l}1,164 \\
1,162 \\
1,178 \\
1,161 \\
1,157 \\
1,161 \\
1,170 \\
1,186 \\
1,195 \\
1,190 \\
1,198 \\
1,182\end{array}$ & $\begin{array}{l}73 \\
72 \\
75 \\
75 \\
75 \\
72 \\
73 \\
76 \\
74 \\
71 \\
70 \\
65\end{array}$ & $\begin{array}{l}3,531 \\
3,493 \\
3,521 \\
3,509 \\
3,551 \\
3,564 \\
3,590 \\
3,636 \\
3,671 \\
3,649 \\
3,643 \\
3,580\end{array}$ \\
\hline 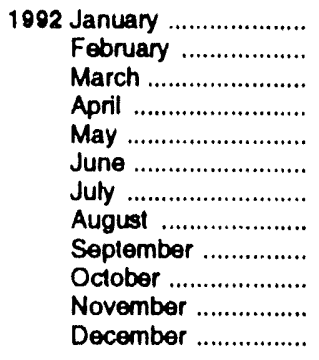 & $\begin{array}{r}117 \\
111 \\
111 \\
111 \\
R^{1} \\
108 \\
112 \\
110 \\
113 \\
110 \\
108 \\
110 \\
107\end{array}$ & $\begin{array}{l}149 \\
145 \\
142 \\
140 \\
147 \\
148 \\
146 \\
150 \\
148 \\
148 \\
149 \\
146\end{array}$ & $\begin{array}{l}293 \\
303 \\
303 \\
307 \\
311 \\
307 \\
299 \\
303 \\
299 \\
302 \\
306 \\
310\end{array}$ & $\begin{array}{r}167 \\
172 \\
P_{169} \\
165 \\
171 \\
P_{166} \\
166 \\
169 \\
{ }^{169} \\
165 \\
166 \\
172 \\
174\end{array}$ & $\begin{array}{l}601 \\
596 \\
586 \\
578 \\
588 \\
583 \\
586 \\
604 \\
608 \\
613 \\
611 \\
603\end{array}$ & $\begin{array}{l}116 \\
118 \\
115 \\
115 \\
115 \\
114 \\
120 \\
117 \\
112 \\
113 \\
116 \\
113\end{array}$ & $\begin{array}{l}1,610 \\
1,588 \\
1,571 \\
1,583 \\
1,602 \\
1,603 \\
1,620 \\
1,621 \\
1,636 \\
1,640 \\
1,636 \\
1,592\end{array}$ & $\begin{array}{r}1,168 \\
1,181 \\
A_{1,162} \\
1,172 \\
1,189 \\
A_{1,190} \\
1,182 \\
1,211 \\
A_{1,194} \\
A_{1,201} \\
A_{1,207} \\
\text { P } 1,210^{1}\end{array}$ & $\begin{array}{l}68 \\
66 \\
66 \\
62 \\
63 \\
69 \\
67 \\
69 \\
69 \\
69 \\
71 \\
67\end{array}$ & 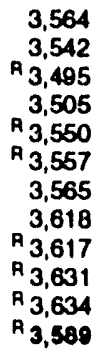 \\
\hline 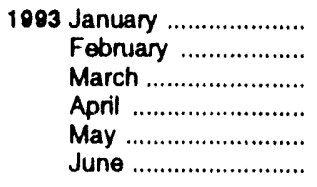 & $\begin{array}{r}110 \\
106 \\
R_{107} \\
110 \\
R \quad 110 \\
108\end{array}$ & $\begin{array}{l}148 \\
142 \\
138 \\
139 \\
145 \\
139\end{array}$ & $\begin{array}{r}319 \\
317 \\
\mathbf{R}^{311} \\
311 \\
320 \\
310\end{array}$ & $\begin{array}{l}171 \\
163 \\
156 \\
158 \\
164 \\
164\end{array}$ & $\begin{array}{r}614 \\
606 \\
R_{593} \\
584 \\
R_{592} \\
601\end{array}$ & $\begin{array}{l}120 \\
120 \\
120 \\
116 \\
117 \\
119\end{array}$ & $\begin{array}{l}1,611 \\
1,595 \\
1,584 \\
1,611 \\
1,643 \\
1,660\end{array}$ & $\begin{array}{l}A_{1,231} \\
R_{1,213} \\
P_{1,192} \\
P_{1,186} \\
P_{1,201} \\
1,183\end{array}$ & $\begin{array}{l}68 \\
68 \\
66 \\
73 \\
68 \\
69\end{array}$ & $\begin{array}{l}A_{3,634} \\
A_{3,588} \\
A_{3,542} \\
A_{3,564} \\
A_{3,614} \\
3,621\end{array}$ \\
\hline
\end{tabular}

- Through December 1990, the data for Germany are for the former West Germany only. Beginning with January 1991, the data for Germany are for the unified Germany, 1.e. the former East Germany and West Germany.

b "OECD Europe" consists of Austria, Belgium, Denmark, Finland, France, Germany, Greece, Iceland, Ireland, lialy, Luxembourg, the Netherlands, Norway, Portugal, Spain, Sweden, Switzerland, Turkey, and the United Kingdom.

c "Other OECD" consists of Australia, New Zealand, and the U.S. Territories.

$R=$ Revised data.

Notes: - Petroleum stocks include crude oil (including strategic resenves), unfinished oits, natural gas plant liquids, and relined products. Petroleum stocks include all nonmilitary petroloum held for storage, regardless of ownership, within each couniry in bulk terminals, refinery tanks, pipeline tankage, intercoastal tankers, tankers in port, and inland ship bunkers. Data exclude oll held in pipelines (except for those in the United States), rail and Iruck cars, sea-going ships' bunkers, service stations, relail stores, and tankers at sea. - The Organization for Economic Cooperation and Development (OECD) consists of Canada, Japan, and the United Siates, as well as "OECD Europe" and "Other OECD." - U.S. geographic coverage is the 50 States and the District ut Columbia. - Totals may not equal sum of components due to independent rounding. - In the United States in January 1975, 1981, and 1983, numerous respondents were added to bulk terminal and pipeline surveys, thereby affecting subsequent stocks reported. Newbasis end-of-year U.S. stocks, in million barrels, would have been 1,121 in 1974, 1,425 in 1980, and 1,461 in 1982. Data through 1990 are linal. Subsequent data are preliminary.

Sources: - United States: Table 3.1a. - All Other Data: International Energy Agency, quarterty and monthly computer tapes supporting Quarterly Oil STatistics and Energy Balances. 
Figure 10.5 Nuclear Electricity Gross Generation

(Billion Kilowatthours)

U.S. and Total* Generation, 1973-1992

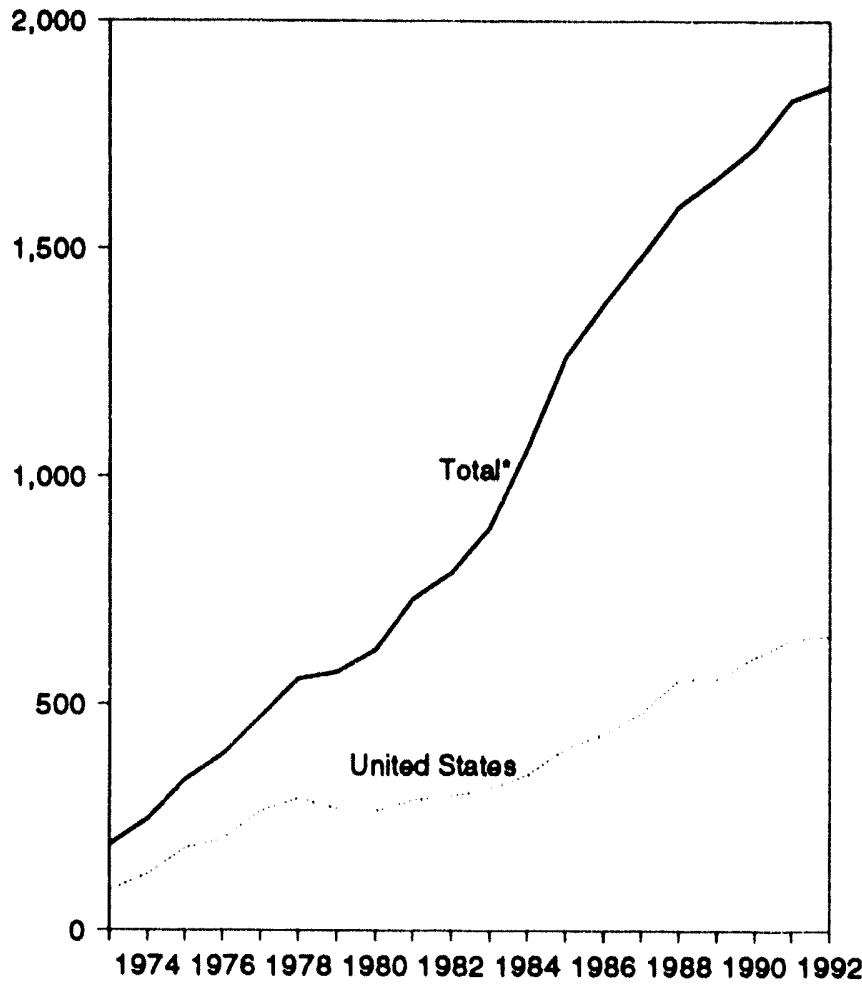

Total* Generation

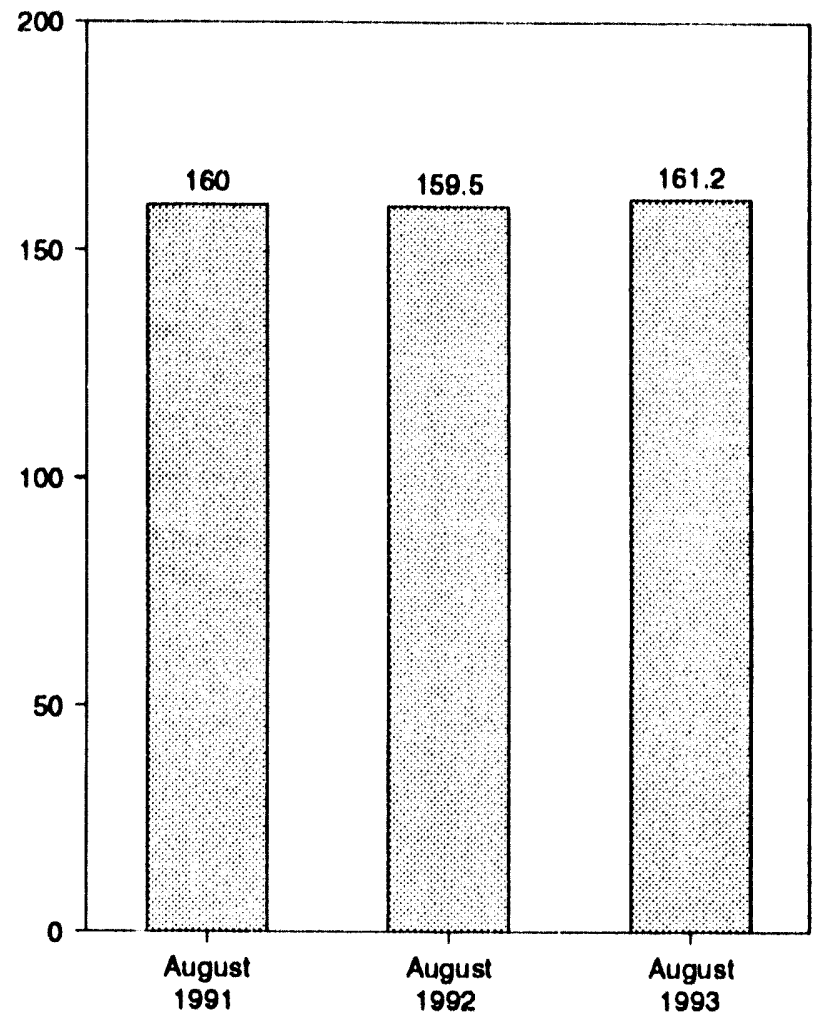

Generation by Selected Country, August 1993

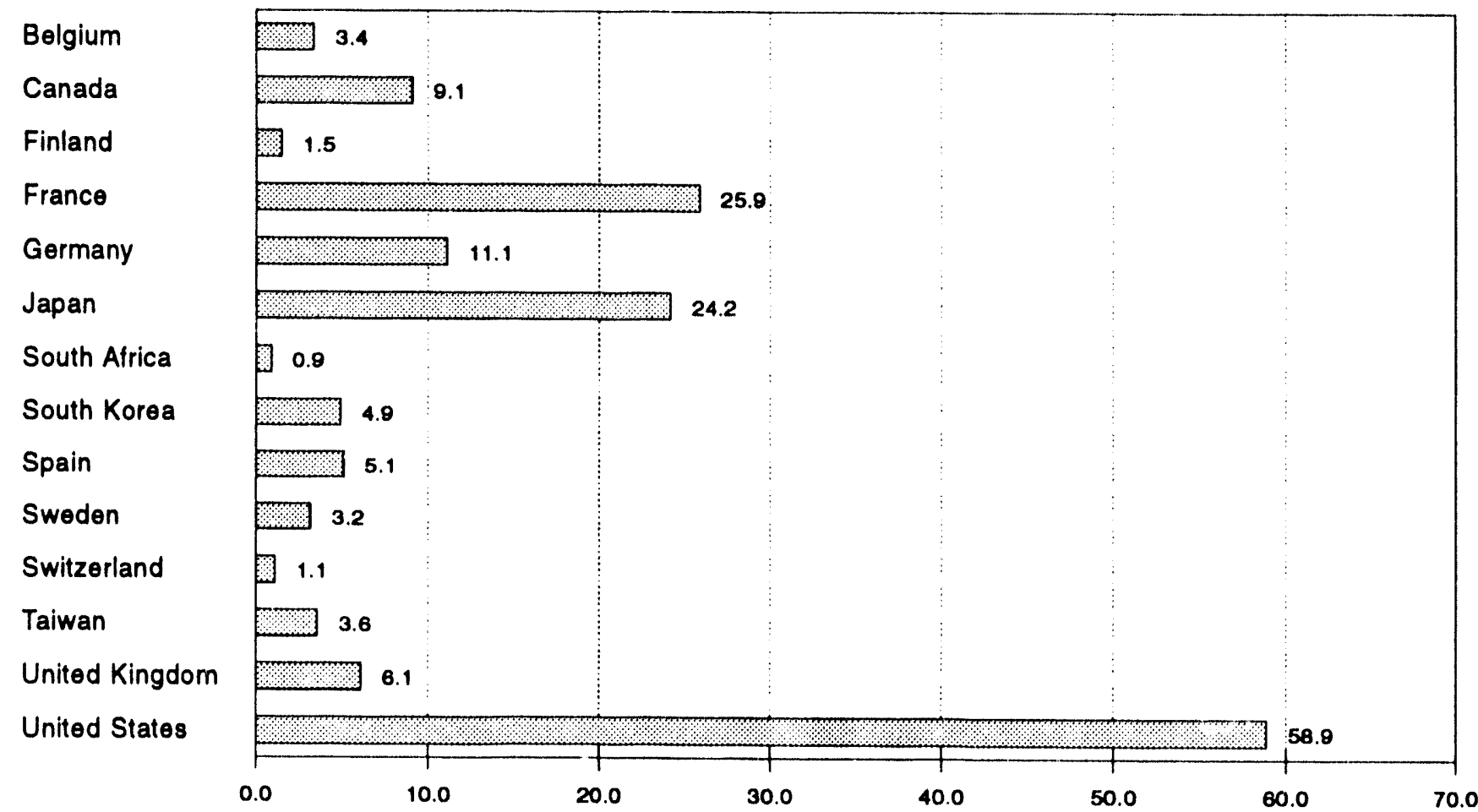

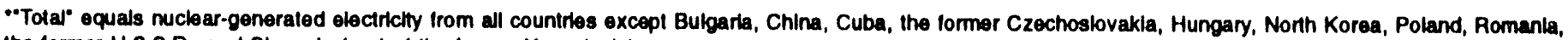
the former U.S.S.R., and Slovenia (part of the former Yugoslavia).

Note: Because vertical scales ditier, graphs should not be compared. 
Table 10.4a Nuclear Electriclty Gross Generation: Argentina Through India (Billion Kilowatthours)

\begin{tabular}{|c|c|c|c|c|c|c|c|c|}
\hline & Arecntina & Belglum & Breall & Ceneda & Finlend & France & Oermany" & Indle \\
\hline 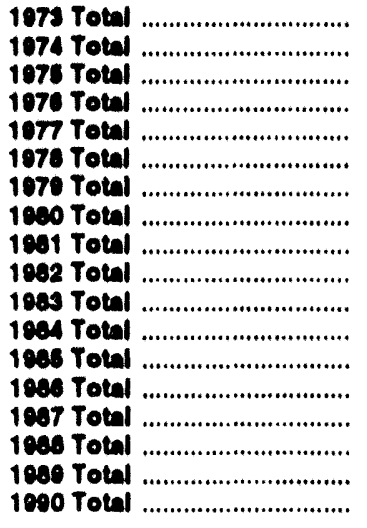 & $\begin{array}{l}0.0 \\
1.0 \\
2.8 \\
2.0 \\
1.0 \\
2.0 \\
2.7 \\
2.3 \\
2.8 \\
1.0 \\
3.4 \\
4.6 \\
8.8 \\
8.7 \\
6.2 \\
5.1 \\
5.0 \\
7.4\end{array}$ & $\begin{array}{r}0.0 \\
.1 \\
10.8 \\
10.0 \\
11.0 \\
12.8 \\
11.4 \\
12.8 \\
12.8 \\
15.0 \\
24.1 \\
27.7 \\
34.8 \\
38.4 \\
11.6 \\
43.1 \\
41.2 \\
42.7\end{array}$ & $\begin{array}{l}0.0 \\
.0 \\
.0 \\
.0 \\
.0 \\
.0 \\
.0 \\
.0 \\
.0 \\
.1 \\
.2 \\
2.1 \\
1.4 \\
.1 \\
1.0 \\
.3 \\
1.0 \\
2.0\end{array}$ & 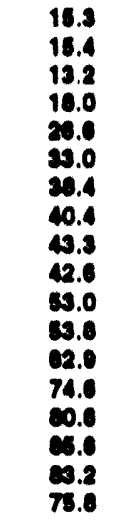 & $\begin{array}{r}0.0 \\
.0 \\
.0 \\
.0 \\
2.7 \\
3.3 \\
6.7 \\
7.0 \\
14.8 \\
10.8 \\
17.4 \\
10.8 \\
10.8 \\
18.8 \\
10.4 \\
10.3 \\
18.0 \\
18.0\end{array}$ & $\begin{array}{r}14.7 \\
14.7 \\
18.3 \\
18.0 \\
17.0 \\
30.0 \\
30.0 \\
101.2 \\
105.2 \\
106.0 \\
144.2 \\
101.2 \\
224.0 \\
254.3 \\
205.8 \\
274.8 \\
302.8 \\
314.1\end{array}$ & $\begin{array}{r}11.0 \\
12.0 \\
21.7 \\
21.8 \\
30.0 \\
38.7 \\
42.2 \\
43.7 \\
83.4 \\
93.4 \\
88.0 \\
92.0 \\
128.6 \\
118.8 \\
130.2 \\
145.2 \\
149.8 \\
147.2\end{array}$ & $\begin{array}{l}2.8 \\
1.0 \\
2.0 \\
3.2 \\
2.0 \\
2.2 \\
9.2 \\
2.0 \\
3.1 \\
2.2 \\
2.0 \\
4.1 \\
4.0 \\
1.1 \\
6.8 \\
1.1 \\
4.0 \\
6.3\end{array}$ \\
\hline 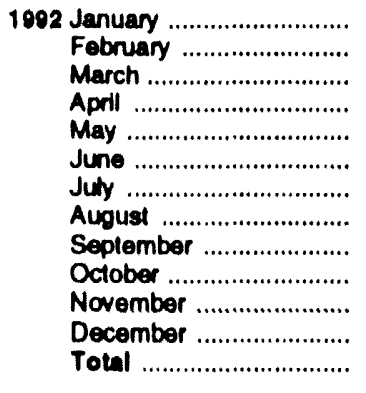 & $\begin{array}{r}.6 \\
.7 \\
.6 \\
.6 \\
.5 \\
.6 \\
.7 \\
.7 \\
.7 \\
.3 \\
.4 \\
\mathbf{E} \\
\mathbf{E} \\
\mathbf{7 . 1}\end{array}$ & $\begin{array}{r}4.3 \\
4.0 \\
4.0 \\
3.4 \\
3.8 \\
3.6 \\
3.1 \\
3.4 \\
3.1 \\
3.6 \\
3.3 \\
3.9 \\
43.8\end{array}$ & $\begin{array}{l}.0 \\
.0 \\
.0 \\
.0 \\
.0 \\
.1 \\
.3 \\
.4 \\
.3 \\
.1 \\
.3 \\
.1 \\
1.0\end{array}$ & $\begin{array}{l}6.9 \\
6.4 \\
7.4 \\
6.4 \\
4.8 \\
5.8 \\
7.2 \\
6.9 \\
6.9 \\
7.2 \\
7.4 \\
8.0 \\
8.4\end{array}$ & $\begin{array}{r}1.8 \\
1.7 \\
1.8 \\
1.7 \\
1.3 \\
1.4 \\
1.8 \\
1.4 \\
1.3 \\
1.6 \\
1.7 \\
1.8 \\
19.0\end{array}$ & $\begin{array}{l}33.5 \\
29.8 \\
30.7 \\
28.0 \\
25.6 \\
22.4 \\
23.7 \\
24.6 \\
25.6 \\
28.5 \\
29.5 \\
33.1 \\
337.6\end{array}$ & $\begin{array}{l}15.6 \\
15.2 \\
15.8 \\
14.1 \\
11.8 \\
11.8 \\
12.0 \\
10.9 \\
11.6 \\
13.2 \\
13.0 \\
13.8 \\
158.8\end{array}$ & $\begin{array}{l}.5 \\
.5 \\
.5 \\
.4 \\
.4 \\
.3 \\
.4 \\
.5 \\
.5 \\
.6 \\
.7 \\
.8 \\
.8\end{array}$ \\
\hline 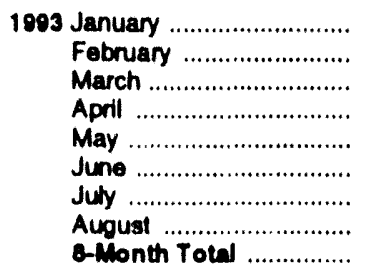 & $\begin{array}{r}.6 \\
.4 \\
.6 \\
.7 \\
.7 \\
E .7 \\
E .7 \\
E .7 \\
\mathrm{E} .3\end{array}$ & $\begin{array}{r}4.3 \\
3.7 \\
3.4 \\
3.3 \\
3.1 \\
3.0 \\
3.2 \\
3.4 \\
27.4\end{array}$ & $\begin{array}{r}.2 \\
.2 \\
(a) \\
.0 \\
.0 \\
.0 \\
.0 \\
.0 \\
.4\end{array}$ & $\begin{array}{r}8.2 \\
7.4 \\
7.8 \\
7.3 \\
6.7 \\
7.1 \\
9.3 \\
9.1 \\
62.0\end{array}$ & $\begin{array}{r}1.8 \\
1.6 \\
1.8 \\
1.7 \\
1.3 \\
1.6 \\
1.8 \\
1.5 \\
13.0\end{array}$ & $\begin{array}{r}36.3 \\
32.7 \\
34.3 \\
30.5 \\
26.9 \\
25.4 \\
26.9 \\
25.9 \\
238.0\end{array}$ & $\begin{array}{r}15.1 \\
13.9 \\
14.2 \\
12.4 \\
11.8 \\
12.0 \\
12.3 \\
11.1 \\
102.8\end{array}$ & $\begin{array}{l}.7 \\
.6 \\
.6 \\
.2 \\
.4 \\
.5 \\
.7 \\
.5 \\
4.3\end{array}$ \\
\hline 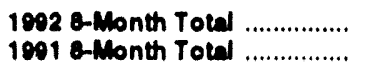 & $\begin{array}{l}8.0 \\
8.3\end{array}$ & $\begin{array}{l}29.6 \\
20.3\end{array}$ & 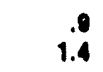 & $\begin{array}{l}81.7 \\
\infty 0.0\end{array}$ & $\begin{array}{l}12.7 \\
12.8\end{array}$ & $\begin{array}{l}218.3 \\
214.5\end{array}$ & $\begin{array}{r}107.2 \\
97.7\end{array}$ & $\begin{array}{l}3.7 \\
3.6\end{array}$ \\
\hline
\end{tabular}

- Through December 1990, the data for Germany are lor the former West Germany only. Beginning with January 1991, the data for Germany are lor the unitied Germany, 1.e., the former East Germany and West Germany.

Ex=Estimale.

Notes: - Net figures are generally lese than grose floures by aboun 5 percent, the difference being the energy consumed by the generating plants themselves. - U.S. geographic coverage is the 50 Stales and the District of Columbia. - Monthly data may not sum to annual totals due to independent rounding and because precommercial generation is included in some annual totals but not in the monthly data.

Source: McGraw-Hill Publishing Company, Nucleonics Woek. 
Table 10.4b Nuclear Electricity Gross Generation: Italy Through Spain (Billion Kilowatthours)

\begin{tabular}{|c|c|c|c|c|c|c|c|c|}
\hline & Maly & Jepan & Menbe & Nothertando & Pokleten & South Alites & south Koren & Epoln \\
\hline 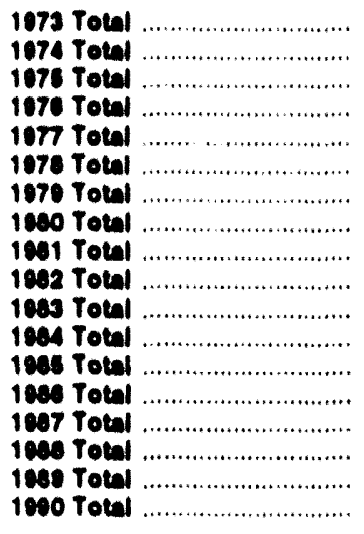 & $\begin{array}{l}3.1 \\
3.4 \\
3.0 \\
3.0 \\
3.4 \\
4.0 \\
2.0 \\
2.2 \\
2.7 \\
6.0 \\
. .0 \\
.0 \\
7.0 \\
.7 \\
2 \\
.0 \\
.0 \\
.0\end{array}$ & 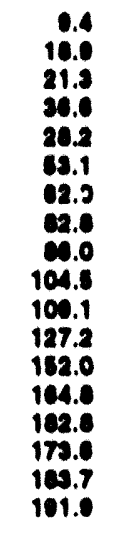 & $\begin{array}{l}0.0 \\
.0 \\
.0 \\
.0 \\
.0 \\
.0 \\
.0 \\
.0 \\
.0 \\
.0 \\
.0 \\
.0 \\
.0 \\
.0 \\
.0 \\
.0 \\
.0 \\
2.1\end{array}$ & 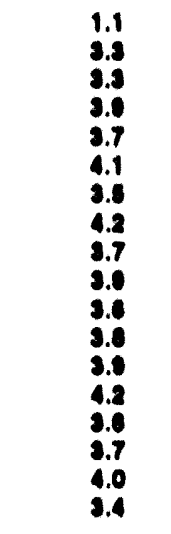 & $\begin{array}{r}0.6 \\
.0 \\
.6 \\
.3 \\
.2 \\
(0) \\
.1 \\
.2 \\
.1 \\
.2 \\
.2 \\
. \\
.0 \\
.3 \\
2 \\
.1 \\
.4\end{array}$ & $\begin{array}{r}0.0 \\
.0 \\
.0 \\
.0 \\
.0 \\
.0 \\
.0 \\
.0 \\
.0 \\
.0 \\
.0 \\
4.2 \\
8.0 \\
1.8 \\
1.0 \\
11.1 \\
11.7 \\
0.0\end{array}$ & $\begin{array}{r}0.0 \\
.0 \\
.0 \\
.0 \\
.1 \\
2.3 \\
3.2 \\
3.8 \\
2.0 \\
3.0 \\
0.0 \\
11.0 \\
11.0 \\
20.1 \\
97.0 \\
24.7 \\
17.2 \\
62.0\end{array}$ & $\begin{array}{r}6.8 \\
7.2 \\
7.8 \\
7.6 \\
6.8 \\
7.6 \\
6.7 \\
6.2 \\
0.4 \\
6.8 \\
10.7 \\
29.1 \\
28.0 \\
97.5 \\
41.2 \\
60.4 \\
6.1 \\
4.3\end{array}$ \\
\hline 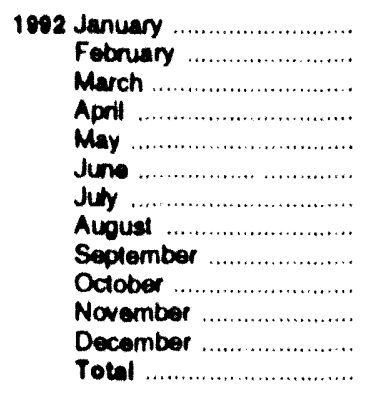 & $\begin{array}{l}.0 \\
.0 \\
.0 \\
.0 \\
0 \\
.0 \\
.0 \\
.0 \\
.0 \\
.0 \\
.0 \\
.0 \\
.0\end{array}$ & $\begin{array}{l}18.5 \\
17.1 \\
17.9 \\
16.0 \\
16.3 \\
17.1 \\
21.1 \\
23.1 \\
17.2 \\
16.2 \\
16.3 \\
19.1 \\
218.0\end{array}$ & $\begin{array}{r}.5 \\
.4 \\
.5 \\
.5 \\
.5 \\
.3 \\
.3 \\
.2 \\
.0 \\
(3) \\
.4 \\
.4 \\
.0\end{array}$ & $\begin{array}{r}.4 \\
.3 \\
.1 \\
.1 \\
.3 \\
.3 \\
.4 \\
.4 \\
.4 \\
.4 \\
.4 \\
.4\end{array}$ & $\begin{array}{l}\text { (a) } \\
.0 \\
(a) \\
(a) \\
(0) \\
.1 \\
.1 \\
.1 \\
.1 \\
.1 \\
.1 \\
.1 \\
.0\end{array}$ & $\begin{array}{r}.0 \\
.4 \\
.4 \\
.4 \\
.7 \\
1.2 \\
1.3 \\
1.0 \\
1.1 \\
1.0 \\
.6 \\
.8 \\
.0\end{array}$ & $\begin{array}{l}4.6 \\
4.0 \\
4.2 \\
1.5 \\
4.5 \\
4.5 \\
5.3 \\
5.4 \\
4.6 \\
4.0 \\
4.7 \\
5.1 \\
4.4\end{array}$ & $\begin{array}{l}5.4 \\
4.6 \\
4.2 \\
3.6 \\
4.3 \\
4.5 \\
5.0 \\
5.2 \\
4.2 \\
5.0 \\
4.4 \\
5.4 \\
6.8\end{array}$ \\
\hline 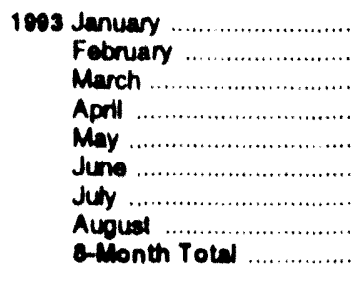 & $\begin{array}{l}0 \\
0 \\
0 \\
0 \\
.0 \\
.0 \\
.0 \\
.0 \\
.0\end{array}$ & $\begin{array}{l}19.5 \\
17.4 \\
18.9 \\
17.6 \\
17.4 \\
17.9 \\
22.3 \\
24.2 \\
188.3\end{array}$ & $\begin{array}{r}.5 \\
.3 \\
.1 \\
.5 \\
.5 \\
.5 \\
.5 \\
.5 \\
.3\end{array}$ & $\begin{array}{r}.4 \\
.3 \\
.1 \\
.1 \\
.4 \\
.4 \\
.4 \\
.4\end{array}$ & $\begin{array}{l}\text { (a) } \\
.1 \\
.1 \\
.1 \\
(8) \\
(8) \\
.1 \\
\text { (s) } \\
.3\end{array}$ & $\begin{array}{r}.6 \\
.6 \\
.5 \\
.6 \\
.8 \\
.5 \\
1.0 \\
.0 \\
.0\end{array}$ & $\begin{array}{r}4.8 \\
4.5 \\
4.6 \\
4.8 \\
5.3 \\
5.1 \\
5.5 \\
4.9 \\
30.0\end{array}$ & $\begin{array}{r}5.4 \\
4.3 \\
4 . \\
4.2 \\
4.1 \\
4.4 \\
5.0 \\
5.1 \\
37.3\end{array}$ \\
\hline 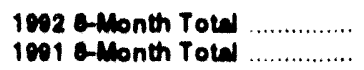 & $\begin{array}{l}.0 \\
.0\end{array}$ & $\begin{array}{l}147.0 \\
130.8\end{array}$ & $\begin{array}{l}3.1 \\
3.8\end{array}$ & $\begin{array}{l}2.3 \\
2.4\end{array}$ & $\begin{array}{l}.3 \\
.3\end{array}$ & $\begin{array}{l}6.4 \\
5.0\end{array}$ & $\begin{array}{l}37.1 \\
36.7\end{array}$ & $\begin{array}{l}34.7 \\
37.4\end{array}$ \\
\hline
\end{tabular}

(s) = Leas than 0.05 billon Wlowatthours.

Noles: - Nel ligures are generally lases than orose ligures by abou 5 percent, the difference being the eneroy consumed by the generaling plante themsetves. US. geographic coverage is the 50 sistes and the Distict of
Columbia. - Monthy dafa may not sum to annual lotals due to independer rounding and becuuse precommencial generation b included in some anrual tolals but not in the monthy data.

Source: McGraw-Hill Publishing Company, Nucleonica Woek. 
Table 10.4c Nuclear Electricity Gross Generation: Sweden Through United States and Total

(Billion Kilowatthours)

\begin{tabular}{|c|c|c|c|c|c|c|c|}
\hline & Imoden & swleerlend & Takwan & $\begin{array}{l}\text { Untted } \\
\text { Kingedem" }\end{array}$ & $\begin{array}{l}\text { Total' } \\
\text { Ixeluding U.8. }\end{array}$ & $\begin{array}{l}\text { United } \\
\text { sentes }\end{array}$ & Tolal' \\
\hline 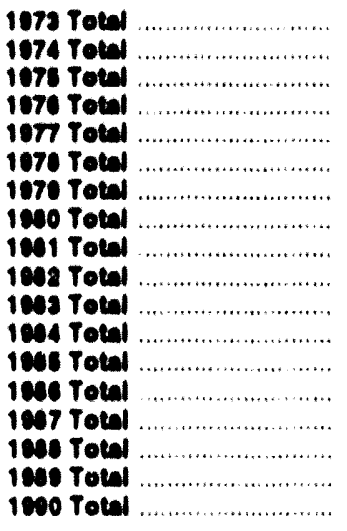 & 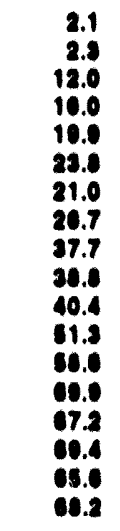 & 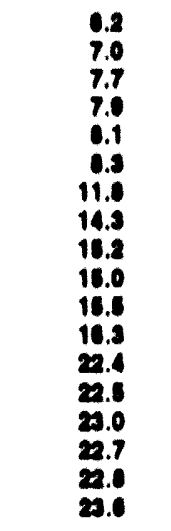 & $\begin{array}{r}0.0 \\
.0 \\
.0 \\
.0 \\
.1 \\
2.7 \\
0.3 \\
0.2 \\
10.7 \\
13.1 \\
10.0 \\
34.3 \\
28.7 \\
28.0 \\
33.1 \\
29.0 \\
20.3 \\
22.0\end{array}$ & $\begin{array}{l}20.2 \\
39.0 \\
30.0 \\
30.0 \\
30.1 \\
30.0 \\
30.8 \\
37.2 \\
30.0 \\
44.1 \\
40.0 \\
64.1 \\
30.7 \\
60.2 \\
60.2 \\
30.4 \\
71.6 \\
60.1\end{array}$ & 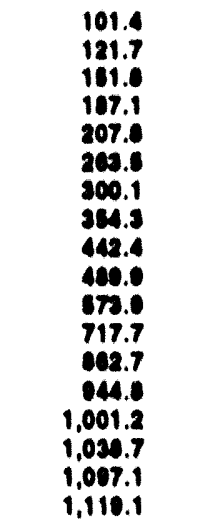 & $\begin{array}{r}17.0 \\
124.2 \\
102.9 \\
201.4 \\
214.2 \\
202.4 \\
270.4 \\
201.4 \\
208.8 \\
204.8 \\
313.4 \\
343.8 \\
402.7 \\
434.1 \\
470.8 \\
384.1 \\
587.0 \\
603.4\end{array}$ & 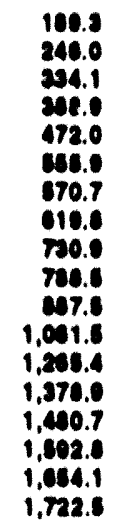 \\
\hline 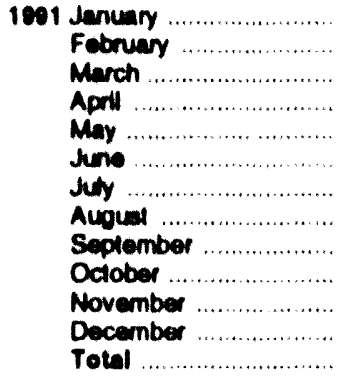 & $\begin{array}{l}7.6 \\
6.9 \\
7.6 \\
6.9 \\
5.7 \\
4.7 \\
1.6 \\
5.2 \\
5.5 \\
7.2 \\
7.3 \\
7.6 \\
70.0\end{array}$ & $\begin{array}{r}2.3 \\
2.1 \\
2.3 \\
2.2 \\
2.0 \\
1.1 \\
1.5 \\
1.0 \\
1.8 \\
2.3 \\
2.2 \\
2.3 \\
22.8\end{array}$ & $\begin{array}{r}2.4 \\
2.2 \\
2.9 \\
2.5 \\
2.8 \\
3.2 \\
3.2 \\
3.6 \\
3.1 \\
3.1 \\
3.0 \\
3.2 \\
38.3\end{array}$ & $\begin{array}{l}6.6 \\
6.8 \\
6.7 \\
5.0 \\
1.5 \\
6.1 \\
5.1 \\
5.4 \\
6.6 \\
5.9 \\
5.2 \\
6.6 \\
70.4\end{array}$ & $\begin{aligned} 111.2 \\
101.1 \\
100.3 \\
80.6 \\
87.3 \\
87.0 \\
86.4 \\
80.6 \\
86.3 \\
101.2 \\
101.7 \\
110.5 \\
1.182 .2\end{aligned}$ & $\begin{array}{l}56.6 \\
50.2 \\
51.6 \\
43.8 \\
49.2 \\
56.0 \\
63.7 \\
61.4 \\
54.4 \\
50.2 \\
48.7 \\
56.3 \\
543.0\end{array}$ & $\begin{array}{l}167.8 \\
151.3 \\
154.0 \\
133.4 \\
136.6 \\
143.9 \\
159.1 \\
160.0 \\
140.7 \\
151 . \\
150.4 \\
166.8 \\
1.025 .2\end{array}$ \\
\hline 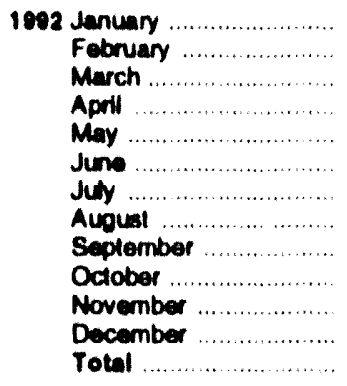 & $\begin{array}{l}7.6 \\
6.8 \\
7.1 \\
6.7 \\
4.7 \\
3.9 \\
3.6 \\
3.5 \\
3.0 \\
5.2 \\
5.2 \\
5.4 \\
03.6\end{array}$ & $\begin{array}{l}2.3 \\
2.1 \\
2.2 \\
1.9 \\
1.9 \\
1.3 \\
1.7 \\
1.1 \\
2.0 \\
2.3 \\
2.2 \\
2.3 \\
23.4\end{array}$ & $\begin{array}{l}3.1 \\
2.2 \\
2.2 \\
2.6 \\
2.6 \\
2.9 \\
3.3 \\
3.6 \\
2.8 \\
2.9 \\
3.2 \\
2.6 \\
33.8\end{array}$ & $\begin{array}{r}6.5 \\
6.3 \\
8.3 \\
5.0 \\
6.0 \\
7.0 \\
4.9 \\
5.5 \\
6.9 \\
5.7 \\
6.1 \\
10.4 \\
70.8\end{array}$ & $\begin{array}{r}113.1 \\
102.8 \\
107.8 \\
95.8 \\
90.1 \\
88.9 \\
96.0 \\
97.9 \\
93.2 \\
98.8 \\
99.9 \\
114.1 \\
1,206.0\end{array}$ & $\begin{array}{l}60.6 \\
55.4 \\
48.3 \\
44.3 \\
48.1 \\
53.7 \\
50.0 \\
61.6 \\
53.2 \\
51.5 \\
53.2 \\
61.0 \\
650.0\end{array}$ & $\begin{array}{r}173.7 \\
158.1 \\
156.1 \\
140.2 \\
138.2 \\
142.7 \\
155.0 \\
159.5 \\
146.4 \\
150.3 \\
153.1 \\
6175.1 \\
61,060.0\end{array}$ \\
\hline 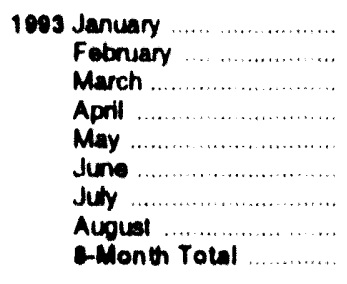 & $\begin{array}{r}5.8 \\
5.8 \\
7.1 \\
6.6 \\
4.6 \\
4.7 \\
3.1 \\
3.2 \\
41.1\end{array}$ & $\begin{array}{r}2.3 \\
2.1 \\
2.3 \\
2.0 \\
1.9 \\
1.2 \\
1.8 \\
1.1 \\
14.7\end{array}$ & $\begin{array}{r}3.0 \\
2.7 \\
2.8 \\
2.8 \\
2.7 \\
2.6 \\
3.4 \\
3.6 \\
23.5\end{array}$ & $\begin{array}{l}7.6 \\
7.9 \\
8.3 \\
7.7 \\
6.0 \\
8.1 \\
6.3 \\
6.3 \\
6.1 \\
80.0\end{array}$ & $\begin{array}{l}117.0 \\
108.0 \\
112.3 \\
103.2 \\
91.6 \\
806.4 \\
104.1 \\
102.3 \\
1836.1\end{array}$ & $\begin{array}{l}61.8 \\
53.7 \\
49.8 \\
45.4 \\
52.7 \\
55.4 \\
58.9 \\
58.9 \\
436.7\end{array}$ & $\begin{array}{r}178.9 \\
160.6 \\
162.1 \\
148.7 \\
147.3 \\
E 150.8 \\
E 163.1 \\
161.2 \\
E 1.272 .8\end{array}$ \\
\hline $\begin{array}{l}1902 \text { E-Month Total } \\
1001 \text { E-Month Total }\end{array}$ & $\begin{array}{l}43.0 \\
40.1\end{array}$ & $\begin{array}{l}14.6 \\
14.3\end{array}$ & $\begin{array}{l}22.4 \\
22.0\end{array}$ & $\begin{array}{l}40.5 \\
46.2\end{array}$ & $\begin{array}{l}702.3 \\
773.8\end{array}$ & $\begin{array}{l}431.1 \\
433.4\end{array}$ & $\begin{array}{l}1,223.3 \\
1,206.0\end{array}$ \\
\hline
\end{tabular}

- Monthly data for the United Kingdom are totals for 4 or 5-weok reponting periods, nol calendar monitis.

- Tolar equals nuclear-generated edectricity from al courtries except Bulgaria. China, Cuba, the former Czechoslovakia. Hungary. Nonth Korea, Poland, Romania, the former U.S.S R., and Slovenia (pant of the former Yugosiavia).

Ex Esimalo.

Notes: - Nex figures are generally less than grose ligures by about 5

percent, the dilference being the exergy consumed by the generaling plants themeotve. U.S. geopraptic coverage is the 50 Stales and the Dielrict of Columbia. Monthy dala may not sum to arniual totals due to independent rounding and because precommercial generation is included in some unnual lotale but nol in the monthly dala. - Dala for countries may nol sum to world totals due to independent rounding.

Source: McGraw.HIII Publishing Company, Nucleonics Woek 


\section{Sources for Tables 10.1a and $10.1 \mathrm{~b}$}

- United States: Table 3.1a.

- Other Countriest Annual Data: 1973-1979-liner. gy Information Administration (EIA), International Energy Annual 1981, Table 8. 1980-EIA. Internation. al Energy Annual 1989. Table 1. 1981-IIIA. International Energy Annual 1990. Table 1. 1982. 1991-EIA. International Energy Annual 1991. Table 1.
1992-Average of monthly data. Monthly Data: Pesroleum Intelligence Weekly, the Oil and Gas Journal. and other industry sources.

- World: Annual Datu: 1973-1979-EIA, Insema. tional Energy Annual 1981. Table 8. 1980-EIA, Intemational Energy Annual 1989. Table 1. 1981.liı. International Energy Annual 1990. Table 1. 1982.1991-BIA. International Energy Annual 1991, Table 1. 1992-Average of monthly data. Monthly Duta-EIA. International Petroleum Statistics Report. sum of all countrics' monthly data. 


\section{Appendix A. Thermal Conversion Factors}

The thermal conversion factors presented in the following eight tables can be used to estimate the heat content in British thermal units (Btu) of a given anount of energy measured in physical units, such as barrels or cuble feet. For example, 10 barrels of asphalt have a heat content of approximately 66.36 million Btu (10 harrels $\times 6.636$ million Btu/barrel $=$ 66.36 million B(u).

Thermal conversion factors for hydrocarbon mixes (Table A1) are weighted averages of the thermal conversion factors for each hydrocarbon included in the mix. For example. in calculating the thermal conversion factor for a $60-40$ butane-propane mixture, the thermal conversion factor for butane is weighted 1.5 times more heavily than the thermal conversion factor for propane.

In general, the annual thermal conversion factors presented in Tables $A 1$ through $A 8$ are computed from final annual data. However, if the current year's final data are not available in time for publication, thermal conversion factors for the currelli year are computed from the best available data and are laheled "preliminary." The source of each faclor is described in the section entitled "Thermal Conversion Factor Source Documentation," which follows Table $A 8$ in this appendix.

\section{Table A1. Approximate Heat Content of Petroleum Products}

(Million Blu per Barrel)

\begin{tabular}{|c|c|c|c|}
\hline Petroleum Produet & Heat Content & Petroleum Product & Hout Content \\
\hline Apphal .................... & 6.038 & Pelrochemical Feedistocks & \\
\hline 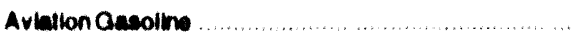 & 5.048 & Nephtha Lese Than $\mathbf{4 0 1 ^ { \circ }} \mathrm{F} \ldots \ldots \ldots \ldots$ & 5240 \\
\hline 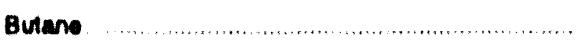 & 4.326 & Other Oth Equal to or Oreater $\operatorname{Than} 401^{\circ} \mathrm{F} .$. & 5.825 \\
\hline Butane-Propene Mixturn"... & 4.130 & sin Cas & 6000 \\
\hline Dwtilate fuet on & 5.825 & Petrobum Coke .................. & 0.024 \\
\hline 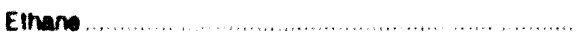 & 3082 & Plant Condenale. & 5418 \\
\hline Elnenepropane Moture & 3300 & 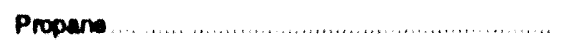 & $3 \mathbf{2 3 6}$ \\
\hline 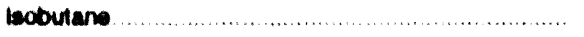 & 3074 & Peskdual Fued ON $\ldots \ldots \ldots \ldots \ldots \ldots \ldots$ & 6287 \\
\hline Jet Fuel, Kerosene Type .................. & 5670 & Road on $\ldots \ldots$ & 6.630 \\
\hline Jel Fuel, Nephtha Typo .......... & 5.355 & Spectal Naphthas. & 5248 \\
\hline Kerosene $\ldots \ldots \ldots \ldots \ldots \ldots$ & 5670 & Sill Oas $\ldots \ldots \ldots \ldots$ & 6000 \\
\hline 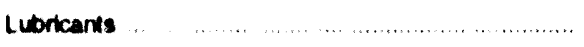 & 6065 & Untinimed Ols $\ldots . . . . . . . . .$. & 5825 \\
\hline 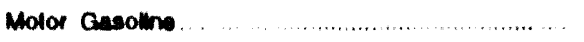 & 5.253 & Unitradionated Stream & 5418 \\
\hline Nalural Gasoline and teopentars ....................... & 4.620 & 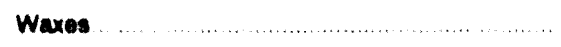 & 5537 \\
\hline Pentanes Plus & 4620 & Mtacellaneous. & 5796 \\
\hline
\end{tabular}

- 60 percenl butane and 40 percent propan

70 percent athano and 30 percent propane

Source See Thermal Corversion Factor Source Documentation." which lollows Table AB 
Tablo A2. Approximate Hoat Contont of Crudo Oll, Crude Oll and Producte, and Nafural Cas Plant Llquids

(Million Blu per Barrol)

\begin{tabular}{|c|c|c|c|c|c|c|}
\hline & \multicolumn{3}{|c|}{ Cructs on } & \multicolumn{2}{|c|}{ Cruts Oll and Producte } & \multirow{2}{*}{$\begin{array}{c}\text { Nefurd en } \\
\text { Plent } \\
\text { Lleplits }\end{array}$} \\
\hline & Produaton & Impente & Exponst & himone & Enporte & \\
\hline 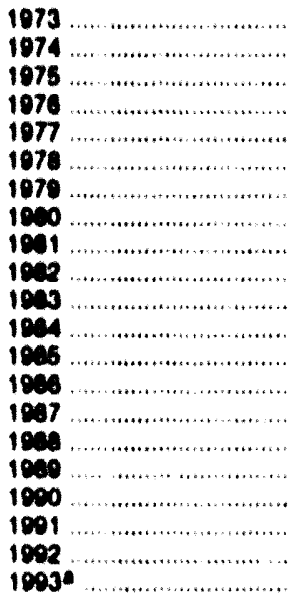 & 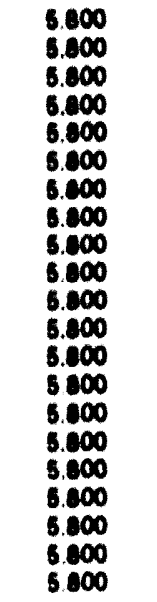 & 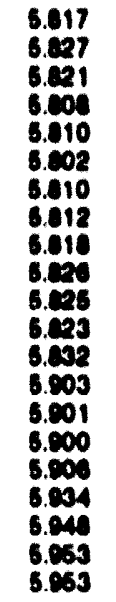 & 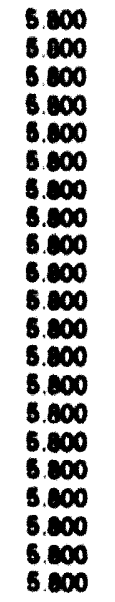 & 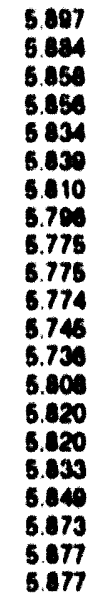 & $\begin{array}{l}5.752 \\
6.774 \\
5.746 \\
5.745 \\
5.707 \\
6.009 \\
5.002 \\
6.020 \\
6.421 \\
5.020 \\
5.000 \\
5.850 \\
5.814 \\
5.032 \\
5.858 \\
5.840 \\
5.857 \\
5.033 \\
5.023 \\
5.711 \\
5.717\end{array}$ & $\begin{array}{l}4.040 \\
4.011 \\
3.084 \\
3.804 \\
3.041 \\
3.025 \\
3.054 \\
3.014 \\
3.000 \\
3.012 \\
3.030 \\
3.812 \\
3.815 \\
3.707 \\
3.004 \\
3.000 \\
3.020 \\
3.022 \\
3.007 \\
3.004 \\
3.004\end{array}$ \\
\hline
\end{tabular}

- Preliminary.

Nole: Crude of inchdes toese condenate.

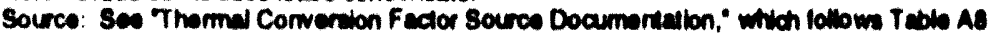

Table A3. Approximate Heat Content of Petroleum Product Wolghted Averages (Million Btu per Barrel)

\begin{tabular}{|c|c|c|c|c|c|c|c|c|}
\hline & & & Conumpilon & & & & & \\
\hline & $\begin{array}{l}\text { Rooldentil } \\
\text { end } \\
\text { Commorolet }\end{array}$ & Induetriel & Trmpopenten & $\begin{array}{l}\text { Eloctris } \\
\text { Elunbe }\end{array}$ & Totel & Importe & Expons & $\begin{array}{c}\text { LPO } \\
\text { Consumption }\end{array}$ \\
\hline 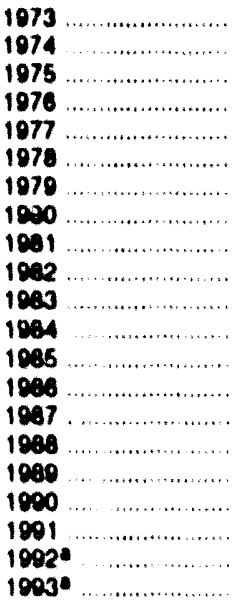 & $\begin{array}{l}5.397 \\
6.377 \\
5.368 \\
5.393 \\
5.389 \\
5.342 \\
5.471 \\
5.498 \\
5.400 \\
5.392 \\
5.286 \\
5.394 \\
5.328 \\
5.357 \\
5.318 \\
5.323 \\
5.280 \\
5.212 \\
5.163 \\
5.158 \\
5.158\end{array}$ & 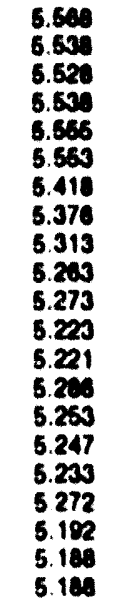 & $\begin{array}{l}5.305 \\
5.304 \\
5.392 \\
5.306 \\
6.400 \\
5.404 \\
5.420 \\
5.440 \\
6.432 \\
6.492 \\
5.416 \\
5.422 \\
5.423 \\
5.427 \\
5.400 \\
5.434 \\
5.440 \\
5.446 \\
5.442 \\
5.444 \\
5.444\end{array}$ & 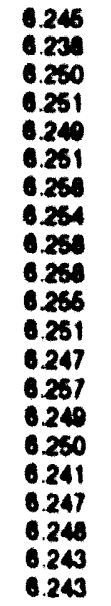 & $\begin{array}{l}5.515 \\
5.504 \\
6.404 \\
5.504 \\
5.518 \\
5.510 \\
5.494 \\
5.470 \\
5.440 \\
5.415 \\
5.408 \\
5.306 \\
5.397 \\
5.418 \\
5.403 \\
5.410 \\
5.410 \\
5.411 \\
5.394 \\
5.376 \\
5.376\end{array}$ & $\begin{array}{l}5.003 \\
5.059 \\
5.035 \\
6.090 \\
5.909 \\
5.055 \\
5.811 \\
5.749 \\
5.050 \\
5.694 \\
5.677 \\
5.613 \\
5.572 \\
5.624 \\
5.590 \\
5.618 \\
5.641 \\
5.614 \\
5.636 \\
5.623 \\
5.623\end{array}$ & $\begin{array}{l}5.752 \\
5.773 \\
5.747 \\
5.743 \\
5.796 \\
5.814 \\
5.844 \\
5.841 \\
5.837 \\
5.820 \\
5.800 \\
5.867 \\
5.810 \\
5.830 \\
5.860 \\
5.842 \\
5.860 \\
5.238 \\
5.827 \\
5.774 \\
5.114\end{array}$ & $\begin{array}{l}3740 \\
3.730 \\
3.715 \\
3.711 \\
3.677 \\
3.680 \\
3.680 \\
3.674 \\
3.643 \\
3.615 \\
3.614 \\
3.590 \\
3.603 \\
3.640 \\
3.650 \\
3.652 \\
3.683 \\
3.625 \\
3.614 \\
3.624 \\
3.624\end{array}$ \\
\hline
\end{tabular}

- Preliminary.

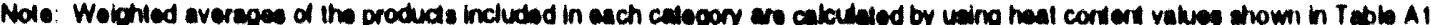

Sounce: See Thermal Corversion Factor Source Documentation, "which lotowe Teble $\mathrm{As}$. 
Table A4. Approximate Hoat Content of Natural Gas (Btu por Cuble Fool)

\begin{tabular}{|c|c|c|c|c|c|c|c|}
\hline & \multicolumn{2}{|c|}{ Productan } & \multicolumn{3}{|c|}{ Consumpilen } & \multirow[b]{2}{*}{ Import } & \multirow[b]{2}{*}{ Exporte } \\
\hline & Dry & (Nile) & Chnom & Wintiles & Tetel & & \\
\hline 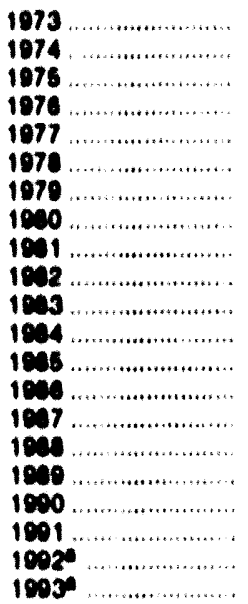 & $\begin{array}{l}1,021 \\
1,024 \\
1,021 \\
1,020 \\
1,021 \\
1,010 \\
1,021 \\
1,021 \\
1,027 \\
1,020 \\
1,031 \\
1,031 \\
1,020 \\
1,030 \\
1,031 \\
1,020 \\
1,031 \\
1,031 \\
1,030 \\
1,030 \\
1,030\end{array}$ & 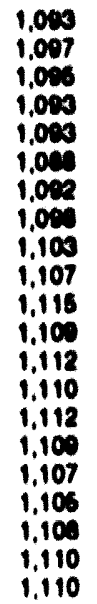 & 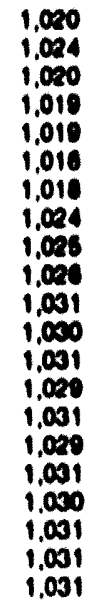 & 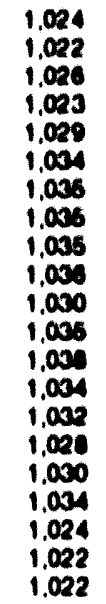 & $\begin{array}{l}1,021 \\
1,024 \\
1,021 \\
1,020 \\
1,021 \\
1,010 \\
1,021 \\
1,020 \\
1,021 \\
1,020 \\
1,031 \\
1,001 \\
1,020 \\
1,000 \\
1,031 \\
1,000 \\
1,031 \\
1,031 \\
1,000 \\
1,030 \\
1,030\end{array}$ & $\begin{array}{l}1,020 \\
1,021 \\
1,020 \\
1,025 \\
1,028 \\
1,030 \\
1,031 \\
1,022 \\
1,014 \\
1,018 \\
1,024 \\
1,005 \\
1,002 \\
001 \\
000 \\
1.002 \\
1,004 \\
1.012 \\
1.014 \\
1,011 \\
1,011\end{array}$ & $\begin{array}{l}1,023 \\
1,018 \\
1,014 \\
1,013 \\
1,013 \\
1,013 \\
1,013 \\
1,013 \\
1,011 \\
1,011 \\
1,010 \\
1,010 \\
1,011 \\
1,009 \\
1,011 \\
1,010 \\
1.010 \\
1,018 \\
1,022 \\
1,018 \\
1,018\end{array}$ \\
\hline
\end{tabular}

- Proliminery.

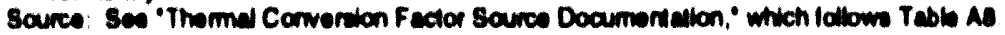

Table A5. Approximate Heat Content of Coal (Million Btu per Short Ton)

\begin{tabular}{|c|c|c|c|c|c|c|c|c|}
\hline & \multirow[b]{2}{*}{ Production } & \multicolumn{5}{|c|}{ Convumption } & \multirow[b]{2}{*}{ Importe } & \multirow[b]{2}{*}{ Exponte } \\
\hline & & Cond & $\begin{array}{l}\text { Cono } \\
\text { Pinnin }\end{array}$ & Onduer & $\begin{array}{l}\text { Eleotris } \\
\text { Uallileoto }\end{array}$ & Totel & & \\
\hline 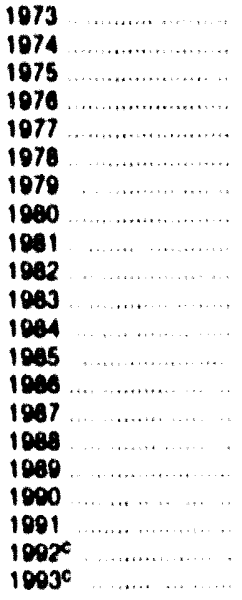 & $\begin{array}{l}23.376 \\
23.072 \\
22.007 \\
22.055 \\
22.507 \\
22.248 \\
22.454 \\
22.415 \\
22.309 \\
22230 \\
22.052 \\
22.010 \\
21.070 \\
21.013 \\
21.022 \\
21.023 \\
21.765 \\
21.022 \\
21.681 \\
21.675 \\
21675\end{array}$ & $\begin{array}{l}22.031 \\
22.470 \\
22.281 \\
22.774 \\
22.010 \\
22.493 \\
22.242 \\
22.543 \\
22.474 \\
22.005 \\
22775 \\
22.844 \\
22.049 \\
22.047 \\
23.04 \\
23.571 \\
23.650 \\
23.137 \\
23114 \\
23197 \\
23.197\end{array}$ & 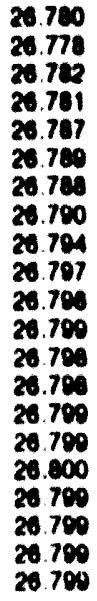 & $\begin{array}{l}22.598 \\
22.410 \\
22.436 \\
22.530 \\
22.322 \\
22.207 \\
22.452 \\
22.690 \\
22.595 \\
22.712 \\
22.691 \\
22.543 \\
22.020 \\
22.108 \\
22.391 \\
22.390 \\
22.347 \\
22.457 \\
22.490 \\
22.313 \\
22.313\end{array}$ & $\begin{array}{l}22.246 \\
21.781 \\
21.042 \\
21.670 \\
21.508 \\
21.275 \\
21.384 \\
21.205 \\
21.085 \\
21.104 \\
21.133 \\
21.101 \\
20.050 \\
21.084 \\
21.136 \\
20.000 \\
20848 \\
20.020 \\
20.755 \\
20804 \\
20.804\end{array}$ & $\begin{array}{l}23.057 \\
22.677 \\
22.500 \\
22.489 \\
22.265 \\
22.017 \\
22.100 \\
21.947 \\
21.713 \\
21.074 \\
21.576 \\
21.573 \\
21.366 \\
21.462 \\
21.517 \\
21.328 \\
21.272 \\
21.331 \\
21.146 \\
21.164 \\
21.164\end{array}$ & $\begin{array}{l}25.000 \\
25.000 \\
25.000 \\
25.000 \\
25.000 \\
25.000 \\
25.000 \\
25.000 \\
25.000 \\
25.000 \\
25.000 \\
25.000 \\
25.000 \\
25.000 \\
25.000 \\
25.000 \\
25000 \\
25.000 \\
25000 \\
25.000 \\
25000\end{array}$ & $\begin{array}{l}20.506 \\
26.700 \\
26.592 \\
26.601 \\
26.549 \\
26.478 \\
26.548 \\
26.344 \\
26.160 \\
26.223 \\
26.201 \\
26.402 \\
26307 \\
26.202 \\
26.291 \\
26290 \\
28.160 \\
26.202 \\
26.188 \\
26.162 \\
26.162\end{array}$ \\
\hline
\end{tabular}

- Inchudes lraneponalion

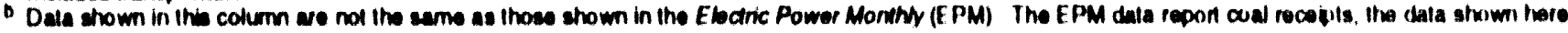
represent coll consumplion

- Preliminary

Sourca: See "Themal Conversion F actor Sounce Documentation," which lollow Table A8 
Table A6. Approximate Heat Content of Bltuminous Coal and Lignite (Million Blu por Shon Ton)

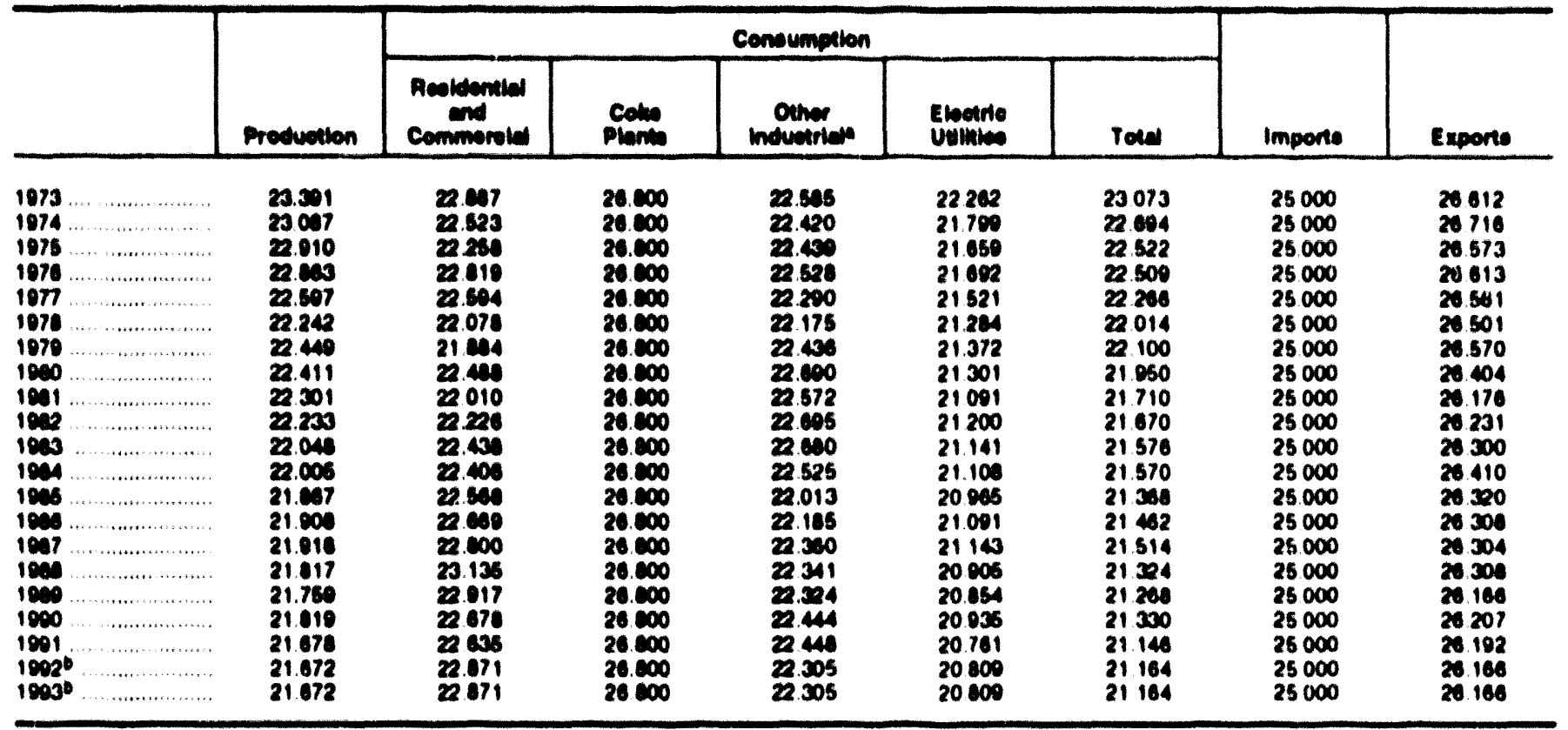

- Inchucise iranaporation.

- Proliminary

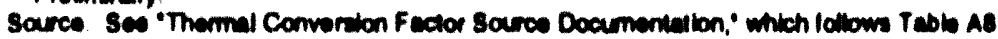

Table A7. Approximate Heat Content of Anthracite and Coal Coke (Million Btu per Shon Ton)

\begin{tabular}{|c|c|c|c|c|c|c|}
\hline & \multirow[b]{3}{*}{ Production } & \multicolumn{4}{|c|}{ Anthresile } & \multirow{3}{*}{$\begin{array}{l}\text { Cenl Cols } \\
\text { Imports } \\
\text { end } \\
\text { Exports }\end{array}$} \\
\hline & & \multicolumn{3}{|c|}{ Conounption } & \multirow{2}{*}{$\begin{array}{l}\text { Inports } \\
\text { end } \\
\text { Export }\end{array}$} & \\
\hline & & Vituibe & Eloctrte Uulinbe & Total & & \\
\hline 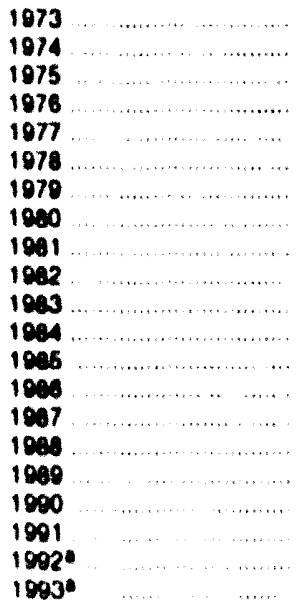 & $\begin{array}{l}22.132 \\
21711 \\
21582 \\
22.045 \\
22.681 \\
23.070 \\
23170 \\
22809 \\
23.201 \\
23.289 \\
22.734 \\
23.107 \\
22.420 \\
23.084 \\
23.100 \\
23.265 \\
23.395 \\
22.574 \\
22.573 \\
22571 \\
22.571\end{array}$ & $\begin{array}{l}22.674 \\
22.300 \\
22.272 \\
22.818 \\
24.101 \\
24.398 \\
24.272 \\
22.710 \\
23.710 \\
24.578 \\
24.539 \\
25.120 \\
23.031 \\
24.300 \\
26.203 \\
26.021 \\
27.198 \\
25.100 \\
25.268 \\
24.680 \\
24660\end{array}$ & $\begin{array}{l}17.020 \\
17.200 \\
17.084 \\
17.520 \\
17.244 \\
17.104 \\
17.464 \\
17.052 \\
18.168 \\
18.160 \\
16.518 \\
17.018 \\
16.704 \\
16.570 \\
15.092 \\
17.312 \\
16.310 \\
16.140 \\
15.058 \\
16.808 \\
16.898\end{array}$ & $\begin{array}{l}21.464 \\
20.910 \\
20.762 \\
21.254 \\
22.066 \\
22.398 \\
22.069 \\
21.405 \\
22.080 \\
22518 \\
21.583 \\
22.322 \\
20.817 \\
21.512 \\
22.435 \\
22.423 \\
22.623 \\
21.668 \\
21.410 \\
21.278 \\
21278\end{array}$ & $\begin{array}{l}25.400 \\
45.400 \\
25.400 \\
25.400 \\
25.400 \\
25.400 \\
25.400 \\
25.400 \\
25.400 \\
25.400 \\
25.400 \\
25.400 \\
25.400 \\
25.400 \\
25.400 \\
25.400 \\
25.400 \\
25.400 \\
25.400 \\
25.400 \\
25.400\end{array}$ & $\begin{array}{l}24.800 \\
24.800 \\
24.800 \\
24.800 \\
24.800 \\
24.800 \\
24.800 \\
24.800 \\
24.800 \\
24.800 \\
24.800 \\
24.000 \\
24.000 \\
24.800 \\
24.800 \\
24.800 \\
24.800 \\
24.800 \\
24.800 \\
24.000 \\
24.800\end{array}$ \\
\hline
\end{tabular}

- Proliminary

Source See "Thermy Conversion F ector Source Documentalion," which lollow Table 48 
Tablo A8. Approximate Hoat Rates for Eloctriclty

(Blu per Kilowatthour)

\begin{tabular}{|c|c|c|c|c|}
\hline & \multicolumn{3}{|c|}{ Eleatriatly Cenerdion } & \multirow[b]{2}{*}{$\begin{array}{l}\text { Electirtoly } \\
\text { Consumption }\end{array}$} \\
\hline & 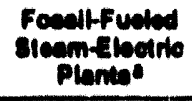 & 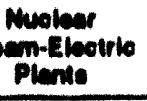 & $\begin{array}{l}\text { Coothormel } \\
\text { Enoroy } \\
\text { Piente }\end{array}$ & \\
\hline 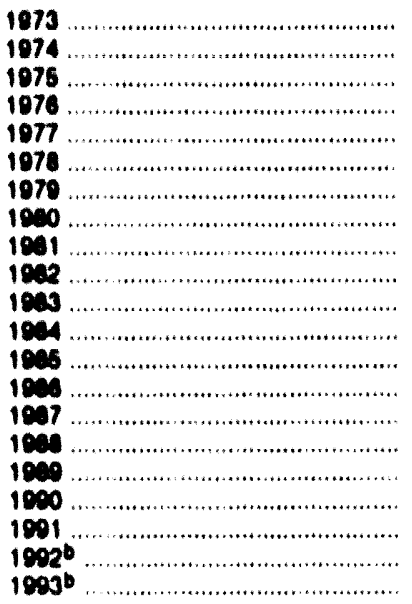 & $\begin{array}{l}10,309 \\
10,440 \\
10,408 \\
10,373 \\
10,495 \\
10,391 \\
10,349 \\
10,389 \\
10,460 \\
10,484 \\
10,520 \\
10,440 \\
10,447 \\
10,440 \\
10,410 \\
10,324 \\
10,317 \\
10,335 \\
10,352 \\
10,352 \\
10,352\end{array}$ & 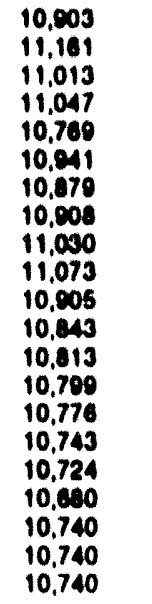 & $\begin{array}{l}21,674 \\
21,674 \\
21,611 \\
21,611 \\
21,611 \\
21,611 \\
21,545 \\
21,630 \\
21,630 \\
21,620 \\
21,290 \\
21,303 \\
21,263 \\
21,263 \\
21,263 \\
21,008 \\
21,096 \\
21,008 \\
20,097 \\
20,997 \\
20,007\end{array}$ & $\begin{array}{l}3,412 \\
3,412 \\
3,412 \\
3,412 \\
3,412 \\
3,412 \\
3,412 \\
3,412 \\
3,412 \\
3,412 \\
3,412 \\
3,412 \\
3,412 \\
3,412 \\
3,412 \\
3,412 \\
3,412 \\
3,412 \\
3,412 \\
3,412 \\
3,412\end{array}$ \\
\hline
\end{tabular}

- This thamel corwerion taclor b used lor hydrosbcirlc power coneration and lor mood and waste, whd, pholovoltaic, and solar thermal energy conaumed at olectic viliniss.

- Preliminey.

Source: See "Thermal Comurion Fector Sounce Documentation," which lollows this lable.

\section{Thermal Conversion Factor Source Documentation}

\section{Approximate Heat Content of Petroloum and Natural Gas Plant Liquids}

Asphalt. The Energy Information Administration (EIA) adopted the thermal conversion factor of 6.636 million British thermal units (Btu) per barrel as estimated by the Bureau of Mines and first published in the Petroleum Statement. Annual. 1956.

Aviation Gasoline. EIA adopted the Bureau of Mines therrnal conversion factor of 5.048 million Btu per harrel as published for "Gasoline, Aviation" by the Texas Eastern Transmission Corporation in Appendix $\checkmark$ of Competition and Grow'th in American Energy Markets 1947.1985, a 1968 release of historical and projected statistics.

Hutune. I:IA adopted the Bureau of Mines thermal conversion factor of 4.326 million Btu per barrel as published in the California Oil World and Petroleum Industry, First Issuc, April 1942.

Hutune-Propane Mixture. EIA adopted the Bureau of Mines calculation of 4.130 million Biu per barrel based on an assumed mixture of 60 percent butane and 40 percent propane. Sec Butune and Propane.

Crude Oil, Exports. Assurned hy lilA to he 5.800 million Betu per harrel or equal to the thermal conversion factor for crude oil produced in the United States. Sec Crude Oil and lease Condensate, Production.

Crude Oil, Imports. Calculated annually hy EIA by weighting the thermal conversion factor of each type of crude oil imported by the quantity imported. Thermal conversion factors for each lype were calculated on a foreign country basis, by determining the average American Petroleum Institute (API) gravity of crude imported from each foreign country from form ERA-60 in 1977 and converting average API gravity to average Btu content hy using Netional Bureau of Standards, Miscellaneous Publication No. 97. Thermal Properties of Petroleum Products. 1933.

Crude Oil and Lease Condensute, Production. BIA adopted the thermal conversion factor of 5,800 million Blu per barrel as reported in a Bureau of Mines internal memorandum, "Bureau of Mines Standard Average Ileating Values of Various liucls, adopted January 3, 19.50."

Crude OII and Petroleum Products, Exports. Calculated annually by Iil $A$ as the average of the thermal conversion factors for each petroleum product 
exported and crude oil exported weighted by the quantity of each petroleum product and crude oil exported. Sce Crude OII, Exports and Petroleum Products, Exports.

Crude Oll and Petroleum Products, Imports. Calculated annually by EIA as the average of the thermal conversion factors for each petroleum product and each type of crude oil imported weighted by the quantity of each petroleum product and each type of crude oil imported. Sce Crude OII, Imports and Petroleum Products, Imports.

Distillate Fuel OII. EIA adopted the Burcau of Mines thermal conversion factor of 5.825 million Biu per harrel as reported in a Bureau of Mines internal memorandum. "Bureau of Mines Standard Average Heating Value of Various Fuels, adopted January 3 , 1950."

Ethane. EIA adopted the Bureau of Mines thermal conversion factor of 3.082 million Biu per barrel as published in the California Oil World and Petroleum Indusiry, First Issue, April 1942.

Ethane-Propane Mixture. ElA calculated 3.308 million Btu per barrel based on an assumed mixture of 70 percent ethane, and 30 percent propane. See Ethane and Propane.

Isobutane. ElA adopted the Bureau of Mines thermal conversion factor of 3.974 million Btu per barrel as published in the California Oil World and Petroleum Industry. First Issue, April 1942.

Jet Fuel, Kerosene Type. EIA adopted the Bureau of Mines thermal conversion factor of 5.670 million Btu per barrel as published for "Jet Fuel, Commercial" by the Texas Eastern Transmission Corporation in Appendix $\mathrm{V}$ of Competition and Growth in American Energy Markets 1947.1985, a 1968 release of historical and projected statistics.

Jet Fuel, Naphtha Type. ElA adopted the Bureau of Mines thermal conversion factor of 5.355 million Btu per barrel as published for "Jet Fuel, Military" by the Texas Eastern Transmission Corporation in Appendix $\checkmark$ of Competition and Growth in American Energy Markets 1947.1985, a 1968 release of historical and projected statistics.

Kerosene. EIA adopted the Bureau of Mines thermal conversion factor of 5.670 million Btu per barrel as reported in a Bureau of Mines internal memorandum, "Bureau of Mines Standard Average Heating Values of Various Fuels, adopted January 3, 1950."

Liquefled Petroleum Gases (LPG) Consumption. Calculated annually by EIA as the average of the thermal conversion factors of each liquefied petroleum gas consumed, weighted by the quantity of each liquefied petroleum gas consumed.
Lubricants. BIA adopied the thermal conversion factor of 6.065 million Btu per harrel as estimated by the Bureau of Mines and first published in the Petroleum Statement, Annual, 1956.

Miscellaneous Products. EIA adopied the thermal conversion factor of 5.796 million Blu per barrel as cstimated by the Rureau of Mines and first published in the Petroleum Statement, Annual, 1956.

Motor Gasoline. EIA adopted the Burcau of Mines thermal conversion factor of 5.253 million Btu per barrel as published for "Gasoline, Motor Fucl" by the Texas Eastern Transmission Corporation in Appendix $V$ of Competition and Growth in American Energy Markets 1947.1985, a 1968 release of historical and projected statistics.

Natural Gas Plant I.lquida, Production. Calculated annually by EIA as the average of the thermal conversion factors of each natural gas plant liquid produced weighted by the quantity of each natural gas plant liquid produced.

Natural Gasoline. EIA adopted the thermal conversion factor of 4.620 million Blu per barrel as estimated by the Bureau of Mines and first published in the Petroleum Statement, Annual, 1956.

Pentunes Plus. EIA assumed the thermal conversion factor to be 4.620 million Btu per barrel or equal to that for natural gasoline. See Natural Gasoline.

Petrochemical Feedstocks, Naphtha Leas Than 401 Degrees Fahrenheit. Assumed by EIA to be 5.248 million Btu per barrel, equal to the thermal conversion factor for special naphtha. Sce Special Naphtha.

Petrochemical Feedstocks, Olls Fqual to or Greater Than 401 Degrees Fahrenheit. Assumed by EIA to be 5.825 million Btu per barrel, equal to the thermal conversion factor for distillate fuel oil. See Distlliate Fuel OII.

Petrochemical Feedstocks, StiII Gas. Assumed by EIA to be 6.000 million Btu per barrel, equal to the thermal conversion factor for still gas. See Still Gas.

Petroleum Coke. ElA adopted the thermal conversion factor of 6.024 million Btu per barrel as reported in Btu per short ton in the Bureau of Mines internal memorandum, "Bureau of Mines Standard Average Heating Value of Various Fuels, adopted January 3, 1950." The Bureau of Mines calculated this factor by dividing $30,120,000$ Btu per short ton, as given in the referenced Bureau of Mines intemal memorandum, by 5.0 barrels per short ton, as given in the Bureau of Mines Form 6.1300-M and successor EIA forms.

Petroleum Products, Total Consumption. Calculated annually by EIA as the average of the 
thermal conversion factors for all petroleum products consumed, weighted by the quantity of each petroleum product consumed.

Petroleum Products, Consumption by Electric Utilities. Calculated annually by EIA as the average of the thermal conversion factors for all petroleum products consumed at electric utilities, weighted by the quantity of each petroleum product consumed at electric utilities. The quantity of petroleum consumed is estimated in the State Energy Data System as documented in the State Energy Data Report.

Petroleum Products, Consumption by Industrial Users. Calculated annually by EIA as the average of the thermal conversion factors for all petroleum products consumed in the industrial sector, weighted by the estimated quantity of each petroleum product consumed in the industrial sector. The quantity of petroleum products consumed is estimated in the State Energy Data System as documented in the State Energy Data Report.

Petroleum Products, Consumption by Residential and Commercial Users. Calculated annually by EIA as the average of the thermal conversion factors for all petroleum products consumed by the residential and commercial sector, weighted by the estimated quantity of each petroleum product consumed in the residential and commercial sector. The quantity of petroleum products consumed is estimated in the State Energy Data System as documented in the State Energy Data Report.

Petroleum Products, Consumption by Transportation Users. Calculated annually by EIA as the average of the thermal conversion factor for all petroleum products consumed in the transportation sector, weighted by the estimated quantity of each petroleum product consumed in the transportation sector. The quantity of petroleum products consumed is estimated in the State Energy Data System as documented in the State Energy Data Report.

Petroleum Products, Exports. Calculated annually by EIA as the average of the thermal conversion factors for each petroleum product, weighted by the quantity of each petroleum product exported.

Petroleum Products, Imports. Calculated annually by EIA as the average of the thermal conversion factors for each petroleum product imported, weignted by the quantity of each petroleum product imported.

Plant Condensate. Estimated to be 5.418 million Btu per barrel by EIA from data provided by McClanahan Consultants, Inc., Houston, Texas.

Propane. EIA adopted the Bureau of Mines thermal conversion factor of 3.836 million Btu per barrel as published in the California Oil World and Petroleum Industry, First Issue, April 1942.
Residual Fuel Oil. EIA adopted the thermal conversion factor of 6.287 million Btu per barrel as reported in the Bureau of Mines internal memorandum, "Bureau of Mines Standard Average Heating Values of Various Fuels, adopted January 3, 1950."

Road Oil. EIA adopted the Bureau of Mines thermal conversion factor of 6.636 million Btu per barrel, which was assumed to be equal to that of asphalt (see Asphalt) and was first published by the Bureau of Mines in the Petroleum Statement, Annual, 1970.

Special Naphtha. EIA adopted the Bureau of Mines thermal conversion factor of 5.248 million Btu per barrel, which was assumed to be equal to that of total gasoline (aviation and motor) factor and was first published in the Petroleum Statement, Annual, 1970.

Still Gas. EIA adopted the Bureau of Mines estimated thermal conversion factor of 6.000 million Btu per barrel and first published in the Petroleum Statement, Annual, 1970.

Unfinished Oil. EIA assumed the thermal conversion factor to be 5.825 million Btu per barrei or equal to that for distillate fuel oil (see Distillate Fuel Oil) and first published in the Annual Report to Congress, Volume 3, 1977.

Unfractionated Stream. EIA assumed the thermal conversion factor to be 5.418 million Btu per barrel or equal to that for plant condensate (see Plant Condensate) and first published in the Annual Report to Congress, Volume 2, 1981.

Waxes. EIA adopted the thermal conversion factor of 5.537 million Btu per barrel as estimated by the Bureau of Mines and first published in the Petroleum Statement, Annual, 1956.

\section{Approximate Heat Content of Natural Gas}

Natural Gas, Total Consumption. 1973-1979: EIA adopted the thermal conversion factor calculated annually by the American Gas Association (AGA) and published in Gas Facts, an AGA annual publication. 1980 forward: Calculated annually by EIA by dividing the total heat content of natural gas consumed by the total quantity of natural gas consumed. The heat content and quantity consumed are from Form EIA-176. Published sources are: 1980-1990: EIA, Natural Gas Annual 1990, Volume 2, Table 15. 1991 forward: 1990 value used as an estimate.

Natural Gas, Consumption by Electric Utilities. Calculated annually by EIA by dividing the total heat content of natural gas received at electric utilities by the total quantity recuived at electric utilities. The heat contents and receipts are from Form FERC-423 and predecessor forms. 
Natural Gas, Consumption by Sectors Other Than Electric Utilities. Calculated annually by EIA by dividing the heat content of all natural gas consumed less the heat content of natural gas consumed at electric utilities by the quantity of all natural gas consumed less the quantity of natural gas consumed at electric utilities. Data are from Forms EIA-176, FERC-423, EIA-759, and predecessor forms.

Natural Gas, Exports. Calculated annually by EIA by dividing the heat content of exported natural gas by the quantity of natural gas exported, both reported on Form FPC-14.

Natural Gas, Imports. Calculated annually by EIA by dividing the heat content of imported natural gas by the quantity of natural gas imported, both reported on Form FPC-14.

Natural Gas Production, Dry. Assumed by EIA to be equal to the thermal conversion factor for the consumption of dry natural gas. See Natural Gas Consumption.

Natural Gas Production, Marketed (Wet). Calculated annually by EIA by adding the heat content of dry natural gas production and the total heat content of natural gas plant liquids production and dividing this sum by the total quantity of marketed (wet) natural gas production.

\section{Approximate Heat Content of Coal and Coal Coke}

Anthracite, Total Consumption. Calculated annually by EIA by dividing the sum of the heat content of anthracite consumed by electric utilities and all other sectors combined by the total quantity of anthracite consumed.

Anthracite, Consumption by Electric Utilities. Calculated annually hy EIA by dividing the heat content of anthracite receipts at electric utilities by the quantity of anthracite received at electric utilities. Heat contents and receipts are from Form FERC-423 and predecessor forms.

Anthracite, Consumption by Sectors Other Than Electric Utilities. Calculated annually by EIA by dividing the beat content of anthracite production less the heat content of the anthracite consumed at electric utilities, net exports, and shipments to U.S. Armed Forces overseas by the quantity of anthracite consumed by sectors other than electric utilities less the quantity of anthracite stock changes, losses, and "unaccounted for."

Anthracite, Imports and Exports. EIA assumed the anthracite imports and exports to be freshly mined anthracite having an estimated heat content of 25.40 million Btu per short ton.
Anthracite, Production. Calculated annually by EIA by dividing the sum of the heat content of freshly mined anthracite (estimated to have an average heat content of 25.400 million Btu per short ton) and the heat content of anthracite recovered from culm banks and river dredging (estimated to have a heat content of 17.500 million Btu per short ton) by the total quantity of anthracite production.

Bituminous Coal and Lignite, Total Consumption. Calculated annually by EIA by dividing the sum of the heat content of bituminous coal and lignite consumed by electric utilities, coal coke plants, other industrial plants, the residential and commercial sector, and the transportation sector by the sum of their respective tonnages.

Bituminous Coal and Lignite, Consumption by Coke Plants. Estimated by EIA to be 26.800 million Btu per short ton on the basis of an input/output analysis of coal carbonization.

Bituminous Coal and Lignite, Consumption by Electric Utilities. Calculated annually by EIA by dividing the total heat content of bituminous coal and lignite received at electric utilities by the total quantity received at electric utilities. Heat contents and receipts are from Form FERC-423 and predecessor forms.

Bituminous Coal and Lignite, Consumption by Other Industrial and Transportation Users. 1973: Calculated by EIA through regression analysis measuring the difference between the average Btu value of coal consumed by other industrial users and that of coal consumed at electric utilities in the 1974-1982 period. 1974 forward: Calculated annually by EIA by assuming that the bituminous coal and lignite delivered to other industrial users from each coal-producing area (reported on Form EIA-6 and predecessor Bureau of Mines Form 6-1419-Q) contained a heat value equal to that of bituminous coal and lignite received at electric utilities from each of the same coal-producing areas (reported on Form FERC-423). The average Biu value of coal by coal-producing area was applied to the volume of deliveries to other industrial users from each coal-producing area, and the sum total of the heat content was divided by the total volume of deliveries. Coal-producing areas are the Bureau of Mines coal-producing districts for 1974 through 1989 and coal-producing States for 1990 forward.

Bituminous Coal and Lignite, Consumption by Residential and Commercial Users. 1973: Calculated by EIA through regression analysis measuring the difference between the average Btu value of coal consumed by residential and commercial users and that of coal consumed by electric utilities in the 1974-1982 period. 1974 forward: Calculated annually by EIA by assuming that the bituminous coal and lignite delivered to residential and commercial 
users from each coal-producing area (reported on Form EIA-6 and predecessor Bureau of Mines Form 6-1419-Q) contained a heat value equal to that of bituminous coal and lignite received at electric utilities from each of the same coal-producing areas (reported on Form FERC-423). The average Btu value of coal by coal-producing area was applied to the volume of deliveries to residential and commercial users from each coal-producing area, and the total of the heat value was divided by the total volume of deliveries. Coal-producing areas are the Bureau of Mines coal-producing districts for 1974 through 1989 and coal-producing States for 1990 forward.

Bituminous Coal and Lignite, Exports. Calculated annually by EIA by dividing the sum of the heat content of exported metallurgical coal (estimated to average 27.000 million Btu per short ton) and the heat content of exported steam coal (estimated to have an average thermal content of 25.000 million Btu per short ton) by the total quantity of bituminous coal and lignite exported.

Bituminous Coal and Lignite, Imports. EIA estimated the average thermal conversion factor to be 25.000 million Btu per short ton.

Bituminous Coal and Lignite, Production. Calculated annually by EIA by dividing the sum of the heat content of bituminous coal and lignite consumption, net exports, stock changes, and unaccounted for by the sum of their respective tonnages. Consumers' stock changes by sectors were assumed to have the same conversion factor as that of the consumption sector. Producers' stock changes and unaccounted for were assumed to have the same conversion factor as that for consumption by all users.

Coal, Consumption. Calculated annually by EIA by dividing the sum of the heat content of bituminous coal and lignite and anthracite consumption by the sum of their respective tonnages.

Coal, Consumption by Electric Utilities. Calculated annually by EIA by dividing the sum of the heat content of bituminous coal and lignite and anthracite received at electric utilities by the sum of their respective tonnages received.

Coal, Consumption by Sectors Other Than Electric Utilities. Calculated annually by EIA by dividing the sum of the heat content of bituminous coal and lignite and anthracite consumed by sectors other than electric utilities by the sum of their respective tonnages.

Coal, Exports. Calculated annually by EIA by dividing the sum of the heat content of bituminous coal and lignite and anthracite exported by the sum of their respective tonnages.

Coal, Imports. Calculated annually by EIA by dividing the sum of the heat content of bituminous coal and lignite and anthracite imported by the sum of their respective tonnages.

Coal, Production. Calculated annually by EIA by dividing the sum of the total heat content of bituminous coal and lignite and anthracite production by the sum of their respective tonnages.

Coal Coke, Imports and Exports. EIA adopted the Bureau of Mines estimate of 24.800 million Btu per short ton.

\section{Approximate Heat Rates for Electricity}

Fossil-Fueled Steam-Electric Plant Generation. There is no generally accepted practice for measuring the thermal conversion rates for power plants that generate electricity from hydroelectric, wood and waste, wind, photovoltaic, or solar thermal energy sources. Therefore, EIA has selected a rate that is equal to the prevailing annual average heat rate factor for fossil-fueled steam-electric power plants in the United States. By using that factor, it is possible to evaluate fossil fuel requirements for replacing those sources during periods of interruption such as droughts. The heat content of a kilowatthour of electricity produced, regardless of the generation process, is 3,412 Btu per kilowatthour. 1973-1990: The weighted annual average heat rate for fossil-fueled steam-electric power plants in the United States, as published by EIA in Electric Plant Cost and Power Production Expenses 1990, Table 11. 1991 forward: 1990 value used as an estimate.

Geothermal Energy Plant Generation. 1973-1981: Calculated annually by EIA by weighting the average annual heat rates of operating geothermal units by the installed nameplate capacities as reported on Form FPC-12. 1982 forward: Estimated annually by EIA on the basis of an informal survey of relevant plants.

Nuclear Steam-Electric Plant Generation. Calculated annually by EIA by dividing the total heat content consumed in nuclear generating units by the total (net) electricity generated by nuclear generating units. The heat content and electricity generation are reported on Form FERC-1, Form EIA-412, and predecessor forms. The factors, beginning with 1982 data, are published in the following EIA reports-1982: Historical Plant Cost and Annual Production Expenses for Selected Electric Plants 1982, page 215. 1983-1990: Electric Plant Cost and Power Production Expenses 1990, Table 15. 1991 forward: 1990 value used as an estimate. 


\section{Appendix B. Metric and Other Physical Conversion Factors}

Data presented in the Monthly Energy Review and in other Energy Information Administration publications are expressed in units, such as British thermal units, barrels, cubic feet, and short tons, that historically have been used in the United States. However, because U.S. activities involve foreign nations, most of which use metric units of measure, the United States is committed to making the transition to the metric system.

The metric conversion factors presented in Table B 1 can be used to calculate the metric-unit equivalents of values expressed in U.S. customary units. For example, 500 short tons are the equivalent of $\mathbf{4 5 3 . 6}$ metric tons $(500$ short tons $X 0.9071847$ metric tons/short ton $=453.6$ metric tons). Most of the metric units shown in Table B1 belong to the International System of Units.

The conversion factors presented in Table B2 can be used to calculate equivalents in various physical units commonly used in energy analyses. For example, 10 barrels are the equivalent of 420 U.S. gallons (10 barrels $X 42$ gallons/barrel $=\mathbf{4 2 0}$ gallons).

In the metric system of weights and measures, designations of multiples and subdivisions of any unit may be arrived at by combining the name of the unit with prefixes, such as deka, hecto, and kilo, meaning, respectively, 10, 100, and 1,000 , and deci, centi, and milli, meaning, respectively, one-tenth, one-hundredth, and one-thousandth. Common metric prefixes can be found in Table B3.

\section{Table B1. Metric Conversion Factors}

\begin{tabular}{|c|c|c|c|c|}
\hline Type of Unit & U.S. Unit & & Conversion Factor & Metric Unit \\
\hline Mass & $\begin{array}{l}\text { short tons }(2,000 \mathrm{lb}) \\
\text { short tons uranium oxide }\left(\mathrm{U}_{3} \mathrm{O}_{8}\right) \\
\text { short tons uranium fluoride }\left(\mathrm{UF}_{6}\right) \\
\text { long tons } \\
\text { pounds (lb) } \\
\text { pounds uranium oxide }\left(\mathrm{lb} \mathrm{U}_{3} \mathrm{O}_{8}\right) \\
\text { ounces, avoirdupois (avdp oz) }\end{array}$ & $\begin{array}{l}x \\
x \\
x \\
x \\
x \\
x \\
x\end{array}$ & $\begin{array}{l}0.9071847 \\
0.769^{\mathrm{a}} \\
0.613^{\mathrm{a}} \\
1.016047 \\
0.45359237^{\mathrm{b}} \\
0.384645^{\mathrm{a}} \\
28.34952\end{array}$ & $\begin{array}{ll}= & \text { metric tons }(t) \\
= & \text { metric tons uranium }(t U) \\
= & \text { metric tons uranium }(t U) \\
= & \text { metric tons }(t) \\
= & \text { kilograms }(\mathrm{kg}) \\
= & \text { kilograms uranium }(\mathrm{kgU}) \\
=\quad \operatorname{grams}(\mathrm{g})\end{array}$ \\
\hline Volume & $\begin{array}{l}\text { barrels of oil (bbl) } \\
\left.\text { cubic yards ( } \mathrm{yd}^{3}\right) \\
\text { cubic feet (fts) } \\
\text { U.S. gallons (gal) } \\
\text { ounces, fluid (fl oz) } \\
\text { cubic inches (in } \text { in }^{3} \text { ) }\end{array}$ & $\begin{array}{l}x \\
x \\
x \\
x \\
x \\
x\end{array}$ & $\begin{array}{l}0.1589873 \\
0.764555 \\
0.02831685 \\
3.785412 \\
29.57353^{a} \\
16.387064\end{array}$ & $\begin{array}{l}=\quad \text { cubic meters }\left(\mathrm{m}^{3}\right) \\
=\quad \text { cubic meters }\left(\mathrm{m}^{3}\right) \\
=\quad \text { cubic meters }\left(\mathrm{m}^{3}\right) \\
=\quad \text { liters }(\mathrm{L}) \\
=\quad \text { milliliters }(\mathrm{mL}) \\
=\quad \text { milliliters }(\mathrm{mL})\end{array}$ \\
\hline Length & $\begin{array}{l}\text { miles (mi) } \\
\text { yards (yd) } \\
\text { foet (ft) } \\
\text { inches (in) }\end{array}$ & $\begin{array}{l}x \\
x \\
x \\
x\end{array}$ & $\begin{array}{l}1.609344^{b} \\
0.9144^{b} \\
0.3048^{b} \\
2.54^{b}\end{array}$ & $\begin{array}{ll}= & \text { kilometers }(\mathrm{km}) \\
= & \text { meters }(\mathrm{m}) \\
= & \text { meters }(\mathrm{m}) \\
= & \text { contimeters }(\mathrm{cm})\end{array}$ \\
\hline Area & $\begin{array}{l}\text { acres } \\
\text { square miles }\left(\mathrm{mi}^{2}\right) \\
\text { square yards }\left(\mathrm{yd}^{2}\right) \\
\text { square feet }\left(\mathrm{fl}^{2}\right) \\
\text { square inches }\left(\mathrm{in}^{2}\right)\end{array}$ & $\begin{array}{l}x \\
x \\
x \\
x \\
x\end{array}$ & $\begin{array}{l}0.40469 \\
2.589988 \\
0.8361274 \\
0.09290304^{b} \\
6.4513^{b}\end{array}$ & $\begin{array}{l}=\text { hectares (ha) } \\
=\quad \text { square kilometers }\left(\mathrm{km}^{2}\right) \\
=\quad \text { square meters }\left(\mathrm{m}^{2}\right) \\
=\quad \text { square meters }\left(\mathrm{m}^{2}\right) \\
=\quad \text { square centimeters }\left(\mathrm{cm}^{2}\right)\end{array}$ \\
\hline Temperature & degrees Fahrenheit $\left({ }^{\circ} \mathrm{F}\right)$ & $x$ & $5 / 9$ (after subtracting 32$)^{b}$ & $=\quad$ degrees Celsius $\left({ }^{\circ} \mathrm{C}\right)$ \\
\hline Energy & $\begin{array}{l}\text { British thermal units (Btu) } \\
\text { calories (cal) } \\
\text { kilowatthours (kWh) }\end{array}$ & $\begin{array}{l}x \\
x \\
x\end{array}$ & $\begin{array}{c}1,055.05585262^{b, d} \\
4.1868^{d} \\
3.6\end{array}$ & $\begin{array}{ll}= & \text { joules }(J) \\
= & \text { joules }(J) \\
= & \text { megajoules (MJ) }\end{array}$ \\
\hline
\end{tabular}

Cakculated by the Energy information Administralion.

tExact converston.

To corvert degrees Celshus ( $C$ ) 10 degrees Fahrentill ${ }^{\circ} F$ ) exadly, mulliply by $9 / 5$, then add 32.

The International Table corversion (5th international Conference on the Properties of Steam, London, 1956).

Sources: - General Services Adninistralion, Federal Standard 376B, preprint copy of Prefermed Metric Unils for General Use by the Federal Government Wastington, DC, January 27, 1993), pp. 2-11, 13, and 16. - National insthute of Standards and Technology, Speclal Publlcatlons 330, 811, and 814. - Amertcan

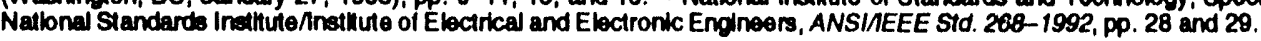


Table B2. Other Physical Conversion Factors

\begin{tabular}{|c|c|c|c|c|}
\hline Energy Source & Original Unh & & Converslon Factor & Final Unt \\
\hline $\begin{array}{l}\text { Crude Oll } \\
\text { (Average Gravity) }\end{array}$ & barrels (bbl) & $x$ & $42^{n}$ & U.S. galtons (gal) \\
\hline Coal & $\begin{array}{l}\text { short tons } \\
\text { long tons } \\
\text { motric tons (1) }\end{array}$ & $\begin{array}{l}x \\
x \\
x\end{array}$ & $\begin{array}{l}2,000^{\infty} \\
2,240^{\infty} \\
1,000^{a}\end{array}$ & $\begin{array}{ll}= & \text { pounds }(\mathrm{lb}) \\
= & \text { pounds }(\mathrm{lb}) \\
= & \text { kilograms }(\mathrm{kg})\end{array}$ \\
\hline $\begin{array}{l}\text { Wood (Average } \\
\text { Dry Hardwood) }\end{array}$ & $\begin{array}{l}\text { cords (cd) } \\
\text { cords (cd) }\end{array}$ & $\begin{array}{l}x \\
x\end{array}$ & $128^{1.25^{b}}$ & $\begin{array}{l}=\quad \text { chort tons } \\
=\quad \text { cublc teot }\left(t^{3}\right)\end{array}$ \\
\hline
\end{tabular}

Exact converston.

"Calculated by the Energy Information Adminiatration.

Source: Nallonal Instkute of Standards and Tectnology. NIST Handbook 44 (1993 Ecition) (Washington, DC, Odober 1992), Pp. C-17 and C-21.

Table B3. Metric Prefixes

\begin{tabular}{|c|c|c|c|c|c|}
\hline $\begin{array}{l}\text { Unit } \\
\text { Multiple }\end{array}$ & Prefix & Symbol & $\begin{array}{l}\text { Unit } \\
\text { Subdiviaion }\end{array}$ & Profix & Symbol \\
\hline $\begin{array}{l}10^{24} \\
10^{21} \\
10^{18} \\
10^{15} \\
10^{12} \\
10^{9} \\
10^{6} \\
10^{3} \\
10^{2} \\
10^{1}\end{array}$ & $\begin{array}{l}\text { yotta } \\
\text { zetta } \\
\text { exa } \\
\text { peta } \\
\text { tera } \\
\text { giga } \\
\text { mega } \\
\text { kilo } \\
\text { hecto } \\
\text { deka }\end{array}$ & $\begin{array}{c}Y \\
Z \\
E \\
P \\
T \\
G \\
M \\
k \\
h \\
d a\end{array}$ & $\begin{array}{l}10^{-1} \\
10^{-2} \\
10^{-3} \\
10^{-6} \\
10^{-0} \\
10^{-12} \\
10^{-15} \\
10^{-18} \\
10^{-21} \\
10^{-24}\end{array}$ & $\begin{array}{l}\text { deci } \\
\text { centi } \\
\text { milli } \\
\text { micro } \\
\text { nano } \\
\text { pico } \\
\text { femto } \\
\text { atto } \\
\text { zepto } \\
\text { yocto }\end{array}$ & $\begin{array}{l}d \\
c \\
m \\
\mu \\
n \\
p \\
t \\
a \\
z \\
y\end{array}$ \\
\hline
\end{tabular}

Source: Nallonal Institute of Standarde and Technology, NIST Spoclal Publlcation 330 (Wechinglon, DC, Auguat 1981$)$. p. 10.

For information regarding the International System of Units, contact Dr. Barry N. Taytor at Building 221. Room B160, National Institute of Standards and Technology. Gaithersburg, MD 20899, or on telephone number 301-875-4220. 


\section{Appendix C. List of Features}

The following is a complete list of features that have appeared in the Monshly Energy Review since the first issue was published in October 1974. There are four categories of features on the list. "Articles" cover a wide range of energyrelated subjects in depth. "Ilighlights" summarize the most important information presented in the subject Energy Information Administration (EIA) report. "Energy Previews" provide brief overviews of EIA preliminary energy data on a given topic. "IIA Data News" items present information on recent changes in the scope, design, methodology, and findings of the EIA's energy surveys and data bases. Questions and comments about features may be directed to Barhara T. Fichman by telephone on 202-586-5737 or by fax on 202-586-0018.
Feature

1993

Energy Preview: Residential Transportation Energy Consumption Survey,

Preliminary Estimates, 1991

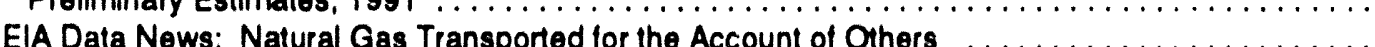

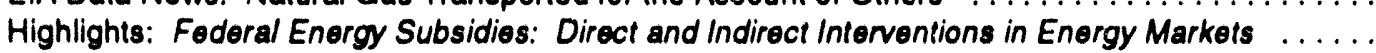
Highlights: Household Energy Consumption and Expenditures $1990 \ldots \ldots \ldots \ldots \ldots \ldots \ldots$ Anticle: Demand, Supply, and Price Outlook for Low-Sulfur Diesel Fuel . . . . . . . . . . . . Energy Preview: Manufacturing Energy Consumption Survey, Preliminary Estimates, 1991 . . . . Highlights: Natural Gas 1992: Issues and Trends $\ldots \ldots \ldots \ldots \ldots \ldots \ldots \ldots \ldots \ldots \ldots \ldots$ Highlights: International Enengy Outlook 1993

1992

Energy Preview: Residential Energy Consumption and Expenditures

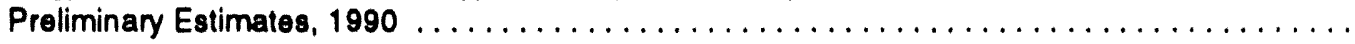

EIA Data News: Oxygenate Data Collection Begins $\ldots \ldots \ldots \ldots \ldots \ldots \ldots \ldots \ldots \ldots \ldots \ldots$

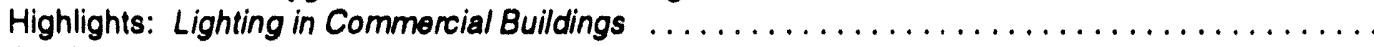
Article: Demand, Supply, and Price Outlook for Oxgenated Gasoline, Winter 1992-1993 . . . . . EIA Data News: EIA Statistics on Electric Utility Demand-Side Management $\ldots \ldots \ldots \ldots \ldots$. . . ElA Data News: ElA Statistics on Nonutility Power Producers $\ldots \ldots \ldots \ldots \ldots \ldots \ldots \ldots$ Highlights: Derived Annual Estimates of Manufacturing Energy Consumption, 1974-1988 . . . . . Article: Energy Efficiency in the Manufacturing Sector

\section{1}

Highlights: U.S. Energy Industry Financial Developments, 1990 Fourth Quarter . . . . . . . . . . Anticle: U.S. Wholesale Electricity Transactions

1990

Article: Refining Results Highlight Energy Companies' First-Haff Profit Pertormance $\ldots . . \ldots \ldots$ Highlights: U.S. Oil and Gas Reserves by Year of Field Discovery

\section{9}

Article: A Review of Valdez Oil Spill Market Impacts $\ldots \ldots \ldots \ldots \ldots \ldots \ldots \ldots \ldots \ldots \ldots \ldots$

Anticle: Monthly U.S. Crude Oil Production Estimates $\ldots \ldots \ldots \ldots \ldots \ldots \ldots \ldots \ldots \ldots \ldots \ldots$

Article: Superconductivity and Energy Production and Consumption $\ldots \ldots \ldots \ldots \ldots \ldots \ldots$

Highlights: Commercial Buildings Consumption and Expenditures 1986

Article: Higher Prices Yield Improved Energy Industry Financial Results

in the First Half of 1989

Article: The Future Structure of the U.S. Commercial Nuclear Power Equipment

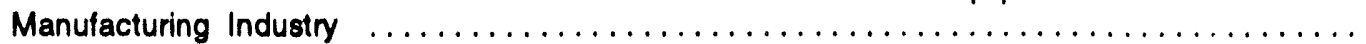

Highlights: Potential Costs of Restricting Chlorofluorocarton Use $\ldots \ldots \ldots \ldots \ldots \ldots \ldots$

Highlights: Manufacturing Energy Consumption Survey: Changes in

Energy Efficiency, 1980-1985

Highlights: Household Energy Consumption and Expenditures 1987 Part 1 . National Data $\ldots \ldots$ Article: Improved Energy Profits Offset by Refining Results in $1989 \ldots \ldots \ldots \ldots \ldots \ldots \ldots$.

\section{Cover Date}

January 1993

Fobruary 1993

July 1993

August 1993

August 1993

September 1993

September 1993

October 1993

April 1992

May 1992

June 1992

August 1992

September 1992

October 1992

November 1992

December 1992

March 1991

April 1991

June 1990

August 1990

March 1989

March 1989

May 1989

May 1989

June 1989

July 1989

September 1989

October 1989

November 1989

December 1989 
Highlights: Characteristica of Commorcial Bulldinge 1988 June 1988

Article: The U.S. Energy Induatry's Financial Recovery Continued in the Firat Half of 1988 . . . . . .

June 1988

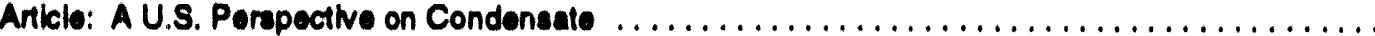

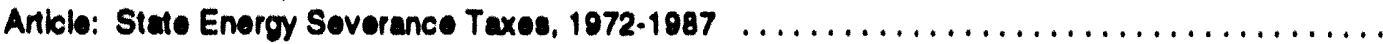

Highlights: Manufacturing Energy Consumption Survoy: Consumption of Enongy, $1985 \ldots \ldots \ldots$.

Highlights: Profiles of Foreign Direct Investment in U.S. Energy 1987 . . . . . . . . . . . . . . . . . .

Highllghte: Manufacturing Energy Consumption Survoy: Fuel Switching, 1985 . . . . . . . . . . . .

Article: Increased Refining incomo Lod U.S. Energy Industry Financial Rocovery in 1988

June 1988

July 1988

September 1088

October 1988

November 1988

December 1988

1987

Articlo: Manufacturing Sector Enorgy Consumption, 1985 Provisional Estimates

January 1087

Highllghte: Consumption and Expenditures, April 1084 Through March 1085,

Part 1: National Date

Highlights: Consumption and Expendihures, April 1984 Through March 1985,

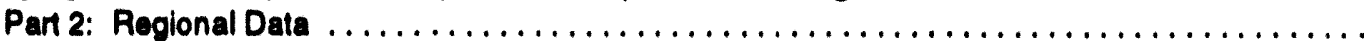

Article: U.S. Energy Induatry Financial Dovolopmente, 1987 Second Quarter . . . . . . . . . . . . .

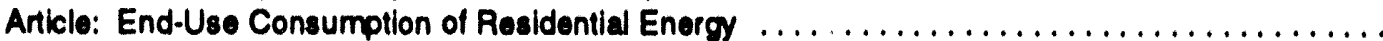

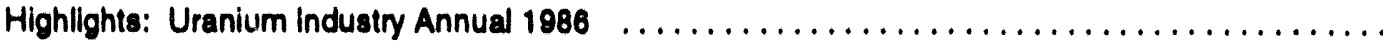

Highlights: Potential Oil Production from ANWR

Highlights: Profiles of Foreign Direct Investment in U.S. Energy 1986

Anticle: The U.S. Energy Industry in 1987: A Slow Recovery

April 1987

May 1987

June 1987

July 1987

September 1987

October 1987

November 1987

December 1987

1986

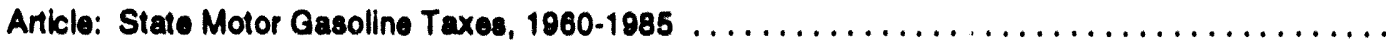

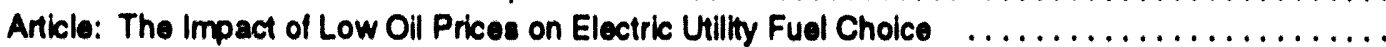

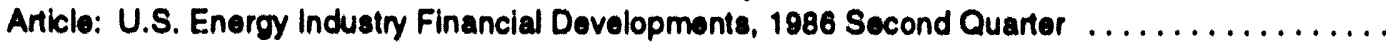

Highlights: International Energy Annual 1985

Article: U.S. Energy Industry Financial Developments, 1986

March 1988

June 1986

June 1986

September 1908

December 1988

1985

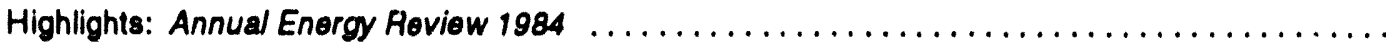

Highlights: Pertormance Profiles of Major Energy Producers $1983 \ldots \ldots \ldots \ldots \ldots \ldots \ldots$

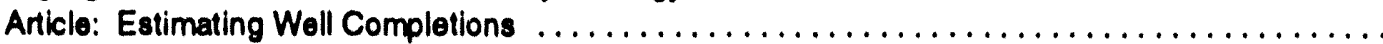

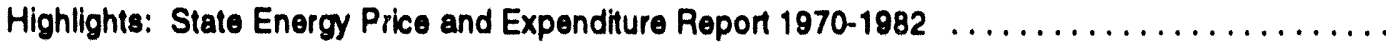

Highlights: State Energy Data Repont, Consumption Estimates, 1960.1983 ................

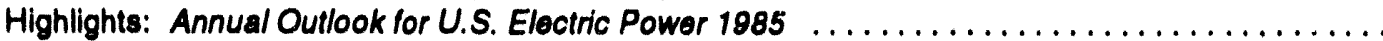

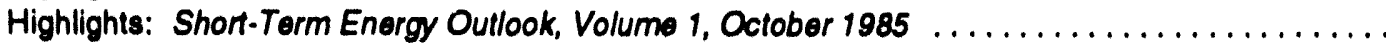

Highlights: Analysis of Growth in Eloctricity Domand, 1980-1984 ........................

Highlights: Profiles of Foreign Direct Investment in U.S. Energy 1984 . . . . . . . . . . . . . . . . .

Highlights: Performance Profiles of Major Energy Producers 1984

January 1985

Fobruary 1985

March 1985

March 1985

April 1985

June 1985

August 1985

August 1985

November 1985

December 1985

\section{4}

Highlights: Annual Energy Roview 1983

Highlights: Annual Energy Outlook 1983

Highlights: State Energy Data Report Consumption Estmates, 1900-1982 ...................

Highlights: State Energy Price and Expenditure Report 1970-1981 ...

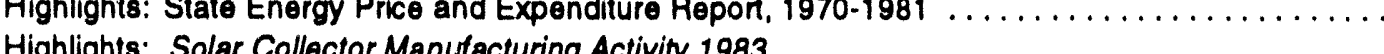

Highlights: International Energy Annual 1983

Highlights: Estimates of U.S. Wood Energy Consumption, 1980-1983

Highlights: Energy Conservation Indicators 1983 Annual Report

Highlights: Annual Energy Outlook 1984

Fobruary 1984

March 1984

March 1984

May 1984

June 1984

September 1984

September 1984

November 1984

December 1984 


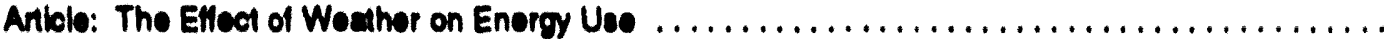

Aprll 1083

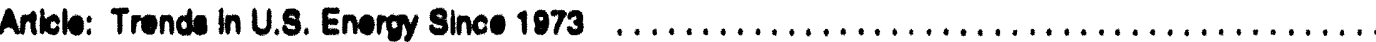

May 1083

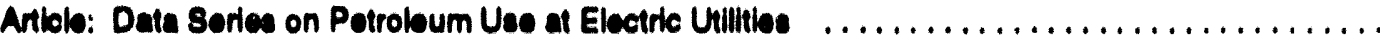

July 1983

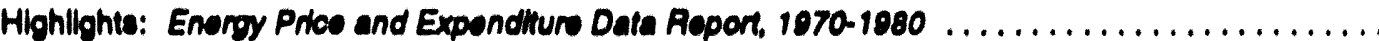

July 1983

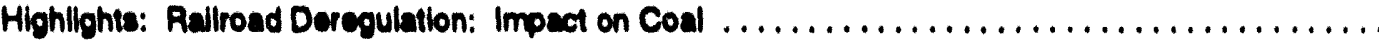

Auguat 1083

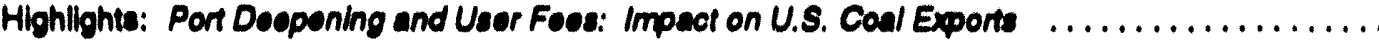

Highlights: U.S. Crude Oll, Natural Cas, and Natural Gas Llquides Reserves,

1982 Annual Report

Articlo: Roaldential Energy Coneumption, 1078 Through $1081 \quad \ldots \ldots \ldots \ldots \ldots \ldots \ldots \ldots \ldots \ldots$

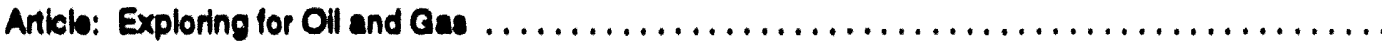

Article: The Influence of Federal Actione on Petroloum Exploration $\ldots \ldots \ldots \ldots \ldots \ldots \ldots \ldots$

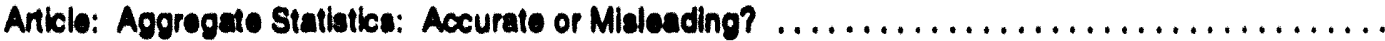

Auguat 1083

September 1003

Soplember 1983

November 1983

Decomber 1983/2]

December 1983[3]

\section{2}

Artcle: The Interetate and Intrastate Natural Gas Markets $\ldots \ldots \ldots \ldots \ldots \ldots \ldots \ldots \ldots$

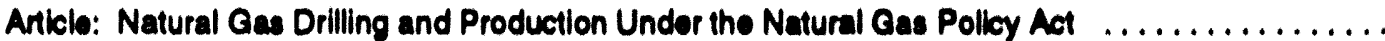
Highlights: U.S. Crude Oil, Natural Gas, and Natural Gas Llquids Roserves, 1981 Annual Report .. Article: Impacts of Financial Conatrainte on the Electric Unility Induatry ................... Highlighte: Enorgy Company Dovelopmont Pattoms in the Poetombargo Era ...............

January 1982

Fobruary 1082

September 1082

October 1982

November 1982

\section{1}

Article: Changes in 1981 Pelroloum Data Soribs $\ldots \ldots \ldots \ldots \ldots \ldots \ldots \ldots \ldots \ldots \ldots \ldots$

Article: Information Sorvices of the Enorgy Information Adminibtration $\ldots \ldots \ldots \ldots \ldots \ldots \ldots$

Article: An Ovenviow of Natural Gas Markots

May 1981

September 1981

December 1981

Fobruary 1980

March 1980

June 1080

Auguat 1980

October 1980

November 1980

December 1980

July 1979

October 1979

December 1979

May 1978

January 1977

July 1977 
Aviblo: Curtallmente of Natural Oas sorvibe

January 1970

Artib: Home Heating Coneorvation Arernatives and the Soler Collector Induatry

March 1070

Antolo: Trends in Uniled States Petroleum Imports

1075

Artble: Enoray Consumption

March 1076

Article: Nucloar Power

Artble: The Price of Cruds Oll

April 1078

Arible: U.S. Coul Resources

Artele: Propane-A National Eneroy Resoures

June 1075

July 1078

Artble: Shon-Term Energy Supply and Demand Foreeding at FEA

Septomber 1076

October 1075 


\section{Glossary}

Anthracite: $A$ hard, black, lustrous coal containing a high percentage of fixed carbon and a low percentage of volatile matter. Onen referred to as hard coal. It conforms to ASTM Specificalion D388.84 for anthracite, meta-anthracite, and scmianthracite.

Asphalt: A dark-brown-to-black cement-like material containing bitumens as the predominant constituents obtained by petroleum processing. The definition includes crude asphalt as well as the following finished products: cements, nuxes, the asphalt content of emulsions (exclusive of water), and petroleum distillates blended with asphalt to make cutback asphalts.

ASTM: The American Society for Testing and Materials.

Aviation Gasoline Blending Components: Naphthas that are used for blending or compounding into finished aviation gasoline (e.g. suraight-nun gasoline, alkylate, and reformate). Excludes oxygenates (alcohols and ethers), butane, and pentanes plus.

Aviatlon Gasoline, Finished: All special grades of gasoline for use in aviation reciprocating engines, as given in ASTM Specification D910 and Military Specification MIL-G-5572. Excludes blending components that will be used in blending or compounding into finished aviation gasoline.

Barrel (petroleum): A unit of volume equal to 42 U.S. gallons.

Base (Cushlon) Gas: The volume of gas needed as a permanent inventory to maintain adequate underground storage reservoir pressures and deliverability rates throughout the withdrawal season. All native gas is included in the base gas volume.

Bltuminous Coal: A dense black coal, often with well-defined bands of bright and dull material, with a moisture content usually less than 20 percent. Often referred to as soft coal. It is the most common coal and is used primarily for generating electricity, making coke, and space heating. It conforms to ASTM Specification D388-84 for bituminous coal. In this report, bituminous coal includes subbituminous coal.

British Thermal Unit (Btu): The quantity of heat needed to raise the temperature of 1 pound of water by $1^{\circ} \mathrm{F}$ at or near $39.2^{\circ} \mathrm{F}$. See Heat Content of a Quantity of Fucl, Gross and Heat Content of a Quantity of Fuel, Net.
Butanet $A$ normally gaseous straight-chain of branched-chain hydrocarbon $\left(C, 11_{10}\right)$. II is extracted from natural gas of refinery gas streams. It includes Isobutane and normal butane and is designated in ASTM Specificalion DI8.35 and Gas Processors Association Specifications for commerclal butane.

- Isobulane: A normally gasecous branched-chain hydrocarbon. It is a colorless paraffinic gas that boils at a temperature of $10.9 \mathrm{~F}$. It is extracted from nalural gas of refinery gas streams.

- Normal Buiane: A normally gaseous straight-chain hydrocarbon. It is a colorless paraffinic gas that boils at a temperature of $31.1^{\circ} \mathrm{F}$. II is extracted from natural gas or refinery gas suteams.

Butylene: $A$ n olefinic hydrocarbon $\left(C_{4} I_{1}\right)$ recovered from refinery processes.

Capacity Factor: The ratio of the electrical energy produced by a generating unit for a given period of time to the electrical energy that could have been produced at continuous full-power operation during the same period.

CIF: Sce Cost, Insurance, Frelght.

City Gate: $\wedge$ point or measuring station at which a distribution gas utility receives gas from a natural gas pipeline company or transmission system.

Coal: A black or brownish-black solid, combustible substance formed by the partial decomposition of vegetable matter without access to air. The rank of coal, which includes anthracite, bituminous coal, subbituminous coal, and lignite, is based on fixed carbon, volatile matter, and heating value. Coal rank indicates the progressive alteration, or coalification, from lignite 10 anthracilc. Lignite contains approximately 9 to 17 million Btu per ton. The heat contents of subbituminous and bituminous coal range from 16 to 24 million Btu per ton, and from 19 to 30 million Btu per ton, respectively. Anthracite contains approximately 22 to 28 million Btu per ton.

Coal Coke: $\mathrm{A}$ hard, porous product made from baking bituminous coal in ovens at temperatures as high as $2,000^{\circ} \mathrm{F}$. It is used both as a fuel and as a reducing agent in smelting iron or: in a blast fumace.

Commerclal Sector: The commercial sector, as defined economically, consists of business establishments that are not engaged in transportation or in manufacluring or other types of industrial activity (agriculture, mining, or construction). Commercial establishments include hotels, motels, 
restaurants, wholesale businesses, retall stores. laundries, and other service enterprises; religious and nonprofit organizations; health, socia, and educutional Institutions; and Federal, State, and local governments. Street lights, pumps, bridges, and public scrvices are aso included if the establishment operating them is considered commercial.

Completions The installation of permanent equipment for the production of oll or eas. If a well is equipped to produce only oll or gas from one zone or reservolr. the definition of a well (classined a an oll well or gas well) and the definition of a completion are identical. However, if well is equipped to produce oll and/or gas separately from more than one reservolr. a well is not synonymous with a completion.

Convernton Fector: A number that translates units of one system into corresponding values of another system. Conversion factors can be used to translate physical units of measure for various fuels into Btu equivalents.

Cost, Insurance, Prelght (CIF): A type of sale in which the buyer of the product agrees to pay anit price that includes the f.o.b. value of the product at the point of origin plus all costs of Insurance and uransportation. This type of transaction differs from a "delivered" purchase in that the buyer accepts the quantity as detertmined at the loading port (as certified by the Bill of Loading and Quality Report) rather than pay on the basis of the quantity and quality ascertained at the unloading port. It is similar to the teruns of an f.o.b. sale, except that the seller, as a service for which be is compensated, arranges for transportation and insurance.

Crude OII P.o.b. Price: The crude oil price actually charged at the oil-producing country's port of loading. Includes deductions for any rebates and discounts or additions of premiums, where applicable. It is the actual price paid with no adjustment for credit terms.

Crude Oll (Including lease Condensate): A mixture of hydrocarbons that exists in liquid phase in underground reservoirs and remains liquid at atmospheric pressure after passing through surface separating facilities. Included are lease condensate and liquid hydrocarbons produced from tar sands, gilsonite, and oil shale. Drip gases are also included, but topped crude oil (residual oil) and other unfinished oils are excluded. Where identifiable, liquids produced at natural gas processing plants and mixed with crude oil are likewise excluded.

Crude OII Landed Cost: The price of crude oil at the port of discharge, including charges associated with the purchase, transporting, and insuring of a cargo from the purchase point to the port of discharge. The cost does not include charges incurred at the discharge pon (e.g.. impont tariffs or fees, wharfage charges, and demurrage).
Crude (oll Kefinery Input: the total crude ofl put into processing units at renneries.

Crude OII Stockst Slocks of crude oil and lease condensate held at refinertes, in pipelines, at pipeline terminals, and on leases.

Crude OII Uned Directly: Crude oil consumed as fuel by crude oll pipelines and on crude ofl leases.

Cuble Foot (natural gan)! A unit of volume equal to 1 cuble loot at a pressure base of 14.73 pounds standard per square inch absolute and cemperature base of $60^{\circ} \mathrm{F}$.

Degree-Day Normalas Simple arithmette averages of monthly or annual degree-days over a long period of lime (usually the 30-year period 1951-1980). The averages may be simple degree-day normals or population-weighted degree-day normals.

Degree-Daya, Cooling (CDD): The number of degrees per day that the daily average temperature is above $65^{\circ} \mathrm{F}$. The dally average temperature is the mean of the maximum and minimum temperatures for a 24-bour period.

Degree.Days, Heatling (HDD): The number of degrees per day that the daily average temperature is below $65^{\circ} \mathrm{F}$. The dally average temperature is the mean of the maximum and minimum temperalures for 24-bour period.

Degree-Days, Populetlon-Welghted: Heating or cooling degree-days weighted by the population of the area in which the degree-days are recorded. To compute State population-weighted degree-days, each State is divided into from one 10 nine climatically homogeneous divisions, which are assigned weights based on the ratio of the population of the division to the total population of the State. Degree-day readings for each division are multiplied by the corresponding population weight for each division and those products are then summed to arrive at the State population-weighted degree-day figure. To compute national population-weighted degree-days, the Nation is divided into nine Census regions comprised of from three to eight States, which are assigned weights hased on the ratio of the population of the region to the total population of the Nation. Degree-day readings for each region are multiplied by the corresponding population weight for each region and those products are then summed to arrive at the national population-weighted degree-day nigure.

Dealgn Electrical Rutlng, Net: The nominal net electrical output of a nuclear unit as specified by the electric utility for the purpose of plant design.

Development Well: $A$ well drilled within the proved area of an oil or gas reservoir to the depth of a stratigraphic horizon known to he productive. 
Dutiliate Fuel Olli $A$ gencral classification for one of the petroleum fractions produced in conventional distillation operations. Included are products known as No. 1. No. 2, and No. 4 fuel olls and No. 1, No. 2. and No. 4 diesel fuels. If is used primarily for space heating. on. and off-highway diesel engine fuel (including railroad engine fuel and fuel for agricultural machinery), and electric power generation.

Dry Holes An explonatory of development well found to be incapable of producing elther oil or gas in sufficient quantities to justify completion as an oll or gas well.

Dry Natural Gas Productlon (wa a decremont from gas reserves): The volume of natural gas withdrawn from reservoirs during the report year less (1) the volume relurned to such reservoirs in cycling. repressuring of oll reservoirs, and conservation operations: (2) shrinkage resulting from the removal of lease condensate and plant liquids: and (3) nonhydrocarton gases, where they occur in sumcient quantity to render the gas unmarketable. Volumes of gas withdrawn from gas storage reservoirs and native gas that has been transferred to the storage calegory are not considered production. This is not the same as marketed production. since the latter also excludes vented and nared gas but contains liquids.

Dry Natural Gas Production (an an Increment to gas supply): Gross withdrawals from production reservoirs less gas used in reservoir repressuring. amounts vented and fared, nonhydrocarbons removed. and various natural gas constituents, such as ethane, propane, and butane, removed at natural gas processing plants. The parameters for measurement are $60^{\circ} \mathrm{F}$ and 14.73 pounds standard per square inch absolute.

Electrical System Energy Losses: The amount of energy lost during generation, transmission, and distribution of electricity, including plant and unaccounted-for uses.

Electricity Generation: The process of producing electric energy or transforming other forms of energy into electric energy. Also the amount of electric energy produced or expressed in watthours (Wh).

Electricity Generation, Grosa: The total amount of electric energy produced by the generating station or stations, measured at the generator terminals.

Electricity Generation, Net: Gross generation less electricity consumed at the generating plant for station use. Electricity required for pumping at pumped-storage plants is regarded as plant use and is deducted from gross generation.

Electriclty Production: Net electricity (gross electricity output measured at generator terminals minus power plant use) generated by publicly and privately owned electric utilities. Excludes industrial electricity generation (except autogeneration of hydroelectric power).

Blectriclty Salos: The amount of kilowatthours sold in a given pertod of time; usually grouped hy classes of service, such as residential, commercial, Industrial, and other. "Other" sales Include sules for public street and bigbway lighting and other sales to public auboorilies, sales 10 rallroads and railways, and interdepertunental sales.

Electric Power Plant: A station containing prime movers, electric sencrators, and auxiliary equipment for converting mechanical, chemical, and/or fission energy into electric energy.

Electrle Utility: A corporalion, person, agency, authority, or other legal entity of instrumentality that owns and/or operates facilities for the generation, transmission, distribution, or sale of electric energy. primarily for use by the public, and that nles forms listed in the Code of Federal Regulations, Title 18. Part 141. Facilities that qualify as cogenerators or small power producers under the Public Utility Regulatory Policies Act are not considered electric utilities.

Electric Utility Sector: The electric vility sector consists of privalely and publicly owned establishments that generate, transmit, distribute, or sell electricity primarily for use by the public and that meet the definition of an electric utility. Nonutility power producers are not included in the electric utility sector.

End-Use Sectors: The residential, coinmercial, industrial, and transportation sectors of the economy.

Enerzy: The capacity for doing work as measured by the capability of doing wort (potential energy) of the conversion of this capability to motion (kinetic energy). Energy uas several forns, some of which are easily convertible and can be changed to another form useful for work. Most of the world's convertible energy comes from fossil fuels that are burned to produce heat that is then used as a transfer medium to mechanical or other means in order to accomplish tasks. Electrical energy is usually measured in kilowatthours, while heat energy is usually measured in British thermal units.

Energy Consumption: The use of energy as a source of beat or power or as an input in the manufacturing process.

Energy Consumption, End-Use: Primary end.use energy consumption is the sum of fossil fuel consumption by the four end use sectors (residential, commercial, industrial, and (ransportation) and seneration of hydroclectric power by nonelectric utilities. Net end-use energy consumption includes 
electric utility stes to those sectors but excludes electrical system energy losess. Total end-use energy consumption includes both electric utility sales to the four end-use sectors and electrical system eneigy losses.

Eneray Conaumption, Total: The sum of fossil fuel consumption by the five sectors (residential. commercial, industrial, transportation, and electric utility) plus bydroelectric power, nuclear electric power, net imports of coal coke, and electricity generated for distribution from wood, waste, geothermal, wind, photovoltaic, and solar thermal energy.

Energy Sourcet A substance, such as petroleum, natural sas, or coal. that supplies heat or power. In Energy Information Administration reports, electricity and renewable forms of energy, such as blomass, geothermal, wind, and solar, are considered to be cnergy sources.

Etbanet A normally gaseous straight-chain hydrocarbon $\left(\mathrm{C}_{2} \mathrm{H}_{6}\right)$. If is a colorless, paraminic gas that boils at a temperature of $-127.48^{\circ} \mathrm{F}$. It is extracted from natural gas and refinery gas streams.

Ethylene: An olefinic hydrocarbon $\left(\mathrm{C}_{2} \mathrm{H}_{4}\right)$ recovered from refinery processes or petrochemical processes.

Exploratory Well: A well drilled to find and produce oil or gas in an unproved area. to find a new reservoir in a field previously found to be productive of oil or gas in another reservoir, or to extend the limit of a known oil or gas reservoir.

Exports: Shipments of goods from the 50 States and the District of Columbia to foreign countries and to Puerto Rico, the Virgin Islands, and other U.S. possessions and territories.

P.ass.: See Free Alongslde Shlp.

Federal Energy Regulatory Commisalon (FERC): The Federal agency with jurisdiction over interstate electricity sales, wholesale electric rates, hydroelectric licensing, natural gas pricing, oil pipeline rates, and gas pipeline certification. FERC is an independent regulatory agency within the Department of Energy and is the successor to the Federal Power Commission.

Federal Power Commission (FPC): The predecessor agency of the Federal Energy Regulatory Commission. The Federal Power Commission was created by an Act of Congress under the Federal Water Power Act on June 10, 1920. Il was charged originally with regulating the electric power and natural gas industries. It was abolished on September 30, 1977. when the Department of Energy was created. Its functions were divided between the Department of
Energy and the Federal Energy Regulatory Commission, an independent regulatory agency.

Firat Purchase Price: The markeled first sales price of domestic crude oil, consistent with the removal price defined by the provisions of the Windfall Pronts Tux on Domestic Crude Oll (Public Law 96-223, Sec. 4998 (c)).

Flared Natural Gas: Natural gas bumed in flares on the base site or at gas processing plants.

\section{f.o.b.s See Free on Bourd.}

Footage Drilleds Total footage for wells in various categories, as reported for any specified period, Includes (1) the deepest total depth (length of well bores) of all wells drilled from the surface. (2) the total of all bypassed tootage drilled in connection with reported wells, and (3) all new fontage drilled for directional sidetrack wells. Footage reported for directional sidetrack wells does not include footage in the common bore. which is reported as footage for the original well. In the case of old wells drilled deeper, the reported footage is that which was drilled below the lotal depth of the old well.

Former U.S.S.R.: See U.S.S.R.

Fomill Fuel: Any naturally occurring organic fucl, such as petrolcum, coal, and natural gas.

Fossll Fuel Steam-Filectrlc Power Plant: An electricity generation plant in which the prime mover is a turbine rotated by high-pressure stcam produced in a boller by heat from buming fossil fuels.

Free Alongside Shlp (f.a.s.): The value of a commodity at the port of exportation, generally including the purchase price, plus all charges incurred in placing the commodity alongside the carrier at the port of exportation.

Free on Board (f.o.b.): A transaction whereby the seller makes the product available within an agreed-on period at a given port at a given price. It is the responsibility of the buyer to arrange for the uansportation and insurance.

Fuel Ethanol: An anhydrous, denatured aliphatic alcohol $\left(\mathrm{C}_{2} \mathrm{H}, \mathrm{OH}\right)$ intended for motor gasoline blending. See Oxygenates.

Full-Power Operation: Operation of a nuclear generating unit at 100 percent of its design capacity. Full-power operation precedes commercial operation.

Gasohol: A blend of finished motor gasoline (leaded or unleaded) and alcohol (generally ethanol but sometimes methanol) limited to 10 percent by volume 
of alcobol. Gasohol is included in nnished leaded and unleaded motor gasoline.

Gus-Turbine Electric Power Plant: $A$ plant in which the prime mover is a gas turbine. A gas turbine typically consists of an axial-flow air compressor, one or more combustion chambers where liquid or gaseous fuel is bumed and the bot gases expand to drive the generator and then are used to nun the compressor.

Gas Woll: A well completed for the production of natural gas from one or thore gas zones or reservoirs. (Wells producing both crude oll and natural gas are classified as oil wells.)

Geothermal Energy: Energy from the internal heat of the Earth, which may be residual heat, friction heat, or a result of radioactive decay. The heat is found in rocks and huids at various depths and can be extracted hy drilling and/or pumping.

(ieothermal Energy (as uned at electric utilitiea): llot water or steam extracted from geothermal rescrvoirs in the Earth's crust and supplied to steam turhines at electric utilities that drive generators to produce electricity.

(iross Domestic Product (GDP): The total value of goods and services produced by labor and property located in the United States. As long as the labor and propertv are located in the United States, the supplier (that is, the workers and, for property, the owners) may be either U.S. residents or residents of foreign countries.

Heat Content of a Quantity of Fuel, Gross: The total amount of heat released when a fuel is burned. Coal, crude oil, and natural gas all include chemical compounds of carbon and hydrogen. When those fuels are burned, the carbon and hydrogen combine with oxygen in the air to produce carton dioxide and water. Some of the energy released in burning goes into transforming the water into steam and is usually lost. The amount of heat spent in transforming the water into steam is counted as pan of gross heat content but is not counted as part of net beat content. Also referred to as the higher beating value. Btu conversion factors typically used in EIA represent gross beat content.

Heat Content of a Quantity of Fuel, Net: The amount of usable beat energy released when a fuel is burned under conditions similar to those in which it is normally used. Also referred to as the lower heating value. Btu conversion factors typically used in EIA represent gross heat content.

Hesvy Oil: The fuel oils remaining after the lighter oils have been distilled off during the refining process. Except for start-up and flame stabilization, virtually all petroleum used in steam-electric power plants is heavy oil.
Hydrocarbon: An organic chemical compound of hydrogen and carbon in the gaseous, llquid, or solid phase. The molecular structure of hydrocarbon compounds varies from the simplest (methane, the primary constituent of natiral gas) to the very heavy and very complex.

Hydrowlectric Power: The production of electricity from the kinetic energy of falling water.

Hydroelectrle Power Planti A plant in which the turbine generators are driven by falling water.

Imports: Receipts of goods into the $\mathbf{5 0}$ States and the District of Columbia from foreign countries and from Puerto Rico, the Virgin Islands, and other U.S. possessions and territories.

Induatrial Sector: The industrial sector comprises manufacturing industries, which make up the largest part of the sector, along with mining. construction, agriculture, nsheries, and forestry. Establishments in the sector range from steel mills, to small farms, to companies assembling electronic components.

Internal Combustion Electric Power Plant: A power plant in which the prime mover is an internal combustion engine. Diesel or gas-fired engines are the principal types used in electric power plants. The plant is usually operated during periods of high demand for electricity.

Jet Fuel: The term includes kerosene-type jet fuel and naphtha-type jet fuel. Kerosene-type jet fuel is a kerosene-quality product used primarily for commercial turbojet and turboprop aircraft engines. Naphtha-type jet fuel is a fuel in the heavy naphthas range used primarily for military turbojet and turboprop aircraft engines.

Kerosene: A petroleum distillate that has a maximum distillation temperature of $401^{\circ} \mathrm{F}$ at the 10-percent recovery point a final boiling point of $572^{\circ} \mathrm{F}$, and a minimum nash point of $100^{\circ} \mathrm{F}$. Included are the two grades designated in ASTM D3699 (No. 1.K and No. 2-K) and all grades of kerosene called range or stove oil. Kerosene is used in space beaiers, cook stoves, and water heaters; it is suitable for use as an illuminant when bumed in wick lan.ps.

Lease and Plant Fuel: Natura gas used in well, field, and lease operations (such as gas used in drilling operations, beaters, debydrators, and field compressors), and us fuel in natural gas processing plants.

Lease Condensate: A natural gas liquid recovered from gas well gas (associated and non-associated) in lease separators or natural gas field facilities. Lease condensate consists primarily of pentanes and beavier hydrocarbons. 
Light Oil: Lighter fuel oils distilled off during the refining process. Virtually all petroleum used in internal combustion and gas-turbine engines is light oil.

Lignite: A brownish-black coal of low rank with a high content of moisture and volatile matter. Often referred to as brown coal. It is used almost exclusively for electric power generation. It conforms to ASTM Specification D388-84 for lignite.

Liquefied Natural Gas (LNG): Natural gas (primarily m(thane) that has been liquefied by reducing its temperature to $-260^{\circ} \mathrm{F}$ at atmospheric pressure.

Liquefied Petroleum Gases (LPG): Ethane, ethylene, propane, propylene, normal butane, butylene, and isobutane produced at refineries or natural gas processing plants, including plants that fractionate new natural gas plant liquids.

Low-Power Testing: The period of time between a nuclear generating unit's initial fuel loading date and the issuance of its operating (full-power) license. The maximum level of operation during that period is 5 percent of the unit's design thermal rating.

Lubricants: Substances used to reduce friction between bearing surfaces or as process materials either incorporated into other materials used as processing aids in the manufacturing of other products or as carriers of other materials. Petroleum lubricants may be produced either from distillates or residues. Other substances may be addec' to impart or improve certain required properties. Excluded are byproducts of lubricating oil refining, such as aromatic extracts derived from solvent extraction or tars derived from deasphalting. Included are all grades of lubricating oils from spindle oil to cylinde: oil and those used in greases. Lubricant categories are paraffinic and naphthenic.

Marketed Production: Gross withdrawals less gas used for repressuring, quantities vented and flared, and nonhydrocarbon gases removed in treating or processing operations. Includes all quantities of gas used in field and processing operations.

Methanol: A light, volatile alcohol $\left(\mathrm{CH}_{3} \mathrm{OH}\right)$ eligible for motor gasoline blending. See Oxygenates.

Miscellaneous Petroleum Products: All finished petroleum products not classified elsewhere-for example, petrolatum, lube refining byproducts (aromatic extracts and tars), absorption oils, ram-jet fuel, petroleum rocket fuels, synthetic natural gas feedstocks, and specialty oils.

Motor Gasoline Blending Components: Naphthas that will be used for blending or compounding into finished motor gasoline (e.g., straight-run gasoline, alkylate, reformate, benzenc, toluene, and zylene).
Excluded are oxygenates (alcohols and ethers), butane, and pentanes plus.

Motor Gasoline, Finished: A complex mixture of relatively volatile hydrocarbons, with or without small quantities of additives, that has been blended to form a fuel suitable for use in spark-ignition engines. Motor gasoline, as given in ASTM Specification D439 or Federal Specification VV-G-1690B, includes a range in distillation temperatures from 122 to $158^{\circ} \mathrm{F}$ at the 10-percent recovery point and from 365 to $374^{\circ} \mathrm{F}$ at the 90-percent recovery point. Motor gasoline includes reformulated motor gasoline, oxygenated motor gasoline, and other finished motor gasoline. Blendstock is excluded until blending has been completed.

- Reformulated Motor Gasoline: Motor gasoline, formulated for use in motor vehicles, the composition and properties of which are certified as "reformulated motor gasoline" by the Environmental Protection Agency.

- Oxygenated Motor Gasoline: Motor gasoline, formulated for use in motor vehicles, that has an oxygen content of 1.8 percent or higher by weight.

- Other Finished Motor Gasoline: Motor gasoline that is not included in the reformulated or oxygenated categories.

Motor Gasoline, Finished Gasohol: A blend of finished motor gasoline (leaded or unleaded) and alcohol (generally ethanol, but sometimes methanol) in which 10 percent or more of the product is alcohol.

Motor Gasoline, Finished Leaded: Motor gasoline that contains more than 0.05 gram of lead per gallon or more than 0.005 gram of phosphorus per gallon. Premium and regular grades are included, depending on the octane rating. Includes leaded gasohol. Blendstock is excluded until blending has been completed. Alcohol that is to be used in the blending of gasohol is also excluded.

Motor Gasoline, Finished Leaded Premium: Motor gasoline having an antiknock index, calculated as $(\mathrm{R}+\mathrm{M}) / 2$, greater than 90 and containing more than 0.05 gram of lead per gallon or more than 0.005 gram of phosphorus per gallon.

Motor Gasoline, Finished Leaded Regular: Motor gasoline having an antiknock index, calculated as $(R+M) / 2$, greater than or equal to 87 and less than or equal to 90 and containing more than $0.05 \mathrm{gram}$ of lead or 0.005 gram of phosphorus per gallon.

Motor Gasoline, Finished Unleaded: Motor gasoline containing not more than 0.05 gram of lead per gallon and not more than $0.005 \mathrm{gram}$ of phosphorus per gallon. Premium and regular grades are included, depending on the octane rating. Includes unleaded gasohol. Blendstock is excluded until blending has 
been completed. Alcohol that is to be used in the blending of gasohol is also excluded.

Motor Gasoline, Finished Unleaded Midgrade: Motor gasoline having an antiknock index, calculated as $(\mathrm{R}+\mathrm{M}) / 2$, greater than or equal to 88 and less than or equal to 90 and containing not more than $0.05 \mathrm{gram}$ of phosphorus per gallon.

Motor Gasoline, Finished Unleaded Premium: Motor gasoline having an antiknock index, calculated as $(\mathrm{R}+\mathrm{M}) / 2$, greater than 90 and containing not more than $0.05 \mathrm{gram}$ of lead or $0.005 \mathrm{gram}$ of phosphorus per gallon.

Motor Gasoline, Finished Unleaded Regular: Motor gasoline having an antiknock index, calculated as $(R+M) / 2$, of 87 containing not more than 0.05 gram of lead per gallon and not more than 0.005 gram of phosphorus per gallon.

Motor Gasoline Retail Prices: Motor gasoline prices calculated each month by the Bureau of Labor Statistics (BLS) in conjunction with the construction of the Consumer Price Index (CPI). Those prices are collected in 85 urban areas selected to represent all urban consumers-about 80 percent of the total U.S. population. The service stations are selected initially, and on a replacement basis, in such a way that they represent the purchasing habits of the CPI population. Service stations in the current sample include those providing all types of service (i.e., full-, mini-, and self-service).

Motor Gasoline, Total: Includes finished leaded motor gasoline (premium and regular), finished unleaded motor gasoline (premium, midgrade, and regular), motor gasoline blending components, and gasohol.

MTBE (Methyl Tertiary Butyl Ether): An ether, $\left(\mathrm{CH}_{3}\right)_{3} \mathrm{COCH}_{3}$, intended for motor gasoline blending. See Oxygenates.

Naphtha: A genetic term applied to a petroleum fraction with an approximate boiling range between 122 and $400^{\circ} \mathrm{F}$.

Natural Gas: A mixture of hydrocarbons (principally methane) and small quantities of various nonhydrocarbons existing in the gaseous phase or in solution with crude oil in underground reservoirs.

Natural Gas, Dry: The marketable portion of natural gas production, which is obtained by subtracting extraction losses, including natural gas liquids removed at natural gas processing plants, from total production.

Natural Gas Marketed Production: Gross withdrawals of natural gas from production reservoirs, less gas used for reservoir repressuring; nonhydrocarbon gases removed in treating and processing operations; and quantities vented and flared.

Natural Gas Plant Liquids (NGPL): Natural gas liquids recovered from natural gas in processing plants and, in some situations, from natural gas field facilities, as well as those extracted by fractionators. Natural gas plant liquids are defined according to the published specifications of the Gas Processors Association and the American Society for Testing and Materials as follows: ethane, propane, normal butane, isobutane, pentanes plus, and other products from natural gas processing plants (i.e., products meeting the standards for finished petroleum products produced at natural gas processing plants, such as finished motor gasoline, finished aviation gasoline, special naphthas, kerosene, distillate fuel oil, and miscellaneous products).

Natural Gas Wellhead Price: The wellhead price of natural gas is calculated by dividing the total reported value at the wellhead by the total quantity produced as reported by the appropriate agencics of individual producing States and the U.S. Minerals Management Service. The price includes all costs prior to shipment from the lease, including gathering and compression costs, in addition to State production, severance, and similar charges.

Natural Gas, Wet: Natural gas prior to the extraction of liquids and other miscellaneous products.

\section{Net Consumption: See Energy Consumption,} End-Use.

Nonhydrocarbon Gases: Typical nonhydrocarbon gases that may be present in reservoir natural gas are carbon dioxide, helium, hydrogen sulfide, and nitrogen.

Nuclear Electric Power: Electricity generated by an electric power plant whose turbines are driven by steam generated in a reactor by heat from the fissioning of nuclear fueí.

Nuclear Electric Power Plant: A single-unit or multiunit facility in which heat produced in one or more reactors by the fissioning of nuclear fuel is used to drive one or more steam turbines.

Nuclear Reactor: An apparatus in which the nuclear fission chain can be initiated, maintained, and controlled so that energy is released at a specific rate. The reactor includes fissionable material (fuel), such as uranium or plutonium; fertile material; moderating material (unless it is a fast reactor); a heavy-walled pressure vessel; shielding to protect personnel; provision for heat removal; and control elements and instrumentation. 
Oftshore: That geographic area that lies seaward of the coastline. In general, the coastline is the line of ordinary low water along with that portion of the coast that is in direct contact with the open sea or the line marking the seaward limit of inland water.

Oil: See Crude Oll (Including Lease Condensate).

Oil Well: A well completed for the production of crude oil from one or more oil zones or reservoirs. Wells producing both crude oil and natural gas are classitied as oil wells.

Operable (nuclear): A U.S. nuclear generating unit is considered operable after it completes low-power testing and is issued a full-power operating license by the Nuclear Regulatory Commission. A foreign nuclear generating unit is considered operable once it has generated electricity to the grid.

Organization for Economic Cooperation and Development (OECD): Current members are Australia, Austria, Belgium, Canada, Denmark, Finland, France, Greece, Iceland, Ireland, Italy, Japan, Luxembourg, the Netherlands, New Zealand, Norway, Portugal, Spain, Sweden, Switzerland, Turkey, the United Kingdom, the United Siates and its territories (Guam, Puerto Rico, and the Virgin Islands), and Germany.

Organization of Petroleum Exporting Countries (OPEC): Countries that have organized for the purpose of negotiating with oil companies on matters of oil production, prices, and future concession rights. Current members are Algeria, Gabon, Indonesia, Iran, Iraq, Kuwait, Libya, Nigeria, Qatar, Saudi Arabia, the United Arab Emirates, and Venezuela.

Oxygenated Motor Gasoline: See Motor Gasoline, Finished.

Oxygenates: Any substance which, when added to motor gasoline, increases the amount of oxygen in that motor gasoline blend. Through a series of waivers and interpretive rules, the Environmental Protection Agency (EPA) has determined the allowable limits for oxygenates in unleaded gasoline. The "Substantially Similar" Interpretive Rules (56 FR [February 11, 1991]) allows blends of aliphatic alcohols other than methanol and aliphatic ethers, provided the oxygen content does not exceed 2.7 percent by weight. The "Substantially Similar" Interpretive Rules also provide for blends of methanol up to 0.3 percent by volume exciusive of other oxygenates, and butanol or alcohols of a higher molecular weight up to 2.75 percent by weight. Individual waivers pertaining to the use of oxygenates in unleaded motor gasoline have been issued by the EPA. They include:

- Fuel Ethanol. Blends of up to 10 percent by volume anhydrous ethanol (200 proof).

- Methanol. Blends of methanol and gasoline-grade tertiary butyl alcohol (GTBA) such that the total oxygen content does not exceed 3.5 percent by weight and the ratio of methanol to GTBA is less than or equal to 1 . It is also specified that this blended fuel must meet ASTM volatility specifications.

Blends of up to 5.0 percent by volume methanol with a minimum of 2.5 percent by volume cosolvent alcohols having carbon number of 4 or less (i.e., ethanol, propanol, butanol, and/or GTBA). The total oxygen must not exceed 3.7 percent by weight, and the blend must meet ASTM volatility specifications as well as phase separation and alcohol purity specifications.

- MTBE (Methyl tertiary butyl ether). Blends up to 15.0 percent by volume MTBE that must meet the ASTM D4814 specifications. Blenders must take precautions that the blends are not used as base gasolines for other oxygenated blends.

Pentanes Plus: A mixture of hydrocarbons, mostly pentanes and heavier, extracted from natural gas. Includes isopentane, natural gasoline, and plant condensate.

Petrochemical Feedstocks: Chemical feedstocks derived from petroleum principally for the manufacture of chemicals, synthetic rubber, and a variety of plastics. The categories reported are naphthas less than $401^{\circ} \mathrm{F}$ endpoint and other oils equal to or greater than $401^{\circ} \mathrm{F}$ endpoint.

Petroleum: A generic term applied to oil and oil products in all forms, such as crude oil, lease condensate, unfinished oils, petroleum products, natural gas plant liquids, and nonbydrocarbon compounds blended into finished petroleum products.

Petroleum Coke: A residue that is the final product of the condensation process in cracking. The product is either marketable petroleum coke or catalyst petroleum coke.

Petroleum Coke, Catalyst: The carbonaceous residue that is deposited on and deactivates the catalyst used in many catalytic operations (e.g., catalytic cracking). Carbon is deposited on the catalyst, thus deactivating the catalyst. The catalyst is reactivated by burning off the carbon, which is used as a fuel in the refining process. That carbon or coke is not recoverable in a concentrated form.

Petroleum Coke, Marketable: Those grades of coke produced in delayed or fluid cokers that may be recovered as relatively pure carbon. Marketable petroleum coke may be sold as is or further purified by calcining.

Petroleum Consumption: The sum of all refined petroleum products supplied. For each refined petroleum product, the amount supplied is calculated by adding production and imports, then subtracting 
changes in primary stocks (net withdrawals are a plus quantity and net additions are a minus quantity) and exports.

Petroleum Imports: Imports of petroleum into the $\mathbf{5 0}$ States and the District of Columbia from foreign countries and from Puerto Rico, the Virgin Islands, and other U.S. territories and possessions. Included are imports for the Strategic Petroleum Reserve and withdrawals from bonded warehouses for onshore consumption, offshore bunker use, and military use. Excluded are receipts of foreign petroleum into bonded warehouses and into U.S. territories and U.S. Foreign Trade Zones.

Petroleum Products: Products obtained from the processing of crude oil (including lease condensate), natural gas, and other hydrocarbon compounds. Petroleum products include unfinished oils, liquefied petroleum gases, pentanes plus, aviation gasoline, motor gasoline, naphtha-type jet fuel, kerosene-type jet fuel, kerosene, distillate fuel oil, residual fuel oil, petrochemical feedstocks, special naphthas, lubricants, waxes, petroleum coke, asphalt, road oil, still gas, and miscellaneous products.

\section{Petroleum Products Supplled: See Petroleum Consumption.}

Petroleum Stocks, Primary: For individual products, quantities that are held at refineries, in pipelines, and at bulk terminals that have a capacity of 50,000 barrels or more, or that are in transit thereto. Stocks held by product retailers and resellers, as well as tertiary stocks held at the point of consumption, are excluded. Stocks of individual products held at gas processing plants are excluded from individual product estimates but are included in other oils estimates and total.

Photovoltaic and Solar Thermal Energy (as used at electric utilities): Energy radiated by the sun as electromagnetic waves (electromagnetic radiation) that is converted at electric utilities into electricity by means of solar (photovoltaic) cells or concentrating (focusing) collectors.

Pipeline Fuel: Gas consumed in the operation of pipelines, primarily in compressors.

Primary Consumption: See Energy Consumption, End-Use.

Propane: A normally gaseous straight-chain hydrocarbon $\left(\mathrm{C}_{3} \mathrm{H}_{8}\right)$. It is a colorless paraffinic gas that boils at a temperature of $-43.67^{\circ} \mathrm{F}$. It is extracted from natural gas or refinery gas streams. It includes all products designated in ASTM Specification D1835 and Gas Processors Association Specifications for commercial propane and HD-5 propane.

Propylene: An olefinic hydrocarbon $\left(\mathrm{C}_{3} \mathrm{H}_{6}\right)$ recovered from refinery or petrochemical processes.
Refiner Acquisition Cost of Crude Oil: The cost of crude oil to the refiner, including transportation and fees. The composite cost is the weighted average of domestic and imported crude oil costs.

Refinery (petroleum): An installation that manufactures finished petroleum products from crude oil, unfinished oils, natural gas liquids, other hydrocarbons, and alcohol.

Renewable Energy: Energy obtained from sources that are essentially inexhaustible (unlike, for example, the fossil fuels, of which there is a finite supply). Renewable sources of energy include wood, waste. photovoltaic, and solar thermal energy.

Repressuring: The injection of a pressurized fluid (such as air, gas, or water) into oil and gas reservoir formations to effect greater ultimate recovery.

Residential Sector: The residential sector is considered to consist of all private residences, whether occupied or vacant, owned or rented, including single-family homes, multifamily housing units, and mobile homes. Secondary homes, such as summer homes, are also included. Institutional housing, such as school dormitories, hospitals, and military barracks, generally are not included in the residential sector; they are included in the commercial sector.

Residual Fuel Oll: The heavier oils that remain after the distillate fuel oils and lighter hydrocarbons are distilled away in refinery operations and that conform to ASTM Specifications D396 and 975. Included are No. 5, a residual fuel oil of medium viscosity; Navy Special, for use in steam-powered vessels in government service and in shore power plants; and No. 6, which includes Bunker $C$ fuel oil and is used for commercial and industrial heating, electricity generation, and to power ships. Imports of residual fuel oil include imported crude oil burned as fuel.

Road Oil: Any heavy petroleum oil, including residual asphaltic oil used as a dust palliative and surface treatment on roads and highways. It is generally produced in six grades, from 0 , the most liquid, to 5 , the most viscous.

Rotary Rig: A machine used for drilling wells that employs a rotating tube attached to a bit for boring holes through rock.

Short Ton (coal): A unit of weight equal to 2,000 pounds.

SIC: See Standard Industrial Classification.

Solar Energy: The radiant energy of the sun, which can be converted into other forms of energy, such as heat or electricity. 
Standard Industrial Classification (SIC): A set of codes developed by the Office of Management and Budget which categorizes industries into groups with similar economic activities.

Startup Test Phase of Nuclear Power Plant: A nuclear power plant that has been licensed by the Nuclear Regulatory Commission to operate but is still in the initial testing phase, during which the production of electricity may not be continuous. In general, when the electric utility is satisfied with the plant's performance, it formally accepts the plant from the manufacturer and places it in commercial operation status. A request is then submitted to the appropriate utility rate commission to include the power plant in the rate base calculation.

Steam-Electric Power Plant: A plant in which the prime mover is a steam turbine. The steam used to drive the turbine is produced in a boiler where fossil fuels are burned.

Strategic Petroleum Reserve (SPR): Petroleum stocks maintained by the Federal Government for use during periods of major supply interruption.

Supplemental Gaseous Fuels: Any gaseous substance that, introduced into or commingled with natural gas, increases the volume available for disposition. Such substances irclude, but are not limited to, propane-air, refinery gas, coke oven gas, still gas, manufactured gas, biomass gas, or air or inert gases added for Btu stabilization.

Synthetic Natural Gas (SNG): A manufactured product chemically similar in most respects to natural gas, resulting from the conversion or reforming of petroleum hydrocarbons. It may easily be substituted for, or interchanged with, pipeline quality natural gas. Also referred to as substitute natural gas.

Total Consumption: See Energy Consumption, End-Use.

Transportation Sector: The transporation sector consists of private and public vehicles that move people and commodities. Included are automobiles, trucks, buses, motorcycles, railroads and railways (including streetcars), aircraft, ships, barges, and natural gas pipelines.

Unaccounted-for Crude Oil: Arithmetic difference between the calculated supply and the calculated disposition of crude oil. The calculated supply is the sum of crude oil production and imports, less changes in crude oil stocks. The calculated disposition of crude oil is the sum of crude oil input to refineries, crude oil exports, crude oil burned as fuel, and crude oil losses.
Underground Storage: The storage of natural gas in underground reservoirs at a different location from which it was produced.

United States: Unless otherwise noted, "United States" in this publication means the 50 States and the District of Columbia. U.S. exports include shipments to U.S. territories, and imports include receipts from U.S. territories.

U.S.S.R.: The Union of Soviet Socialist Republics consisted of 15 constituent republics: Armenia, Azerbaijan, Belorussia, Estonia, Georgia, Kazakhstan, Kirghizia, Latvia, Lithuania, Moldavia, Russia, Tadzhikistan, Turkmenistan, Ukraine, and Uzbekistan. As a political entity, the U.S.S.R. ceased to exist as of December 31, 1991.

Vented Natural Gas: Gas released into the air on the base site or at processing plants.

Wellhead Price: The value of crude oil or natural gas at the mouth of the well.

Well Servicing Unit: Truck-mounted equipment generally used for downhole services after a well is drilled. Services include well completions and recompletions, maintenance, repairs, workovers, and well plugging and abandonments. Jobs range from minor operations, such as pulling the rods and rod pumps out of an oil well, replacing the pump and rerunning the assemblage into the well, to major workovers, such as milling out and repairing collapsed casing. Well depth and characteristics determine the type of equipment used.

Wind Energy (as used at electric utilities): The kinetic energy of wind converted at electric utilities into mechanical energy by wind turbines (i.e., blades rotating from a hub) that drive generators to produce electricity for distribution.

Wood and Waste (as used at electric utilities): Wood energy, garbage, bagasse, sewerage gas, and other industrial, agricultural, and urban refuse used to generate electricity for distribution.

Wood Energy: Wood and wood products used as fuel, including round wood (cord wood), limb wood, wood chips, bark, sawdust, forest residues, charcoal, pulp waste, and spent pulping liquor.

Working Gas: The gas in a reservoir that is in addition to the base (cushion) gas. It may or may not be completely withdrawn during any particular withdrawal season. Conditions permitting, the total working capacity could be used more than once during any given season. 


\section{Publication Order Form \\ The Changing Structure of the U.S. Coal Industry: An Update}

Published: July 1993

Energy Information Administration

GPO Stock No. 061-003-008-119

Price per copy: $\$ 2.75$

Company or Personal Name:

Additional Address/Attention Line:

Street Address:

City, State, Zip Code:

Daytime Phone Number (area code first):

Purchase Order No:

May we make your name and address available to other mailers?

yes

no

\section{Please include payment with this order form. Allow a minimum of 4 weoks for domestic dolivery and an additional 6 weeks for international dellvery.}

Quantity $\times \$ 2.75=\$$ (total due). (International customers add 25\%.)

$\square$ Check payable to Superintend
$\square$ GPO Deposit Account No.
$\square$ VISA or MasterCard Account of Documents

Authorizing Signature Credit Card Expiration Date

Note: Price includes regular domestic postage and handling. It is subject to change.

Mail order form to: U.S. Government Printing Office

P.O. Box 371954

Pittsburgh, PA 15250-7954

Or fax order form to: $202-512-2250$ 


\title{
Publication Order Form \\ State Energy Price and Expenditure Report 1991
}

\author{
Published: September 1993 \\ Energy Information Administration \\ GPO Stock No. 061-003-00818-6 \\ Price per copy: $\$ 18.00$
}

Company or Personal Name:

Additional Address/Attention Line:

Street Address:

City, State, Zip Code:

Daytime Phone Number (area code first):

Purchase Order No:

May we make your name and address available to other mailers?

yes

no

The State Energy Price and Expenditure Report 1991 presents energy price and expenditure estimates for the 50 States, the District of Columbia, and the United States for 1970, 1975, 1980, and 1985 through 1991. The estimates are provided by energy source (petroleum, natural gas, coal, and electricity) and by major consuming sector (residential, commercial, industrial, transportation, and electric utilities). The 290-page report includes technical documentation describing the data sources and estimation procedures used.

Please include payment with this order form. Allow a minimum of 4 woeks for domestic delivery and an additional 6 weoks for international delivery.

Quantity $x \$ 18.00=\$$ (total due). (International customers add 25\%.)

Check payable to Superintendent of Documents

GPO Deposit Account No.

$\square$ VISA or MasterCard Account

Authorizing Signature

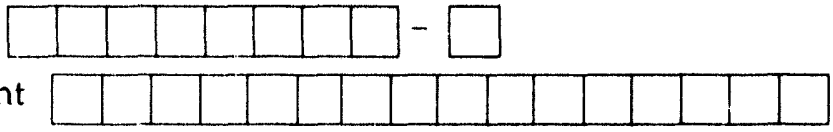

Credit Card Expiration Date

Note: Price includes regular domestic postage and handling. It is subject to change.

Mail order form to: U.S. Government Printing Office

P.O. Box 371954

Pittsburgh, PA 15250-7954

Or fax order form to: $202-512-2250$ 


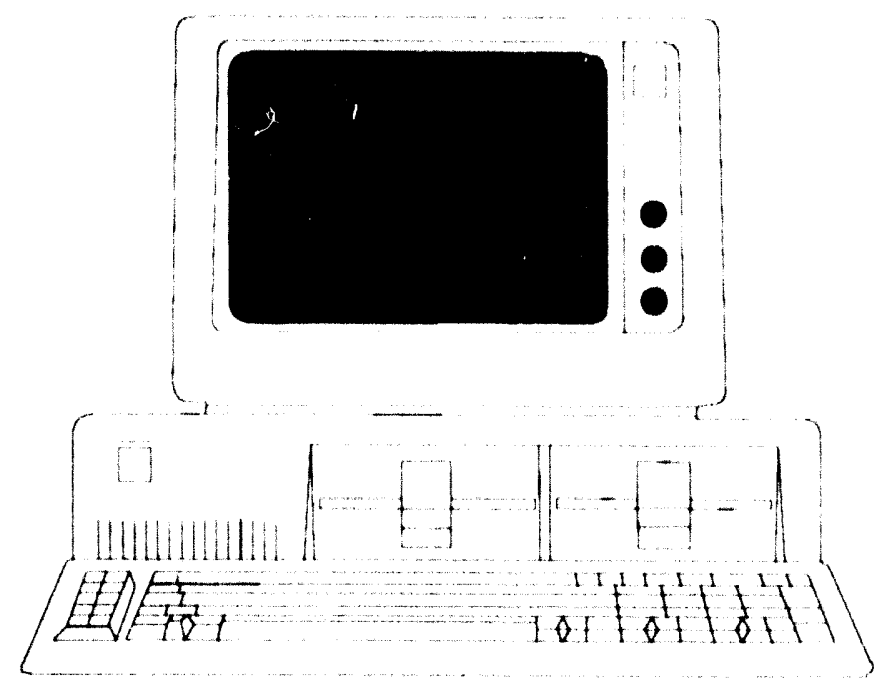

\section{State Energy Data on Diskettes}

\section{Available from GPO and NTIS}

- For IBM-PC and compatible microcomputers

- 5 1/4-inch double-sided high-density diskettes

- ASCII comma-delimited format

- Can easily be imported into Lotus ${ }^{\circ} 1-23^{\circ}$ or dBASE using utilities that are included on the diskettes

State Energy Data System (SEDS) diskettes contain the data for 1960 through 1991 as published in Tables 12 through 323 of the State Energy Data Report 1991, Consumption Estimates. State Energy Price and Expenditure System (SEPEDS) diskettes contain the data for 1970 through 1991 as published in the "Statistical Tables" section of the State Energy Price and Expenditure Report 1991. Although the published tables present data in rounded form, the diskettes contain data in the fullest precision available. Diskettes containing data for all the States within a Census region, the U.S. data, documentation, and utilities can be purchased separately or in a complete set. For prices and more information, contact:

Superintendent of Documents U.S. Government Printing Office P.0. Box 37082

Washington, DC 20402

Att: Esther Edmonds

202-512-1530
Order Control

National Tochnical Information Service 5285 Port Royal Road

Springfield, VA 22161

703-487-4650 
$Y$
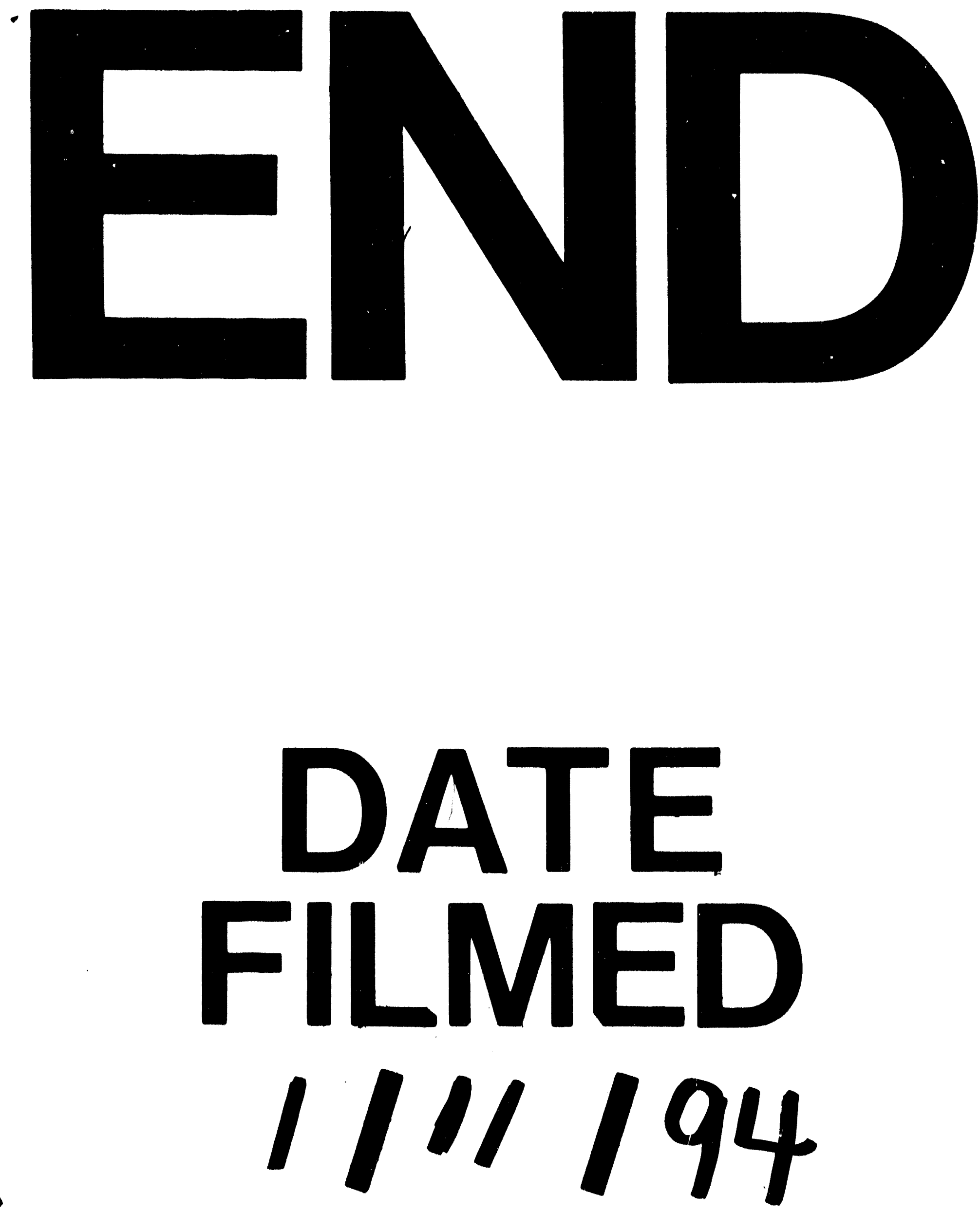

1 
